\title{
Größen des Wasserhaushaltes verschiedener Böden unter landwirtschaftlicher Nutzung im klimatischen Grenzraum des Mitteldeutschen Trockengebietes - Ergebnisse der Lysimeterstation Brandis -
}

\author{
Dissertation \\ zur Erlangung des Doktorgrades \\ doctora scientiarum agrariarum \\ Dr. sc. agr. \\ der Fakultät für Agrarwissenschaften \\ der Georg-August-Universität Göttingen \\ von Ulrike Haferkorn \\ Diplom-Hydrologin
}

Gutachter: Prof. Dr. B. Meyer, Universität Göttingen

Prof. Dr. W. Ehlers, Universität Göttingen

Tag der mündlichen Prüfung: 22.06.2000 


\begin{abstract}
Die Wirkung bodenphysikalischer und -morphologischer Eigenschaften natürlicher landwirtschaftlich genutzter Böden auf den Wasserhaushalt wird durch die Witterung, das Wurzelwachstum, die Wasseraufnahme der Pflanzen und Bearbeitungsmaßnahmen beeinflusst. Um den Einfluss dieser Faktoren auf die Höhe der Verdunstung, die Bodenwasserentnahme durch die Pflanzen, die Erträge, die Sickerwasserbildung und den Stickstoffaustrag analysieren zu können, wurden acht typische Böden Mitteldeutschlands (mit jeweils drei Wiederholungen) unter gleichen Witterungsbedingungen und gleicher Bewirtschaftung hinsichtlich Fruchtart, Düngung, Bodenbearbeitung und Pflege mittels 24 wägbarer, 3 m tiefer Lysimeter untersucht.
\end{abstract}

Die Auswahl der Böden beinhaltet sowohl Standorte mit Sandlöß über Schmelzwassersanden, Sandlöß über Geschiebelehm als auch Standorte mit Geschiebemergel und tiefgründigem Löß.

Auf Grundlage der im Verlauf von 17 Beobachtungsjahren am Untersuchungsstandort Brandis unter den klimatischen Bedingungen am Rande des Mitteldeutschen Trockengebietes (hohe Variabilität von Wasser- und Energieangebot, hohes klimatologisches Wasserdefizit in den Sommermonaten, geringe Winterniederschläge) ermittelten Datenbasis, konnten sowohl Aussagen zum Schwankungsverhalten der untersuchten Größen infolge Witterung und Bewirtschaftung (angebaute Fruchtart) getroffen werden, als auch die Wirkung der verschiedenen Böden im Verlauf der einzelnen Anbaujahre auf die Wasserhaushaltsgrößen ermittelt werden.

Einfluss auf die Sickerwassermenge haben die Höhe der sommerlichen Bodenwasserausschöpfung und die Höhe der Niederschläge in den darauffolgenden Herbst- und Wintermonaten. Daraus resultiert, in Abhängigkeit von der Kapazität der Bodenwasserspeicher: eine hohe Variabilität der vergleichsweise geringen Sickerwassermengen (im Mittel der Jahre $175 \mathrm{~mm}$ bei Böden mit geringem nutzbaren Wasservorrat und $50 \mathrm{~mm}$ bei Lößböden), ein geringer Zusammenhang zwischen Wasserverbrauch der Pflanzen und Sickerwassermenge sowie zwischen Jahresniederschlag und Sickerwassermenge. Die Böden mit geringer und mittlerer Wasserspeicherkapazität können trotz hoher jährlicher Inanspruchnahme der Vorräte den Wasserbedarf der Pflanzen in der überwiegenden Anzahl der Jahre nicht decken. Die limitierte Wasserversorgung führt zu geringeren Erträgen und einem unwirtschaftlichen Umgang der Pflanzen mit dem verfügbaren Bodenwasser. Nur auf den Lößböden mit einem nutzbaren Wasservorrat von mehr als $400 \mathrm{~mm}$ sind die Voraussetzungen für eine kulturartenspezifische maximale Verdunstung gegeben. Geringe Sickerwasserbildung in Trockenjahren führt zu einer Akkumulation von Stickstoffbilanzüberschüssen in den Böden, Feuchtjahre zu erhöhten Stickstoffausträgen mit dem Sickerwasser. Im Mittel einer Periode intensiver landwirtschaftlicher Bewirtschaftung zeigte sich bei den Böden mit geringer Wasserspeicherkapazität ein mittlerer Nitratgehalt von $100 \mathrm{mg} / \mathrm{l}$ im Sickerwasser. In den seltenen Perioden mit Sickerwasserbildung wird selbst bei den Lößböden mit $60 \mathrm{mg} / \mathrm{l}$ die lt. E.G.-Trinkwasserrichtlinie zulässige Höchstkonzentration überschritten.

Am Beispiel dieser ausgewählten Ergebnisse wird deutlich - werden in der landwirtschaftlichen und ingenieurhydrologischen Praxis Mittelwerte verwendet - führt dies auf Grund der hohen witterungsbedingten Variabilität der Wasserhaushaltsgrößen, die sich auf allen Böden zeigte, zu erheblichen Fehleinschätzungen. In Abhängigkeit von der klimatologischen Repräsentanz des 17jährigen Untersuchungszeitraumes, beschreibt die Arbeit Zusammenhänge zwischen den Einflußfaktoren und den Wasserhaushaltsgrößen der verschiedenen Böden und liefert konkrete Angaben zu den möglichen Spannbreiten innerhalb derer sich die Wasserhaushaltsgrößen Verdunstung, Sickerwasserbildung und Bodenwasserausschöpfung im Untersuchungsgebiet bewegen.

Weitere Untersuchungen führten zu der Erkenntnis, dass ähnliche Böden, trotz Abweichungen in der Horizontabfolge, in Bezug auf den Bodenwasserhaushalt keine Unterschiede aufweisen, die über die natürliche Variabilität der Standorte hinausgehen. Deshalb konnten die acht analysierten Böden zu vier Pedo-Hydrotopen zusammengefasst werden. 


\section{Inhaltsverzeichnis}

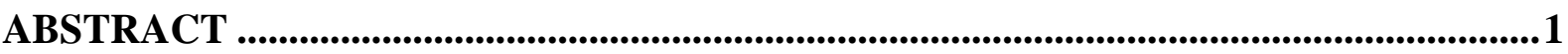

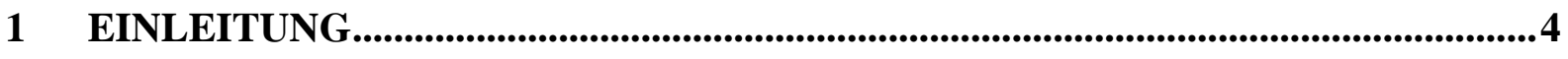

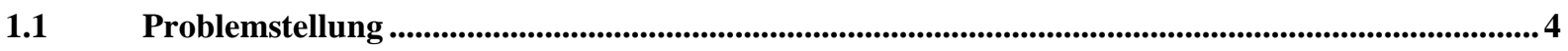

$1.2 \quad$ Ziel der Untersuchungen..........................................................................................................................5

$1.3 \quad$ Ausgangspunkt und Lösungsweg ......................................................................................................6

$2 \quad$ MATERIAL UND METHODEN....................................................................................9

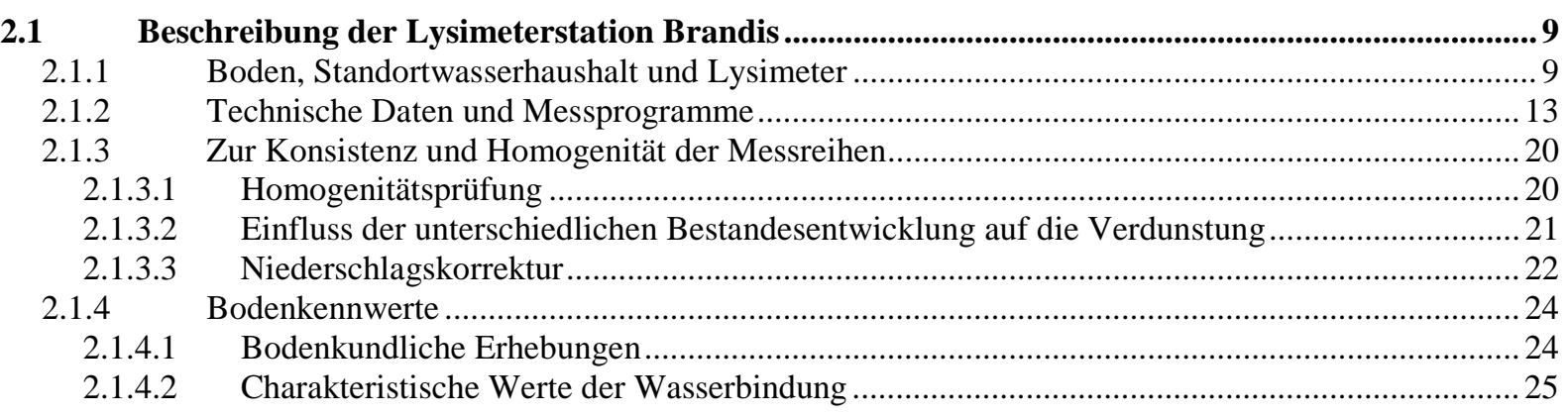

$2.2 \quad$ Die Herkunftsflächen der Lysimeterböden ......................................................................................28

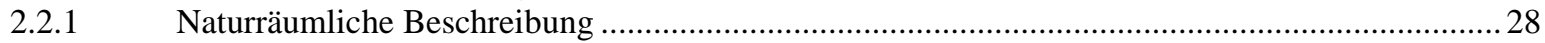

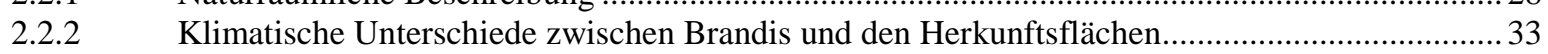

\begin{tabular}{|c|c|}
\hline 2.3 & 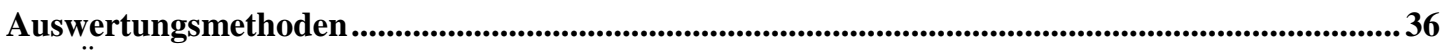 \\
\hline 2.3 .1 & Überblick. \\
\hline 2.3 .2 & ng der pflanzenverfügbaren Bodenwassermenge \\
\hline 2.3 .3 & Ermittlung der Ausschöpfungstiefe \\
\hline 2.3 .4 & Potentielle und reale Verdunstung - Wasserverbrauch der Pflanzen. \\
\hline 2.3 .5 & Sickerwassermenge und Verlagerungsrisiko von Nitrat..... \\
\hline 2.3 .6 & Untersuchungen zur hydrologischen Wirksamkeit der verschiedenen Böden.. \\
\hline
\end{tabular}

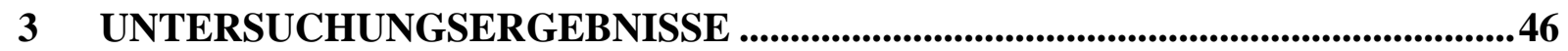

$3.1 \quad$ Niederschlagshöhe und -verteilung am Untersuchungsstandort ........................................................46

3.2 Vergleichsrechnungen zur Ermittlung der potentiellen Verdunstung..........................................47

3.2.1 Verwendete Verfahren zur Berechnung der potentiellen Verdunstung ......................................47

$3.2 .2 \quad$ Verfügbare Klimadaten ................................................................................... 50

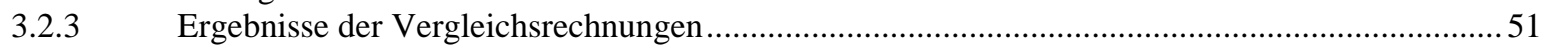

3.3 Klimatische Wasserbilanz......................................................................................................................55

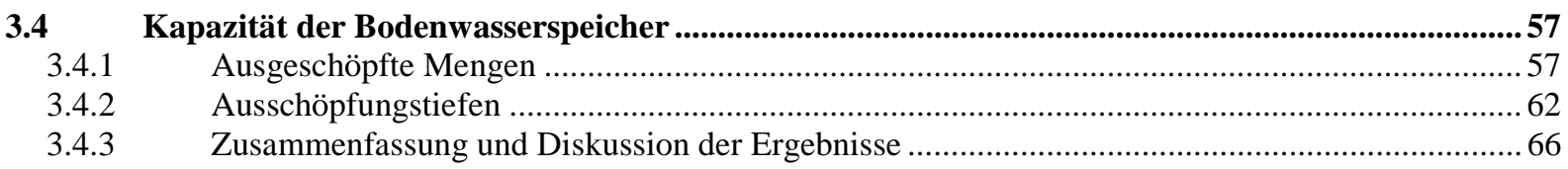

$3.5 \quad$ Verdunstung in Abhängigkeit von Witterung, Boden und Bewirtschaftung ...................................67

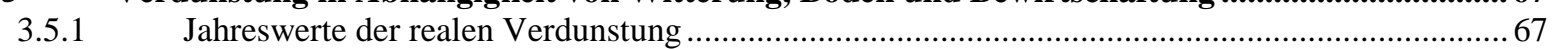

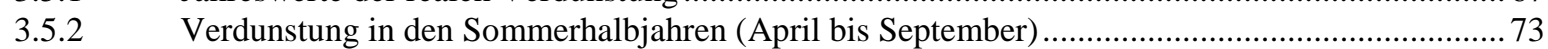

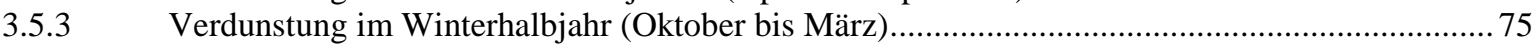

3.5.4 Ermittlung von Jahreswerten mit dem Verfahren von RENGER\&WESSOLEK .....................76 


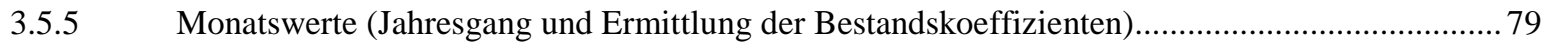

3.5.6 Einfluss von Witterung und Boden auf den Wasserverbrauch der Pflanzen und

die Ertragsbildung.................................................................................................... 83

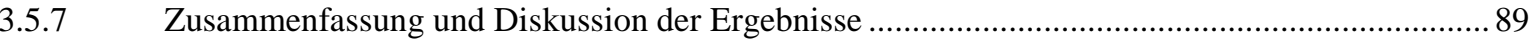

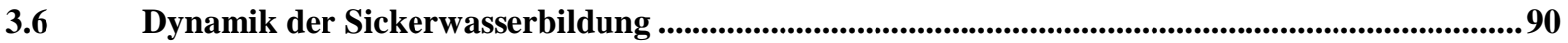

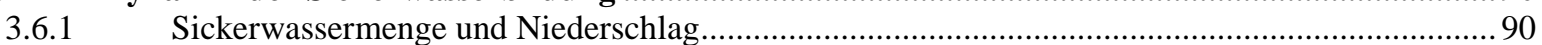

3.6.2 Verlauf der Sickerwasserperiode ................................................................................... 96

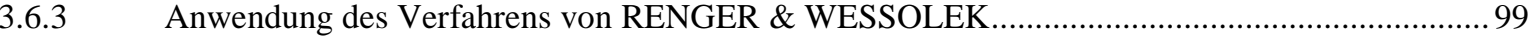

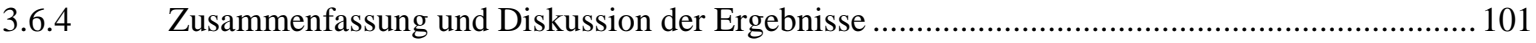

3.7 Beziehung zwischen Bewirtschaftung, Bodenwasserhaushalt und Stickstoff-Austrag................ 104

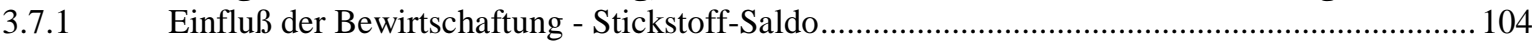

3.7.2 Einfluss der jährlichen Inanspruchnahme des Bodenwasservorrates auf die

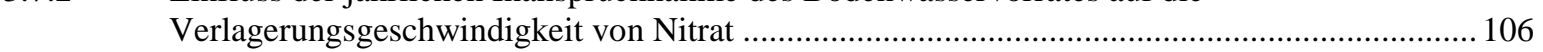

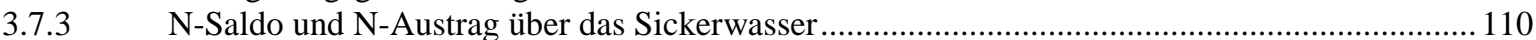

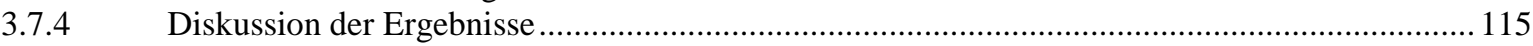

\section{ZUR REPRÄSENTANZ DER LYSIMETERUNTERSUCHUNGEN ......................116}

\begin{tabular}{|c|c|}
\hline 4.1 & suchungen zur räumlichen Repräsentanz ..........................................................................116 \\
\hline 4.1 .1 & Hydrologische Wirksamkeit verschiedener Böden ........................................................ 116 \\
\hline 4.1 .2 & Vergleichbare Standorte im Freistaat Sachsen \\
\hline 4.1 .3 & nenge und Grundwasserneubildung. \\
\hline 4.1 .3 .1 & Problemstellung. \\
\hline 4.1 .3 .2 & hungen im Einzugsgebiet der Parthe \\
\hline
\end{tabular}

4.2 Zeitliche Repräsentanz der Messreihen von 1981 bis 1997 .............................................................128

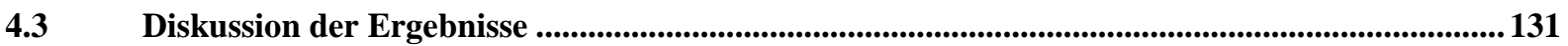

5 SCHLUSSFOLGERUNGEN......................................................................................133

6 LITERATURVERZEICHNIS ....................................................................................138

$7 \quad$ ABKÜRZUNGEN UND FORMELZEICHEN ........................................................147

$8 \quad$ VERZEICHNIS DER ABBILDUNGEN ...........................................................149

9 VERZEICHNIS DER TABELLEN ...............................................................................152

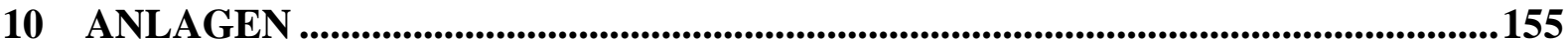




\section{Einleitung}

\subsection{Problemstellung}

Mit Beginn seiner Entwicklung übernimmt der Boden in den Einzugsgebieten die Transformation der Niederschläge in Grund- und Oberflächenwasserabflüsse. Die differenzierte zeitliche und örtliche Verteilung der Wasserressourcen und ihre Befrachtung mit (anthropogenen) Inhaltsstoffen sind Folge der auf und im Boden ablaufenden Prozesse.

War der Boden in Mitteldeutschland ursprünglich ausschließlich Standort von Wäldern, so sind in der heutigen mitteldeutschen Agrar- und Industrieregion mit ausgedehnten Tagebauund Tagebaurekultivierungsflächen kaum noch ursprüngliche Standortbedingungen zu finden.

Die ökologischen und wirtschaftlichen Folgen/Auswirkungen, die durch den totalen Verlust oder durch Veränderungen/Beeinträchtigungen der natürlichen Bodenfunktionen auftreten, sind im Rahmen der Planungs- und Genehmigungsverfahren (u. a. Landes-, Regional- und Bauleitplanung) abzuwägen und zu berücksichtigen. In einem regional unterschiedlich stark wachsenden Konflikt zwischen Nutzung und Erhalt/Schutz der Ressourcen Wasser und Boden nehmen Landwirtschaft und Wasserwirtschaft als flächen- und mengenmäßig größte Nutzer eine besondere Stellung ein. Letztlich dienen beide Wirtschaftszweige der Grundversorgung der Menschen und ihrem Lebenserhalt.

Voraussetzung für optimale Wachstumsbedingungen der Kulturpflanzen ist eine bedarfsgerechte Wasserversorgung und Zuführung von Nährstoffen. Für die Wasserwirtschaft ist das von den Flächen verdunstende Wasser eine negative Bilanzgröße. Der Anteil des Niederschlages, der als Sickerwasser das Grundwasser erreicht, belastet dieses in Abhängigkeit vom standort- und nutzungsbedingten Verlagerungsrisiko (HENNINGS \& SCHEFFER, 1999) mit leicht aus dem Bodenkörper auswaschbaren Pflanzennährstoffen.

Zum Schutz des Grundwassers werden Nutzungsbeschränkungen (z. B. in TrinkwasserSchutzgebieten) erteilt, Fonds eingerichtet (Wasserpfennig) sowie Ausgleichszahlungen (für Auflagen bei der landwirtschaftlichen Bewirtschaftung) und Entschädigungen in beträchtlicher Höhe geleistet. Da sich die verschiedenen Einflussfaktoren (Klima, Landnutzung, Boden) überlagern und die Prozesse (Stoffumsatz, Sickerwasserverlagerung) sehr langsam ablaufen, ist der tatsächliche Erfolg dieser Maßnahmen zum Schutz der Bodenfunktionen und der Wasserressourcen schwer zu quantifizieren.

Hinzu kommt, dass in den vergangenen Jahrzehnten gerade in Mitteldeutschland durch gravierende Landnutzungsänderungen (Flächenstilllegung und -versiegelung, Einstellung von Grundwasserabsenkungen, Entstehung von Wasserflächen infolge Braunkohlentagebau und Kiesabbau) eine Überprüfung der derzeit gültigen wasserwirtschaftlichen Mittel- und Extremwerte erforderlich wird.

Typisch für das Klima im Mitteldeutschen Raum sind vergleichsweise geringe Jahresniederschläge zwischen 450 und $600 \mathrm{~mm}$ (DWD, Agrameteorologische Monatsberichte) bei einem relativ hohen Verdunstungsanspruch der Atmosphäre von mehr als 600 mm (MÜLLER et al., 1989). Trocken- und Feuchtjahre - die sich deutlich im Verlauf der Grundwasserstände und in den Abflussschwankungen der Vorfluter widerspiegeln - umfassen Perioden von 3 bis 7 Jahren (GRAHMANN, 1943). Es handelt sich um ein subhumides Klima (LIEDKE \& MARCINEK, 1995) mit mindestens 6 wasserdefizitären Monaten. Das sub- 
humide Klima (= zeitweise Trockenheit im humiden Klimagebiet) ist repräsentativ für nahezu alle Gebiete der neuen Bundesländer (außer Harz, Mittelgebirge und deren Vorländer), es ist in den alten Bundesländern nur im Oberrheingraben und in der südlichen Hessischen Senke anzutreffen. Unter diesen klimatischen Bedingungen ist vor allem in den Sommermonaten mit einem angespannten Wasserhaushalt zu rechnen.

Die Hinweise zahlreicher Autoren (u. a. RAPP \& SCHÖNWIESE, 1996; SEIDLER, 1999; PFISTER, 1999) auf mögliche Klimaveränderungen werfen darüber hinaus neue Fragestellungen auf. So wird das Jahrzehnt 1988-1997 von PFISTER (1999) im Rahmen der Wetterbeobachtungen der letzten 500 Jahre als außergewöhnlich warm charakterisiert. Auch aus diesen Gründen ist künftig mit Wasserhaushaltsgrößen zu rechnen, die von existierenden Erfahrungswerten abweichen.

So benötigen sowohl die Institutionen der Landwirtschaft als auch der Wasserwirtschaft eine exakte und aktuelle Datenbasis in hoher zeitlicher Auflösung. Einverständnis besteht darüber, dass zur Beantwortung komplexer Fragestellungen zum Einfluß von Klima, Witterung und landwirtschaftlicher Nutzung auf den Bodenwasserhaushalt der Einsatz mathematischer Modelle erforderlich ist. Diese sind zahlreich verfügbar, benötigen aber vielfältige Modellparameter und Vergleichswerte, um derartige nichtlineare Umweltsysteme adäquat widerspiegeln zu können (ALY, 1988; FUNK, 1993; PESCHKE, 1999). Mit der Aufbereitung und Interpretation von Versuchsreihen soll die vorliegende Arbeit sowohl mit der Bereitstellung von Zahlenmaterial als auch mit der Beschreibung von Zusammenhängen zwischen Witterung, Boden, Pflanze und Wasserhaushalt einen Beitrag liefern.

\subsection{Ziel der Untersuchungen}

Gegenstand der Untersuchungen ist der Wasserhaushalt und der Stickstoffaustrag über das Sickerwasser von acht repräsentativen Ackerböden Mitteldeutschlands unter den klimatischen Bedingungen im Grenzraum des mitteldeutschen Trockengebietes (Leipziger Tieflandsbucht). Die Auswahl grundwasserfreier Böden beinhaltet sowohl Standorte mit Sandlöß über Schmelzwassersanden, Sandlöß über Geschiebelehm als auch Standorte mit Geschiebemergel und tiefgründigem Löß.

Für diese Böden soll der Wasserhaushalt unter gebietstypischer landwirtschaftlicher Bewirtschaftung ermittelt werden. Der Einfluss der jährlichen Witterung und der jeweils angebauten Pflanzen auf unterschiedlichen Böden ist schwer zu quantifizieren, da entsprechende Messreihen an verschiedenen Böden selten unter gleichen Witterungsbedingungen und/oder gleicher Bewirtschaftung gewonnen werden.

Die hier vorgestellten Untersuchungen an verschiedenen Böden wurden unter gleichen Klimaund Witterungsbedingungen und gleicher Bewirtschaftung hinsichtlich Fruchtart, Düngemenge, Bodenbearbeitung und Pflege durchgeführt. Somit bestand die Möglichkeit zur Identifikation der einzelnen Einflussfaktoren auf den Bodenwasserhaushalt. Im Einzelnen sollten folgende Fragen beantwortet werden:

- Wie hoch ist die sommerliche Ausschöpfung der Wasservorräte der Böden unter dem Einfluss der sommerlichen Trockenheit in den verschiedenen Anbaujahren? Aus welchen Tiefen wird das Bodenwasser von den Pflanzen entnommen? 
- Wie groß ist die reale Verdunstung (als dominierende Wasserhaushaltsgröße) und welchen Einfluss haben Witterung und Boden auf den Wasserverbrauch der Pflanzen und die Ertragsbildung?

- Welchen Einfluss haben Niederschlag und Bodenwasserausschöpfung auf die Sickerwasserbildung?

- Wie eng ist der Zusammenhang zwischen Bewirtschaftung und Stickstoffaustrag mit dem Sickerwasser?

Auf Basis der Messreihen von acht Böden sollte ferner untersucht werden, wie signifikant sich Wasserhaushaltsgrößen verschiedener Böden tatsächlich voneinander unterscheiden.

Letztlich konnten Hilfsmittel für die Wasserwirtschaft zur Ableitung der Bilanzgrößen Verdunstung und Sickerwasserbildung bereitgestellt und Aussagen zur Repräsentanz von Standortuntersuchungen für den Gebietswasserhaushalt getroffen werden.

\subsection{Ausgangspunkt und Lösungsweg}

Mit dem Bau der Lysimeterstation Brandis (zur Stationslage s. Abb. 1) wurde in den Jahren 1976/79 eine Anlage geschaffen (MORITZ et al., 1991), mit der die genannten Zielstellungen realisiert werden konnten. Nach umfangreichen Voruntersuchungen im Einzugsgebiet der Parthe (PRETSCHOLD, 1963; THOMAS \& HAASE, 1970; SPENGLER, 1973), die zur Ausgrenzung von Hydrotopen (,Flächen gleichen hydrologischen Verhaltens“) führten, wurden für ausgewählte Hydrotope wägbare Lysimeter gebaut. Dabei sollten die Bodenmonolithe nach MORITZ \& SÄMISCH \& SPENGLER (1991), über das Parthegebiet hinaus zur Ergänzung gleichartiger Stationen (in der Letzlinger Heide, Eberswalde seit 1929, Groß-Lüsewitz bei Rostock und Großobringen bei Jena) die wichtigsten Standortformen Mitteldeutschlands - nämlich Acker auf Löß, Geschiebelehm und -sand erfassen.“ Auf Grund dieses hohen Anspruches wurden auch Böden aus dem Raum Halle, Wittenberg und Oschatz in der Lysimeterstation Brandis installiert.

$\mathrm{Zu}$ diskutieren sind die in der Literatur vorgebrachten Vorbehalte gegen die Lysimetertechnik. Dem gegenüber stehen die Vorteile von Lysimetern geeigneter Bauweise und Ausstattung, die von kaum einer anderen Messtechnik erreicht werden. Die Eignung von Lysimetern zur Beschreibung des Standortwasser- und Stoffhaushaltes geht u. a. aus Veröffentlichungen von AMBERGER \& SCHWEIGER (1978), KATZUR \& MROSKO (1983), GUTSER et al. (1987), KATZUR et al. (1989), MÜLLER et al. (1991), GÜNTHER \& KNOBLAUCH (1993) hervor.

Von Vorteil ist, dass sich bei Lysimeteruntersuchungen in idealer Weise die Möglichkeiten von Labor- (gesicherte Messreihen aller Komponenten der Wasserhaushaltsgleichung in hoher zeitlicher Auflösung) und Felduntersuchungen (reale Freilandbedingungen) verbinden lassen. Für den im Lysimeter befindlichen Boden können für beliebige zeitliche Abschnitte (in Brandis $\geq 1 \mathrm{Tag}$ ) Wasserhaushaltsbilanzen erstellt werden.

Lysimeter von $1 \mathrm{~m}^{2}$ Fläche und $3 \mathrm{~m}$ Tiefe, wie dies in Brandis der Fall ist, kompensieren bereits zahlreiche kleinräumige Inhomogenitäten der Böden. Damit bieten sich günstigere Voraussetzungen für eine Übertragung in die Fläche als dies im Ergebnis von punktuellen Untersuchungen mittels Tensiometern, TDR-Sonden und Saugkerzen im Gelände möglich ist.

Die Konzentration verschiedener Bodenmonolithe an einem Standort ermöglicht eine intensive messtechnische Betreuung, so auch an Sonn- und Feiertagen, in der vegetations- 
losen Zeit und über die Wintermonate. Ein kontinuierlicher Stationsbetrieb gewährleistet die Beibehaltung einheitlicher Bewirtschaftungs-, Mess- und Berechnungsmethoden, was für die Konsistenz der Daten von außerordentlicher Bedeutung ist.

Da die in Brandis untersuchten Böden von teils weit entfernt liegenden Herkunftsflächen stammen, werden die klimatischen Unterschiede zwischen Brandis und diesen Flächen mit in die Untersuchungen einbezogen. Eine Beschreibung der Entstehung und der natürlichen Ausstattung der Herkunftsflächen dient dem Verständnis der im Lysimeter ablaufenden Prozesse und der Rückführung der standortbezogenen Untersuchungsergebnisse in den Gesamtzusammenhang (= Einzugs- oder Teileinzugsgebiet).

Die tägliche Verdunstung wird mittels wägbarer Lysimeter als Restgröße über die Wasserhaushaltsgleichung aus den täglichen Messwerten von

- Niederschlag,

- Sickerwasser am Lysimeterauslauf und

- Bodenwasservorratsänderung (= Gewicht des Vortages minus Gewicht des Messtages)

berechnet. Der Niederschlag wird in Brandis nicht direkt am Lysimeter, sondern mittels Regenmesser nach HELLMANN (200 $\mathrm{cm}^{2}$ Auffangfläche) in $1 \mathrm{~m}$ Aufstellhöhe und parallel dazu bodengleich ermittelt. Standardaufstellung im Niederschlagsmessnetz des Deutschen Wetterdienstes ist $1 \mathrm{~m}$ über dem Erdboden. Bekannt ist, dass ausgerechnet die Ermittlung des Niederschlages als größte Komponente des Wasserhaushaltes mit beachtlichen Fehlern verbunden ist (u. a. GOLF, 1982; SOKOLLEK, 1983; DYCK \& PESCHKE, 1995; DISSE, 1995). Die von den Autoren genannten Fehler liegen in der Größenordnung zwischen $10 \%$ und $20 \%$ im Jahresmittel und bis zu $100 \%$ an wind- oder schneereichen Einzeltagen und monaten, wobei die Niederschlagsmessung mit allen Standardgeräten zu geringe Werte liefert. So mussten auch die Niederschläge für den Standort Brandis korrigiert werden.

Weil verschiedene Ansätze zur Berechnung der potentiellen Verdunstung zum Teil stark differierende Ergebnisse liefern (z. B. bei SPONAGEL, 1980 und GENID, 1982), war es auf Grund der Bedeutung dieser Einflussgröße erforderlich, für den Standort Brandis vergleichende Berechnungen durchzuführen.

Das anschließende Kapitel widmet sich sehr ausfühlich dem Stationsbetrieb und der Vorgehensweise bei der Auswertung der Messreihen, da - neben der Bereistellung konkreter Untersuchungsergebnisse - mit dieser Arbeit auch gezeigt werden sollte, wie komplex der Beitrag ist, den Lysimeterstationen zur Problematik Bodenwasserhaushalt liefern können.

Um bei dem erheblichen Umfang der Meßergebnisse (8 verschiedene Böden mit jeweils 3 Wiederholungen, 8 Fruchtarten, 17 Untersuchungsjahre) in jedem Fall eine anschauliche Ergebnisdarstellung zu erreichen, musste ein Arbeitsergebnis - die Zusammenfassung von 8 verschiedenen Böden zu vier „Pedo-Hydrotopen“ (Kap. 4.1.1) bereits in den vorstehenden Kapiteln verwendet werden. 


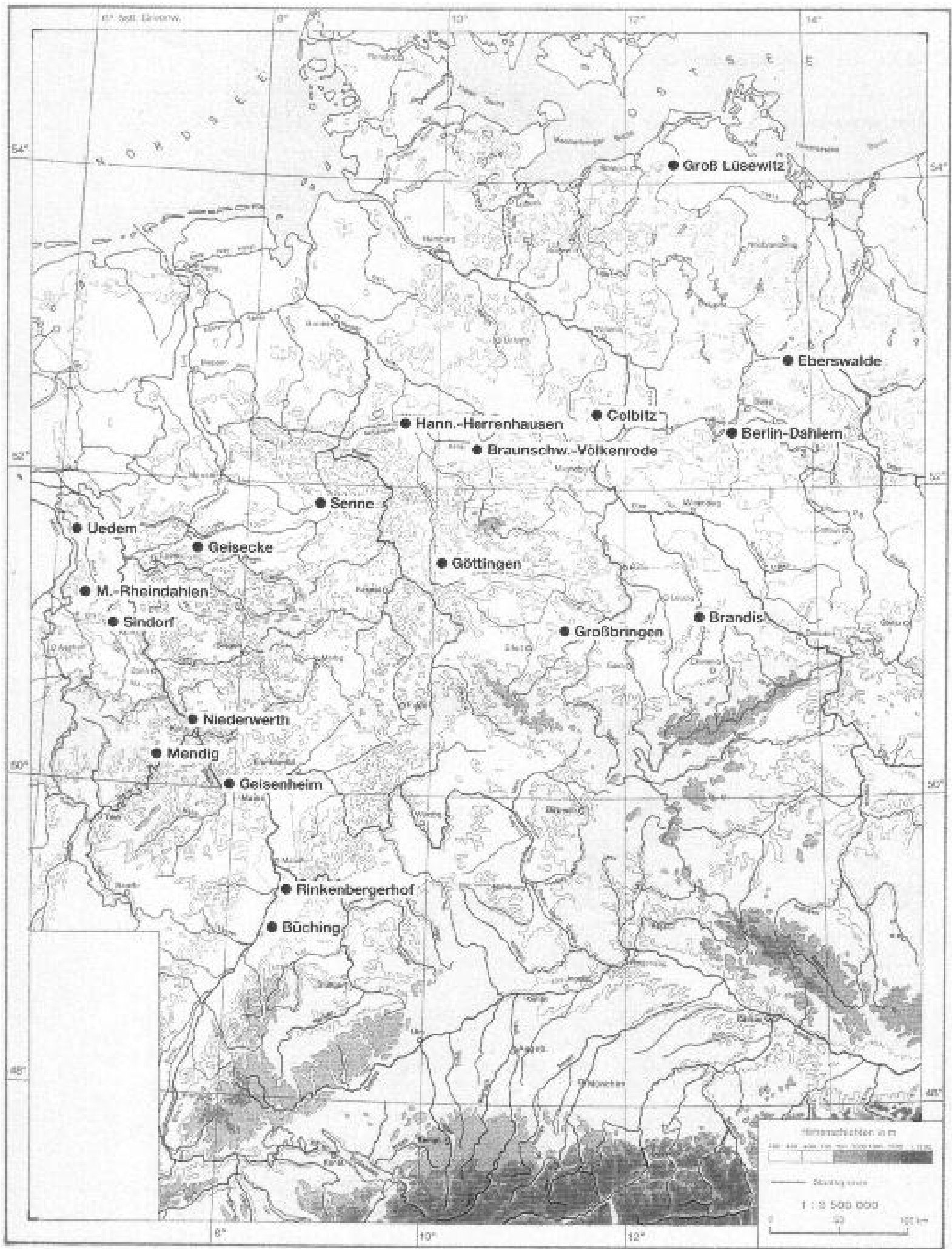

Abb. 1: Lage der Lysimeterstation Brandis und weiterer wägbarer Lysimeteranlagen der Bundesrepublik Deutschland (aus DVWK, 1996) 


\section{Material und Methoden}

\subsection{Beschreibung der Lysimeterstation Brandis}

\subsubsection{Boden, Standortwasserhaushalt und Lysimeter}

Mittels Lysimeter wird der Bodenwasserhaushalt eines bekannten Volumens der ungesättigten Bodenzone ermittelt, wobei im Vergleich zu anderen Freilanduntersuchungen eine eindeutige Bilanzierung der Wasserhaushaltsgrößen erfolgen kann. Konstruktive Maßnahmen beim Bau der Lysimeter (z. B. Größe der Lysimeter, Art der Gewinnung der Lysimeterböden, Realisierung des Sickerwasserauslaufes, Nutzung der Lysimeteroberfläche) führen zu Unterschieden zwischen Standortwasserhaushalt im Freiland und im Lysimeter. Deshalb werden einige Gesichtspunkte zu Wasserhaushalt und Boden sowie wichtige Aspekte, die zur Erzielung möglichst natürlicher Standortbedingungen im Lysimeter zu beachten sind, nachfolgend kurz dargelegt und diskutiert.

Der Begriff „Boden“ ist in den verschiedenen Wissenschaftsdisziplinen mit sehr unterschiedlichen Definitionen belegt.

So ist in der traditionellen Bodenkunde das Produkt gemeint, das aus dem komplexen $\mathrm{Zu}$ sammenspiel bodenbildender Faktoren wie Muttergestein, Relief, Klima, Organismen und Zeit entstanden ist. Ausgangs-(Mutter-)gestein der hier untersuchten Böden sind Sedimente, die während der Weichsel-, Saale- und Elsterkaltzeit zur Ablagerung kamen. In die Betrachtungen der Bodenkunde werden in der Regel nur die Bodenhorizonte bis etwa 1,2 m unter Gelände einbezogen. Damit liegt der Schwerpunkt der Untersuchungen auf Bodenmerkmalen, die als Ergebnis der bodenbildenden Prozesse gelten.

Bei Untersuchungen zum Bodenwasserhaushalt (als Teil des regionalen Wasserkreislaufes) gewinnen in Abhängigkeit von der Lage des Grundwasserspiegels und der Entfernung zum Vorfluter auch die Eigenschaften der tiefer liegenden Ausgangssedimente an Bedeutung. In diesem Zusammenhang wird der Boden nach der Wasserbindung und -bewegung bis zu seiner unteren Berandung, dem Grundwasserspiegel, gegliedert in eine

1. obere, verdunstungsbeeinflusste Zone, in der Infiltration, Wasserentzug durch die Pflanzen und Versickerung zu stark wechselnden Wassergehalten führen,

2. darunter folgende Dränzone und

3. Kapillarwasserzone über der Grundwasseroberfläche.

Bei dieser Betrachtungsweise umfasst der Begriff „Boden“ den gesamten Bereich der „ungesättigten Zone“ (auch als „Aerationszone“ bezeichnet). In diesem Zusammenhang kann der Boden (= ungesättigte Zone) nach DIN 4049 (1994) auch als „Grundwasserüberdeckung“ behandelt werden.

Im Vergleich dieser beiden Betrachtungsweisen ist der Boden im engeren Sinne der bodengenetischen Betrachtung (als durchwurzelter und durch Kleintiere belebter oberster Bereich der Erdkruste) an Standorten mit flurfernen Grundwasserständen nur ein räumlich nicht scharf abgrenzbarer Teilbereich der ungesättigten Zone.

Untersuchungen zum Bodenwasserhaushalt sollten sich auf die gesamte räumlich sowie zeitlich in sehr unterschiedlicher Mächtigkeit vorhandene ungesättigte Zone erstrecken. Insbesondere an grundwasserfernen Standorten, die hier Gegenstand der Untersuchungen sind, beeinflussen auch die strukturellen Besonderheiten des Ausgangsgesteins die Wasserspeicherung und -durchlässigkeit der Böden. Diese Schichten sind zudem Umsatzräume für Stoffe, die mit dem Sickerwasser in Richtung Grundwasser transportiert werden. 
In der weiteren Bearbeitung erfolgt die Verwendung des Begriffes „Boden“ nun stets im Sinne von „ungesättigter Zone“ (DBG, 1994).

Am Beispiel einer 3 m mächtigen Bodensäule im Lysimeter Abb. 2 wird nachfolgend der Bodenwasserhaushalt eines ebenen, vegetationsbedeckten, grundwasserfernen Standortes beschrieben.

Unter Berücksichtigung der am Lysimeter tatsächlich messbaren Wasserhaushaltsgrößen gilt für diese Standortverhältnisse folgende Wasserhaushaltsgleichung:

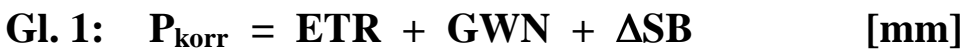

$\mathrm{P}_{\text {korr }}$ ist der mittels HELLMANN-Messer ermittelte und anschließend korrigierte Niederschlag (s. Kap. 2.1.3.3). Das Niederschlagswasser wird auf dem Lysimeter zwischengespeichert (Interzeption, Muldenspeicherung, Schneeakkumulation) um dann $\mathrm{zu}$ infiltrieren oder $\mathrm{zu}$ verdunsten. Ein oberflächliches Abfließen ist auf Grund des Lysimeterrandes nicht möglch.

Die reale Verdunstung (ETR) als Gesamtverdunstung beinhaltet die Teilkomponenten Transpiration und Evaporation sowie die Interzeptionsverdunstung als Teil der Evaporation.

Wie im Freiland kommt es im Sommerhalbjahr in den 3 m mächtigen Lysimeterböden sowohl zu aufwärts (infolge der Bodenwasserentnahme durch die Pflanzen) als auch zu abwärts gerichteten Wasserbewegungen und damit zur Ausbildung einer hydraulischen Wasserscheide unterhalb des Hauptwurzelhorizontes. Der innerjährliche Verlauf und die jährliche maximale Ausdehnung der verdunstungsbeeinflussten Zone sind in Abhängigkeit von Bodenart, Witterung, Wurzeldichte und -tiefe sehr variabel. Insbesondere bei bindigen Böden erreicht die maximale Ausdehnung der verdunstungsbeeinflussten Zone (tiefste Lage der Wasserscheide) infolge der kapillaren Nachlieferung aus dem Unterboden in Trockenjahren auch Bereiche weit unterhalb der Wurzelzone (zum Verlauf der Ausschöpfungstiefen s. Kap. 3.4.2).

Der zwischen permanentem Welkepunkt (PWP) und Feldkapazität (FK) schwankende, aktuelle Wassergehalt $(\theta)$ in der verdunstungsbeeinflussten Bodenzone ergibt sich aus dem Zusammenspiel von Infiltration, Versickerung, Wasseraufnahme der Pflanzenwurzeln und kapillarem Aufstieg aus tieferen Bodenschichten. Er unterliegt einer großen jahreszeitlichen Dynamik.

Die Dränwasserzone unterhalb der verdunstungsbeeinflussten Bodenzone ist durch eine ausschließlich abwärtsgerichtete Wasserbewegung gekennzeichnet, die auch zum Stillstand kommen kann und bei geringen Änderungen der Saugspannung, des Wasser- und Luftgehaltes stattfindet (DBG, 1994). In Teufen größer $5 \mathrm{~m}$ liegen quasistationäre Sickerverhältnisse vor (VOIGT, 1990). Sickerwasser, das in mehr als $2 \mathrm{~m}$ Tiefe die Dränwasserzone passiert, dient dort nur noch in geringem Umfang zur Auffüllung von Bodenwasserdefiziten und kann nicht mehr in die verdunstungsbeeinflusste Zone gehoben werden. Es wird als potentielle Grundwasserneubildung $(\mathrm{GWN})$ betrachtet.

Die Bodenwasservorratsänderung ( $\Delta \mathrm{SB})$ im 3 m mächtigen Lysimeter resultiert somit aus der Niederschlagsmenge $\left(\mathrm{P}_{\text {korr }}\right)$, der Höhe der realen Verdunstung (ETR) und der am Lysimeterauslauf im Bilanzzeitraum registrierten Wassermenge (GWN). 


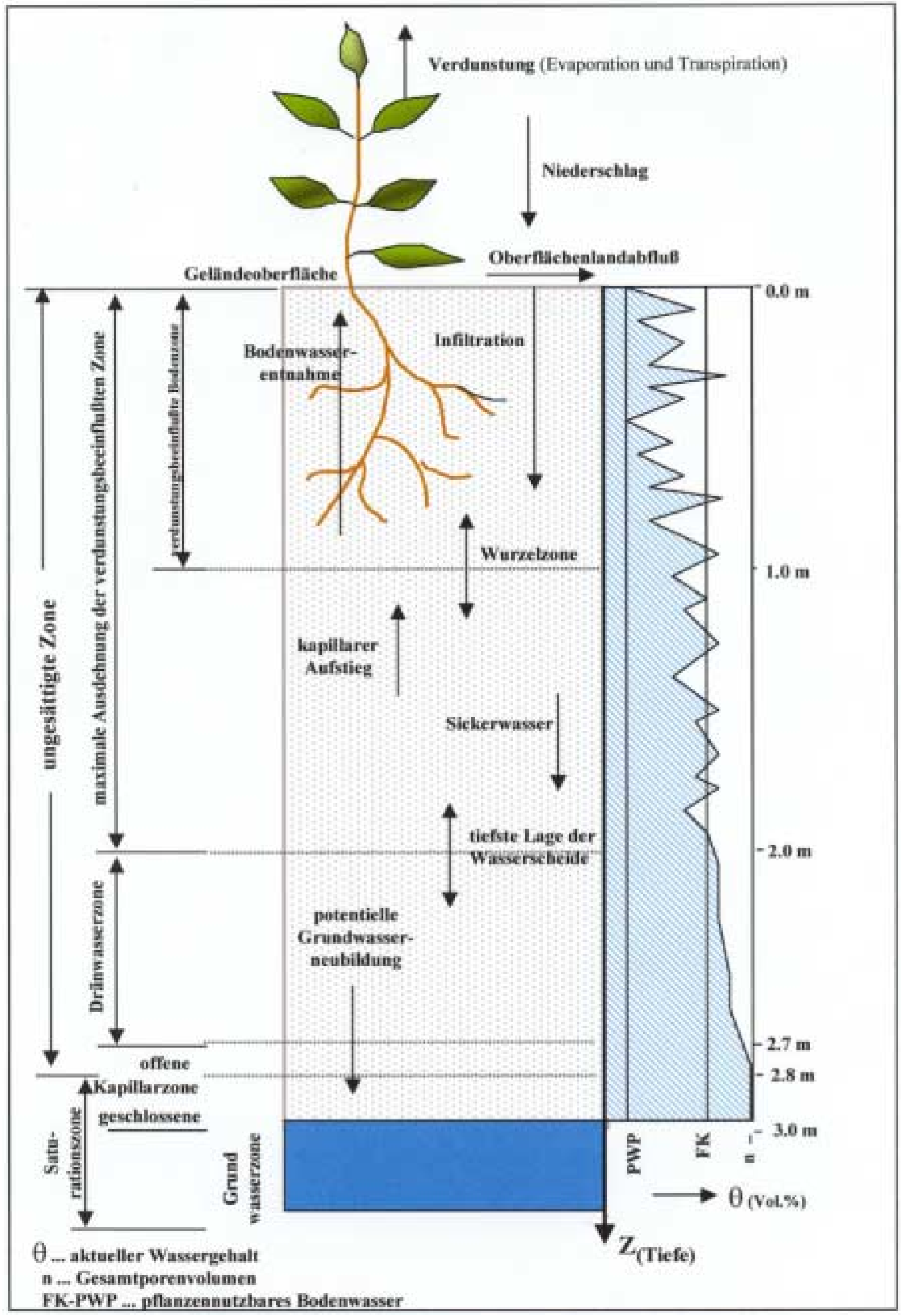

Abb. 2: Standortwasserhaushalt: Schematische, auf ein $3 \mathrm{~m}$ tiefes Lysimeter bezogene Darstellung der ungesättigten Zone am Beispiel eines ungeschichteten Bodenprofils 
Der Grundwasserspiegel im Freiland unterliegt in Abhängigkeit von der Höhe der Grundwasserneubildung, dem Kapillaraufstieg, Zu- und Abflüssen (einschließlich künstlicher Entnahmen und Einleitungen), seiner Tiefenlage, Porosität, Mächtigkeit und Neigung der Sohle erheblichen jahreszeitlichen Schwankungen. Der Einfluß des fluktuierenden Verhaltens des Grundwasserspiegels fehlt im Lysimeter.

Weitere Unterschiede die aus dem Einbau der Böden in die Lysimeterbehälter resultieren:

- An der Erdoberfläche wird der horizontale Wasser-/Stoffzu- und -abfluß verhindert, da die Wand der Brandiser Lysimeterbehälter höher ist als die Oberfläche der Bodenmonolithe.

Beurteilung dieses Fehlers: In den reliefarmen Lockergesteinsbereichen der Mitteldeutschen Tiefebene tritt großräumig Oberflächenlandabfluss nur bei Bodenfrost und nach größeren Niederschlagsereignissen (Sättigungsflächen) auf, wobei dieser Abflussanteil die Grundwasserneubildung nur dann verringert, wenn er ein Gewässer erreicht. Nach SPENGLER (1973) ist sein Anteil auf den Herkunftsflächen der Lysimeterböden kleiner $10 \%$ des Gesamtabflusses (langjähriger Mittelwert).

Beispiele dafür, dass mit Lysimetern auch der Oberflächenlandabfluss (,runoff“ = RO) gemessen werden kann, sind die Lysimeteranlagen der Stadtwerke Mönchengladbach (HELLEKES, 1985) und der United States Department of Agriculture in der Nähe von Coshocton in Ohio (BONTA et al., 1989).

- Die Seitenwände der Lysimeterbehälter $\mathrm{Abb}$. 3 verhindern den lateralen bodeninneren Fluss (RH) über Stauhorizonte. Dies hat zur Folge, dass im Lysimeter die gesamte Wassermenge, die infiltriert und nicht durch die Evapotranspiration aufgebraucht wird, als Gesamtdargebot den Lysimeterboden erreicht.

Beurteilung dieses Fehlers: Der dadurch entstehende Bilanzfehler ist gering, denn aufgrund des geringen Gefälles und der schlechten horizontalen Leitfähigkeiten erreichen die lateralen Wasserflüsse im Lockergesteinsbereich Mittel- und Norddeutschlands eine sehr geringe Reichweite. Nur wenn laterale Abflüsse im unmittelbaren Uferbereich eines Vorfluters $(<500$ m) entstehen, werden sie diesen auch erreichen (s. auch Kap. 4.1.3). 


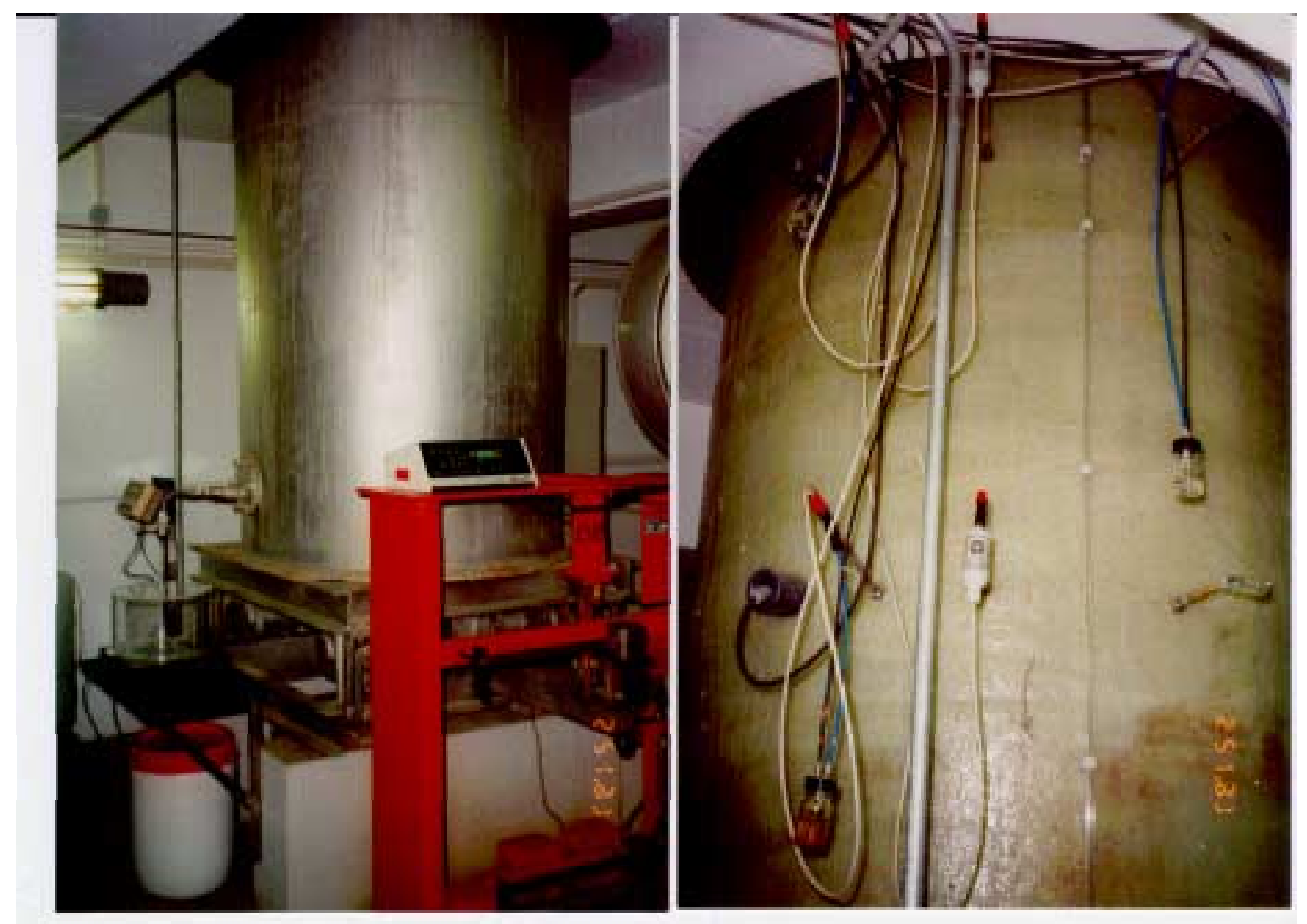

Abb. 3: Einzellysimeter mit Wägeeinrichtung, Sickerwasserauslauf, Saugkerzen, Tensiometern, TDR-Sonden und Sonden zur Bodenluftentnahme

- An der Sohle des Lysimeters wird die natürliche Bodenzone in ihrer vertikalen Ausdehnung unterbrochen und damit die Tiefenfunktion von Wassergehalt und Saugspannung verändert (SCHRÖDER, 1980; KLAGHOFER, 1991)

Beurteilung dieses Fehlers: Die Veränderungen, die hervorgerufen werden, sind um so kleiner, je tiefer das Lysimeter ist. Die ,ausreichende“ Lysimetertiefe resultiert aus den klimatischen Bedingungen, der Durchwurzelungstiefe der Pflanzen und den bodenphysikalischen Parametern. KLAGHOFER (1991) rechnet z. B. bei Sand mit $2 \mathrm{~m}$ und bei Löß mit $4 \mathrm{~m}$ erforderlicher Lysimetertiefe. Diese Aussagen werden im Ergebnis der Untersuchungen zur Ausschöpfungstiefe in Kap. 3.4.2 unter Berücksichtigung der Mehrschichtigkeit der Böden präzisiert.

Zur Vermeidung von Stauwassereinfluss bei nicht ausreichender Lysimetertiefe wird der Einsatz von Absaugvorrichtungen über dem Lysimeterboden (Unterdrucklysimeter) empfohlen (KLAGHOFER, 1991; ROTH et al., 1994), wobei der ,richtige“, am Lysimeter anzulegende Unterdruck durch zeitgleiche Freilandmessungen zu ermitteln ist.

\subsubsection{Technische Daten und Messprogramme}

Die Messreihen (1981-97) der Lysimeterstation Brandis werden im Rahmen dieser Arbeit erstmals zusammenfassend und themenübergreifend (zur Meteorologie, Bodenkunde, Landwirtschaft und Hydrologie) ausgewertet. Untersucht wird der Wasserhaushalt acht 
typischer Böden mit jeweils drei Wiederholungen (= 24 wägbare Lysimeter). Zur Verfügung standen Tageswerte der Wasserhaushaltsgrößen und Klimadaten, Monatswerte der Stickstoffgehalte im Niederschlag und Sickerwasser sowie Bodenkennwerte, Angaben zu Witterungserscheinungen und Bodennutzung (Vegetationsentwicklung, Düngung, agrotechnische Maßnahmen, Ernteerträge).

Auf Grund des erheblichen Umfanges des für die Auswertungen zur Verfügung stehenden Datenmaterials und der Länge des Beobachtungszeitraumes erscheint eine ausführliche Beschreibung des Stationsbetriebes (der von mir in den vergangenen 8 Jahren mit gestaltet werden konnte) erforderlich. So verweist dieses Kapitel auf die Konzeption, die dem Stationsbetrieb zu Grunde liegt und - mit Bezug zum vorstehende Kapitel - auf die Bemühungen zur Realisierung eines möglichst fehlerfreien Standortwasserhaushaltes in den Brandiser Lysimetern.

Die Station liegt 136 m über NN etwa $15 \mathrm{~km}$ südöstlich von Leipzig Abb. 1 am südlichen Ortsrand der Stadt Brandis (geographische Breite 51,32 ${ }^{\circ} \mathrm{N}$; geographische Länge $12,62^{\circ} \mathrm{E}$ ).

Es werden 24 wägbare Lysimeter sowie 19 alte Versickerungsmesser betreut, die bereits in den 60er Jahren an ihren Gewinnungsorten im Parthegebiet betrieben wurden. Die 19 Versickerungsmesser sind mit $205 \mathrm{~cm}$ und $265 \mathrm{~cm}$ unterschiedlich tief. Für die wägbaren Lysimeter wurden einheitlich Bodenprofile mit einer Mächtigkeit von $300 \mathrm{~cm}$ gewonnen. Jeder der 43 Bodenmonolithe hat eine Oberfläche von $1 \mathrm{~m}^{2}$. Die Messergebnisse der Versickerungsmesser werden im Rahmen dieser Arbeit nicht ausgewertet (s. hierzu KEESE \& KNAPPE, 1995).

Die Entnahme der Bodenmonolithe für die wägbaren Lysimeter erfolgte in den Jahren 197678, die kontinuierlich geführten Messreihen beginnen im November 1980 (MORITZ \& SÄMISCH \& SPENGLER, 1991). Bei der Gewinnung der Bodenmonolithe wurden von den Herkunftsflächen Profilbeschreibungen angefertigt und bodenphysikalische Parameter ermittelt (s. Kap. 2.1.4).

Für die Entnahme „ungestörter“ Monolithe wurde entsprechend der Methode nach FRIEDRICH-FRANZEN ein Stahlzylinder durch Auflast vorsichtig in den Boden gepresst. Gleichlaufend dazu entstand durch das Freigraben des eingetriebenen Behälters eine Baugrube, die es dann ermöglichte, den Lysimeterbehälter mit einer Bodenplatte zu verschließen und nach Brandis zu transportieren. Das Behältermaterial besteht aus verzinktem Stahlblech.

Die Entnahmeorte wurden so ausgewählt, dass an der Sohle der Lysimeter von Natur aus mindestens $50 \mathrm{~cm}$ gut durchlässiges Lockergestein ansteht. In einem Bereich von 10 bis 20 $\mathrm{cm}$ über der Sohle wurde horizontal ein geschlitztes, mit Glaswolle gefülltes, doppeltes Stahlfilterrohr eingebaut (Abb. 4). 


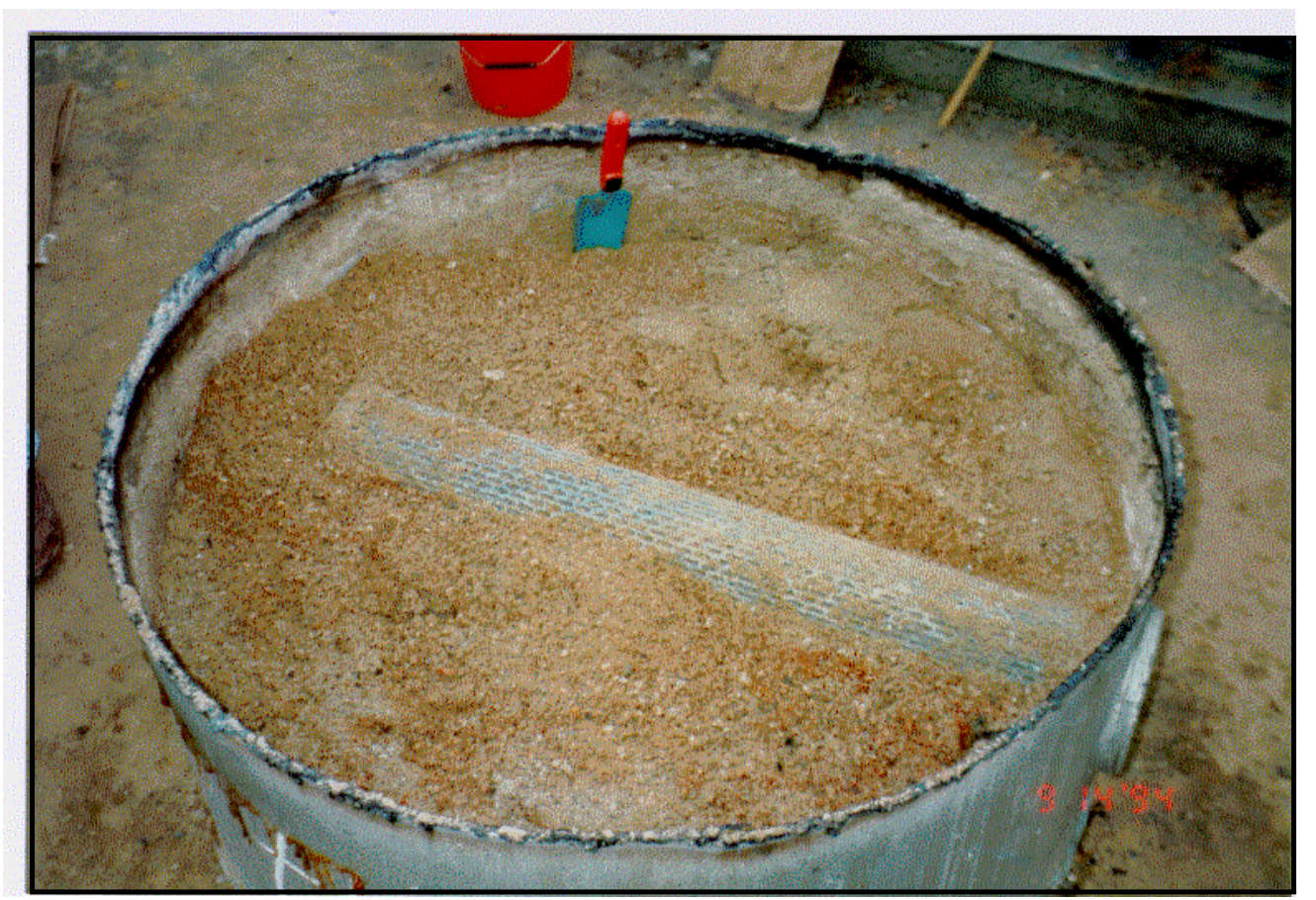

Abb. 4: Filterrohr für den Sickerwasserablauf am Lysimeterboden

Auf diese Weise wird ein ungestörtes Abfließen des im Bereich des Lysimeterbodens eintreffenden Sickerwassers ermöglicht und verhindert, dass Wasser aus dem Staubereich am Lysimeterboden wieder kapillar in die verdunstungsbeeinflusste Zone gehoben wird (s. Kap. 3.4.2).

In Anlehnung an die Richtlinien des DWD beginnt ein Messtag im Lysimeterkeller um 7.30 Uhr und endet um 7.30 Uhr des folgenden Tages.

Die Sickerwassermengen werden täglich ermittelt. Die Beprobung der Sickerwässer erfolgt in der Regel monatlich.

Durch die tägliche Wägung der Lysimeter wird die Gewichtsdifferenz zum Vortag mit einer Genauigkeit von $100 \mathrm{~g}=0,1 \mathrm{~mm}$ Wasser ermittelt. Seit 1995 erfolgt die Gewichtsaufnahme kontinuierlich mittels Druckmesszelle, wobei auch die Voraussetzung für die On-lineKopplung an einen PC gegeben ist.

Die Konstruktion der $230 \mathrm{~m}^{2}$ großen Kellerdecke erlaubte eine 1,2 m mächtige Kulturbodenüberdeckung und damit die gleiche Art der Bewirtschaftung wie auf den darin befindlichen Lysimetern (Verringerung der Randeffekte). Die Station und das Lysimeterfeld befinden sich inmitten einer landwirtschaftlichen Nutzfläche.

Zum Messprogramm auf dem Lysimeterfeld gehören Pflanzenbonituren und die jährliche Beprobung des oberen Bodenhorizontes. Das Lysimeterfeld dient darüber hinaus der Bestimmung der meteorologischen Eingangsgrößen und der Bodentemperaturen in verschiedenen Tiefen. Der Niederschlag wird täglich sowohl bodengleich als auch in einem HELLMANN-Messer in $1 \mathrm{~m}$ Aufstellhöhe ermittelt (s. Kap. 2.1.3.3). Die Standardwetterhütte und die Bodenthermometer wurden 1992 durch eine automatische Klimastation ersetzt, die alle verdunstungswirksamen Elemente in einem Zeitintervall von 10 Minuten auf data-Logger erfasst. Ergänzt wird das Messprogramm durch Wetterbeobachtungen und Messgeräte zur Ermittlung der atmosphärischen Deposition.

Die reale Evapotranspiration der 24 wägbaren Lysimeter wird nach Gl. 1 auf der Basis der täglichen Niederschläge, Sickerwassermengen und Gewichtsänderungen (= Bodenwasservorratsänderungen) berechnet (s. Kap. 2.3.4). 
In den sächsischen Wassereinzugsgebieten stellen landwirtschaftliche Nutzflächen prozentual den höchsten Flächenanteil. Deshalb wählte man für die Lysimeter und den umliegenden Schlag eine landwirtschaftliche Bewirtschaftung in ortsüblicher Weise (Tab. 1). Ziel dieser Strategie sollte sein

- Schaffung gebietstypischer Bedingungen für die Grundwasserneubildung und die reale Verdunstung,

- Nachweis der Effizienz der mineralischen Düngung und der Stickstoff-Entzüge durch die Pflanzen,

- Ermittlung der potentiellen Stickstoff-Auswaschung verschiedener Böden und der Gefahr für das Grundwasser.

Um zu vergleichbaren Aussagen zu gelangen, wurden alle Lysimeter bzw. Bodenarten gleich behandelt. Aus versuchstechnischen Gründen wurde bis 1997 generell mineralischer Dünger eingesetzt. Eine Unterbrechung des in Tab. 1 aufgezeigten Stickstoff-Düngungsregimes erfolgte nur auf den Lysimetern mit pseudovergleyter Braunerde-Fahlerde. Hier kam in den Jahren von 1982 bis 1990 auch Gülle zum Einsatz. Dabei wurde die Düngermenge in Mineraldüngeäquivalenten der jährlichen Mineraldüngung der anderen Lysimeterböden angeglichen.

Die landwirtschaftliche Bearbeitung, Bestellung und Ernte des Lysimeterfeldes wurde mit Kleintraktor, Anhängegeräten und Kleintechnik durchgeführt. Die Bewirtschaftung der Lysimeter selbst erfolgte per Hand. Für alle Feldarbeiten auf den Herkunftsflächen wurde seit 1978 übliche Landtechnik eingesetzt.

Im Unterschied $\mathrm{zu}$ den Herkunftsflächen war es auf den Lysimetern selten notwendig, Pflanzenschutzmittel (PSM) einzusetzen, da Bestände manuell gepflegt wurden. Die Liste der eingesetzten PSM liegt in der Station Brandis vor.

Im Herbst 1992 wurde in Zusammenarbeit mit dem Umweltforschungszentrum Leipzig-Halle und der Universität Halle-Wittenberg das beschriebene Bewirtschaftungsregime beendet. Nach Flächenstilllegungsmaßnahmen in den Jahren 1993-95 (selbstbegrünte Brache nach Stoppelsturz im September 1992; April 1993 Saatbettbereitung und Aussaat einer Mischung verschiedener Gräser; mehrere Schnitte; im Frühjahr 1995 Umbruch und Aussaat von Rotklee) wurde 1996 auf den Lysimetern und dem umliegenden 0,66 ha großen Lysimeterfeld mit einer „umweltgerechten, nachhaltigen feldbaulichen Nutzung“ begonnen. Zielstellung ist ein „optimaler Flächenertrag ohne (bzw. mit geringer) Umweltbelastung“ - eine Bewirtschaftung nach den Prinzipien des geschlossenen Stoffkreislaufes eines Landwirtschaftsbetriebes (Ökologischer Landbau). Im Einzelnen heißt das:

- ökologisch vertretbares, standortbezogenes Düngeniveau, keine synthetischen Düngemittel,

- Einsatz von Wirtschaftsdünger, Gründüngung und Leguminosen,

- Ausschaltung der Unkrautkonkurrenz durch mechanische Pflegearbeiten, Einsatz von biologischen Pflanzenschutzmitteln, kein Einsatz von synthetischen Pflanzenschutzmitteln,

- Wahl einer geeigneten Fruchtfolge zur Minimierung des N-Austrages.

Seit 1999 wird auf Grundlage der Empfehlungen der Sächsischen Landesanstalt für Landwirtschaft zur „Umweltgerechten Landwirtschaft in Sachsen“ bewirtschaftet (SML, 1995). 
Tab. 1: Fruchtfolge, Stickstoff-(N)-Einträge und Niederschläge der Jahre 1980-98

\begin{tabular}{|llccc|}
\hline $\begin{array}{l}\text { Kalender- } \\
\text { jahr }\end{array}$ & $\begin{array}{l}\text { Fruchtart } \\
\text { (Lysimeter } \\
\text { und Feld) }\end{array}$ & $\begin{array}{c}\text { mineralische } \\
\text { N-Düngung } \\
{[\mathrm{kg} / \mathrm{ha} / \mathrm{a}]}\end{array}$ & $\begin{array}{c}\text { N-Immission als } \\
\text { nasse Deposition } \\
{[\mathrm{kg} / \mathrm{ha} / \mathrm{a}]}\end{array}$ & $\begin{array}{c}\text { Niederschlag } \\
\text { (n.HELLMANN) } \\
\text { [mm/a] }\end{array}$ \\
\hline 1980 & Mais & 140 & 44 & 657 \\
1981 & Zuckerrüben & 160 & 53 & 727 \\
1982 & Winterweizen & 120 & 28 & 390 \\
1983 & Wintergerste & 120 & 33 & 672 \\
1984 & Weidelgras & 175 & 42 & 536 \\
1985 & Kartoffel & 100 & 69 & 477 \\
1986 & Winterweizen & 120 & 35 & 581 \\
1987 & Kartoffel & 100 & 37 & 629 \\
1988 & Winterweizen & 140 & 46 & 574 \\
1989 & Wintergerste & 120 & 46 & 546 \\
1990 & Zuckerrüben & 140 & 44 & 579 \\
1991 & Winterweizen & 140 & 37 & 417 \\
1992 & Wintergerste & 120 & 29 & 583 \\
Mittel der Jahre 1980-92 & $\mathbf{1 3 0}$ & $\mathbf{4 1}$ & $\mathbf{5 6 8}$ \\
1993 & Grünbrache & 0 & 26 & 669 \\
1994 & Grünbrache & 0 & 32 & 660 \\
1995 & Rotklee & 0 & 18 & 664 \\
1996 & Kartoffel & 0 & 19 & 500 \\
1997 & Sommerweizen & 60 (Stalldung)* & 22 & 505 \\
1998 & Winterroggen & 0 & 35 & 691 \\
\hline
\end{tabular}

$* \cong 200 \mathrm{dt} / \mathrm{ha}$

Für einen Vergleich mit der Praxis sind die Hektarerträge der einzelnen Kulturpflanzen (mit dem Hinweis auf den hohen Grad der Pflege und die geringen Ernteverluste auf den Lysimetern) zu korrigieren. So empfiehlt z. B. GLUGLA (1993), bei der Übertragung von Ernteerträgen auf landwirtschaftliche Schläge für Getreide minus $5 \%$, für Feldgras, Grünland und Hackfrüchte minus $10 \%$ der Lysimetererträge anzusetzen.

In Bezug auf die Pflanzenentwicklung und Bestandesdichte auf den Brandiser Lysimetern konnte durch die phänologischen Beobachtungen von SÄMISCH (1990) allerdings eine weitgehende Übereinstimmung von Lysimeter und Vergleichsfläche festgestellt werden. Weitere Hinweise liefern Vergleiche der Ernteergebnisse zwischen Lysimeter und Freiland.

In Anlage 1 sind für alle Lysimetergruppen die Stickstoffentzüge durch die jeweiligen Kulturpflanzen und die jährlich über das Sickerwasser ausgetragenen Stickstofffrachten zusammengestellt.

Die Bemühungen zur Realisierung eines möglichst fehlerfreien Standortwasserhaushaltes in den Brandiser Lysimetern lassen sich wie folgt zusammenfassen:

- Untersuchung von acht für Mitteldeutschland aus bodenkundlicher und hydrogeologischer Sicht repräsentativer Ackerstandorte mittels wägbarer Lysimeter in jeweils 3 Wiederholungen;

- Über der Bodenplatte der Lysimeter befindet sich stets gut durchlässiges kiesiges Material mit geringem Kapillarhub. Auf diese Weise soll ausgeschlossen werden, dass sich verdunstungsbeeinflusste Zone und Kapillarwasserzone über der Bodenplatte des Lysimeters gegenseitig beeinflussen. Ziel ist, mit den 3 m tiefen Lysimetern grundwasserferne Standorte zu simulieren, da diese repräsentativ für den mitteldeutschen Lockergesteinsbereich sind. 
- Entsprechend der seit dem Bau der Station bestehenden Aufgabenstellung, Böden unter landwirtschaftlicher Nutzung untersuchen zu wollen, werden sowohl die Lysimeterböden als auch das umliegende Feld gleichartig dieser Nutzungsform unterzogen. Der oft diskutierte „Oaseneffekt" soll damit vermieden werden.

- Für ein flächenadäquates Pflanzenwachstum darf die Lysimeteroberfläche nicht kleiner als $1 \mathrm{~m}^{2}$ sein, für Hackfrüchte besser $2-3 \mathrm{~m}^{2}$ (GLUGLA, 1998). Doch auch auf $1 \mathrm{~m}^{2}$ konnte bisher bei jeder der angebauten Pflanzenarten der übliche Reihen- und Pflanzenabstand eingehalten werden (z. B. 4 - 5 Knollen bei Kartoffeln und $9-10$ Pflanzen bei Zuckerrïben).

- Wichtig ist, dass zwischen der Bodentemperatur im Freiland und im Lysimeterboden keine großen Differenzen bestehen. Dies wird weitgehend dadurch erreicht, dass die Kellerdecke ca. 1,2 $\mathrm{m}$ unter der Erdoberfläche liegt und der Spalt zwischen den Lysimetern und der sie umgebenden Führung so klein wie möglich gehalten wird. Ein Vergleich zwischen der Lufttemperartur im Lysimeterkeller und den in verschiedenen Tiefen gemessenen Bodentemperaturen (im Klimagarten unter Gras) zeigt, dass eine temperaturbedingte Beeinflussung der Bodenmonolithe nahezu auszuschließen ist. Die Kellertemperatur verläuft synchron zur Bodentemperatur in $1 \mathrm{~m}$ Tiefe $A b b$. 5. In den Sommermonaten ist sie niedriger als diese und in den Wintermonaten höher, liegt dann aber noch unter der Bodentemperatur in 2,5 m Tiefe. Im Frühjahr und Herbst sind Kellertemperatur und Bodentemperatur in $1 \mathrm{~m}$ Tiefe fast identisch.

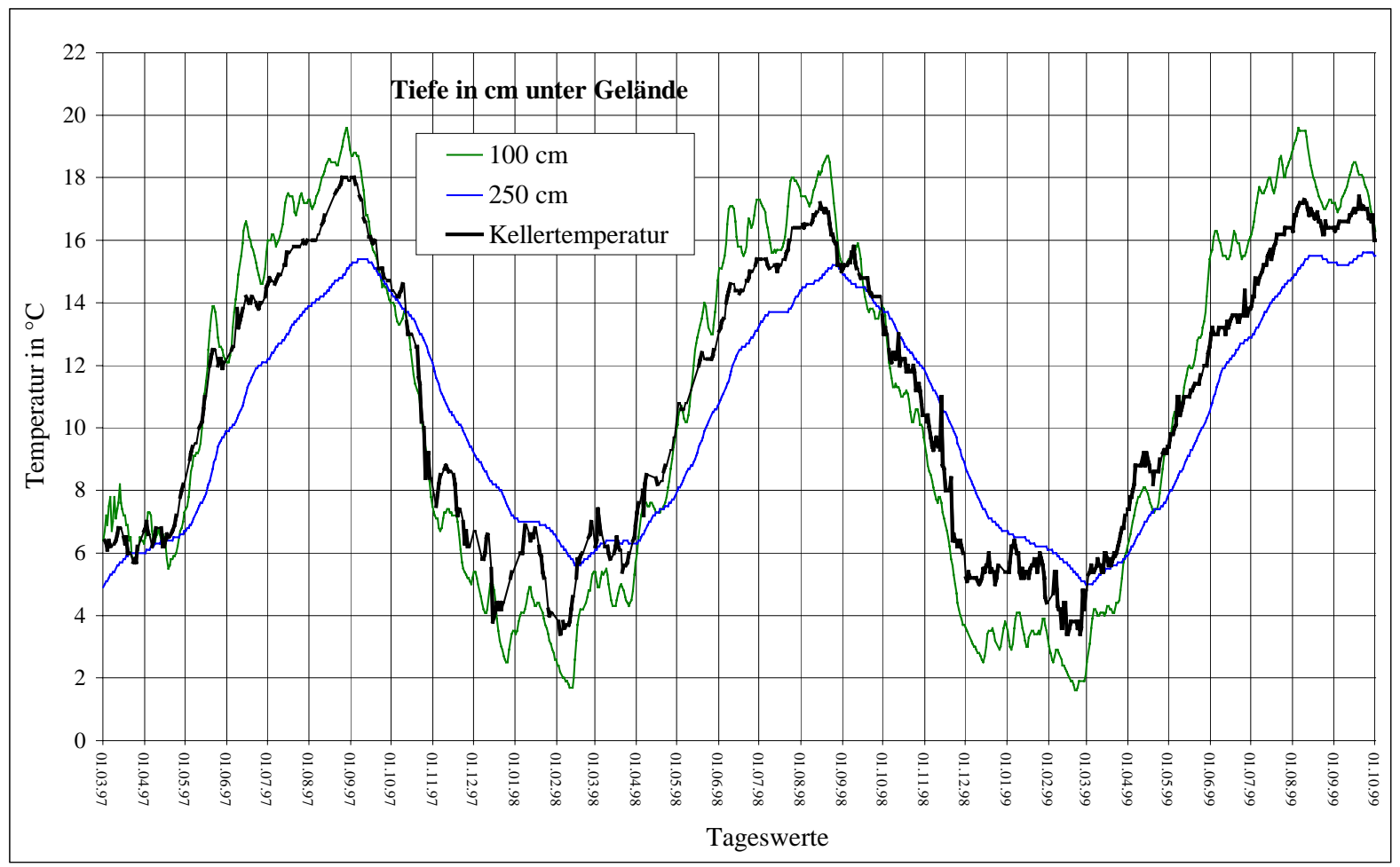

Abb. 5: Vergleich der in verschiedenen Tiefen gemessenen Bodentemperaturen mit der im Lysimeterkeller gemessenen Lufttemperatur am Beispiel der Jahre 1997-99 (Tageswerte)

- Alle Bodenmonolithe sind, wie für Altpleistozänlandschaften typisch, mehrschichtig Tab. \$). Es handelt sich um ungestört gewonnene Bodenmonolithe. So gehen die schwer nachzubildenden Heterogenitäten eines Bodens, wie

$\Rightarrow$ Wurzelgänge, 
$\Rightarrow$ Hohlräume infolge der Tätigkeit höherer Lebewesen,

$\Rightarrow$ Schrumpfrisse,

$\Rightarrow$ Horizonte, die u. a. durch Stoffverlagerungsprozessen entstande sind

$\Rightarrow$ und geologische Schichtgrenzen,

die den Wasser- und Stoffhaushalt wesentlich beeinflussen, nicht verloren.

- Als Basis für die Einbindung der Lysimeterergebnisse in den Gebietswasserhaushalt werden im Einzugsgebiet der Parthe (KEESE et al., 1996) Wasserhaushaltsuntersuchungen durchgeführt (s. auch Kap. 4.1).

Tensiometer, Saugkerzen, TDR-Sonden, Temperaturfühler und Sonden zur Entnahme von Bodenluft (s. Abb. 3 in jeweils drei Ebenen im Lysimeter liefern zusätzliche Informationen über die im Bodenprofil ablaufenden Prozesse. Die Auswertung der seit 1996 vorliegenden Messreihen ist jedoch nicht Gegenstand dieser Arbeit. Gründe dafür sind:

1. Die Instrumente liefern Messwerte einer anderen Maßstabsebene (Lysimeter = makroskopische Ebene; Tensiometer, Saugkerzen und TDR-Sonden = mikroskopische Ebene). In den teils sehr heterogenen Böden ist damit zu rechnen, dass Infiltrationsereignisse zur Aktivierung bevorzugter Fließpfade führen, deren Erfassung mit der o. g. Messtechnik zum Zufallsereignis wird. Die eingebauten Messgeräte werden u. a. zur Kalibrierung von Modellen verwendet.

2. Eine Erweiterung des auszuwertenden, ohnehin sehr umfangreichen Datenmaterials sollte nicht erfolgen.

Nach der langjährigen Nutzung der Lysimetermonolithe ergab sich die Frage, inwieweit Oaseneffekte, nutzungs- und lysimeterspezifische Einflüsse zu Veränderungen im Boden und damit zu Problemen bei der Interpretation und Verwendung der Lysimeterergebnisse führen. Deshalb wurden 1994 drei der langjährig genutzte Lysimetermonolithe zerlegt. Parallel dazu wurden auf den Herkunftsflächen der Lysimeterböden Profile aufgenommen und mit gleichem Untersuchungsprogramm analysiert (KEESE \& KNAPPE, 1996).

Die 1994 durchgeführten Untersuchungen zeigten u. a., dass:

$\rightarrow$ die Möglichkeit eines bevorzugten Sickerwasserablaufes entlang der Lysimeterwände OLBERTZ (1957) weitgehend ausgeschlossen werden kann, was bereits durch LIEBSCHER (1970) bestätigt wurde.

$\rightarrow$ die zur Herkunftsfläche abweichende Form der landwirtschaftlichen Bewirtschaftung, d. h. die jahrelange Bearbeitung per Hand im Lysimeter im Unterschied zur Bewirtschaftung mit schwerer Technik auf den Herkunftsflächen nur im bewirtschafteten Bodenhorizont Veränderungen in Bezug auf Lagerungsdichte, Durchwurzelung, Belüftung und biogene Aktivität bewirkt. Allerdings ist noch der Nachweis zu erbringen, wie signifikant sich diese Veränderungen tatsächlich auf den Bodenwasserhaushalt auswirken.

$\rightarrow$ Das zerlegte Lysimeter 7-6 war in Bezug auf Sickerwasserrate und Höhe der Verdunstung ein „Ausreißer“ (vgl. Tab. 41 in Kap. 4.1.1). Das abweichende Verhalten konnte eindeutig auf natürliche Inhomogenitäten der Herkunftsfläche zurückgeführt werden (KEESE et al., 1997). Keines der drei zerlegten Lysimeter zeigte messtechnisch bedingte Fehler am Lysimeterauslauf, wie zunächst vermutete wurde. 


\subsubsection{Zur Konsistenz und Homogenität der Messreihen}

\subsubsection{Homogenitätsprïfung}

Vor Beginn der Auswertung musste geprüft werden, ob die Messreihen (Tageswerte von Niederschlag, Sickerwasser und Gewichtsänderung) konsistent und homogen sind.

Konsistent sind die Daten, wenn sie nicht durch Schreib- und Ablesefehler oder Gerätefehler beeinflusst sind. Fehler dieser Art werden in der Regel bei der Berechnung der Verdunstung entdeckt und korrigiert. Besondere Vorsorge wird durch regelmäßiges Eichen der Waagen getroffen. Problematisch sind die Fehler, die bei der Ermittlung des Niederschlages auftreten (zur Beschreibung der Korrektur der Niederschlagsmesswerte s. Kap. 2.1.3.3).

Kann von der Konsistenz aller Messreihen ausgegangen werden, muss sich eine Homogenitätsprüfung anschließen. Ziel dieser Untersuchungen ist es, festzustellen, ob eine dauerhafte Beeinflussung des untersuchten Regimes (hier Wasserhaushalt der Lysimeterböden) vorliegt. Eine Inhomogenität der Messreihen liegt vor, wenn u. a. Baumaßnahmen oder Bepflanzungen im Umfeld der Lysimeter $\mathrm{zu}$ Veränderungen der klimatischen Bedingungen führten, ein Nutzungswechsel erfolgte oder z. B. fortschreitende Verockerung den Sickerwasserauslauf behindert. Wird eine Beeinflussung festgestellt, gilt es die Dauer und die Art der Beeinflussung festzustellen. Ein Verfahren, mit dem diese Fragen beantwortet werden können, ist die Doppelsummenanalyse (HANSEL \& SCHÄFER, 1970). Eine Doppelsummenkurve ergibt sich, wenn die fortlaufenden Werte (hier Monatswerte) einer zu prüfenden Beobachtungsreihe (hier Verdunstung oder Sickerwasser) gegen die im gleichen Zeitrhythmus aufsummierten Werte einer Bezugsreihe (hier Niederschlag) aufgetragen werden. Bei ausreichender Korrelation der beiden Reihen lässt sich im linearen Maßstab die Doppelsummenkurve als Gerade anpassen. Ist eine Veränderung in der Steigung der Geraden erkennbar, ist die Zeitreihe inhomogen. So bewirkt das Vorhandensein eines Trends eine Krümmung, ein plötzlicher einmaliger Eingriff (z. B. das Fällen aller Bäume auf einem Waldlysimeter) verursacht einen Knick in der Doppelsummenkurve (SCHRÖDER, 1980).

Die Homogenitätsprüfung erfolgte für alle 49 Messreihen $(24 *$ Sickerwasser, $24 *$ Verdunstung, $1 *$ Niederschlag). Im Ergebnis der Untersuchungen zeigte sich, dass sich alle Summenlinien mit einem Bestimmtheitsmaß von $99 \%$ durch eine Gerade anpassen ließen. Damit kann von der Homogenität der Messreihen ausgegangen werden. Abb. 5 zeigt die Doppelsummenkurven am Beispiel der Lysimetergruppen 5 und 10. Die Unterschiede, die sich in Bezug auf die Sickerwassermenge und die Höhe der Verdunstung bei den drei Lysimetern einer Herkunftsfläche (= Lysimetergruppe) zeigen, resultieren aus der Heterogenität der jeweiligen Herkunftsfläche.

Somit kann von der Konsistenz und Homogenität der Messreihen ausgegangen werden. Infolge der unterschiedlichen Bestandsentwicklung auf den einzelnen Lysimetern existiert aber noch eine Zufallsgröße, deren Einfluss nur beschrieben, aber nicht korrigiert werden kann. Der Einfluss, den diese Einflussgröße auf die Genauigkeit der Messreihen insbesondere die der Verdunstung nimmt, wird im nachfolgenden Kapitel beschrieben. 

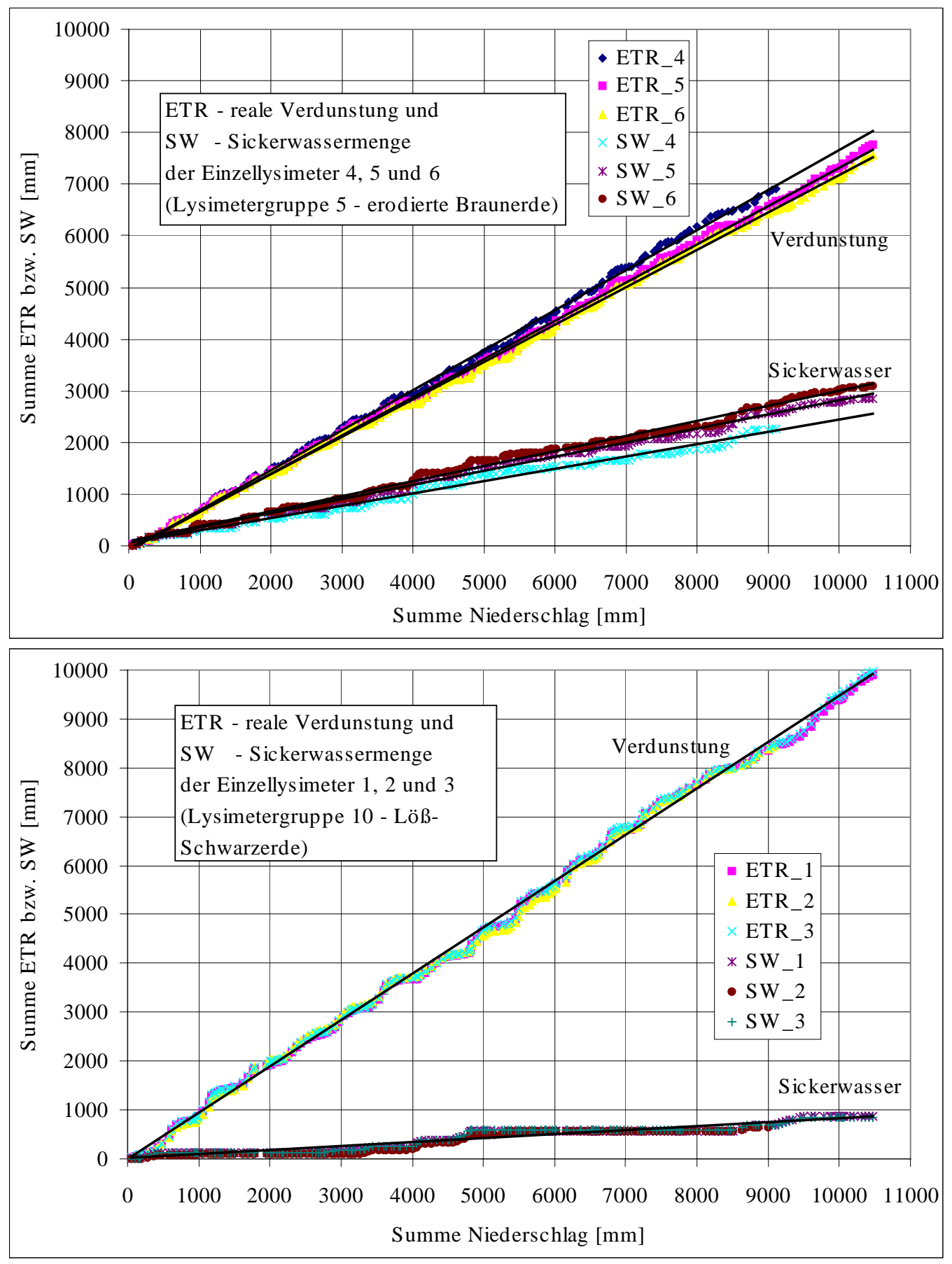

Abb. 6: Doppelsummenanalyse am Beispiel der Lysimetergruppe 5 (erodierte Braunerde) und Lysimetergruppe 10 (Löß-Schwarzerde) auf Basis der Monatswerte der Jahre 1981-97

\subsubsection{Einfluss der unterschiedlichen Bestandesentwicklung auf die Verdunstung}

Hinsichtlich der Repräsentativität der mittels Lysimeter ermittelten Verdunstung ist sicherzustellen, dass Pflanzenentwicklung, Bestandsdichte und Höhe der Erträge mit denen auf den Herkunftsflächen weitgehend übereinstimmen.

Damit die Zufallsgröße „Bestandsentwicklung“ möglichst gering ist, sollten Pflanzenentwicklung und Bestandsdichte der jeweils drei Lysimeter mit gleichem Boden keine wesentlichen Unterschiede aufweisen. Es wurde angestrebt, dies durch sorgfältige Bewirt- 
schaftung zu gewährleisten, d. h., alle Bearbeitungs- und Pflegemaßnahmen wurden völlig gleichartig durchgeführt. Trotzdem zeigte sich infolge kleinräumiger Bodenunterschiede sowie unterschiedlicher Vegetationsentwicklung und Reaktion der Pflanzenbestände auf Krankheiten und Schädlingsbefall eine Abweichung der Einzelwerte der Verdunstung vom Gruppenmittel. Zur Beschreibung dieser zufälligen Reststreuung (als Ausdruck der Versuchsgenauigkeit) wurde für alle Lysimetergruppen die Standardabweichung der Dekaden- und Monatswerte von 1981-97 berechnet, wobei diese erwartungsgemäß bei allen Böden einen Jahresgang zeigt (Tab. 2). Die Standardabweichung der Dekadenwerte ist höher als die der Monatswerte. Ein Zusammenhang zwischen Bodentyp und Höhe der Streuung der Verdunstung der Einzellysimeter ist nicht zu erkennen.

Tab. 2: Standardabweichung der Verdunstung der Einzellysimeter vom Gruppenmittel [mm/Monat] als mittlerer Jahresgang

\begin{tabular}{|c|cccccc|cccccc|c|}
\hline $\begin{array}{c}\text { Monat } \\
\text { Lys.-Gr. }\end{array}$ & $\mathbf{1 1}$ & $\mathbf{1 2}$ & $\mathbf{1}$ & $\mathbf{2}$ & $\mathbf{3}$ & $\mathbf{4}$ & $\mathbf{5}$ & $\mathbf{6}$ & $\mathbf{7}$ & $\mathbf{8}$ & $\mathbf{9}$ & $\mathbf{1 0}$ & Jahr \\
\hline $\mathbf{5 - D 3}$ & 1,4 & 1,9 & 1,6 & 1,5 & 2,7 & 2,5 & 5,8 & 6,0 & 4,5 & 2,7 & 2,5 & 1,5 & $\mathbf{2 , 9}$ \\
\hline $\mathbf{4}-\mathbf{D 5}$ & 1,4 & 1,3 & 1,1 & 1,4 & 1,8 & 2,8 & 4,2 & 4,7 & 4,2 & 3,2 & 2,1 & 1,3 & $\mathbf{2 , 5}$ \\
$\mathbf{8}-\mathbf{D 3}$ & 1,4 & 1,1 & 1,5 & 1,6 & 1,7 & 1,8 & 5,0 & 8,1 & 7,3 & 3,3 & 2,5 & 1,6 & $\mathbf{3 , 1}$ \\
\hline $\mathbf{1 - D 6}$ & 1,2 & 1,1 & 1,6 & 1,1 & 2,0 & 3,3 & 6,6 & 7,5 & 6,1 & 3,5 & 2,3 & 2,3 & $\mathbf{3 , 2}$ \\
$\mathbf{7 - D 4}$ & 1,3 & 1,3 & 1,8 & 2,4 & 4,0 & 5,2 & 8,7 & 7,9 & 5,5 & 4,3 & 2,7 & 2,5 & $\mathbf{4 , 0}$ \\
$\mathbf{1 1}-\mathbf{D 3}$ & 1,6 & 1,4 & 2,2 & 2, & 1,6 & 4,7 & 6,3 & 5,9 & 3,6 & 4,4 & 2,5 & 2,1 & $\mathbf{3 , 2}$ \\
\hline $\mathbf{9}-\mathbf{L} \ddot{3} 3$ & 0,9 & 1,0 & 1,8 & 1,3 & 2,1 & 1,7 & 4,4 & 3,6 & 3,4 & 2,3 & 2,5 & 1,8 & $\mathbf{2 , 2}$ \\
\hline $\mathbf{1 0}-\mathbf{L} \ddot{\mathbf{o 1}}$ & 1,8 & 1,6 & 1,9 & 2,1 & 1,5 & 2,9 & 7,9 & 11,3 & 7,3 & 5,6 & 3,5 & 1,8 & $\mathbf{4 , 1}$ \\
\hline
\end{tabular}

Zu Lysimetergruppe, Bodenform und Standorteinheit (D3-D6, Lö1, Lö3) s. Tab. 6.

Eine Beeinflussung der Verdunstung infolge der Lysimetertechnik, z. B. durch den „Blumentopfeffekt“ oder eine bessere Wasserversorgung der Pflanzen infolge kapillaren Aufstieges von Stauwasser bei zu flachen Lysimetern, ist im Ergebnis der in Kap. 3.4.2 geführten Diskussion zur Lysimetertiefe nicht zu vermuten.

\subsubsection{Niederschlagskorrektur}

Die Niederschlagsmessung hat bereits eine lange Tradition, wobei die Methodik der Niederschlagsmessung seit rd. 2000 Jahre prinzipiell unverändert geblieben ist (RODDA, 1971). So kann die Erfassung der Niederschlagsmengen auch heute trotz vielfältiger zusätzlicher Messtechnik nicht fehlerfrei durchgeführt werden. Wesentliche Ursachen hierfür sind (DISSE, 1995):

- Deformationen des Windfeldes in der Höhe der Sammelgefäße (mögliche Fehlergröße je nach Stationslage: $2 \%$ bis $10 \%$ bei Regen und $15 \%$ bis $35 \%$ bei Schnee)

- Benetzungsverdunstung (5\% bis $10 \%$ Fehler)

- Verdunstung aus dem Sammelgefäß (1 \% bis $3 \%$ Fehler).

Diese Fehler addieren sich und haben als Verluste einen erheblichen Einfluss auf die Höhe der realen Verdunstung, da diese als Restgröße mittels der Wasserhaushaltsgleichung berechnet wird. Erfolgt die Messung des Niederschlages in Erdbodenhöhe, reduzieren sich die Messfehler in den Sommermonaten auf Benetzungs- und Verdunstungsverluste.

In Brandis wird der Niederschlag bodengleich (für die Wasserhaushaltsbilanzen der Lysimeter) und in $1 \mathrm{~m}$ Höhe (zur Anbindung an das Messnetz des DWD) gemessen. Beide 
Niederschlagsreihen müssen nach RICHTER (1995) korrigiert werden. Dabei war es von Vorteil, dass in der Vergangenheit neben der Niederschlagsmenge auch Witterungserscheinungen und die Niederschlagsart notiert wurden. Die Korrektur aller Tageswerte beider Niederschlagsreihen erfolgte 1997 im Rahmen eines DFG-Projektes (BfG 1997) durch den DWD in Potsdam (Tab. 3):

1. Korrektur der in $1 \mathrm{~m}$ Höhe gemessenen Niederschläge beim DWD nach RICHTER (1995). Bei diesem Verfahren werden Wind-, Benetzungs- und Verdunstungsfehler zusammengefasst und in Abhängigkeit von der Niederschlagshöhe betrachtet. Für die Niederschlagsstationen erfolgt entsprechend ihrer Windexposition eine Einteilung in vier Gruppen von „frei“, „leicht geschützt“, „mäßig geschützt“ bis „,stark geschützt“”. Des weiteren berücksichtigen die Korrekturfaktoren einen innerjährlichen Gang und die Art des Niederschlages wie Regen, Mischniederschlag und Schnee. Die Station Brandis wurde bezüglich der Windexposition als Standort in „leicht geschützter Lage“ eingestuft.

2. Korrektur der Benetzungs- und Verdunstungsverluste beim bodengleichen Niederschlag in Abhängigkeit von der Jahreszeit und von der Art des Niederschlages (flüssig, fest oder Mischniederschlag) erfolgt durch Addition von Korrekturwerten (Tab. 8).

Tab. 3: Korrekturwerte des bodengleichen Niederschlages nach RICHTER (1995)

\begin{tabular}{|c|c|c|c|c|c|}
\hline \multirow{2}{*}{\multicolumn{2}{|c|}{$\begin{array}{c}\text { Sommer } \\
\text { (April bis September) }\end{array}$}} & \multicolumn{4}{|c|}{$\begin{array}{c}\text { Winter } \\
\text { (Oktober bis März) }\end{array}$} \\
\hline & & \multicolumn{2}{|c|}{ Niederschlagsart 4 oder 8} & \multicolumn{2}{|c|}{ Niederschlagsart 7} \\
\hline $\begin{array}{l}\text { Niederschlags- } \\
\text { klasse }\left[\mathrm{mm} \mathrm{d}^{-1}\right]\end{array}$ & $\begin{array}{c}\text { Korrektur } \\
{\left[\mathrm{mm} \mathrm{d}^{-1}\right]}\end{array}$ & $\begin{array}{l}\text { Niederschlags- } \\
\text { klasse }\left[\mathrm{mm} \mathrm{d}^{-1}\right]\end{array}$ & $\begin{array}{l}\text { Korrektur } \\
{\left[\mathrm{mm} \mathrm{d}^{-1}\right]}\end{array}$ & $\begin{array}{l}\text { Niederschlags- } \\
\text { klasse }\left[\mathrm{mm} \mathrm{d}^{-1}\right]\end{array}$ & $\begin{array}{l}\text { Korrektur } \\
\text { [mm d-1] }\end{array}$ \\
\hline $0,1-0,4$ & $+0,1$ & $0,2-1,7$ & $+0,1$ & $\geq 0,5$ & $+0,1$ \\
\hline $0,5-1,7$ & $+0,2$ & $1,8-6,7$ & $+0,2$ & & \\
\hline $1,8-4,4$ & $+0,3$ & $\geq 6,8$ & $+0,3$ & & \\
\hline $4,5-8,7$ & $+0,4$ & & & & \\
\hline$\geq 8,8$ & $+0,5$ & & & & \\
\hline
\end{tabular}

Ergänzung:

\begin{tabular}{cl} 
Schlüsselziffer & Niederschlagsart \\
\hline 0 & kein Niederschlag \\
4 & flüssiger Niederschlag \\
7 & fester Niederschlag \\
8 & Mischniederschlag
\end{tabular}

3. Weitere Niederschlagskorrekturen erfolgten unter Berücksichtigung der täglichen Lysimeterwägungen insbesondere in den Wintermonaten. Diese Korrekturen wurden im Rahmen des o. g. DFG-Projektes in der Bundesanstalt für Gewässerkunde, Außenstelle Berlin, (JANKIEWCZ, 1998) ausgeführt. Im Ergebnis der nachfolgend beschriebenen Handlungen ergaben sich teils unterschiedliche Niederschlagshöhen für die einzelnen Lysimeter:

- Vorrangig bei festen Niederschlägen können aufgrund der räumlichen Entfernung der Niederschlagsmessung vom Lysimeter Abweichungen zwischen gemessenem, korrigiertem Niederschlag und der tatsächlich das Lysimeter erreichenden Wassermenge auftreten. Unter der Annahme, dass die Messung der Bodenwasservorratsänderung mit größter Sorgfalt erfolgte, wurde diese als maßgebliche Messgröße zur Niederschlagsermittlung verwendet. 
- An Schneedeckentagen wird deshalb der bereits korrigierte Niederschlag noch um den Parameter „Schneeumlagerung“ ergänzt. Damit wird gewährleistet, dass der am Ende einer Schneedeckenperiode aufsummierte gemessene Niederschlag einschließlich positiver und negativer Schneeumlagerung der Wassermenge entspricht, die beim Tauprozess in den Boden des Lysimeters gelangt.

- Auch an schneefreien Tagen mit Niederschlag, an denen eine negative Verdunstung errechnet wurde und die positive Bodenwasservorratsänderung am Lysimeter bis zu $5 \mathrm{~mm}$ (Wert aus Daten abgeleitet) über dem korrigierten Niederschlag liegt, wird der korrigierte Niederschlag grundsätzlich auf den Wert der positiven Bodenwasservorratsänderung gesetzt. Die im Niederschlagsmesser erfasste Wassermenge wird demnach als zu niedrig angesehen.

Zwischen unkorrigiertem Niederschlag nach HELLMANN in $1 \mathrm{~m}$ Höhe $(572 \mathrm{~mm})$ und korrigiertem Niederschlag $(655 \mathrm{~mm})$ wurde ein jährlicher Unterschied von rd. $12 \%$ ermittelt (Tab. 4).

Tab. 4: Unkorrigierter und korrigierter Niederschlag [mm/a] am Standort Brandis (1981-97)

\begin{tabular}{|c|c|c|c|c|c|c|c|c|c|}
\hline & \multicolumn{3}{|c|}{ Niederschlag (HELLMANN-Messung) } & \multirow{2}{*}{\multicolumn{6}{|c|}{$\begin{array}{c}\text { Korrigierter Niederschlag } \\
\text { der einzelnen Lysimetergruppen: }\end{array}$}} \\
\hline & \multicolumn{2}{|c|}{ unkorrigiert } & \multirow[t]{2}{*}{ korrigiert } & & & & & & \\
\hline & 1m Höhe & bodengleich & & $\begin{array}{ccc}5 & 4 & 8 \\
\text { D3 } & \text { D5 } & \text { D3 }\end{array}$ & $\begin{array}{c}1 \\
\text { D6 }\end{array}$ & $\begin{array}{c}7 \\
\text { D4 }\end{array}$ & $\begin{array}{l}11 \\
\text { D3 }\end{array}$ & $\begin{array}{c}9 \\
\text { Lö3 }\end{array}$ & $\begin{array}{l}10 \\
\text { Lö1 }\end{array}$ \\
\hline Winter & 259 & 283 & 302 & & & & & & \\
\hline Sommer & 313 & 332 & 353 & & & & & & \\
\hline Jahr & 572 & 615 & 655 & $652655 \quad 655$ & 652 & 656 & 656 & 659 & 659 \\
\hline
\end{tabular}

Zu Lysimetergruppe, Bodenform und Standorteinheit (D3-D6, Lö1, Lö3) s. Tab. 6.

Der Unterschied zwischen bodengleichem Niederschlag $(615 \mathrm{~mm})$ und korrigiertem Niederschlag beträgt rd. $6 \%$. Die Korrekturen von JANKIEWICZ (1998) führten mit max. 2 $\%$ zu einer weiteren jährlichen Erhöhung der Niederschlagswerte. Diese weitere Erhöhung ist gering, aber in ihrer zeitlichen Auflösung für die richtige Berechnung der täglichen Verdunstungswerte von Bedeutung. Da für bisherige Auswertungen der Lysimetermessungen stets der unkorrigierte bodengleiche Niederschlag verwendet wurde, muss auf entsprechende Abweichungen zu bisherigen Veröffentlichungen hingewiesen werden.

Alle Korrekturen bewirkten insgesamt eine durchschnittliche jährliche Erhöhung der Verdunstung um rd. $50 \mathrm{~mm}$ im Vergleich zu den bisher veröffentlichten Werten, denen der unkorrigierte bodengleiche Niederschlag zu Grunde lag.

\subsubsection{Bodenkennwerte}

\subsubsection{Bodenkundliche Erhebungen}

Zur Ermittlung der physikalischen Eigenschaften der Böden wurden bei der Lysimetergewinnung in den Jahren 1978/79 entsprechende Untersuchungen auf den Lysimeterherkunftsflächen durchgeführt. Es handelt sich dabei um Profilbeschreibungen (Anlage 2) und die horizontweise Bestimmung der Bodenarten. Des weiteren liegen von jeder der acht Herkunftsflächen Angaben zur/zum 
- Korngrößenverteilung der Ton-, Schluff- und Sandfraktionen (Feinboden $<=2$ $\mathrm{mm})$

- $\quad$ Skelett (Grobboden > $2 \mathrm{~mm}$ )

- Porengrößenverteilung aus Wasserspannungskurven (pF-Kurven), deren experimentelle Bestimmung allerdings nicht mehr nachvollziehbar ist

- Trockenrohdichte, effektiven Lagerungsdichte

- gesättigten Leitfähigkeit sowie

- Humusgehalt, pH-Wert, Carbonat (CaCO3)-Gehalt, Sorption und

- Basenaustauschkapazität

aus den einzelnen Bodenhorizonten vor. Anlage $\mathbf{3}$ enthält eine Tabelle mit den Ergebnissen der bodenphysikalischen Untersuchungen.

\subsubsection{Charakteristische Werte der Wasserbindung}

Für die acht verschiedenen Böden (Tab.5) werden

$$
\begin{array}{lll}
\Theta_{\mathrm{PWP}} & =\text { Volumenfeuchte für den permanenten Welkepunkt } & \text { [Vol.-\%] } \\
\Theta_{\mathrm{FK}} & =\text { Volumenfeuchte für Feldkapazität } & \text { [Vol.-\%] } \\
\mathrm{nFK}=\Theta_{\mathrm{FK}}-\Theta_{\mathrm{PWP}} & =\text { nutzbare Feldkapazität } & \text { [Vol.-\%] }
\end{array}
$$

auf Grundlage der Angaben aus den Jahren 1978/79 wie folgt ermittelt:

I. aus der Bodenart (s. KA 4, 1994), die Ergebnisse sind in Tab. 5 unter „I. aus der Körnung“" zusammengestellt.

II. aus den $\mathrm{pF}-$ Kurven und der daraus abgeleiteten Porengrößenverteilung, wobei PWP = $\mathrm{FP}, \mathrm{FK}=\mathrm{FP}+\mathrm{MP}+\mathrm{GP}_{\mathrm{e}}$ und $\mathrm{nFK}=\mathrm{MP}+\mathrm{GP}_{\mathrm{e}}$ sind. Die Ergebnisse sind unter " $\boldsymbol{I I}$. aus der pF-Kurve" ersichtlich.

Die „Urdaten“ aus der Zeit der Lysimetergewinnung (Anlage 3) enthielten einige Unsicherheiten, die mit eckigen Klammern gekennzeichnet sind. Diese Kennzeichnung wurde in Tab. 5 übernommen. Es zeigten sich teilweise sehr große Unterschiede zwischen den unter „I“" und „II“" ermittelten Werten.

Auf Grundlage der Angaben zum Feinbodenanteil (Körnung) und zur effektiven Lagerungsdichte in Anlage $\mathbf{3}$ erfolgte eine Überprüfung (und bedarfsweise Korrektur der Bodenarten) und die Ableitung der Feldkapazität und der nutzbaren Feldkapazität. Da bei der Klassifizierung der Bodenarten nur die Anteile der Ton-, Schluff- und Sandfraktionen eingehen, mußten die in der Bodenkundlichen Katieranleitung (KA 4, 1994) aufgeführten Werte bei Böden, die Grobbodenanteile (Skelett) aufweisen, noch korrigiert werden. Die Kennwerte (Feldkapazität und nutzbare Feldkapazität) wurden um einen Faktor $F=(100-$ Skelett[Vol.-\%])/100 abgemindert. Des Weitern wurden Zuschläge in Abhängigkeit vom Gehalt an organischer Substanz (Humus) vorgenommen. 
Tab. 5: Zusammenstellung charakteristischer Werte der Wasserbindung [Vol.\%]

\begin{tabular}{|c|c|c|c|c|c|c|c|c|c|c|c|c|c|c|}
\hline \multirow{2}{*}{\begin{tabular}{|c}
$\begin{array}{c}\text { Tiefe } \\
\text { [cm u. Gel. }\end{array}$ \\
Lysimeter
\end{tabular}} & $\begin{array}{l}\text { Bodenart } \\
\text { dv-Kurzz. }\end{array}$ & \multicolumn{5}{|c|}{$\begin{array}{l}|\mathrm{PV}| \mathrm{LK}|\mathrm{FK}| \mathrm{nFK}|\mathrm{PWP}| \\
\text { I. aus der Körnung }\end{array}$} & \multicolumn{5}{|c|}{$\begin{array}{l}\text { PV }|\mathrm{LK}| \mathrm{FK}|\mathrm{nFK}| \mathrm{PWP} \\
\text { II. aus der pF-Kurve }\end{array}$} & \multicolumn{3}{|c|}{$\begin{array}{l}\text { FK } \mid \text { nFK } \mid \text { PWP } \\
\text { endgültige Werte* }^{*}\end{array}$} \\
\hline & gruppe 5 & rodi & rte & Brat & nerc & & & & & & & & & \\
\hline $0-35$ & $\mathrm{Su} 3 / \mathrm{S} 13$ & 36 & 7 & 29 & 21 & 8 & 40 & 10 & 30 & 23 & 7 & 25,6 & 16,8 & 8,8 \\
\hline $35-175$ & $\mathrm{mSgs}$ & 36 & 23 & 13 & 9 & 4 & 36 & 14 & 22 & 20 & 2 & 8,1 & 5,9 & 2,2 \\
\hline $175-300$ & $\mathrm{mSfs}$ & 42 & 23 & 19 & 14 & 5 & 40 & [16] & 24 & 20 & 4 & 17,2 & 13,2 & 3,9 \\
\hline \multicolumn{15}{|c|}{ Lysimetergruppe 4 - Braunerde-Fahlerde } \\
\hline $0-30$ & Su $4 /$ Slu & 45 & 10 & 35 & 26 & 9 & 45 & 10 & 35 & 29 & 6 & 38,0 & 26,6 & 11,4 \\
\hline $30-55$ & Us/Uls & 44 & 9 & 35 & 25 & 9 & 41 & 9 & 32 & 25 & 7 & 31,4 & 21,9 & 9,5 \\
\hline $55-120$ & St2 & 40 & 16 & 24 & 15 & 9 & 36 & 13 & 22 & 13 & 9 & 15,2 & 9,3 & 5,9 \\
\hline $120-300$ & $\mathrm{mS}$ & 42 & 28 & 14 & 9 & 5 & 45 & [23] & 22 & 21 & 1 & 12,4 & 8,3 & 4,1 \\
\hline \multicolumn{15}{|c|}{ Lysimetergruppe 8 - Parabraunerde-Braunerde } \\
\hline $0-25$ & $\mathrm{Su} 4$ & 42 & 10 & 32 & 25 & 7 & 38 & 3 & 35 & 30 & 5 & 29,3 & 22,6 & 6,7 \\
\hline $25-70$ & $\mathrm{Su} 4$ & 41 & 9 & 32 & 25 & 7 & 42 & 8 & 34 & 28 & 6 & 29,3 & 22,6 & 6,7 \\
\hline $70-150$ & $\mathrm{~S} 13 / \mathrm{S} 14$ & 41 & 12 & 29 & 19 & 10 & [40] & [10] & 30 & 24 & [6] & 23,4 & 14,4 & 9,0 \\
\hline $150-300$ & $\mathrm{mSfs}$ & 41 & 23 & 18 & 14 & 4 & 43 & [20] & 23 & 22 & 1 & 19,0 & 12,5 & 6,5 \\
\hline \multicolumn{15}{|c|}{ Lysimetergruppe 1- lessivierter Braunerde-Pseudogley } \\
\hline $0-25$ & Uls & 48 & 8 & 40 & 26 & 14 & 43 & 3 & 40 & 31 & 9 & 46,1 & 28,0 & 18,1 \\
\hline $25-40$ & Uls & 43 & 7 & 36 & 24 & 12 & 39 & 4 & 35 & 27 & 8 & 32,1 & 20,9 & 11,2 \\
\hline 40 & Uls & 40 & 7 & 33 & 22 & 11 & & & & & & 30,0 & 20,0 & 10,0 \\
\hline 50 & $\mathrm{~L}$ & 34 & 4 & 30 & 15 & 15 & 34 & 2 & 32 & 14 & 18 & 25,8 & 11,5 & 14,3 \\
\hline $120-300$ & $\mathrm{mSgs}$ & 34 & 23 & 11 & 8 & 3 & 30 & 20 & 10 & 9 & 1 & 4,1 & 3,0 & 1,1 \\
\hline \multicolumn{15}{|c|}{ Lysimetergruppe 7 - Braunerde-Pseudogley } \\
\hline $0-35$ & Slu & 37 & 7 & 30 & 20 & 11 & 35 & 2 & 33 & 24 & 9 & 34,3 & 21,6 & 12,7 \\
\hline $35-50$ & Slu & 36 & 11 & 25 & 17 & 8 & 40 & 5 & 35 & 26 & 8 & 26,9 & 18,1 & 8,8 \\
\hline $50-135$ & Ls4 & 32 & 5 & 27 & 16 & 12 & 31 & 1 & 30 & 11 & 19 & 26,9 & 12,0 & 14,9 \\
\hline $135-220$ & Ls3 & 32 & 7 & 25 & 17 & 8 & 29 & 0 & 29 & 8 & [21] & 28,1 & 12,6 & 15,5 \\
\hline $220-300$ & $\mathrm{mSfs}$ & 42 & 23 & 19 & 14 & 5 & 36 & 16 & 20 & 19 & 1 & 12,2 & 9,5 & 2,7 \\
\hline \multicolumn{15}{|c|}{ Lysimetergruppe 11 - pseudovergleyte Braunerde-Fahlerde } \\
\hline $0-20$ & $\mathrm{Su} 2 / \mathrm{Su} 3$ & 37 & 11 & 26 & 19 & 7 & 36 & 6 & 30 & 26 & 4 & 21,9 & 15,7 & 6,2 \\
\hline $20-35$ & $\mathrm{Su} 2 / \mathrm{Su} 3$ & 37 & 11 & 26 & 19 & 7 & 36 & 6 & 30 & 26 & 4 & 20,6 & 15,3 & 5,3 \\
\hline $35-55$ & $\mathrm{Su} 2$ & 34 & 13 & 21 & 15 & 6 & 34 & 0 & 10 & 21 & 4 & 19,6 & 14,6 & 5,0 \\
\hline $55-90$ & Ls4 & 34 & 4 & 30 & 15 & 15 & 31 & 8 & 23 & 5 & 18 & 26,9 & 12,0 & 14,9 \\
\hline $90-240$ & S14 & 32 & 5 & 27 & 15 & 12 & 29 & 2 & 27 & 13 & 14 & 24,5 & 13,9 & 10,6 \\
\hline $240-300$ & $\mathrm{mSfs}$ & 38 & 23 & 15 & 12 & 3 & 40 & 20 & 20 & 20 & 0 & 19,0 & 14,5 & 4,5 \\
\hline
\end{tabular}

*mit Berücksichtigung des Humus- und Skelettgehaltes 
Fortsetzung Tabelle 5

\begin{tabular}{|c|c|c|c|c|c|c|c|c|c|c|c|c|c|c|}
\hline \multirow{2}{*}{$\begin{array}{c}\begin{array}{c}\text { Tiefe } \\
{[\mathrm{cm} \text { u. Gel }}\end{array} \\
\text { Lysimete }\end{array}$} & $\begin{array}{l}\text { Bodenart } \\
\text { dv-Kurzz. }\end{array}$ & \multicolumn{5}{|c|}{$\begin{array}{l}\text { PV } \mid \text { LK } \mid \text { FK } \mid \text { nFK } \mid \text { PWP } \\
\text { I. aus der Körnung }\end{array}$} & \multicolumn{5}{|c|}{$\begin{array}{l}\text { PV } \mid \text { LK }|\mathrm{FK}| \mathrm{nFK} \mid \mathrm{PWP} \\
\text { II. aus der pF-Kurve }\end{array}$} & \multicolumn{3}{|c|}{$\begin{array}{l}\text { FK } \mid \text { nFK } \mid \text { PWP } \\
\text { endgültige Werte* }\end{array}$} \\
\hline & gruppe 9 - & $\ddot{\sigma} \beta-I$ & ara & brau & erc & & & & & & & & & \\
\hline $0-30$ & $\mathrm{Ut} 3 / \mathrm{Ut} 4$ & 43 & 5 & 38 & 23 & 15 & 36 & 0 & 36 & 22 & 14 & 40,0 & 22,0 & 18,0 \\
\hline $30-60$ & Ut4 & 44 & 6 & 38 & 23 & 15 & 43 & 2 & 41 & 25 & 15 & 38,5 & 21,0 & 17,5 \\
\hline $60-170$ & Ut3 & 40 & 4 & 36 & 24 & 12 & 38 & 0 & 38 & 26 & 12 & 36,0 & 23,5 & 12,5 \\
\hline $170-205$ & Ut3 & 40 & 4 & 36 & 24 & 12 & 39 & 0 & 39 & 27 & 12 & 35,6 & 23,2 & 12,4 \\
\hline $205-225$ & Uls & 42 & 6 & 36 & 22 & 14 & 36 & 0 & 36 & 22 & 14 & 31,0 & 20,7 & 10,3 \\
\hline $225-300$ & $\mathrm{mS}$ & 40 & 28 & 12 & 8 & 4 & 40 & [16] & 24 & [22] & 2 & $\mathbf{9 , 9}$ & 6,7 & 3,2 \\
\hline \multicolumn{15}{|c|}{ Lysimetergruppe 10 - Löß-Schwarzerde } \\
\hline $0-25$ & Ut4 & 44 & 6 & 38 & 21 & 17 & 44 & 3 & 41 & 25 & 16 & 39,9 & 21,9 & 18,0 \\
\hline $25-45$ & Ut4 & 48 & 9 & 39 & 22 & 17 & 48 & 2 & 46 & 30 & 16 & 43,0 & 23,5 & 19,5 \\
\hline $45-60$ & $\mathrm{Ut} 4 / \mathrm{Ut} 3$ & 47 & 9 & 38 & 24 & 15 & 48 & 4 & 44 & 32 & 12 & 41,5 & 22,5 & 19,0 \\
\hline $60-220$ & Ut2Ut3 & 47 & 9 & 38 & 27 & 11 & 49 & 10 & 39 & 31 & 8 & 34,7 & 24,0 & 10,7 \\
\hline $220-260$ & $\mathrm{mS}$ & 40 & 22 & 18 & 12 & 6 & [44] & 25 & 19 & 19 & 0 & 13,5 & $\mathbf{9 , 0}$ & 4,5 \\
\hline $260-300$ & St2 & 40 & 22 & 18 & 12 & 6 & 40 & 15 & 25 & 19 & 6 & 10,7 & 5,8 & 4,9 \\
\hline
\end{tabular}

*mit Berücksichtigung des Humus- und Skelettgehaltes 


\subsection{Die Herkunftsflächen der Lysimeterböden}

\subsubsection{Naturräumliche Beschreibung}

Die Bodenmonolithe für die Lysimeterstation wurden in den jetzigen Bundesländern Sachsen und Sachsen-Anhalt gewonnen Abb. 7). Fünf Entnahmeorte liegen im Einzugsgebiet der Parthe (Sachsen) sowie jeweils ein Entnahmeort im Raum Wittenberg (Sachsen-Anhalt), Oschatz (Sachsen) und Halle (Sachsen-Anhalt). Großräumig gesehen liegen alle acht Herkunftsorte im „Norddeutschen Tiefland“ (MEYNEN \& SCHMITHÜSEN, 1953).

Sachsen läßt sich vereinfacht in drei Naturregionen unterteilen: das lößfreie Tiefland (Altmoränengebiet) mit $18 \%$, die Lößgebiete mit $49 \%$ und die sächsischen Mittelgebirge mit $33 \%$ der Gesamtfläche Sachsens. Letztere und ihre Randbereiche sind als "Festgesteinsanteil" Sachsens nicht Gegenstand der nachfolgenden Beschreibungen. Abb. 7 zeigt in großer Vereinfachung die Abgrenzungen dieser drei Regionen und die drei Entnahmeorte von Lysimeterböden außerhalb des Partheeinzugsgebietes.

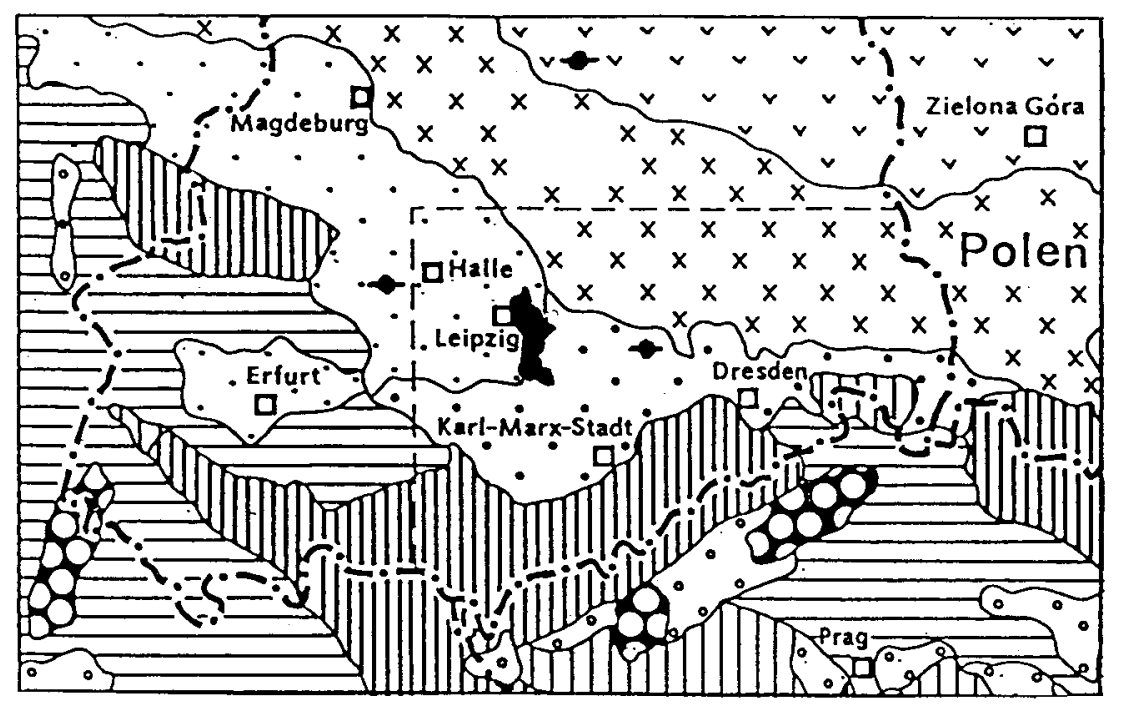

Herkunftsflächen außerhaib des Parthegebietes

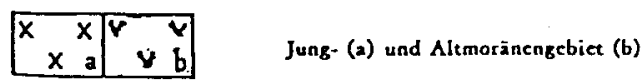

. Niederschlagsarme (2) und nicderschlagsreidere Löbgebiete (b) am Nordrand der Miteclgebirge
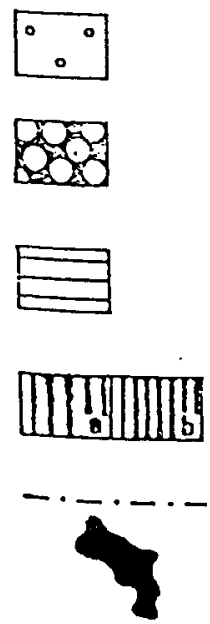

LöGgebiete und Becken der Mittelgebirgssdiwelle

Tertiazr-Vulkanit-Gebiege

Bergland und Mittelgebirge in Schiditgesteinen des Tafelstockwerks

Bergland und Mitcelgebirge in Gesteinen des Grundgebirgs- und Mulassc-Stodkwcrks (a), mit markanter Hōhenstufung (b) Staatsgrenzen

Einzugsgebiet der Parthe

Abb. 7: Skizze zur Lage der Naturräume in Sachsen (ergänzt aus MEYNEN \& SCHMITHÜSEN, 1953)

Wichtigste Prägung für die heutige naturräumliche Ausstattung dieses Gebietes sind die quartären Lockersedimente - hier als kuppige Moränenlandschaft des Drenthe-Stadiums der Saalekaltzeit. Die Herkunftsflächen der Lysimeter (außer die der Lysimetergruppe 11 aus dem Altmoränengebiet im Raum Wittenberg) repräsentieren aus hydrogeologischer Sicht die komplizierten Lagerungsverhältnisse des Randpleistozäns (EISSMANN, 1970).

Die quartären Lockersedimente dieser hydrogeologischen Einheiten wurden im Verlauf der Elster- und Saalekaltzeit als Folge der glazialen Serie Grundmoräne, Endmoräne und Sander abgelagert. Kennzeichnend für das Randpleistozän ist die Verzahnung der Inlandeisablagerungen mit fluviatilen und äolischen Bildungen oder auch mit fossilen Böden. Das heißt, Geschiebemergel und -lehme sowie Schmelzwassersande (und -tone) der Eisrandlagen befinden sich in komplizierter Wechsellagerung mit den aus Süden kommenden saalekaltzeitlichen Flussschottern. 
Diese bereits äußerst inhomogenen Bildungen erhielten dann im oberflächennahen Bereich während des periglazialen Milieus der Weichsel-Kaltzeit im wesentlichen ihre heutige Prägung. Die Dauerfrostböden unterlagen durch wechselndes Gefrieren und Auftauen, Umlagerung (Bodenfließen), Abtrag und Akkumulation nochmals entscheidenden Veränderungen. Zeugen davon sind Sandbänder, Sandkeile (ehemalige Eiskeile) und Sandlinsen bis zu einer Tiefe von mindestens 2 bis $3 \mathrm{~m}$ unter Flur. Eingelagerte Sandkeile und Sandlinsen wirken insbesondere in den Geschiebelehmpaketen als natürliche Dränagen (EISSMANN, 1970).

In der Weichsel-Kaltzeit folgten dann vor allem äolische Ablagerungen (Sandlöß). Diese sind im Partheeinzugsgebiet von geringerer Mächtigkeit (im Mittel knapp $1 \mathrm{~m}$, oft nur 0,6 m und weniger, selten $2 \mathrm{~m}$ ) und bestehen aus einem Gemenge von Treibsand und Flugstaub, auch mit Feinschluff- und Tonanteilen. Sie bestimmen für alle fünf Herkunftsflächen aus dem Parthegebiet weitgehend die Fruchtbarkeit der heutigen Bodendecke.

Im östlichen Harzvorland (Lysimetergruppe 10) und am Nordrand der Mittelgebirge (Lysimetergruppe 9) erreichen die Lößablagerungen dagegen große Mächtigkeiten und prägen damit wesentlich den Charakter der Grundwasserleiterdeckschichten.

Nachfolgend wird eine kurze Beschreibung der acht verschiedenen Herkunftsflächen vorgenommen (MEYNEN \& SCHMITHÜSEN, 1953; EISSMANN, 1970; PESTER, 1978):

\section{Lysimetergruppe 5: Kiesige Mittel- und Grobsande ohne bindige Deckschichten}

Diese Herkunftsfläche befindet sich im Bereich eines spornartigen Ausläufers des breiten Geschiebesandrückens zwischen Beucha und Brandis, einem Teilgebiet der staunässebeeinflussten Altmoränenplatte Borsdorf - Wurzen (Muldentalkreis).

Den angeschnittenen Grundwasserleiter (GWL) 1.6 (GWL-Bezeichnung nach PESTER, 1978) bilden fluviatile bis glazifluviatile spätelsterkaltzeitliche Sande und Kiese, die im Zusammenhang mit dem Abschmelzen des Inlandeises der Elsterkaltzeit (letzter Vorstoß) zur Ablagerung kamen. Die Mächtigkeit des GWL 1.6 ist mit 2 - 15 m sehr unterschiedlich. Von Bedeutung sind vor allem die Ablagerungen in Schmelzwasserrinnen, die sich teilweise recht tief in die ältere quartäre und tertiäre Schichtfolge eingeschnitten haben und dadurch zu hydraulischen Verbindungen mit anderen Grundwasserleitern führen. Diese Rinnen wurden teilweise durch Schmelzwasserablagerungen wieder aufgefüllt. Die weitere Füllung erfolgte nach bzw. während des Abschmelzens des Eises durch Ablagerung von Schmelzwassersedimenten, teilweise aber auch durch Flussablagerungen (Rückzugsschotter).

Die Sedimente des GWL 1.6 sind sehr inhomogen. Sie bestehen hauptsächlich aus feinkiesigen Mittel- und Grobsanden bzw. grobsandigen Feinkiesen mit vereinzelten Steinen. Häufig wurden Schlufflagen nachgewiesen (LfUG \& IBGW, 1994). Die darüber liegenden Schichten wurden aus umgelagerten Geschiebesanden wechselnder Mächtigkeit gebildet.

Der oberste Horizont besteht aus solifluidal umgelagertem Sandlöß, wobei auch Material aus der darunter liegenden Schicht eingearbeitet wurde. Aus diesem vorwiegend sandhaltigen Ausgangssubstrat entstanden durch Verbraunung Braunerden.

\section{Lysimetergruppe 4 und 8: Gebänderte Mittel- und Feinsande über Grobsand und Kies ohne bindige Deckschichten}

Die Herkunftsfläche der Lysimetergruppe 4 liegt auf der fast ebenen „Pomßener Geschiebesandhochfläche" (Muldentalkreis).

Bei dem angeschnittenen Grundwasserleiter handelt es sich um intersaalekaltzeitliche fluviatile und glazifluviatile Sande und Kiese, die im Anschluss an den ersten Vorstoß des 
Eises der Saalekaltzeit abgelagert wurden. Diese Schmelzwassersande (GWL 1.4) mit einer Mächtigkeit von 6 - $13 \mathrm{~m}$ bestehen aus wechselnd sandigen Fein- bis Mittelkiesen. Es kommen auch untergeordnet Feinsande und schluffige Sande vor. Über diesen Bildungen lagern glazigen oder periglazial umgelagerte Sande und Kiese in wechselnder Korngrößenzusammensetzung (Geschiebesande bzw. Rückzugsschotter). Diese Formation wird durch eine relativ kiesige Sandlößdecke der Weichselkaltzeit abgeschlossen, auf der sich eine durch starke Tonverlagerung gekennzeichnete Braunerde-Fahlerde ausbildete.

Im Bereich der Herkunftsfläche der Lysimetergruppe 8 (Südost-Ausläufer der Brehnaer Platte) ist wiederum der GWL 1.4 angeschnitten. Im Vergleich zur Lysimetergruppe 4 wird der GWL 1.4 an diesem Standort durch fluviatile kiesige Mittel- und Grobsande in starker Wechsellagerung (z. T. in Kreuzschichtung) mit lehmigen Sanden gebildet. Einzelne Horizonte sind taschenartig erhalten. Die darüber lagernde Sandlößdecke wurde solifluidal umgelagert und mit dem darunterliegenden grobsandigen Mittelsand vermengt. Sie enthält auch einzelne Kiese. Auf diesem Ausgangsmaterial entwickelte sich eine ParabraunerdeBraunerde.

\section{Lysimetergruppe 1,7 und 11: Geschiebelehm (-mergel) über Sanden oder Kiesen}

Die Herkunftsfläche der Lysimetergruppe 1 befindet sich im Kernraum der 10 bis $20 \mathrm{~m}$ mächtigen „Naunhofer Schotterebene“. Es handelt sich um eine Hauptterrasse mit frühsaalekaltzeitlichen fluviatilen Ablagerungen (GWL 1.5 - Muldeschotter). Dieser Grundwasserleiter besteht hauptsächlich aus stark sandigem Fein- bis Mittelkies mit einem schwankenden, aber nicht homogenen Anteil an Grobkies. Darüber lagert geringmächtig Geschiebelehm $(<1 \mathrm{~m})$ der Saalegrundmoräne und Sandlöß der Weichselkaltzeit. Die Böden dieser Herkunftsfläche (Braunerde-Pseudogley) repräsentieren eine Besonderheit. Sie lagerten über Gley und hatten früher Anschluss an das Grundwasser. Mit Inbetriebnahme der Wasserwerke Naunhof im Jahre 1895 sank der Grundwasserspiegel dauerhaft ab und auch in den $3 \mathrm{~m}$ tiefen Lysimetern bleibt dieser Boden weiterhin ohne Grundwassereinfluss.

Im Bereich der Herkunftsfläche der Lysimetergruppe 7 (Südostausläufer der Brehnaer Platte) wurde gleichfalls der GWL 1.5 angetroffen. Er ist hier durch geschichtete Sande wechselnder Korngrößenzusammensetzung gekennzeichnet. Darüber lagert in diesem Fall bis $\mathrm{zu}$ 2,70 m mächtiger Geschiebelehm der Saalegrundmoräne. Kennzeichnend für die Geschiebelehmschicht ist ihre Inhomogenität, die durch wiederholte Entmischung, Auffrieren und Entwässerung entstanden ist. Typisch sind kleine Sandlinsen und Eisrisswerke (Klüfte). Abgedeckt wird dieser Komplex durch eine solifluidal und periglazial umgelagerte kies- und sandhaltige Sandlößdecke. Typisch für das sächsische Mittelgebirgsvorland sind die darauf entstandenen Sandlößtieflehm-Staugley-Böden (Braunerde-Pseudogley), die durch temporär und periodisch auftretendes Stauwasser geprägt sind.

Die Herkunftsfläche der Lysimetergruppe 11 liegt am Südhang des Fläming zum Elbtal, nördlich von Wittenberg. In MEYNEN \& SCHMITHÜSEN (1953) wird dieses Gebiet als Roßlau-Wittenberger-Vorfläming beschrieben und zählt damit nicht mehr zum Randpleistozän. Es handelt sich um ein Altmoränengebiet, vorwiegend um eine größere Eisrandlage der Saale-Vereisung (Endmoräne mit dazugehörigen Sander- und Grundmoränenflächen). Typisch für diesen Teil des Altmoränengebietes ist, dass sich Sanderflächen, Stauchmoränen und übersandete Grundmoränenflächen engräumig ablösen, teilweise auch mit tertiären Ablagerungen verzahnen. Im Bereich der Herkunftsfläche werden Schmelzwassersande (z. T. geschichtet) von einem reichlich $2 \mathrm{~m}$ mächtigen Geschiebemergelpaket überlagert. Hinsichtlich ihres hydrogeologischen Aufbaues ist diese Herkunftsfläche durchaus vergleichbar mit der Lysimetergruppe 7 aus dem Bereich des Randpleistozäns. 
Den oberen Horizont bilden wenige Dezimeter mächtige Geschiebesande der Weichselkaltzeit. Das Altmoränengebiet des Fläming liegt außerhalb der Lößlandschaften, so dass die Böden - hier pseudovergleyte Braunerde-Fahlerde - durch Nährstoffarmut und intensive Verwitterung gekennzeichnet sind.

\section{Lysimetergruppe 9 und 10: Mächtige Lößschichten über Sanden und Kiesen}

Die Herkunftsfläche der Lysimetergruppe 9 bei Sornzig im Kreis Oschatz zählt zum niederschlagsreicheren Mittelsächsischen Lößhügelland, dem Kerngebiet des „Sächsischen Lößgefildes“ am Nordrand der Mittelgebirge. Die Lößdecke ist nahezu durchgängig mehrere Meter mächtig. Für die Lysimeter wurde ein Standort mit einer rd. $2 \mathrm{~m}$ mächtigen Lößschicht gewählt. Darunter lagern Schmelzwassersande, die „saale- (vor allem warthe-) oder auch elsterkaltzeitlichen Abschnitten des Pleistozäns angehören“" (MEYNEN \& SCHMITHÜSEN, 1953). Für diese Region sind die fruchtbaren Löß-Parabraunerden (mit schwacher Tonverlagerung) typisch.

Die Herkunftsfläche der Lysimetergruppe 10 liegt im Mitteldeutschen Schwarzerdegebiet, genauer im östlichen Harzvorland bei Etzdorf (Saalkreis). Auch diese Lößgebiete bilden „einen Raum von großer morphologischer Einheitlichkeit. Der in der Weichselkaltzeit in großer Mächtigkeit angelagerte Löß (im Bereich der Herkunftsfläche > $2 \mathrm{~m}$ ) verdeckt die Bildungen einer alten Phase der Saalekaltzeit, bestehend aus Geschiebemergel, einigen verwaschenen Moränenzügen und flachen, oasenartigen Sandrücken“ (MEYNEN \& SCHMITHÜSEN, 1953). Für die warmen und niederschlagsarmen Gebiete des Halleschen Lößhügellandes sind Löß-Schwarzerdeböden typisch. Diese Böden sind aufgrund ihres optimalen Luft- und Wasserhaushaltes bestens für eine landwirtschaftliche Nutzung geeignet. Besondere Merkmale sind Krotowinen sowie Carbonat-Konkretionen und -Ausblühungen (Lößkindel).

Zusammenfassend enthält Tab. 6 Informationen zur Bodenform, zum Entnahmeort und zum Profilaufbau der Lysimetergruppen.

Anlage 2 und 3 beschreiben die acht Bodenprofile, wie sie zur Zeit der Lysimetergewinnung am Entnahmeort angetroffen wurden und enthalten Angaben zu wichtigen Eigenschaften der Lysimeterböden, wie u. a. Korngrößenverteilung, Porosität und gesättigte Wasserleitfähigkeit. 
Tab. 6: Bodenform, Entnahmeorte und Profilaufbau der Brandiser Lysimeter

\begin{tabular}{|c|c|c|c|c|}
\hline Gruppe & $\begin{array}{l}\text { Bodenform } \\
\text { [Entnahmeorte] }\end{array}$ & Tiefe $(\mathrm{cm})$ & Profilaufbau & $\begin{array}{l}\text { tandort- } \\
\text { einheit }\end{array}$ \\
\hline 5 & $\begin{array}{l}\text { Erodierte Braunerde geringer } \\
\text { Entwicklungstiefe aus Sandlöß } \\
\text { über kiesführendem Fluvisand } \\
\text { [Brandis, Muldentalkreis] }\end{array}$ & $\begin{array}{r}0-35 \\
35-170 \\
170-300\end{array}$ & $\begin{array}{l}\text { Sandlöß } \\
\text { kiesiger Mittel- u.Grobsand } \\
\text { Schmelzwassersand, locker }\end{array}$ & D3 \\
\hline 4 & $\begin{array}{l}\text { Braunerde-Fahlerde mittlerer } \\
\text { Entwicklungstiefe aus Sandlöß } \\
\text { über kiesführendem Fluvisand } \\
\text { [Pomßen, Muldentalkreis] }\end{array}$ & $\begin{array}{r}0-30 \\
30-130 \\
130-300\end{array}$ & $\begin{array}{l}\text { Sandlöß, schwach kiesig } \\
\text { lehmiger Sand, kornverkittet } \\
\text { Schmelzwassersande u. -kiese }\end{array}$ & D5 \\
\hline 8 & $\begin{array}{l}\text { Parabraunerde-Braunerde } \\
\text { mittlerer Entwicklungsstufe } \\
\text { aus Sandlöß über tiefem, } \\
\text { kiesführendem Moränenlehm } \\
\text { [westl. Brandis, Muldentalkreis] }\end{array}$ & $\begin{array}{r}0-70 \\
70-150 \\
150-300\end{array}$ & $\begin{array}{l}\text { Sandlöß } \\
\text { Fließerde (sandiger Lehm) } \\
\text { Mittel- u. Feinsand }\end{array}$ & D3 \\
\hline 1 & $\begin{array}{l}\text { lessivierter Braunerde- } \\
\text { Pseudogley } \\
\text { mittlerer Entwicklungstiefe } \\
\text { über fossilem Gley im nahen } \\
\text { Untergrund aus Sandlöß über } \\
\text { kiesführenden Moränenlehm } \\
\text { [Naunhof, Muldentalkreis] }\end{array}$ & $\begin{array}{r}0-50 \\
50-120 \\
120-300\end{array}$ & $\begin{array}{l}\text { Sandlöß } \\
\text { Geschiebelehm } \\
\text { kiesiger Mittel- u. Grobsand }\end{array}$ & D6 \\
\hline 7 & $\begin{array}{l}\text { Braunerde-Pseudogley } \\
\text { mittlerer Entwicklungstiefe aus } \\
\text { Sandlöß über kiesführendem } \\
\text { Moränenlehm } \\
\text { [Beucha, Muldentalkreis] }\end{array}$ & $\begin{array}{r}0-50 \\
50-220 \\
220-300\end{array}$ & $\begin{array}{l}\text { Sandlöß } \\
\text { Geschiebelehm } \\
\text { Schmelzwassersand }\end{array}$ & D4 \\
\hline 11 & $\begin{array}{l}\text { pseudovergleyte Braunerde- } \\
\text { Fahlerde mittlerer Entwicklungs- } \\
\text { tiefe aus Geschiebedecksand } \\
\text { über Geschiebemergel } \\
\text { [Rahnsdorf, Kreis Wittenberg] }\end{array}$ & $\begin{array}{r}0-20 \\
20-240 \\
240-300\end{array}$ & $\begin{array}{l}\text { Geschiebesand } \\
\text { Geschiebemergel } \\
\text { Schmelzwassersand }\end{array}$ & D3 \\
\hline 9 & $\begin{array}{l}\text { Parabraunerde mittlerer } \\
\text { Entwicklungstiefe aus Löß } \\
\text { [Sornzig, Kreis Oschatz] }\end{array}$ & $\begin{array}{r}0-205 \\
205-300\end{array}$ & $\begin{array}{l}\text { fahlbrauner lehmiger Schluff } \\
\text { Schmelzwassersand }\end{array}$ & Lö3 \\
\hline 10 & $\begin{array}{l}\text { Schwarzerde mittlerer } \\
\text { Entwicklungstiefe aus Löß } \\
\text { [Etzdorf, Saalkreis] }\end{array}$ & $\begin{array}{r}0-220 \\
220-250 \\
250-300\end{array}$ & $\begin{array}{l}\text { dunkelbrauner, humoser } \\
\text { Schluff } \\
\text { Geschiebemergel } \\
\text { Schmelzwassersand }\end{array}$ & Lö1 \\
\hline
\end{tabular}

NStE - Natürliche Standorteinheiten (KUNDLER et al., 1989) beschreiben Entstehungsgeschichte und Zustand der Böden, wobei

- „D“ (Diluvium - $\mathrm{D}_{1}$ bis $\mathrm{D}_{6}$. ) für Böden auf Ablagerungen infolge der Inlandeisvorstöße steht. Hohe Indizes verweisen auf eine hohe Bodenfruchtbarkeit.

- „ „Lö“ (Löß - Lö $\ddot{O}_{1}$ bis L $\left.\ddot{O}_{6}\right)$ steht für die angewehten Feinerden mit besonderer Fruchtbarkeit. Hier zeigen kleine Indizes eine hohe Bodenfruchtbarkeit an. 


\subsubsection{Klimatische Unterschiede zwischen Brandis und den Herkunftsflächen}

Brandis und die Herkunftsflächen der Lysimeterböden liegen in der nördlichen gemäßigten Zone, in der Übergangszone zwischen maritimem und kontinentalem Klima (bereits etwas regenreichere Sommer und regenärmere Winter). Es herrscht ein ausgesprochenes Jahreszeitenklima mit überwiegend westlichen Winden. Die kalte Jahreszeit hat ein sehr uneinheitliches Gepräge: „Vorstöße maritimer Warmluft wechseln mit Einbrüchen arktischer Kaltluft ab“(GOLDSCHMIDT, 1950).

Hervorgerufen durch die Hauptwindrichtungen SW, W und NW und die vorhandenen Gebirge ist das Klima im Untersuchungsgebiet relativ engräumig gegliedert (Abb. 8). Aufgrund der vorherrschenden feuchten Winde aus westlicher Richtung bedingt die Leewirkung des Harzes eine ,weite Ausdehnung der trockenen und mäßig trockenen Form der unteren Lagen nach Südosten“ (NATURRÄUME, 1986). Es zeigt sich ein deutliches NW-SO-Profil vom östlichen Harzvorland bis zum Erzgebirge.

Für eine detaillierte Beschreibung der klimatischen Unterschiede zwischen Brandis und den Herkunftsflächen stehen die Stationen Wittenberg, Halle-Kröllwitz, Leipzig-Schkeuditz und Oschatz des DWD zur Verfügung.

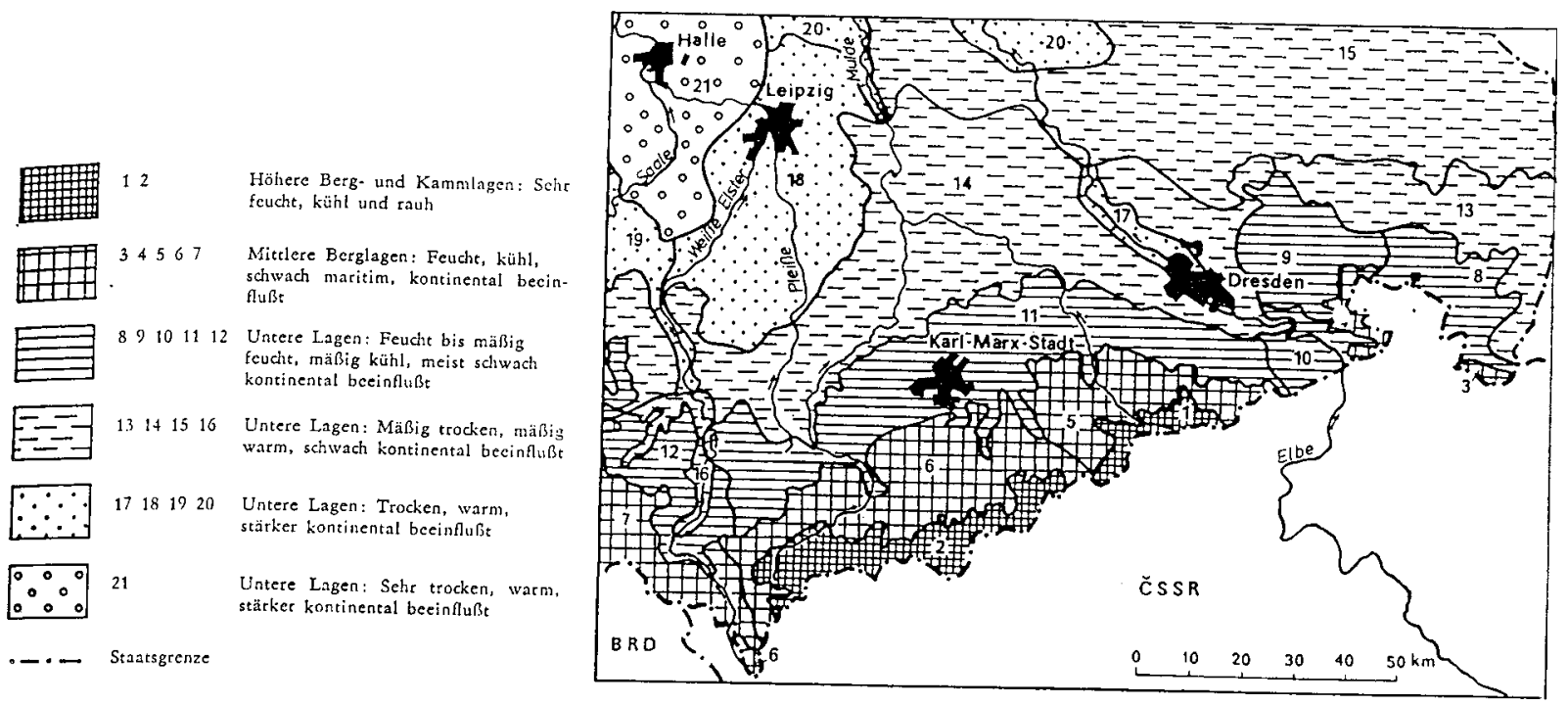

Abb. 8: Klimastufen- und gebiete (aus „Naturräume der sächsischen Bezirke“, 1986)

Mit dem Klimagebiet 21 ist in Abb. 8 der südöstliche Teil des mitteldeutschen Trockengebietes dargestellt. Hier steht mittleren Jahresniederschlägen von 460 bis $550 \mathrm{~mm}$ eine reale Verdunstung von mehr als $570 \mathrm{~mm}$ gegenüber. Entsprechend der größer werdenden Entfernung vom Mitteldeutschen Trockengebiet ist das Untersuchungsgebiet von West nach Süd-Ost durch eine deutliche Zunahme der Niederschläge und Abnahme der Lufttemperatur gekennzeichnet (vgl. Tab. 7).

Die mittlere innerjährliche Verteilung der Niederschlagshöhe ist durch ein Maximum in den Sommermonaten (Höchstwerte im Juni und August) und einem Minimum im Februar bei relativ geringen Unterschieden zwischen Oktober und März gekennzeichnet.

Ein Vergleich der Monatsniederschläge zwischen Brandis und den o. g. Stationen des DWD ergab zwar unterschiedlich hohe Monatssummen, aber gleiche Zeitpunkte für das Auftreten von Monaten mit geringen bzw. hohen Niederschlägen. Für Monatswerte der potentiellen Verdunstung wurden Korrelationskoeffizienten zwischen 0,97 und 0,98 berechnet. Für den 
Niederschlag schwanken die Koeffizienten zwischen 0,79 (Brandis-Wittenberg) und 0,89 (Brandis-Oschatz). Ursache für den schlechteren Zusammenhang der Monatsniederschläge der verschiedenen Stationen sind vor allem die hohen, lokal sehr unterschiedlich auftretenden Sommerniederschläge. Die potentielle Evapotranspiration variiert regional deutlich weniger als der Niederschlag.

Tab. 7: Monatsmittelwerte ausgewählter Klimagrößen für die Jahre 1981-97 von Brandis und Klimastationen des DWD (Hydrologisches Jahr; Niederschlag unkorrigiert)

\begin{tabular}{|c|c|c|c|c|c|c|}
\hline & & Wittenberg & $\begin{array}{l}\text { Halle- } \\
\text { Kröllwitz } \\
\end{array}$ & $\begin{array}{l}\text { Leipzig- } \\
\text { Schkeuditz } \\
\end{array}$ & Brandis & Oschatz \\
\hline Stationslage [m ü NN & & 105 & 96 & 131 & 136 & 150 \\
\hline Niederschlag $(\mathrm{P})$ & $\mathrm{Wi}$ & 260 & 185 & 224 & 259 & 262 \\
\hline [Summe in mm] & & 285 & 267 & 289 & 313 & 308 \\
\hline & Wi/So & 0,91 & 0,69 & 0,77 & 0,83 & 0,85 \\
\hline & Jahr & 556 & 452 & 513 & 572 & 570 \\
\hline Lufttemperatur & $\mathrm{Wi}$ & 3,1 & 3,5 & 3,3 & 3,1 & 3,2 \\
\hline [Mittel in $\left.{ }^{\circ} \mathrm{C}\right]$ & So & 15,1 & 15,1 & 15,1 & 15,0 & 14,9 \\
\hline & Jahr & 9,1 & 9,3 & 9,2 & 9,0 & 9,0 \\
\hline Sonnenschein- & $\mathrm{Wi}$ & 528 & 528 & 512 & 494 & 531 \\
\hline dauer [Summe in $\mathrm{h}$ ] & So & 1148 & 1127 & 1099 & 1107 & 1114 \\
\hline & Jahr & 1677 & 1655 & 1611 & 1601 & 1645 \\
\hline potentielle & $\mathrm{Wi}$ & 140 & 151 & 151 & 136 & 140 \\
\hline Verdunstung (ETP*) & So & 519 & 513 & 507 & 494 & 506 \\
\hline [Summe in $\mathrm{mm}$ ] & Jahr & 659 & 664 & 658 & 630 & 646 \\
\hline $\mathbf{a}=\mathbf{P} / \mathbf{E T P}$ & Jahr & $\mathbf{0 , 8 2}$ & 0,68 & 0,78 & $\mathbf{0 , 9 1}$ & $\mathbf{0 , 8 8}$ \\
\hline $\mathbf{k W B}[\mathrm{mm}]$ & Jahr & -116 & -212 & -143 & -58 & -76 \\
\hline
\end{tabular}

*ETP nach TURC (s. Gl. 15)

kWB - klimatische Wasserbilanz

Der mittlere Jahresverlauf von Niederschlag und potentieller Verdunstung Abb. 9 zeigt, dass nur in den Monaten November, Dezember und Januar das Wasserdargebot wesentlich höher ist als das Energieangebot.

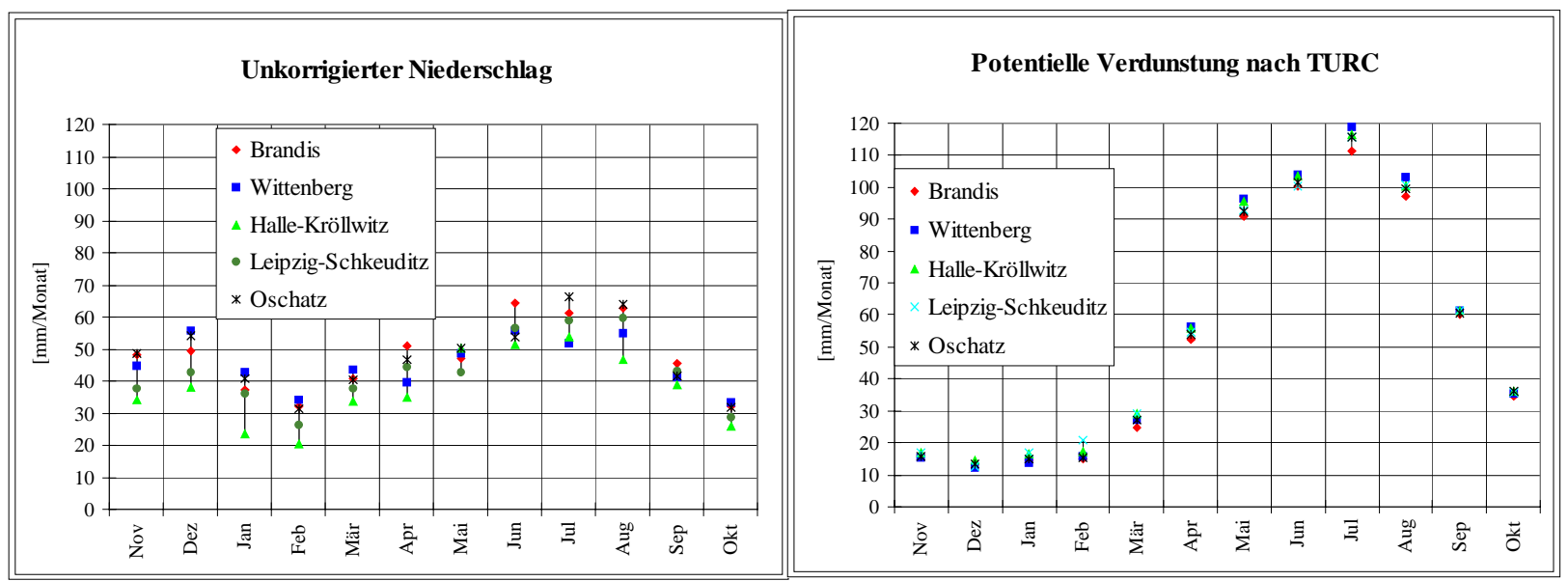

Abb. 9: Monatswerte von unkorrigiertem Niederschlag und potentieller Verdunstung nach TURC für ausgewählte Klimastationen des DWD und Brandis (Reihe 1981-97)

Das Verhältnis zwischen Winter- und Sommerniederschlägen ist nicht an allen Stationen gleich. Im Hinblick darauf, dass insbesondere die Winterniederschläge zur Grundwasserneubildung beitragen, fällt das Verhältnis Winter- zu Sommerniederschlägen für die Station 
Halle-Kröllwitz besonders ungünstig aus (s. Tab. 7). Der Ariditätskoeffizient a aus dem Verhältnis der Jahressummen von Niederschlag und potentieller Verdunstung gibt einen Hinweis auf aride $(\mathrm{a}<1)$ bzw. humide $(\mathrm{a}>1)$ Witterungsbedingungen.

Einen weiteren Hinweis auf klimatisch bedingte Unterschiede zwischen den einzelnen Stationen liefern die kWB und eine Häufigkeitsanalyse der Monatssummen des Niederschlages (Tab. 8).

Tab. 8: Relative Häufigkeit monatlicher Niederschlagssummen in Prozent und ihre jahreszeitliche Differenzierung für ausgewählte Stationen (Wasserhaushaltsjahre 1981-96)

\begin{tabular}{|l|rrr|rrr|rrr|}
\hline \multirow{2}{*}{ Station } & \multicolumn{3}{|c|}{ Winter } & \multicolumn{3}{c|}{ Sommer } & \multicolumn{3}{c|}{ Mittel } \\
\cline { 2 - 11 } & $\mathrm{t}$ & $\mathrm{n}$ & $\mathrm{f}$ & $\mathrm{t}$ & $\mathrm{n}$ & $\mathrm{f}$ & $\mathrm{t}$ & $\mathrm{n}$ & $\mathrm{f}$ \\
\hline Wittenberg & 48 & 42 & 10 & 42 & 49 & 9 & 45 & 45 & 10 \\
\hline Halle-Kröllwitz & 78 & 19 & 3 & 43 & 47 & 10 & 60 & 33 & 7 \\
\hline Leipzig-Schkeuditz & 62 & 32 & 6 & 44 & 47 & 9 & 52 & 40 & 8 \\
\hline Brandis & 53 & 38 & 9 & 39 & 46 & 15 & 46 & 42 & 12 \\
\hline Oschatz & 45 & 46 & 9 & 41 & 43 & 16 & 43 & 44 & 13 \\
\hline
\end{tabular}

$\mathrm{t} \quad$ trockene Monate $<40 \mathrm{~mm}$

n normale Monate $40-80 \mathrm{~mm}$

f feuchte Monate $>80 \mathrm{~mm}$

Langanhaltende zyklonale Niederschläge sind an Wetterfronten gebunden, so dass bei diesen Niederschlagsereignissen kaum mit Unterschieden zwischen Brandis und den Herkunftsflächen der Lysimeterböden zu rechnen ist. Problematischer sind konvektive Niederschläge, z. B. sommerliche Gewitter, die räumlich sehr begrenzt sind, dafür aber mit großen Intensitäten auftreten.

Zusammenfassend wird eingeschätzt, dass nur zwischen dem Herkunftsort Etzdorf (Lysimetergruppe 10), der durch die Station Halle-Kröllwitz $(\mathrm{kWB}=-212 \mathrm{~mm} / \mathrm{Jahr})$ repräsentiert wird und Brandis wesentliche klimatische Unterschiede bestehen. Diese Unterschiede müssen bei der Interpretation der Lysimeterergebnisse berücksichtigt werden. Sie stehen einer direkten Übertragung der in Brandis gewonnenen Erkenntnisse auf die Herkunftsflächen entgegen. Eine Einordnung der Beobachtungsreihe 1981-97 in das langjährige Klimageschehen erfolgt durch einen Vergleich mit den „Normalwerten“ der Reihen 1901-50 und 1951-80 in Kapitel 4.2. 


\subsection{Auswertungsmethoden}

\subsection{1 Überblick}

Ziel der beiden vorstehenden Kapitel war es, das Verständnis für die Enstehung der Lysimetermessreihen herzustellen und mögliche Fehler und Randbedingungen aufzuzeigen, die bei Untersuchungen zum Standortwasserhaushalt mittels Lysimeter zu beachten sind.

Ziel der Untersuchungen ist die Feststellung von generellen Zusammenhängen zwischen Witterung, Boden, Vegetation und den Wasserhaushaltsgrößen am Standort. Lassen sich daraus entsprechende Regressionen ableiten, so können diese für eine Übertragung auf andere Standorte genutzt werden.

Die Beschreibung der Entstehung und der naturräumlichen Ausstattung der Herkunftsflächen dient vordergründig dem Verständnis der im Bodenprofil (Lysimeter) ablaufenden Prozesse. Mit dem Vergleich der klimatischen Unterschiede zwischen Brandis und den Herkunftsflächen wurde - mit Ausnahme der Schwarzerdeböden aus der Region östlich von Halle - der Nachweis erbracht, dass am Entstehungsort der Böden und am Untersuchungsstandort in Brandis mittel- und langfristig keine abweichenden Ergebnisse zu erwarten sind. Dies ist eine wesentliche Voraussetzung für die Akzeptanz der Untersuchungen.

Für alle Auswertungen werden jeweils die Gruppenmittel der drei Lysimeter einer Herkunftsfläche verwendet. Unterschiede der Messwerte von drei Lysimetern einer Gruppe (= eines Bodens) sind ausschließlich Gegenstand der Varianzanalyse zur ,hydrologischen Wirksamkeit“" der Böden in Kapitel 4.1.1.

Die Bewertung der eigenen Ergebnisse erfolgt auf Basis von Messwerten anderer Lysimeterstationen und mit Hilfe von Vergleichsrechnungen. Hierzu wurden verschiedene Berechnungsverfahren zur Ermittlung der potentiellen Verdunstung sowie die Verfahren von RENGER \& WESSOLEK (1990) und SPONAGEL (1980) zur Ermittlung der Sickerwasserraten bzw. der Verdunstung verwendet.

Für die Varianzanalyse (Kap. 4.1.1) kam das Programm STATISTICA/W (StatSoft 5.0, USA) zum Einsatz. Die Auswertungen wurden in der Arbeitsgruppe Biometrie und Agrarinformatik der Landwirtschaftlichen Fakultät der Martin-Luther-Universität Halle-Wittenberg durchgeführt.

Die methodischen Grundlagen u. a. zur Bestimmung der pflanzenverfügbaren Bodenwassermengen und der Ausschöpfungstiefen bedürfen einer kurzen Beschreibung. Dies gilt auch für die Ermittlung der Bestandskoeffizienten und der Evapotranspirationskoeffizienten, insbesondere aber für die Varianzanalyse. Diese Beschreibungen sind Gegenstand der nachfolgenden Kapitel 2.3.2 - 2.3.5.

Eine Erläuterung der in den Tabellen und Abbildungen verwendeten Abkürzungen und Formelzeichen enthält Kapitel 7. 


\subsubsection{Bestimmung der pflanzenverfügbaren Bodenwassermenge}

Für Standorte mit flurfernen Grundwasserständen wird die pflanzenverfügbare Wassermenge $\left(\mathrm{nFK}_{\mathrm{We}}\right)$ im Wurzelraum nach RENGER et al. (1974) aus dem Produkt von nutzbarer Feldkapazität (nFK) und ,effektiver“ Durchwurzelungstiefe (We) ermittelt.

Zur Ermittlung der nFK ist die Beziehung zwischen Wasserspannung und Wassergehalt (pFKurve) ein häufig benutztes Hilfsmittel. Da einzelne pF-Kurven nicht selten mit Messfehlern behaftet sind und/oder nur einen sehr kleinen, wenig repräsentativen Ausschnitt (Stechzylinderprobe) eines Bodenprofils beschreiben, werden näherungsweise (durch vergleichende pF-Messungen gestützte) Beziehungen zwischen Bodenart (Körnung), Lagerungsdichte, Humusgehalt und mittlerer Porenraumgliederung (FK, PWP, nFK) verwendet (HARTGE \& HORN, 1991).

Die Durchwurzelungstiefe ist abhängig von den Bodeneigenschaften, vom Witterungsverlauf, der Art und dem Entwicklungszustand der Pflanzen. In der Regel werden für die Mächtigkeit des Hauptwurzelraumes einjähriger landwirtschaftlicher Nutzpflanzen aber Mittelwerte verwendet, die nur vom Boden abhängig sind (RENGER et al., 1974; KA4, 1994). Dadurch erhält die nutzbare Feldkapazität im effektiven Wurzelraum $\left(\mathrm{nFK}_{\mathrm{We}}\right)$ den Status eines Bodenkennwertes.

Auf Basis der Lysimeteruntersuchungen kann die tatsächliche jährliche Bodenwasserentnahme durch die Pflanzen ermittelt werden, wobei die unterschiedliche Reaktion der Pflanzen auf die von Jahr zu Jahr unterschiedlichen Witterungsbedingungen Berücksichtigung findet. Gegenstand der Auswertungen ist also die Ermittlung des jährlichen Verlaufes der Ausschöpfung sowie der maximalen Entnahmemenge bei verschiedenen Böden während der 17 Beobachtungsjahre bei unterschiedlichem Bewuchs.

Die Bodenwasserentnahme durch die Pflanzen und die Wiederauffüllung der Defizite wurde auf Basis der korrigierten Niederschläge und der aus allen Korrekturen resultierenden Verdunstung ermittelt. Bei bekanntem Anfangszustand (Sättigung) erhält man die bis zum aktuellen Zeitpunkt j tatsächlich entnommene Bodenwassermenge als Ergebnis der fortlaufenden Bilanzierung von

\begin{tabular}{|c|c|}
\hline Gl. 2: & $\mathbf{S B}_{\mathbf{j}}=\mathbf{S B}_{\mathbf{j}-1}+\left(\mathbf{P}_{\mathrm{korr} \mathrm{j}}-\mathbf{E T R}_{\mathbf{j}}\right)-\mathbf{S}^{*}$ \\
\hline $\mathrm{j}$ & $=$ Zeitindex $($ Dekaden $)$ \\
\hline $\mathrm{SB}_{\mathrm{j}-1}$ & $\begin{array}{l}=\text { Summe der bis zur Dekade j-1 seit Beginn der Ausschöpfung } \\
\text { entnommenen Bodenwassermenge }\end{array}$ \\
\hline$P_{\text {korr } j}$ & $=$ korrigierter Niederschlag der aktuellen Dekade \\
\hline $\mathrm{ETR}_{\mathrm{j}}$ & $=$ mittels Lysimeter ermittelte Verdunstung der aktuellen Dekade \\
\hline $\mathrm{S}^{*}$ & $\begin{array}{l}=\text { Versickerung bei zwischenzeitlicher Wiederauffüllung } \\
\text { aus der verdunstungsbeeinflussten Zone in die Dränwasserzone }\end{array}$ \\
\hline
\end{tabular}

Diesen Bilanzrechnungen liegt das Konzept der Kapazitäts- oder Speichermodelle wie z. B. bei GERIES (1989) oder den Modellen VERMO (GLUGLA, 1993), CANDY (FRANKO et al. 1995) und NUBALYS (HERSEMANN, 1997) zu Grunde. Bei diesem Ansatz steht nicht der Prozess der Sickerwasserbewegung im Vordergrund, sondern der Boden wird als ein Speicher angesehen, wobei Niederschlags- und Schneeschmelzmengen den Speicher auffüllen und Verdunstung sowie Abflusskomponenten (Versickerung) den Speicher entleeren.

Die Dynamik ist hauptsächlich auf den oberen Bereich des Speichers, auf die durchwurzelte (verdunstungsbeeinflusste) Zone beschränkt. Versickerung $\left(\mathrm{S}^{*}\right)$ aus der verdunstungsbeein- 
flussten Zone erfolgt erst nach ihrer Wiederauffüllung über die Feldkapazität (RENGER et al., 1974), in diesem Fall nach Erreichen des Ausgangszustandes.

Bei der Ermittlung der Bodenwasserausschöpfung wurde wie folgt vorgegangen:

- Die Berechnungen beginnen jeweils im Frühjahr, wenn auf Grund fehlender Vegetation und ausreichendem Niederschlag eine Auffüllung aller Böden bis zur Feldkapazität (oder bei Frühjahrsfeuchte mit Stauwasser auch darüber) erreicht ist. Bei den Löß-Böden wird eine vollständige Auffüllung nicht in jedem Frühjahr erreicht. Auf diesen Sachverhalt wird noch gesondert eingegangen.

Eine kontinuierliche Gewichtsabnahme (und damit der Beginn der Ausschöpfung durch die Pflanzen) ist zu verzeichnen, wenn $\left(\mathrm{P}_{\text {korr } \mathrm{j}}-\mathrm{ETR}_{\mathrm{j}}\right)$ im Verlauf mehrerer aufeinanderfolgender Zeitschritte negativ ist. Die Versickerung aus der verdunstungsbeeinflussten Zone ist mit Beginn der Ausschöpfung in der Regel abgeschlossen. Noch am Lysimeterauslauf auftretendes Sickerwasser resultiert aus dem relativ langen Sickerweg bis in $3 \mathrm{~m}$ Tiefe.

- Bei häufigen und starken Frühjahrsniederschlägen beginnt die Zehrung erst lange nach Pflanzenaufgang. Ist $\left(\mathrm{P}_{\text {korr }} \mathrm{j}-\mathrm{ETR}_{\mathrm{j}}\right)$ nach bereits begonnener Zehrung $>0$, erhöht diese Überschusswassermenge den Bodenwasservorrat. Wird dabei wieder der Ausgangswassergehalt (= Ausgangsgewicht $=$ Feldkapazität) erreicht, kommt es zur Versickerung aus dem Wurzelraum in den Unterboden. Nur besonders große Niederschläge führen infolge Druckfortpflanzung auch in $3 \mathrm{~m}$ Tiefe zur erhöhten Sickerwasserbildung (im Verlauf der in den meisten Jahren noch nicht abgeschlossenen Sickerwasserperiode).

- Da eine laterale Wasserbewegung im Lysimeter ausgeschlossen ist, müssen alle Wassermengen $\left(\mathrm{P}_{\text {korr } \mathrm{j}}-\mathrm{ETR}_{\mathrm{j}}\right)>0$, die nach Erreichen der Feldkapazität (=Ausgangsgewicht) infolge starker Sommerniederschläge im Verlauf der Ausschöpfung auftreten, zur Versickerung kommen. Dieses Wasser $\left(\mathrm{S}^{*}\right)$ führt zur Erhöhung des Wassergehaltes in der Dränwasserzone. In wenigen Fällen kommt es auch im Sommer zu einem (erhöhten) Sickerwasserauslauf in $3 \mathrm{~m}$ Tiefe wie z. B. infolge des Extremniederschlages im August 1983. Unklar ist, welchen Anteil die Pflanzen von dem schnell beweglichen Sickerwasser nutzen können. Bei der beschriebenen Vorgehensweise wird davon ausgegangen, dass die Pflanzen dieses Wasser nicht nutzen.

- Nach der Ernte beginnt in der Regel die Wiederauffüllung. Wenn Niederschlag fällt, ist $\left(\mathrm{P}_{\text {korr } \mathrm{j}}-\mathrm{ETR}_{\mathrm{j}}\right)$ meistens $>0$. Die Summierung von $\mathrm{SB}_{\mathrm{j}}=\mathrm{SB}_{\mathrm{j}-1}+\left(\mathrm{P}_{\mathrm{j}}-\mathrm{ETR}_{\mathrm{j}}\right)$ wird bis zur Wiederauffüllung (= Ausgangsgewicht) durchgeführt. Der Zeitpunkt für das Ende der Ausschöpfung ist sowohl im Fall der verschiedenen Böden als auch von Jahr zu Jahr sehr unterschiedlich und wird bei den Löß-Böden in einigen Jahren nicht erreicht.

Die Berechnungen wurden auf Basis der zu Dekadenwerten summierten Tageswerte durchgeführt, wobei auch eine Bilanzierung auf der Basis von Tageswerten möglich wäre.

Wichtiges Ergebnis dieser Bilanzrechnungen ist die in jedem Jahr und für jeden Boden ermittelte Endsumme der Ausschöpfung. Es handelt sich dabei um die von der Pflanze unter den gegebenen Einflussfaktoren (Bodenart, Pflanzenart und -entwicklung, Niederschlag und Verdunstungsanspruch der Atmosphäre) tatsächlich entnommene Bodenwassermenge.

Von besonderem Interesse ist die in den 17 Untersuchungsjahren auf den einzelnen Böden jeweils in einem Trockenjahr ermittelte größte (maximal) ausgeschöpfte Wassermenge (s. Kap. 3.4.1), die der nutzbaren Feldkapazität im effektiven Wurzelraum ( $\mathrm{nFK}_{\mathrm{We}}$ ) nach KA4 (1994) zu vergleichen ist. 


\subsubsection{Ermittlung der Ausschöpfungstiefe}

Nach der Ermittlung der tatsächlich entnommenen Bodenwassermenge interessiert, aus welcher Tiefe das Bodenwasser von der jeweiligen Pflanze im jeweiligen Boden und Jahresverlauf entnommen wurde. Erforderte die bisherige Modellvorstellung nur eine Diskretisierung der Zeit (Tages- bzw. Dekadenwerte), so ist es nun erforderlich, auch eine räumliche Diskretisierung vorzunehmen. Es erfolgte eine vertikale Aufteilung der Bodenprofile in einzelne Schichten von 0,5 dm. Auf Grundlage der vorhandenen Bodenkennwerte (Kap. 2.1.4) wurde für jede Schicht die nFK ermittelt. Mittels der Dekadensummen der Bodenwasserentnahme kann eine Abschätzung der Entnahmetiefen vorgenommen werden.

Es wird vereinfachend davon ausgegangen, dass die Wurzeln in jeder Bodenschicht gleichmäßig verteilt sind und nacheinander in jeder Schicht $100 \%$ der nFK ausgeschöpft werden. Des weiteren wird angenommen, dass jede neu erschlossene Bodenschicht eine Bodenfeuchte im Bereich der Feldkapazität hat. Zur rechentechnischen Realisierung der Berechnung von $\mathrm{We}_{\mathrm{j}}$ aus $\mathrm{SB}_{\mathrm{j}}$ wurde für jeden Boden für die Beziehung zwischen Profiltiefe We und $\Sigma \mathrm{nFK}$ eine Polynomfunktion erstellt. Mittels dieser Kurven erfolgte dekadenweise die Berechnung der entsprechenden Entnahmetiefen:

\begin{tabular}{|c|c|c|}
\hline Gl. 3: & $\mathrm{We}_{\mathbf{j}}=\mathrm{SB}_{\mathbf{j}} / \Sigma(\mathrm{nFK} * \mathbf{0 , 5})_{\mathrm{i}}$ & [dm] \\
\hline $\mathrm{j}$ & $=$ Zeitindex (Dekaden $)$ & {$[-]$} \\
\hline $\mathrm{i}$ & $=$ Laufindex über alle Bodenhorizonte bis $\mathrm{We}_{\text {eff }}$ & {$[-]$} \\
\hline $\mathrm{We}_{\mathrm{j}}$ & $=$ seit Beginn der Ausschöpfung erreichte Entnahmetiefe & {$[\mathrm{dm}]$} \\
\hline $\mathrm{SB}_{\mathrm{j}}$ & $\begin{aligned}= & \text { Summe der entnommenen Bodenwassermenge } \\
& \text { seit Beginn der Ausschöpfung }\end{aligned}$ & {$[\mathrm{mm}]$} \\
\hline $\mathrm{nFK}_{\mathrm{i}}$ & $=$ nutzbare Feldkapazität in der i-ten Schicht & {$[\mathrm{mm} / \mathrm{dm}]$} \\
\hline 0,5 & $=$ Schichtmächtigkeit & {$[\mathrm{dm}]$} \\
\hline
\end{tabular}

Auf diese Weise wird für jede Vegetationsperiode und für jeden Boden der Verlauf der Ausschöpfungstiefen ermittelt. Dabei werden jährlich sehr unterschiedliche Tiefen erreicht. Letztlich entspricht die im Verlauf der 17 Untersuchungsjahre absolut tiefste Ausschöpfung in einem Trockenjahr der effektiven Durchwurzelungstiefe ( $\left.\mathrm{We}_{\text {eff }}\right)$. Tatsächlich wird die Ausschöpfung so wie in Abb. 10 dargestellt verlaufen, so dass die tiefste Lage der Wasserscheide um einen hier nicht auszuweisenden Betrag (in $\mathrm{dm}$ ) unter der effektiven Durchwurzelungstiefe liegen wird. Die drei Tensiometer, die sich in Tiefenlagen von ca. $5 \mathrm{dm}, 15 \mathrm{dm}$ und $25 \mathrm{dm}$ in den Lysimetern befinden, reichten nicht aus, um den tatsächlichen Verlauf der Wasserscheide zu identifizieren.

Problematisch ist die Abschätzung der Entnahmetiefen bei den Löß-Böden, wenn die Winterniederschläge nicht ausreichten, die im vorangegangenen Sommerhalbjahr entstandenen Bodenwasserdefizite aufzufüllen und deshalb zu Beginn der Vegetationsperiode noch ein erhebliches Wasserdefizit aus dem Vorjahr zu verzeichnen ist (vgl. Tab. 16 in Kap. 3.4.1). Bei der Verteilung der Winterniederschläge im wasserdefizitären Bodenprofil wird angenommen, dass zunächst die oberen Bodenschichten aufgefüllt werden, während in den unteren Schichten ein Defizit verbleibt. 


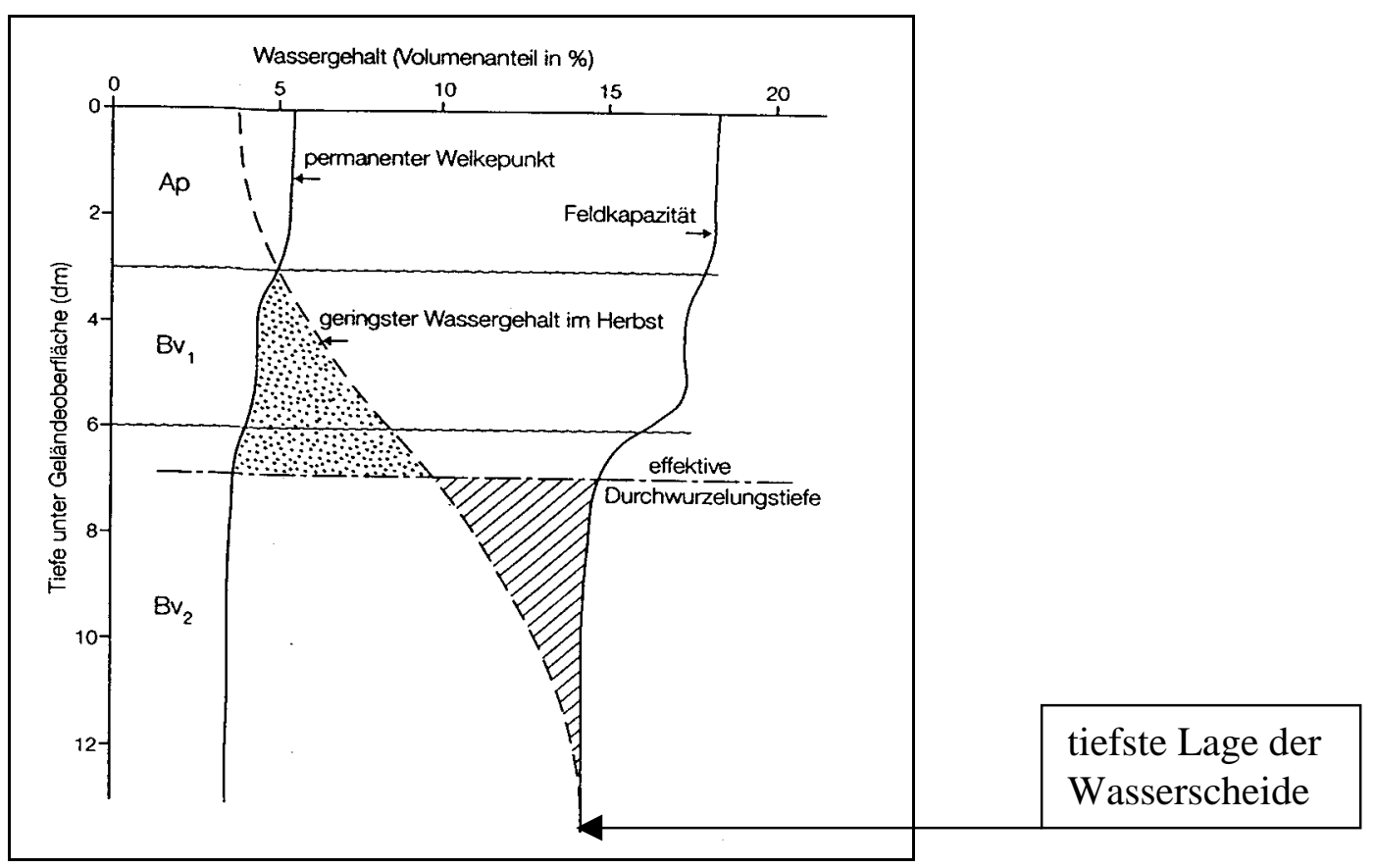

Abb. 10: Beispiel für die Ermittlung des effektiven Wurzelraumes (We eff) aus Feldkapazität, permanentem Welkepunkt und aktuellem Wassergehalt, dargestellt für den Herbst eines Trockenjahres auf einem Boden mit Feinsand (aus KA4, 1995 und SCHEFFER \& SCHACHTSCHABEL, 1992)

Bei der Beurteilung der Ergebnisse zum Verlauf der Ausschöpfungstiefen (Kap. 3.4.2) ist zu hinterfragen, ob die effektive Durchwurzelungstiefe (oder effektiver Wurzelraum) tatsächlich ein Bodenkennwert ist oder ob eine Abhängigkeit von den klimatischen Bedingungen am jeweiligen Untersuchungsstandort besteht.

In diesen Zusammenhang stellt sich auch die Frage nach den Kriterien eines „Trockenjahres“. Enthält der Untersuchungszeitraum 1981-97 tatsächlich alle regionaltypischen Witterungsextreme des Standortes? Die Beantwortung dieser Frage wird in den Kapiteln 3.3 und 4.2 erfolgen.

\subsubsection{Potentielle und reale Verdunstung - Wasserverbrauch der Pflanzen}

Verdunstung (Evapotranspiration) ist die pro Flächen- und Zeiteinheit in die Atmosphäre transferierte Wassermenge. Die Atmosphäre nimmt auf Grund ihres aktuellen Zustandes stets nur eine bestimmte Menge Wasserdampf auf. Dieser obere Grenzwert, der Verdunstungsanspruch der Atmosphäre (potentielle Verdunstung), wird in der Regel unter der Annahme unbegrenzter Wasserverfügbarkeit aus meteorologischen Größen berechnet. Das Attribut potentiell wird dabei sowohl auf Evaporation und Transpiration gesondert als auch auf Evapotranspiration insgesamt bezogen (DVWK, 1996). Kann eine potentielle Verdunstung infolge von Wassermangel (mangelnde Bodenfeuchte) nicht realisiert werden, resultiert daraus die reale Verdunstung, wobei für Evaporation und Transpiration unterschiedliche Abhängigkeiten von der Bodenfeuchte bestehen.

Evaporation ist die Verdunstung von der unbewachsenen oder versiegelten Bodenoberfläche (auch Schnee- und Eisverdunstung) und von freien Wasserflächen (Gewässerverdunstung) sowie von der Oberfläche der Pflanzen (Interzeptionsverdunstung). 
Der Begriff Transpiration steht für die Pflanzenverdunstung (Wasserdampfabgabe aus dem Pflanzenorganismus heraus in die Atmosphäre). Der Wassertransport zur Pflanzenwurzel, die Wasseraufnahme sowie der Transport durch die Pflanze und die Wasserabgabe an der Pflanzenoberfläche werden vorrangig durch die zumeist bestehende Potentialdifferenz zwischen Boden und Atmosphäre gesteuert (EHLERS, 1996; SEIDLER, 1999). Verschiedene Widerstände behindern diese Prozesse (KUNDLER, 1989). Umfang und zeitlicher Verlauf dieser Einflussnahme hängen, neben Pflanzenart und Bodentyp, ab

- vom aktuellen Matrix-(Wasser)potential im Boden und der hydraulischen Leitfähigkeit

- vom Zustand der Atmosphäre, wie Strahlungsbilanz (Nettostrahlung), Sättigungsdefizit und Wärmeadvektion (Turbulenz),

- von den Pflanzen selbst (Wachstum der Wurzeln und der oberen Pflanzenteile, Regulierung der Wasserabgabe durch Öffnen und Schließen der Stomata, Veränderung der effektiven Blattoberfläche z. B. durch Blattrollen),

- von der Nährstoffversorgung der Pflanzen.

Transpiration und Evaporation sind an der Verdunstung (potentiell und/oder real) in unterschiedlichen Teilraten beteiligt, deren Anteil im Verlauf der Vegetationsentwicklung stark variiert. Im Sommerhalbjahr bei geringer Feuchte im Oberboden oder dichtem Bestand geht die Verdunstung von der Bodenoberfläche gegen Null. Bei spärlichem Bewuchs nimmt dagegen der Anteil der Transpiration an der Gesamtverdunstung stark ab. Wie bereits beschrieben, werden mittels der Brandiser Lysimeter Tageswerte der (Gesamt-)Verdunstung (Evapotranspiration) ermittelt. Es kann also nicht zwischen den beiden Teilkomponenten Transpiration und Evaporation unterschieden werden. Dies ist insbesondere bei der Ermittlung der Transpirationskoeffizienten (Kap. 3.5.7) von Bedeutung.

Obwohl bereits sehr detaillierte Modelle zur Berechnung der realen Verdunstung existieren, ist es weiterhin sehr schwierig, die erforderlichen Parameter $\mathrm{zu}$ quantifizieren (DIEKKRÜGER, 1992). Deshalb kommen zur Berechnung der Verdunstung häufig vereinfachte Modelle zur Anwendung, die von der Berechnung der potentiellen Verdunstung eines über das ganze Jahr einheitlichen Grasbestandes $\left(\mathrm{ETP}_{\mathrm{Gras}}\right)$ ausgehen und anschließend mittels Bestandeskoeffizienten $\left(\mathrm{k}_{\mathrm{c}}\right)$ die kulturartenspezifische potentielle Verdunstung

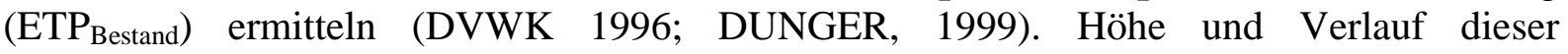
landnutzungsabhängigen maximalen Verdunstung $\left(\mathrm{ETP}_{\text {Bestand }}=\mathrm{k}_{\mathrm{c}} * \mathrm{ETP}_{\text {Gras }}\right)$ resultiert aus dem Entwicklungsstand der jeweiligen Fruchtart bei ausreichendem Wasserangebot des Bodens (SOKOLLEK, 1983; DUNGER, 1999; SAMBALE \& PESCHKE, 2000). Die Koeffizienten $\mathrm{k}_{\mathrm{c}}$ berücksichtigen die Bestandshöhe und den Bedeckungsgrad des Bodens mit Pflanzen. Bedeckt der Bestand den Boden noch nicht vollständig, kann $\mathrm{k}_{\mathrm{c}}$ kleiner als 1 sein und bei voll entwickeltem Bestand bis etwa 1,5 ansteigen (SPONAGEL, 1980; GÜNTHER, 1999). Dabei bedeutet $k_{c}=1$, dass die Bestandesverdunstung gleich der von Gras ist.

In Kapitel 3.5 erfolgt die Berechnung von $\mathrm{k}_{\mathrm{c}}$-Werten für die verschiedenen Fruchtarten aus potentieller Verdunstung und der Verdunstung der Lößböden und ein Vergleich mit entsprechenden Werten aus der Literatur. Auf diese Weise kann geprüft werden, ob die Verdunstung der Lysimeter mit Löß-Schwarzerde und Löß-Parabraunerde (beide mit einer hohen kapillaren Wasseranlieferung aus dem Unterboden) einer kulturartenspezifischen potentiellen Verdunstung entspricht. 
Kenntnisse über die kulturartenspezifische potentielle Verdunstung (=potentielle Wasserbedarf) werden vom Landwirt für bestandsbildende Maßnahmen hauptsächlich für die Hauptvegetationsperiode (von Bestandsschluss bis beginnende Reife) benötig (GÜNTHER, 1999). In diesem Zusammenhang können die Messreihen der Lysimeter mit Löß-Schwarzerde und Löß-Parabraunerde zur Bereitstellung von $\mathrm{k}_{\mathrm{c}}$-Werten (ergänzend $\mathrm{zu}$ den Literaturwerten) genutzt werden.

Des weiteren interessiert die Effizienz, mit der die Pflanzen das aufgenommene Wasser zur Ertragsbildung genutzt haben. Zur Bildung von $1 \mathrm{~kg}$ Trockenmasse (TM) werden je nach Pflanzenart und Umweltbedingungen rd. 200 - 6001 Wasser benötigt (SCHILLING, 1982; ROTH et. al, 1988; LARCHER, 1994). Das Verhältnis von Wasserverbrauch zur Trockenmasse wird als Transpirationskoeffizient bezeichnet, von EHLERS (1996) besser als Evapotranspirationskoeffizient, weil i. a. beide Komponenten einfließen, die insbesondere bei Lysimetermessungen nicht getrennt ermittelt werden. Die Ermittlung und Beschreibung von Evapotranspirationskoeffizienten erfolgt in Kapitel 3.5.7.

Ausgehend von der kulturartenspezifischen potentiellen Verdunstung wird anschließend der Wert der realen Verdunstung (ETR) ermittelt. Dabei sind bei bewachsenen Standorten die aktuelle Bodenfeuchte, ihre vertikale Verteilung sowie die Tiefe und Dichteverteilung der Pflanzenwurzeln von besonderer Relevanz. KOITZSCH (1977) und KOITZSCH (1980) entwickelten ein Modell, das den Wasserentzug (Verdunstung) für Pflanzen mit beliebiger Wurzelverteilung in Abhängigkeit von der Vegetationsüppigkeit und der Bodenfeuchte beschreibt. Eine noch einfachere Modellvorstellung besteht darin, alle Einflüsse, die zu einer Reduzierung der Verdunstung gegenüber dem potentiellen Wert von ETP $_{\text {Bestand }}$ führen, zu einem Abminderungsfaktor $\phi$ zusammenzufassen, der direkt als Funktion (fkt) der verfügbaren Bodenfeuchte dargestellt werden kann:

$$
\phi=\mathrm{ETR} / \mathrm{ETP}_{\mathrm{Bestand}}=\mathrm{fkt}(\Theta)
$$

Hierbei ist $\Theta$ die aktuelle Bodenfeuchte bzw. der relative Bodenwasservorrat in $\% \mathrm{nFK}_{\mathrm{We}}$. Eine übersichtliche Darstellung von Berechnungsformeln enthält DISSE (1995).

Mittels wägbarer Lysimeter kann die tägliche Verdunstung aus der Wasserhaushaltsgleichung berechnet werden. Für die Entwicklung von Verdunstungsmodellen ist dies eines der wichtigsten Resultate der Lysimeteruntersuchungen. Aus den Messungen können reale Verdunstung und gleichzeitig der entsprechende Verlauf der Bodenfeuchte (= relativer Bodenwasservorrat in $\% \mathrm{nFK}_{\mathrm{We}}$ ) ermittelt werden. Somit kann die Reaktion der Pflanzen in den einzelnen Aufwuchsstadien (die in Modellen schwer nachzubilden ist) auf den aktuellen Zustand der Atmosphäre und die Wasser- und Nährstoffentwicklung in den verschiedenen Böden analysiert und beschrieben werden. Die Funktion $\phi$ kann für die untersuchten Fruchtarten und Böden abgeleitet und mit bereits vorliegenden Ergebnissen aus der Literratur verglichen werden. Dies erfolgt an einem Beispiel in Kapitel 3.5.

Zur Berechnung der potentiellen Verdunstung stehen zahlreiche Verfahren zur Verfügung. Sie beziehen sich in der Regel (Ausnahmen: Verdunstungskessel s. GÜNTHER, 1999; Keramische Scheibe nach CZERATZKI s. GENID, 1982) auf das ganze Jahr kurz gehaltene, gut mit Wasser versorgte Grasflächen und berücksichtigen die meteorologischen Einflussfaktoren in unterschiedlicher Anzahl und Genauigkeit. So liefern alle Ansätze unterschiedliche Ergebnisse, wobei einige Verfahren häufig zur Anwendung kommen und damit regional unterschiedlich Einfluss auf die Verdunstungsmodelle und somit auf die Untersuchungsergebnisse in der Land- und Wasserwirtschaft genommen haben. Ein Vergleich der wichtigsten Verfahren erfolgt in Kapitel 3.2. 


\subsubsection{Sickerwassermenge und Verlagerungsrisiko von Nitrat}

Zur Abschätzung der vom Pflanzennährstoff Stickstoff $(\mathrm{N})$ ausgehenden Gefährdung für die Gewässer, hat die N-Bilanzmethode eine breite Anwendung gefunden (WALTHER et al., 1985; FREDE \& DABBERT, 1999). Bei der schlagbezogenen N - Bilanz - Methode wird ein N-Saldo nach folgendem Kalkulationschema berechnet:

\section{N-Saldo = mineralische N-Düngung plus bilanzwirksamer organischer Düngerstickstoff minus $\mathbf{N}$-Abfuhr durch Ernteprodukte in $\mathrm{kg} / \mathrm{ha}$}

Eine Berücksichtigung von N-Transformationen (Immobilisierung, Mobilisierung) erfolgt bei dieser Betrachtungsweise in der Regel nicht. Es wird davon ausgegangen, dass die NImmobilisierung und N-Mobilisierung im Boden im Gleichgewicht stehen. Das N-Saldo liefert einen Hinweis auf das ,nutzungsbedingte Verlagerungsrisiko“, wobei angenommen wird, dass durch die Nutzung insbesondere der Pool an mobilen und damit leicht verlagerbaren Stoffen beeinflusst wird (HENNINGS \& SCHEFFER, 1999).

Zur Abschätzung des ,standörtlichen Verlagerungsrisikos“ kommt häufig eine Methode zur Anwendung, die von einer vereinfachenden Vorstellung der in der Dränwasserzone ablaufenden Wassertransport- und -austauschprozesse ausgeht (DIN 19732). Mit Kenntnis der Sickerwassermenge und der Feldkapazität der Bodenschichten wird die Verlagerungsgeschwindigkeit für den kapillaren Wasserfluss abgeschätzt (AG Bodennutzung, 1992; DVWK, 1994; FREDE \& DABBERT, 1999):

\section{Sickerwasserrate ( $\mathrm{mm} /$ Zeitintervall)

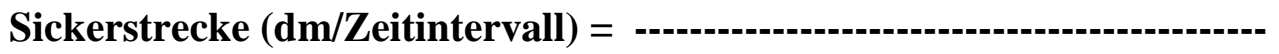 \\ Feldkapazität (mm/dm).}

Diese Abschätzung gilt auch für die Verlagerungsgeschwindigkeit von Nitrat, das eine hohe Löslichkeit besitzt und in der Regel nicht mit der Bodenmatrix reagiert. Damit kann ein konvektiver Massentransport vorausgesetzt werden. Bevorzugte Fließwege und Prozesse wie Diffusion und Dispersion finden keine Berücksichtigung. Bei diesem Ansatz zur Abschätzung der vertikalen Verlagerung gelöster Stoffe, wird dem Fließquerschnitt mit dem Wassergehalt bei Feldkapazität entsprochen und somit ein möglicher ,immobiler“ Bodenwasseranteil, wie u. a. von DUYNISVELD (1984) und ZEPP (1988) beschrieben, ausgeschlossen.

Seit Beginn der Messungen im Jahr 1980 wurde das Sickerwasser kontinuierlich auf den Gehalt an Stickstoffkomponeten untersucht. Des weiteren erfolgte die Ermittlung der NEinträge über die Niederschläge (nasse Deposition), die Höhe der mineralischen N-Düngung sowie der N-Abfuhr durch das Erntegut. Deshalb ist es möglich, für die verschiedenen Böden unter den gegebenen Witterungsbedingungen am Standort Brandis einen Zusammenhang zwischen N-Saldo, Fruchtart, Ertrag und N-Austrag über das Sickerwasser in $3 \mathrm{~m}$ Tiefe herzustellen (s. Kap. 3.7.2). Es galt zu prüfen, ob auf Basis der jährlich ermittelten Austauschhäufigkeit des Wassers im effektiven Wurzelraum und der Sickerwasser-Verlagerungsgeschwindigkeit in der Dränwasserzone, Erklärungen zum Verbleib der ermittelten NBilanzüberschüsse gefunden werden können. 


\subsubsection{Untersuchungen zur hydrologischen Wirksamkeit der verschiedenen Böden}

Eine übliche Herangehensweise bei der Übertragung vom Daten des Standortes auf das Einzugsgebiet ist die Unterteilung des Gebietes in Flächen, die bezüglich ihrer hydrologischen Eigenschaften homogen sind. Berücksichtigt werden in der Regel Klima, Relief, Boden, Grundwasserflurabstand und Landnutzung. Mittels GIS-Modulen entsteht in Abhängigkeit von der räumlichen Variabilität dieser Eigenschaften ein Flächenmosaik von meist sehr vielen Teilflächen, auf die ein am Standort geeichtes Berechnungsverfahren angewendet wird.

Die große Anzahl von Teilflächen, die aus der Verschneidung o. g. Standorteigenschaften eines Gebietes resultiert, hat ihre Ursache vorwiegend in der Anzahl verschiedener Böden, die auf Grund ihrer unterschiedlichen Prozesswirksamkeit berücksichtigt werden sollen. Untersuchungsergebnisse u. a. von GERIES (1989), HOSANG (1995) und SCHEUERER (1998) deuten darauf hin, dass der Einfluss verschiedener Böden mit flurfernen Grundwasserständen auf den Wasserhaushalt durchaus geringer ist, als bisher angenommen. So wird angestrebt, verschiedene Böden (bei gleicher Flächennutzung und flurfernen Grundwasserständen) zu Pedo-Hydrotopen „Areale gleichen bodenwasserhaushaltlich wirksamen pedophysikalischen Aufbaues“ (JORDAN, 1978) bzw. zu Flächen gleicher hydrologischer Wirksamkeit (ETZENBERG, 1998) zusammenfassen.

Auf Basis der vorliegenden Messreihen von Verdunstung und Sickerwasserbildung der acht verschiedenen Böden wurde geprüft, ob eine Zusammenfassung von Böden zu o. g. PedoHydrotopen möglich ist.

Witterung und Bewirtschaftung sind im „Versuchsaufbau“ der Station Brandis für alle Böden (Standorte) gleich, so dass ideale Voraussetzungen bestanden, die Reaktionen der acht verschiedenen Böden - messbar an den Wasserhaushaltsgrößen Verdunstung und Sickerwasserbildung - vergleichend zu analysieren. Um Hinweise auf Heterogenitäten innerhalb eines Bodens zu erhalten, wurden die drei Einzellysimeter „Wiederholungen“ von jeder der acht Herkunftsflächen in die Untersuchungen einbezogen. Die Variabilität, die sich im Verlauf der Messreihen von Verdunstung und Sickerwasserbildung dieser drei Wiederholungen zeigt, liefert trotz der geringen Anzahl der Wiederholungen Hinweise auf die Wirksamkeit von Bodenheterogenitäten auf die Prozesse Verdunstung und Sickerwasserbildung. Damit stellten sich zwei Fragen:

$\rightarrow$ Zeigen die drei Bodenmonolithe (Wiederholungen) eines Entnahmeortes infolge der räumlichen Heterogenität der Bodenparameter eine größere Varianz der Meßgrößen (Merkmale) als die Meßgrößen verschiedener Herkunftsflächen?

$\rightarrow$ Wie groß sind die Unterschiede, die acht verschiedene Böden (= acht Lysimetergruppen) bei gleicher Witterung und Bewirtschaftung auf den Verlauf der Wasserhaushaltsgrößen Verdunstung und Sickerwassermenge ausüben?

Für diese Untersuchungen wurden die Meßreihen aller 24 wägbaren Lysimeter verwendet. Ausgangspunkt bildeten die Jahresmittelwerte von Verdunstung und Sickerwassermenge bis zur Lysimeterzerlegung im Jahr 1994. Es zeigte sich erwartungsgemäß eine Rangfolge der Böden entsprechend der Höhe ihrer Versickerungsleistung und Verdunstung (Tab. 41 in Kap. 4.1.1). Auf der Grundlage dieser Rangfolge und unter Berücksichtigung der bodenkundlichen und hydrogeologischen Beschreibung der acht untersuchten Herkunftsorte erfolgte eine Zusammenfassung der acht Böden (Lysimetergruppen) zu vier Pedo-Hydrotopen, die mit Top a bis d bezeichnet wurden (Tab. 9). Zu prüfen war, ob sich diese Gruppierung für die Messwerte (Prüfmermale) Sickerwasserbildung und Verdunstung statistisch belegen lässt. 
Grundlage der Untersuchungen bildeten die Monatssummen der Jahre 1981-94. Die Auswertung erfolgte als einfache Varianzanalyse mit ungleichen Wiederholungen (BÄTZ et al., 1982):

- Prüffaktor = acht verschiedene Böden Tab.9,

- Prüfmerkmale $=$ Verdunstung und Sickerwassermenge,

- $\quad$ konstante Faktoren = Acker- und pflanzenbauliche Maßnahmen,

- Zufallseinflüsse = Witterungsschäden und Krankheitsbefall der Pflanzen,

- exogene Faktoren = Witterung, Fruchtfolge .

Geprüft wird, welche Auswirkungen die unterschiedlichen Abstufungen des Prüffaktors (Boden) auf die beiden Prüfmerkmale Verdunstung und Sickerwassermenge haben. Der Grundgedanke der Varianzanalyse besteht darin, den Einfluss des Prüffaktors auf die Varianz der untersuchten Merkmale zu erfassen und von den Zufallseinflüssen zu trennen. Der Einfluss des Prüffaktors ist statistisch gesichert, wenn die durch ihn verursachte Varianz im Prüfmerkmal wesentlich größer als die Restvarianz ist. Die Signifikanz der Unterschiede wurde mittels F-Test ermittelt.

Tab. 9: Anzahl der Bodentypen und Wiederholungen je Pedo-Hydrotop

\begin{tabular}{|l|c|c|c|c|}
\hline Pedo-Hydrotop & a & b & c & d \\
\hline Anzahl der Böden & 1 & 2 & 3 & 2 \\
(Lys.-Gr. 5 ) & $\begin{array}{c}\text { (Lys.-Gr. 4 } \\
\text { und 8) }\end{array}$ & $\begin{array}{c}\text { (Lys.-Gr. 1, 7 } \\
\text { und 11) }\end{array}$ & $\begin{array}{c}\text { (Lys.-Gr. 9 } \\
\text { und 10) }\end{array}$ \\
$\begin{array}{l}\text { Anzahl der Wieder- } \\
\text { holungen }\end{array}$ & 3 & 6 & 9 & 6 \\
\hline
\end{tabular}

Es wird davon ausgegangen, dass eine Zusammenfassung verschiedener Böden zu einem Pedo-Hydrotopen nur dann erfolgen sollte, wenn sich die Variabilität von Sickerwassermenge und Verdunstung der Böden eines Pedo-Hydrotopes nicht vom Resteinfluß unterscheidet (pLevel > 0,05). Es wurden also die Monate ausgewiesen (verworfen), in denen sich die Varianz der „Prüfmerkmale“ (Sickerwasserhöhe oder Verdunstung) zwischen den Böden eines Pedo-Hydrotops signifikant von der Variabilität innerhalb der Böden unterscheidet. Dies waren von 168 Monaten nur wenige \% (s. Kap. 4.1.1). Für den Sandboden (Top a) erfolgten keine Berechnungen, da dieser Pedo-Hydrotop nur durch eine Lysimetergruppe vertreten ist. Des weiteren wurde geprüft, ob möglicherweise noch die Pedo-Hydrotopen „,b“ und „,c“ zusammengefasst werden können (Ergebnisse und Schlussfolgerungen s. Kap. 4.1.1). 


\section{Untersuchungsergebnisse}

\subsection{Niederschlagshöhe und-verteilung am Untersuchungsstandort}

Höhe und innerjährliche Verteilung des Niederschlages sind Ausdruck des bereits kontinental beeinflussten Klimas am Standort Brandis. Wie aus Tab. 10 ersichtlich, schwanken die mittleren korrigierten Jahresniederschläge zwischen 508 mm (1991) und 808 mm (1994).

Von den $655 \mathrm{~mm}$ Jahresniederschlag fallen $54 \%$ im Sommerhalbjahr. Dabei beinhaltet das Verhältnis zwischen Sommer- und Winterniederschlag eine größere Variabilität als der Jahresniederschlag. Die Monate Juni und August sind im Mittel der Untersuchungsperiode mit reichlich $70 \mathrm{~mm}$ die niederschlagsreichsten Monate. Wie aus Abb. 11 hervorgeht ist der Niederschlag im Mittel der Jahre von April bis Oktober gleich oder geringer als die potentielle Verdunstung. Deshalb wirkt sich die Höhe der Sommerniederschäge unmittelbar auf die Intensität der Bodenwasserentnahme aus (s. Kap. 3.4.1).

Tab. 10: Korrigierter Niederschlag [mm] am Standort Brandis und statistische Maßzahlen (Wasserhaushaltsjahre 1981-97)

\begin{tabular}{|c|c|c|c|c|c|c|c|c|c|c|c|c|c|c|c|c|}
\hline JAHR & NOV & DEZ & JAN & FEB & MÄR & APR & MAI & JUN & JUL & AUG & SEP & OKT & JAHR & WI & SO & \begin{tabular}{|l|} 
So/Wi \\
\end{tabular} \\
\hline 1981 & 61 & 39 & 58 & 27 & 97 & 96 & 40 & 58 & 100 & 40 & 67 & 71 & 753 & 377 & 375 & 0.99 \\
\hline 1982 & 81 & 69 & 36 & 9 & 23 & 29 & 66 & 79 & 50 & 29 & 8 & 49 & 528 & 247 & 281 & 1.14 \\
\hline 1983 & 23 & 41 & 83 & 27 & 50 & 129 & 75 & 58 & 28 & 173 & 43 & 10 & 739 & 353 & 387 & 1.10 \\
\hline 1984 & 35 & 46 & 39 & 39 & 9 & 69 & 58 & 68 & 65 & 83 & 78 & 42 & 630 & 237 & 393 & 1.66 \\
\hline 1985 & 46 & 29 & 36 & 22 & 43 & 72 & 30 & 62 & 61 & 74 & 37 & 10 & 521 & 247 & 273 & 1.10 \\
\hline 1986 & 39 & 76 & 51 & 19 & 55 & 46 & 88 & 65 & 50 & 54 & 37 & 79 & 658 & 286 & 373 & 1.31 \\
\hline 1987 & 15 & 101 & 76 & 56 & 22 & 38 & 76 & 84 & 72 & 89 & 62 & 12 & 702 & 307 & 395 & 1.29 \\
\hline 1988 & 61 & 55 & 45 & 71 & 67 & 12 & 23 & 82 & 60 & 34 & 46 & 30 & 585 & 311 & 275 & 0.88 \\
\hline 1989 & 73 & 115 & 30 & 45 & 37 & 86 & 19 & 50 & 49 & 63 & 34 & 43 & 642 & 384 & 257 & 0.67 \\
\hline 1990 & 87 & 64 & 16 & 55 & 25 & 49 & 3 & 147 & 23 & 86 & 81 & 25 & 661 & 296 & 365 & 1.23 \\
\hline 1991 & 94 & 41 & 23 & 20 & 30 & 44 & 45 & 110 & 20 & 42 & 22 & 18 & 508 & 253 & 255 & 1.01 \\
\hline 1992 & 46 & 63 & 59 & 49 & 93 & 32 & 28 & 37 & 119 & 53 & 47 & 60 & 685 & 342 & 343 & 1.00 \\
\hline 1993 & 47 & 48 & 77 & 35 & 22 & 36 & 87 & 101 & 123 & 64 & 74 & 33 & 747 & 265 & 482 & 1.82 \\
\hline 1994 & 54 & 89 & 49 & 22 & 125 & 80 & 90 & 23 & 36 & 144 & 60 & 37 & 808 & 418 & 390 & 0.93 \\
\hline 1995 & 52 & 47 & 49 & 52 & 42 & 107 & 59 & 98 & 58 & 112 & 101 & 11 & 786 & 348 & 438 & 1.26 \\
\hline 1996 & 72 & 33 & 6 & 25 & 22 & 27 & 77 & 50 & 123 & 40 & 58 & 51 & \begin{tabular}{|l|l|}
583 \\
\end{tabular} & 185 & 399 & 2.16 \\
\hline 1997 & 70 & 33 & 18 & 64 & 45 & 50 & 45 & 54 & 117 & 16 & 35 & 49 & 595 & 279 & 316 & 1.13 \\
\hline Mittel & 56 & 58 & 44 & 37 & 47 & 59 & 53 & 72 & 68 & 70 & 52 & 37 & 655 & 302 & 353 & 1.17 \\
\hline Max. & 94 & 115 & 83 & 71 & 125 & 129 & 90 & 147 & 123 & 173 & 101 & 79 & 808 & 418 & 482 & 2.16 \\
\hline Min. & 15 & 29 & 6 & 9 & 9 & 12 & 3 & 23 & 20 & 16 & 8 & 10 & 508 & 185 & 255 & 0.67 \\
\hline Varianz & 477 & 625 & \begin{tabular}{|l|}
489 \\
\end{tabular} & 326 & 1001 & 1029 & 722 & 894 & 1271 & 1742 & 555 & 458 & 8699 & 3809 & 4426 & 0.13 \\
\hline$[\mathrm{mm}]$ & 21.8 & 25.0 & 22.1 & 18.1 & 31.6 & 32.1 & 26.9 & 29.9 & 35.7 & 41.7 & 23.6 & 21.4 & 93 & 62 & 67 & 0.37 \\
\hline $\mathrm{c}_{\mathrm{v}}[\%]$ & 39 & 43 & 50 & 48 & 67 & 54 & 50 & 41 & 53 & 59 & 45 & 58 & 14 & 20 & 19 & 31 \\
\hline
\end{tabular}

$s_{x}$ - Standardabweichung, $c_{v}$-Variationskoeffizient

Die vergleichsweise geringe Anzahl von Tagen mit messbarem Niederschlag in den Sommermonaten Abb. 11 ist ein Hinweis darauf, dass hohe Monatssummen vielfach aus Starkregen resultieren. Am niederschlagsärmsten sind mit rd. $35 \mathrm{~mm}$ die Monate Februar und Oktober. Sehr gering (4\%) ist der Anteil des festen Niederschlages am Gesamtniederschlag. 

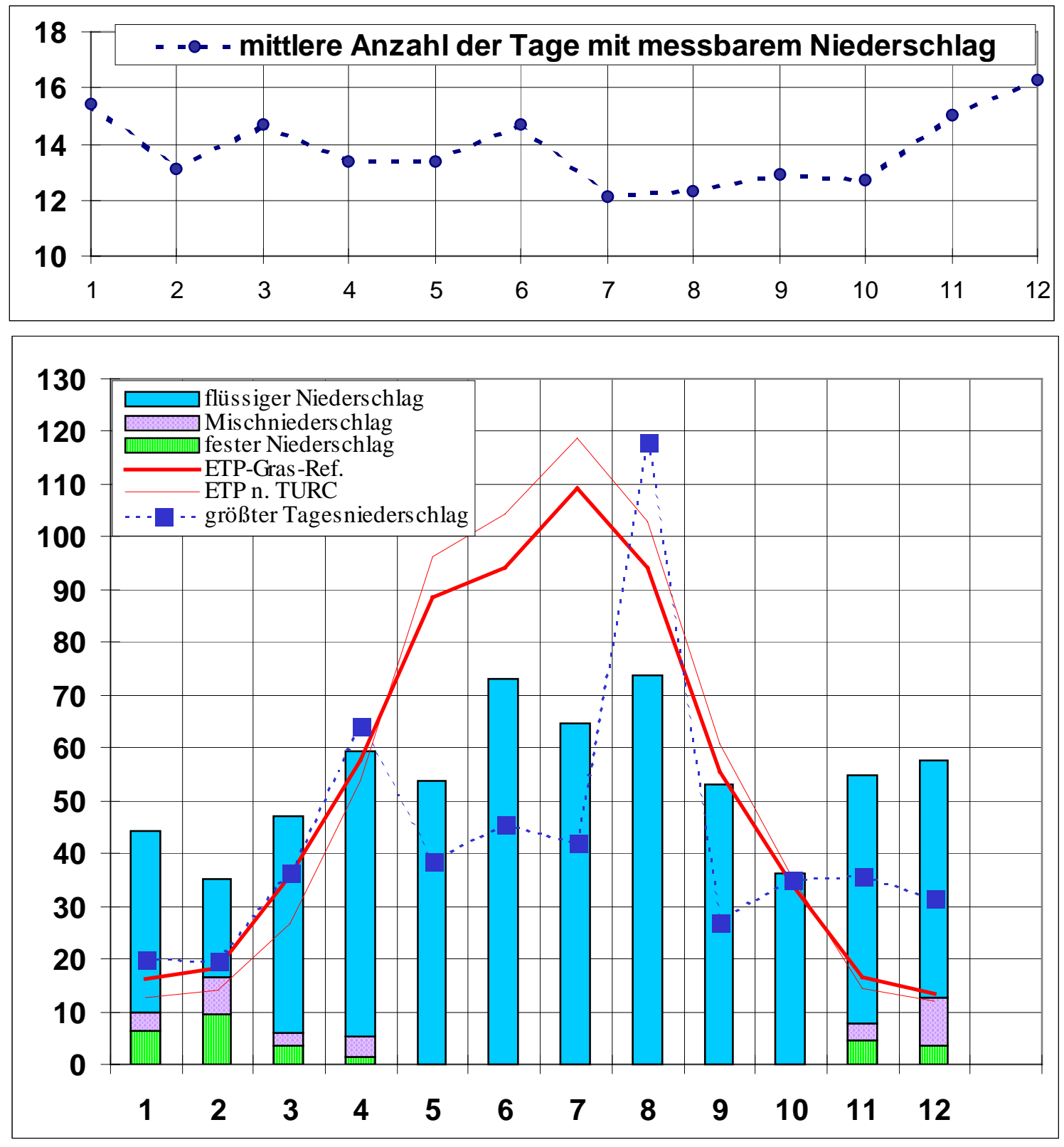

Abb. 11: Mittlere Anzahl der Tage mit messbarem Niederschlag, sowie potentielle Verdunstung (ETP) und Niederschlagsregime der Station Brandis (Reihe 1981-97, korrigierte Niederschläge) [Angaben in $\mathrm{mm}$ ]

\subsection{Vergleichsrechnungen zur Ermittlung der potentiellen Verdunstung}

\subsubsection{Verwendete Verfahren zur Berechnung der potentiellen Verdunstung}

Die potentielle Verdunstung wurde in den alten Bundesländern bisher meist nach HAUDE und in den neuen Bundesländern nach TURC/IVANOV oder WENDLING berechnet. Die zum Teil stark differierenden Ergebnisse dieser und anderer empirischer Verfahren haben sich in der Vergangenheit zunehmend als problematisch erwiesen.

Zur Nivellierung der teils auf sehr verschiedenen Annahmen beruhenden Berechnungsansätze (s. DVWK, 1996) wurde vom DWD die von der FAO als Standard empfohlene „GrasReferenzverdunstung" eingeführt (WENDLING, 1995). 
Auf Grund der Bedeutung der verschiedenen Berechnungsverfahren für Bodenwasserhaushaltsuntersuchungen erscheint es notwendig, für den Standort Brandis vergleichende Berechnungen durchzuführen. Nachfolgend werden die für diese Vergleichsrechnungen ausgewählten Verfahren erläutert. Zur Erklärung der Formelzeichen s. Kapitel 7.

Die höchsten Anforderungen an das meteorologische Datenmaterial stellt das Verfahren von PENMAN-MONTEITH (Gl. 4). Eigene Untersuchungen wurden mit diesem Verfahren nicht durchgeführt. Zur Anwendung kam die Gras-Referenzverdunstung und die von WENDLING et al. (1991) erarbeitete Modifizierung der PENMAN-Formel (Gl. 5).

Das Verfahren von TURC/IVANOV (Gl. 6 und Gl. 7) und die ebenfalls von WENDLING et al. (1991) modifizierte TURC-Formel (Gl. 9) benötigen Tagesmittel der Temperatur, Luftfeuchte, Globalstrahlung und/oder Sonnenscheindauer, aber keine Angaben zur Windgeschwindigkeit. Die HAUDE-Formel Gl. 10 basiert ausschließlich auf Meßwerten der Lufttemperatur und -feuchte (Sättigungsdefizit) um 14 Uhr.

\section{Das Verfahren von PENMAN-MONTEITH}

beruht auf einer rein physikalischen Betrachtungsweise und ist deshalb auch das aufwendigste Verfahren. Es bildet die Grundlage für viele abgeleitete, halbempirische Verfahren (z. B. WENDLING, 1991). Bei Kenntnis der entsprechenden Albedo, der Pflanzenhöhe, des Bedeckungsgrades und der verminderten Wasserverfügbarkeit (Stomatawiderstand $>70 \mathrm{~s} / \mathrm{m}$ ) kann mit der PENMAN-Gleichung neben der kulturartenspezifischen maximalen auch die tatsächliche (reale) Verdunstung eines beliebigen Pflanzenbestandes berechnet werden:

$$
\text { Gl. 4: } \quad \quad \quad E T P=1 / \lambda \cdot\left(s \cdot\left(R_{n}-G\right)+p_{L} \cdot c_{p} \cdot\left(\left(E_{2 m}-e_{2 m}\right) / r_{a}\right)\right) /\left(s+\gamma \cdot\left(1+\left(r_{s} / r_{a}\right)\right)\right)
$$

Die PENMANMONTEITH-Gleichung „bildet heute die Basis für die modellmäßige Behandlung des Verdunstungsprozesses im System-Boden-Pflanze-Atmosphäre unter Berücksichtigung des gesamten Netzwerkes der Widerstände, die dem Wasser- bzw. Wasserdampftransport vom Boden durch die Pflanze und die Grenzschicht zwischen Pflanzenbestand und Atmosphäre entgegenstehen, so dass sowohl die aerodynamischen Einflüsse auf den Transport von fühlbarer und latenter Wärme als auch pflanzenphysiologische und bestandespezifische Merkmale der Vegetation erfasst werden können“ (SCHRÖDTER, 1985; DISSE, 1995).

\section{Die Gras-Referenzverdunstung}

Sie wird mit der PENMAN-MONTEITH-Gleichung für eine Gras-Referenzfläche nach ALLEN et al. (1994) mit

- Albedo $\alpha=23 \%$ für Gras

- aerodynamischer Widerstand $r_{d}$ bei einer Grashöhe von $12 \mathrm{~cm}$ und einem Bedeckungsgrad von 1

- minimaler Stomatawiderstand $r_{\mathrm{s}, \min }=70 \mathrm{~s} / \mathrm{m}$ bei ausreichender Wasserverfügbarkeit (für Pflanzen ohne Wasserstreß)

berechnet.

\section{Das Verfahren von WENDLING nach PENMAN}

Für die klassische PENMAN-Beziehung sind die erforderlichen Eingangsdaten (s. Gl. 4) oft nicht vorhanden. Deshalb veröffentlichte WENDLING (1991) nach umfangreichen Vergleichen mit anderen Berechnungsverfahren und mit Lysimetermessungen eine vereinfachte Beziehung zur Berechnung von Tageswerten der potentiellen Verdunstung. Im 
Vergleich zu TURC werden hier auch die relevanten Einflussgrößen Luftfeuchte (bei TURC nur im Korrekturglied) und Windgeschwindigkeit berücksichtigt.

Gl. 5: $\quad \mathbf{E T P}=\mathrm{g} *\left[\left(\mathbf{R}_{\mathrm{G}} / \mathbf{4 1 0}\right)+\left(0.5+0.54 * \mathbf{v}_{2 \mathrm{~m}}\right) *\left(100-\mathrm{U}_{2 \mathrm{~m}}\right) *(\mathrm{~h} / \mathrm{905})\right] \quad[\mathrm{mm} / \mathrm{Tag}]$

mit $\mathrm{g}=2.4 *(\mathrm{~T}+22) /(\mathrm{T}+123)$ und $\mathrm{h}=$ Tageslänge in Stunden.

\section{Das Verfahren nach TURC/IVANOV}

ist eine empirische Formel, die in Frankreich mittels Lysimetermessungen auf Grünland hergeleitet wurde. In den neuen Bundesländern ist dieses Verfahren Grundlage vieler Berechnungsmethoden zur Ermittlung des verfügbaren Grundwasserdargebotes. Mit Bezug auf die Beschreibung des Verfahrens in DVWK, 1996 gilt

für $\mathbf{T}_{2 \mathrm{~m}}>\mathbf{5}^{\circ} \mathrm{C}$ :

Gl. 6: $\quad \mathbf{E T P}=0.0031 * \mathbf{C} *\left(\mathbf{R}_{\mathbf{G}}+209\right) * \mathbf{T}_{2 m} /\left(\mathbf{T}_{2 \mathrm{~m}}+15\right)$.

$[\mathrm{mm} / \mathrm{Tag}]$

Ist die relative Luftfeuchtigkeit $\mathrm{U}_{2 \mathrm{~m}}<50 \%$, ist Gl. (6) mit dem Korrekturglied $\mathrm{C}=1+\left(\left(50-\mathrm{U}_{2 \mathrm{~m}}\right) / 70\right)$ zu multiplizieren, $\mathrm{C}=1$ bei $\mathrm{U}_{2 \mathrm{~m}}>=50 \%$.

Für $\mathbf{T}_{\mathbf{2 m}}<=\mathbf{5}^{\circ} \mathbf{C}$ außerhalb der Vegetationsperiode können Dekaden- und Monatssummen nach IVANOV (mit der Korrektur von WENDLING \& MÜLLER, 1984) berechnet werden mit:

Gl. 7:

$$
\begin{array}{ll}
\mathbf{E T P}=\mathbf{0 , 0 0 0 3 6} *\left(\mathbf{T}_{\mathbf{2 m}}+\mathbf{2 5}\right)^{2} *\left(\mathbf{1 0 0}-\mathbf{U}_{\mathbf{2 m}}\right) & {[\mathbf{m m} / \text { Dekade }]} \\
=0,011 *\left(\mathrm{~T}_{2 \mathrm{~m}}+25\right)^{2} *(100-\mathrm{U}) & {[\mathrm{mm} / \text { Monat }]}
\end{array}
$$

Während der Tage mit Schneedecke wird in DYCK \& PESCHKE (1995) empfohlen, die ETP auf die Hälfte zu reduzieren. Für Tagesmittel mit ETP $<0,1 \mathrm{~mm} / \mathrm{d}$ und $\mathrm{T}<5^{\circ} \mathrm{C}$ sollte mit $\mathrm{ETP}=0,1 \mathrm{~mm} / \mathrm{d}$ gerechnet werden.

Oft ist statt der Globalstrahlung $\mathrm{R}_{\mathrm{G}}$ nur die tatsächliche tägliche Sonnenscheindauer $\mathrm{S}$ verfügbar. Dann ist es möglich, die Globalstrahlung nach der Formel von ANGSTRÖM zu schätzen:

Gl. 8: $\quad \mathbf{R}_{\mathbf{G}}=\mathbf{R}_{\mathbf{0}} *\left(\mathbf{0 . 1 9}+\mathbf{0 . 5 5} *\left(\mathbf{S} / \mathbf{S}_{\mathbf{0}}\right)\right) \quad\left[\mathbf{J} * \mathbf{c m}^{-2}\right]$

Da das TURC-Verfahren für Deutschland etwas zu niedrige Werte liefert, wird nach DVWK (1996) ein Korrekturfaktor von 1,1 angewendet.

\section{Das Verfahren von WENDLING nach TURC}

Es handelt sich um eine einfache Beziehung, die der von TURC ähnlich ist und die sich einheitlich sowohl für $\mathrm{T}>5^{\circ} \mathrm{C}$ als auch $\mathrm{T}<=5^{\circ} \mathrm{C}$ anwenden lässt (DVWK, 1996):

Gl. 9: $\quad E T P=\left(R_{G}+F\right) \cdot\left(T_{2 m}+22\right) /\left(150 \cdot\left(T_{2 m}+123\right)\right) \quad[\mathrm{mm} / \mathrm{d}]$

$\mathrm{F}=54$ als Konstante für den $30 \mathrm{~km}$ breiten Küstenstreifen der Ostsee und

$=93$ für das übrige Flachland bis $400 \mathrm{~m}$ ü NN .

\section{Das empirische Verfahren von HAUDE}

wurde in den Jahren 1952/53 auf der Grundlage von Messungen mit sehr kleinen Lysimetern (Grastöpfen) in einer Landschaft nördlich von Hannover abgeleitet (HAUDE, 1955). Es ist das in den alten Bundesländern am weitesten verbreitete Verfahren.

Gl. 10: $\quad \mathrm{ETP}=\mathrm{f} *\left(\mathrm{E}_{2 \mathrm{~m}}-\mathrm{e}_{2 \mathrm{~m}}\right)_{14 \mathrm{Uhr}}<=7 \quad[\mathrm{~mm} / \mathrm{d}]$ 
mit $\mathrm{f}$ in $\mathrm{mm} / \mathrm{hPa}$ als HAUDE-Faktor zur Berechnung der möglichen Verdunstung von Gras, $\mathrm{f}_{1}$ für mittlere Tageswerte und $\mathrm{f}_{2}$ für Monatssummen (aus DVWK, 1996):

\begin{tabular}{|ccccccccccccc|}
\hline Monat & $\mathbf{1}$ & $\mathbf{2}$ & $\mathbf{3}$ & $\mathbf{4}$ & $\mathbf{5}$ & $\mathbf{6}$ & $\mathbf{7}$ & $\mathbf{8}$ & $\mathbf{9}$ & $\mathbf{1 0}$ & $\mathbf{1 1}$ & $\mathbf{1 2}$ \\
\hline $\mathrm{f}_{\mathbf{1}}$ & 0,22 & 0,22 & 0,22 & 0,29 & 0,29 & 0,28 & 0,26 & 0,25 & 0,23 & 0,22 & 0,22 & 0,22 \\
\hline $\mathrm{f}_{\mathbf{2}}$ & 6,82 & 6,82 & 6,82 & 8,70 & 8,99 & 8,40 & 8,06 & 7,75 & 6,90 & 6,82 & 6,60 & 6,82 \\
\hline
\end{tabular}

Der Sättigungsdampfdruck E wird in Abhängigkeit von der Lufttemperatur um 14 Uhr MEZ nach DVWK (1996) wie folgt berechnet:
Gl. 11:
$\mathbf{E}\left(\mathbf{T}_{14}\right)=\mathrm{C} 1 * \exp \left(\left(\mathrm{C} 2 * \mathbf{T}_{14}\right) /\left(\mathrm{C} 3+\mathbf{T}_{14}\right)\right)$
[hPa]

Für die Koeffizienten C1, C2 und C3 gilt:

\begin{tabular}{|c|c|c|}
\hline & über Eis bei $\mathbf{- 6 5}$ bis $+\mathbf{0 , 0 1}{ }^{\circ} \mathbf{C}$ & sonst bei $-\mathbf{4 5}$ bis $+\mathbf{6 0}^{\circ} \mathbf{C}$ \\
\hline C1 & 6,11 & 6,11 \\
C2 & 22,46 & 17,62 \\
C3 & 272,62 & 243,12 \\
\hline
\end{tabular}

Der aktuelle Dampfdruck e errechnet sich aus Temperatur- und Feuchtemessungen der Luft um 14 Uhr MEZ:
Gl. 12:$$
\mathbf{e}=\mathbf{E}\left(\mathbf{T}_{14}\right) * \mathbf{U}_{14} / 100
$$
[hPa]

\subsubsection{Verfügbare Klimadaten}

Seit Juni 1992 wird zur Erfassung des Wärmehaushaltes auf dem Lysimeterfeld eine automatische Klimastation betrieben. Für den Zeitraum vor Juni 1992 standen neben dem Niederschlag am Standort Brandis lediglich folgende Tageswerte zur Verfügung:

- Sonnenscheindauer seit 01.01.1984

- Beobachtungen der Witterung seit 1978

Deshalb musste der Datenbestand bis 1981 rückwirkend ergänzt werden. Zur Verfügung standen die Daten der Stationen Schkeuditz und Oschatz des DWD. Eine statistische Untersuchung der Daten zur Lufttemperatur und zur Sonnenscheindauer von DÖRING (1994) lieferte folgende Ergebnisse:

Bei einem Vergleich statistischer Parameter auf der Basis von Monatswerten zwischen Brandis und Schkeuditz bzw. Brandis und Oschatz lassen sich keine signifikanten Unterschiede erkennen.

Ein Vergleich der auf langjährige Mittelwerte reduzierten Daten ergibt bei der Lufttemperatur für Brandis und Oschatz und bei der Sonnenscheindauer für Brandis und Schkeuditz, die besten Annäherungen.

Auf der Basis von Regressionsbeziehungen zwischen Brandis und Schkeuditz bzw. Oschatz für die Sonnenscheindauer und die Lufttemperatur konnten die Klimadaten für den Standort Brandis für den Zeitraum vor 1992 ergänzt werden. Die Ermittlung der Windgeschwindigkeit 
und die Berechnung der Referenzverdunstung erfolgte im Rahmen des DFG-Projektes „Wasserhaushaltsverfahren“ (BfG, 1977).

\subsubsection{Ergebnisse der Vergleichsrechnungen}

Der nachfolgende Vergleich der verschiedenen Berechnungsverfahren wurde für den Zeitraum Juni 1992 bis Dezember 1997, also nach Inbetriebnahme der Klimastation Brandis, vorgenommen. Die Berechnungen erfolgten auf der Basis von Tageswerten, die anschließend zu Dekadenwerten verdichtet wurden.

Zunächst wurde mittels Regressionsanalyse festgestellt, dass die Verfahren von

- TURC/IVANOV (Gl. 6 und Gl.7) und WENDLING nach TURC (Gl.9) sowie die

- „Gras-Referenzverdunstung“ (GL.9) und das Verfahren von WENDLING nach PENMAN (Gl.5)

bei einem Bestimmtheitsmaß von rd. $99 \%$ bzw. rd. $96 \%$ jeweils nahezu identische Werte lieferten Abb. 12 und Abb. 13. Daraus resultiert eine weitere Bestätigung der von WENDLING et al. (1991) erarbeiteten Modifikationen der Ausgangsformeln.

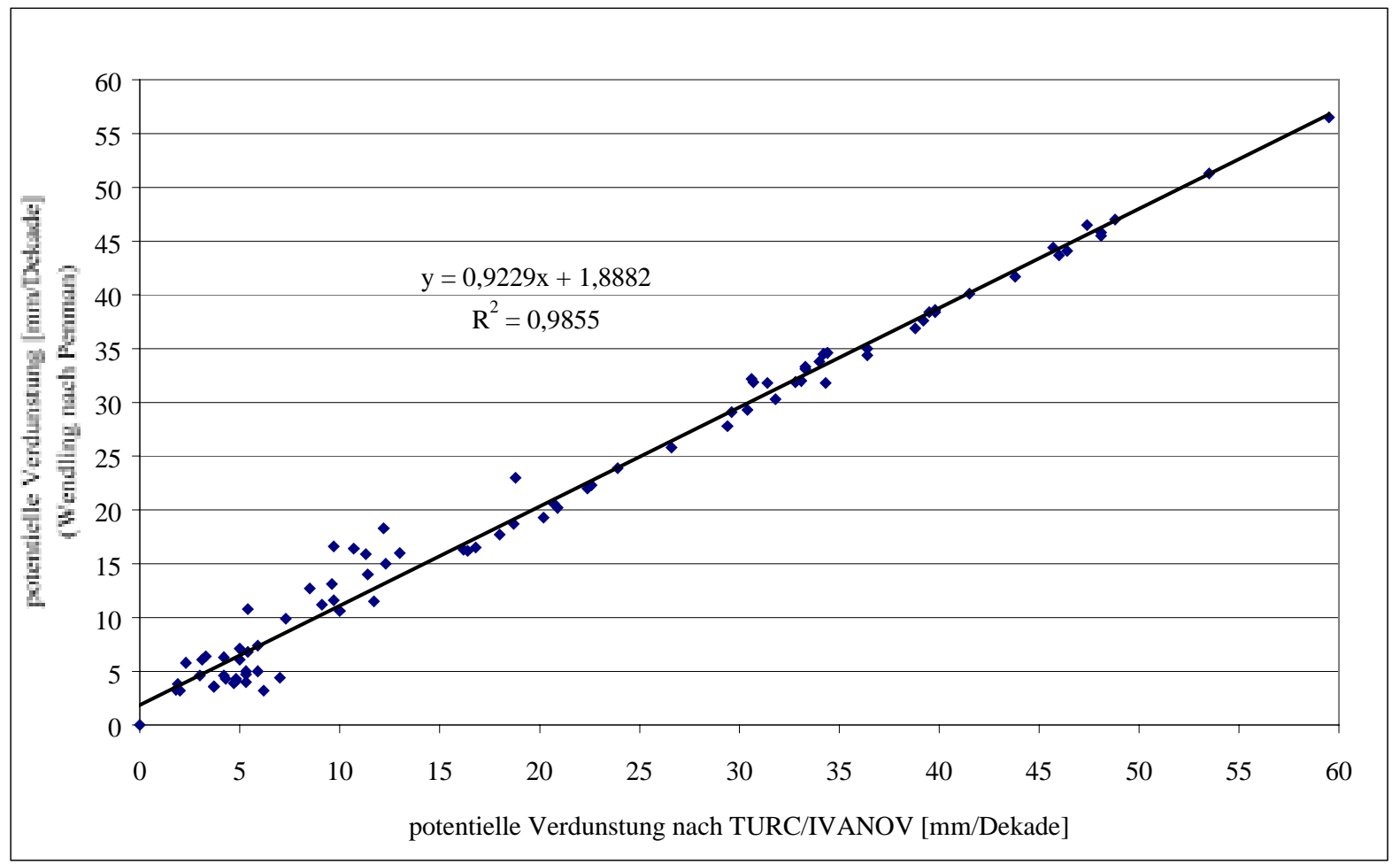

Abb. 12: Regressionsgerade: Dekadenwerte der potentiellen Verdunstung von TURC/IVANOW (Gl. 6 und Gl. 7) und WENDLING nach TURC (Gl.9) 


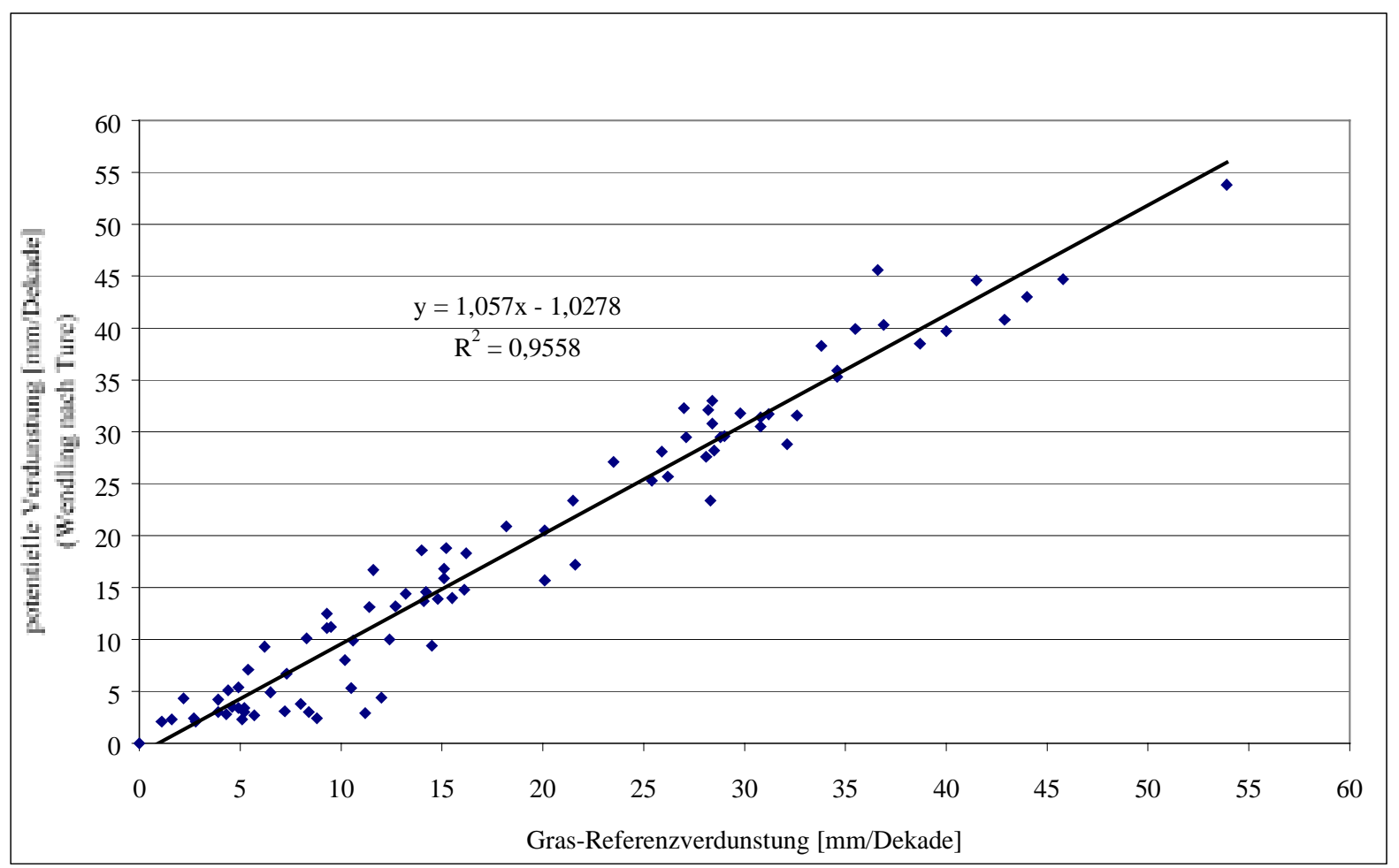

Abb. 13: Regressionsgerade: Dekadenwerte der Gras-Referenzverdunstung und der potentiellen Verdunstung von WENDLING nach PENMAN (Gl. 5

Weitere Vergleiche Abb. 14 basieren deshalb auf Berechnungsergebnissen der Verfahren von HAUDE (Gl.10), TURC/IVANOV (Gl. 6 und Gl. 7) und der Gras-Referenzverdunstung. Vergleichsweise herangezogen wurden der korrigierte Niederschlag sowie die Messergebnisse der Löß-Parabraunerde (Lysimetergruppe 9) und die Messergebnisse der Braunerde aus Sandlöß - des Bodens mit dem geringsten Wasserspeichervermögen. Die Verdunstung der Lößböden wurde dargestellt, da diese unter der Voraussetzung ausreichender Wasserversorgung (s. Kap. 3.5.6) eine „kulturartenspezifische maximale“ Verdunstung liefern.

Bezieht man die Ergebnisse der Vergleichsrechnungen auf die „Gras-Referenzverdunstung“, so ergeben sich für den Untersuchungszeitraum folgende Unterschiede (Tab. 11):

Tab. 11: Berechnete und mittels Lysimeter gemessene Jahresverdunstung, bezogen auf die Gras-Referenzverdunstung (= $100 \%)$ am Standort Brandis in verschiedenen Zeitabschnitten

\begin{tabular}{|l|c|c|c|c|c|c|c|}
\hline \multicolumn{1}{|c|}{ Verfahren } & \multirow{2}{*}{$\mathbf{R}^{\mathbf{2}}$} & \multicolumn{2}{c|}{ Juni 1992 - Dez. 1997 } & \multicolumn{2}{c|}{$\mathbf{1 9 9 3}$} & \multicolumn{2}{c|}{1997} \\
& {$[\%]$} & {$[\mathrm{mm}]$} & {$[\%]$} & {$[\mathrm{mm}]$} & {$[\%]$} & {$[\mathrm{mm}]$} & {$[\%]$} \\
\hline Gras-Referenzverdunstung & - & 3491 & 100 & 621 & 100 & 646 & 100 \\
TURC/IVANOV & 95 & 3755 & 108 & 651 & 105 & 668 & 103 \\
HAUDE & 90 & 3952 & 113 & 634 & 102 & 758 & 117 \\
Löß-Parabraunerde & 64 & 3575 & 102 & 608 & 98 & 661 & 102 \\
Braunerde aus Sandlöß & 53 & 2935 & 84 & 564 & 91 & 477 & 74 \\
korrigierter Niederschlag & - & 3706 & 106 & 754 & 121 & 539 & 83 \\
\hline
\end{tabular}

HAUDE liefert für den gesamten Untersuchungszeitraum Juni 1992 bis Dezember 1997 mit $13 \%$ etwas größere Abweichungen von der Gras-Referenzverdunstung als TURC/IVANOV $(8 \%)$. Erwartungsgemäß zeigt das Bestimmtheitsmaß $\left(\mathrm{R}^{2}\right)$ u. a., dass auf Grund der Bestandsunterschiede zwischen Gras und den Ackerkulturen nur $64 \%$ der Variation der Dekadenwerte 
der (kulturartenspezifischen maximalen) Verdunstung der Lößböden durch die GrasReferenzverdunstung beschrieben werden können.

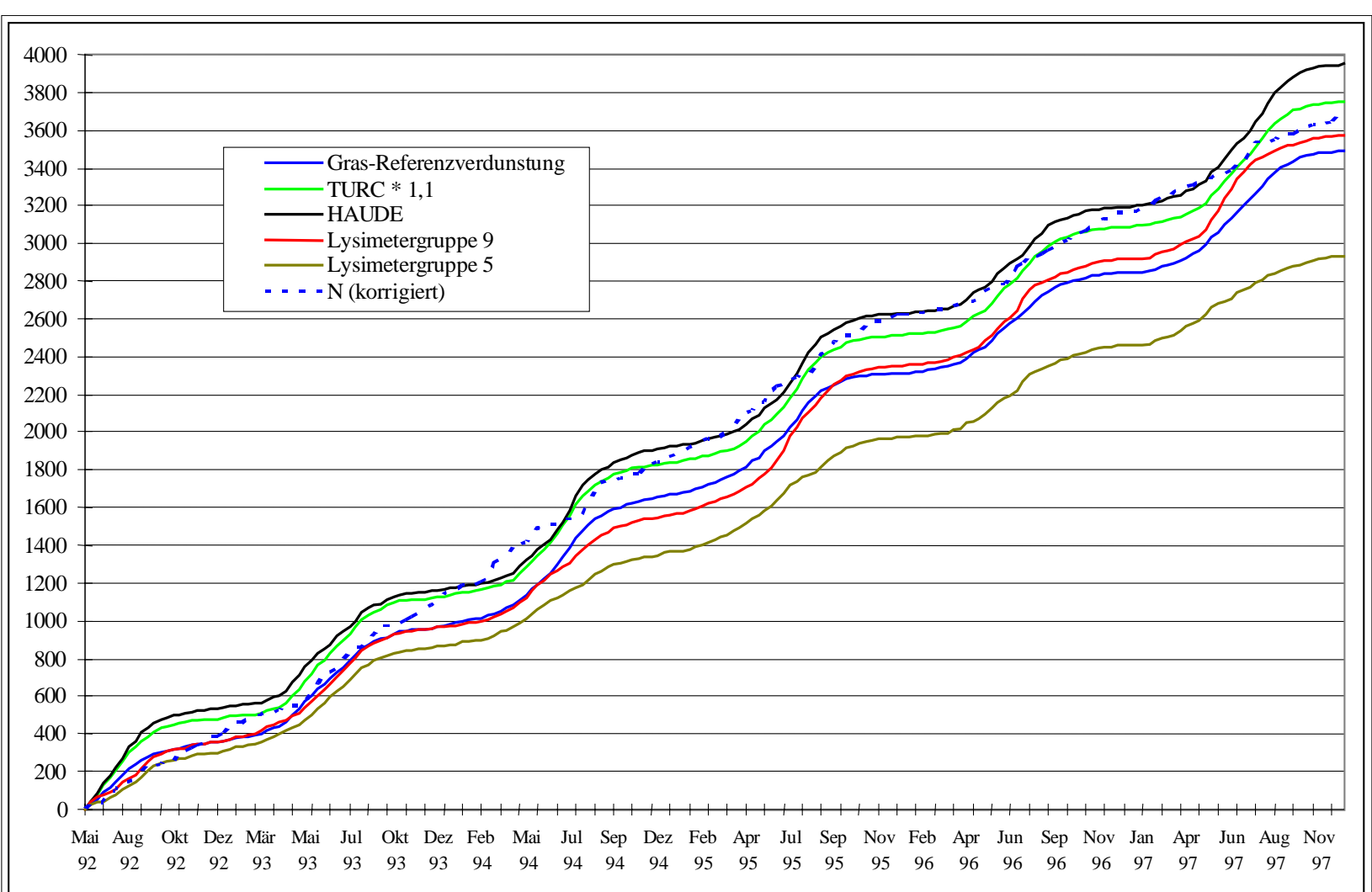

Abb. 14: Summenkurve der Dekadenwerte des korrigierten Niederschlages und der mittels verschiedener Verfahren ermittelten Verdunstung [mm]

Am Beispiel der Jahre 1993 und 1997 wird deutlich (Tab. 11), dass die Vergleichszahlen in Abhängigkeit von der Witterung starken Schwankungen unterliegen. Weitere Untersuchungen bei höherer zeitlicher Auflösung (Tages- und Dekadenwerte) zeigten zunehmende Unterschiede zwischen den einzelnen Verfahren (s. Anlage 4). Auffallend sind die großen Abweichungen der HAUDE-Verdunstung von der Gras-Referenzverdunstung im Verlauf heißer und trockener Sommerdekaden, obwohl an 53 Tagen berechnete Werte $>7 \mathrm{~mm}$ auf $7 \mathrm{~mm}$ korrigiert wurden.

GÜNTHER (1999) verweist im Ergebnis seiner Untersuchungen für den Zeitraum von 198388 an der Station Großobringen (Freistaat Thüringen) auf besonders große Abweichungen der HAUDE-Verdunstung zu anderen Verfahren, wobei hier mit HAUDE tendenziell zu niedrige Werte ermittelt wurden. Auch SPONAGEL (1980) ermittelte im regenreichen Jahr 1972 sehr geringe HAUDE-Verdunstungswerte. Er erklärt dies mit dem Hinweis darauf, dass sich das HAUDE-Verfahren ausschließlich auf das Sättigungsdefizit der Luft um 14 Uhr stützt. In den Untersuchungsjahren 1973 und 1974 finden sich dagegen bei den Untersuchungen von SPONAGEL (1980) Monate mit außergewöhnlich hoher HAUDE-Verdunstung. Diese wurden wie in Brandis im Verlauf sehr warmer und trockener Witterung ermittelt.

Die bei höherer zeitlicher Auflösung (Tages-, Dekaden- und Monatswerte) sichtbaren Abweichungen der HAUDE-Verdunstung im Verlauf sehr trockener oder feuchter Perioden gleichen sich über einen längeren Zeitraum aus. Folgen aber insbesondere mehrere überdurchschnittlich trockene Perioden aufeinander, wie im Fall der Brandiser Untersuchungen, so führt das Verfahren zu einer Überschätzung der potentiellen Verdunstung auch im Verlauf längerer Zeiträume. 
Für die heiße und trockene Periode Ende April bis Anfang Mai 1993 liefert die GrasReferenzverdunstung Werte, die deutlich über denen der mit „Gras“ bewachsenen LößSchwarzerdeböden liegen (s. Anlage 4a). Nach Ernte der Wintergerste und dem Stoppelsturz im September im Jahr 1992 sollte sich die Schwarzbrache auf den Lysimetern selbst begrünen. U. a. auf Grund der geringen Niederschläge im Frühjahr 1993 etablierte sich nur ein sehr mangelhafter Bestand mit entsprechend geringer Verdunstung. Ab Ende Mai - mit Beginn der Niederschläge - unterscheiden sich die Ergebnisse der Berechnungsverfahren dann nur noch wenig voneinander. Unter diesen Witterungs- und Bewuchsbedingungen liefert auch die „leichte“ Braunerde (Lysimetergruppe 5) eine vergleichsweise hohe Verdunstung, die sich wenig von der Löß-Parabraunerde unterscheidet.

Anlage 4b zeigt den Verlauf der Mess- und Berechnungsgrößen (Dekadenwerte) im Jahr 1997 mit Sommerweizen. Bis Jahresmitte unterscheiden sich Gras-Referenzverdunstung und HAUDE-Verdunstung zunächst relativ wenig voneinander. Beide sind deutlich geringer als die Verdunstung auf der Löß-Parabraunerde mit Sommerweizen. Erst Ende Juli - mit Beginn einer trockenen und warmen Periode - wird mittels HAUDE wieder eine sehr hohe potentielle Verdunstung berechnet.

Unterschiede der Berechnungsverfahren zur kulturartenspezifischen maximalen Verdunstung der Löß-Parabraunerde (Lysimetergruppe 9) resultieren aus den Bestandesunterschieden zwischen Gras und Winterweizen. Die auf Grund von Wassermangel erheblich reduzierte (reale) Verdunstung einer Braunerde wird am Beispiel der Lysimetergruppe 5 demonstriert.

Im Ergebnis der Vergleichsrechnungen wurde beschlossen, in dieser Arbeit für alle weiteren Untersuchungen zur Beschreibung des Verdunstungsanspruchs der Atmosphäre die GrasReferenzverdunstung als die Bezugsgröße zu verwenden. Berechnungen mit dem Verfahren nach TURC/IVANOV oder HAUDE dienen dem Vergleich zu bereits vorhandenen Untersuchungsergebnissen. 


\subsection{Klimatische Wasserbilanz}

Im langjährigen Mittel ist der korrigierte Niederschlag im Untersuchungsgebiet nur geringfügig höher als der Verdunstungsanspruch der Atmosphäre, d. h. im langjährigen Mittel ist kaum ein Wasser-Überschuss vorhanden $\left(\mathrm{P}_{\text {korr }} / \mathrm{ETP} \cong 1\right)$. Andererseits bewirkt die erhebliche Schwankungsbreite der Niederschläge Abb. 15 in Einzeljahren ein hohes Wasserdefizit, das letztlich nur aus dem Bodenwasservorrat gedeckt werden kann.
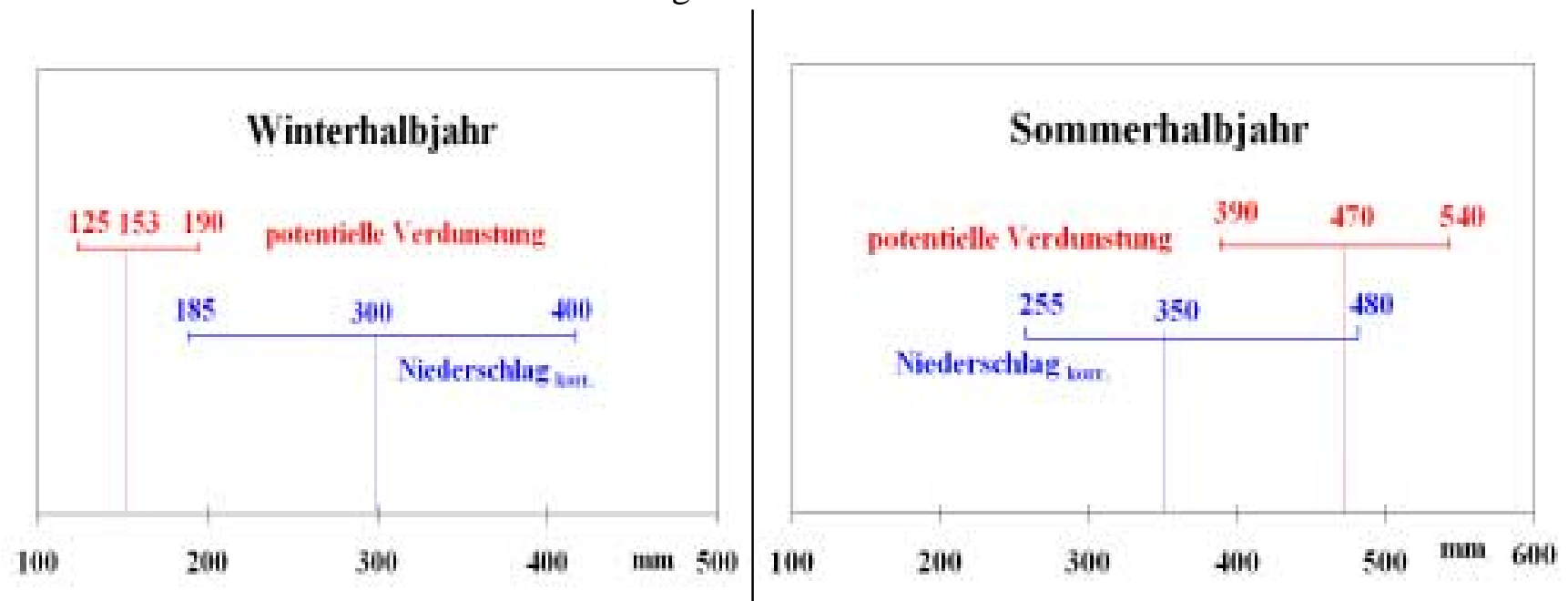

Abb. 15: Niederschlag und potentielle Verdunstung der Winter- und Sommerhalbjahre am Standort Brandis (Minimum, Mittelwert und Maximum der Reihe 1981-97)

In der Agrarmeteorologie und gleichermaßen in der Wasserwirtschaft wird die klimatische Wasserbilanz (kWB) als Differenz von unkorrigiertem Niederschlag (in $1 \mathrm{~m}$ Höhe nach HELLMANN gemessen) und potentieller Verdunstung von Gras als Standortcharakteristik definiert. Mittels der kWB läßt sich die Witterung einzelner Jahre an einem Untersuchungsstandort beschreiben (Tab. 12). Für die Genauigkeit und Vergleichbarkeit der ermittelten kWB mit anderen Gebieten sind entscheidend:

- die Genauigkeit der Niederschlagsmessung und

- die Art des verwendeten Verfahrens zur Berechnung der potentiellen Verdunstung.

Als ETP wurde hier die Gras-Referenzverdunstung verwendet. Bei der Berechnung nach TURC ergab sich im Mittel der Jahre eine ETP von $645 \mathrm{~mm}$.

Tab. 12: Niederschlag, potentielle Verdunstung und klimatische Wasserbilanz der Jahre 1981-97 am Standort Brandis [mm/a]

\begin{tabular}{|c|c|c|c|c|c|}
\hline & \multicolumn{3}{|c|}{$\begin{array}{c}\text { Niederschlag (Hellmann-Messung) } \\
{[\mathrm{mm}]}\end{array}$} & \multirow[t]{3}{*}{$\begin{array}{l}\text { ETP } \text { Gras-Ref. } \\
{[\mathrm{mm}]}\end{array}$} & \multirow[t]{3}{*}{$\begin{array}{l}\mathbf{k W B} \\
{[\mathrm{mm}]}\end{array}$} \\
\hline & \multicolumn{2}{|c|}{ unkorrigiert } & \multirow[t]{2}{*}{ korrigiert } & & \\
\hline & 1 m Höhe & bodengleich & & & \\
\hline Winter & 259 & 283 & 302 & 153 & 106 \\
\hline Sommer & 313 & 332 & 353 & 470 & -157 \\
\hline Jahr & 572 & 615 & 655 & 623 & -51 \\
\hline
\end{tabular}

Mit der klimatischen Wasserbilanz werden die wesentlichsten meteorologischen Einflussgrößen auf den Bodenwasserhaushalt beschrieben. Um eine gute Übereinstimmung zwischen - mittels Lysimeter ermittelter tatsächlicher Bodenwasserentnahme und dem mittels kWB ermittelten Wasserdefizit - herstellen zu können, wird die kWB in den nachfolgenden Darstel- 
lungen (abweichend zur bisher üblichen Verfahrensweise) auf Basis der korrigierten Niederschläge ermittelt. Durch die Verwendung des korrigierten Niederschlages wandelt sich Brandis von einem leicht ariden Standort in einen Standort mit einer positiven kWB von 32 mm (Reihe 1981-97). Wie aus Abb. 16 zu ersehen ist, reicht die Spannbreite von sehr feuchten (1981 und 1987) bis zu sehr trockenen Jahren (1982 und 1991).

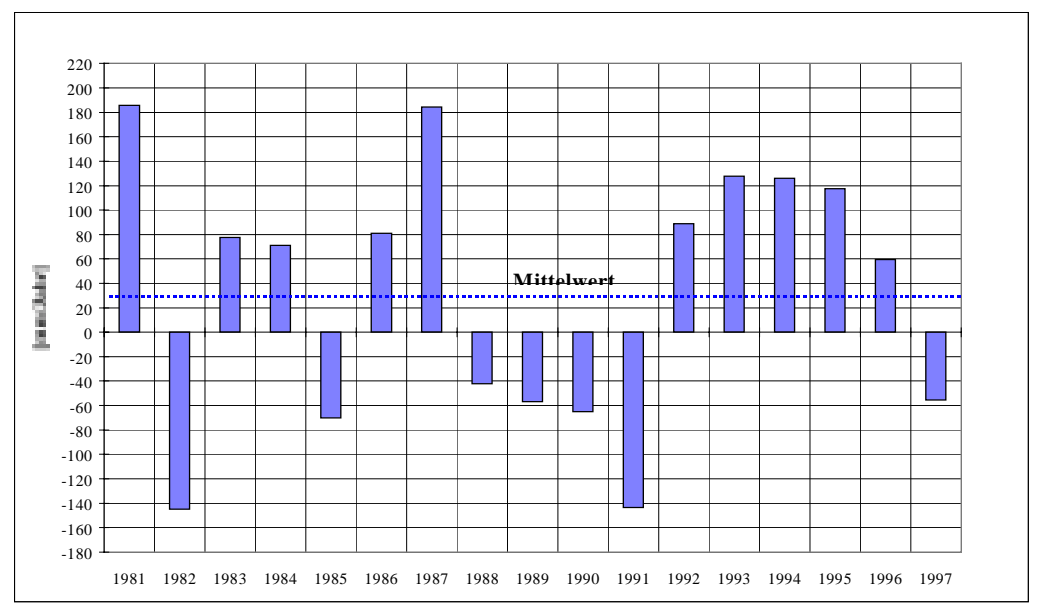

Abb. 16: Jahreswerte der klimatischen Wasserbilanz am Standort Brandis in [mm/a]

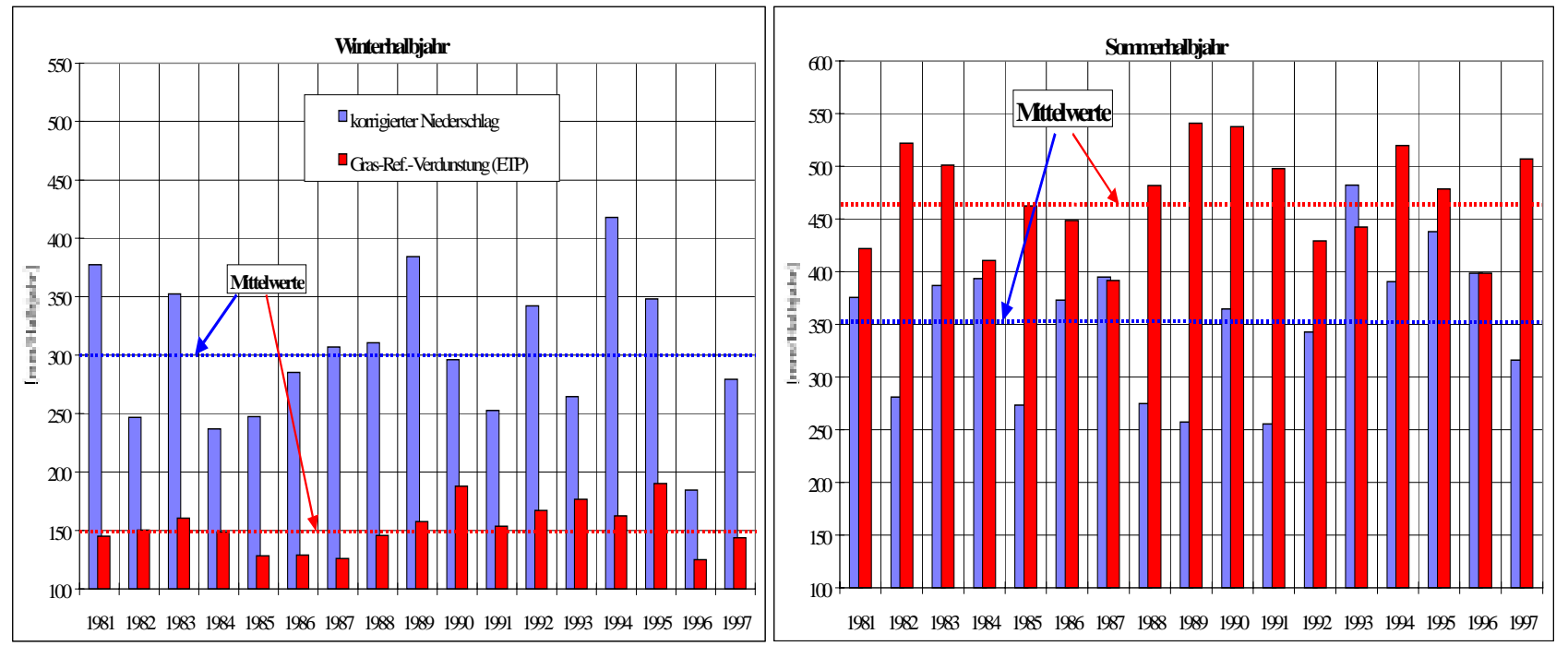

Abb. 17: Niederschlag (korrigiert) und Gras-Referenzverdunstung der Winter- und Sommerhalbjahre am Standort Brandis

Für den Wasserhaushalt sind vor allem eine Folge von trockenen bzw. feuchten Jahren und die Verteilung des Niederschlages auf die Winter- und Sommerhalbjahre von Bedeutung. Abb. 17 zeigt, dass die Trockenheit im Verlauf der Jahre 1988-91 vorwiegend aus zu geringen Sommerniederschlägen und einem überdurchschnittlich hohen Verdunstungsanspruch der Atmosphäre resultierte. Geht man davon aus, dass der Verlauf der "mittleren“ kWB das „klimatische Normaljahr“ der Reihe 1981-97 charakterisiert, kann eine detaillierte Einschätzung der einzelnen Wasserhaushaltsjahre erfolgen. Es fällt auf, dass die Beobachtungsreihe kaum ein Jahr enthält, das dem „klimatischen Normaljahr“ entspricht (Abb. 16). Typisch für die Beobachtungsreihe sind eine große Anzahl von zu feuchten Winter- und zu trockenen Sommerhalbjahren. Auch mit Blick auf Abb. 11 wird noch einmal deutlich, dass im Verlauf der Sommerhalbjahre ein überwiegend sehr angespannter Wasserhaushalt zu verzeichnen ist. Es ist zu erwarten, dass die Verdunstung und Pflanzenproduktion am Unter- 
suchungsstandort nicht durch die Strahlung, sondern durch das mangelnde Niederschlagsdargebot begrenzt wird, falls dieses Defizit nicht durch pflanzenverfügbares Bodenwasser gedeckt werden kann.

\subsection{Kapazität der Bodenwasserspeicher}

\subsubsection{Ausgeschöpfte Mengen}

Vergleicht man die durchschnittlichen Entnahmemengen der einzelnen Fruchtarten, so ergibt sich für die verschiedenen Böden eine Reihenfolge (Tab. 13, wobei die höchste Entnahme mehrheitlich in den Jahren mit Zuckerrüben oder Wintergetreide erfolgt.

Tab. 13: Bodenwasserentnahme in Abhängigkeit von den Fruchtarten, ohne Rotklee (Reihenfolge nach der Entnahmemenge)

\begin{tabular}{|l|l|}
\hline \multicolumn{1}{|c|}{ Bodentyp (Lys.-Gr.) } & \multicolumn{1}{c|}{ Reihenfolge } \\
\hline Erodierte Braunerde (5) & Zu.-Rü. $\rightarrow$ Wi.-Wei. $\rightarrow$ Wi.-Ger. $\rightarrow$ Gras $\rightarrow$ Kart. \\
\hline Braunerde-Fahlerde (4) & Zu.-Rü. $\rightarrow$ Wi.-Wei. $\rightarrow$ Wi.-Ger. $\rightarrow$ Gras $\rightarrow$ Kart. \\
Parabraunerde-Braunerde (8) & Wi.-Ger. $\rightarrow$ Wi.-Wei. $\rightarrow$ Zu.-Rü. $\rightarrow$ Gras $\rightarrow$ Kart. \\
\hline $\begin{array}{l}\text { Lessivierter Braunerde- } \\
\text { Pseudogley (1) }\end{array}$ & Zu.-Rü. $\rightarrow$ Wi.-Wei. $\rightarrow$ Wi.-Ger. $\rightarrow$ Gras $\rightarrow$ Kart. \\
Braunerde-Pseudogley (7) & Wi.-Wei. $\rightarrow$ Wi.-Ger. $\rightarrow$ Zu.-Rü. $\rightarrow$ Gras $\rightarrow$ Kart. \\
Pseudovergleyte Braunerde- & Zu.-Rü. $\rightarrow$ Wi.-Ger. $\rightarrow$ Wi.-Wei. $\rightarrow$ Gras $\rightarrow$ Kart. \\
Fahlerde (11) & \\
\hline $\begin{array}{l}\text { Parabraunerde aus Löß (9) } \\
\text { Schwarzerde aus Löß (10) }\end{array}$ & Wi.-Wei $\rightarrow$ Wi.-Ger. $\rightarrow$ Zu.-Rü. $\rightarrow$ Kart. $\rightarrow$ Gras \\
\hline
\end{tabular}

Ursache für diese Reihenfolge sind in erster Linie das Vermögen der Pflanze, vorhandenes Wasser zu nutzen (z. B. lange Vegetationszeit bei Zuckerrüben), der Verdunstungsanspruch der Atmosphäre im Anbaujahr und die physikalischen Eigenschaften der Böden.

Es ist davon auszugehen, dass Witterung und/oder wechselnder Bewuchs auch bei gleichen Böden von Jahr zu Jahr zu einer unterschiedlichen Inanspruchnahme der Bodenwasservorräte führen. In der Zusammenstellung der jährlichen (boden- und kulturartenspezifischen) Bodenwasserentnahmen in Tab. 14 zeigen erwartungsgemäß deutliche Abhängigkeiten von der kWB im Sommerhalbjahr. So wird bei fast allen Böden eine besonders hohe Ausschöpfung in den Jahren 1982 und 1989 mit einer negativen kWB von -241 mm bzw. -284 mm erreicht. Erstaunlich ist das Vermögen der Lößböden, mehrere Jahre hintereinander jährlich mehr als $200 \mathrm{~mm}$ Bodenwasser für die Verdunstung bereit stellen $\mathrm{zu}$ können. Die geringste Ausschöpfung war in den Jahren 1987, 1993 und $1996(\mathrm{kWB}=+4 \mathrm{~mm}, 0 \mathrm{~mm}$ und +40 mm).

Die Zusammenstellung der statistischen Kennwerte in Tab. 15 zeigt eine erhebliche Spannbreite und Variation der jährlichen Bodenwasserentnahme im Verlauf der 17 Untersuchungsjahre. Ursache dafür ist die zufällige Kombination von Witterung und Fruchtart in den Einzeljahren. Die maximale Entnahmemenge $\left(\mathbf{S B}_{\max }\right)$, die im Verlauf der 17 Untersuchungsjahre für jeden Boden ermittelt wurde, wird mit der pflanzenverfügbaren

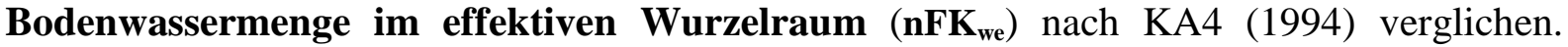
Spannbreite und Variationskoeffizient $\left(\mathrm{c}_{\mathrm{v}}\right)$ der tatsächlichen jährlichen Bodenwasserentnahme durch die Pflanzen (SB) wachsen proportional mit der Größe der $\mathrm{nFK}_{\mathrm{We}}\left(=\mathrm{SB}_{\max }\right)$. 
Tab. 14: Boden- und kulturartenspezifische Bodenwasserentnahme [mm]

\begin{tabular}{|c|c|c|c|c|c|c|c|c|c|c|}
\hline \multirow[t]{2}{*}{ Jahr } & \multirow[t]{2}{*}{ Fruchtart } & \multirow{2}{*}{$\begin{array}{c}\text { kWB } \\
\text { Sommer- } \\
\text { halbjahr }\end{array}$} & \multicolumn{8}{|c|}{$\begin{array}{l}\text { Bodenwasserentnahme durch die Pflanzen (SB) } \\
{[\mathrm{mm}]}\end{array}$} \\
\hline & & & $\begin{array}{c}\text { Lys } 5 \\
\text { D3 } \\
\end{array}$ & $\begin{array}{c}\text { Lys } 4 \\
\text { D5 } \\
\end{array}$ & \begin{tabular}{|c} 
Lys 8 \\
D3 \\
\end{tabular} & $\begin{array}{c}\text { Lys 1 } \\
\text { D6 } \\
\end{array}$ & \begin{tabular}{|c} 
Lys 7 \\
D4 \\
\end{tabular} & \begin{tabular}{|c|} 
Lys 11 \\
D3 \\
\end{tabular} & $\begin{array}{c}\text { Lys } 9 \\
\text { Lö3 }\end{array}$ & \begin{tabular}{|c} 
Lys 10 \\
Lö1 \\
\end{tabular} \\
\hline 1981 & Zu.-Rüben & -47 & 74 & 120 & 107 & 152 & 146 & 157 & 240 & 246 \\
\hline 1990 & Zu.-Rüben & -173 & 73 & 124 & 113 & 136 & 128 & 147 & 159 & 191 \\
\hline $\mathbf{m m}$ & Mittelwert & -110 & 74 & 122 & 110 & 144 & 137 & 152 & 200 & 219 \\
\hline $\mathrm{mm}$ & $\mathrm{s}$ & 89 & 0,7 & 2,8 & 4,2 & 11,3 & 12,7 & 7,1 & 57,3 & 38,9 \\
\hline$\%$ & $c v$ & -81 & 1,0 & 2,3 & 3,9 & 7,9 & 9,3 & 4,7 & 28,7 & 17,8 \\
\hline 1985 & Kartoffeln & -189 & 56 & 95 & 89 & 93 & 108 & 88 & 136 & 107 \\
\hline 1987 & Kartoffeln & 4 & 41 & 65 & 66 & 58 & 80 & 55 & 69 & 51 \\
\hline 1996 & Kartoffeln & 0 & 49 & 79 & 73 & 80 & 82 & 78 & 115 & 90 \\
\hline $\mathbf{m m}$ & Mittelwert & -62 & 49 & 80 & 76 & 77 & 90 & 74 & 107 & 83 \\
\hline $\mathrm{mm}$ & S & 110 & 7,5 & 15,0 & 11,8 & 17,7 & 15,6 & 16,9 & 34,3 & 28,7 \\
\hline$\%$ & $c v$ & -179 & 15,4 & 18,8 & 15,5 & 23,0 & 17,4 & 23,0 & 32,1 & 34,7 \\
\hline 1982 & i.-Weizen & -241 & 74 & 127 & 118 & 164 & 156 & 144 & 251 & 260 \\
\hline 1986 & zen & -76 & 68 & 112 & 111 & 115 & 159 & 108 & 214 & 198 \\
\hline 1988 & Wi.-Weizen & -207 & 73 & 124 & 108 & 120 & 174 & 126 & 271 & 211 \\
\hline 1991 & Wi.-Weizen & -243 & 72 & 117 & 119 & 143 & 153 & 131 & 200 & 215 \\
\hline $\mathbf{m m}$ & Mittelwert & -192 & 72 & 120 & 114 & 136 & 161 & 127 & 234 & 221 \\
\hline $\mathrm{mm}$ & $\mathrm{S}$ & 79 & 2,6 & 6,8 & 5,4 & 22,6 & 9,3 & 14,9 & 32,7 & 26,9 \\
\hline$\%$ & $C v$ & -41 & 3,7 & 5,7 & 4,7 & 16,7 & 5,8 & 11,7 & 14,0 & 12,2 \\
\hline 1983 & Wi.-Gerste & -114 & 66 & 120 & 111 & 134 & 142 & 131 & 193 & 197 \\
\hline 1989 & Wi.-Gerste & -284 & 74 & 126 & 124 & 140 & 162 & 141 & 301 & 252 \\
\hline 1992 & Wi.-Gerste & -87 & 68 & 120 & 116 & 138 & 158 & 130 & 170 & 193 \\
\hline 1997 & Wi.-Gerste & -191 & 67 & 107 & 116 & 127 & 151 & 120 & 204 & 194 \\
\hline $\mathbf{m m}$ & Mittelwert & -169 & 69 & 118 & 117 & 135 & 153 & 131 & 217 & 209 \\
\hline $\mathrm{mm}$ & $\mathrm{S}$ & 88 & 3,6 & 8,0 & 5,4 & 5,7 & 8,8 & 8,6 & 57,8 & 28,7 \\
\hline$\%$ & $C v$ & -52 & 5,2 & 6,8 & 4,6 & 4,3 & 5,7 & 6,6 & 26,6 & 13,7 \\
\hline 1984 & Weidelgras & -17 & 53 & 82 & 81 & 92 & 100 & 104 & 113 & 104 \\
\hline 1993 & Grünbrache & 40 & 62 & 92 & 82 & 90 & 93 & 65 & 72 & 63 \\
\hline 1994 & Grünbrache & -130 & 60 & 85 & 89 & 94 & 143 & 99 & 134 & 85 \\
\hline $\mathbf{m m}$ & Mittelwert & -36 & 58 & 86 & 84 & 92 & 112 & 89 & 106 & 84 \\
\hline $\mathrm{mm}$ & $\mathrm{S}$ & 87 & 4,7 & 5,1 & 4,4 & 2,0 & 27,1 & 21,2 & 31,5 & 20,5 \\
\hline$\%$ & $C v$ & -243 & 8,1 & 5,9 & 5,2 & 2,2 & 24,2 & 23,8 & 29,7 & 24,4 \\
\hline 1995 & Rotklee & -41 & 64 & 114 & 94 & 120 & 104 & 95 & 204 & 256 \\
\hline $\mathbf{m m}$ & $\begin{array}{c}\text { Mittel } \\
\text { 1981-97 }\end{array}$ & -117 & 64 & 106 & 101 & 117 & 132 & 113 & 179 & 171 \\
\hline $\mathbf{m m}$ & $\mathbf{S}$ & 99 & 10 & 19 & 18 & 29 & 31 & 30 & 67 & 72 \\
\hline$\%$ & $c v$ & -85 & 15 & 18 & 18 & 24 & 23 & 26 & 37 & 42 \\
\hline
\end{tabular}

Zu Lysimetergruppe (Lys.), Bodenform und Standorteinheit (D3-D6, Lö1, Lö3) s. Tab. 6. 
Für die Böden mit geringer bis mittlerer $\mathrm{nFK}_{\mathrm{We}}$, deren sommerliches Defizit jährlich wieder aufgefüllt wird (Lysimetergruppe 5, 4, 8, 1, 7 und 11), enthält Tab. 15 unter $\mathrm{SB}_{\text {Mittel }}, \mathrm{SB}_{\text {min }}$ und $\mathrm{SB}_{\max }$ die jeweils mittlere, geringste und höchste sommerliche Ausschöpfung der 17 Untersuchungsjahre.

Tab. 15: Statistische Kennwerte der tatsächlichen Bodenwasserentnahme für die Böden der Station Brandis im Vergleich zu $n F K_{\text {we }}$ und We nach KA4 (1994)

\begin{tabular}{|c|c|c|c|c|c|c|c|c|}
\hline \multirow{2}{*}{$\begin{array}{c}\text { Lys.-Gr. } \\
- \\
\text { Standort } \\
\text { - } \\
\text { Einheit }\end{array}$} & \multicolumn{2}{|c|}{$\begin{array}{c}\text { Nach KA4 } \\
\text { (1994) }\end{array}$} & \multicolumn{6}{|c|}{$\begin{array}{c}\text { Mittels Lysimeter ermittelte } \\
\text { Bodenwasserentnahmemenge } \\
{[\mathrm{mm} / \mathrm{Jahr}]}\end{array}$} \\
\hline & $\begin{array}{c}\text { nFK }_{W e} \\
{[\mathrm{~mm}]}\end{array}$ & $\begin{array}{c}\text { We } \\
{[\mathrm{dm}]}\end{array}$ & SB $_{\text {Mittel }}$ & $\begin{array}{c}\mathbf{c}_{\mathrm{v}} \\
{[\%]}\end{array}$ & $\mathbf{S B}_{\text {min }}$ & $\begin{array}{c}\mathrm{SB}_{\max } \\
\left(\mathrm{nFK}_{\mathrm{We}}\right)\end{array}$ & \begin{tabular}{|l} 
Spann \\
-breite
\end{tabular} & $\begin{array}{c}\text { Einstufung } \\
{\text { der } \mathrm{SB}_{\max }}^{\left(\mathrm{nFK}_{\mathrm{We}}\right)}\end{array}$ \\
\hline $5-D 3$ & 68 & 5 & 64 & 15,6 & 41 & 75 & 34 & gering \\
\hline 4-D5 & 148 & 7 & 106 & 17,9 & 65 & 127 & 62 & gering \\
\hline $8-D 3$ & 158 & 7 & 101 & 17,8 & 66 & 124 & 58 & gering \\
\hline 1 -D6 & 179 & 10 & 117 & 24,8 & 58 & 164 & 106 & mittel \\
\hline 7 - D4 & 163 & 10 & 132 & 23,4 & 80 & 174 & 94 & mittel \\
\hline $11-D 3$ & 140 & 10 & 113 & 26,5 & 55 & 160 & 105 & mittel \\
\hline 9- Lö3 & 246 & 11 & 179 & 41,6 & 69 & $301(450)$ & 232 & sehr hoch \\
\hline 10 - Lö1 & 256 & 11 & 171 & 41,5 & 51 & $260(410)$ & 209 & sehr hoch \\
\hline
\end{tabular}

Zu Lysimetergruppe, Bodenform und Standorteinheit (D3-D6, Lö1, Lö3) s. Tab. 6:

Bei den Lößböden mit sehr hoher $\mathrm{nFK}_{\mathrm{We}}$ (Lysimetergruppe 9 und 10) wurden im Untersuchungszeitraum drei Perioden (1982 bis 1984, 1989 bis 1994 und 1996 bis 1998) beobachtet, in deren Verlauf zwar jedes Jahr Bodenwasser entnommen, aber zwischenzeitlich keine vollständige Auffüllung erreicht wurde (s. Tab. 16 und Anlage 5).

Tab. 16: Höhe des Bodenwasserdefizits bei den Lößböden zu Beginn der Ausschöpfung (a), am 1.4. des Jahres (b), Höhe der jährlichen Bodenwasserentnahme (c) und Wasserdefizit ermittelt auf Basis der kWB - zu Beginn der Ausschöpfung $(d)$ in [mm] am Standort Brandis

\begin{tabular}{|lc|c|c|c|c|c|c|c|c|c|c|c|c|c|c|c|c|c|}
\hline JAHR & $\mathbf{1 9 8 1}$ & $\mathbf{- 8 2}$ & $\mathbf{- 8 3}$ & $\mathbf{- 8 4}$ & $\mathbf{- 8 5}$ & $\mathbf{- 8 6}$ & $\mathbf{- 8 7}$ & $\mathbf{- 8 8}$ & $\mathbf{- 8 9}$ & $\mathbf{- 9 0}$ & $\mathbf{- 9 1}$ & $\mathbf{- 9 2}$ & $\mathbf{- 9 3}$ & $\mathbf{- 9 4}$ & $\mathbf{- 9 5}$ & $\mathbf{- 9 6}$ & $\mathbf{1 9 9 7}$ \\
\hline Lys.- & a) & - & 29 & 67 & 27 & - & - & - & - & 65 & 219 & 250 & 246 & 257 & - & - & 103 & 52 \\
Gr.9 & b) & - & 45 & 143 & 67 & - & - & - & - & 75 & 235 & 259 & 245 & 281 & 8 & - & 111 & 56 \\
& c) & 240 & 251 & 193 & 113 & 136 & 214 & 69 & 271 & 301 & 166 & 200 & 170 & 72 & 134 & 204 & 115 & 203 \\
\hline Lys.- & a) & - & 60 & 111 & 68 & - & - & - & - & 7 & 127 & 195 & 211 & 245 & - & - & 220 & 148 \\
Gr. 10 b) & - & 74 & 185 & 107 & 33 & - & - & - & 17 & 142 & 203 & 211 & 259 & - & - & 228 & 151 \\
& c) & 246 & 260 & 197 & 104 & 107 & 198 & 51 & 211 & 252 & 191 & 215 & 193 & 63 & 85 & 256 & 90 & 194 \\
\hline kWB & d) & - & - & 80 & 96 & 15 & 32 & - & - & 50 & 174 & 260 & 304 & 282 & - & - & - & - \\
Defizit
\end{tabular}

Lys.-Gr. = Lysimetergruppe; $\mathrm{kWB}=$ klimatische Wasserbilanz;

So ist z. B. das in Tab. 15 für die Lößböden ausgewiesenen $\mathrm{SB}_{\max }$ von $450 \mathrm{~mm}$ bzw. $410 \mathrm{~mm}$ die Summe aus der Bodenwasserentnahme von $200 \mathrm{~mm}$ bzw. $215 \mathrm{~mm}$ im Jahr 1991 und einem bereits aus den Vorjahren vorhandenen Wasserdefizit von $250 \mathrm{~mm}$ bzw. $195 \mathrm{~mm}$. Die maximale Entnahmemenge von $450 \mathrm{~mm}$ bzw. $410 \mathrm{~mm}$ ist also nicht das Ergebnis der Ausschöpfung nur eines Sommers und steht unter den klimatischen Bedingungen am Untersuchungsstandort nicht jedes Jahr in dieser Höhe zur Verfügung. 
Ein Vergleich zwischen $\mathrm{SB}_{\max }$ und der nach Bodenkundlicher Kartieranleitung (KA4, 1994) ermittelten $\mathrm{nFK}_{\mathrm{we}}$ ergab folgende Unterschiede (Tab. 15):

Im Fall der erodierten Braunerde mit sehr geringer Sandlößauflage (Lysimetergruppe 5) verweist die mittels Lysimeter ermittelte, etwas höhere pflanzenverfügbaren Wassermenge auf die Bildung von Stauwasser an der Grenze zu den darunter liegenden Mittel- und Grobsanden. Dieses führte zu einer etwas besseren Wasserversorgung der Pflanzen. Bei den Böden der Lysimetergruppe 4 und 8 werden mittels Lysimetermessung geringere pflanzenverfügbare Wassermengen ermittelt, als sich aus der Bodenkundlichen Kartieranleitung ableiten läßt. Eine mögliche Ursache für diese Unterschiede ist die Nährstoffversorgung der Lysimeterböden, die insbesondere bei den leichten Böden keine standorttypische Pflanzenentwicklung ermöglichte (Wasser- und Nährstoffmangel). Andererseits verweisen MÜLLER et al. (1996) auf eine mögliche Überschätzung des Wasserretentionsvermögens schwach lehmiger grundwasserferner Böden durch die Richtwerte der Bodenkundlichen Kartieranleitung (1994).

Bei den Böden der Lysimeter 1 und 7 (Sandlöß über Geschiebelehm) sind die Unterschiede gering. Abweichungen zeigen sich bei der pseudovergleyten Braunerde-Fahlerde (Lysimetergruppe 11), die ursprünglich auf Grund der fehlenden Sandlößauflage durch Nährstoffarmut und intensive Verwitterung gekennzeichnet sind. Auf diesen Böden kam in den Jahren 198290 Gülle zum Einsatz, was offensichtlich zu einer erheblichen Verbesserung der Nährstoffversorgung und des Wasserhaltevermöges führte (s. Tab. 15.

Deutlich größere Unterschiede zeigen sich bei den Lößböden (Lysimetergruppe 9 und 10). Bei diesen Böden bewirken - unter den klimatischen Bedingungen am Standort Brandis - eine ausgezeichnete kapillare Wasser- und Nährstoffanlieferung aus dem Unterboden, auch in niederschlagsarmen Jahren eine meist optimale Pflanzenentwicklung (s. Kap. 3.5.6). Es wird davon ausgegangen, dass noch bei hohen Saugspannungswerten bis $\mathrm{pF}$ 3,2 eine gute kapillare Leitfähigkeit gegeben ist. Die daraus resultierende sehr hohe pflanzenverfügbaren Wassermenge von $450 \mathrm{~mm}$ bzw. $410 \mathrm{~mm}$ findet sich bei ähnlichen klimatischen Bedingungen auch bei den Böden der Lysimeterstation Großobringen (Thüringen). Für die hier untersuchte tiefgründige Löß-Braunschwarzerde wurde ein ebenso hohes Bereitstellungsvermögen ermittelt (ROTH et al., 1995). Ein weiteres Beispiel liefern die Untersuchungen von OLIKOVA \& SYCHEVA (1996) in der Strelitzensteppe in Russland, wo für Schwarzerden eines tiefgründigen Löß mit tief liegendem Grundwasserstand eine vergleichbare Beanspruchung des Bodenwasservorrates registriert wurde.

Dazu abweichende Befunde zeigen sich in niederschlagsreicheren Gebieten. Die Untersuchungen von HELLEKES (1985) im Raum Mönchengladbach (Niederrheinisches Tiefland) ergaben bei Niederschlägen von 785 mm (1983) und 894 mm (1984) im Fall der untersuchten $2 \mathrm{~m}$ mächtigen Parabraunerde aus Löß eine vergleichsweise geringe Inanspruchnahme der Bodenwasservorräte. Gleiches gilt für die von GERIES (1989) in Dassensen (Raum Göttingen) untersuchten tiefgründigen Lößböden.

In Abb. 18 ist die tatsächliche jährliche Bodenwasserentnahme der untersuchten Böden noch einmal anschaulich zusammengestellt. Im Interesse der Übersichtlichkeit wurde die Zusammenfassung zu 4 Pedo-Hydrotopen (s. Kap. 4.1.1) gewählt. Bei Top a-c handelt es sich um die Böden mit geringer bis mittlerer $\mathrm{nFK}_{\mathrm{We}}$, bei denen der Bodenwasservorrat in jedem Jahr wieder aufgefüllt wird. Die dargestellte Bodenwasserentnahme entspricht der tatsächlichen jährlichen Entnahmemenge. Die entsprechende Säule für den Top d (Löß) setzt sich dagegen aus der jährlichen Bodenwasserentnahme und einem Defizit aus dem Vorjahr zusammen, das in einigen Jahren zu Beginn der Ausschöpfung noch anzutreffen ist (s. auch Tab. 16. 
Bezeichnend ist die jährlich gleiche Rangfolge der „Tope“. Geringe Unterschiede im Wasserverbrauch der Böden (oder eine andere Rangfolge) sind nur im Verlauf sehr feuchter Sommer (1987, 1993 und 1996) zu verzeichnen, in denen die unterschiedliche Kapazität der Bodenwasserspeicher nicht wirksam wird oder in Jahren mit schlechter Bestandsentwicklung infolge extremer Trockenheit wie im Jahr 1994.

Ursache für die überwiegend hohe Inanspruchnahme der Bodenwasservorräte ist die Höhe und der Verlauf der klimatischen Wasserbilanz (rote Säule). Mit der Summenkurve von $\mathrm{P}_{\text {korr }}$ minus Gras-Referenzverdunstung wird der potentielle Bodenwasserbedarf am Standort beschrieben (RENGER et al., 1974). So verweist auch die kWB in einigen Jahren zu Beginn der Ausschöpfung auf ein Bodenwasserdefizit aus dem Vorjahr (Tab. 16). Der Verlauf der kWB (=potentieller Bodenwasserbedarf) bzw. die den Lößböden (Top d + Defizit) entnommenen Bodenwassermengen entsprechen dem Bodenwasservorrat, der für eine kulturartenspezifische maximale Verdunstung am Standort Brandis verfügbar sein sollte.

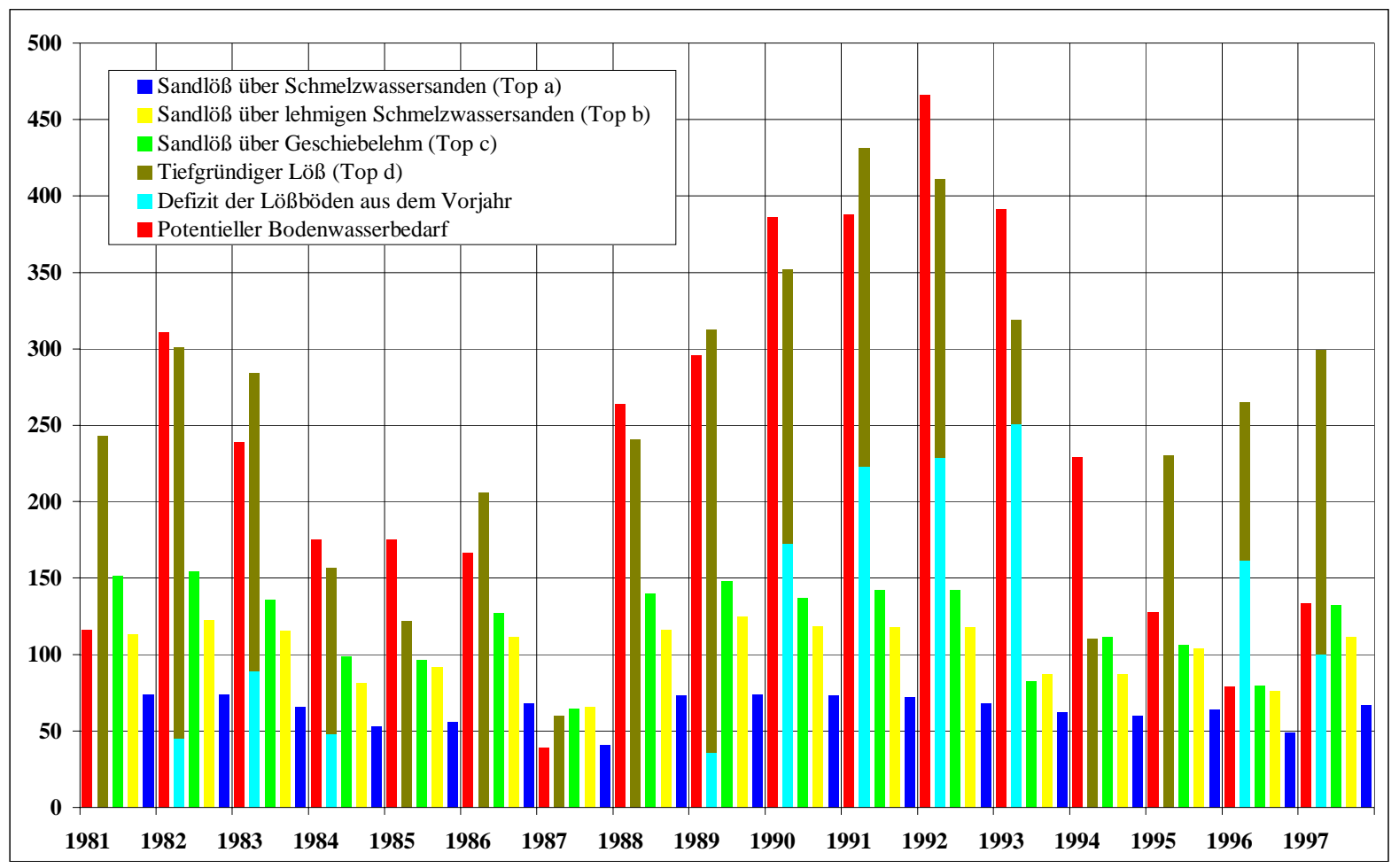

Abb. 18: Vergleich zwischen potentiellem Bodenwasserbedarf und tatsächlicher jährlicher Bodenwasserentnahme [mm/a]

Die jährliche Differenz zum Bodenwasserverbrauch der Böden mit reduzierter Verdunstung entspricht annähernd der Wassermenge, die zusätzlich für eine optimale Versorgung der Pflanzen auf diesen Böden benötigt wird. In der überwiegenden Anzahl der untersuchten Jahre sind diese Fehlmengen sehr hoch. Dabei ist zu berücksichtigen, dass eine optimale Bestandsentwicklung neben der optimalen Wasserversorgung auch aus einer optimalen Nährstoffversorgung resultiert. Diese scheint bei den Lößböden gesichert, bei den anderen Böden dagegen nicht über die gesamte Vegetationsperiode gegeben zu sein, da mit einer eingeschränkten Wasserversorgung auch eine mangelhafte Anlieferung von Nährstoffen verbunden ist.

Abschließend soll auf die Abweichungen eingegangen werden, die sich zwischen dem auf Basis der kWB ermittelten potentiellen Bodenwasserbedarf und der tatsächlichen jährlichen Bodenwasserentnahme (+ Defizit aus dem Vorjahr) auf den Lößböden ergeben. So lag die Wasserentnahme der Zuckerrüben 1981 und des Rotklees 1995 deutlich über dem potentiellen Bodenwasserdefizit. Auf Grund der hohen Wasserentnahme des Rotklees ist auf den Löß- 
böden zu Beginn der Jahre 1996 und 1997 noch ein Bodenwasserdefizit zu verzeichnen, das durch die kWB nicht ausgewiesen wird. 1994 lag die Verdunstung dagegen infolge des schlechten Grünbrachebestandes auf den Lößböden deutlich niedriger als die GrasReferenzverdunstung. Die Folge war eine vergleichsweise geringe Bodenwasserentnahme.

\subsubsection{Ausschöpfungstiefen}

In Abhängigkeit von den Ausschöpfungsmengen zeigen auch die jährlich erreichten Ausschöpfungstiefen (zur Begriffsbestimmung s. Kap. 2.3.3) eine hohe witterungsbedingte, boden- und kulturartenabhängige Variabilität.

Die größte Ausschöpfungstiefe $\left(\mathrm{We}_{\max }\right.$ ), die im Verlauf der 17 Untersuchungsjahre für die Böden der Lysimetergruppen 5, 4, 8, 1, 7 und 11 in einem Trockenjahr ermittelt wurde, entspricht der effektiven Durchwurzelungstiefe (We $\mathrm{Weff}_{\mathrm{ff}}$ u. a. nach SCHEFFER \& SCHACHTSCHABEL (1992) und KA4 (1994) (s. auch Abb. 10). Ausgangspunkt für die jährliche Ausschöpfung waren in jedem Fall Bodenwassergehalte zwischen Feldkapazität und Sättigung. Abweichend davon resultiert $\mathrm{We}_{\max }$ bei den Lößböden (Lysimetergruppe 9 und 10) aus der Ausschöpfung mehrerer aufeinanderfolgender Jahre (1988-91) bei fehlender Auffüllung der Böden zu Beginn des Frühjahrs dieser Untersuchungsjahre.

$\mathrm{We}_{\text {Mittel }}$ ist das arithmetische Mittel (Tab. 17) der in den 17 Einzeljahren erreichten Ausschöpfungstiefen. Die geringsten Ausschöpfungstiefen $\left(\mathrm{We}_{\min }\right)$ wurden auf allen Böden im Jahr 1987 mit Kartoffeln registriert, da Kartoffeln einen vergleichsweise geringen Wasserverbrauch haben und in diesem Sommerhalbjahr ausreichend Niederschlag fiel.

Tab. 17: Maximale, minimale und durchschnittliche Entnahmetiefen von Bodenwasser in den Jahren 1981-97 und Angaben zu deren Streuung (Angaben zu We hier ohne Berücksichtigung des Stein- und Humusgehaltes)

\begin{tabular}{|l|c|c|c|c|c|c|}
\hline Bodenform (Lysimetergruppe) & $\begin{array}{c}\boldsymbol{s}_{\boldsymbol{x}} \\
{[\mathbf{c m}]}\end{array}$ & $\begin{array}{c}\boldsymbol{c}_{\boldsymbol{v}} \\
{[\%]}\end{array}$ & $\begin{array}{c}\mathbf{W e}_{\text {Mittel }} \\
{[\mathbf{c m}]}\end{array}$ & $\begin{array}{c}\mathbf{W e}_{\mathbf{m i n}} \\
{[\mathbf{c m}]}\end{array}$ & \multicolumn{2}{|c|}{$\begin{array}{c}\mathbf{W e}_{\mathbf{m a x}} \\
{[\mathbf{c m}]}\end{array}$} \\
\hline Erodierte Braunerde (5) & 5 & 18,2 & 26 & 15 & 31 & 1981 \\
\hline Braunerde-Fahlerde (4) & 7 & 18,6 & 40 & 24 & 45 & 1992 \\
Parabraunerde-Braunerde (8) & 7 & 18,9 & 38 & 24 & 47 & 1989 \\
\hline lessivierter Braunerde-Pseudogley (1) & 15 & 29,1 & 51 & 22 & 76 & 1982 \\
Braunerde-Pseudogley (7) & 17 & 28,4 & 60 & 32 & 84 & 1988 \\
pseudovergleyte Braunerde-Fahlerde 11) & 18 & 27,5 & 65 & 31 & 92 & 1981 \\
\hline Parabraunerde aus Löß (9) & 45 & 40,2 & 109 & 23 & $189^{*}$ & 1991 \\
Schwarzerde aus Löß (10) & 43 & 39,4 & 112 & 31 & $173^{*}$ & 1991 \\
\hline
\end{tabular}

*Ergebnis der Ausschöpfung mehrerer aufeinanderfolgender Jahre (1988-91) bei fehlender Auffüllung der Böden zu Beginn des Frühjahrs

Im Fall der erodierten Braunerde (Lysimetergruppe 5) ist anzunehmen, dass keine Wasseranlieferung aus dem IICv-Horizont erfolgt (s. hierzu auch Anlage 2), d. h. daß nur das Bodenwassser der rd. $35 \mathrm{~cm}$ mächtigen Sandlößauflage pflanzenverfügbar ist.

Die für Lysimetergruppe 4 (Braunerde-Fahlerde) und Lysimetergruppe 8 (ParabraunerdeBraunerde) ermittelten $\mathrm{We}_{\max }$ deuten darauf hin, dass auch bei diesen Böden die Pflanzen nur das Wasser innerhalb der hier etwas mächtigeren $(45 \mathrm{~cm}-55 \mathrm{~cm}$ ), teils taschenartig (bis 90 $\mathrm{cm})$ ausgebildeten Sandlößauflage nutzen.

Anders bei den Böden der Lysimetergruppe 1, 7 und 11 - hier erfolgt eine Bodenwasserentnahme auch unterhalb der Sandlößauflage aus dem Geschiebelehm bzw. -mergel der Saale-Kaltzeit. Obwohl die Geschiebelehm- bzw. -mergelablagerungen unterschiedlich 
mächtig sind (Reichweite von 1,20 m bis 2,40 m unter Gelände), wird bei allen Lysimetern ein relativ einheitliches $\mathrm{We}_{\max }\left(=\mathrm{We}_{\text {eff }}\right)$ zwischen $76 \mathrm{~cm}$ und $92 \mathrm{~cm}$ erreicht. Auf Grund der kleinräumigen Hetorogenitäten (große Speicher- und Leitfähigkeitsunterschiede) ist tatsächlich in diesen Böden mit einer sehr unregelmäßigen Ausbildung der Wasserscheide im Profil zu rechnen, die zum Teil weit unterhalb der geschätzten Ausschöpfungstiefen $\mathrm{We}_{\max }$ liegen wird (s. Beschreibung der Herkunftsflächen in Kap. 2.2.1 und Profilbeschreibung in Anlage 2: Feinwurzelbahnen auf Klüften bis 1,35 m unter Gelände).

Zwischen Unterkante der Geschiebelehm- bzw. -mergelschicht und der in $3 \mathrm{~m}$ Tiefe befindlichen Bodenplatte lagern in den Lysimetern in jedem Fall mindestens $60 \mathrm{~cm}$ (maximal 180 $\mathrm{cm})$ gut durchlässige Schmelzwassersande $\left(\mathrm{k}_{\mathrm{f}} \approx 1-2,9 * 10^{-4}\right)$ mit geringer kapillarer Steighöhe.

Auf Grund der ermittelten Ausschöpfungstiefen (= effektiven Durchwurzelungstiefen) und der vorliegenden Schichtungen wird eingeschätzt, dass ein Einfluss der in $3 \mathrm{~m}$ Tiefe befindlichen Bodenplatte auf den Verdunstungsprozess in den Lysimetern der Gruppen 5, 4, 8, 1, 7 und 11 nicht vorliegt.

Bei den Lößböden (Lysimetergruppe 9 und 10) reicht die effektive Durchwurzelungstiefe vermutlich bis zur Schichtgrenze Löß-Schmelzwassersand bei ca. 2,0 - 2,2 m unter Gelände (einzelne Wurzelbahnen bis 1,7 m). Auch eine Anlieferung von Stauwasser aus dem Bereich der Bodenplatte an diese Schichtgrenze kann bei den Lysimetern der Gruppe 9 und 10 nicht ausgeschlossen werden.

Tab. 18 zeigt die Spannbreite möglicher We mit Bezug zu den angebauten Fruchtarten. Zu beachten ist die geringe Anzahl von Anbaujahren, woraus zufällige Kombinationen von Kulturart und Witterung im entsprechenden Anbaujahr resultieren (z. B. Anbau von Kartoffeln nur in relativ feuchten Jahren). Unter Berücksichtigung dieser Einschränkung zeigt sich, dass Fruchtarten wie Kartoffeln und Gras prinzipiell auf allen Böden weniger Bodenwasser verbrauchen als Zuckerrüben und Getreide. Ursachen dafür sind die kurze Vegetationszeit und die geringe Wurzelbildung der Kartoffeln. Die teils geringe Ausschöpfungstiefe bei Gras resultiert aus der schlechten Bestandsentwicklung im Untersuchungszeitraum.

Tab. 18: Ausschöpfungstiefen [cm] der verschiedenen Kulturarten (Anzahl der Anbaujahre) in Abhängigkeit vom Boden (Lysimetergruppe)

\begin{tabular}{|c|c|c|c|c|c|c|}
\hline \multirow{2}{*}{$\begin{array}{l}\text { Lys.-Gr. } \\
\text { - Standort } \\
\text { einheit }\end{array}$} & \multicolumn{6}{|c|}{ Fruchtart (Anzahl der Anbaujahre) } \\
\hline & $\begin{array}{c}\text { Zu.-Rüben } \\
\text { (2) }\end{array}$ & $\begin{array}{c}\text { Kartoffeln } \\
\text { (3) }\end{array}$ & \begin{tabular}{|c|} 
Wi.-Weizen \\
$(4)$
\end{tabular} & \begin{tabular}{|c}
$\begin{array}{c}\text { Wi.-Gerste } \\
(4)\end{array}$ \\
\end{tabular} & $\begin{array}{c}\text { Gras/Grünbrache } \\
\text { (3) }\end{array}$ & $\begin{array}{c}\text { Rotklee } \\
(1)\end{array}$ \\
\hline $5-D 3$ & $30-31$ & $15-22$ & $28-31$ & $27-31$ & $20-25$ & 26 \\
\hline 4 - D5 & $40-42$ & $24-33$ & $42-45$ & $42-47$ & $30-33$ & 35 \\
\hline 8 - D3 & $45-47$ & $24-35$ & $40-48$ & $40-48$ & $30-34$ & 43 \\
\hline 1 - D6 & $60-69$ & $22-38$ & $51-76$ & $55-62$ & $36-38$ & 51 \\
\hline 7 - D4 & $56-67$ & $32-46$ & $72-84$ & $65-76$ & $38-70$ & 44 \\
\hline $11-D 3$ & $86-92$ & $31-50$ & $62-84$ & $69-82$ & $36-60$ & 54 \\
\hline 9 - Lö3 & $104-134$ & $23-130$ & $84-173$ & $109-169$ & $37-130$ & 128 \\
\hline 10 - Lö1 & $107-164$ & $31-97$ & $95-189$ & $115-177$ & $58-146$ & 90 \\
\hline
\end{tabular}

Zu Lysimetergruppe (Lys.-Gr.), Bodenform und Standorteinheit (D3-D6, Lö1, Lö3) s. Tab. 6 Angaben zu We ohne Berücksichtigung des Steingehaltes 
Analog zu den bereits diskutierten Unterschieden bei den Ausschöpfungsmengen sind auch die mittels Lysimeter ermittelten $W_{\text {eff }}$ der leichten Böden geringer und die der Lößböden größer als die mittels KA4 (1994) ausgewiesenen Werte.

Nachfolgend soll am Beispiel der Jahre 1981 und 1990 mit Zuckerrüben (s. Abb. 19) der innerjährliche Verlauf von sommerlicher Ausschöpfung und Wiederauffüllung beschrieben werden. Diese Form der Darstellung ist praktisch für alle Böden und alle Anbaujahre möglich.

Der zeitliche Beginn der sommerlichen Ausschöpfung ist bei allen Böden gleich. Er hängt, da zumindestens der Oberboden voll gesättigt ist und die Pflanzen noch keinen oder nur geringen Einfluss auf die Verdunstung haben, nur vom Witterungsverlauf ab. Zu Beginn der Vegetationsperiode im Jahr 1990 (Aussaat der Zuckerrüben bereits am 30. März) bestand in den unteren Bodenschichten der Lysimetergruppen 9 und 10 noch ein Wasserdefizit von 219 $\mathrm{mm}$ bzw. $127 \mathrm{~mm}$. Die Wasseraufnahme der Zuckerrüben erfolgte zunächst aus dem bereits aufgefüllten Oberboden. Mit wachsendem Wasserbedarf der Zuckerrüben (spätestens ab Mai) erreichten die Pflanzenwurzeln Bereiche mit unvollständiger Auffüllung, so dass schnell tiefere Schichten in Anspruch genommen werden mussten. Trotz des Wasserdefizites aus dem Vorjahr wurden 1990 auf den Böden der Lysimetergruppe 10 ebenso hohe Erträge (bei gleichen ETK = Evapotranspirationskoeffizienten, s. Kap. 3.5.6) wie 1981 erzielt. Auf den Böden der Lysimetergruppe 9 waren die Erträge und ETK 1990 ungünstiger, da das Bodenwasserdefizit aus dem Vorjahr deutlich größer war (Tab. 16.

Das Maximum der Ausschöpfung wurde bei den Böden mit geringer und mittlerer $\mathrm{nFK}_{\mathrm{We}} \mathrm{im}$ Jahr 1990 nahezu gleichzeitig bereits in der ersten Augustdekade und bei den Lößböden (Lysimetergruppe 9 und 10) Mitte August erreicht. Ursache dafür war die lang anhaltende Trockenheit von Ende August bis Ende Oktober. Geerntet wurde am 04.10.1990. Im Jahr 1981 dauerte die Phase der Ausschöpfung dagegen bis Mitte September (zwei Dekaden vor der Ernte). Der Zeitpunkt der maximalen Ausschöpfung hängt u. a. auch davon ab, ob Niederschlagsereignisse im Juli, August und September noch einen Wachstumsschub bei den Zuckerrüben bewirken können oder nicht.

Ist der Niederschlag nach der Ernte größer als die Evaporation der Böden, beginnt die Wiederauffüllung. Der Zeitpunkt, zu dem die Wiederauffüllung abgeschlossen ist, hängt von der Witterung nach der Ernte und von der Höhe der sommerlichen Ausschöpfung ab. 1981 war bei allen Böden (außer bei den Lößböden) Ende November die Wiederauffüllung abgeschlossen. Im Jahr 1991 war dies Ende Dezember der Fall, bei der Lysimetergruppe 11 erst im März und bei den Lößböden erfolgte keine Wiederauffüllung. 

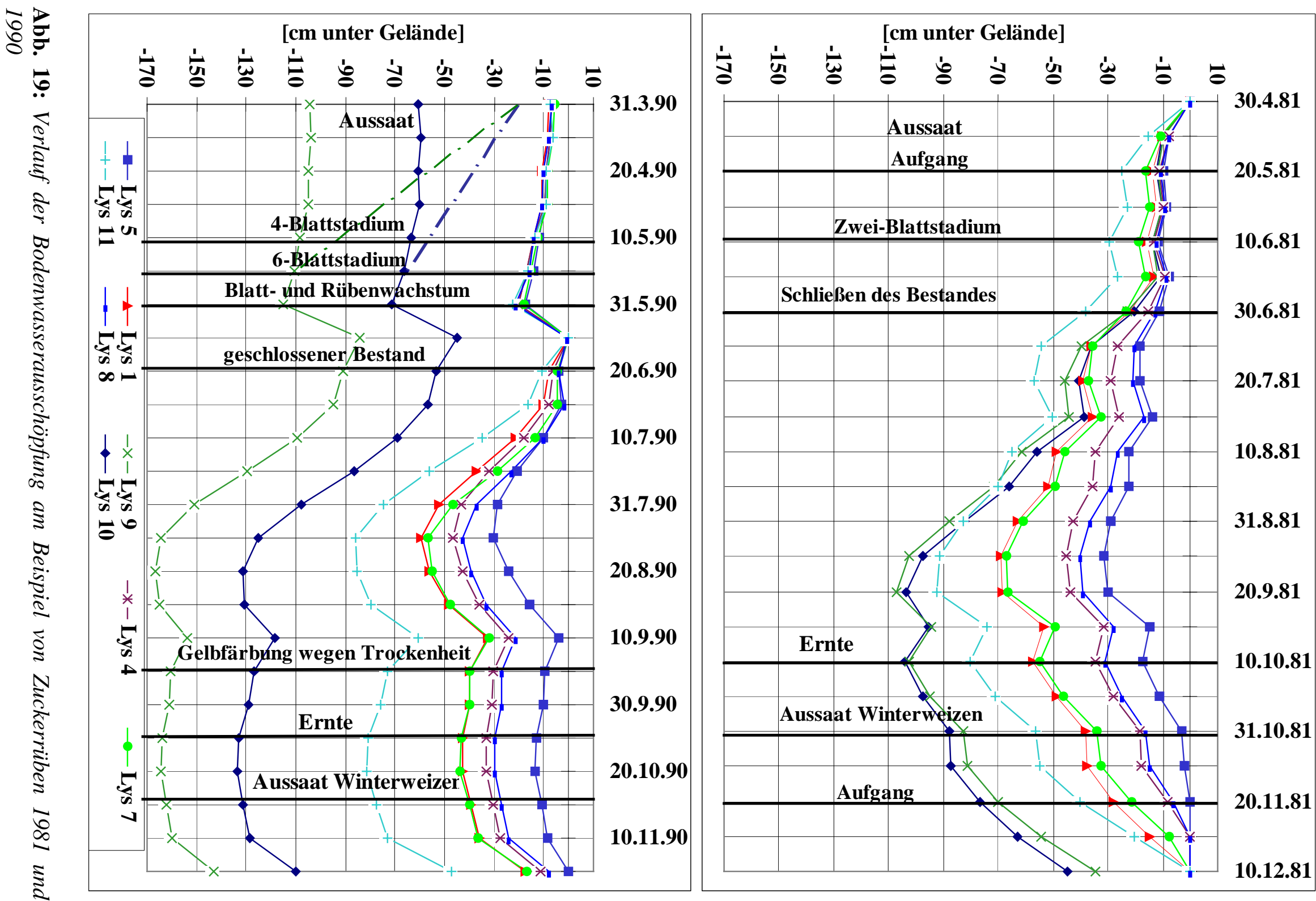


\subsubsection{Zusammenfassung und Diskussion der Ergebnisse}

Der Wasserhaushalt des Untersuchungsgebietes ist - klimatisch bedingt - sehr angespannt. Stehen sich im Mittel der Jahre Verdunstungsanspruch der Atmosphäre (potentielle Verdunstung als Gras-Referenzverdunstung) und Wasserdargebot (korrigierter Niederschlag) relativ ausgeglichen gegenüber, so zeigen sich in Einzeljahren (z. B. 1982 und 1991: kWB = $145 \mathrm{~mm}$ ) und im Verlauf der Sommermonate (Sommerhalbjahr 1989: kWB $=-284 \mathrm{~mm}$ ) hohe Wasserbilanzdefizite. Die Wasserhaushaltssituation ist besonders kritisch, wenn mehrere Jahre mit negativer kWB nacheinander auftreten, wie zum Beispiel im Zeitraum von 1988-91. Diese klimatischen Bedingungen sind typisch für den Grenzraum des Mitteldeutschen Trockengebietes. Sie finden sich prinzipiell in nahezu alle Gebiete der neuen Bundesländer (außer Harz, Mittelgebirge und deren Vorländer) und sind in den alten Bundesländern nur im Oberrheingraben und in der südlichen Hessischen Senke anzutreffen.

Auf Grund der klimatischen Bedingungen kommt der Speicherkapazität der Böden im Untersuchungsgebiet eine hervorragende Rolle zu. Steht für die Vegetation bei hohem Verdunstungsanspruch der Atmosphäre nicht ausreichend Wasser aus Niederschlägen zur Verfügung, entscheidet die Höhe des pflanzenverfügbaren Bodenwasservorrates über die Höhe der realen Verdunstung.

Untersucht wurden Böden mit geringer, mittlerer und sehr hoher nutzbarer Feldkapazität. Bei allen Böden zeigten sich im Verlauf der 17 Untersuchungsjahre in Abhängigkeit von kWB, Witterung und Fruchtart, große Unterschiede in der Höhe der jährlichen Bodenwasserentnahme. Wie hoch der Bodenwasserbedarf der Pflanzen für eine kulturartenspezifische Verdunstung und Ertragsbildung am Untersuchungsstandort tatsächlich ist, wird am Beispiel der Lößböden deutlich. Sie stellen mehrere Jahre hintereinander 150-200 mm Bodenwasser für die Verdunstung zur Verfügung und garantieren - ohne dass es im Frühjahr zu einer Auffüllung bis zur Feldkapazität kommt - eine nahezu optimale Wasserversorgung der Pflanzen. So wurde ein Wasserbereitstellungsvermögen von mehr als $400 \mathrm{~mm}$ ermittelt, das aus der Bodenwasserentnahme von rd. $200 \mathrm{~mm}$ im Jahr 1991 und einem bereits aus den Vorjahren vorhandenen Wasserdefizit in gleicher Höhe resultiert. Nach GÜNTHER (2000) konnte bei Lysimeterversuchen mit Schwarzerden in Großobringen für tiefwurzelnde Fruchtarten (z. B. Wintergetreide) bei Beregnung keine Verbesserung der Bestandsentwicklung erreicht werden. Inwieweit die Lößböden eine optimale Wasserversorung auch bei Flachwurzlern wie Kartoffeln realisieren, konnte in Brandis nicht geklärt werden, da es sich bei den Anbaujahren mit Kartoffeln stets um Feuchtjahre handelte.

Die Böden mit geringer $\mathrm{nFK}_{\mathrm{We}}$ (Sandlöß über Schmelzwassersanden: Lysimetergruppe 5, 4 und 8), aber auch die Böden der Lysimetergruppen 1, 7 und 11 (Sandlöß über Geschiebelehm bzw. - mergel) mit mittlerer $\mathrm{nFK}_{\mathrm{We}}$ können den Wasserbedarf der Pflanzen am Untersuchungsstandort in der überwiegenden Anzahl der Jahre nicht decken. Die Differenz zum Bodenwasserverbrauch der Pflanzen auf den Lößböden zeigt an, wie hoch der Wasserbedarf für eine optimale Versorgung der Pflanzen auf diesen Böden tatsächlich ist.

Auf die Trockenheitsgefährdung des Untersuchungsgebietes infolge ungünstiger Niederschlagsbedingungen und der weit verbreiteten sehr heterogenen, schlecht wasserspeichernden Böden der Moränengebiete verweisen u. a. auch LINKE (1991) und SEIDLER (1999). Diese Trockenheitsgefährdung war Ursache dafür, daß sich in der Vergangenheit zahlreiche Untersuchungen der Beregnungsbedürftigkeit grundwasserferner Böden widmeten. Stellvertretend genannt werden die Arbeiten von WEISE \& WENDLING (1974).

In Abhängigkeit von der angebauten Frucht zeigen auch die Entnahmetiefen für Bodenwasser eine boden- und witterungsbedingte Spannbreite. So wurden z. B. in unterschiedlichen An- 
baujahren für Winterweizen auf den Lößböden Entnahmetiefen zwischen $95 \mathrm{~cm}$ (1986) und $190 \mathrm{~cm}$ (1991) erreicht. Auf den Böden mit geringer $\mathrm{nFK}_{\mathrm{We}}$ zeigten sich beim Vergleich einzelner Jahre bei Winterweizen kaum Unterschiede, da der verfügbare Bodenspeicher immer vollständig geleert wurde.

Für jeden Boden wurde im Sinne der „effektiven Durchwurzelungstiefe“ (We $\mathrm{Weff}_{\text {f }}$ die im Verlauf der 17 Untersuchungsjahre absolut tiefste Ausschöpfung in einem Trockenjahr ermittelt.

Im Fall der leichten Böden mit geringer $\mathrm{nFK}_{\mathrm{We}}$ (Sandlöß über Schmelzwassersanden $=$ Lysimetergruppe 5, 4 und 8) blieb die Wasserentnahme der Pflanzen auf den Bereich der Sandlößauflage beschränkt ( $\mathrm{rd} .35 \mathrm{~cm}$ und $55 \mathrm{~cm}$ taschenartig bis ca. $90 \mathrm{~cm}$ ). We eff ist damit kleiner als die entsprechenden Richtwerte in der Bodenkundlichen Kartieranleitung (1994).

Bei den Böden mit mittlerer $\mathrm{nFK}_{\mathrm{We}}$ (Sandlöß über Geschiebelehm bzw. -mergel = Lysimetergruppe 1, 7 und 11) erfolgt eine Bodenwasserentnahme auch unterhalb der Sandlößauflage aus dem Geschiebelehm bzw. -mergel der Saale-Kaltzeit $(76 \mathrm{~cm}-92 \mathrm{~cm})$. Diese Böden sind sehr heterogen mit großen Speicher- und Leitfähigkeitsunterschieden, woraus eine sehr unregelmäßige Wasseranlieferung im Profil resultiert (Feinwurzelbahnen auf Klüften bis $1,35 \mathrm{~m}$ unter Gelände).

Die effektive Durchwurzelungstiefe der Lößböden beschreibt den Wurzelraum und einen Raum kapillarer Nachlieferung der in Trockenzeiten in Anspruch genommen wird. Dieser reicht bis zur Schichtgrenze Löß-Schmelzwassersand bei ca. 2,0 - 2,2 m unter Gelände (einzelne Wurzelbahnen bis 1,7 m).

Mit Kenntnis der Ausschöpfungstiefen im Lysimeter wird bei den Böden mit geringer und mittlerer $\mathrm{nFK}_{\mathrm{We}}$ davon ausgegangen, daß sich verdunstungsbeeinflußte Zone und der Kapillarsaum über dem Lysimeterboden nicht beeinflussen. Damit kann eine Veränderung der Verdunstung und Sickerwasserbildung im Vergleich zum Freiland weitgehend ausgeschlossen werden. Für die Untersuchungsergebnisse der Lößböden muß dagegen einschränkend festgestellt werden, dass sie nur für Standorte mit Lößauflagen ähnlicher Mächtigkeit gelten. Im Verlauf der Trockenperioden wird das Stauwasser über der Bodenplatte nahezu aufgebraucht.

\subsection{Verdunstung in Abhängigkeit von Witterung, Boden und Bewirtschaftung}

\subsubsection{Jahreswerte der realen Verdunstung}

Die Darstellung von Jahres-, Halbjahres- und Monatswerten bedient zunächst wasserwirtschaftliche Interessen. Deshalb wurde als Bilanzjahr das Wasserhaushaltsjahr von November bis Oktober zu Grunde gelegt. Für detaillierte Betrachtungen zur Verdunstung der Pflanzenbestände werden kürzere Zeitintervalle (Dekaden) gewählt (Kap. 3.5.6).

Die Verdunstung ist sowohl Teil der Energie- als auch der Wasserbilanz. Somit bestand die Möglichkeit, die Höhe der potentiellen (maximalen) Verdunstung mit zwei verschiedenen und unabhängigen Methoden zu ermitteln:

a) aus meteorologischen Daten als potentielle Verdunstung (hier als Gras-Referenzverdunstung) und 
b) mittels Wasserhaushaltsgleichung auf Basis von Lysimetermessungen mit Lößböden (sehr hohe $\mathrm{nFK}_{\mathrm{We}}$ ).

Im Vergleich der Ergebnisse auf Basis der langjährigen Mittelwerte zeigt (Tab. 19, dass die Verdunstung der Lößböden (= landnutzungsabhängige potentielle Verdunstung) mit rd. 630 $\mathrm{mm} / \mathrm{Jahr}$ ebenso hoch ist, wie die Gras-Referenzverdunstung. Die Abweichungen, die in Einzeljahren auf Grund der Bestandsunterschiede zwischen Gras und den angebauten Ackerkulturen auftreten, scheinen sich im Mittel der Jahre auszugleichen. Auch GÜNTHER (1999) ermittelte für die Lößböden der Lysimeterstation Großobringen im Mittel der Jahre 1983-96 geringe Unterschiede zwischen berechneter und mittels Lysimeter (bei nahezu uneingeschränkter Wasserversorgung) gemessener potentieller Verdunstung.

So konnte die maximale Verdunstung des Untersuchungsstandortes mittels zweier unabhängiger Methoden in guter Übereinstimmung ermittelt werden.

Auf den Böden mit mittlerer und geringer $\mathrm{nFK}_{\mathrm{We}}$ ist die ermittelte Verdunstung infolge Wassermangel deutlich geringer als die maximale Verdunstung. So wurde für die Böden mit geringer $\mathrm{nFK}_{\mathrm{We}}$ (Lysimetergruppe 5, 4 und 8) ein Jahresmittel der Verdunstung von rd. 520 $\mathrm{mm}$ und für die Böden mit mittlerer $\mathrm{nFK}_{\mathrm{We}}$ (Lysimetergruppe 1, 7 und 11) von rd. $564 \mathrm{~mm}$ ermittelt. Nur in feuchten, strahlungsarmen Jahren wie z. B. 1987 wird auch auf diesen Böden eine potentielle (maximale) Verdunstung realisiert.

Bei vergleichbaren Niederschlägen berechneten MÜLLER et al. (1996) für grundwasserferne Böden des Pleistozäns mit geringem Haftwasser in Nord- und Mitteldeutschland eine reale Verdunstung von rd. $500 \mathrm{~mm}$. Deutliche Unterschiede ergeben sich $\mathrm{zu}$ den realen Verdunstungshöhen, die von KUNKEL \& WENDLAND (1998) im Rahmen von Wasserhaushaltsuntersuchungen für den deutschen Teil des Elbeeinzugsgebietes (Jahresreihe 196190) ermittelt wurden. Die für das Untersuchungsgebiet ermittelten Verdunstungshöhen sind um 50-70 mm geringer als die mittels Lysimeter ermittelten Werte.

Ursachen dafür sind u. a. die klimatischen Unterschiede zwischen den Beobachtungsreihen 1961-90 und 1981-97 (s. Kap. 4.2) und die Tatsache, dass den Berechnungen von KUNKEL \& WENDLAND (1998) vermutlich unkorrigierte Niederschläge zu Grunde liegen.

Die Ergebnisse in Tab. 19 führten für den Standort Brandis zu folgenden Erkenntnissen:

- Die in Einzeljahren durch unterschiedliche Witterung bei gleichen Ackerfrüchten hervorgerufene Variation der Jahresverdunstung ist teils geringer (bei Zuckerrüben, Wintergetreide) und teils größer (bei Kartoffeln, Gras/Klee) als die bodenabhängigen Differenzierungen.

- Die Unterschiede in der jährlichen Höhe der Verdunstung, die durch verschiedene Witterung und Fruchtarten im Verlauf der 17 Beobachtungsjahre hervorgerufen werden, sind bei Variationskoeffizienten zwischen 8 und $12 \%$ auf allen Böden ähnlich. Eine Ausnahme bildet mit $17 \%$ die Lysimetergruppe 10 (Lö1) infolge der außergewöhnlich hohen Verdunstung des Rotklees im Jahr 1995. 
Tab. 19: Jahreswerte der Gras-Referenzverdunstung (ETP) und der realen Verdunstung für die 8 verschiedenen Böden (Gruppenmittel) in Reihung nach der Vegetation

\begin{tabular}{|c|c|c|c|c|c|c|c|c|c|c|c|}
\hline \multirow[t]{2}{*}{ Jahr } & \multirow[t]{2}{*}{ Fruchtart } & \multirow[t]{2}{*}{ ETP } & \multicolumn{8}{|c|}{$\begin{array}{c}\text { reale Verdunstung [mm/Jahr] } \\
\text { (von November - Oktober) }\end{array}$} & \\
\hline & & & $\begin{array}{c}\text { Lys } 5 \\
\text { D3 }\end{array}$ & $\begin{array}{c}\text { Lys } 4 \\
\text { D5 }\end{array}$ & \begin{tabular}{|c} 
Lys 8 \\
D3
\end{tabular} & \begin{tabular}{|l} 
Lys 1 \\
D6
\end{tabular} & \begin{tabular}{|l} 
Lys 7 \\
D4
\end{tabular} & $\begin{array}{l}\text { Lys } 11 \\
\text { D3 }\end{array}$ & $\begin{array}{c}\text { Lys } 9 \\
\text { Lö3 }\end{array}$ & $\begin{array}{l}\text { Lys } 10 \\
\text { Lö1 }\end{array}$ & $\begin{array}{l}\mathrm{cv} \\
{[\%}\end{array}$ \\
\hline 1981 & Zu.-Rübe & 567 & 514 & 565 & 559 & 608 & 608 & 634 & 703 & 719 & 11 \\
\hline 1990 & Zu.-Rübe & 726 & 534 & 596 & 592 & 618 & 604 & 644 & 686 & 720 & 9 \\
\hline$[\mathrm{mm}]$ & Mittelwert & 646 & 524 & 580 & 575 & 613 & 606 & 639 & 694 & 720 & \\
\hline & Standardabw. & 112 & 14 & 22 & 23 & 7 & 3 & 8 & 12 & 0 & \\
\hline $\begin{array}{l}c_{\mathrm{v}} \\
{[\%]}\end{array}$ & Varianzkoeff. & 17 & 3 & 4 & 4 & 1 & 1 & 1 & 2 & 0 & \\
\hline 1985 & Kartoffel & 591 & 417 & 446 & 444 & 456 & 471 & 446 & 490 & 495 & 6 \\
\hline 1987 & Kartoffel & 518 & 495 & 521 & 512 & 531 & 550 & 512 & 530 & 505 & 3 \\
\hline 1996 & Kartoffel & 524 & 485 & 515 & 521 & 526 & 530 & 526 & 563 & 546 & 4 \\
\hline$[\mathrm{mm}]$ & Mittelwert & 544 & 466 & 494 & 492 & 504 & 517 & 495 & 527 & 515 & \\
\hline & Standardabw. & 41 & 43 & 42 & 42 & 42 & 41 & 42 & 37 & 27 & \\
\hline $\begin{array}{l}\mathrm{c}_{\mathrm{v}} \\
{[\%]}\end{array}$ & Varianzkoeff. & 7 & 9 & 9 & 9 & 8 & 8 & 9 & 7 & 5 & \\
\hline 1982 & Wi.-Weizen & 673 & 433 & 486 & 483 & 535 & 531 & 499 & 612 & 627 & 13 \\
\hline 1986 & Wi.-Weizen & 578 & 440 & 511 & 510 & 530 & 567 & 514 & 627 & 614 & 11 \\
\hline 1988 & Wi.-Weizen & 627 & 431 & 468 & 484 & 492 & 558 & 483 & 637 & 579 & 13 \\
\hline 1991 & Wi.-Weizen & 651 & 457 & 500 & 505 & 529 & 541 & 501 & 590 & 607 & 9 \\
\hline$[\mathrm{mm}]$ & Mittelwert & 632 & 440 & 491 & 496 & 522 & 549 & 499 & 616 & 607 & \\
\hline & Standardabw. & 41 & 12 & 19 & 14 & 20 & 16 & 13 & 21 & 20 & \\
\hline $\begin{array}{l}\mathbf{c}_{\mathrm{v}} \\
{[\%]}\end{array}$ & Varianzkoeff. & 6 & 3 & 4 & 3 & 4 & 3 & 3 & 3 & 3 & \\
\hline 1983 & Wi.-Gerste & 662 & 510 & 586 & 562 & 620 & 618 & 616 & 654 & 653 & 8 \\
\hline 1989 & Wi.-Gerste* & 699 & 513 & 554 & 558 & 572 & 595 & 587 & 731 & 703 & 13 \\
\hline 1992 & Wi.-Gerste** & 596 & 505 & 561 & 549 & 550 & 595 & 587 & 624 & 652 & 8 \\
\hline 1997 & So.-Weizen & 651 & 489 & 518 & 522 & 534 & 560 & 560 & 675 & 643 & 12 \\
\hline$[\mathrm{mm}]$ & Mittelwert & 652 & 504 & 555 & 548 & 569 & 592 & 587 & 671 & 663 & \\
\hline & Standardabw. & 42 & 11 & 28 & 18 & 37 & 24 & 23 & 45 & 27 & \\
\hline $\begin{array}{l}c_{\mathrm{v}} \\
{[\%]}\end{array}$ & Varianzkoeff. & 7 & 2 & 5 & 3 & 7 & 4 & 4 & 7 & 4 & \\
\hline 1984 & Weidelgras & 559 & 494 & 518 & 522 & 543 & 555 & $\overline{553}$ & 573 & 567 & 5 \\
\hline 1993 & Grünbrache & 619 & 572 & 606 & 602 & 640 & 616 & 605 & 619 & 611 & 3 \\
\hline 1994 & Grünbrache & 682 & 484 & 506 & 508 & 531 & 599 & 546 & 580 & 512 & 7 \\
\hline 1995 & Rotklee & 669 & 615 & 701 & 640 & 710 & 650 & 679 & 796 & 938 & 15 \\
\hline$[\mathrm{mm}]$ & Mittelwert & 632 & 541 & 583 & 568 & 606 & 605 & 596 & 642 & 657 & \\
\hline & Standardabw. & 56 & 63 & 91 & 64 & 85 & 39 & 62 & 104 & 192 & \\
\hline $\begin{array}{l}c_{\mathrm{v}} \\
{[\%]}\end{array}$ & Varianzkoeff. & 9 & 12 & 16 & 11 & 14 & 7 & 10 & 16 & 29 & \\
\hline$[\mathrm{mm}]$ & \begin{tabular}{|l|} 
Mittel \\
1981-97
\end{tabular} & 632 & 493 & 539 & 534 & 560 & 573 & 558 & 629 & 629 & \\
\hline $\mathbf{S}$ & Standardabw. & 61 & 51 & 61 & 48 & 61 & $\overline{43}$ & 64 & $\overline{75}$ & 106 & \\
\hline $\begin{array}{l}\mathrm{c}_{\mathrm{v}} \\
{[\%]}\end{array}$ & Varianzkoeff. & 10 & 10 & 11 & 9 & 11 & 8 & 11 & 12 & 17 & \\
\hline
\end{tabular}

*und als Zweitfrucht Ölrettich; **anschließend Grünbrache 
Als Ursache für die geringen Unterschiede in der Streuung der Jahresverdunstung gleicher Fruchtarten auf den verschiedenen Böden wurde der Einfluss der Winterverdunstung vermutet, die ja auf allen Böden ähnlich ist. Weiterführende Untersuchungen zu dieser Problematik werden in Kap. 3.5.6 beschrieben.

Hinweise auf einen engen Zusammenhang zwischen Boden (verfügbarem Bodenwasservorrat), Fruchtart und Jahresverdunstung liefert Abb. 20. Auch in dieser Darstellung zeigen sich die größten bodenabhängigen Differenzierungen im Mittel der Jahre bei Zuckerrüben und Wintergetreide und die geringsten bei Gras (ohne Rotklee) und Kartoffeln.

Diese Beziehung kann bei Kenntnis der entsprechenden Bodennutzung für eine Abschätzung der Wasserhaushaltsgröße Verdunstung verwendet werden. Sie verdeutlicht noch einmal die Spannbreite der möglichen Jahresverdunstung auf den untersuchten Böden am Standort Brandis.

$\mathrm{Zu}$ beachten ist, dass der verfügbare Bodenwasservorrat der Lößböden (Top d) aus der Bodenwassermenge (200 mm bzw. $215 \mathrm{~mm}$ ) resultiert, die im Jahr 1991 durch den Winterweizen verdunstet wurde, zuzüglich einem bereits aus dem Vorjahr vorhandenen Bodenwasserdefizit von $250 \mathrm{~mm}$ bzw. $195 \mathrm{~mm}$. Es wird angenommen, dass die Lößböden prinzipiell in der Lage sind, bei entsprechenden meteorologischen Bedingungen bedürftigen Kulturen in einer Vegetationsperiode eine Bodenwassermenge von $450 \mathrm{~mm}$ bzw. $410 \mathrm{~mm}$ zur Verfügung zu stellen. Auf vergleichbare Untersuchungsergebnisse von OLIKOVA \& SYCHEVA (1996) wurde bereits in Kap. 3.4 verwiesen.

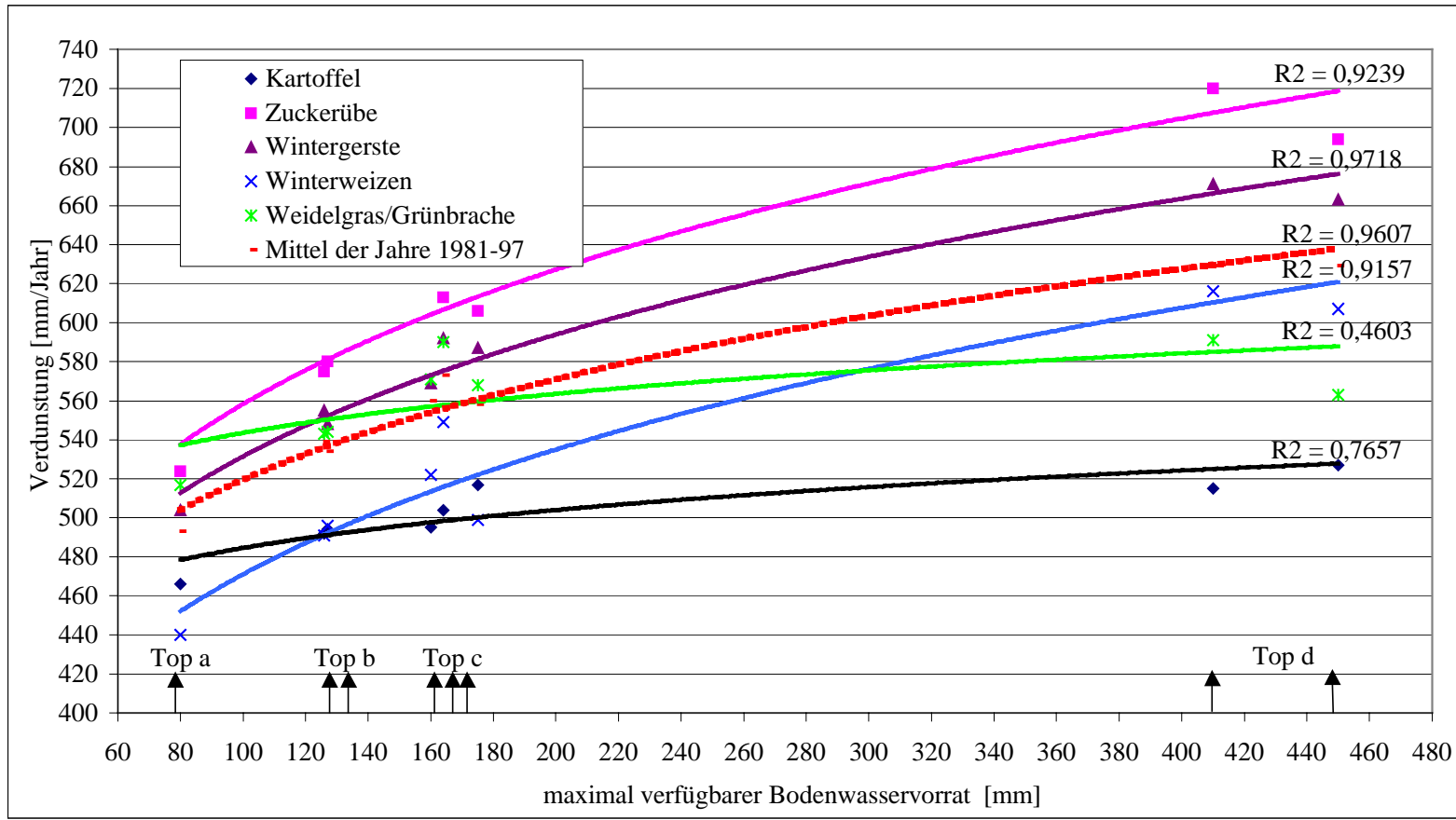

Top a: Erodierte Braunerde; Top b: Braunerde-Fahlerde und Parabraunerde-Braunerde; Top c: lessivierter Braunerde-Pseudogley, Braunerde-Pseudogley und pseudovergleyte Braunerde-Fahlerde; Top d: Parabraunerde aus Löß und Schwarzerde aus Löß; Top = Pedo-Hydrotop; R2 = Bestimmtheitsmaß;

Abb. 20: Mittlere Jahresverdunstung (1981-97) in Abhängigkeit vom pflanzenverfügbaren Bodenwasservorrat für Wintergetreide, Kartoffeln, Zuckerrüben und Gras/Klee sowie Mittel der Jahre 1981-97 (ohne Klee) unter den klimatischen Bedingungen am Standort Brandis

Die enge Beziehung zwischen Verdunstung und Trockenmasseertrag ermöglicht eine Abschätzung der durchschnittlichen Jahresverdunstung von landwirtschaftlichen Nutzflächen für das Untersuchungsgebiet, ohne dass Kenntnisse über den Boden vorhanden sein müssen. In Abb. 21 wurde die mittlere Jahresverdunstung der hydrologischen Jahre (November bis 
Oktober) mit Wintergetreide, Gras/Klee, Kartoffeln und Zuckerrüben der acht untersuchten Böden (Lysimetergruppen) den entsprechenden Trockenmasseerträgen aus Tab. 23 gegenübergestellt. Für Gras, Klee, Zuckerrüben (Rübenkörper mit Blatt) und Getreide wurden die Gesamtmasseerträge zu Grunde gelegt, bei Kartoffeln nur die Knollen. Eine Unterteilung zwischen Wintergerste und Winterweizen erfolgte nicht, da sich im Verlauf der Kurven nur geringe Unterschiede zeigten. Bei Rotklee im Jahr 1995 steht den Trockenmasseerträgen (24 $\mathrm{dt} / \mathrm{ha}$ - Lysimetergruppe 5 und $64 \mathrm{dt} / \mathrm{ha}$ - Lysimetergruppe 10) eine vergleichsweise hohe Verdunstung gegenüber. Dieser Effekt wird durch die Jahre mit Weidelgras und Grünbrache ausgeglichen, so dass eine Zusammenfassung für Abschätzungen auf Jahresebene gerechtfertigt ist.

Auch die nachfolgenden Untersuchungen zur Verdunstung in den Sommer- und Winterhalbjahren dienen zur Abschätzung der Wasserhaushaltgröße „Verdunstung“ im Rahmen wasserwirtschaftlicher Planungen. Der jahreszeitlich spezifische Wasserverbrauch der unterschiedlichen Kulturpflanzen auf den verschiedenen Böden ist Gegenstand von Kap. 3.5.7. 
Wintergetreide

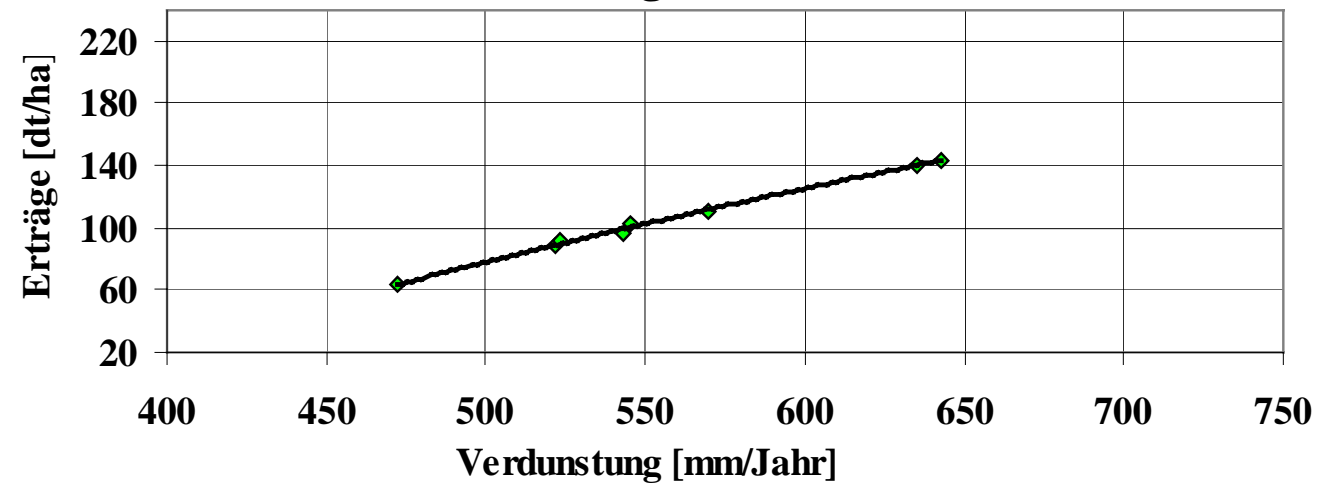

Gras/Klee

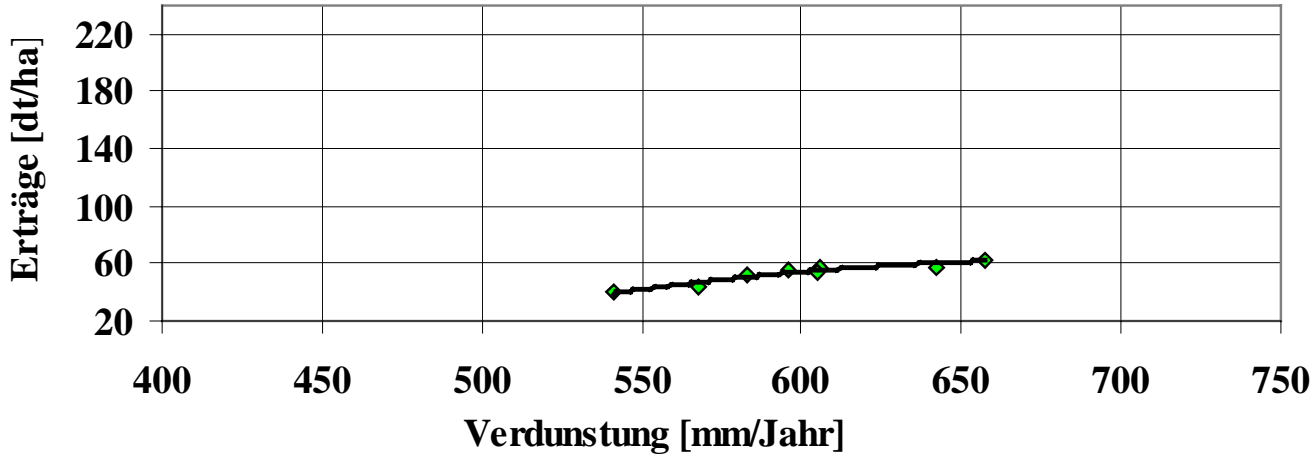

Kartoffel

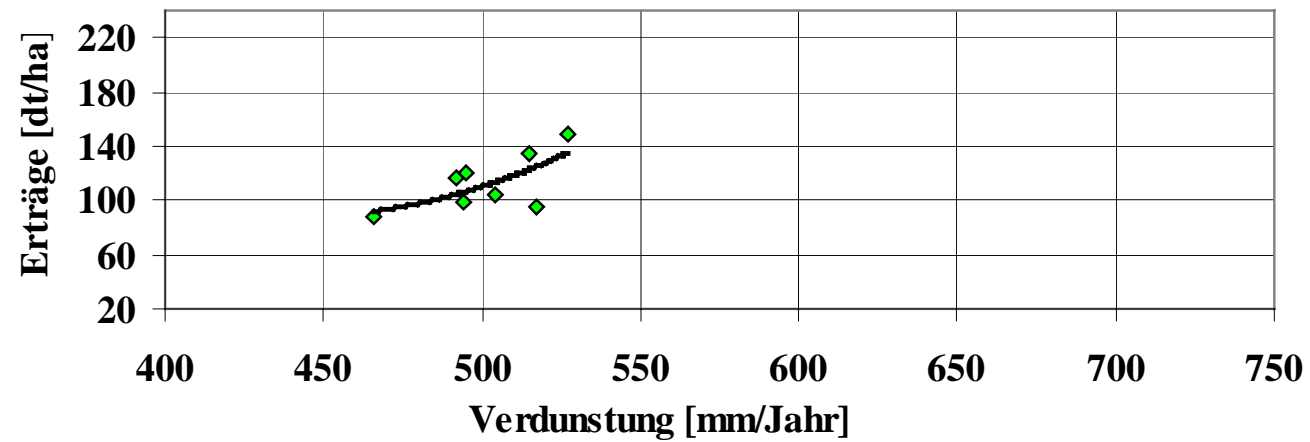

\section{Zuckerrüben}

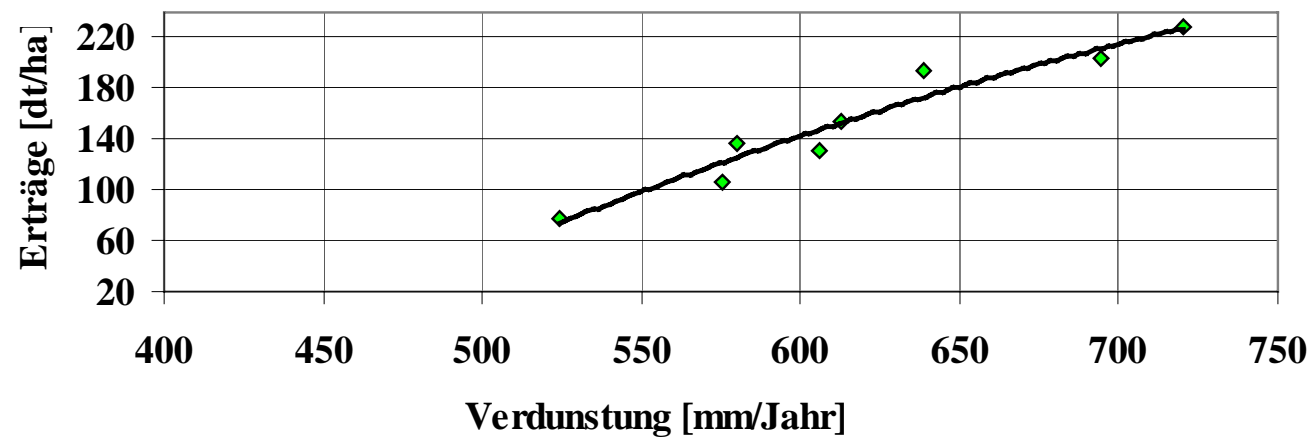

Abb. 21: Beziehung zwischen Trockenmasseerträgen und Jahresverdunstung am Beispiel von Wintergetreide, Gras/Klee, Kartoffeln und Zuckerrüben am Standort Brandis 


\subsubsection{Verdunstung in den Sommerhalbjahren (April bis September)}

In den Nomogrammen zur Jahresverdunstung der einzelnen Kulturarten wird der Einfluss der Witterung infolge Mittelwertbildung vernachlässigt. Bei den nachfolgenden Untersuchungen steht der Witterungseinfluss im Vordergrund. Sommerniederschlag und potentielle Verdunstung (= Gras-Referenzverdunstung) beschreiben das witterungsbedingte Wasser- und Strahlungsangebot des Sommerhalbjahres, wobei eindeutig die Jahre mit klimatischem Wasserdefizit überwiegen $\left(\mathrm{P}_{\mathrm{so}} / \mathrm{ETP}_{\mathrm{so}}<1\right)$. Die tatsächliche Verdunstungsleistung der Pflanzen auf den verschieden Böden (Top a bis d) spiegelt sich im Verhältnis zwischen realer und potentieller Verdunstung $\left(\mathrm{ETR}_{\mathrm{so}} / \mathrm{ETP}_{\mathrm{so}}\right)$ wider. Sie ist um so höher, je größer der verfügbare Bodenwasservorrat ist.

So berücksichtigt Abb. 22 die Standorteigenschaften Niederschlag, Boden (repräsentiert durch die vier Pedo-Hydrotope) und Vegetation (Landnutzung in Form von Winterweizen und Wintergerste, Kartoffeln, Zuckerrüben, Gras und Klee). Bei Kenntnis des korrigierten Sommerniederschlags und der Gras-Referenzverdunstung $\left(\mathrm{ETP}_{\text {so }}\right)$ kann aus den Nomogrammen für beliebige Sommerhalbjahre die reale Verdunstung abgeschätzt werden.

Von Nachteil bei der Aufstellung war, dass nur die Jahre mit Wintergetreide die mögliche Spannbreite von sehr trockenen Jahren $\left(\mathrm{P}_{\mathrm{so}} / \mathrm{ETP}_{\mathrm{so}}<0,5\right)$ und relativ feuchten Jahren $\left(\mathrm{P}_{\mathrm{so}} / \mathrm{ETP}_{\mathrm{so}}>1\right)$ repräsentieren. Für die anderen Ackerkulturen sind weitere Messjahre erforderlich, um die Gültigkeit der Nomogramme erweitern zu können. Der Verlauf der Kurve für die Lößböden (Typ d bei Gras/Klee) in Abb. 22 wird wesentlich durch die hohe Verdunstung des Rotklees im Jahr 1995 beeinflusst.

Die Jahre 1983 und 1987 sind durch hohe Niederschläge und eine vergleichsweise geringe potentielle Verdunstung im Sommerhalbjahr gekennzeichnet. So führen feuchte Witterung und mangelndes Energieangebot auch auf den Lößböden zu einer geringen Verdunstung und Ertragsbildung (s. Tab. 23).

Der überwiegend parallele Verlauf der Kurven ist überraschend. Erwartet wurde, dass zumindestens die Lößböden mit sehr hohem verfügbaren Bodenwasservorrat in Bezug auf die Verdunstungsleistung eine andere (= geringere) Abhängigkeit vom Wasserdargebot zeigen als die leichten Sandböden. Andererseits verweisen u. a. FREDE et. al (1985) und EHLERS (1996) auf den Einfluss des Temperaturverlaufes und des Sättigungseinflusses der Luft auf die Transpiration und Ertragsbildung. So führen niedrige Lufttemperaturen und ein geringes Sättigungsdefizit auch bei geringen Bodenwassergehalten zu einer ausreichenden Wasserversorgung der Pflanze, wobei dies im umgekehrten Fall (hohe Lufttemperartur und hohes Sättigungsdefizit) selbst bei hohen Bodenwassergehalten nicht gelingt. Somit wird der Einfluss der Böden (und der $\mathrm{nFK}_{\mathrm{We}}$ ) auf die Transpiration durch das aktuelle Witterungsgeschehen überlagert.

Eine weitere Ursache ist der gewählte Bilanzzeitraum (Sommerhalbjahr), in dessen Verlauf nicht nur die Verdunstungsleistung der Pflanzen Berücksichtigung findet, sondern auch die teils vegetationslosen Zeiträume nach der Ernte. In Kap. 3.5.6 wird geprüft, ob ein differenzierteres Bild entsteht, wenn nur die Vegetationsperiode betrachtet wird. 

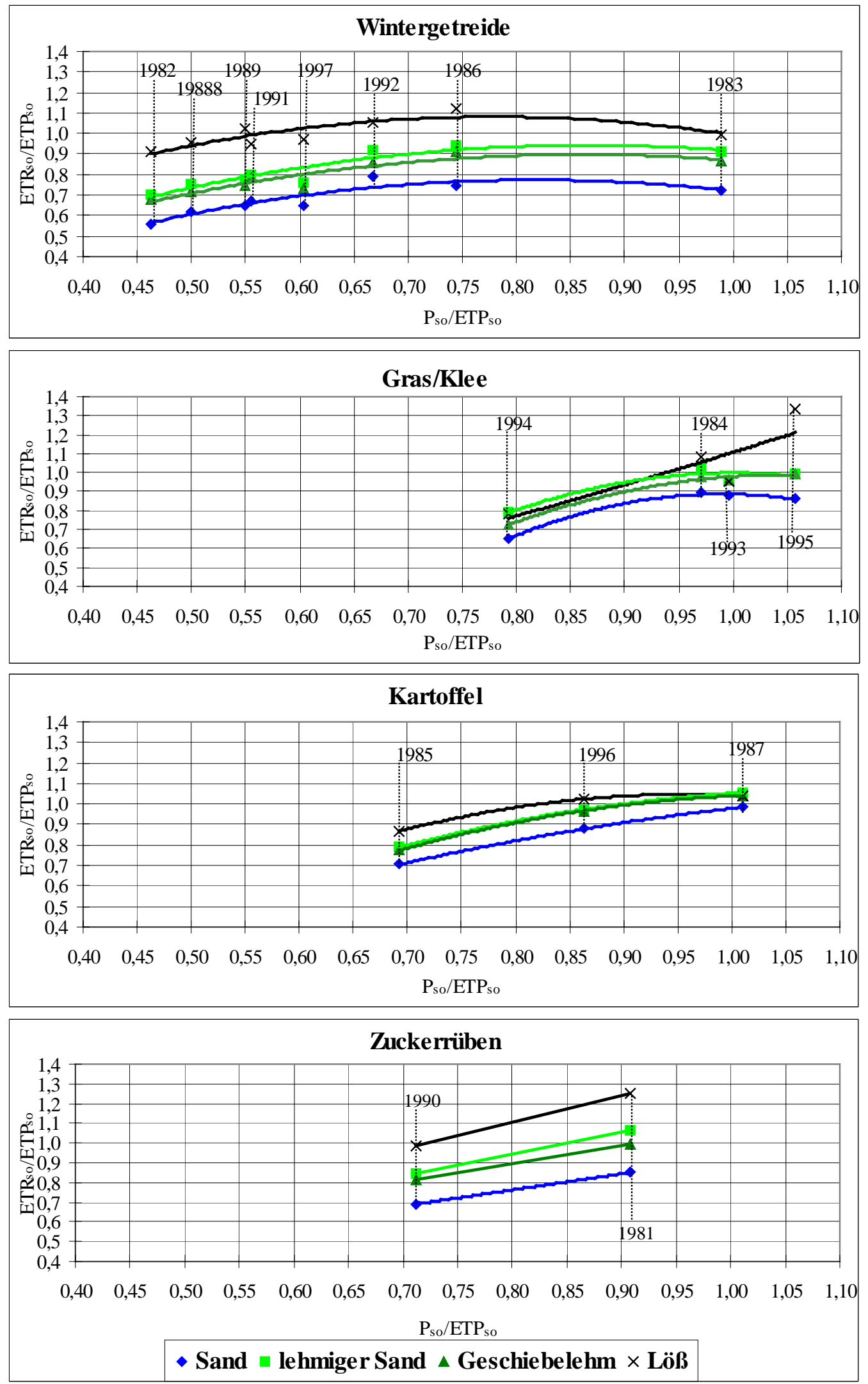

Abb. 22: Einfluss von Niederschlag und potentieller Verdunstung im Sommerhalbjahr $(P / E T P)$ auf die Verdunstungsleistung (ETR/ETP) von Wintergetreide, Gras/Klee, Kartoffeln und Zuckerrüben in Abhängigkeit von der Bodenart 


\subsubsection{Verdunstung im Winterhalbjahr (Oktober bis März)}

Im Winterhalbjahr war der Boden vegetationslos. Eine Ausnahme bilden die Winterhalbjahre mit Wintergetreide, Gras oder Klee. Die Vegetationsruhe dauert in der Regel von November bis Februar, wurde aber im Verlauf der warmen Winter häufig unterbrochen. Eine über längere Zeit geschlossene Schneedecke > 5 cm war nur im Winter 1986/87 zu verzeichnen.

Bei nicht gefrorenen Böden ist neben Evaporation von der Boden- oder Schneeoberfläche mit einem Wasserentzug aus dem Boden zu rechnen. Die Höhe dieses Wasserentzuges ist nach WOHLRAB et al. (1992) von der Häufigkeit der Niederschläge sowie von der Infiltrationsund Wasserleitfähigkeit der Böden abhängig. Diese Differenzierungen der Evaporation können durch die Brandiser „Winter-Messungen“" nur an wenigen Beispielen bestätigt werden. In der Regel reichen die Niederschläge aus, um den geringen Verdunstungsanspruch der Luft zu decken, auch wenn ein Teil dieses Niederschlages noch zur Füllung des Bodenwasservorrates verwendet wird.

Im Mittel der Winterhalbjahre sind reale Verdunstung und Gras-Referenzverdunstung bei allen Böden gleich groß (rd. $150 \mathrm{~mm}$ ). Abweichungen zeigen sich in den Jahren mit Kartoffeln (1985, 1987 und 1996) und Wintergerste (1983, 1989, 1992 und 1997). In den Jahren mit Kartoffeln ist die reale Verdunstung auf Grund der winterlichen Brache rd. 15-20 $\mathrm{mm}$ niedriger als die Gras-Referenzverdunstung. Fallen eine gute Entwicklung der Wintergerste und sehr warmes Wetter im März und April zusammen, ist die reale Verdunstung der Wintergerste auf allen Böden ca. 15 - $40 \mathrm{~mm}$ höher als die GrasReferenzverdunstung (Abb. 23. Unter Berücksichtigung dieser Abweichungen kann die Gras-Referenzverdunstung zur Abschätzung der Verdunstung im Winterhalbjahr verwendet werden.

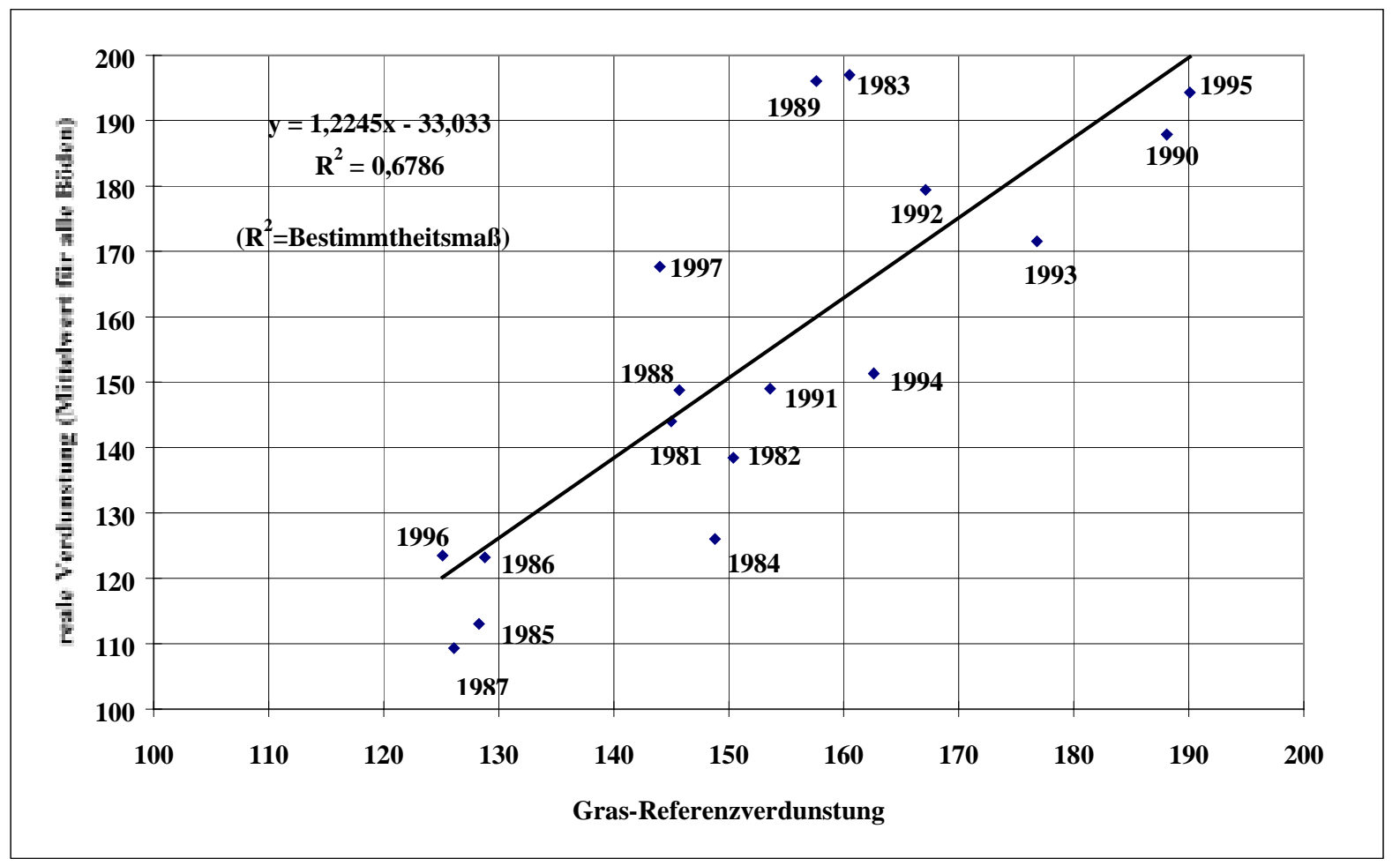

Abb. 23: Beziehung zwischen realer und potentieller Verdunstung im Winterhalbjahr (Nov. April) [ $\mathrm{mm}$ ] (Halbjahresmittel über alle 8 Lysimeterböden) 


\subsubsection{Ermittlung von Jahreswerten mit dem Verfahren von RENGER\&WESSOLEK}

Mit dem Verfahren von RENGER \& WESSOLEK (1990) kann die reale Verdunstung ebener Standorte und Böden auf Lockergestein ermittelt werden. Es hat in der wasserwirtschaftlichen Praxis breite Anwendung gefunden und soll deshalb zur Beurteilung der mittels Lysimeter ermittelten Jahresverdunstung herangezogen werden. Die Berechnung der realen Verdunstung (ETR) in [mm] als Summe vom April bis zum März des Folgejahres erfolgt nach der Formel:

$$
\begin{array}{ll}
\text { Gl. 13: } & \text { ETR }=\mathbf{a} * \mathbf{P}_{\mathbf{S o}}+\mathbf{b} * \mathbf{P}_{\mathbf{w i}}+\mathbf{c} * \log \mathbf{W}_{\mathbf{p f l}}+\mathbf{d} * \mathbf{E T P}+\mathbf{e} \\
\mathrm{P}_{\mathrm{SO}} & =\text { Sommerniederschlag von April bis September } \\
\mathrm{P}_{\mathrm{wi}} & =\text { Winterniederschlag von Oktober bis März des Folgejahres } \\
\mathrm{W}_{\text {pfl }} & =\text { pflanzenverfügbare Bodenwassermenge (s. Tab. 15] Tand Tab. 20 } \\
\text { ETP } & =\text { potentielle Evapotranspiration nach HAUDE } \\
& \text { (April bis März des Folgejahres) } \\
\text { a,b,c,d,e } & =\text { landnutzungsspezifische Regressionskoeffizienten }
\end{array}
$$

\begin{tabular}{|l|c|c|c|c|c|}
\hline Bodennutzung & a & b & c & d & e \\
\hline Ackerland & 0,39 & 0,08 & 153 & 0,12 & -109 \\
Grünland & 0,48 & 0,10 & 286 & 0,10 & -330 \\
\hline
\end{tabular}

Die Konstanten a bis e sind abhängig von Klima und Standortbedingungen. Sie wurden über Berechnungen der realen Verdunstung in Tagesschritten nach dem Rijtema-Ansatz (RIJTEMA, 1965) ermittelt. Die Kalibrierung der Modellrechnungen erfolgte an Hand von Geländemessungen in Norddeutschland (Raum Hannover). Die o.g. Konstanten gelten für norddeutsche Bedingungen, so dass für den Standort Brandis prinzipiell Abweichungen zu erwarten sind. Mit Anwendung des Verfahrens kann also auch seine Gültigkeit für das Untersuchungsgebiet geprüft werden.

Die Berechnungen wurden für die Einzeljahre der Reihe 1981-97 durchgeführt. Folgende Änderungen wurden vorgenommen:

- Die potentielle Verdunstung nach HAUDE wurde durch die Gras-Referenz-Verdunstung ersetzt.

- Als Sommer- und Winterniederschlag wurde der nach RICHTER korrigierte Niederschlag verwendet.

Diese Veränderungen hatten wenig Einfluss auf die Berechnungsergebnisse. Die Verwendung des korrigierten Niederschlages führte zu einer geringfügigen Erhöhung der berechneten Verdunstung von $1-2 \%$ gegenüber der Berechnung mit unkorrigierten Werten. Die Einführung der Gras-Referenzverdunstung bewirkte dagegen eine Verringerung der Berechnungswerte zwischen 0 und $3 \%$. Von Bedeutung ist die Tatsache, dass die Lößböden nicht in jedem Frühjahr bis Feldkapazität aufgefüllt sind, denn dies ist eine wesentliche Voraussetzung für die Gültigkeit des Verfahrens. Bei den anderen Böden existieren in einzelnen Jahren Anfang April nur geringe Wasserdefizite (max. $25 \mathrm{~mm}$ bei Wintergetreide). 
Tab. 20: Vergleich zwischen mittels Lysimeter gemessener und nach RENGER\&WESSOLEK (1990) berechneter Jahresverdunstung in [mm] und in [\% Abweichung] der Jahre 1981-97

\begin{tabular}{|c|c|c|c|c|c|c|}
\hline $\begin{array}{c}\text { Lysimeter } \\
\text {-gruppe }\end{array}$ & nFK $_{\text {We }}$ & \multicolumn{3}{|c|}{$\begin{array}{c}\text { reale Verdunstung } \\
\text { (ETR) }\end{array}$} & \multicolumn{3}{|l|}{ ETR $_{\text {berechnet }}$ ETR $_{\text {gemessen }}$} \\
& & berechnet & gemessen & [\%] & s(x) & cv \\
\hline $\mathbf{5}$ & 75 & 436 & 491 & 89 & 7,4 & 8,3 \\
\hline $\mathbf{4}$ & 127 & 478 & 536 & 89 & 9,8 & 11,3 \\
$\mathbf{8}$ & 124 & 476 & 532 & 89 & 9,4 & 10,5 \\
\hline $\mathbf{1}$ & 164 & 498 & 558 & 89 & 9,6 & 10,8 \\
$\mathbf{7}$ & 175 & 503 & 571 & 88 & 9,9 & 11,2 \\
$\mathbf{1 1}$ & 160 & 496 & 556 & 89 & 10,5 & 11,7 \\
\hline $\mathbf{9}$ & 450 & 580 & 627 & 93 & 17,3 & 18,7 \\
$\mathbf{1 0}$ & 410 & 574 & 627 & 92 & 19,8 & 21,7 \\
\hline
\end{tabular}

mit $\quad \mathrm{P}_{\text {Sommer }}$ (korrigiert) $=375 \mathrm{~mm} ; \quad \mathrm{P}_{\text {Winter }}$ (korrigiert) $=275 \mathrm{~mm}$

Gras-Referenzverdunstung $=613 \mathrm{~mm}$

$\mathrm{s}_{\mathrm{x}}$ - Standardabweichung [mm] und

$\mathrm{c}_{\mathrm{v}}$ - Variationskoeffizient [\%] in Bezug auf die Unterschiede in den Einzeljahren.

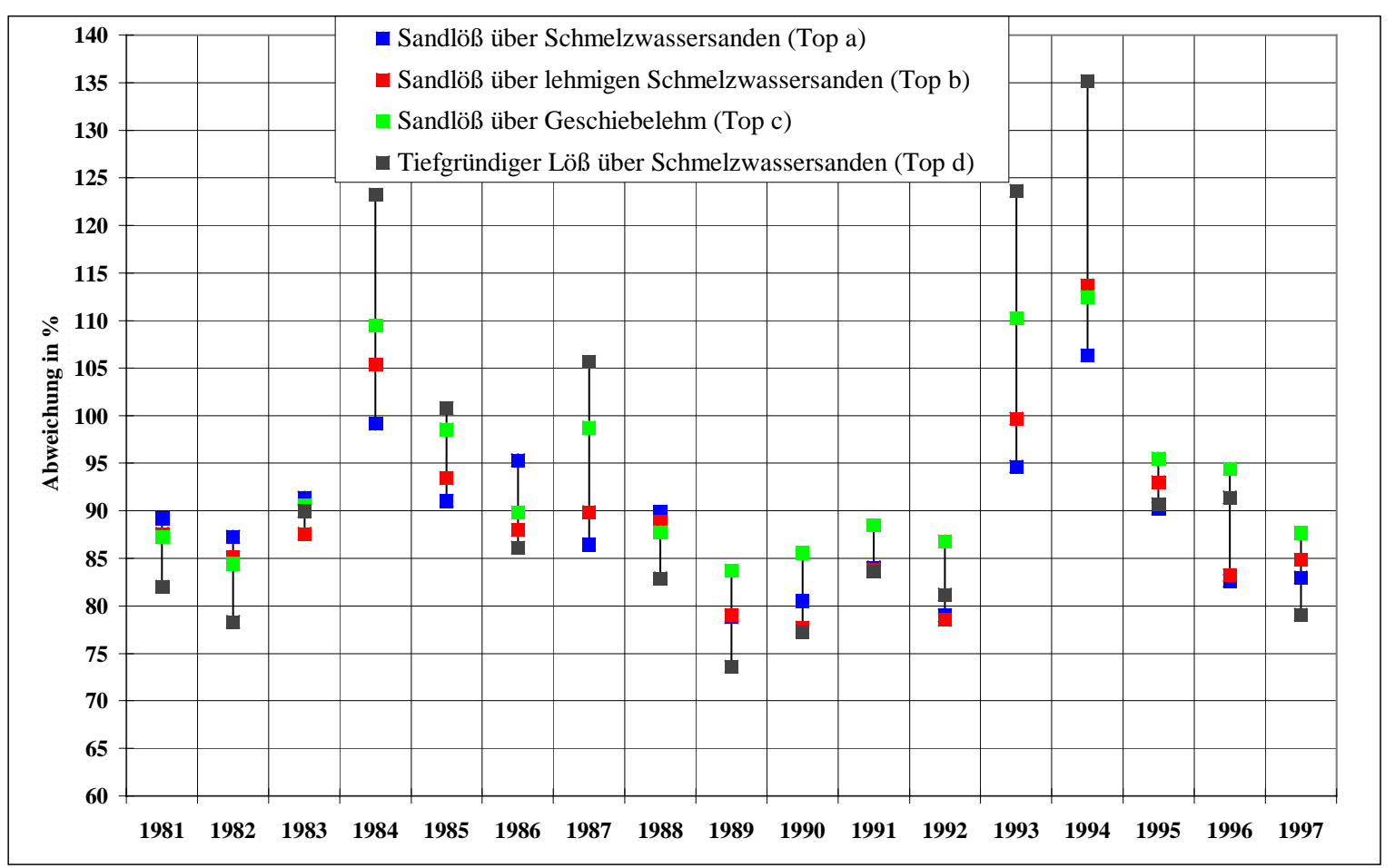

Abb. 24: Prozentuale Abweichung zwischen der nach RENGER \& WESSOLEK (1990) berechneten und der mittels Lysimeter gemessenen Jahresverdunstung (=100\%)

Im Mittel der Jahre wird die Verdunstung der Böden mit geringer und mittlerer $n$ FK ${ }_{W e}$ (Top a bis c) um $11 \%$ und die der Lößböden (pflanzenverfügbare Bodenwassermenge $=410 \mathrm{~mm}$ bzw. $450 \mathrm{~mm}$ ) um $8 \%$ unterschätzt (Гab. 20). Diese prinzipiellen Abweichungen können im Ansatz des Verfahrens begründet sein. Hier wird die reale Verdunstung mit Hilfe der klimatischen Wasserbilanz aus der potentiellen Verdunstung abgeleitet. Werden der klimatischen Wasserbilanz die unkorrigierten Niederschläge zu Grunde gelegt, so wird das Bodenwasserdefizit überschätzt und eine zu geringe reale Verdunstung ermittelt. Hinzu kommt, dass am Standort Brandis (wie auch auf den Herkunftsflächen der Böden) neben einer höheren potentiellen Verdunstung auch der Anteil der Sommerniederschläge am Gesamtniederschlag höher ist als im Raum Hannover. 
Unterschiede zeigten sich auch in den Einzeljahren. Erwartungsgemäß überschätzt wird die Verdunstung in den Jahren mit Gras (1984, 1993-94) und geringer Bodenwasserausschöpfung, dabei insbesondere im Jahr 1994 auf Grund der schlechten Entwicklung der Grünbrache. Im feuchten und strahlungsarmen Jahr $1987\left(\mathrm{P}_{\text {korr }} / \mathrm{ETP}_{\mathrm{Gras}}>1\right)$ wird die Verdunstung auf den Lößböden überschätzt, sicherlich infolge der extrem geringen Inanspruchnahme der $\mathrm{nFK}_{\mathrm{We}}$.

Die Jahre, für die eine zu geringe Verdunstung ermittelt wird, zählen zu den Trockenjahren (1982, 1988-92 und 1997). Mit $\mathrm{P}_{\text {korr }} / \mathrm{ETP}_{\text {Gras }}<=0,5$ wird in diesen Jahren ein Grad der Aridität erreicht, der in Nordwestdeutschland kaum auftritt. Unter diesen Bedingungen kommt der $\mathrm{nFK}_{\mathrm{We}}$ eine höhere Bedeutung zu. Der Variationskoeffizient, der die Unterschiede in den Einzeljahren beschreibt, steigt mit der $n_{F K} K_{W e}$ Je größer die $n_{F K} K_{W e}$, um so größer auch die möglichen Abweichungen.

Die in einigen Untersuchungsjahren fehlende Auffüllung der Lößböden bis zum April war für die Berechnung der Verdunstung ohne Einfluss, da in jedem Fall ausreichend Bodenwasser für die Verdunstung zur Verfügung stand. 


\subsubsection{Monatswerte (Jahresgang und Ermittlung der Bestandskoeffizienten)}

\section{a) Mittlerer Jahresgang der Verdunstung von Wintergetreide, Hackfrüchten und Gras/Grünland}

Die Art der Bewirtschaftung im Verlauf der 17jährigen Beobachtungsreihe kann als ein Rotationssystem mit $47 \%$ Wintergetreide, $27 \%$ Hackfrucht (Kartoffeln/Zuckerrüben) und $27 \%$ Futter (Gras/Klee) beschrieben werden.

Unter diesen Bedingungen erreichen die Lößböden im Sommerhalbjahr (April bis Oktober) eine Verdunstung von $480 \mathrm{~mm}$, die leichten Sandböden dagegen nur eine Verdunstung von 344 mm. Das sind $102 \%$ bzw. $73 \%$ der Gras-Referenzverdunstung und $93 \%$ bzw. $68 \%$ der potentiellen Verdunstung nach TURC.

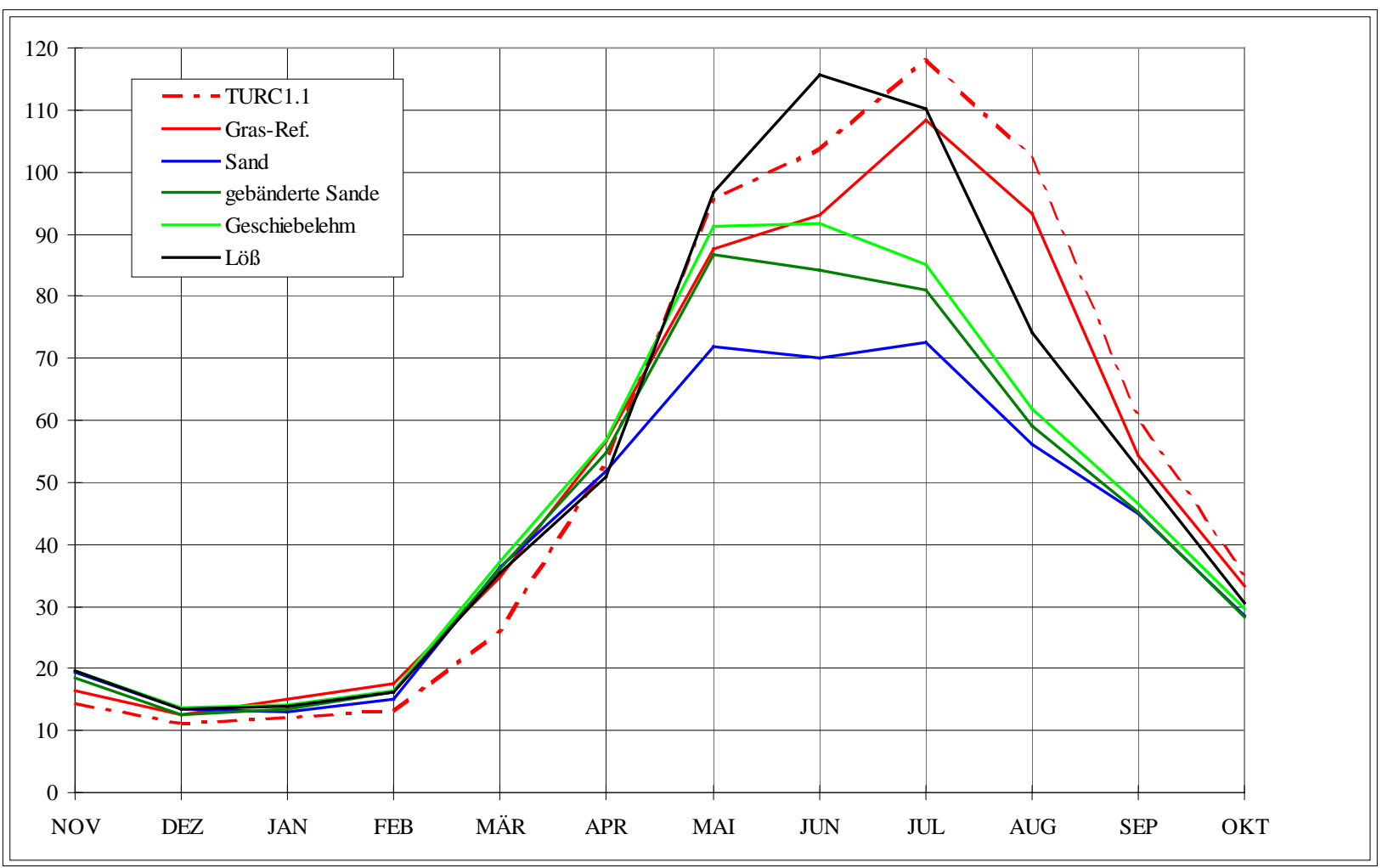

Abb. 25: Mittlere Monatssummen der potentiellen Verdunstung nach TURC, der GrasReferenzverdunstung und der realen Verdunstung der untersuchten Böden am Standort Brandis bei landwirtschaftlicher Bewirtschaftung (Reihe 1981-97)

Die TURC-Verdunstung ist in den Wintermonaten etwas geringer, dafür aber im Verlauf der Sommermonate höher als die Gras-Referenzverdunstung (Abb. 25). In der hohen JuniVerdunstung der Lößböden spiegelt sich der hohe Anteil der Jahre mit Wintergetreide (8 Jahre) wider. Nach der Gelbreife und Ernte ist die landnutzungsabhängige maximale Verdunstung dann geringer als die Gras-Referenzverdunstung. Die Verdunstung der Böden mit geringer und mittlerer $\mathrm{nFK}_{\mathrm{We}}($ Sand, gebänderte Sande und Geschiebelehm = Top a-c) wird im Mittel der Jahre auf Grund des Wassermangels bereits ab Mai eingeschränkt.

Nachfolgend soll neben Bodenart auch der Einfluß der einzelnen Fruchtarten auf den mittleren Jahresgang der Verdunstung betrachtet werden. Da Kartoffeln und Zuckerrüben nur durch jeweils 3 bzw. 2 Anbaujahre repräsentiert werden, sind sie unter „Hackfrüchte“ zusammengefasst, obwohl ihre Wachstumsperioden unterschiedlich verlaufen. Des weiteren wird zwischen Wintergetreide (8 Anbaujahre) und Gras/Grünland (4 Anbaujahre) unter- 
schieden. Eine differenzierte Betrachtung der Verdunstung aller untersuchten Fruchtarten erfolgt im nächsten Kapitel.

Tab. 21: Mittlere Monatswerte der potentiellen Verdunstung nach TURC, der Gras-Referenzverdunstung und der realen Verdunstung in Abhängigkeit von Bodenart und Vegetation abgeleitet aus den Lysimetermessungen der Station Brandis (Reihe 1981-97)

\begin{tabular}{|c|c|c|c|c|c|c|c|c|c|c|c|c|c|c|c|}
\hline Kultur, Anbaujahre & NOV & DEZ & JAN & FEB & MÄR & APR & MAI & JUN & JUL & AUG & SEP & OKT & Wi & So & Jahr \\
\hline \multicolumn{16}{|l|}{ Fruchtfolge, 1981-97 } \\
\hline TURC1.1 & 14 & 11 & 12 & 13 & 26 & 53 & 96 & 104 & 118 & 102 & 60 & 35 & 130 & 515 & 645 \\
\hline Gras-Referenzverdunstung. & 17 & 12 & 15 & 17 & 35 & 57 & 88 & 93 & 108 & 93 & 54 & 33 & 153 & 470 & 623 \\
\hline Sand & 19 & 14 & 13 & 15 & 36 & 52 & 72 & 70 & 73 & 56 & 45 & 29 & 149 & 344 & 493 \\
\hline gebänderte Sande & 19 & 13 & 13 & 16 & 36 & 55 & 87 & 84 & 81 & 59 & 45 & 28 & 152 & 384 & 536 \\
\hline Geschiebelehm & 20 & 14 & 14 & 17 & 37 & 57 & 91 & 92 & 85 & 62 & 47 & 30 & 158 & 406 & 564 \\
\hline Löß & 20 & 13 & 14 & 16 & 35 & 51 & 97 & 116 & 110 & 74 & 52 & 31 & 149 & 480 & 629 \\
\hline \multicolumn{16}{|c|}{ Wintergetreide 1982, 83, 86, 88, 89, 91, 92, 97} \\
\hline TURC1.1 & 14 & 11 & 12 & 13 & 27 & 49 & 99 & 111 & 122 & 105 & 66 & 35 & 127 & 538 & 665 \\
\hline Gras-Referenzverdunstung. & 18 & 12 & 14 & 17 & 36 & 55 & 92 & 99 & 112 & 96 & 59 & 34 & 151 & 491 & 642 \\
\hline Sand & 21 & 13 & 14 & 15 & 37 & 56 & 83 & 61 & 60 & 46 & 38 & 28 & 157 & 315 & 472 \\
\hline gebänderte Sande & 20 & 12 & 15 & 16 & 37 & 60 & 107 & 82 & 59 & 46 & 39 & 28 & 161 & 362 & 522 \\
\hline Geschiebelehm & 21 & 13 & 16 & 16 & 40 & 61 & 113 & 93 & 64 & 47 & 39 & 30 & 167 & 386 & 553 \\
\hline Löß & 21 & 13 & 15 & 16 & 37 & 56 & 130 & 138 & 95 & 49 & 41 & 29 & 158 & 481 & 639 \\
\hline \multicolumn{16}{|c|}{ Hackfrucht 1981, 85, 87, 90, 96} \\
\hline TURC1.1 & 15 & 10 & 9 & 13 & 24 & 55 & 92 & 95 & 108 & 96 & 55 & 35 & 127 & 480 & 607 \\
\hline Gras-Referenzverdunstung. & 14 & 11 & 11 & 18 & 33 & 56 & 84 & 86 & 99 & 89 & 51 & 33 & 143 & 442 & 585 \\
\hline Sand & 20 & 12 & 6 & 13 & 33 & 46 & 56 & 76 & 92 & 61 & 47 & 26 & 130 & 358 & 489 \\
\hline gebänderte Sande & 19 & 12 & 6 & 14 & 34 & 49 & 59 & 86 & 112 & 67 & 45 & 25 & 134 & 393 & 527 \\
\hline Geschiebelehm & 20 & 13 & 6 & 16 & 34 & 50 & 62 & 91 & 113 & 72 & 49 & 26 & 139 & 412 & 551 \\
\hline Löß & 20 & 13 & 7 & 14 & 33 & 42 & 55 & 94 & 136 & 95 & 58 & 29 & 129 & 466 & 596 \\
\hline \multicolumn{16}{|c|}{ Gras/Klee/Grünland 1984, 1993-95 } \\
\hline TURC1.1 & 14 & 12 & 16 & 13 & 27 & 57 & 93 & 101 & 124 & 105 & 55 & 35 & 140 & 512 & 652 \\
\hline Gras-Referenzverdunstung. & 16 & 16 & 23 & 18 & 36 & 61 & 84 & 91 & 113 & 95 & 49 & 31 & 170 & 463 & 632 \\
\hline Sand & 15 & 16 & 19 & 18 & 39 & 51 & 70 & 81 & 74 & 70 & 56 & 34 & 158 & 384 & 541 \\
\hline gebänderte Sande & 15 & 14 & 19 & 20 & 37 & 53 & 80 & 88 & 86 & 75 & 57 & 33 & 157 & 418 & 575 \\
\hline Geschiebelehm & 16 & 16 & 20 & 19 & 37 & 57 & 85 & 90 & 91 & 79 & 59 & 35 & 164 & 438 & 602 \\
\hline Löß & 16 & 15 & 20 & 20 & 35 & 51 & 82 & 99 & 109 & 98 & 68 & 37 & 156 & 493 & 649 \\
\hline
\end{tabular}

Die in Tab. 21 zusammengestellten Ergebnisse zeigen:

1. Das Wintergetreide hat schon im März zu Beginn der Vegetationsperiode einen nahezu bodendeckenden Bestand mit gut ausgebildetem Wurzelsystem, so dass eine intensive Nutzung der hohen Frühjahrsfeuchte für den Wachstums- und Transpirationsprozess stattfindet. Die realen Verdunstungshöhen (ETR) liegen im Bereich der potentiellen Verdunstung (ETP). Im Mai wird dann die ETP deutlich überschritten, mit Ausnahme beim Sandboden. Auf dem Löß liegt die ETR auch noch im Juni um mehr als $20 \mathrm{~mm}$ über der ETP, während auf den anderen Böden der Rückgang des Bodenwasservorrates bereits zur Einschränkung der ETR führt. Von 7 Anbaujahren mit Wintergetreide erfolgte die Ernte in 4 Fällen Anfang August, in 2 Fällen Anfang Juli und im Trockenjahr 1992 bereits im Juni.

Nach der Ernte, im Verlauf der Monate August und September, bewirken die verschiedenen Böden kaum eine Variation der Verdunstungshöhen, wobei die ETR bis einschließlich Oktober (Ende der Vegetationsperiode) z. T. weit unter der ETP liegt.

Die Getreidejahre zeichnen sich mit $551 \mathrm{~mm}$ durch eine überdurchschnittlich hohe ETP und auch durch eine hohe ETR aus.

2. Die Aussaat der Zuckerrüben und das Stecken der Kartoffeln erfolgte (bis auf 1990) erst Mitte Mai. Infolge dessen liegt die ETR bis Juni bei den Hackfrüchten deutlich unter der ETP der Anbaujahre. Mit zunehmendem Pflanzenwachstum steigt die ETR, übersteigt aber nur im Juli und nur auf dem Löß deutlich die ETP. Die Ernte der Kartoffeln erfolgte in der 
Regel Mitte August, die der Zuckerrüben dagegen erst Anfang Oktober. Dies ist bei Nutzung der Verdunstungswerte der entsprechenden Monate zu berücksichtigen.

\section{Gras/Grünland}

Eine starke Generalisierung enthält die Zusammenführung von Gras und Klee. Die Verdunstung im Jahr 1994 ist bei allen Böden durch den schlechten Aufgang der Günbrache geprägt, wobei dies durch die hohe Verdunstung des Rotklees im Jahr 1995 wieder ausgeglichen wird. Im Vergleich $\mathrm{zu}$ den anderen Fruchtarten sind Differenzierungen der ETR zwischen den einzelnen Böden weniger ausgeprägt. Dies verweist auf eine geringere Bedeutung unterschiedlicher $\mathrm{nFK}_{\mathrm{We}}$ für die Wasseraufnahme durch Gras/Klee.

Die vorstehend beschriebenen Monatswerte der Verdunstung lassen erkennen:

- die Bedeutung der pflanzenverfügbaren Bodenwasservorräte für die Verdunstung,

- die phänologischen Effekte infolge des standorttypischen Wassermangels und

- die Unterschiede in der Bestandsentwicklung zwischen dem stets kurzgehaltenen Gras und den Ackerfrüchten.

Wie bereits erwähnt, ist bei der Verwendung der in Tab. 21 dokumentierten Verdunstungshöhen zur Berechnung von Bestandskoeffizienten zu beachten, dass die für die einzelnen Fruchtarten vergleichsweise geringe Anzahl von Beobachtungsjahren noch nicht alle am Standort Brandis möglichen Witterungsabläufe widerspiegelt (Abb. 22). Die Jahre mit Hackfrüchten waren im Vergleich zum „Durchschnitts- oder Normaljahr“ zu feucht und zu kühl, die Jahre mit Wintergetreide enthielten einige sehr trockene Jahre.

\section{b) Bestandskoeffizienten $\mathbf{k}_{\mathbf{c}}$}

Die Höhe der Erträge hängt von der Wasserversorgung der Pflanzen während der einzelnen Aufwuchsstadien ab. Höhe und Verlauf der landnutzungsabhängigen maximalen Verdunstung $\left(\mathrm{ETP}_{\text {Bestand }}=\mathrm{k}_{\mathrm{c}} * \mathrm{ETP}_{\text {Gras }}\right)$ können in diesem Zusammenhang wichtige Hinweise auf den möglichen Entwicklungsstand der jeweiligen Fruchtart bei ausreichendem Wasserangebot liefern.

Auf der Basis von Monatswerten wurden die $\mathrm{k}_{\mathrm{c}}$ aus der Verdunstung der Lößböden (= Mittel der Verdunstung von Lysimetergruppe 9 und 10) und der Grasreferenzverdunstung berechnet (s. hierzu auch Kap. 2.3.2) und Bestandskoeffizienten gegenübergestellt, die aus der Literatur (nach verschiedenen Autoren DVWK, 1996) entnommen wurden (Tab. 22). Im Vergleich der Mittelwerte konnte festgestellt werden, dass die Verdunstung der Lößböden bei allen untersuchten Fruchtarten (außer bei Gras) einer kulturartenspezifischen potentiellen Verdunstung entspricht. Im Vergleich der Mittelwerte einzelner Monate, insbesondere bei Wintergetreide zeigen die Brandiser Lysimeter eine höhere Verdunstungsleistung, wobei die von GÜNTHER (1999) in Großobringen ermittelten $\mathrm{k}_{\mathrm{c}^{-}}$Werte (1,7 für Winterweizen, 1,7 .. 1,8 für Zuckerrüben und 1,4 .. 1,5 für Kartoffeln), die sich auch auf die Gras-Referenzverdunstung beziehen, nur in Einzeljahren erreicht werden.

Unterschiede zu den in DVWK (1996) dokumentierten $\mathrm{k}_{\mathrm{c}}$-Werten ergeben sich auf Grund der frühen Erntetermine in Brandis, die sich vor allem in Trockenjahren nach dem Reifestadium der Früchte auf den leichten Böden richteten. Eine Differenzierung der Erntetermine für die verschiedenen Böden erfolgte nicht. So lagen die Erntetermine für Winterweizen meist Anfang August. Damit erklären sich der niedrige kc-Wert von 0,44 Tab. 22 . Auch der $\mathrm{k}_{\mathrm{c}^{-}}$ Wert für Kartoffeln ist durch die Erntetermine im August beeinflusst (s. hierzu auch Tab. 23). 
Die hohe Verdunstung der Lößböden in den Monaten November und Dezember resultiert aus der Verdunstung der Grünbrache oder des aufgehenden Wintergetreides im Verlauf überdurchschnittlich warmer und feuchter Witterungsperioden.

Tab. 22: Bestandskoeffizienten kc $\left(\right.$ ETR $_{\text {Löß }} /$ ETP $\left._{\text {Gras }}\right)$ im Vergleich zu Angaben aus DVWK (1996)

\begin{tabular}{|c|c|c|c|c|c|c|c|c|c|c|c|c|}
\hline Fruchtart & NOV & DEZ & JAN & FEB & MÄR & APR & MAI & JUN & JUL & AUG & SEP & OKT \\
\hline \multicolumn{13}{|c|}{ Beobachtungsjahr } \\
\hline & & & & & & & & & & & & \\
\hline \multicolumn{13}{|l|}{ Winterweizen } \\
\hline 1982 & 1,37 & 1,00 & 1,39 & 0,78 & 0,87 & 0,56 & 1,30 & 1,61 & 1,16 & 0,33 & 0,18 & 0,95 \\
\hline 1986 & 0,80 & 1,10 & 0,82 & 0,68 & 0,99 & 1,00 & 1,21 & 1,64 & 1,19 & 0,60 & 0,76 & 0,77 \\
\hline 1988 & 0,92 & 1,41 & 1,04 & 0,88 & 1,20 & 0,88 & 1,16 & 1,50 & 1,06 & 0,41 & 0,65 & 0,89 \\
\hline 1991 & 1,44 & 0,67 & 1,01 & 0,52 & 0,98 & 0,99 & 1,51 & 1,48 & 1,00 & 0,43 & 0,28 & 0,44 \\
\hline Mittel & 1,13 & 1,04 & 1,06 & 0,72 & 1,01 & 0,86 & 1,30 & 1,56 & 1,10 & 0,44 & 0,47 & 0,77 \\
\hline$D V W K(1996)$ & - & - & - & - & 0,90 & 0,95 & 1,15 & 1,35 & 1,30 & 1,00 & - & - \\
\hline & & & & & & & & & & & & \\
\hline \multicolumn{13}{|l|}{ Wintergerste } \\
\hline 1983 & 0,95 & 1,44 & 1,08 & 1,00 & 1,13 & 1,51 & 1,99 & 1,39 & 0,31 & 0,61 & 0,76 & 0,77 \\
\hline 1989 & 1,24 & 1,44 & 1,24 & 0,95 & 0,96 & 1,56 & 1,76 & 1,22 & 0,39 & 0,54 & 0,99 & 0,91 \\
\hline 1992 & 1,08 & 1,09 & 0,93 & 1,07 & 1,23 & 0,98 & 1,22 & 0,91 & 0,75 & 0,93 & 2,02 & 1,14 \\
\hline 1997 & 1,47 & 1,29 & 0,90 & 1,40 & 1,10 & 0,90 & 1,34 & 1,42 & 1,03 & 0,39 & 0,59 & 0,99 \\
\hline Mittel & 1,18 & 1,32 & 1,04 & 1,10 & 1,10 & 1,24 & 1,58 & 1,23 & 0,62 & 0,62 & 1,09 & 0,95 \\
\hline DVWK (1996) & - & - & - & - & 0,95 & 1,00 & 1,30 & 1,40 & 1,35 & - & - & - \\
\hline & & & & & & & & & & & & \\
\hline \multicolumn{13}{|l|}{ Zuckerrüben } \\
\hline 1981 & 1,23 & 1,23 & 1,00 & 1,05 & 1,27 & 0,53 & 0,72 & 0,94 & 1,75 & 1,80 & 1,81 & 1,69 \\
\hline 1990 & 1,80 & 1,25 & 0,93 & 0,90 & 0,86 & 0,91 & 0,25 & 1,21 & 1,25 & 1,11 & 1,31 & 0,63 \\
\hline Mittel & 1,52 & 1,24 & 0,97 & 0,98 & 1,06 & 0,72 & 0,49 & 1,08 & 1,50 & 1,45 & 1,56 & 1,16 \\
\hline$D V W K(1996)$ & - & - & - & - & - & 0,50 & 0,75 & 1,10 & 1,30 & 1,25 & 1,10 & 0,85 \\
\hline & & & & & & & & & & & & \\
\hline \multicolumn{13}{|l|}{ Kartoffel } \\
\hline 1985 & 1,08 & 1,11 & 1,00 & 0,72 & 0,83 & 0,87 & 0,56 & 1,09 & 1,16 & 0,70 & 0,70 & 0,33 \\
\hline 1987 & 0,99 & 0,98 & 1,00 & 0,63 & 0,98 & 0,73 & 0,94 & 1,20 & 1,15 & 1,13 & 0,95 & 0,75 \\
\hline 1996 & 2,46 & 1,84 & 1,05 & 0,60 & 1,07 & 0,66 & 1,11 & 1,00 & 1,64 & 0,67 & 0,91 & 1,14 \\
\hline Mittel & 1,51 & 1,31 & 1,02 & 0,65 & 0,96 & 0,76 & 0,87 & 1,10 & 1,31 & 0,84 & 0,85 & 0,74 \\
\hline$D V W K(1996)$ & - & - & - & - & - & 0,50 & 0,90 & 1,10 & 1,40 & 1,20 & 0,90 & - \\
\hline & & & & & & & & & & & & \\
\hline \multicolumn{13}{|l|}{ Gras } \\
\hline 1984 & 0,65 & 1,00 & 0,78 & 0,94 & 0,76 & 0,84 & 1,33 & 1,23 & 0,99 & 0,98 & 1,13 & 0,98 \\
\hline 1993 & 1,11 & 1,55 & 1,09 & 1,37 & 1,18 & 0,56 & 0,81 & 1,05 & 1,11 & 1,11 & 1,09 & 0,95 \\
\hline 1994 & 1,17 & 0,66 & 0,71 & 0,72 & 1,16 & 0,97 & 0,97 & 0,67 & 0,54 & 0,79 & 1,03 & 0,90 \\
\hline Mittel & 0,98 & 1,07 & 0,86 & 1,01 & 1,03 & 0,79 & 1,04 & 0,98 & 0,88 & 0,96 & 1,08 & 0,94 \\
\hline$D V W K(1996)$ & - & - & - & - & 1,00 & 1,00 & 1,05 & 1,10 & 1,10 & 1,05 & 1,05 & 1,00 \\
\hline \multicolumn{13}{|l|}{ (Mähweide) } \\
\hline & & & & & & & & & & & & \\
\hline \multicolumn{13}{|l|}{ Rotklee } \\
\hline 1995 & 1,49 & 0,31 & 0,22 & 1,16 & 0,94 & 0,86 & 1,49 & 1,91 & 0,79 & 0,41 & 0,73 & 1,95 \\
\hline
\end{tabular}

In den Einzeljahren werden bei allen Fruchtarten die witterungsbedingten Unterschiede in der Entwicklung deutlich, so dass sich eine Berechnung der Bestandskoeffizienten mit Bezug zu den Entwicklungsphasen des Bestandes empfiehlt. Entsprechende phänologische Beobachtungen liegen in Brandis vor, wurden aber im Rahmen dieser Arbeit nicht ausgewertet.

Im Ergebnis der Untersuchungen konnte der Nachweis erbracht werden, dass die Verdunstung der Lößböden trotz der hohen Bodenwasserdefizite im Verlauf der Jahre 1981-97 bis auf wenige Monate einer landnutzungsabhängigen maximalen Verdunstung entspricht. 


\subsubsection{Einfluss von Witterung und Boden auf den Wasserverbrauch der Pflanzen und die Ertragsbildung}

Der Schwerpunkt bisheriger Auswertungen zur Verdunstung landwirtschaftlich genutzter Böden in den Bilanzzeiträumen von Jahren, Halbjahren oder Monaten bediente vorrangig wasserwirtschaftliche Interessen (u. a. Lieferung von Daten für die Bilanzierung). In der Landwirtschaft interessiert jedoch die zur pflanzlichen Produktion benötigte Wassermenge. Ziel ist, mit möglichst wenig Wasser hohe Erträge zu erzielen. Das Verhältnis zwischen Wasserverbrauch in der Vegetationsperiode und Trockenmasseproduktion wird mit dem Evapotranspirations-Koeffizienten (ETK) angegeben (s. Kap. 2.3.4), wobei auf Einschränkungen hinzuweisen ist, die bei der Bewertung von ETK zu berücksichtigen sind:

- In die Berechnung geht die gesamte Trockenmasse ein (z. B. bei Getreide keine Trennung zwischen Stroh und Korn).

- Der ETK erfasst die ganze Vegetationszeit (Aufgang bis Ernte) und gibt damit keine Auskunft über den Wasserverbrauch der Pflanzen im Verlauf ihrer verschiedenen Entwicklungsstadien. Bei Wintergetreide wird der Wasserverbrauch von Aufgang bis Vegetationsende im Jahr der Aussaat vernachlässigt.

- Mittels Lysimeter (in der Regel auch bei Felduntersuchungen) wird neben der Transpiration als Wasserverbrauch zwangsläufig auch die Bodenevaporation mit gemessen.

Unter Berücksichtigung dieser Einschränkungen liefert der ETK wichtige Hinweise auf den Einfluss von Standorteigenschaften (Verfügbarkeit von Wasser und Nährstoffen) und Zustand der Atmosphäre auf die Effizienz des Wasserverbrauches verschiedener Fruchtarten.

Die in Tab. 23 vorgenommene Zusammenstellung zeigt für jede Fruchtart erwartungsgemäß einen engen Zusammenhang zwischen Wasserverbrauch, Trockenmasseertrag und Bodentyp: je höher die $\mathrm{nFK}_{\mathrm{We}}$ der untersuchten Böden um so höher auch der Wasserverbrauch und die Erträge. In Bezug auf die Effizienz des Wasserverbrauches der verschiedenen Fruchtarten zeigt sich: je höher die $\mathrm{nFK}_{\mathrm{We}}$ um so günstiger sind in der Regel auch die ETK.

Wie bereits in den vorangegangenen Kapiteln beschrieben, können nur die Lößböden (Lysimetergruppe 9 und 10) mit einer sehr hohen verfügbaren Bodenwassermenge eine landnutzungsabhängige maximale Verdunstung realisieren. Mit Ausnahme weniger feuchter Jahre (z. B. 1987) ist das Wasserangebot auf den Böden mit geringer und mittlerer nFK $\mathrm{We}_{\mathrm{e}}$ (eventuell auch eine zu geringe Nährstoffversorgung) der limitierende Faktor für die Transpiration und Ertragsbildung. Wie die Untersuchungsergebnisse zeigen, resultiert aus der mangelnden Wasserversorgung der Pflanzenbestände ein unwirtschaftlicher Umgang mit dem Wasser. In trockenen Jahren $\left(\mathrm{P}_{\text {korr }} / \mathrm{ETP}<0,65\right)$ kommt der Einfluss der Bodeneigenschaften besonders zum Tragen. Hinweise darauf liefern die Variationskoeffizienten (cv) in Гab. 23, die den Einfluss des Bodens beschreiben. Dabei zeigt sich, dass die von vielen Faktoren abhängigen Erträge im gleichen Anbaujahr deutlich stärker variieren als der Wasserverbrauch der Pflanzen. So reduziert z. B. Nährstoffmangel die Erträge ungleich stärker als den Wasserverbrauch (ROTH, 1988).

Die zwischen Fruchtart und Höhe des Wasserverbrauches bestehende Abhängigkeit resultiert aus dem unterschiedlichen Vermögen der Pflanzen, sich das vorhandene Wasser nutzbar zu machen. So fällt der Wasserverbrauch in der Reihenfolge: Rotklee $\rightarrow$ Zuckerrübe $\rightarrow$ Winterweizen $\rightarrow$ Wintergerste $\rightarrow$ Kartoffel. Diese Reihenfolge gilt für alle Böden, sie wird durch die Größe des Wurzelsystems, Blattflächenindex und Vegetationsdauer der jeweiligen Fruchtart bestimmt. 
Tab. 23: Höhe der Verdunstung im Verlauf der Vegetationsperiode (Aufgang bis Ernte), Trockenmasseerträge und Evapotranspirationskoeffizienten der Jahre 1981-97 für acht verschiedene Böden der Station Brandis

\begin{tabular}{|c|c|c|c|c|c|c|c|c|c|c|c|c|c|c|}
\hline \multirow[t]{2}{*}{ Jahr } & \multirow[t]{2}{*}{ Fruchtart } & \multirow[t]{2}{*}{\begin{tabular}{|c|} 
Zeitraum \\
Aufgang bis \\
Ernte \\
\end{tabular}} & \multirow[t]{2}{*}{$\begin{array}{c}\mathbf{P}_{\text {korr }} \\
{[\mathrm{mm}]}\end{array}$} & \multirow[t]{2}{*}{$\begin{array}{c}\text { ETP }_{\text {Gras }} \\
{[\mathrm{mm}]}\end{array}$} & \multirow[t]{2}{*}{$\begin{array}{c}\mathbf{P}_{\text {korr }} / \\
\text { ETP }_{\text {Gras }}\end{array}$} & \multicolumn{8}{|c|}{$\begin{array}{c}\text { Verdunstung } \\
{\left[\mathbf{1} / \mathbf{m}^{2}\right]}\end{array}$} & \multirow[t]{2}{*}{$\operatorname{cv}[\%]$} \\
\hline & & & & & & Lys.5 & Lys.4 & Lys.8 & Lys1 & Lys.7 & Lys.11 & Lys.9 & Lys.10 & \\
\hline 1984 & Weidelgras & $15.05-12.09$ & 306 & 332 & 0,92 & 300 & 323 & 326 & 337 & 359 & 337 & 385 & 372 & 8 \\
\hline 1993 & Grünbrache & $28.04-25.10$ & 482 & 454 & 1,06 & 408 & 438 & 428 & 464 & 441 & 443 & 451 & 456 & 4 \\
\hline 1994 & Grünbrache & $04.03 .-15.11$. & 601 & 620 & 0,97 & 408 & 461 & 453 & 484 & 518 & 498 & 434 & 443 & 8 \\
\hline 1995 & Rotklee & 20.04.-32.10. & 454 & 501 & 0,91 & \begin{tabular}{l|l}
439 \\
\end{tabular} & 536 & 476 & 543 & 470 & 506 & 628 & \begin{tabular}{l|l}
753 \\
\end{tabular} & 19 \\
\hline 1985 & Kartoffel & $20.05 .-24.09$ & 255 & 370 & 0,69 & 258 & 286 & 281 & 287 & 298 & 295 & 339 & 342 & 10 \\
\hline 1987 & Kartoffel & 27.05.-19.08. & 239 & 223 & 1,07 & 227 & 253 & 264 & 251 & 269 & 248 & 279 & 256 & 6 \\
\hline 1996 & Kartoffel & 10.05.-21.08. & 248 & 284 & 0,87 & 262 & 300 & 297 & 299 & 298 & 308 & 339 & 321 & 7 \\
\hline Mittel & Kartoffel & & 247 & 292 & $\mathbf{0 , 8 8}$ & 249 & 280 & 281 & 279 & 288 & 284 & 319 & 306 & 7 \\
\hline \multicolumn{2}{|c|}{ Standardabw. } & & 8 & 74 & 0,19 & 19 & 24 & 17 & 25 & 17 & 32 & 35 & 45 & \\
\hline \multicolumn{2}{|c|}{ Varianzkoeff. } & & 3 & 25 & 22 & 8 & \begin{tabular}{l|l}
9 \\
\end{tabular} & 6 & 9 & 6 & 11 & 11 & 15 & \\
\hline 1983 & Wintergerste & 06.03.-06.07. & 313 & 276 & 1,13 & 286 & 341 & 330 & 355 & 364 & 358 & 416 & \begin{tabular}{l|l}
419 \\
\end{tabular} & 12 \\
\hline 1989 & Wintergerste & 08.03.-05.07. & 198 & 317 & 0,62 & 251 & 293 & 297 & 306 & 338 & 321 & 479 & 416 & 22 \\
\hline 1992 & Wintergerste & 28.02.-08.07. & 245 & 314 & 0,78 & 226 & 269 & 273 & 291 & 311 & 284 & 323 & 337 & 12 \\
\hline Mittel & Wintergerste & & 252 & 302 & $\mathbf{0 , 8 5}$ & 254 & 301 & 300 & 317 & 338 & 321 & 406 & 391 & 15 \\
\hline \multicolumn{2}{|c|}{ Standardabw. } & & 58 & 23 & 0,26 & 30 & 37 & 29 & 33 & 27 & 37 & 78 & \begin{tabular}{l|l}
47 \\
\end{tabular} & \\
\hline \multicolumn{2}{|c|}{ Varianzkoeff. } & & 23 & 8 & 31 & 12 & 12 & 10 & 11 & 8 & 12 & 19 & 12 & \\
\hline 1982 & Winterweizen & 25.03.-04.08. & 225 & 400 & 0,56 & 279 & 329 & 323 & 361 & 364 & 340 & 465 & 469 & 18 \\
\hline 1986 & Winterweizen & 30.03.-04.08. & 259 & 348 & 0,74 & 270 & 343 & 342 & 347 & 391 & 338 & 448 & 432 & 16 \\
\hline 1988 & Winterweizen & 31.03.-02.08. & 180 & 362 & 0,50 & 245 & 281 & 293 & 297 & 345 & 299 & 444 & 385 & 20 \\
\hline 1991 & Winterweizen & 10.03.-14.08. & 280 & 408 & 0,69 & 330 & 372 & 376 & 399 & 413 & 373 & 460 & 475 & 12 \\
\hline Mittel & \multicolumn{2}{|c|}{ Winterweizen } & 236 & 380 & 0,62 & 281 & 331 & 334 & 351 & 378 & 338 & 454 & 440 & 16 \\
\hline \multicolumn{2}{|c|}{ Standardabw. } & & 44 & 29 & 0,11 & 36 & 38 & 35 & 42 & 30 & 30 & 10 & 41 & \\
\hline \multicolumn{2}{|c|}{ Varianzkoeff. } & & 19 & 8 & 18 & 13 & 11 & 10 & 12 & 8 & 9 & 2 & 9 & \\
\hline 1981 & Zuckerrüben & $17.05-08.10$ & 303 & 356 & 0,85 & 327 & 372 & 359 & 404 & 390 & 403 & 515 & 529 & 18 \\
\hline 1990 & Zuckerrüben & 24.04.-04.10. & 356 & 510 & 0,70 & 342 & 408 & 394 & 419 & 416 & 453 & 493 & 526 & 13 \\
\hline Mittel & Zuckerrüben & & 330 & 433 & 0,77 & 335 & 390 & 377 & 412 & 403 & 42 & 504 & 528 & 15 \\
\hline \multicolumn{2}{|c|}{ Standardabw. } & & 37 & 109 & 0,11 & 11 & 25 & 25 & 11 & 18 & 35 & 16 & 2 & \\
\hline \multicolumn{2}{|c|}{ Varianzkoeff. } & & 11 & 25 & 14 & 3 & 7 & 7 & 3 & 5 & 8 & 3 & \begin{tabular}{l|l}
0 \\
\end{tabular} & \\
\hline 1997 & Sommerweize & 03.04.-11.08. & 268 & 389 & 0,69 & 268 & 313 & 311 & 334 & 350 & 332 & 464 & 424 & 18 \\
\hline
\end{tabular}


Tab. 23 (Fortsetzung)

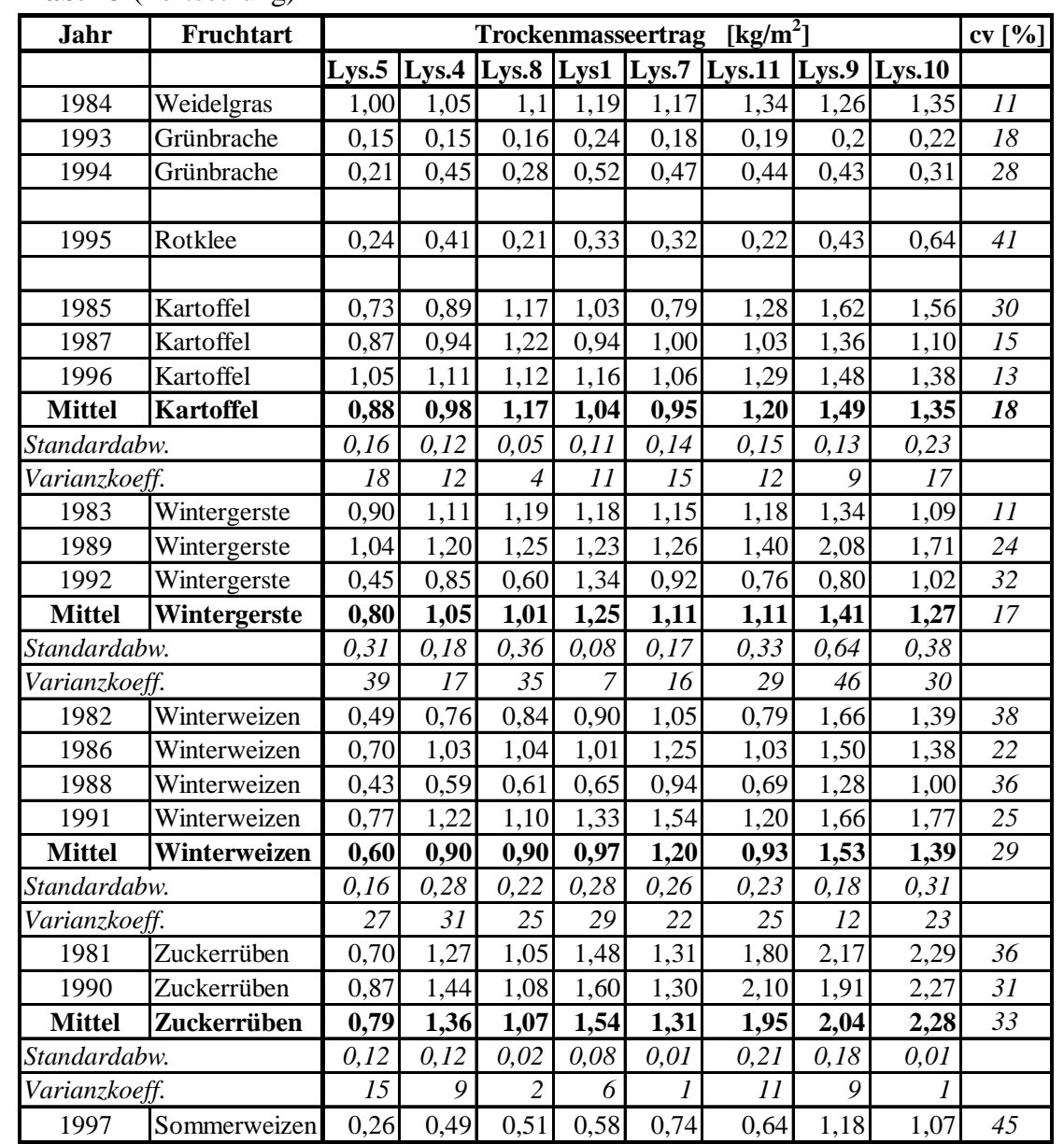

\begin{tabular}{|c|c|c|c|c|c|c|c|c|c|c|c|c|c|c|}
\hline \multirow[t]{2}{*}{ Jahr } & \multirow[t]{2}{*}{ Fruchtart } & \multirow[t]{2}{*}{\begin{tabular}{|c|} 
Zeitraum \\
Aufgang bis \\
Ernte \\
\end{tabular}} & \multirow[t]{2}{*}{$\begin{array}{c}\mathbf{P}_{\text {korr }} \\
{[\mathrm{mm}]}\end{array}$} & \multirow[t]{2}{*}{$\begin{array}{l}\mathbf{E T P}_{\text {Gras }} \\
{[\mathrm{mm}]}\end{array}$} & \multirow[t]{2}{*}{$\begin{array}{c}\mathbf{P}_{\text {korr }} l \\
\mathbf{E T P}_{\text {Gras }}\end{array}$} & \multicolumn{8}{|c|}{$\begin{array}{c}\text { Evapotranspirationskoeffizient } \\
{[1 / \mathrm{kg}]}\end{array}$} & \multirow[t]{2}{*}{\begin{tabular}{|c} 
cv \\
{$[\%]$}
\end{tabular}} \\
\hline & & & & & & Lys.5 & $\begin{array}{l}\text { Lys.4 } \\
\end{array}$ & \begin{tabular}{|l|l|} 
Lys.8 & \\
\end{tabular} & Lys1 & Lys.7 & Lys.11 & \begin{tabular}{|l|l|} 
Lys.9 \\
\end{tabular} & Lys.10 & \\
\hline 1984 & \begin{tabular}{|l|} 
Weidelgras \\
\end{tabular} & $\begin{array}{l}15.05-12.09 \\
\end{array}$ & 306 & 332 & 0,92 & 300 & 308 & 296 & 283 & 307 & 251 & 306 & 276 & \\
\hline 1993 & \begin{tabular}{|l|} 
Grünbrache \\
\end{tabular} & 28.04-25.10. & 482 & 454 & 1,06 & 2720 & 2920 & \begin{tabular}{|l|}
2675 \\
\end{tabular} & 1933 & 2450 & 2332 & 2255 & 2073 & 14 \\
\hline 1994 & \begin{tabular}{|l|} 
Grünbrache \\
\end{tabular} & $04.03 .-15.11$. & 01 & 620 & 0,97 & \begin{tabular}{|l|l|}
1943 \\
\end{tabular} & 1024 & \begin{tabular}{|l|}
1618 \\
\end{tabular} & 931 & 1102 & 1132 & \begin{tabular}{|l|}
1009 \\
\end{tabular} & 1429 & 28 \\
\hline 1995 & Rotklee & $2004-3210$ & 54 & 501 & 091 & $\mid 1829$ & 1307 & & 45 & 69 & ond & $60 \mathrm{~L}$ & $177 \mid$ & 25 \\
\hline & & & & & & & & & & & & & & \\
\hline 1985 & Kartoffel & 20.05.-24.09. & 55 & 370 & 0,69 & 353 & 321 & 240 & 279 & 377 & 230 & 209 & 219 & 23 \\
\hline 1987 & Kartoffel & 27.05.-19.08. & 239 & 223 & 1,07 & 261 & 269 & 216 & 267 & 269 & 241 & 205 & 233 & 10 \\
\hline 1996 & Kartof & $10.05 .-21.08$ & 248 & 284 & 0,87 & 250 & 270 & 265 & 258 & 281 & 239 & 229 & 233 & 7 \\
\hline Mittel & Kartoffel & & 247 & 292 & 0,88 & 288 & 287 & 241 & 268 & 309 & 237 & 214 & 228 & 13 \\
\hline tandardabw. & & & 8 & 74 & 0,19 & 57 & 30 & 24 & 10 & 59 & 5 & 13 & 8 & \\
\hline Varianzkoeff. & & & 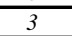 & 25 & 21,79 & 20 & 10 & 10 & 4 & 19 & 2 & 6 & 3 & \\
\hline 1983 & Wintergerste & 06.03.-06.07. & 313 & 276 & 1,13 & 318 & 307 & 277 & 301 & 317 & 303 & 310 & 384 & 10 \\
\hline 1989 & Wintergerste & $08.03 .-05.07$ & 198 & 317 & 0,62 & 241 & 244 & 238 & 249 & 268 & 229 & 230 & 243 & 5 \\
\hline 1992 & Wintergerste & 28.02.-08.07. & 245 & 314 & 0,78 & 502 & 316 & 455 & 217 & 338 & 374 & 404 & 330 & 24 \\
\hline Mittel & Wintergerste & & 252 & 302 & 0,85 & 354 & 289 & 323 & 256 & 308 & 302 & 315 & 319 & \\
\hline tandardabw. & & & 58 & 23 & 0,26 & 134 & 39 & 116 & 42 & 36 & 72 & 87 & 71 & \\
\hline Varianzkoeff. & & & 23 & 8 & 30,85 & 38 & 14 & 36 & 17 & 12 & 24 & 28 & 22 & \\
\hline 1982 & Winterweizen & 25.03.-04.08. & 225 & 400 & 0,56 & 569 & 433 & 385 & 401 & 347 & 430 & 280 & 337 & 22 \\
\hline 1986 & Winterweizen & 30.03.-04.08. & 259 & 348 & 0,74 & 386 & 333 & 329 & 344 & 313 & 328 & 299 & 313 & 8 \\
\hline 1988 & Winterweizen & 31.03.-02.08. & 180 & 362 & 0,50 & 570 & 476 & 480 & 457 & 367 & 433 & 347 & 385 & 17 \\
\hline 1991 & Winterweizen & \begin{tabular}{|l}
$10.03 .-14.08$ \\
\end{tabular} & 280 & 408 & 0,69 & 429 & 305 & 342 & 300 & 268 & 311 & 277 & 268 & 17 \\
\hline Mittel & Winterweizen & & 236 & 380 & 0,62 & 488 & 387 & 384 & 375 & 324 & 376 & 301 & 326 & 16 \\
\hline tandardabw. & & & 44 & 29 & 0,11 & 95 & 81 & 69 & 68 & 43 & 65 & 32 & 49 & \\
\hline Varianzkoeff. & & & 19 & 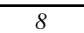 & 18,12 & 20 & 21 & 18 & 18 & 13 & 17 & 11 & 15 & \\
\hline 1981 & Zuckerrüben & $\begin{array}{l}17.05-08.10 . \\
\end{array}$ & 303 & 356 & 0,85 & 467 & 293 & 342 & 273 & 298 & 224 & 237 & 231 & 27 \\
\hline 1990 & Zuck & 24.04.-04.10. & 356 & 510 & 0,7 & 393 & 283 & 365 & 262 & 320 & 216 & 258 & 232 & 22 \\
\hline Mittel & \begin{tabular}{|l|} 
Zuckerrïben \\
\end{tabular} & & 330 & 433 & 0,77 & 430 & 288 & 353 & 267 & 309 & 220 & 248 & 231 & 24 \\
\hline tandardabw. & & & 37 & 109 & 0,11 & 52 & 7 & 16 & 8 & 16 & 6 & 15 & 1 & \\
\hline Varianzkoeff. & & & 11 & 25 & 13,97 & 12 & 2 & 5 & 3 & 5 & 3 & 6 & 0 & \\
\hline 1997 & Sommerweizen & 03.04.-11.08. & 268 & 389 & 0,69 & \begin{tabular}{|l|}
1031 \\
\end{tabular} & 639 & 610 & 576 & 473 & 519 & 393 & 396 & 35 \\
\hline
\end{tabular}


Auch die ETK zeigen eine Abstufung nach den Fruchtarten: Im Mittel der Anbaujahre sind die ETK bei Kartoffeln am günstigsten und bei Winterweizen am ungünstigsten. Dies scheint u. a. ursächlich aus der Witterung der Anbaujahre zu resultieren - der Anbau von Winterweizen erfolgte zufällig nahezu ausschließlich in Trockenjahren, der von Kartoffeln in Feuchtjahren. Ursache für die auffallend ungünstigen ETK von Grünbrache und Rotklee sind im Fall der Grünbrache u. a. die schlechte Bestandsentwicklung, im Fall des Rotklees steht einem geringen Trockemasseertrag auf Grund der großen Blattfläche eine sehr hohe Verdunstung gegenüber.

Eine gute Übereinstimmung zeigen die ermittelten ETK für Zuckerrüben ( 248 1/kg bzw. 231 1/kg - Düngung $150 \mathrm{~kg} \mathrm{~N} / \mathrm{ha}$ ) und die von WEINZIERL (1984) im Lößareal des Leinetalgrabens bei Göttingen im Freilandversuch ermittelten ETK von 229 1/kg (Düngung $180 \mathrm{~kg} \mathrm{~N} / \mathrm{ha}$ ) und 213 1/kg (Düngung $160 \mathrm{~kg} \mathrm{~N} / \mathrm{ha}$ ). In der gleichen Größenordnung liegen die ETK für Zuckerrüben von EHLERS (1989) mit 210 1/kg und ROTH (1993) mit 188 1/kg, wobei es sich bei den Angaben von ROTH (1993) ebenfalls um Ergebnisse von Lysimetermessungen (Löß-Braunschwarzerde) handelt. Mit Brandis vergleichbare Ergebnisse für die Lößböden finden sich bei ROTH (1993) auch für Kartoffeln (190 1/kg) und Winterweizen (308 1/kg).

Angaben zu ETK von pseudovergleyten Parabraunerden auf Geschiebemergel liefern Untersuchungen aus der Lysimeterstation Großlüsewitz bei Rostock (GALL et al., 1994). Von den Autoren wird auf die starke Streuung bei gleichen Fruchtarten in verschiedenen Anbaujahren (Kartoffel: 155 - 171 1/kg, Winterweizen: 272 - 331 1/kg) verwiesen, die allerdings geringer ist als die der eigenen Untersuchungen.

Wasserverbrauch und Erträge, so auch die ETK, variieren für jede Kultur in einer bestimmten Spannbreite, die wesentlich durch die Witterung der Anbaujahre (und durch unterschiedliche Aussaattermine) beeinflusst wird. Wie bereits in Kap. 4.5.2 diskutiert, wurde erwartet, dass die entsprechenden Variationskoeffizienten, die für verschiedene Böden bei gleichen Fruchtarten berechnet wurden, eine Abhängigkeit von der $\mathrm{nFK}_{\mathrm{We}}$ zeigen - mit zunehmender $\mathrm{nFK}_{\mathrm{We}} \mathrm{u}$. a. Wasserdefizite besser ausgeglichen werden und deshalb geringere $\mathrm{c}_{\mathrm{v}} \mathrm{zu}$ verzeichnen sind.

Diese Abhängigkeiten konnten anhand der Variationskoeffizienten (s. in Tab. 23) nicht eindeutig nachgewiesen werden. Die leichten Sandböden (Lysimetergruppe 5) zeigen eindeutig die höchste witterungsbedingte Varianz von Wasserverbrauch, Ertragsbildung und ETK. Für keine der untersuchten Fruchtarten läßt sich eindeutig ausweisen, dass diese Varianz bei den Böden mit mittlerer und sehr hoher $\mathrm{nFK}_{\mathrm{We}}$ in jedem Fall geringer ist.

Dieses Ergebnis verweist auf den Einfluss den der aktuelle Witterungsverlauf auf die Transpiration und die Ertragsbildung ausübt. Während die mögliche Wasserbereitstellung durch den Boden (Höhe der $\mathrm{nFK}_{\mathrm{We}}$ ) das entsprechende Niveau der Versorgungslage der Pflanze mit Wasser festlegt, bestimmt der aktuelle Zustand der Atmosphäre (Sättigungsdefizit, Lufttemperatur) die aktuelle Produktivität der Transpiration. So ist die Reaktion der Pflanze auf extrem günstige oder ungünstige Witterungsabläufe (Einfluß des Wasserdampf-Sättigungsdefizits der Luft) zeitgleich und auf allen Böden ähnlich.

Nach DENMEAD \& SHAW (1962) wird die Transpiration bei einem hohen Verdunstungsanspruch der Luft (z. B. 6 bis $7 \mathrm{~mm} / \mathrm{Tag}$ ) schon bei relativ hohen pflanzennutzbaren Bodenwassergehalten eingeschränkt, während an einem kühlen, wolkenreichen Tag die Transpiration erst bei relativ niedrigen Bodenwassergehalten vermindert wird.

Diese Zusammenhänge sollen nachfolgend an einem Beispiel demonstriert werden. Im Jahr 1996 (Anbaujahr mit Kartoffel) führten Niederschläge im Mai und Juli bei allen Böden nach niederschlagsarmen Perioden kurzzeitig zu einer guten Wasserverfügbarkeit, so dass der hohe 
Verdunstungsanspruch der Atmospäre gleichzeitig auf allen Böden (hier am Beispiel Lysimetergruppen 4, 1 und 9) zu einer überdurchschnittlichen Verdunstungsleistung führte.

Als Basis für den Vergleich zwischen „mittlerer“ und „überdurchschnittlicher“ Verdunstungsleistung dienen die von SPONAGEL (1980) ermittelten Faktoren. Das Verfahren von SPONAGEL (1980) basiert auf Regressionsbeziehungen zwischen dem Quotient aus ETR [nach RIJTEMA (1965) berechnet] und ETP nach HAUDE sowie dem prozentualen Grad der Ausschöpfung der nFK (aus Messungen mit Gammadoppelsonden).

In Abb. 26 wurden für das o. g. Beispiel die im Untersuchungsjahr mittels Lysimetermessungen ermittelten Bodenfeuchtewerte, die entsprechenden ETR/ETP Haude $_{\text {nach }}$ SPONAGEL und die aus den Messwerten für die aktuellen Dekaden im Jahr 1996 berechneten ETR/ETP ${ }_{\text {Haude }}$ aufgetragen.

Für alle drei untersuchten Böden konnte nachgewiesen werden, dass der aus den Lysimeteruntersuchungen resultierende Zusammenhang zwischen aktueller Bodenfeuchte und dem Quotienten ETR/ETP ${ }_{\text {Haude }}$ nicht so eng ist, wie bei dem Verfahren von SPONAGEL. Im Mai und Juli zeigt sich auf allen drei Böden die vergleichsweise zu den SPONAGEL-Faktoren hohe Verdunstungsleistung, die aus den o.g. Witterungsbedingungen resultiert.

Die daraus resultierenden Abweichungen zwischen den gemessenen und berechneten Verdunstungshöhen (Tab. 24 werden noch durch Fehler der HAUDE-Verdunstung beinflusst (s. hierzu auch Anlage 3). Weitere Abweichungen zeigen sich nach der Ernte der Kartoffeln, da bei den SPONAGEL-Faktoren von einem Erntetermin Ende September ausgegangen wird.

Tab. 24: Dekadenwerte der Verdunstung von Kartoffeln im Jahr 1996 (April bis September)

\begin{tabular}{|c|c|c|c|c|c|c|c|}
\hline \multicolumn{2}{|c|}{$\begin{array}{l}\text { Monat } \\
\text { Dekade }\end{array}$} & \multicolumn{2}{|c|}{ Lysimetergruppe 4} & \multicolumn{2}{|c|}{ Lysimetergruppe 1} & \multicolumn{2}{|c|}{ Lysimetergruppe 9} \\
\hline April & $\begin{array}{l}1 . \\
2 . \\
3 .\end{array}$ & $\begin{array}{l}11.4 \\
24.3 \\
17.6 \\
\end{array}$ & $\begin{array}{c}6.9 \\
19.4 \\
23.0 \\
\end{array}$ & $\begin{array}{l}12.0 \\
25.3 \\
16.7 \\
\end{array}$ & $\begin{array}{c}6.9 \\
22.2 \\
26.9 \\
\end{array}$ & $\begin{array}{l}11.6 \\
15.8 \\
13.8 \\
\end{array}$ & $\begin{array}{c}5.5 \\
18.0 \\
23.0 \\
\end{array}$ \\
\hline Mai & $\begin{array}{l}1 . \\
2 . \\
3 .\end{array}$ & $\begin{array}{l}12.9 \\
21.9 \\
30.8 \\
\end{array}$ & $\begin{array}{c}8.0 \\
14.8 \\
27.4 \\
\end{array}$ & $\begin{array}{l}13.0 \\
23.3 \\
32.4 \\
\end{array}$ & $\begin{array}{c}8.0 \\
14.0 \\
27.4 \\
\end{array}$ & $\begin{array}{l}18.1 \\
28.2 \\
30.7 \\
\end{array}$ & $\begin{array}{c}7.0 \\
10.7 \\
24.2 \\
\end{array}$ \\
\hline Juni & $\begin{array}{l}1 . \\
2 . \\
3 .\end{array}$ & $\begin{array}{l}36.1 \\
36.0 \\
17.7\end{array}$ & $\begin{array}{l}30.6 \\
23.8 \\
21.0\end{array}$ & $\begin{array}{l}35.4 \\
35.4 \\
19.7\end{array}$ & $\begin{array}{l}32.4 \\
26.9 \\
24.5\end{array}$ & $\begin{array}{l}31.3 \\
35.7 \\
26.8\end{array}$ & $\begin{array}{l}32.4 \\
30.1 \\
24.5\end{array}$ \\
\hline Juli & $\begin{array}{l}1 . \\
2 . \\
3 .\end{array}$ & $\begin{array}{l}33.5 \\
47.4 \\
43.5 \\
\end{array}$ & $\begin{array}{l}29.9 \\
37.7 \\
48.4 \\
\end{array}$ & $\begin{array}{l}33.2 \\
45.5 \\
41.3 \\
\end{array}$ & $\begin{array}{l}28.8 \\
30.5 \\
46.2 \\
\end{array}$ & $\begin{array}{l}42.0 \\
54.3 \\
50.0 \\
\end{array}$ & $\begin{array}{l}26.5 \\
30.5 \\
46.2 \\
\end{array}$ \\
\hline August & $\begin{array}{l}1 . \\
2 . \\
3 .\end{array}$ & $\begin{array}{l}17.8 \\
14.6 \\
17.8 \\
\end{array}$ & $\begin{array}{l}34.7 \\
23.5 \\
26.3 \\
\end{array}$ & $\begin{array}{l}17.6 \\
14.2 \\
18.4 \\
\end{array}$ & $\begin{array}{l}38.8 \\
26.4 \\
30.1 \\
\end{array}$ & $\begin{array}{l}24.1 \\
15.0 \\
18.0 \\
\end{array}$ & $\begin{array}{l}38.8 \\
26.4 \\
30.1 \\
\end{array}$ \\
\hline Sept. & $\begin{array}{l}1 . \\
2 . \\
3 .\end{array}$ & $\begin{array}{l}10.9 \\
14.2 \\
11.6\end{array}$ & $\begin{array}{c}12.5 \\
6.8 \\
4.6 \\
\end{array}$ & $\begin{array}{l}10.7 \\
14.8 \\
11.6 \\
\end{array}$ & $\begin{array}{c}13.6 \\
6.8 \\
5.1 \\
\end{array}$ & $\begin{array}{l}11.2 \\
14.4 \\
12.3 \\
\end{array}$ & $\begin{array}{c}13.6 \\
6.2 \\
4.6\end{array}$ \\
\hline Summ & & 420 & 399 & 420 & 415 & 453 & 398 \\
\hline
\end{tabular}

Der Vergleich zwischen den gemessenen und mittels SPONAGEL-Faktoren ermittelten Dekadenwerte der Verdunstung zeigt, dass es bei der Anwendung des Verfahrens zu erheblichen Abweichungen zur tatsächlichen Verdunstung kommen kann. Gleichzeitig wird noch einmal deutlich, dass diese Abweichungen auf den Lößböden größer sind, aber bei allen drei Böden gleichzeitig auftreten. 

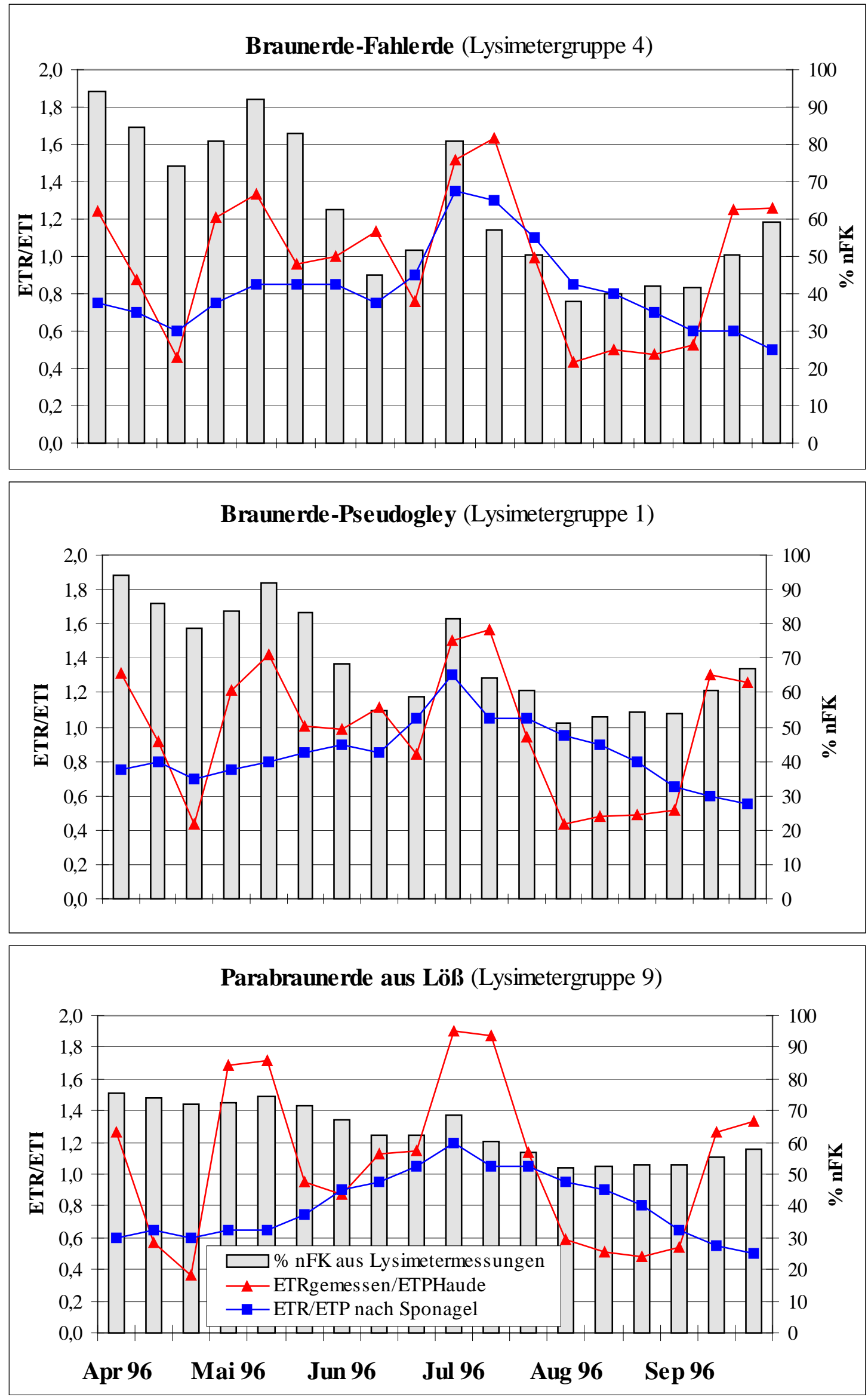

Abb. 26: Vergleich der SPONAGEL-Faktoren mit den mittels Lysimetermessungen in Brandis ermittelten Faktoren am Beispiel von Kartoffeln im Jahr 1996 (Dekadenwerte) 
Wie anhand der langjährigen Lysimetermessungen in Brandis gezeigt werden kann, unterscheiden sich bei gleicher Fruchtart und gleichem Boden Bestandsentwicklung, Wasserverbrauch und Höhe der Erträge von Jahr zu Jahr erheblich (Tab. 23). Deshalb ist die große witterungsbedingte Variabilität der pflanzenspezifischen Parameter, die in den wenigsten Berechnungsverfahren berücksichtigt wird, eine Ursache für die relativ großen Unterschiede zwischen den gemessenen und berechneten Verdunstungshöhen.

\subsubsection{Zusammenfassung und Diskussion der Ergebnisse}

Die Untersuchungen zur Kapazität der Bodenwasserspeicher zeigten, dass nur die Lößböden eine bedarfsgerechte Wasserbereitstellung garantieren können. Die meteorologischen Bedingungen und die schlechte Wasserspeicherkapazität der anderen Böden führen zu einer limitierten Wasserversorgung der Pflanzen. Während auf den Lößböden im Mittel der Untersuchungsperiode rd. $96 \%$ der (korrigierten) Niederschläge verdunsten, sind es auf den Böden mit geringer $\mathrm{nFK}_{\mathrm{We}}$ (Lysimetergruppe 5, 4 und 8) rd. $80 \%$ und auf den Böden mit mittlerer $\mathrm{nFK}_{\mathrm{We}}$ (Lysimetergruppe 1, 7 und 11) rd. $86 \%$. Davon entfallen auf allen Böden rd. $22 \%$ (rd. $150 \mathrm{~mm}$ ) auf das Winterhalbjahr.

Hinsichtlich der Höhe der Verdunstung ist zu beachten, dass die Ermittlung auf Basis der korrigierten Niederschläge nach HELLMANN erfolgte. Vergleiche zu Literaturwerten bestätigten die eigenen Untersuchungsergebnisse (MÜLLER et al., 1996), liefern aber auch geringere Verdunstungsraten, die möglicherweise auf fehlende Niederschlagskorrekturen zurückzuführen sind.

Die Wasserbereitstellung der Lößböden ermöglicht eine kulturartenspezifische maximale Verdunstung. Da sich über einen längeren Zeitraum (hier 17 Beobachtungsjahre) Bestandesunterschiede zwischen Gras-Referenzverdunstung und kulturartenspezifischer Verdunstung ausgleichen, zeigte sich - wie in Großobringen (GÜNTHER, 1999) - eine gute Übereinstimmung zwischen den Jahresmittelwerten der Verdunstung der Lößböden und der Gras-Referenzverdunstung.

Die Verdunstung der Lößböden wurde zur Berechnung der Bestandskoeffizienten verwendet. Diese beschreiben auf der Basis von Monatswerten die unterschiedliche phänologische Entwicklung von Gras und den entsprechenden Fruchtarten. Dabei zeigen sich für gleiche Monate und Fruchtarten in verschiedenen Anbaujahren infolge außergewöhnlicher Witterungserscheinungen, Pflanzenschutzmaßnahmen oder Krankheitsbefall starke Unterschiede. Der Vergleich zu Literaturwerten führte zu dem Schluss, dass eine kritiklose Anwendung für Einzeljahre zu großen Berechnungsfehlern führt, da diese nur standortabhängige Mittelwerte der Bestandsentwicklung widerspiegeln, ein Problem, auf das auch SAMBALE \& PESCHKE (2000) hinweisen.

Auf Grund ihrer Größenordnung ist die Verdunstung neben dem Niederschlag die dominierende Wasserhaushaltsgröße im Untersuchungsgebiet. Dem Bedarf der Wasserwirtschaft an Zahlenwerten für die „Bilanzgröße Verdunstung“ wurde mit der Aufstellung von Nomogrammen für Bilanzzeiträume von Jahren, Halbjahren und Monaten entsprochen. Möglich wurde dies auf Grund entsprechender Korrelationen zwischen Boden $\left(\mathrm{nFK}_{\mathrm{We}}\right)$, Fruchtart, Ertragsbildung, Niederschlag, potentieller und realer Verdunstung. Da es sich bei Bilanzrechnungen im Gebietsmaßstab in der Regel um mittel- und langfristige Aufgabenstellungen handelt, ist es nicht in jedem Fall möglich, aber auch nicht notwendig, auf landwirtschaftlichen Nutzflächen zwischen einzelnen Fruchtarten zu unterscheiden.

Für die ermittelten Evapotranspirationskoeffizienten (ETK) konnte für entsprechende Böden und Fruchtarten eine gute Übereinstimmung mit den Ergebnissen neuerer Untersuchungen festgestellt werden, wobei es sich sowohl um Feld- als auch um Lysimeterversuche handelte. 
Damit bestätigte sich indirekt, dass mittels Lysimeter geeigneter Bauart repräsentative Verdunstungswerte und Erträge bereitgestellt werden können. Prinzipiell konnte gezeigt werden: je limitierter die Versorgung der Pflanzen mit Bodenwasser, um so ungünstiger die ETK. Der Einfluss extremer Witterungsperioden (sehr trocken oder sehr feucht und warm) auf die Verdunstung und Ertragsbildung der einzelnen Fruchtarten ist - unabhängig vom Bodenwassergehalt - auf allen Böden ähnlich.

\subsection{Dynamik der Sickerwasserbildung}

\subsubsection{Sickerwassermenge und Niederschlag}

Wird die Sickerwasserbildung $\left(\mathrm{SW}_{3 \mathrm{~m}}=\mathrm{SW}\right.$ in $3 \mathrm{~m}$ Tiefe $)$ als Ergebnis der Wirkung von Niederschlag, Verdunstung und Bodenwasservorratsänderung, betrachtet
GI. 14:
$\mathrm{SW}_{3 \mathrm{~m}}=\mathbf{P}-\mathbf{E T R}+/-\Delta \mathrm{SB}$
$[\mathrm{mm}]$

so bestimmen absolute Höhe und Varianz dieser Wasserhaushaltsgrößen die Jahresmenge und den jahreszeitlichen Verlauf der Sickerwasserbildung der untersuchten Böden.

Der Variationskoeffizient des Jahresniederschlages liegt bei $14 \%$, die jährliche Höhe der Verdunstung schwankt in Abhängigkeit von Boden und Vegetation in relativ engen Grenzen (max. Variationskoeffizient rd. $12 \%$ ).

In Tab. 25 sind die Jahreswerte der Sickerwasserbildung unter Reihung nach der Vegetation zusammengestellt. Wie in Kap. 3.4.1 gezeigt werden konnte, ist die Höhe der Bodenwasserausschöpfung im aktuellen Jahr abhängig vom Verlauf der kWB (s. Tab. 14) und der angebauten Pflanzenart. Bezüglich der jährlichen Sickerwasserbildung wird der Einfluss der Pflanzen dann aber insbesondere durch die Höhe der Winterniederschläge überdeckt.

Größten Einfluss auf die Variabilität der Sickerwasserbildung der untersuchten Böden haben also Höhe der Bodenwasserausschöpfung und Höhe der Niederschläge im Anschluss an die Vegetationsperiode. Je geringer die Bodenwasserdefizite am Ende des Sommerhalbjahres und je höher die Niederschläge in den darauffolgenden Herbst- und Wintermonaten, um so eher beginnt die Neubildungsperiode und um so höher sind die Neubildungsmengen. Aus diesem jährlichen Wechselspiel zwischen Niederschlagshöhe und Bodenwasserdefizit resultiert die hohe Varianz der Sickerwassermengen.

Die durchschnittliche Sickerwasserleistung der einzelnen Böden schwankt zwischen $175 \mathrm{~mm}$ bei Sandböden (Lysimetergruppe 5) und rd. $50 \mathrm{~mm}$ bei Lößböden. Die Unterschiede in der Sickerwasserleistung resultieren aus der unterschiedlichen Verfügbarkeit von Bodenwasser (max. $75 \mathrm{~mm}$ bei Sandböden und max. $450 \mathrm{~mm}$ bei Lößböden). Unter vergleichbaren klimatischen Bedingungen werden diese geringen Sickerwassermegen u. a. durch Untersuchungen von MÜLLER et al. (1996), DÖRING et al. (1995) und ROTH et al. (1995) bestätigt.

Die leichten Böden der Lysimetergruppe 5 zeigen auf Grund der beschränkten Verfügbarkeit von Bodenwasser für die Verdunstung (geringe $\mathrm{nFK}_{\mathrm{We}}$ ) die geringste Varianz der Sickerwasserbildung (Variationskoeffizient rd. $42 \%$ ). Der Variationskoeffizient der jährlichen Sickerwassermenge der Geschiebelehmböden (mittlere $\mathrm{nFK}_{\mathrm{We}}$ ) beträgt rd. $70 \%$. Bei den Lößböden (sehr hohe $\mathrm{nFK}_{\mathrm{We}}$ ) mit einem Variationskoeffizienten von rd. $130 \%$ ist die Spannbreite der jährlichen Sickerwasserhöhe von 0 bis max. 184 mm im Jahr 1988 am größten. 
Tab. 25: Jahreswerte (November - Oktober) 1981-97 der Sickerwasserbildung für 8 verschiedenen Böden (Gruppenmittel) unter Reihung nach der Vegetation in [mm]

\begin{tabular}{|c|c|c|c|c|c|c|c|c|c|c|}
\hline \multirow[t]{2}{*}{ Jahr } & \multirow[t]{2}{*}{ Fruchtart } & \multirow{2}{*}{$\begin{array}{l}\mathbf{P}_{\text {korr }} \\
{[\mathrm{mm}]}\end{array}$} & \multicolumn{8}{|c|}{$\begin{array}{c}\text { Grundwasserneubildung [mm/Jahr] } \\
\text { (von November - Oktober) }\end{array}$} \\
\hline & & & $\begin{array}{l}\text { Lys } 5 \\
\text { D3 }\end{array}$ & \begin{tabular}{|c|} 
Lys 4 \\
D5
\end{tabular} & $\begin{array}{c}\text { Lys } 8 \\
\text { D3 }\end{array}$ & \begin{tabular}{|c|} 
Lys 1 \\
D6
\end{tabular} & $\begin{array}{l}\text { Lys } 7 \\
\text { D4 }\end{array}$ & $\begin{array}{l}\text { Lys } 11 \\
\text { D3 }\end{array}$ & $\begin{array}{l}\text { Lys } 9 \\
\text { Lö3 }\end{array}$ & $\begin{array}{l}\text { Lys } 10 \\
\text { Lö1 }\end{array}$ \\
\hline 1981 & Zu.-Rüben & 753 & 231 & 201 & 214 & 165 & 135 & 132 & 174 & 115 \\
\hline 1990 & Zu.-Rüben & 661 & 100 & 47 & 53 & 30 & 24 & 26 & 0 & 0 \\
\hline $\mathbf{m m}$ & Mittelwert & 707 & 165 & 124 & 134 & 97 & 79 & 79 & 87 & 57 \\
\hline $\mathrm{mm}$ & Standardabw. & 65 & 93 & 109 & 114 & 95 & 78 & 75 & 123 & 81 \\
\hline$\%$ & Variationskoeff. & 9 & 56 & 88 & 85 & 97 & 99 & 95 & 141 & 141 \\
\hline 1985 & Kartoffeln & 521 & 132 & 113 & 120 & 95 & 67 & 118 & 60 & 33 \\
\hline 1987 & Kartoffeln & 702 & 268 & 254 & 290 & 223 & 161 & 267 & 162 & 132 \\
\hline \multirow[t]{4}{*}{1996} & Kartoffeln & 583 & 98 & 24 & 50 & 7 & 65 & 29 & 0 & 0 \\
\hline & Mittelwert & 602 & 166 & 130 & 153 & 108 & 98 & 138 & 74 & 55 \\
\hline & Standardabw. & 92 & 90 & 116 & 124 & 109 & 55 & 120 & 82 & 69 \\
\hline & Variationskoeff. & 15 & 54 & 89 & 81 & 100 & 56 & 87 & 111 & 125 \\
\hline 1982 & Wi.-Weizen & 528 & 163 & 120 & 124 & 72 & 84 & 71 & 5 & 11 \\
\hline 1986 & Wi.-Weizen & 658 & 197 & 154 & 163 & 129 & 104 & 143 & 99 & 67 \\
\hline 1988 & Wi.-Weizen & 585 & 213 & 208 & 212 & 204 & 152 & 208 & 184 & 184 \\
\hline \multirow[t]{4}{*}{1991} & Wi.-Weizen & 508 & 111 & 62 & 67 & 40 & 34 & 18 & 0 & 0 \\
\hline & Mittelwert & 570 & 171 & 136 & 141 & 111 & 93 & 110 & 72 & 65 \\
\hline & Standardabw. & 68 & 45 & 61 & 61 & 72 & 49 & 83 & 88 & 84 \\
\hline & Variationskoeff. & 12 & 26 & 45 & 43 & 65 & 52 & 75 & 122 & 129 \\
\hline 1983 & Wi.-Gerste & 739 & 210 & 138 & 155 & 91 & 70 & 91 & 0 & 0 \\
\hline 1989 & Wi.-Gerste* & 642 & 134 & 111 & 101 & 95 & 55 & 84 & 0 & 28 \\
\hline 1992 & Wi.-Gerste*** & 685 & 138 & 95 & 101 & 71 & 45 & 66 & 0 & 0 \\
\hline \multirow[t]{4}{*}{1997} & So.-Weizen & 595 & 146 & 98 & 103 & 90 & 101 & 94 & 2 & 0 \\
\hline & Mittelwert & 665 & 157 & 110 & 115 & 87 & 68 & 84 & 1 & 7 \\
\hline & Standardabw. & 61 & 36 & 20 & 27 & 11 & 25 & 13 & 1 & 14 \\
\hline & Variationskoeff. & 9 & 23 & 18 & 23 & 12 & 36 & 15 & 200 & 200 \\
\hline 1984 & Weidelgras & 630 & 96 & 75 & 81 & 69 & 56 & 63 & 2 & 0 \\
\hline 1993 & Grünbrache & 747 & 145 & 72 & 94 & 82 & 55 & 74 & 0 & 0 \\
\hline 1994 & Grünbrache & 808 & 381 & 352 & 351 & 314 & 269 & 292 & 134 & 117 \\
\hline \multirow[t]{7}{*}{1995} & Rotklee & 786 & 217 & 175 & 211 & 150 & 191 & 163 & 144 & 170 \\
\hline & Mittelwert & 743 & 209 & 168 & 184 & 154 & 142 & 148 & 70 & 72 \\
\hline & Standardabw. & 79 & 124 & 131 & 126 & 113 & 106 & 106 & 80 & 85 \\
\hline & Variationskoeff. & 11 & 59 & 78 & 68 & 73 & 74 & 71 & 114 & 119 \\
\hline & Mittel 1981-97 & 655 & 175 & 135 & 146 & 113 & 98 & 114 & 57 & 50 \\
\hline & Standardabw. & 93 & 74 & 83 & 85 & 78 & 65 & 80 & 74 & 66 \\
\hline & Variationskoeff. & 14 & 42 & 61 & 58 & 69 & 66 & 70 & 130 & 133 \\
\hline \multicolumn{2}{|c|}{ Winter } & 302 & 136 & 105 & 113 & 91 & 76 & 93 & 47 & 43 \\
\hline \multicolumn{2}{|c|}{ Sommer } & 353 & 40 & 31 & 33 & 23 & 22 & 21 & 10 & 7 \\
\hline
\end{tabular}




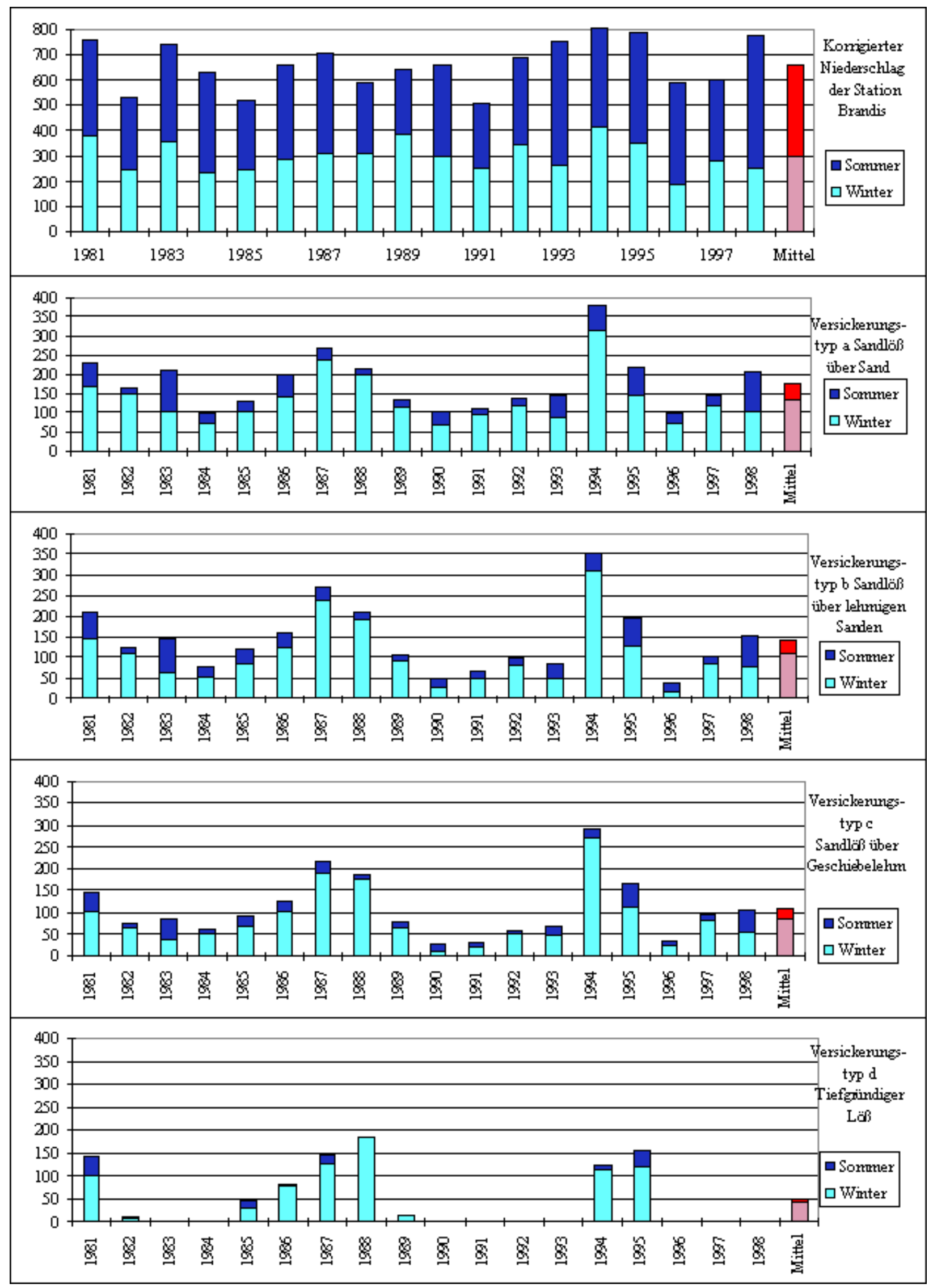

Abb. 27: Jahressummen der Sickerwasserbildung, unterteilt in Sommer- und Wintersumme [mm] 
Über den Beobachtungszeitraum von 17 Jahren, ist ein Wechsel von Jahren mit hoher und niedriger Sickerwasserbildung zu beobachten Abb. 27. Die Trocken- und Feuchtperioden umfassen Zeiträume von 3 bis 6 Jahren und spiegeln sich auch in den Abflussschwankungen der Vorfluter und im Verlauf der Grundwasserstände als kurzfristige, nicht zyklische Persistenz wider (Kap. 4.1). Diese Persistenz ist sowohl Folge des klimatisch bedingten unterschiedlichen Wasser- und Energieangebotes als auch der bereits geschilderten Wirkung des Bodenwasserspeichers. So sind positive Abweichungen vom Mittel seltener, aber meist größer als die negativen Differenzen. D. h. die Jahreswerte der Sickerwasserbildung sind nicht normal verteilt. Unter den gegebenen Bedingungen werden im Untersuchungsgebiet kaum „mittlere“ Grundwasserneubildungswerte und damit auch keine ,mittleren“ Abflüsse und Grundwasserstände auftreten. In Kap. 4.2 wird dieser Frage nachgegangen und insbesondere untersucht, ob die Messreihen in Bezug auf Größe, Dauer und Wiederkehr alle für das Gebiet möglichen Extremereignisse enthalten.

Für eine Charakterisierung der Untersuchungsjahre in Bezug auf Trocken- oder Feuchtjahre wurden die Jahreswerte von Niederschlag (P) und Sickerwasserbildung (SW) der Reihe 198197 für jeden Boden separat in das Wasserhaushaltsdreieck nach GOLF (1993) eingetragen (Anlage 6). Die sich unter der jeweiligen Linie zwischen den Punkten $(0 ; 0)$ und $((\mathrm{SW} / \mathrm{P}) * 700)$ befindenden Jahre werden in Hinsicht auf die Sickerwassermenge als trocken, die darüberliegenden als feucht definiert. Als repräsentativ für mittlere Verhältnisse gelten die Jahre, deren Ordinaten- und Abzissenwerte in der Nähe $(<>10 \%$ Abweichung) der o. g. Linien liegen. Mit dieser Normierung wird für die Einzeljahre eine bodenabhängige Charakterisierung der Beziehung zwischen Niederschlag und Sickerwasserbildung vorgenommen (Tab. 26). Der Wert 700 orientiert sich am größten Jahresniederschlag (hier unkorrigiert) im Beobachtungszeitraum.

Tab. 26: Charakterisierung der Wasserhaushaltsjahre 1981-97 in Abhängigkeit von der Bodenart

\begin{tabular}{|l|l|l|l|l|}
\hline Bodenart & \multicolumn{1}{|c|}{$\begin{array}{c}\text { Top a } \\
\text { Lys.-Gr. 5 }\end{array}$} & \multicolumn{1}{c|}{$\begin{array}{c}\text { Top b } \\
\text { Lys.-Gr. 4, 8 }\end{array}$} & \multicolumn{1}{c|}{$\begin{array}{c}\text { Top c } \\
\text { Lys.-Gr. 1, 7, 11 }\end{array}$} & \multicolumn{1}{c|}{$\begin{array}{c}\text { Top d } \\
\text { Lys.-Gr. 9, 10 }\end{array}$} \\
\hline Feuchtjahre & $1982-83,1986-$ & $1981,1986-88$, & $1981,1986-88$, & $1981,1986-88$, \\
& 88,1994 & $1994-95$ & 1994 & $1994-95$ \\
\hline Normaljahre & 1981,1985, & $1982-83,1985$ & 1985,1997 & 1985 \\
& 1995,1997 & & & $1982-84,1989-93$, \\
\hline Trockenjahre & $1984,1989-93$ & $1984,1989-93$ & $1982-84,1989-93$, \\
& 1996 & $1996-97$ & 1996 & $1996-97$ \\
\hline
\end{tabular}

Lys.-Gr. - Lysimetergruppe

Feuchtjahre sind Jahre mit überdurchschnittlich hoher Sickerwasserbildung in deren Verlauf es in Speisungsgebieten zu einem Ansteigen der Grundwasserstände über den jahreszeitlich bedingten Schwankungsbereich hinaus kommt.

Als Trockenjahre gelten Jahre mit unterdurchschnittlicher bzw. keiner Sickerwasserbildung, die zu einem dauerhaften Absinken der Grundwasserstände unter den sommerlichen Tiefstand führen.

Auf Grund der hohen Inanspruchnahme wird bis zum November in der Regel keine Wiederauffüllung der Bodenwasservorräte erreicht (s. innerjährlicher Verlauf der Sickerwasserperiode). So dienen die zu Beginn des hydrologischen Jahres (November bis Oktober) fallenden Niederschläge in jährlich unterschiedlichem Anteil zunächst der Wiederauffüllung. Deshalb ist mit keiner engen Beziehung zwischen Jahresniederschlag und Sickerwassermenge zu rechnen: für die Lysimetergruppe 5 (erodierte Braunerde) wurde ein Bestimmtheitsmaß 
$\left(\mathrm{R}^{2}\right)$ von $40 \%$ ermittelt, für die anderen Böden mit geringer bis mittlerer $\mathrm{nFK}_{\mathrm{We}}$ ist $\mathrm{R}^{2} \mathrm{rd} .30$ $\%$ und für die Lößböden ist $\mathrm{R}^{2}=16 \%$.

Wenn der Beginn des Jahres auf den 1. April verlegt wird (Lysimeterjahr), verbessert sich die Abhängigkeit zwischen jährlicher Sickerwassermenge und Niederschlag: bei der erodierten Braunerde auf ein $\mathrm{R}^{2}$ von $72 \%$, bei den Böden mit geringer bis mittlerer $n F K_{W e}$ auf $\mathrm{R}^{2}=66$ $\%$ und bei Lößböden auf $\mathrm{R}^{2}=23 \%$ Abb. 28. Zu Beginn des Lysimeterjahres ist die Auffüllung der Bodenwasserdefizite - mit Ausnahme bei den Lößböden - in der Regel abgeschlossen.

Die Höhe der sommerlichen Bodenwasserausschöpfung innerhalb des Lysimeterjahres resultiert nicht allein aus der Höhe der Niederschläge, sondern insbesondere auch aus dem Verdunstungsanspruch der Atmosphäre und der angebauten Fruchtart. Beides findet in den Regressionsbeziehungen (Abb. 28) keine Berücksichtigung. Im Lysimeterjahr 1982 (mit Winterweizen) wurden bei einem $\mathrm{P}_{\text {korr }} / \mathrm{ETP}_{\text {Gras }}=0,77$ sehr geringe Sickerwassermengen gemessen. In den Jahren 1986 (ebenfalls mit Winterweizen bei einem $\mathrm{P}_{\mathrm{korr}} / \mathrm{ETP}_{\text {Gras }}=1,23$ und 1993 (schlecht etablierte Grünbrache) $\mathrm{P}_{\text {korr }} / \mathrm{ETP}_{\text {Gras }}=1,38$ zeigten sich dagegen sehr hohe Sickerwassermengen.

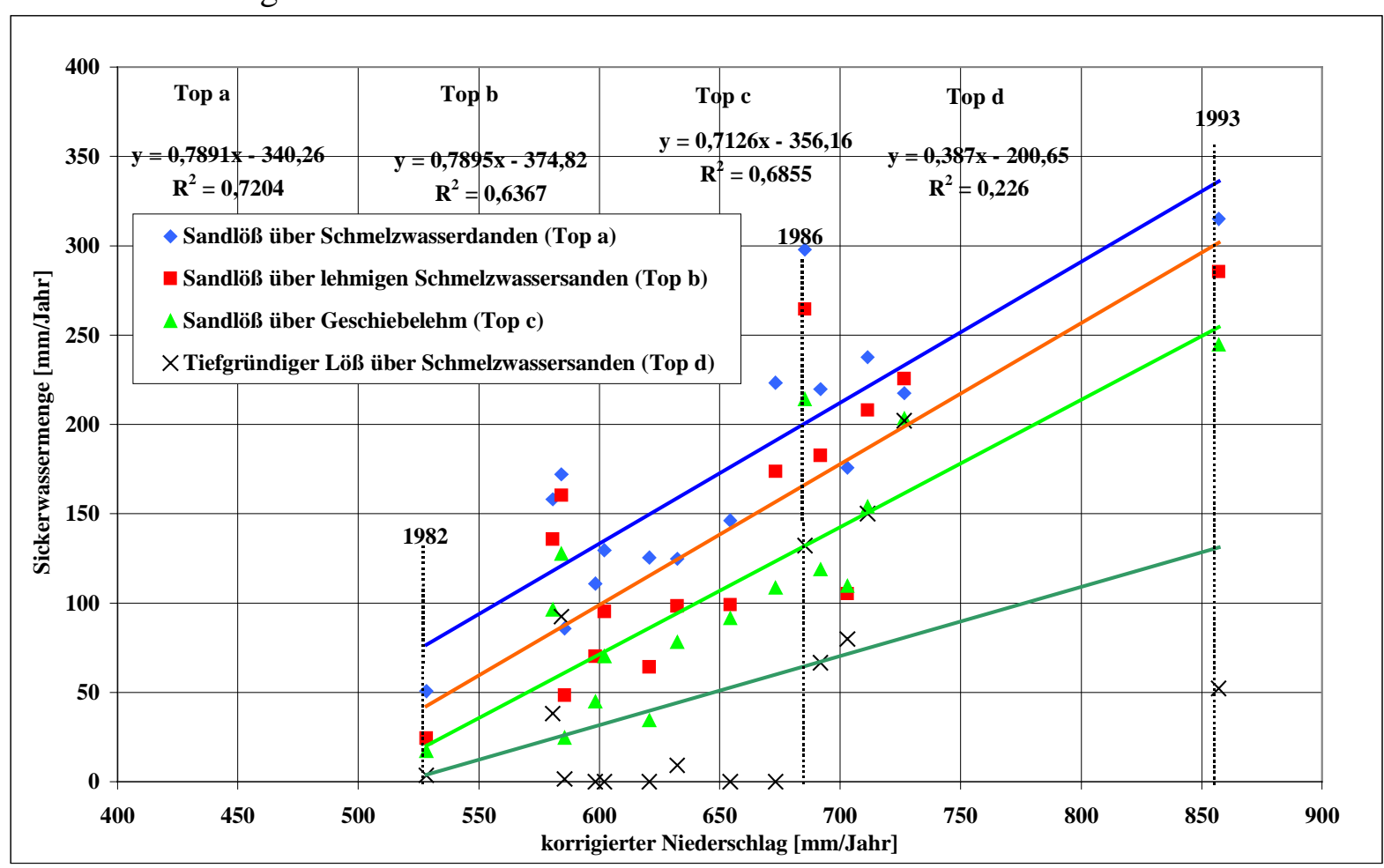

Abb. 28: Abhängigkeit der Sickerwassermengen vom Niederschlag [mm/Jahr] (Beginn des Jahres am 1. April)

Die große Varianz von $\mathrm{P}_{\text {korr }} / \mathrm{ETP}_{\text {Gras }}$ (die sich bei der Betrachtung einzelner Vegetationsperioden noch deutlicher zeigt, s. Tab. 23 scheint die Ursache für die geringe Korrelation zwischen Jahresniederschlag und Sickerwassermenge am Untersuchungsstandort zu sein. Dass in Gebieten mit größeren Wasserbilanzüberschüssen deutlich engere Abhängigkeiten zwischen Jahresniederschlag und Sickerwassermenge bestehen, zeigen die Untersuchungen von DFG (1987) im Raum Hannover, von GERIES (1989) für Lößböden im Raum Göttingen und von PROKSCH (1990).

Das Beispiel der Fruchtart Winterweizen verdeutlicht

Abb. 29 nochmals die große Schwankungsbreite der Sickerwassermengen, die sich am Untersuchungsstandort Brandis bei allen acht untersuchten Böden zeigte. 


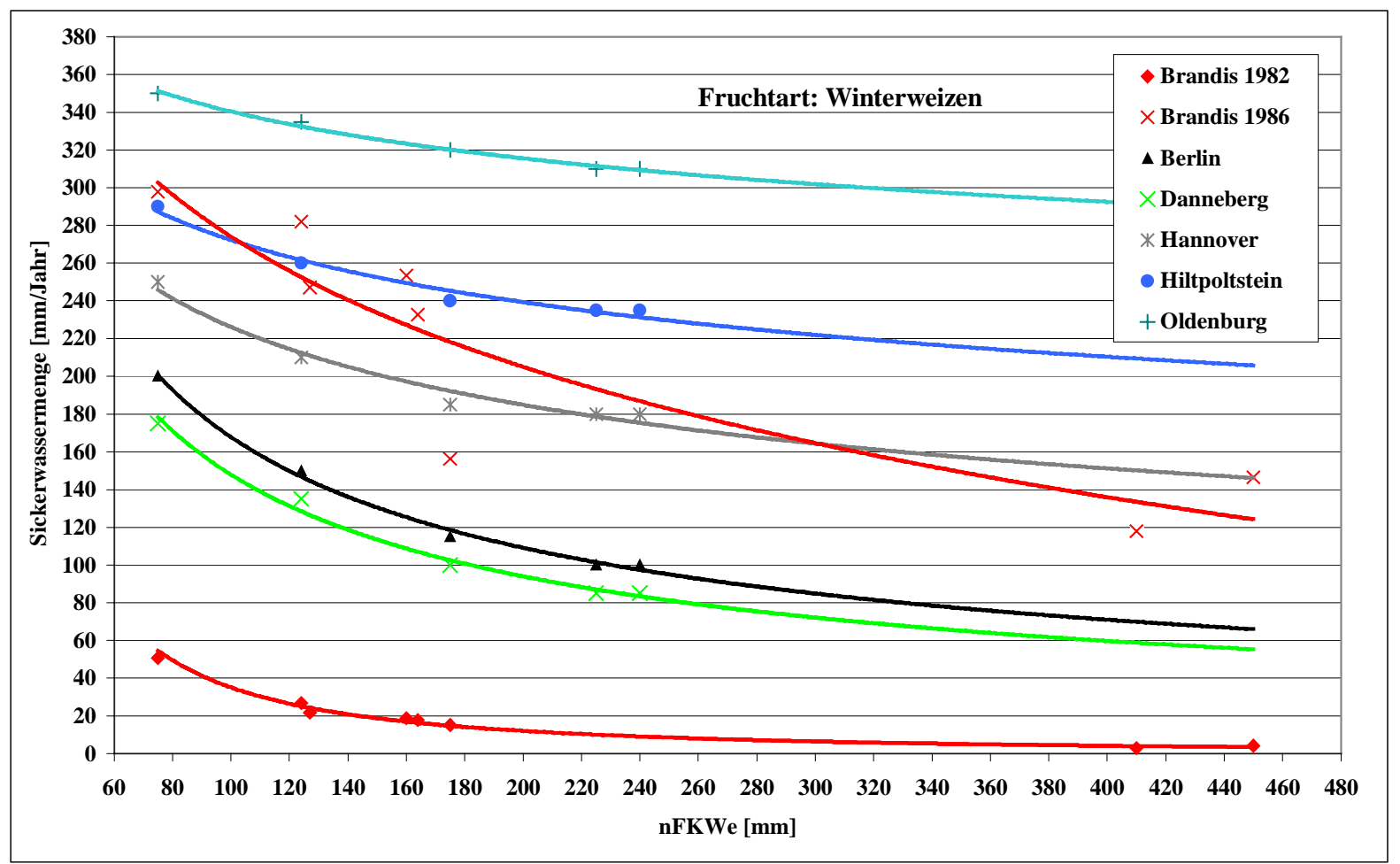

Abb. 29: Beziehung zwischen Sickerwasserrate aus dem Boden und der nutzbaren Feldkapazität im effektiven Wurzelraum $\left(n F K_{W e}\right)$ für unterschiedliche Klimagebiete (ergänzt aus RENGER, 1992)

Die Ergebnisdarstellung in Tab. 27 soll auf die mögliche Schwankungsbreite von Halbjahreswerten der Sickerwasserbildung hinweisen.

Hohe Sickerwassermengen sind zu verzeichnen, wenn hohe Winterniederschläge auf einen vollständig gefüllten Bodenwasserspeicher treffen. Diese außergewöhnlich guten Bedingungen zur Grundwasserneubildung traten am Standort Brandis im Verlauf der 17jährigen Versuchsreihe nur in den Winterhalbjahren 1988 und 1994 auf (für die Lößstandorte nur im Jahr 1988). Unter diesen Bedingungen zeigen die Sickerwasserleistungen zwischen Sand-, Geschiebelehm- und Lößböden nur geringe Unterschiede.

Häufiger sind Jahre mit geringen Sickerwassermengen. Im Verlauf dieser Jahre zeigen sich große Unterschiede in der Höhe der Sickerwassermengen von Sand-, Geschiebelehm- und Lößböden (s. Winterhalbjahre 1990 und 1996).

Die hohen Sickerwassermengen im Sommer 1981 resultieren aus der lang andauernden Sickerwasser-Periode mit noch sehr hohen Neubildungswerten im Mai. Dagegen ist die hohe Sickerwassermenge im Sommerhalbjahr 1983 Folge eines außergewöhnlichen Niederschlagsereignisses im August. Dieses führte bei allen Böden kurzfristig zur Wiederauffüllung und hohen Sickerwassermengen, nur bei den Lößböden reichte dieser Extremniederschlag nicht zur Wiederauffüllung der Bodenwasserdefizite und zu einer Sickerwasserbildung in $3 \mathrm{~m}$ Tiefe.

Der Sommer 1982 zeichnete sich durch ein hohes Strahlungsangebot aus. Auf Grund geringer Niederschläge kam es bei einem Bewuchs mit Winterweizen schon im März zu einer erheblichen Inanspruchnahme von Bodenwasser. Die Sickerwasserperiode endete bereits im März. 
Tab. 27: Mittel der Sickerwasserbildung für die Untersuchungsjahre 1981-97 und maximale bzw. minimale Sickerwasserbildung für die acht Lysimetergruppen

\begin{tabular}{|c|c|c|c|c|c|c|c|c|c|}
\hline \multirow[t]{2}{*}{$\begin{array}{l}\text { Halbjahr } \\
\text { (Jahr) }\end{array}$} & \multirow{2}{*}{$\begin{array}{l}\text { korrigierter } \\
\text { Niederschlag } \\
{[\mathrm{mm}]}\end{array}$} & \multicolumn{8}{|c|}{$\begin{array}{l}\text { Grundwasserneubildung [mm/Halbjahr] } \\
\text { (von November - April, Mai - Oktober) }\end{array}$} \\
\hline & & \begin{tabular}{|c|} 
Lys 5 \\
D3
\end{tabular} & $\begin{array}{c}\text { Lys } 4 \\
\text { D5 }\end{array}$ & $\begin{array}{c}\text { Lys } 8 \\
\text { D3 }\end{array}$ & $\begin{array}{c}\text { Lys } 1 \\
\text { D6 }\end{array}$ & $\begin{array}{c}\text { Lys } 7 \\
\text { D4 }\end{array}$ & $\begin{array}{c}\text { Lys } 11 \\
\text { D3 }\end{array}$ & $\begin{array}{c}\text { Lys } 9 \\
\text { Lö3 }\end{array}$ & $\begin{array}{c}\text { Lys } 10 \\
\text { Lö1 }\end{array}$ \\
\hline Winter $_{\text {Mittel }}$ & & 136 & 105 & 113 & 91 & 76 & 93 & 47 & 43 \\
\hline \multicolumn{10}{|l|}{ Winter $_{\max }$} \\
\hline 1988 & 311 & 198 & 188 & 194 & 189 & 143 & 199 & 182 & 184 \\
\hline 1994 & 418 & 313 & 308 & 314 & 293 & 244 & 275 & 125 & 97 \\
\hline & & & & & & & & & \\
\hline \multicolumn{10}{|l|}{ Winter $_{\min }$} \\
\hline 1990 & 296 & 68 & 33 & 26 & 13 & 6 & 13 & 0 & 0 \\
\hline 1996 & 185 & 73 & 7 & 30 & 2 & 48 & 21 & 0 & 0 \\
\hline Sommer $_{\text {Mittel }}$ & & 40 & 31 & $\overline{33}$ & 23 & 22 & 21 & 10 & 7 \\
\hline \multicolumn{10}{|l|}{ Sommer $_{\max }$} \\
\hline 1983 & 387 & 107 & 83 & 83 & 54 & 37 & 50 & 0 & 0 \\
\hline 1981 & 375 & 63 & 61 & 64 & 50 & 34 & 44 & 54 & 37 \\
\hline \multicolumn{10}{|l|}{ Sommer $_{\min }$} \\
\hline 1982 & 281 & 13 & 12 & 13 & 11 & 9 & 9 & 0 & 0 \\
\hline
\end{tabular}

Sommer (Mai-Oktober), Winter (November-April)

Auch diese Auswertung ergab, dass „,mittlere“ Neubildungsmengen ganz selten auftreten. Deshalb sollten langjährigen Jahres- und Halbjahresmittelwerte nur für langfristige und großräumige Untersuchungen verwendet werden.

\subsubsection{Verlauf der Sickerwasserperiode}

Die winterliche Sickerwasser-Periode beginnt stets unmittelbar nach erfolgter Auffüllung der sommerlichen Bodenwasserdefizite.

Die zum Zeitpunkt der erreichten Wiederauffüllung fallenden Niederschläge bestimmen die Höhe der Sickerwassermengen zu Beginn der Sickerwasser-Periode und ihren weiteren Verlauf.

Der Beginn der Sickerwasser-Periode kann nach den bisherigen Beobachtungen in einem Zeitraum von Oktober bis April liegen (Tab. 28.

- Bei den Sandböden (Top a - b; Lysimetergruppe 5, 4, 8) ist auf Grund der beschränkten Kapazität des Bodenwasserspeichers die Wiederauffüllung in der Regel im Dezember abgeschlossen.

- Bei den Geschiebelehmböden (Top c; Lysimetergruppe 1, 7, 11) ist der Wasserbedarf für die Wiederauffüllung der Bodenwasserdefizite schon erheblich größer (Гab. 28), so dass dieser Prozess in der überwiegenden Anzahl der Jahre erst im Januar abgeschlossen ist, aber auch bis März oder April dauern kann. 
Tab. 28: Häufigkeitsverteilung für den Beginn der jährlichen Sickerwasserperiode auf der Basis der Monatswerte der 17 Abflußjahre 1981-97

\begin{tabular}{|c|c|c|c|c|c|c|c|c|}
\hline Lysimeter- & nFK* & \multicolumn{7}{|c|}{ Anzahl der Jahre } \\
\cline { 3 - 9 } gruppe & {$[\mathrm{mm}]$} & Okt. & Nov. & Dez. & Jan. & Febr. & März & April \\
\hline 5 & 75 & 1 & 1 & 11 & 3 & 1 & & \\
\hline 4 & 127 & 1 & & 5 & 5 & 4 & & 2 \\
8 & 126 & 1 & & 6 & 5 & 3 & 2 & \\
\hline 1 & 164 & & 1 & 5 & 4 & 5 & 1 & 1 \\
7 & 175 & & 1 & 4 & 7 & 2 & 2 & 1 \\
11 & 160 & & 1 & 8 & 4 & 2 & 2 & \\
\hline $9 * *$ & 455 & & & 1 & 3 & 1 & 2 & \\
$10^{* *}$ & 420 & 1 & & 1 & 3 & & 2 & \\
\hline
\end{tabular}

* nFK - als maximal pflanzenverfügbarer Bodenwasservorrat einschließlich kapillarer Nachlieferung aus dem Unterboden, ermittelt auf der Basis der Lysimetermessungen (s. Kap. 3.4.1)

**Die Lysimetergruppen 9 und 10 hatten von 17 Abflußjahren 10 Jahre keine Grundwasserneubildung.

- Bei den Lößböden (Top d; Lysimetergruppe 9, 10) sind die Niederschläge, die nach dem Erreichen des sommerlichen Maximums der Bodenwasserausschöpfung fallen, in 10 von 17 Untersuchungsjahren geringer, als die zur Wiederauffüllung der Bodenwasserdefizite erforderlichen Wassermengen. Deshalb kam es in diesen Jahren zu keiner Sickerwasserbildung.

Das Maximum der jährlichen Neubildungsperiode wird bei allen Böden in der überwiegenden Anzahl der Jahre im März oder April erreicht (Tab. 29, während aus der oberen Bodenzone infolge Verdunstung bereits wieder Bodenwasser entnommen wird.

Tab. 29: Häufigkeitsverteilung für den Eintritt des Maximums der jährlichen Sickerwasserperiode auf der Basis der Monatswerte der 17 Abflußjahre 1981-97

\begin{tabular}{|c|c|c|c|c|c|c|c|c|}
\hline \multirow{2}{*}{$\begin{array}{c}\text { Lysimeter- } \\
\text { gruppe }\end{array}$} & \multicolumn{7}{|c|}{ Anzahl der Jahre } \\
\cline { 2 - 9 } & Okt. & Nov. & Dez. & Jan. & Febr. & März & April & Mai \\
\hline 5 & 1 & & 3 & 4 & 1 & 4 & 4 & \\
\hline 4 & 1 & & & 3 & 3 & 5 & 4 & 1 \\
8 & & & 1 & 2 & 4 & 3 & 7 & \\
\hline 1 & & & & 5 & 1 & 6 & 5 & \\
7 & & & & 4 & 2 & 6 & 4 & 1 \\
11 & & 1 & & & 4 & 7 & 4 & 1 \\
\hline $9 *$ & & & & & 1 & 4 & 2 & \\
$10^{*}$ & & & & & & 4 & 3 & \\
\hline
\end{tabular}

*Die Lysimetergruppen 9 und 10 hatten von 17 Abflußjahren 10 Jahre keine Grundwasserneubildung.

Die Dauer der Sickerwasser-Periode beträgt im Durchschnitt der Jahre (etwas differenziert in Abhängigkeit von der Bodenform) ca. 6 Monate (Tab. 30). Sickerwasser, das im Verlauf der Sommermonate in $3 \mathrm{~m}$ Tiefe das Lysimeter verläßt, resultiert aus der winterlichen Neubildungsperiode. Nur in Ausnahmefällen wie im August 1983 führen im Sommer Starkregenereignisse zur erhöhten Sickerwasserbildung, d. h., Sickerwassermengen, als eine Reaktion auf einen ,auslösenden Niederschlag“ (HELLEKES, 1985), sind nur während der kurzen Phasen vollständiger Auffüllung zu verzeichnen. Erhöhte Sickerwassermengen, die zu Zeiten mit erheblichem Bodenwasserdefizit auftreten, werden als Folge präfentieller Flüsse interpretiert. 
Die Sickerwasserbildung am Auslauf des Lysimeters in $3 \mathrm{~m}$ Tiefe entspricht mengenmäßig der Versickerung unterhalb der verdunstungsbeeinflussten Bodenzone. Der zeitliche Verlauf resultiert aus einer Abflachung und Phasenverschiebung der Sickerwasserwellen auf dem Weg von der Unterkante der verdunstungsbeeinflussten Bodenzone bis zum Lysimeterauslauf. Im Verlauf eines hydrologischen Jahres (November bis Oktober) kann eine Neubildungsperiode vollständig erfasst werden, wobei zu Beginn des hydrologischen Jahres die Wiederauffüllung der Bodenwasservorräte noch nicht abgeschlossen ist.

\section{Monate:}

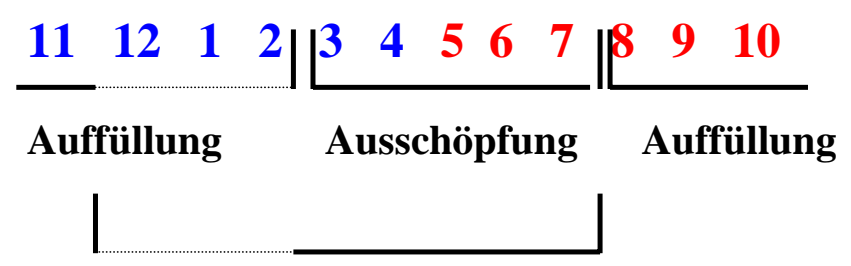

\section{Sickerwasser-Periode}

Bei den Lehm- und Lößböden (Top c und d) liegen zwischen dem Zeitpunkt der Wiederauffüllung und dem Beginn der Periode der Ausschöpfung oft nur eine oder zwei Dekaden. Es kommt auch vor, dass die Wiederauffüllung erst nach dem Beginn der Vegetationsperiode erreicht wird.

Auf Grund der festgestellten Variabilität der innerjährlichen Verteilung der Sickerwasserbildung ist über die Anwendung der Monatsmittelwerte (Tab. 30 für Bilanzrechnungen vor Ort, in Abhängigkeit von der jeweiligen Aufgabenstellung, zu entscheiden.

Tab. 30: Mittlerer Jahresgang der Sickerwasserbildung und des Niederschlages [mm] für Standorte mit Sand, Geschiebelehm und Löß (Wasserhaushaltsjahre 1981-97)

\begin{tabular}{|c|cccccc|ccccccc|c|c|c|}
\hline Lys & NOV & DEZ & JAN & FEB & MÄR & APR & MAI & JUN & JUL & AUG & SEP & OKT & JAHR & WI & SO \\
\hline $\mathbf{5}$ & 2 & 22 & 31 & 24 & 31 & 25 & 12 & 8 & 5 & 7 & 5 & 3 & $\mathbf{1 7 5}$ & 136 & 40 \\
\hline $\mathbf{4}$ & 1 & 7 & 21 & 21 & 29 & 27 & 15 & 6 & 4 & 4 & 1 & 1 & $\mathbf{1 3 5}$ & 105 & 31 \\
$\mathbf{8}$ & 1 & 9 & 23 & 23 & 29 & 28 & 14 & 7 & 4 & 5 & 2 & 1 & $\mathbf{1 4 6}$ & 113 & 33 \\
\hline $\mathbf{1}$ & 1 & 7 & 18 & 19 & 26 & 20 & 9 & 5 & 3 & 3 & 1 & 1 & $\mathbf{1 1 3}$ & 91 & 23 \\
$\mathbf{7}$ & 1 & 6 & 13 & 16 & 21 & 19 & 8 & 6 & 3 & 3 & 1 & 1 & $\mathbf{9 8}$ & 76 & 22 \\
$\mathbf{1 1}$ & 1 & 8 & 18 & 19 & 25 & 22 & 10 & 5 & 2 & 3 & 1 & 0 & $\mathbf{1 1 4}$ & 93 & 21 \\
\hline $\mathbf{9}$ & 0 & 1 & 3 & 10 & 18 & 14 & 8 & 2 & 0 & 0 & 0 & 0 & $\mathbf{5 7}$ & 47 & 10 \\
$\mathbf{1 0}$ & 0 & 4 & 5 & 8 & 15 & 11 & 5 & 1 & 0 & 0 & 1 & 0 & $\mathbf{5 0}$ & 43 & 7 \\
\hline $\mathbf{P}_{\text {korr }}$ & 56 & 58 & 44 & 37 & 47 & 59 & 53 & 72 & 68 & 70 & 52 & 37 & $\mathbf{6 5 5}$ & 302 & 353 \\
\hline
\end{tabular}

Anlage 7 zeigt den aktuellen Verlauf der Monatswerte der Grundwasserneubildung im Vergleich zu den langjährigen Monatsmittelwerten. 


\subsubsection{Anwendung des Verfahrens von RENGER \& WESSOLEK}

Nachfolgend soll untersucht werden, ob mit Hilfe Regressionsgleichungen von RENGER \& WESSOLEK (1990) eine Abschätzung der jährlichen Sickerwassermengen erfolgen kann, da hier $\mathrm{nFK}_{W \mathrm{~W}}$ und der jährliche Verdunstungsanspruch der Atmosphäre Berücksichtigung finden. Wie bereits in Kap. 3.5.4 beschrieben, wurde das Verfahren von RENGER \& WESSOLEK (1990) zur Ermittlung der Jahresverdunstung und der Höhe des jährlichen Gesamtabflusses für grundwasserferne, ebene Flächen entwickelt. Unter der Annahme

$$
\mathrm{R}=\mathrm{SW}=\mathrm{P}_{\mathrm{Wi}}+\mathrm{P}_{\mathrm{So}}-\mathrm{ETR}
$$

mit $\mathrm{R}=$ jährliche Höhe des Gesamtabflusses $=\mathrm{SW}=$ jährliche Sickerwassermenge gilt nun zur Berechnung der Sickerwasserbildung folgende Gleichung:

$$
\text { Gl. 15: } \quad \mathbf{S W}=\mathbf{a} * \mathbf{P}_{\mathbf{w i}}+\mathbf{b} * \mathbf{P}_{\mathbf{S o}}-\mathbf{c} * \log \mathbf{W}_{\mathbf{p f l}}-\mathbf{d} * \mathbf{E T P}+\mathbf{e}
$$

$\mathrm{P}_{\mathrm{Wi}} \quad=$ korrigierter Winterniederschlag von Oktober bis März des Folgejahres

$\mathrm{P}_{\mathrm{SO}} \quad=$ korrigierter Sommerniederschlag von April bis September

$\mathrm{W}_{\mathrm{pfl}} \quad$ = pflanzenverfügbare Bodenwassermenge (s. Tab. 15 und Tab. 20

ETP = Gras-Referenzverdunstung (Oktober bis September des Folgejahres)

a,b,c,d,e = landnutzungsspezifische Regressionskoeffizienten

Für die Konstanten a bis e wurden von RENGER \& WESSOLEK (1990) für nordwestdeutsche Bedingungen folgende Werte bestimmt:

\begin{tabular}{|l|c|c|c|c|c|}
\hline Bodennutzung & a & b & c & d & e \\
\hline Ackerland & 0,92 & 0,61 & 153 & 0,12 & -109 \\
Grünland & 0,90 & 0,52 & 286 & 0,10 & -330 \\
\hline
\end{tabular}

Es werden der korrigierte Niederschlag und statt HAUDE die Gras-Referenzverdunstung verwendet, wodurch es zu relativ geringen Abweichungen der Berechnungsergebnisse kommt (s. Kap. 4.6.3). Der Beginn des Bilanzjahres wurde zunächst auf den 1. Oktober gelegt. Wie aus Tab. 31 hervorgeht, kommt es erwartungsgemäß u. a. auf Grund der noch vorhandenen Bodenwasserdefizite im Ergebnis der Berechnungen zu einer erheblichen Überschätzung der Sickerwassermengen.

Tab. 31: Mittlere Abweichung zwischen gemessener und berechneter Sickerwasserbildung und Bestimmtheitsmaß $\left(R^{2}\right)$ für die Übereinstimmung in den Einzeljahren in [\%] - Beginn des Bilanzjahres am 1. Oktober

\begin{tabular}{|l|c|c|c|c|c|c|c|c|}
\hline Lys.-Gruppe & $\mathbf{5}$ & $\mathbf{4}$ & $\mathbf{8}$ & $\mathbf{1}$ & $\mathbf{7}$ & $\mathbf{1 1}$ & $\mathbf{9}$ & $\mathbf{1 0}$ \\
\hline $\mathrm{W}_{\text {pfl }[\mathrm{mm}]}$ & 75 & 127 & 124 & 164 & 175 & 160 & 450 & 410 \\
\hline berechn./gem. [\%] & 128 & 121 & 133 & 157 & 176 & 156 & 193 & 225 \\
\hline $\mathrm{R}^{2}[\%]$ & 44 & 31 & 34 & 22 & 11 & 23 & 8 & 6 \\
\hline
\end{tabular}

Deshalb wurde von RENGER \& WESSOLEK (1990) der 1. April (Lysimeterjahr) als Beginn des Berechnungsjahres festgelegt. Auch zu diesem Zeitpunkt entspricht der Bodenwassergehalt in einigen Jahren bei den Lößböden noch nicht der FK (s. Tab. 16. Für diese Jahre konnte das Verfahren bei den Lößböden nicht zur Anwendung kommen.

Wie aus Tab. 32 hervorgeht, wurde auch bei Verwendung des Lysimeterjahres keine zufriedenstellende Übereinstimmung zwischen Mess- und Berechnungsgrößen erreicht. 
Große Abweichungen wurden vorwiegend in den Jahren ermittelt, die durch eine hohe Verdunstung während der Vegetationsperiode bei einem $\mathrm{P}_{\text {korr }} / \mathrm{ETP}_{\text {Gras }}<0,9$ gekennzeichnet sind (vgl. hierzu auch Tab. 23. Wie bereits in der Diskussion zur Abschätzung der Verdunstung festgestellt wurde, führt der höhere Verdunstungsanspruch der Atmosphäre im Untersuchungsgebiet zu einer höheren Verdunstungsleistung als im Raum Hannover. Die Höhe der Sommerniederschläge und der potentiellen Verdunstung finden in den Regressionsbeziehungen aber nur wenig Berücksichtigung. Dies ist eine Ursache dafür, dass die Sickerwassermengen insbesondere in warmen Jahren überschätzt wird.

Mit einer Veränderung der für nordwestdeutsche Bedingungen festgelegten Konstanten a bis e kann eine bessere Übereinstimmung von Mess- und Berechnungsergebnissen für den hier untersuchten Standort erreicht werden. Diese Veränderungen sollten aber gemeinsam mit den Urhebern des Verfahrens vorgenommen werden. Für den Ansatz zur Berechnung von Grünland ist eine prinzipielle Überprüfung der Gültigkeit der Koeffizienten für den Untersuchungsstandort erforderlich.

Tab. 32: Vergleich zwischen nach RENGER \& WESSOLEK (1990) berechneten und gemessenen Sickerwassermengen sowie Niederschlag $\left(P_{\text {korr }}\right)$ und Gras-Referenzverdunstung $\left(E T P_{\text {Gras }}\right)$ - Beginn des Bilanzjahres am 1. April

\begin{tabular}{|c|c|c|c|c|c|c|c|c|c|c|c|c|}
\hline \multirow[b]{2}{*}{ Jahr } & \multirow[b]{2}{*}{ Fruchtart } & \multirow[b]{2}{*}{$\overline{\mathbf{E T P}_{\text {Gras }}}$} & \multirow[b]{2}{*}{$\mathbf{P}_{\text {korr }}$} & \multirow[b]{2}{*}{$\begin{array}{c}\mathbf{P}_{\text {korrr }} / \\
\text { ETP }_{\text {Gras }}\end{array}$} & \multicolumn{4}{|c|}{ Nach Renger \&Wessolek } & \multicolumn{4}{|c|}{ gemessen } \\
\hline & & & & & Top a & Top b & Top c & Top d & op a & Top b & Top c & Top d \\
\hline 1981 & & 4,0 & 1,7 & 1,2 & 265,3 & 231,1 & 212,4 & 150,9 & 219,7 & 182,7 & 118,8 & 66,7 \\
\hline 1982 & zen & 7,6 & 528,3 & 0,8 & 145,7 & 111,5 & 92,8 & $\mathrm{n}$ & 50,6 & 24,3 & 17,2 & 3,6 \\
\hline 1983 & i & 1,0 & 673,2 & 1,0 & 209,2 & 175,0 & 156,3 & $\mathrm{n}$ & 223,3 & 173,8 & 108,7 & 0,0 \\
\hline 1984 & - & & & 1,2 & 149,5 & 81,3 & 42,2 & $\mathrm{n}$ & 124,7 & 98,4 & 78,1 & 9,2 \\
\hline 1985 & $\operatorname{artc}$ & 4,5 & 584,2 & 1,0 & 183,1 & 148,9 & 130,2 & 67,9 & 172,0 & 160,4 & 127,6 & 92,4 \\
\hline 1986 & 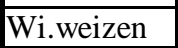 & 558,3 & 685,4 & 1,2 & 280,2 & 246,0 & 227,3 & 163,0 & 297,9 & 264,6 & 214,1 & 132,2 \\
\hline 1987 & Kar & 3,4 & 726,6 & 1,4 & 296,5 & 262,3 & 243,6 & 181,5 & 217,4 & 225,6 & 203,3 & 202,0 \\
\hline 1988 & en & 4,5 & 580,6 & 0,9 & 198,4 & 164,2 & 145,5 & 84,0 & 158,1 & 135,9 & 96,3 & 38,1 \\
\hline 1989 & & & 6 & 0,8 & 0 & 146,8 & 128,2 & $\mathrm{n}$ & 85,9 & 48,3 & 24,6 & 1,5 \\
\hline 1990 & Zu.r & 694,1 & 620,7 & 0,9 & 189,2 & 155,0 & 136,4 & $\mathrm{n}$ & 125,4 & 64,3 & 34,4 & 0,0 \\
\hline 1991 & Wi. & 652,4 & 598,3 & 0,9 & 207,3 & 173,1 & 154,4 & $\mathrm{n}$ & 110,9 & 70,1 & 44,9 & 0,0 \\
\hline 1992 & Wi.g & 596,3 & 602,1 & 1,0 & 206,7 & 172,5 & 153,8 & $\mathrm{n}$ & 129,7 & 95,2 & 70,2 & 0,0 \\
\hline 1993 & Grünbrach & 619,1 & 857,0 & 1,4 & 318,7 & 254,8 & 219,9 & $\mathrm{n}$ & 314,9 & 285,5 & 244,5 & 52,3 \\
\hline 1994 & Grünbrac & 713,6 & 711,4 & 1,0 & 198,2 & 134,3 & 99,4 & $-16,8$ & 237,6 & 207,9 & 153,8 & 150,1 \\
\hline 1995 & Rotklee & 597,5 & 703,0 & 1,2 & 163,8 & 99,9 & 65,0 & $\mathrm{n}$ & 175,8 & 105,2 & 109,6 & 80,0 \\
\hline 1996 & Kartoffel & 551,4 & 654,4 & 1,2 & 242,0 & 207,8 & 189,1 & 127,6 & 146,3 & 99,0 & 91,4 & 0,0 \\
\hline & Mittel & 621,2 & 652,2 & 1,1 & 214,7 & 172,8 & 149,8 & - & 172,6 & 137,7 & 107,8 & 48,8 \\
\hline
\end{tabular}

$\mathrm{n}$ - das Verfahren wurde nicht angewendet 


\subsubsection{Zusammenfassung und Diskussion der Ergebnisse}

Unter den klimatischen Bedingungen am Standort Brandis ist die Sickerwasserbildung der untersuchten Böden durch drei Merkmale gekennzeichnet:

- einem typischen jahreszeitlichen Gang mit relativ hohen Werten im Winter und sehr geringen oder keinen Neubildungsraten im Sommer, wobei die Lößböden auch mehrere Jahre hintereinander kein Sickerwasser (in 3 m Tiefe) liefern,

- einem mehrjährigen Rhythmus von Jahren mit überdurchschnittlich hoher bzw. niedriger Sickerwasserbildung,

- einer sehr hohen Abweichung der Einzelwerte vom langjährigen Mittelwert.

Größten Einfluss auf die Sickerwasserbildung im betrachteten Bilanzjahr haben die Bodenwasserausschöpfung der untersuchten Böden und die Höhe der Niederschläge im Anschluss an die Vegetationsperiode. Aus dem jährlichen Wechselspiel zwischen Niederschlagshöhe und Bodenwasserdefizit resultiert eine hohe Variabilität der jährlichen Sickerwassermengen.

Ursache für die geringen Sickerwasserraten (175 $\mathrm{mm}$ bei Sandlöß über Schmelzwassersanden und rd. $50 \mathrm{~mm}$ beim tiefgründigen Löß) ist der im Vergleich zum Jahresniederschlag vergleichsweise hohe Verdunstungsanspruch der Atmosphäre, insbesondere in den Sommermonaten. Dieser kann zwar nur auf den Lößböden tatsächlich realisiert werden, führt aber auch bei den Böden mit geringer und mittlerer $\mathrm{nFK}_{\mathrm{We}} \mathrm{zu}$ einer vergleichsweise hohen Verdunstung. Die Verteilung der Sommer- zu Winterniederschlägen fällt nach WENDLAND et al. (1993) in den östlichen Bundesländer eindeutig zu Gunsten der Sommerniederschläge aus. Dies ist von Nachteil für die Sickerwasserbildung, da die Sommerniederschläge nahezu vollständig verdunsten Abb. 30 und nur die Winterniederschläge abzüglich der zur Auffüllung der Bodenwasserdefizite erforderlichen Wassermengen zur Sickerwasserbildung beitragen können.

Die zum Referenzgebiet des Verfahrens von RENGER \& WESSOLEK (1990) abweichende Wasserhaushaltssituation wird als Ursache für die schlechte Übereinstimmung von Mess- und Berechnungsergebnissen (in Bezug auf Verdunstung und Sickerwasserbildung) in Einzeljahren am Standort Brandis angesehen. Da das Verfahren auf Grund seiner praktischen Handhabung zunehmend auch in den neuen Bundesländern angewendet wird, besteht dringender Bedarf einer Anpassung der Koeffizienten.

Probleme bei der Aufstellung von Nomogrammen zur Ableitung von Jahreswerten der Sickerwasserbildung aus Niederschlag, Verdunstung und verfügbarem Bodenwasservorrat ergeben sich auch aus der Tatsache, daß keine geeignete Abgrenzung des Bilanzzeitraumes (=Jahres) gelingt. Im Mittel der Jahre sind drei Phasen zu verzeichnen (Abb. 30:

- die Phase der Ausschöpfung von März bis Juli

- die Phase der Wiederauffüllung der Bodenwasservorräte von August bis Dezember (auch bis Januar oder Februar)

- die Phase der Sickerwasserbildung von Dezember (Januar oder Februar) bis Juni. 

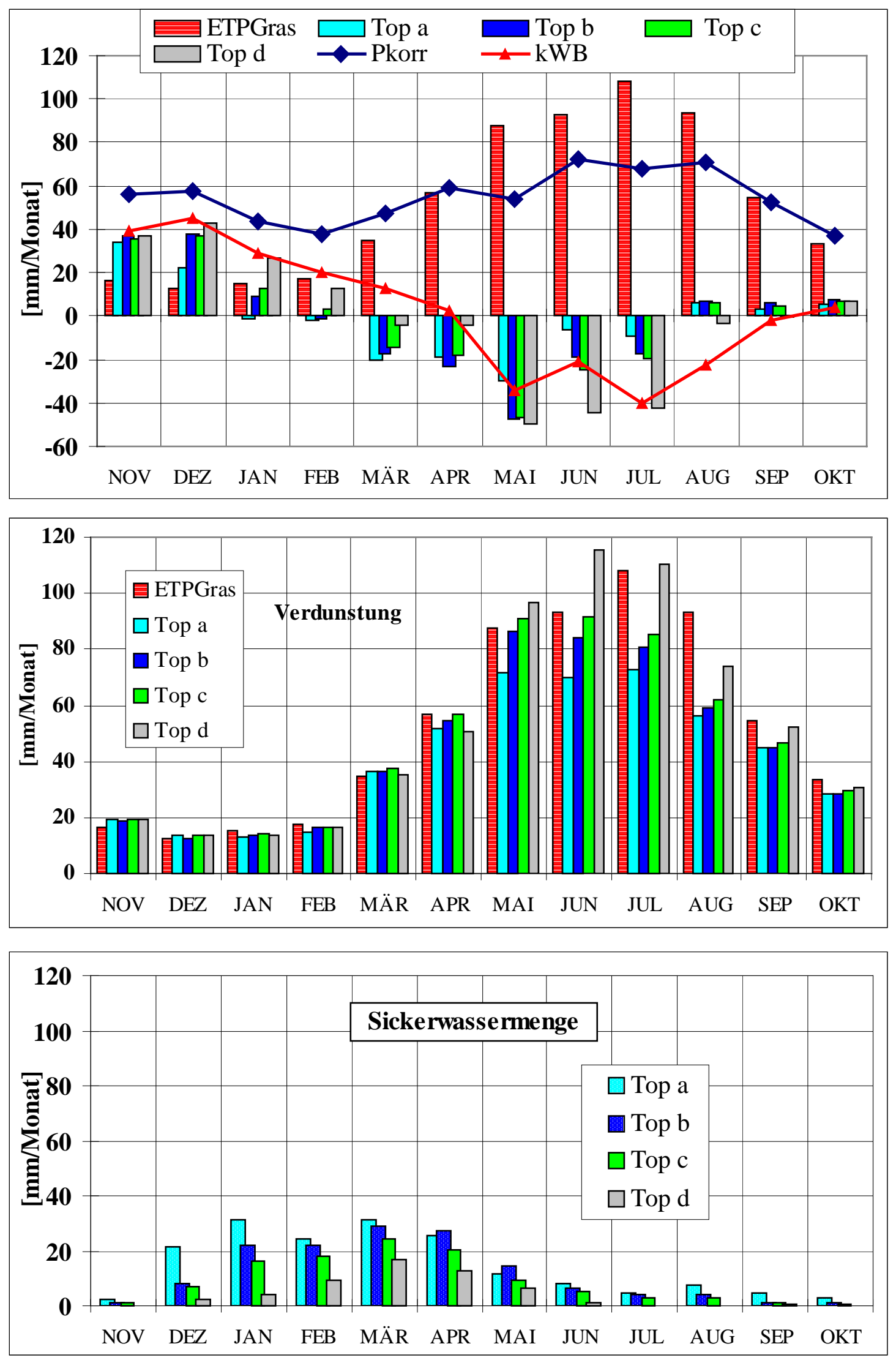

Abb. 30: Mittlerer Jahresgang von Bodenwasservorratsänderung (dSB), Niederschlag $\left(P_{\text {korr }}\right)$, Gras-Referenzverdunstung $\left(\right.$ ETP $\left._{\text {Gras }}\right)$, klimatischer Wasserbilanz $(k W B$, realer Verdunstung und Grundwasserneubildung am Standort Brandis (Reihe 1981-97) 
Auf Grund der jährlichen Variabilität dieser Prozesse läßt sich weder für das hydrologische Jahr (Nov. bis Okt.) noch für das Lysimeterjahr (April bis März) ein zufriedenstellender Zusammenhang zwischen Boden ( $\mathrm{nFK}_{\mathrm{We}}$ ), Jahresniederschlag und Sickerwassermenge in $3 \mathrm{~m}$ Tiefe herstellen. Regressionsbeziehungen für das hydrologische Jahr entstehen erst, wenn das aktuelle Bodenwasserdefizit zu Beginn des entsprechenden Bilanzjahres berücksichtigt wird. Dieses jährliche bodenabhängige Bilanzdefizit kann aus Lysimeter- oder Bodenfeuchtemessungen ermittelt werden. Wird als Beginn des Jahres der April gewählt, ist es auf Grund der Laufzeiten bis in $3 \mathrm{~m}$ Tiefe nicht möglich, eine vollständige Neubildungsperiode zu erfassen.

Unter der Voraussetzung, dass $3 \mathrm{~m}$ tiefe Lysimeter Standortbedingungen mit flurfernen Grundwasserständen repräsentieren (mit Ausnahme tiefgründiger Lößböden), entspricht die ermittelte Sickerwassermenge dem maximal mögliche Abfluss (Gesamtabfluss) des Standortes. Diese Wassermenge gilt als potentielle Speisungsgröße für das Grundwasser. Sie ist deshalb bilanzmäßig von wasserwirtschaftlichem Interesse und gleichzeitig wichtiges Kriterium hinsichtlich der Risikoabschätzung von Stoffeinträgen in das Grundwasser. Ob dieser „potentielle Abfluss“ jedoch der Grundwasserneubildung gleichgesetzt werden kann, hängt von den hydrogeologischen Gegebenheiten der Einzugsgebiete ab (s. Kap. 4.1.3). Diese Fragestellung gilt es insbesondere zu berücksichtigen, wenn unter Standortbedingungen gewonnene Sickerwassermengen mittels GIS-System (oft ohne Interaktion zwischen den benachbarten Flächen) zur Bestimmung von Gebietsabflüssen verwendet werden. 


\subsection{Beziehung zwischen Bewirtschaftung, Bodenwasserhaushalt und Stickstoff-Austrag}

\subsubsection{Einfluß der Bewirtschaftung - Stickstoff-Saldo}

Im Ergebnis der Brandiser Lysimeterversuche stehen folgende Messwerte zur Verfügung:

- mineralische Stickstoff(N)-Düngung und N-Einträge über die Niederschläge (nasse Deposition $=N$-Gehalt des Niederschlagswassers im HELLMANN-Messer)

- N-Abfuhr durch das Erntegut und die

- $\quad$-Austräge über das Sickerwasser in 3 m Tiefe.

Zur Aufstellung der N-Salden werden nachfolgend die Untersuchungsergebnisse der Jahre 1981-95 (1981-92: intensive landwirtschaftliche Bewirtschaftung, 1993-95: Flächenstilllegungsmaßnahmen) verwendet.

\section{N-Einträge [kg/ha/Jahr]:}

Niederschläge (nasse Deposition):

Stickstoffdüngung (nur mineralisch):
Jährliche Mengen (von ... bis) in $\mathrm{kg} / \mathrm{ha}$ der Jahre 1981-92 der Jahre 1993-95

$$
\begin{array}{cc}
30-66 & 13-30 \\
100-160 & 0
\end{array}
$$

N-Abfuhr über das pflanzliche Erntegut $[\mathrm{kg} / \mathrm{ha} / \mathrm{Jahr}]$ :

Tab. 33 zeigt die N-Mengen, die über das Erntegut der verschiedenen Fruchtarten von den acht Lysimeterböden abgeführt wurden (jeweils als Mittel der drei Wiederholungen). Es ist zu beachten, dass die einzelnen Fruchtarten (bis auf das Wintergetreide) innerhalb der Beobachtungsreihe von 1981-95 nur ein- oder zweimal angebaut wurden, die Ergebnisse also stark von der jeweiligen Witterung der Anbaujahre abhängig sind.

Tab. 33: N-Entzüge durch das Erntegut verschiedener Fruchtarten auf acht verschiedenen Böden [kg/ha/Jahr] (Die Lysimetergruppen sind nach fallender Sickerwasserleistung gereiht.)

\begin{tabular}{|l|c|c|c|c|c|c|c|c|}
\hline $\begin{array}{c}\text { Lys.-Gruppe } \\
\text { NStE }\end{array}$ & $\begin{array}{c}\mathbf{5} \\
\mathbf{D 3}\end{array}$ & $\begin{array}{c}\mathbf{8} \\
\mathbf{D 3}\end{array}$ & $\begin{array}{c}\mathbf{4} \\
\mathbf{D 5}\end{array}$ & $\begin{array}{c}\mathbf{1} \\
\mathbf{D 6}\end{array}$ & $\begin{array}{c}\mathbf{7} \\
\mathbf{D 4}\end{array}$ & $\begin{array}{c}\mathbf{1 1} \\
\mathbf{D 3}\end{array}$ & $\begin{array}{c}\mathbf{9} \\
\text { Lö3 }\end{array}$ & $\begin{array}{c}\mathbf{1 0} \\
\text { Lö1 }\end{array}$ \\
\hline Mais & 97 & 139 & 124 & 125 & 159 & 168 & 178 & 148 \\
\hline Wintergetreide & $50-124$ & $63-132$ & $84-138$ & $102-147$ & $87-169$ & $86-157$ & $80-251$ & $115-219$ \\
\hline & & & & & & & & \\
Zuckerrüben & $73 / 131$ & $127 / 142$ & $201 / 211$ & $209 / 214$ & $175 / 199$ & $217 / 250$ & $229 / 240$ & $333 / 423$ \\
Kartoffeln & $111 / 155$ & $117 / 157$ & $89 / 128$ & $103 / 141$ & $79 / 137$ & $128 / 158$ & $162 / 180$ & $158 / 169$ \\
\hline $\begin{array}{l}\text { Weidelgras } \\
\text { Grünbrache* }\end{array}$ & 118 & 133 & 130 & 141 & 138 & 168 & 155 & 175 \\
Rotklee* & 52 & $21 / 23$ & $26 / 35$ & $37 / 39$ & $25 / 38$ & $29 / 33$ & $31 / 32$ & $24 / 36$ \\
\end{tabular}

Lys.-Gruppe = Lysimetergruppe; NStE = natürliche Standorteinheiten; *ohne mineralische Düngung

N-Saldo [kg/ha/Jahr]:

Auf Grundlage von N-Eintrag (nasse Deposition + mineralische Düngung) minus N-Entzug über die Pflanzen sind in Tab. 34 für die Fruchtfolge im Zeitraum von 1981-92, sowie für die Jahre 1993-95 (Gras/Klee mit Umbruch, ohne Düngung) mittlere N-Salden für die verschiedenen Böden zusammengestellt. 
Tab. 34: Mittlere N-Salden in [kg/ha/Jahr](Böden in Reihung nach abnehmender Sickerwasserleistung)

\begin{tabular}{|c|c|c|c|c|c|c|c|c|}
\hline $\begin{array}{c}\text { Lys.-Gruppe } \\
\text { NStE }\end{array}$ & $\begin{array}{c}5 \\
\text { D3 } \\
\end{array}$ & $\begin{array}{c}8 \\
\text { D3 } \\
\end{array}$ & $\begin{array}{c}4 \\
\text { D5 } \\
\end{array}$ & $\begin{array}{c}1 \\
\text { D6 } \\
\end{array}$ & $\begin{array}{c}7 \\
\text { D4 } \\
\end{array}$ & $\begin{array}{l}11 \\
\text { D3 } \\
\end{array}$ & $\begin{array}{c}9 \\
\text { Lö3 } \\
\end{array}$ & $\begin{array}{c}10 \\
\text { Lö1 } \\
\end{array}$ \\
\hline $\begin{array}{l}\text { der Jahre } \\
\text { 1981-92* } \\
\text { [kg/ha/Jahr] }\end{array}$ & 69 & 50 & 40 & 27 & 27 & 18 & -16 & -28 \\
\hline $\begin{array}{l}\text { der Jahre } \\
1993-94 * * \\
1995 * * \\
\text { [kg/ha/Jahr] }\end{array}$ & $\begin{array}{c}-4 \\
-39\end{array}$ & $\begin{array}{c}2 \\
-35\end{array}$ & $\begin{array}{c}-6 \\
-91\end{array}$ & $\begin{array}{l}-14 \\
-74\end{array}$ & $\begin{array}{c}-7 \\
-48\end{array}$ & $\begin{array}{c}-7 \\
-42\end{array}$ & $\begin{array}{c}-8 \\
-98\end{array}$ & $\begin{array}{c}-6 \\
-155\end{array}$ \\
\hline
\end{tabular}

NStE = natürliche Standorteinheiten; *Fruchtwechsel mit Düngung;

**Flächenstilllegung: Gras mit jährlichem Umbruch, ohne Düngung, 1995 Anbau von Leguminosen (Klee);

Die ermittelten N-Salden korrelieren eng mit den entsprechenden Frisch- bzw. Trockenmasseerträgen. Abb. 31 zeigt, wie unterschiedlich die N-Einträge von durchschnittlich 130 $\mathrm{kg} / \mathrm{ha} / \mathrm{Jahr}$ von den Pflanzen auf den verschiedenen Böden im Mittel der Jahre 1981-92 verwendet wurden. Bei hohen Erträgen auf den Lößböden (Lysimetergruppe 9 und 10) beanspruchten die Pflanzen den N-Speicher im Boden über die Düngegabe hinaus, beim Sand (Lysimetergruppe 5) mit deutlich geringeren Erträgen wurde dagegen nur die Hälfte der NEinträge genutzt. Die Grünbrache in den Jahren 1993-94 führte zu leicht negativen N-Salden. Die negativen N-Salden im Jahr 1995 mit Rotklee zeigen, dass infolge der Intensivbewirtschaftung von 1981-92 noch ein erheblicher N-Pool in den einzelnen Böden vorhanden war, wobei aber auch ein Anteil Luftstickstoff (N-Fixierung von Leguminosen) Bestandteil der Bilanz ist.

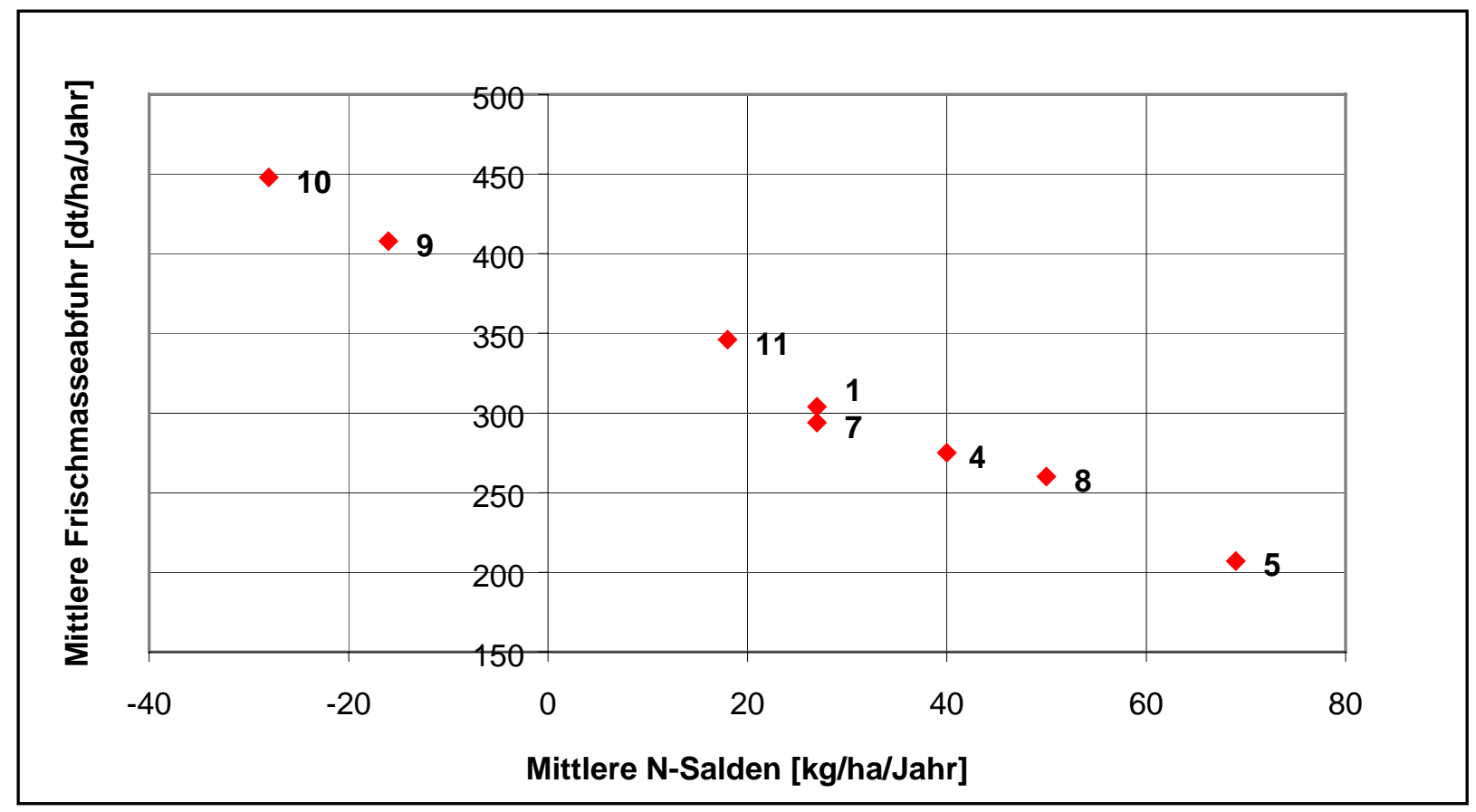

Abb. 31: Jahresmittel 1981-92 der Frischmasseabfuhr und N-Salden der verschiedenen Lysimetergruppen 
Je geringer die Pflanzenerträge, desto höher sind die von der Pflanze nicht genutzten NMengen, die eine Gefahr für das Grundwasser darstellen. Deshalb liefern alle ökologisch vertretbaren acker- und pflanzenbaulichen Massnahmen, die zu hohen Erträgen führen, einen Beitrag zur Verminderung von umweltbelastenden N-Austrägen. Von GUTSER \& HAUCK (1994) wird darauf verwiesen, dass eine N-Düngung, die wesentlich geringer ist als das standortgerechte Düngeniveau, einen N-Entzug bewirkt, der unter dem von optimal ernährten Pflanzen liegt und deshalb längerfristig $\mathrm{zu}$ Ertragsverlusten führt. Die Brandiser Versuchanstellung beinhaltete neben gleicher Bewirtschaftung aller Lysimeter auch die $\mathrm{N}$ Düngung aller Böden auf gleichem Niveau. Damit wurde die Düngung nicht der Ertragserwartung angepaßt. Im Rahmen künftiger Untersuchungen soll geprüft werden, ob durch bedarfsgerechte N-Gaben auf den leichten Böden (Lysimetergruppe 5, 4 und 8) bessere Erträge bei geringeren $\mathrm{N}-$ Salden zu erzielen sind.

\subsubsection{Einfluss der jährlichen Inanspruchnahme des Bodenwasservorrates auf die Verlagerungsgeschwindigkeit von Nitrat}

Die in unterschiedlicher Höhe ermittelten nicht genutzten N-Einträge und die durch Mineralisation (in unbekannter Höhe) im Boden freigesetzten N-Mengen können von den Bodenkolloiden sorbiert oder biologisch in organischer Substanz für einen längeren Zeitraum immobolisiert werden. Nur der in der Bodenlösung verbleibende N-Anteil (vorwiegend der leicht lösliche Nitrat-Stickstoff) kann mit dem Sickerwasser verlagert werden. Im nachfolgenden Kapitel wird das Verlagerungsrisiko der in Bodenlösung befindlichen N-Mengen ermittelt, wobei die - infolge der jährlich sehr variablen Inanspruchnahme des Bodenwasserspeichers durch die Verdunstung - unterschiedlich hohen Sickerwassermengen berücksichtigt werden.

Zunächst erfolgt eine Abschätzung der mittleren Austauschhäufigkeiten von Bodenlösung in der effektiven Wurzelzone auf Basis der Sickerwassermengen (Mittel der Jahre 1981-97) und des Wassergehaltes bei Feldkapazität in der (mittleren) effektiven Wurzelzone.

Bei dieser Abschätzung der Stoffverlagerung (Transport über Konvektion) am Beispiel von Nitrat in den verschiedenen Böden wird von folgenden Annahmen ausgegangen:

- Die Verlagerung des Sickerwassers erfolgt nach dem Verdrängungsprinzip, d. h., in den Boden eindringendes Wasser verdrängt das in darunterliegenden Bodenschichten befindliche Wasser um die Verlagerungsstrecke „VS“.

- Die gesamte im Profil bei Feldkapazität vorhandene Wassermenge nimmt an der Verlagerung teil.

- Direkter Landoberflächenwasserabfluß wird ausgeschlossen.

- Unterhalb der verdunstungsbeeinflußten Zone bleibt der Wassergehalt im Boden konstant.

Mit Hilfe der Austauschhäufigkeit (DVWK, 1994)

Gl. 16: $\quad$ Austauschhäufigkeit $[\% / J a h r]=\left(\right.$ Sickerwasser $\left.[\mathrm{mm} / \mathrm{Jahr}] / \mathrm{FK}_{\mathrm{We}}\right) * \mathbf{1 0 0}$

$\mathrm{FK}_{\mathrm{we}}=$ Feldkapazität im effektiven Wurzelraum [mm] 
wird abgeschätzt, wie häufig im Verlauf eines Jahres ein Austausch der Bodenlösung in der effektiven Wurzelzone erfolgt. Die $\mathrm{FK}_{\mathrm{we}}$ Tab. 35 wurden schichtweise auf Grundlage der in Kap. 2.1.4.2 dokumentierten Bodenkennwerte ermittelt.

Tab. 35: Mittlere Austauschhäufigkeit des Bodenwassers bei Feldkapazität im effektiven Wurzelraum [\%/Jahr] - Reihe 1981-97

\begin{tabular}{|c|c|c|c|c|c|c|}
\hline $\begin{array}{l}\text { Lys.- } \\
\text { Gruppe } \\
\text {-NStE }\end{array}$ & $\begin{array}{l}\mathrm{We}^{*} \\
{[\mathrm{dm}]}\end{array}$ & $\begin{array}{c}\mathrm{nFK}_{\mathrm{We}}{ }^{* *} \\
{[\mathrm{~mm}]}\end{array}$ & $\begin{array}{l}K_{W e} \\
{[\mathrm{~mm}]}\end{array}$ & $\begin{array}{c}\text { Sickerwasser } \\
{[\mathrm{mm} / \mathrm{Jahr}]}\end{array}$ & $\begin{array}{c}\text { Austausch- } \\
\text { häufigkeit } \\
\text { [\%/Jahr] }\end{array}$ & $\begin{array}{l}\text { Bewertung des } \\
\text { standstandörtlichen } \\
\text { Verlagerungsrisikos } * * *\end{array}$ \\
\hline $5-\mathrm{D} 3$ & 2,6 & 64 & 67 & 175 & 263 & groß \\
\hline $4-\mathrm{D} 5$ & 4,0 & 106 & 145 & 135 & 93 & gering \\
\hline $8-\mathrm{D} 3$ & 3,8 & 101 & 111 & 146 & 131 & mittel \\
\hline $1-\mathrm{D} 6$ & 5,1 & 117 & 196 & 113 & 58 & sehr gering \\
\hline $7-\mathrm{D} 4$ & 6,0 & 132 & 187 & 98 & 52 & sehr gering \\
\hline $11-\mathrm{D} 3$ & 6,5 & 113 & 141 & 114 & 81 & gering \\
\hline 9-Lö3 & 10,9 & 257 & 412 & 57 & 14 & äußerst gering \\
\hline 10 - Lö1 & 11,2 & 253 & 428 & 50 & 12 & äußerst gering \\
\hline
\end{tabular}

$* \mathrm{We}=$ mittlere Entnahmetiefe und $* * \mathrm{nFK}_{\mathrm{we}}=$ mittlere jährliche Bodenwasserentnahme jeweils auf Basis der Lysimetermessungen ermittelt (Kapitel 3.4.2); ***Bewertung nach DVWK (1994)

Liegen die Werte für die Austauschhäufigkeit über $100 \%$ (bei Lysimetergruppe 5 und 8), wird die Bodenlösung im Jahresverlauf vollständig ausgetauscht (d. h. im Fall der Lysimetergruppe 5 mehr als 2 mal). Bei der überwiegenden Anzahl der Böden ist der Austausch in Folge der Bodenwasserausschöpfung gering bis sehr gering, die Werte für die Ausstauschrate liegen deutlich unter $100 \%$.

Nach dem Verlassen der Wurzelzone erfolgt der Weitertransport des ausgewaschenen Nitrates in der Dränwasserzone (bis hin zum Grundwasser). Die Verlagerungsgeschwindigkeit wird abgeleitet aus:

$$
\text { Gl. 17: } \quad \text { VG }=\text { VS }=S W / \text { FK }_{\text {Drän. }}
$$

\section{[dm/Jahr]}

\begin{tabular}{|c|c|c|}
\hline VG & $=$ Verlagerungsgeschwindigkeit & {$[\mathrm{dm} / \mathrm{a}]$,} \\
\hline VS & $=$ jährliche Verlagerungsstrecke & {$[\mathrm{dm} / \mathrm{a}]$} \\
\hline $\mathrm{FK}_{\mathrm{I}}$ & $=$ Feldkapazität in der Dränwasserzone & {$[\mathrm{mm} / \mathrm{dm}]$} \\
\hline SW & $=$ Sickerwassermenge & {$[\mathrm{mm}$ oder $\mathrm{mm} / \mathrm{a}]$} \\
\hline
\end{tabular}

Die Dränzone der einzelnen Böden in den Lysimetern endet über der Kapillarwasserzone in ca. $3 \mathrm{~m}$ Tiefe. Ihre Mächtigkeit (Tab. 36 ist in diesem Fall von der mittleren effektiven Wurzeltiefe $\mathrm{We}_{\text {eff }}$ (Tab. 35) abhängig. Die Feldkapazität $\left(\mathrm{FK}_{\text {Drän. }}\right.$ ) in der Dränwasserzone

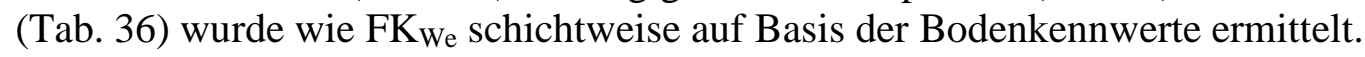

Aus Tab. 36 geht hervor, dass die durchschnittliche Verlagerungsgeschwindigkeit in der Dränwasserzone der Böden in Folge der geringen Sickerwassermengen nur bei Lysimetergruppe 5 und 1 als mittel, bei Lysimetergruppe 4, 8, 7 und 11 aber als gering und sehr gering bei Lysimetergruppe 7, 11, 9 und 10 einzuschätzen ist. So benötigt Sickerwasser, das im Lysimeter 10 die mittlere effektive Wurzelzone verlassen hat, für die Passage in der 18,8 dm mächtigen Dränwasserzone bis zum Lysimeterauslauf im langjährigen Mittel ca. 9 Jahre. 
Tab. 36: Durchschnittliche Verlagerungsgeschwindigkeit [dm/Jahr] und Verweilzeit des Sickerwassers in der Dränwasserzone der Lysimeter - Reihe 1981-97

\begin{tabular}{|c|c|c|c|c|c|}
\hline $\begin{array}{c}\text { Lys.- } \\
\text { Gruppe }\end{array}$ & $\begin{array}{l}\begin{array}{l}\text { Drän- } \\
\text { zone* }\end{array} \\
\text { [dm] }\end{array}$ & $\begin{array}{c}\text { FK }_{\text {Drän. }}{ }^{* *} \\
{[\mathrm{~mm}]} \\
\end{array}$ & $\begin{array}{c}\text { Sicker- } \\
\text { wasser } \\
\text { [mm/Jahr] }\end{array}$ & $\begin{array}{c}\text { Verlagerungs- } \\
\text { geschwindigkeit } \\
\text { in der Dränzone } \\
{[\mathrm{dm} / \mathrm{Jahr}]}\end{array}$ & $\begin{array}{l}\text { Verweilzeit } \\
\text { in der } \\
\text { Dränzone } \\
\text { [Jahre] } \\
\end{array}$ \\
\hline 5 & 27,4 & 351 & 175 & 13,6 & 2,0 \\
\hline 4 & 26,0 & 369 & 135 & 9,5 & 2,7 \\
\hline 8 & 26,2 & 566 & 146 & 6,8 & 3,9 \\
\hline 1 & 24,9 & 251 & 113 & 11,2 & 2,2 \\
\hline 7 & 24,0 & 538 & 98 & 4,4 & 5,5 \\
\hline 11 & 23,5 & 548 & 114 & 4,9 & 4,8 \\
\hline 9 & 19,1 & 481 & 57 & 2,3 & 8,3 \\
\hline 10 & 18,8 & 471 & 50 & 2,0 & 9,4 \\
\hline
\end{tabular}

Dränzone $=$ Lysimetertiefe $(3 \mathrm{~m})$ minus We aus Tab. 35

**schichtweise berechnete FK in der Dränwasserzone des Lysimeters

Die Ergebnisse in Tab. 35 und Tab. 36 verweisen überwiegend auf ein geringes bis sehr geringes standörtliches Verlagerungsrisiko der Böden. Dies gilt allerdings nicht für Einzeljahre. Eine hohe Ausschöpfung im Sommerhalbjahr reduziert die winterliche Sickerwasserhöhe, verzögert den jährlichen Beginn der Sickerwasserbildung und damit den Beginn der Nitratauswaschung aus der Wurzelzone. Diese Folgewirkungen treten in den Einzeljahren extrem in Erscheinung und werden insbesondere bei bindigen Böden durch die Aufeinanderfolge mehrerer Trockenjahre verstärkt. Im umgekehrten Fall bewirken hohe Niederschläge und eine geringe Ausschöpfung im Vorjahr bei den Böden mit geringer und mittlerer $\mathrm{nFK}_{\mathrm{We}}$ bzw. FK $\mathrm{We}_{\mathrm{W}}$ überdurchschnittlich hohe Sickerwasserraten und damit eine intensive Auswaschung aus der effektiven Wurzelzone (Tab. 37 und Tab. 38).

Nachfolgend wird am Beispiel von drei ausgewählten Lysimeterböden gezeigt, wie sich neben bewirtschaftungsbedingten Einflüssen vor allem witterungsbedingte Einflüsse auf das standörtliche Verlagerungsrisiko auswirken. Für die Berechnungen der jährlichen Austauschhäufigkeiten wurden die Angaben $\mathrm{nFK}_{\mathrm{we}}$ und FK aus Tab. 35 verwendet.

Die Berechnungen zeigen, je bindiger die Böden, d. h. je höher der maximal nutzbare Bodenwasservorrat, um so höher ist auch die mögliche Varianz des Verlagerungsrisikos in den Einzeljahren. Besonders hervorzuheben ist, dass in Einzeljahren auch die Geschiebelehmböden (Lysimetergruppe 7) ein mittleres bis großes Verlagerungsrisiko aufweisen.

Die unterschiedlichen Ergebnisse unter Austauschhäufigkeit (1) und (2) in Tab. 37 Tab. 38 und Tab. 39 berücksichtigen die Tatsache, dass den Berechnungen teils die $\mathrm{FK}_{\mathrm{We}}$ (DVWK, 1994) und teils die nFK $_{W e}$ (HÖLTING et al., 1995) zu Grunde liegt. Auf Grund des geringeren Fließquerschnittes bei Ansatz der $\mathrm{nFK}_{\mathrm{We}}$ treten in diesem Fall höhere Austauschhäufigkeiten auf (= schnellere Verlagerung der Bodenlösung). In Bezug auf den Anteil immobilen Wassers, das den Fließquerschnitt für den Wassertransport einschränkt, finden sich in der Literatur unterschiedliche Angaben. Am geringsten ist der Anteil vermutlich bei den Lößböden. So erzielte GERIES (1989) bei Untersuchungen an den südniedersächsischen Lößdecken gute Ergebnisse, ohne dass immobiles Wasser berücksichtigt wurde. 
Tab. 37: Austauschhäufigkeit des Bodenwassers bei Feldkapazität im effektiven Wurzelraum [\%/Jahr] am Beispiel der Lysimetergruppe 5 (D3) in den Jahren 1981-97

\begin{tabular}{|c|c|c|c|l|}
\hline Jahr & $\begin{array}{c}\text { Sicker- } \\
\text { wasser- } \\
\text { menge } \\
\text { [mm/Jahr] }\end{array}$ & $\begin{array}{c}\text { Austausch- } \\
\text { häufigkeit } \\
(1) \\
\text { [\%/Jahr] }\end{array}$ & $\begin{array}{c}\text { Austausch- } \\
\text { häufigkeit } \\
(2) \\
\text { [\%/Jahr] }\end{array}$ & \multicolumn{1}{|c|}{ Fruchtart } \\
\hline 1981 & 231 & 361 & 344 & Zuckerrüben \\
1982 & 163 & 255 & 243 & Winterweizen \\
1983 & 210 & 328 & 313 & Wintergerste \\
1984 & 96 & 150 & 143 & Weidelgras \\
1985 & 132 & 206 & 197 & Kartoffeln \\
1986 & 197 & 308 & 294 & Winterweizen \\
1987 & 268 & 419 & 400 & Kartoffeln \\
1988 & 213 & 333 & 318 & Winterweizen \\
1989 & 134 & 209 & 200 & Wintergerste \\
1990 & 100 & 156 & 149 & Zuckerrüben \\
1991 & 111 & 173 & 166 & Winterweizen \\
1992 & 138 & 216 & 206 & Wintergerste \\
1993 & 145 & 227 & 216 & Grünbrache \\
1994 & 381 & 595 & 568 & Grünbrache \\
1995 & 217 & 339 & 324 & Rotklee \\
1996 & 98 & 153 & 146 & Kartoffeln \\
1997 & 146 & 228 & 218 & Winterweizen \\
\hline Mittel & $\mathbf{1 7 5}$ & $\mathbf{2 7 3}$ & $\mathbf{2 6 1}$ & \\
\hline
\end{tabular}

(1) - Berechnung mit nFK $\quad$ (2) - Berechnung mit $\mathrm{FK}_{\mathrm{we}}$

Tab. 38: Austauschhäufigkeit des Bodenwassers bei Feldkapazität im effektiven Wurzelraum [\%/Jahr] am Beispiel der Lysimetergruppe 7 (D4) in den Jahren 1981-97

\begin{tabular}{|c|c|c|c|l|}
\hline Jahr & $\begin{array}{c}\text { Sicker- } \\
\text { wasser- } \\
\text { menge } \\
\text { [mm/Jahr] }\end{array}$ & $\begin{array}{c}\text { Austausch- } \\
\text { häufigkeit } \\
(1) \\
\text { [\%/Jahr] }\end{array}$ & $\begin{array}{c}\text { Austausch- } \\
\text { häufigkeit } \\
(2) \\
\text { [\%/Jahr] }\end{array}$ & Fruchtart \\
\hline 1981 & 135 & 102 & 72 & Zuckerrüben \\
1982 & 84 & 64 & 45 & Winterweizen \\
1983 & 70 & 53 & 37 & Wintergerste \\
1984 & 56 & 42 & 30 & Weidelgras \\
1985 & 67 & 51 & 36 & Kartoffeln \\
1986 & 104 & 79 & 56 & Winterweizen \\
1987 & 161 & 122 & 86 & Kartoffeln \\
1988 & 152 & 115 & 81 & Winterweizen \\
1989 & 55 & 42 & 29 & Wintergerste \\
1990 & 24 & 18 & 13 & Zuckerrüben \\
1991 & 34 & 26 & 18 & Winterweizen \\
1992 & 45 & 34 & 24 & Wintergerste \\
1993 & 55 & 42 & 29 & Grünbrache \\
1994 & 269 & 204 & 144 & Grünbrache \\
1995 & 191 & 145 & 102 & Rotklee \\
1996 & 65 & 49 & 35 & Kartoffeln \\
1997 & 101 & 77 & 54 & Winterweizen \\
\hline Mittel & $\mathbf{9 8}$ & $\mathbf{7 4}$ & $\mathbf{5 2}$ & \\
\hline
\end{tabular}

(1) - Berechnung mit $\mathrm{nFK}_{\mathrm{we}} \quad$ (2) - Berechnung mit $\mathrm{FK}_{\mathrm{we}}$ 
Tab. 39: Austauschhäufigkeit des Bodenwassers bei Feldkapazität im effektiven Wurzelraum [\%/Jahr] am Beispiel der Lysimetergruppe 9 (Lö3) in den Jahren 1981-97

\begin{tabular}{|c|c|c|c|l|}
\hline Jahr & $\begin{array}{c}\text { Sicker- } \\
\text { wasser- } \\
\text { menge } \\
\text { [mm/Jahr] }\end{array}$ & $\begin{array}{c}\text { Austausch- } \\
\text { häufigkeit } \\
(1) \\
\text { [\%/Jahr] }\end{array}$ & $\begin{array}{c}\text { Austausch- } \\
\text { häufigkeit } \\
(2) \\
\text { [\%/Jahr] }\end{array}$ & \multicolumn{1}{|c|}{ Fruchtart } \\
\hline 1981 & 174 & 68 & 42 & Zuckerrüben \\
1982 & 5 & 2 & 1 & Winterweizen \\
1983 & 0 & 0 & 0 & Wintergerste \\
1984 & 2 & 1 & 1 & Weidelgras \\
1985 & 60 & 23 & 15 & Kartoffeln \\
1086 & 99 & 39 & 24 & Winterweizen \\
1987 & 162 & 63 & 39 & Kartoffeln \\
1988 & 184 & 72 & 45 & Winterweizen \\
1989 & 0 & 0 & 0 & Wintergerste \\
1990 & 0 & 0 & 0 & Zuckerrüben \\
1991 & 0 & 0 & 0 & Winterweizen \\
1992 & 0 & 0 & 0 & Wintergerste \\
1993 & 0 & 0 & 0 & Grünbrache \\
1994 & 134 & 52 & 33 & Grünbrache \\
1995 & 144 & 56 & 35 & Rotklee \\
1996 & 0 & 0 & 0 & Kartoffeln \\
1997 & 2 & 1 & 1 & Winterweizen \\
\hline Mittel & $\mathbf{5 7}$ & $\mathbf{2 2}$ & $\mathbf{1 4}$ & \\
\hline
\end{tabular}

(1) - Berechnung mit $\mathrm{nFK}_{\mathrm{we}} \quad$ (2) - Berechnung mit $\mathrm{FK}_{\mathrm{we}}$

Die Sand- und Geschiebeböden lassen eher vermuten, dass der Wassertransport (in Abhängigkeit von der Höhe der Sickerwassermengen) nicht ausschließlich nach dem Verdrängungsprinzip stattfindet. In den stark strukturierten Böden führen präferentielle Flüsse durchaus zu höheren Transportraten als mit den vorgenommenen Abschätzungen ausgewiesen wird (RUSSOW et al., 1996). Mit dem Einfluss möglicher Makroporen begründen HÖLTING et al. (1995) die mittels $\mathrm{nFK}_{\mathrm{We}}$,eher zurückhaltende Bewertung der Schutzfunktion des Bodens“.

\subsubsection{N-Saldo und N-Austrag über das Sickerwasser}

\section{a) Einzeljahre}

Mit Kenntnis der Dynamik des Wasserumsatzes in der Wurzelzone und der langen Verweilzeit der Bodenlösung in der Dränzone wird erklärlich, warum für einzelne Jahre kein unmittelbarer Zusammenhang zwischen N-Saldo und der Höhe der N-Austräge zu erkennen ist (vgl. Abb. 33), ein Ergebnis, auf das bereits u. a. in Arbeiten von EULENSTEIN \& WURBS (1994) und RICHTER et al. (1994) hingewiesen wird. Auf Grund der durchschnittlichen Verweilzeit des Sickerwassers in der Dränzone von ca. 2 Jahren werden selbst auf dem Sandstandort (Lysimetergruppe 5) N-Überhänge nicht im Jahr ihrer Entstehung bis in eine Tiefe von $3 \mathrm{~m}$ Tiefe ausgewaschen.

Etwas deutlicher ist der Zusammenhang zwischen mittlerer jährlicher Nitrat-Konzentration und Sickerwassermenge Abb. 34 - ein Hinweis darauf, dass die Aussagekraft von jährlichen $\mathrm{N}$-Salden wesentlich von den aktuellen hydrologischen Bedingungen beeinflusst wird. Von den Pflanzen nicht genutzte N-Mengen werden bei unvollständigem Austausch der Bodenlösung im Wurzelraum und/oder stagnierendem Transport in der Dränwasserzone akkumuliert. So ist zu erklären, dass mit steigender Sickerwassermenge bei den Böden mit geringer und mittlerer $\mathrm{nFK}_{\mathrm{We}}$ (Lysimetergruppe 5 und 7) die Nitrat-N-Konzentration 
tendenziell zunimmt. Bei den Löß-Parabraunerden (Lysimetergruppe 9) ist der Zusammenhang auf Grund der geringen Austauschraten nicht so deutlich.

Vor ihrer Entnahme unterlagen die Lysimeterböden auf den Herkunftsflächen anderen Bewirtschaftungsformen als in Brandis. Auf den Herkunftsflächen wurde auch organischer Dünger verwendet und das Niveau der mineralischen Düngung auf den Standort und die Ertragserwartung ausgerichtet. Aus dieser „Erblast“ resultieren die hohen Konzentrationen der Jahre 1981 und 1982 (Abb. 34). Die geringen Konzentrationen in den Jahren 1994-95 sind dagegen bereits eine Folge des Bewirtschaftungswechsels (KNAPPE et al., 1997) im Jahr 1992.

\section{b) Im Mittel der Jahre 1981-92}

Für die Jahre 1981-92 ergeben sich in Abhängigkeit von Boden und Bewirtschaftung mittlere jährliche N-Austräge, die zwischen $7 \mathrm{~kg} / \mathrm{ha}$ auf den ertragreichen, sorptionsstarken LößParabraunerden (Lysimetergruppe 9) und $48 \mathrm{~kg} / \mathrm{ha}$ auf den weniger ertragreichen sandigen Braunerden (Lysimetergruppe 5) liegen (Tab. 40.

Tab. 40: $N$-Auswaschungsverluste über das Sickerwasser in 3m Tiefe [kg/ha/Jahr](Reihung nach abnehmender Sickerwasserleistung)

\begin{tabular}{|l|c|c|c|c|c|c|c|c|}
\hline $\begin{array}{l}\text { Lys.-Gruppe } \\
\text { NStE }\end{array}$ & $\begin{array}{c}\mathbf{5} \\
\mathbf{D 3}\end{array}$ & $\begin{array}{c}\mathbf{8} \\
\mathbf{D 3}\end{array}$ & $\begin{array}{c}\mathbf{4} \\
\mathbf{D 5}\end{array}$ & $\begin{array}{c}\mathbf{1} \\
\text { D6 }\end{array}$ & $\begin{array}{c}\mathbf{7} \\
\mathbf{D 4}\end{array}$ & $\begin{array}{c}\mathbf{1 1} \\
\mathbf{D 3}\end{array}$ & $\begin{array}{c}\mathbf{9} \\
\text { Lo3 }\end{array}$ & $\begin{array}{c}\mathbf{1 0} \\
\text { Lö1 }\end{array}$ \\
\hline $\begin{array}{l}\text { der Jahre 1981- } \\
92 * * \\
{[\mathrm{~kg} / \mathrm{ha} / \mathrm{Jahr}]}\end{array}$ & 48 & 32 & 40 & 33 & 18 & 41 & 7 & $(55)$ \\
\hline $\begin{array}{l}\text { der Jahre 1993- } \\
\begin{array}{l}95^{* * *} \\
{[\mathrm{~kg} / \mathrm{ha} / \mathrm{Jahr}]}\end{array}\end{array}$ & 22 & 15 & 26 & 18 & 3 & 32 & 2 & 7 \\
\hline
\end{tabular}

*wurden auch organisch gedüngt; **Fruchtfolge mit Düngung; ***Gras mit Umbruch ohne Düngung

Wie Abb. 32 hervorgeht, zeigen die Brandiser Untersuchungen auch im Mittel von 12 Untersuchungsjahren (1981-92) nur einen losen Zusammenhang zwischen N-Saldo und NAustrag über das Sickerwasser. Erwartungsgemäß sollte eine enge Korrelation zwischen Boden $\left(\mathrm{nFK}_{\mathrm{We}}\right)$, N-Saldo und N-Austrag über das Sickerwasser bestehen. So sichern die Böden mit großem Bodenwasserspeicher (und hohen Vorräten an pflanzenverfügbaren Nährstoffen) ein gutes Pflanzenwachstum und damit hohe Erträge. Diese bewirken eine effiziente Ausnutzung der N-Gehalte im Boden Tab. 34 und damit geringe NBilanzüberschüsse, im Fall der Lößböden überwiegen sogar die Entzüge.

Für jeden Boden bildete sich im Mittel über die Jahre 1981-92 ein von der Fruchtbarkeit abhängiges Niveau der Nitratgehalte im Sickerwasser heraus. Die Nitrat-N-Konzentrationen der Lysimetergruppen 4, 8, 1 liegen mit $\approx 100 \mathrm{mg} / \mathrm{l} \mathrm{NO}$ auf vergleichbarem Niveau zur Lysimetergruppe 5. Der Braunerde-Pseudogley (Lysimetergruppe 7) zeigt geringere NGehalte im Sickerwasser $(70 \mathrm{mg} / \mathrm{l} \mathrm{NO}$ ). Das wird als Folge der Neigung dieses Bodens zu anaeroben Bedingungen und den daraus resultierenden Denitrifikationsverlusten interpretiert. Die N-Gehalte der Lysimetergruppe 11 (pseudovergleyte Braunerde-Fahlerde) sind infolge der nur auf diesen Lysimetern erfolgten organischen Düngung vergleichsweise sehr hoch (170 $\mathrm{mg} / \mathrm{l} \mathrm{NO} 3$ ). Die fruchtbaren Lößböden (Lysimetergruppe 9) weisen von allen hier untersuchten Böden die geringsten Gehalte auf $\left(60 \mathrm{mg} / \mathrm{l} \mathrm{NO} \mathrm{NO}_{3}\right)$ und scheinen auch intensive landwirtschaftliche Bewirtschaftung relativ gut zu puffern (KNAPPE et al., 1994). 


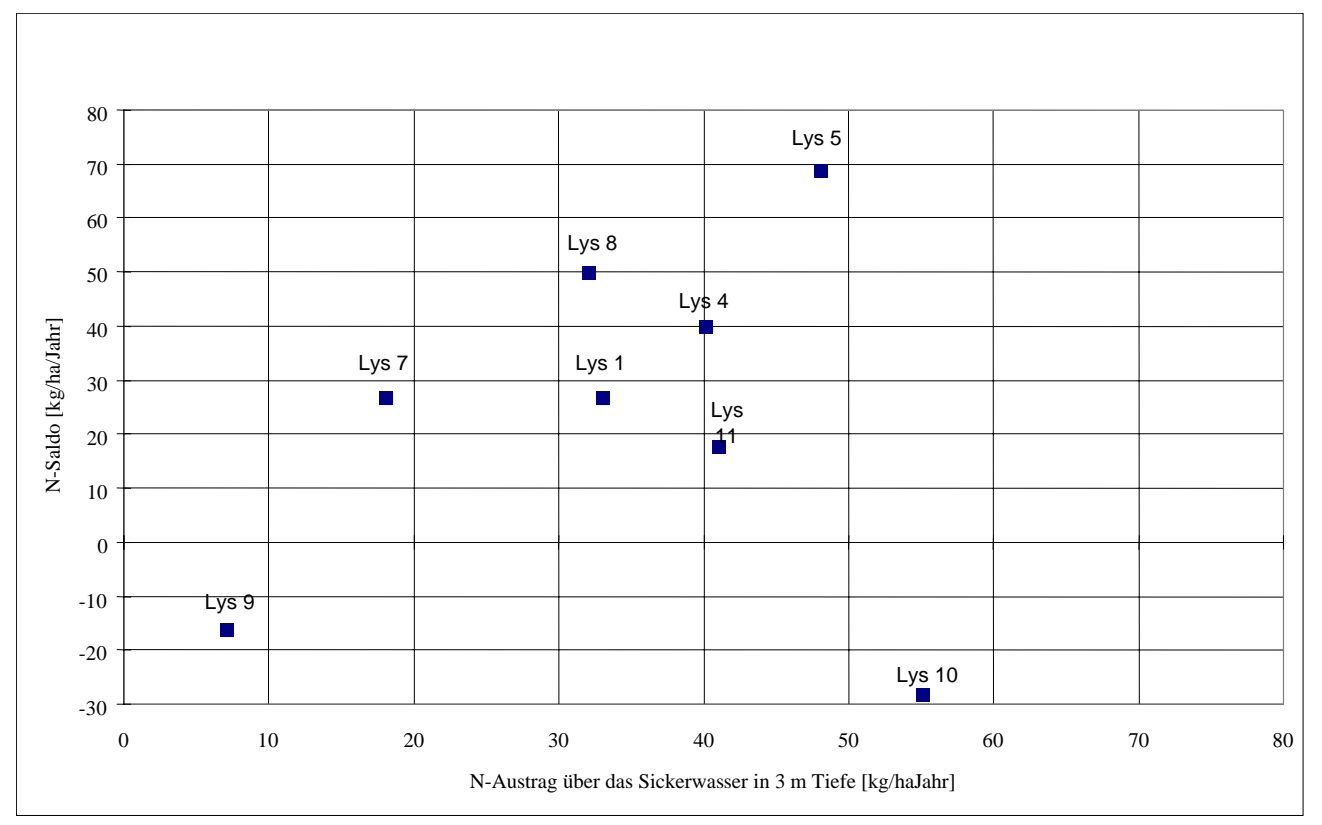

Abb. 32: Jahresmittel 1981-92 der N-Salden und der N-Austräge über das Sickerwasser der verschiedenen Lysimetergruppen ( $s$. Tab. 33

In Bezug auf das ermittelte N-Saldo zeigen insbesondere die Böden der Lysimetergruppen 10 und 11 sehr hohe N-Austräge. Ursache dafür sind folgende Umstände:

- Die vergleichsweise hohen N-Gehalte und N-Austräge Abb. 32 der pseudovergleyte Braunerde-Fahlerde (Lysimetergruppe 11) aus Wittenberg resultieren aus einer organischen Düngung in Form von Rinder- oder Schweinegülle, die von 1982 bis 1990 jährlich nur auf dieser Lysimetergruppe erfolgte. Dabei wurde die Düngermenge in Mineraldüngeäquivalenten der jährlichen Mineraldüngung der anderen Lysimeterböden angeglichen.

- Bei den trotz negativer N-Salden sehr hohen N-Austrägen (55 kg/ha/Jahr) der ertragreichen Schwarzerde (Lysimetergruppe 10) muß davon ausgegangen werden, dass unter den gegebenen Verhältnissen (rd. $100 \mathrm{~mm} / \mathrm{Jahr}$ mehr Niederschlag in Brandis als am Herkunftsort Etzdorf) eine erhebliche Mineralisation organisch gebundenen Boden-Stickstoffs stattfindet (KÖRSCHENS \& MAHN, 1995). Da der hohe N-Austrag der Schwarzerde aus diesem Grund keine standorttypische Reaktion beschreibt, wurde dieser Wert in (Tab. 40) in Klammern gesetzt.

Die Dynamik des monatlichen N-Austrages ist eng mit dem Jahresgang der Sickerwasserbildung Tab. 30 gekoppelt. In den leichten Sandböden (Lysimetergruppe 5) ist die winterliche Wiederauffüllung des Bodenwasservorrates relativ schnell erreicht, so dass in der Regel bereits im Januar ein Maximum der jährlichen Sickerwasserperiode erreicht wird. In gleicher Weise zeigt sich auch die innerjährliche Verteilung der monatlich ausgewaschenen N-Mengen. Die größte N-Menge wird im Mittel der Jahre 1981-92 demzufolge im Januar mit durchschnittlich $10 \mathrm{~kg} / \mathrm{ha}$ ausgewaschen. Beim Geschiebelehm (Lysimetergruppe 7) wird die größte monatliche N-Menge im Durchschnitt der Jahre im März ausgetragen. Für den Lößstandort (Lysimetergruppe 9) liegt das Maximum der Sickerwasser-Periode ebenfalls im März. Auf Grund der sehr geringen N-Konzentrationen im Sickerwasser ist das Maximum der $\mathrm{N}$-Auswaschung mit durchschnittlich 2,2 kg/ha aber erst im Mai zu verzeichnen. 

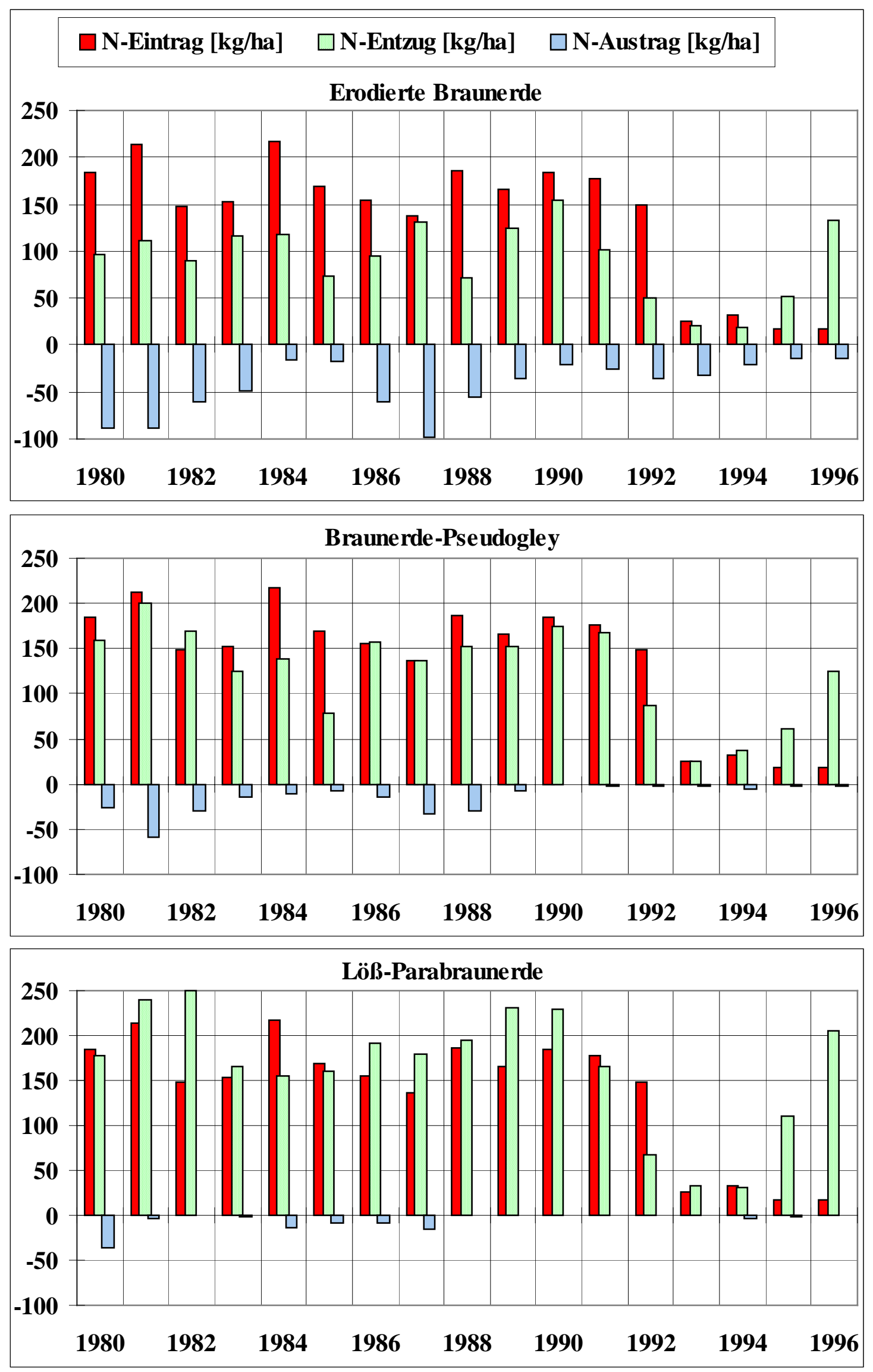

Abb. 33: Gegenüberstellung von N-Eintrag, N-Entzug und N-Austrag am Beispiel von ausgewählten Lysimeterböden (Reihe 1980-96) 

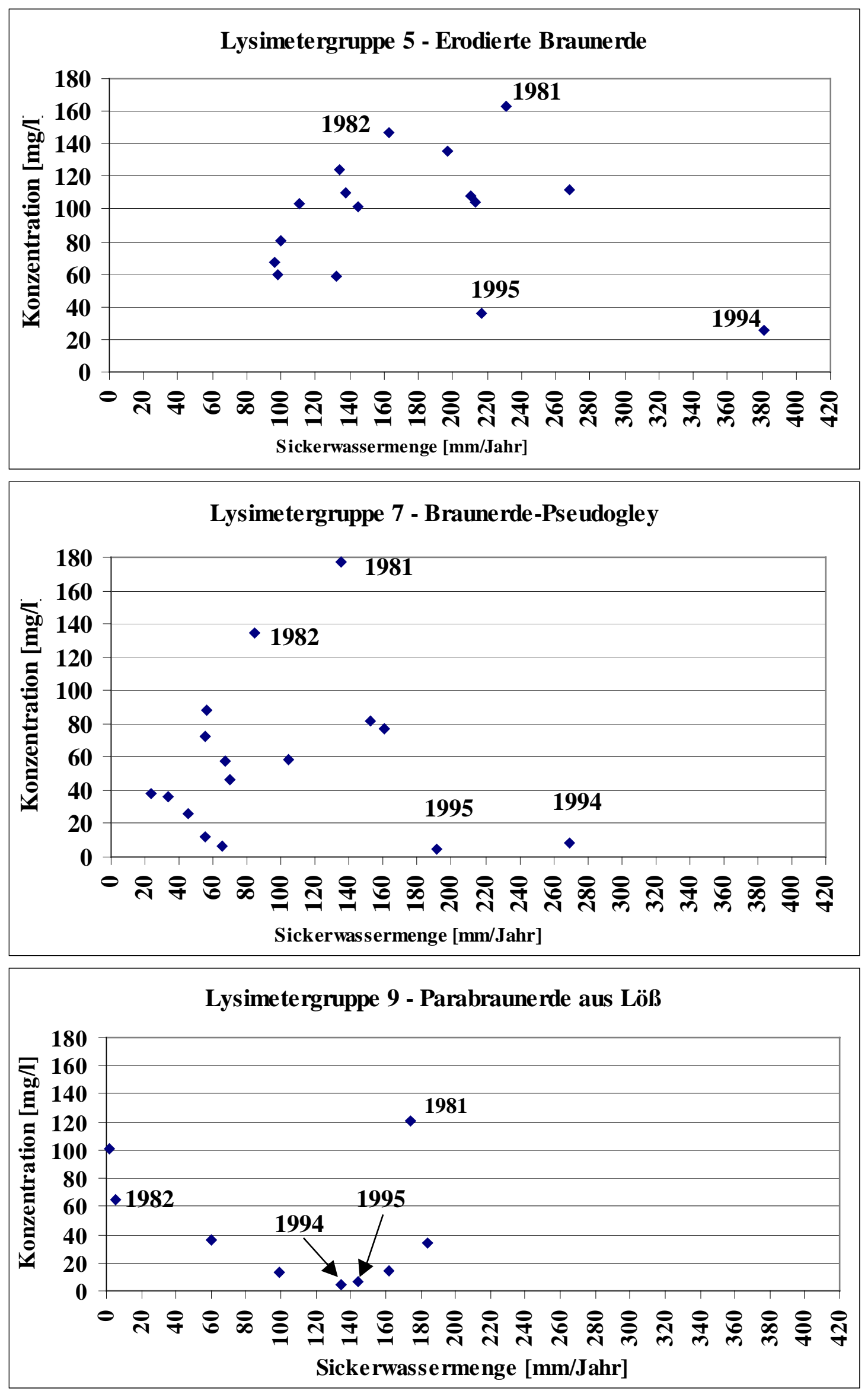

Abb. 34: Nitratkonzentration im Sickerwasser (Jahresmittelwerte) in Abhängigkeit von der Sickerwassermenge 


\subsubsection{Diskussion der Ergebnisse}

Ziel einer grundwasserschonenden Landwirtschaftung sollten möglichst geringe N-Salden sein. Bei der vereinfachten N-Bilanzmethode, wie sie hier zur Ermittlung der N-Salden zur Anwendung kam, werden wesentliche Stickstoffkomponenten und Umwandlungsprozesse nicht erfasst.

So ist damit $\mathrm{zu}$ rechnen, dass im Fall eines positiven N-Saldo nicht der gesamte NÜberschuss die durchwurzelte Zone mit dem Sickerwasser verläßt. Denitrifikation und $\mathrm{NH}_{3}-$ Verflüchtigung mit N-Abgaben an die Atmosphäre führen zu Verlusten (s. BraunerdePseudogley - Lysimetergruppe 7). N-Überschüsse können durch Immobilisation in Form organischer N-Bindung reduziert oder durch Mineralisation organischer N-Vorräte aus dem Boden (s. Lysimetergruppe 11 - pseudovergleyte Braunerde-Fahlerde) erhöht werden.

Andererseits deutet ein negativer $\mathbf{N}$-Saldo lediglich auf eine verhältnismäßig geringe Gefährdung hin, jedoch nicht auf das Fehlen jeglicher N-Auswaschung, wie am Beispiel der Lößböden gezeigt wird. Von Nachteil ist weiterhin, dass auch die N-Fixierung von Leguminosen bei der N-Bilanz-Methode meist vernachlässigt wird (hier im Jahr 1995 mit Gras/Klee).

Tendenziell erhöht sich mit steigenden Bilanzüberschüssen der N-Austrag. Im Mittel der Periode intensiver landwirtschaftlicher Bewirtschaftung stehen bei der flachgründigen Braunerde (geringste Wasserspeicherkapazität), einem 12jährigen N-Saldo (1981-92) von 69 $\mathrm{kg} / \mathrm{ha} * \mathrm{a}$ ein mittlerer Nitratgehalt von $100 \mathrm{mg} / \mathrm{l}$ im Sickerwasser und ein mittlerer N-Austrag von $48 \mathrm{~kg} / \mathrm{ha} * \mathrm{a}$ gegenüber. Dagegen kommen bei den Löß-Parabraunerden (in den seltenen Perioden mit Sickerwasserbildung) $7 \mathrm{~kg} / \mathrm{ha}{ }^{*}$ a zum Austrag, bei einem N-Saldo von -16 $\mathrm{kg} / \mathrm{ha}$ *a und einem mittleren Nitratgehalt von $60 \mathrm{mg} / \mathrm{l}$. Damit wird selbst bei den Lößböden (bei ausschließlich mineralischer Düngung) die 1t. E.G.-Trinkwasserrichtlinie zulässige Höchstkonzentration von $50 \mathrm{mg} \mathrm{NO} / 1$ überschritten.

Permanenter Wassermangel führt auf den leichten Böden zu geringen Erträgen und hohen Bilanzüberschüssen, d. h. je höher die von der Pflanze nicht genutzten N-Restmengen im Boden, um so höher sind auch die N-Austräge. Die ausreichend mit Wasser versorgten LößParabraunerden ermöglichen „Höchsterträge“ und dementsprechende N-Entzüge. Das negative N-Saldo verweist auf erhebliche N-Vorräte im Unterboden, die bei tiefreichender Bodenwasserauschöpfung von den Pflanzen auch genutzt werden.

Von entscheidendem Einfluss auf die N-Verlagerung ist die Sickerwasserbildung. An Hand der überwiegend geringen Austauschhäufigkeiten und der geringen Verlagerungsgeschwindigkeit von Bodenlösung, konnte für alle Böden der mangelnde Zusammenhang zwischen jährlichem N-Saldo und N-Austrag begründet werden. Deutliche Hinweise auf die zeitweise Akkumulation von N-Bilanzüberschüssen in den Böden liefert die Korrelation zwischen Sickerwassermenge und Nitratkonzentration. Bei hohen Sickerwasserraten kommt es nicht $\mathrm{zu}$ einem Verdünnungseffekt, sondern $\mathrm{zu}$ einem verstärkten Austrag von akkumuliertem Stickstoff aus der Wurzelzone. Dieser Effekt ist standortabhängig (Höhe der N-Salden, Sickerwassermenge und Austauschhäufigkeit der Bodenlösung) und resultiert bei (im Mittel der Jahre) vorwiegend geringen Sickerwasserraten aus der Abfolge von Trockenund Feuchtjahren. Trockenjahre sind Jahre der Akkumulation, Feuchtjahre führen zu erhöhtem N-Austrag. Auch KNOBLAUCH et al. (1999) verweisen im Ergebnis ihrer Untersuchungen auf unterschiedlichen Ackerstandorten Thüringens darauf, dass ein Zusammenhang zwischen N-Saldo, Nitratgehalt im Sickerwasser und N-Austrag nur über mehr- und langjährige Messreihen eindeutig erkennbar ist. Nach ihrer Meinung sollte es auf Standorten mit geringen Austauschraten möglich sein, N-Saldo-Überhänge im Folgejahr durch empfehlungskonforme N-Düngung wieder abzubauen, um damit zur Verminderung des $\mathrm{N}$-Verlagerungsrisikos beizutragen. 


\section{Zur Repräsentanz der Lysimeteruntersuchungen}

\subsection{Untersuchungen zur räumlichen Repräsentanz}

\subsubsection{Hydrologische Wirksamkeit verschiedener Böden}

Witterung und Bewirtschaftung (Landnutzung) waren im „Versuchsaufbau“ der Station Brandis für alle acht Böden (Standorte) gleich. Damit waren günstige Voraussetzungen für einen Vergleich der „Reaktionen“ der verschiedenen Böden, messbar an den Wasserhaushaltsgrößen Sickerwasserbildung und Verdunstung, gegeben. Untersucht wurde der Einfluss (= hydrologische Wirksamkeit) der verschiedenen Böden auf die Verdunstung und Sickerwasserbildung, wobei der Begriff der „hydrologischen Wirksamkeit“ hier im Sinne der Pedo-Hydrotopen (Kap. 2.3.6) auch den Anteil des Wasserhaushaltes an der Bodenfruchtbarkeit mit einschließt.

Ausgangspunkt für die in Kap. 2.3.6 beschriebene Varianzanalyse war eine Zusammenstellung der Jahresmittelwerte ausgewählter Untersuchungsergebnisse (Tab. 41). Dabei zeigte sich erwartungsgemäß eine Rangfolge der Böden entsprechend der Höhe ihrer Versickerungsleistung, wobei sich aber die Einzellysimeter nicht immer entsprechend ihrer Zugehörigkeit zu den Lysimetergruppen (Herkunftsflächen) einordneten.

Tab. 41: Nach Jahresmittelwerten der Sickerwasserbildung (SW) geordnete Angaben zur Verdunstung, zu Trockenmasse-Erträgen, Stickstoffentzügen durch die Pflanze und Höhe der Stickstoffausträge über das Sickerwasser der Einzellysimeter (Abflussjahre 1981-94)

\begin{tabular}{|c|c|c|c|c|c|}
\hline $\begin{array}{c}\text { Lysimeter } \\
\text { Nr. }\end{array}$ & $\begin{array}{c}\text { SW } \\
{[\mathrm{mm} / \mathbf{J a h r}]}\end{array}$ & $\begin{array}{c}\text { Verdunstung } \\
\text { [mm/Jahr] }\end{array}$ & $\begin{array}{c}\text { Trockenmasse } \\
\text { [dt/ha/Jahr] }\end{array}$ & $\begin{array}{c}\text { N-Entzug } \\
{[\mathrm{kg} / \mathrm{ha} / J a h r]}\end{array}$ & $\begin{array}{c}\text { N-Austrag } \\
{[\mathrm{kg} / \mathrm{ha} / J a h r]}\end{array}$ \\
\hline $10 / 2 *$ & 47 & 614 & 126 & 182 & 31 \\
\hline $7 / 6 *$ & 51 & 605 & 107 & 134 & 9 \\
\hline $10 / 3$ & 57 & 609 & 142 & 186 & 76 \\
\hline $10 / 1$ & 58 & 612 & 140 & 172 & 35 \\
\hline $9 / 1$ & 62 & 619 & 139 & 168 & 3 \\
\hline $9 / 3$ & 65 & 616 & 139 & 165 & 4 \\
\hline $9 / 2$ & 66 & 619 & 134 & 172 & 11 \\
\hline $11 / 2$ & 111 & 558 & 106 & 129 & 42 \\
\hline $1 / 7$ & 116 & 562 & 106 & 131 & 21 \\
\hline $1 / 6$ & 116 & 559 & 114 & 133 & 29 \\
\hline $7 / 5$ & 118 & 552 & 100 & 127 & 17 \\
\hline $7 / 4$ & 120 & 557 & 103 & 131 & 20 \\
\hline $11 / 1$ & 122 & 553 & 106 & 136 & 40 \\
\hline $11 / 3$ & 131 & 544 & 118 & 149 & 32 \\
\hline $1 / 5$ & 134 & 542 & 92 & 123 & 44 \\
\hline $4 / 8$ & 142 & $\overline{530}$ & 95 & 120 & $\overline{38}$ \\
\hline $4 / 6$ & 146 & 529 & 91 & 116 & 43 \\
\hline $4 / 7$ & 146 & 531 & 92 & 114 & 37 \\
\hline $8 / 5$ & 148 & 538 & 100 & 112 & 26 \\
\hline $8 / 4$ & 160 & 517 & 83 & 107 & 35 \\
\hline $8 / 6$ & 160 & 529 & 92 & 110 & 28 \\
\hline $5 / 4 *$ & 162 & 493 & $\overline{79}$ & 106 & 34 \\
\hline $5 / 5$ & 184 & 486 & 63 & 86 & 48 \\
\hline $5 / 6$ & 198 & 478 & 57 & 83 & 49 \\
\hline
\end{tabular}

*Diese Lysimeter wurden im September 1994 zerlegt. 
Das die Meßergebnisse einzelner Lysimeter als „Ausreißer“, wie im Fall der Lysimeter 7/6 und 5/4 nicht auf Geräte- oder Meßfehlern beruhen, zeigen die Untersuchungsergebnisse der Lysimeterzerlegung (KEESE \& KNAPPE, 1996). Der abweichende Verlauf der Messreihen dieser Lysimeter ist ausschließlich eine Folge der Varianz der bodenphysikalischen Eigenschaften der Herkunftsflächen.

Die geringsten Unterschiede in der Höhe von Sickerwasserbildung und Verdunstung zeigen erwartungsgemäß die Lößböden (Herkunftsflächen = Lysimetergruppe 9 und 10).

Auch die Rangfolge der Lysimeter der Herkunftsflächen 4, 8 und 5 zeigt an, dass die flächenhafte Heterogenität der Böden durch die gewählte Querschnittsfläche der Lysimeter voll erfasst wird. Die Unterschiede in den Jahresmittelwerten der Sickerwasserbildung der Einzellysimeter verweisen jedoch darauf, dass die Schmelzwassersande der Elster- und SaaleKaltzeit aufgrund ihrer glazifluviatilen Entstehung zu starken Inhomogenitäten neigen. Kornform und Korngröße variieren erheblich und damit auch auf die Porosität und Durchlässigkeit.

Noch extremer sind Inhomogenitäten bei den Geschiebemergeln und -lehmen der Grundmoräne ausgebildet. Diese zeigen sowohl hinsichtlich der primären (Korngröße von tonigschluffig bis sandig) als auch hinsichtlich der sekundären Durchlässigkeit (Klüftigkeit aufgrund glazigener Überprägung) große Unterschiede. Infolge dessen treten bei den Lysimetergruppen 1, 7 und 11 "Ausreißer" auf. Dies ist ein Hinweis auf großräumige Strukturelementen, die mittels einzelner Lysimeter nur teilweise und sehr unterschiedlich erfasst werden (z. B. Eiskeile, Sandbänder).

Der Zusammenhang zwischen der Heterogenität der Böden und der geologischen Vorgänge, die zur Ablagerung der Ausgangsgesteine geführt haben, zeigt sich in der Spannbreite der Sickerwasserbildung. So bewirken die äolischen Sedimente bedeutend geringere Varianzen als die glazigenen Bildungen.

Auf der Grundlage der Rangfolge in Tab. 41 sowie eines Vergleiches der Zeitreihen von Monatswerten der Sickerwasserbildung und Verdunstung unter Berücksichtigung der bodenkundlichen und hydrogeologischen Beschreibung der acht untersuchten Herkunftsorte, erfolgte eine Zusammenfassung der acht Böden (Lysimetergruppen) zu vier Versickerungstypen im Sinne von Pedo-Hydrotopen (s. Kap. 2.3.6). Das Ergebnis der Varianzanalyse (Tab. 42) bestätigt die vorgenommene Zusammenfassung.

Tab. 42: Anzahl der Monate mit signifikanten Unterschieden der Verdunstung und Sickerwasserbildung zwischen den Lysimetergruppen (Ergebnisse der Varianzanalyse von 168 Monaten je Einzellysimeter der Jahre 1981-94)

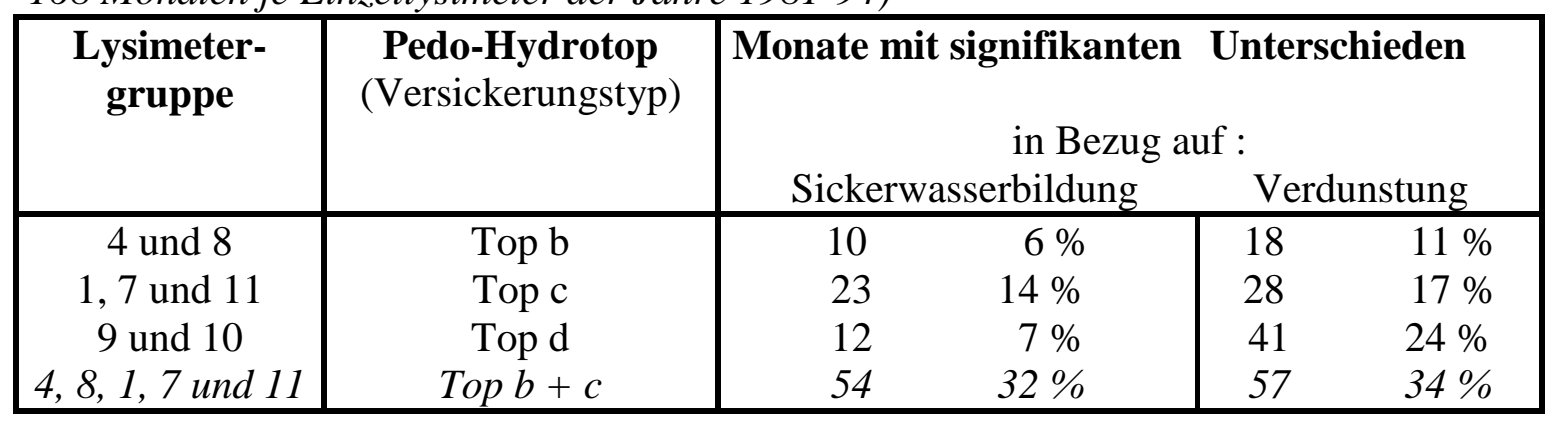

Aus der im Verhältnis geringen Anzahl abgelehnter Monate und der Tatsache, dass es sich dabei überwiegend um Monate mit sehr geringer Sickerwasserbildung handelt, wird die Berechtigung zur Bildung der vier Pedo-Hydrotopen (Tope) abgeleitet. Eine Zusammenfassung der Tope „, b“ und „,c“ wurde verworfen. Tab. 43 liefert eine erste Beschreibung der mit „Tope a bis d“ bezeichneten Versickerungstypen. 
Tab. 43: Langjährige Jahresmittelwerte der Sickerwasserbildung (SW), der realen Verdunstung (ETR), der Trockenmasse-Erträge (TM), Stickstoffentzüge durch die Pflanze (NEntzug) und die Höhe der Stickstoffausträge über das Sickerwasser (N-Austrag) der Versickerungstypen (Wasserhaushaltsjahre 1981-94)

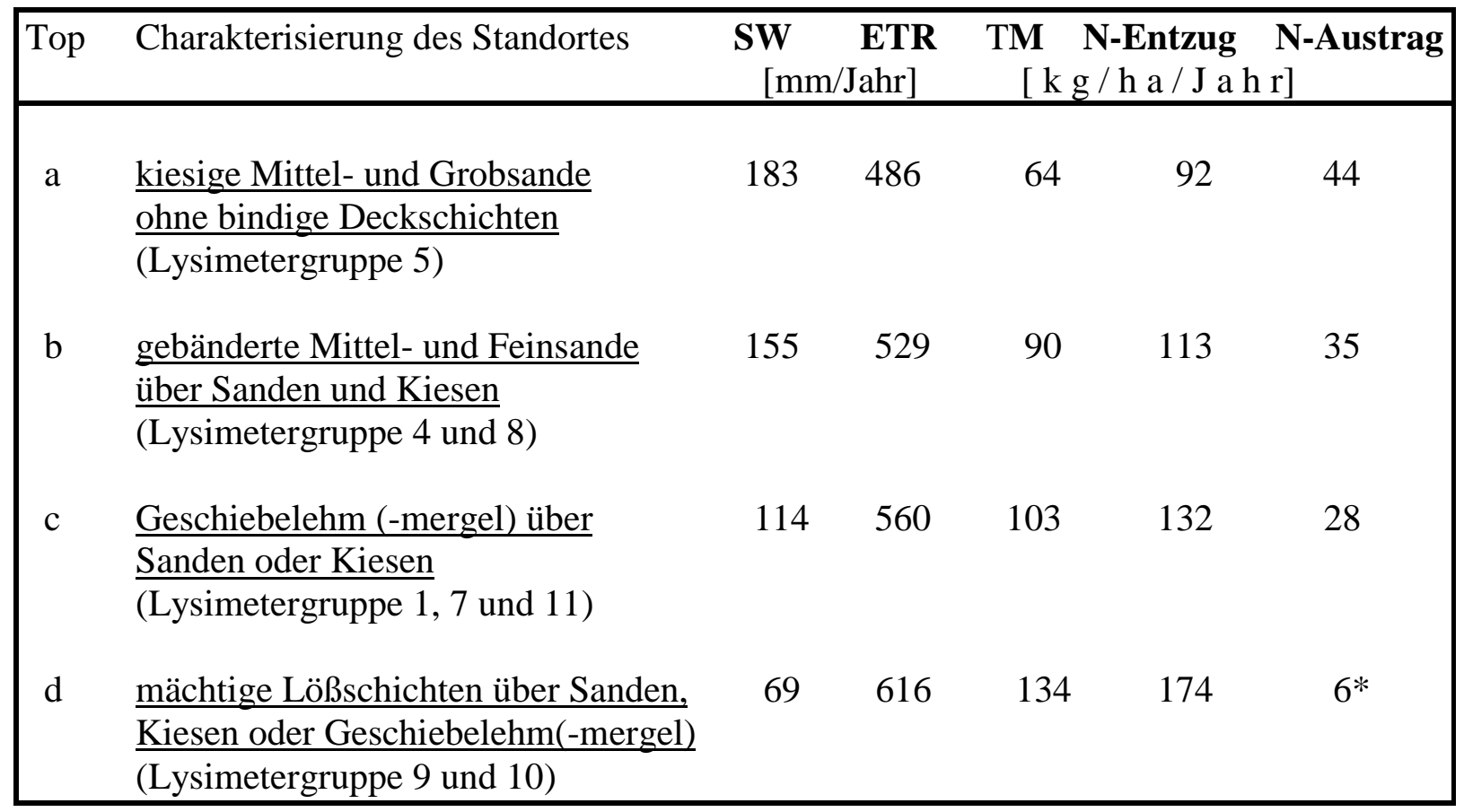

*Ohne Lysimetergruppe 10

Die erzielten Ergebnisse lassen folgende Schlussfolgerungen zu:

- Bei gleicher Witterung und Bewirtschaftung wird die Abflussbildung im Untersuchungsgebiet bei Böden mit flurfernen Grundwasserständen primär durch die Sedimente geprägt, die in der Elster- bzw. Saalekaltzeit (Schmelzwassersand und Geschiebelehm) und der Weichselkaltzeit (Sandlöß und Löß) zur Ablagerung kamen. Die in Folge der bodenbildenden Prozesse wie u. a. Verbraunung, Lessivierung (bei Fahlerden), Vergleyung und Humusakkumulation (bei Schwarzerden) entstandenen Bodentypen haben für die Abflussbildung in $3 \mathrm{~m}$ Tiefe vermutlich nur sekundäre Bedeutung, wobei Ausgangssubstrat und bodenbildende Prozesse in genetischem Zusammenhang stehen.

- Für Gebietsuntersuchungen können die acht analysierten Bodentypen zu vier PedoHydrotopen (auch Versickerungstypen) zusammengefasst werden, ohne dass es dadurch zu großen Fehlern bei der Ermittlung des Gebietsabflusses kommt (Kap. 4.1.3.2).

- Auf den Geschiebelehmböden (Top c) der Grundmoräne, die auf Grund ihrer Entstehungsgeschichte eine große räumliche Diskontinuität aufweisen, ist bei gleicher Witterung und Bewirtschaftung mit einer hohen räumlichen Varianz der Abflussbildung zu rechnen.

- Im Unterschied dazu ist in Gebieten mit Schmelzwassersanden und -kiesen (Top a und b) und mit äolischen Ablagerungen (Top d) bei gleicher Witterung und Bewirtschaftung eine relativ geringe räumliche Varianz der Abflussbildung zu erwarten.

- In Bezug auf die Verdunstung ist die Zuordnung zu den Pedo-Hydrotopen nicht ganz so eindeutig. Hier sind neben den physikalischen Eigenschaften der Ausgangssubstrate auch die Bodenfruchtbarkeit und der aktuelle Zustand des Bestandes von Einfluss. 
Tab. 44: Definition relevanter Pedo-Hydrotope im Untersuchungsgebiet

\begin{tabular}{|c|c|c|c|c|c|c|}
\hline $\begin{array}{l}\text { Lysi- } \\
\text { meter- } \\
\text { gruppe }\end{array}$ & Bodenform & $\begin{array}{l}\text { Wasser- und } \\
\text { Nährstoff- } \\
\text { speicher- } \\
\text { vermögen }\end{array}$ & $\begin{array}{l}\text { Boden- } \\
\text { art* }\end{array}$ & $\begin{array}{l}\text { Hydro- } \\
\text { morphie }\end{array}$ & Schichtung & Top \\
\hline 5 & $\begin{array}{l}\text { erodierte } \\
\text { Braunerde }\end{array}$ & gering & $1 \mathrm{~S}-\mathrm{SL}$ & $\begin{array}{l}\text { sickerwasser- } \\
\text { beeinflusst }\end{array}$ & $\begin{array}{l}\text { Sandlöß über } \\
\text { Schmelzwasser } \\
\text { sanden (locker) }\end{array}$ & $\overline{\mathbf{a}}$ \\
\hline 4 und 8 & $\begin{array}{l}\text { Braunerde- } \\
\text { Fahlerde, } \\
\text { Parabraunerde- } \\
\text { Braunerde } \\
\end{array}$ & gering & $\mathrm{SL}$ & $\begin{array}{l}\text { sickerwasser- } \\
\text { beeinflusst }\end{array}$ & $\begin{array}{l}\text { Sandlöß über } \\
\text { Schmelzwasser } \\
\text { sanden (lehmig) }\end{array}$ & $\mathbf{b}$ \\
\hline $\begin{array}{l}1,7 \text { und } \\
11\end{array}$ & $\begin{array}{l}\text { (lessivierter) } \\
\text { Braun-erde- } \\
\text { Pseudogley, } \\
\text { pseudovergleyte } \\
\text { Braunerde- } \\
\text { Fahlerde }\end{array}$ & mittel & sL & $\begin{array}{l}\text { stauwasser- } \\
\text { beeinflusst, } \\
\text { früher teils } \\
\text { gw- } \\
\text { beeinflusst** }\end{array}$ & $\begin{array}{l}\text { Sandlöß } \\
\text { (Geschiebedeck } \\
\text { sand) über } \\
\text { Geschiebelehm, } \\
\text {-mergel (teils } \\
\text { stark } \\
\text { verdichtet) }\end{array}$ & c \\
\hline 9 und 10 & $\begin{array}{l}\text { Parabraunerde, } \\
\text { Schwarzerde }\end{array}$ & sehr hoch & $\mathrm{U}$ & $\begin{array}{l}\text { starke } \\
\text { Aufwärts- } \\
\text { bewegung } \\
\text { infolge hoher } \\
\text { Verdunstung }\end{array}$ & $\begin{array}{l}\text { Löß über } \\
\text { Schmelzwasser } \\
\text { sanden (sehr } \\
\text { homogen) }\end{array}$ & $\bar{d}$ \\
\hline
\end{tabular}

*nach Bodenschätzung, **grundwasserbeeinflusst

Mit der Zusammenführung der 24 Lysimeter zu 4 Versickerungstypen (Topen) verbessern sich die flächenbezogenen Anwendungsmöglichkeiten von Lysimeterergebnissen wesentlich, wobei diese Generalisierung zunächst nicht bei Beschaffenheitsuntersuchungen vorgenommen werden sollte. 


\subsubsection{Vergleichbare Standorte im Freistaat Sachsen}

Nachdem Standortuntersuchungen abgeschlossen sind, besteht in den meisten Fällen der Wunsch, diese Ergebnisse auf die Fläche zu übertragen. Deshalb wird nachfolgend recherchiert, wie groß im Freistaat Sachsen die Flächenanteile sind, für die auf Grund ihrer Böden und hydrogeologischen Verhältnisse eine Übertragung der Brandiser Untersuchungen denkbar ist. Der sächsische Festgesteinsbereich und seine Randbereiche wurden dabei nicht betrachtet.

Zunächst zeigte sich bei diesen Arbeiten, dass keine der Übersichtskarten die "ungesättigte Zone" und die Reichweite eines Brandiser Lysimeters zufrieden stellend beschreibt. In Abb. $35^{\circ}$ sind von DUTELOFF (1996) anhand eines Beispielprofils die Geltungsbereiche der im LfUG vorhandenen Übersichtskarten schematisch dargestellt.

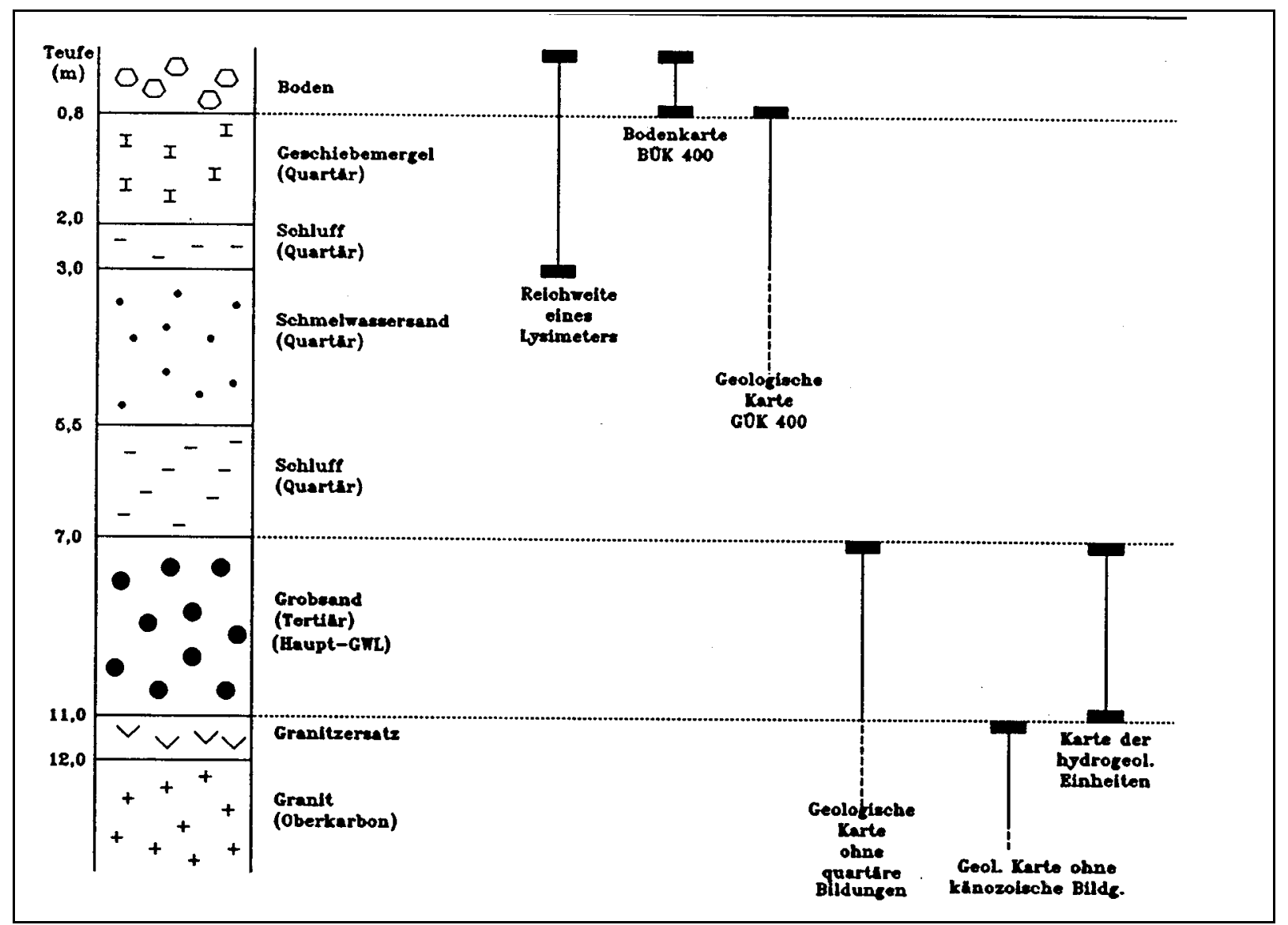

Abb. 35: Schematische Darstellung der Geltungsbereiche geowissenschaftlicher Übersichtskarten des Sächsischen Landesamtes für Geologie (DUTELOFF, 1996)

Werden allein die Übersichtskarten der Böden des Freistaates Sachsen 1 : 400000 (LfUG, 1993) zugrunde gelegt, so repräsentieren die Lysimeterböden die Bodengesellschaften im Verbreitungsgebiet von Löß, Lößderivaten und Sand. Das sind nach MANNSFELD \& RICHTER (1995) rd. 67 \% des sächsischen Lockergesteinsbereiches.

SÄMISCH (1990) hat auf Grundlage der Verteilung der „Natürlichen Standorteinheiten (NStE) in \% der Ackerfläche“ (mit Stand von 1963) nach SCHILLING, BANNORTH \& SCHLICHT (1965) folgende Zahlen erarbeitet: Die Lysimeter repräsentieren acht natürliche Bodenformen und sechs NStE und damit $53 \%$ des Ackerlandes der Neuen Bundesländer, 69 $\%$ des Ackerlandes von Sachsen und $49 \%$ des Ackerlandes von Sachsen - Anhalt. 
Mit Hinweis auf die Stellung der ungesättigten Zone im Gebietswasserhaushalt sind Betrachtungen, die nur bis in $0,8 \mathrm{~m}$ bis $1 \mathrm{~m}$ Tiefe angestrebt werden, nicht ausreichend. Um das Verständnis und einen Flächenbezug für die darunterliegenden hydrogeologischen Formationen zu erhalten, wurden von DUTELOFF (1996) Untersuchungen auf Basis der Geologischen Übersichtskarte $1: 400000$ (GÜK 400, 1992) vorgenommen. Diese Karte stellt die unmittelbar unterhalb der in Zuständigkeit der Bodenkartierung befindlichen Gesteine dar und repräsentiert damit die unteren 2/3 der Lysimetermächtigkeit.

Im Ergebnis einer stark vereinfachenden Betrachtung - bei der davon ausgegangen wird, dass die Unterschiede zwischen den Schmelzwassersanden der Elster-Kaltzeit und der SaaleKaltzeit sowie zwischen den jeweiligen Grundmoränen vernachlässigbar sind - werden den acht Lysimeterböden 3 Gebiete zugewiesen:

- Gebiete mit Schmelzwassersanden (Top a und b)

- Gebiete mit Geschiebelehm/-mergel (Top c)

- Lößgebiete (Top d).

Anhand der GÜK 400 lassen sich die Verbreitungsgebiete von Schmelzwasserablagerungen der Saale- und Elster-Kaltzeit, Grundmoränen der Saale- und Elster-Kaltzeit sowie Löß und Lößlehme ausgrenzen, womit folgende Flächengrößen repräsentiert werden:

\begin{tabular}{ll} 
Schmelzwasssersande & $1731 \mathrm{~km}^{2}$ \\
Grundmoränen & $1026 \mathrm{~km}^{2}$ \\
Löß und Lößlehm & $1766 \mathrm{~km}^{2}$ \\
\hline gesamt & $4523 \mathrm{~km}^{2}$
\end{tabular}

Bei einer Gesamtfläche des Lockergesteinsbereiches im Freistaat Sachsen von 9.887 km² entspricht das etwa $46 \%$ dieser Fläche. Die Untersuchungen ergaben auch, dass folgende geologische Einheiten durch Lysimeter nicht repräsentiert werden, so dass eine Ergänzung der Brandiser Lysimeter aus folgenden hydrogeologischen Einheiten notwendig ist:

- Endmoränen und Eisstausee-Ablagerungen

- Flussterrassen

- Auelehme

- tertiäre Schichten, die an der Oberfläche anstehen sowie

- Gehängelehme. 


\subsubsection{Sickerwassermenge und Grundwasserneubildung}

\subsubsection{Problemstellung}

Obwohl im Ergebnis der Untersuchungen zu den Ausschöpfungstiefen davon ausgegangen werden kann, dass die Lysimetergruppen 5, 4, 8, 1, 7 und 11 Standorte mit flurfernen Grundwasserständen repräsentieren (kein Einfluss der Bodenplatte des Lysimeters auf die verdunstungsbeeinflusste Zone, s. Kap. 3.4.3), wurde trotzdem stets von Sickerwassermenge und nicht von Grundwasserneubildung gesprochen.

Die bei eindimensionaler Betrachtungsweise mittels Lysimeter ermittelte Sickerwassermenge ist der maximal mögliche Abfluß - der Gesamtabfluß eines Standortes. Welcher Anteil davon als Grundwasserneubildung tatsächlich die Grundwasseroberfläche erreicht, hängt von den jeweiligen geographischen und hydrogeologischen Eigenschaften im Einzugsgebiet ab.

Die Ermittlung der Grundwasserneubildung ist eine weitere Aufgabenstellung, die sich der Ermittlung des Gesamtabflusses anschließen sollte. Bei unbedeckten oder bedeckten Grundwasserleitern mit geringer Reliefenergie der Geländeoberfläche (z. B. Urstromtäler und ebene Geschiebelehmdecke auf Talschottern) entspricht die Sickerwasserbildung in der Regel der Differenz zwischen Niederschlag und Verdunstung. Allerdings kann bei Standorten mit flurnaher Grundwasseroberfläche Grundwasser wieder kapillar in die verdunstungsbeeinflusste Bodenzone aufsteigen, je nach Jahreszeit verdunsten und so die $\mathrm{zu}$ anderen Jahreszeiten erfolgte Grundwasserneubildung um diesen Betrag mindern. Dies ist im Untersuchungsgebiet von Bedeutung, da die Jahresverdunstung bei flurnahen Grundwasserständen höher ist als der Jahresniederschlag. Permanente Grundwasserzehrflächen sind Wasserflächen und große Flussauen, da nur hier die erforderliche Wassernachlieferung gewährleistet ist.

Bei bedeckten Grundwasserleitern mit mehreren Stockwerken bzw. mit großer Reliefenergie der Geländeoberfläche tritt Landoberflächen (RO)- und/oder hypodermischer Abfluß $(\mathrm{RH})$ auf, der die Gewässer speist. In diesem Fall muss der Gesamtabfluß ( $\left.\mathrm{P}_{\text {korr }}-\mathrm{ETR}\right)$ um den Abflussanteil $(\mathrm{RO}+\mathrm{RH})$ reduziert werden. Unabhängig davon können laterale Abflüsse, wenn sie den Vorfluter nicht erreichen, weit vom Ort ihrer Entstehung zum Grundwasser gelangen.

Fallende und steigende Grundwasserstände infolge wechselnder Witterung und/oder durch anthropogene Einflüsse verändern die Mächtigkeit der ungesättigten Zone und führen zu veränderten Abflussbedingungen. Standorte mit flurfernen Grundwasserständen werden zu Standorten mit flurnahen Grundwasserständen und umgekehrt. 


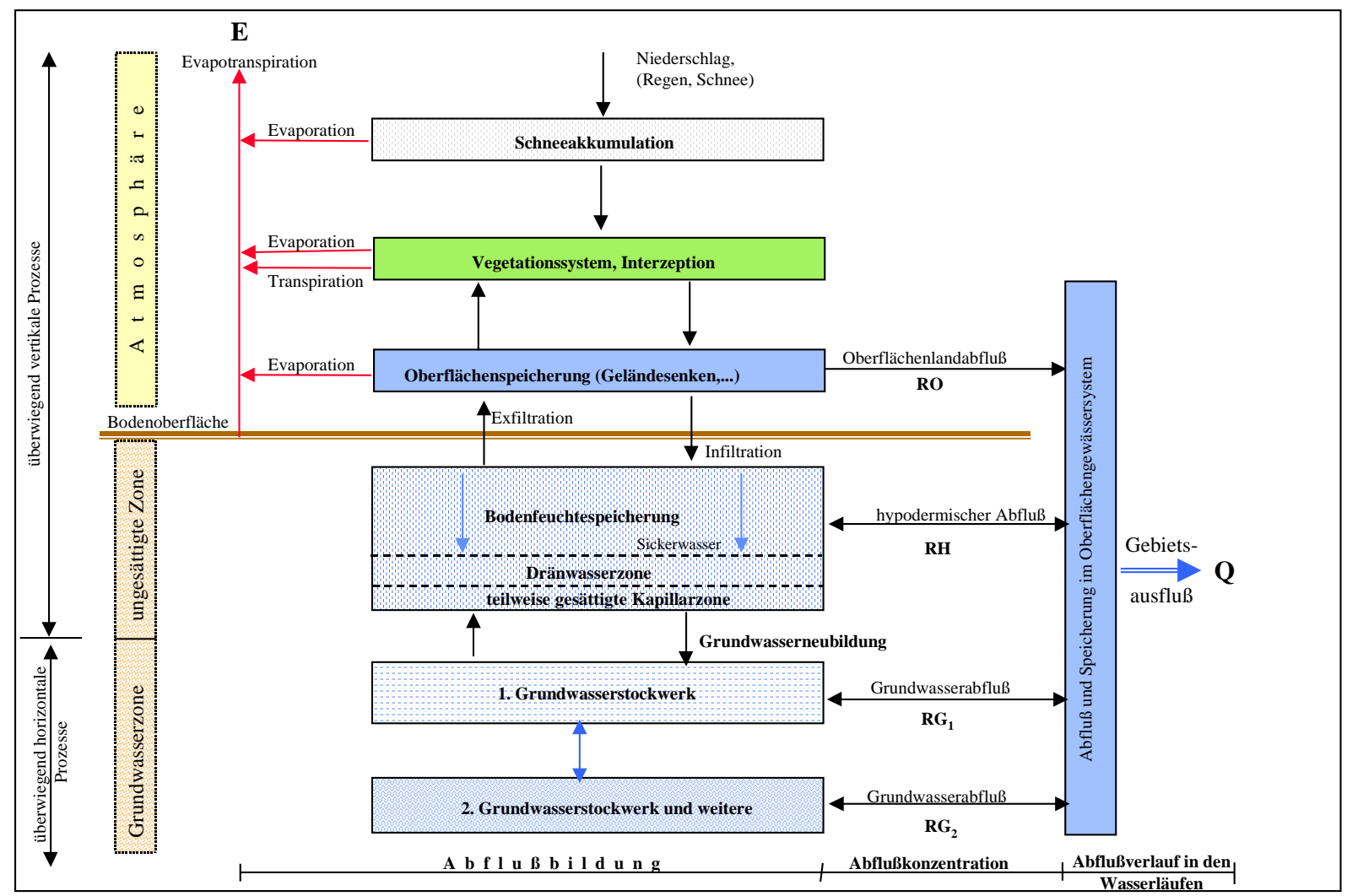

Abb. 36: Gebietswasserhaushalt: Vertikale Gliederung eines Einzugsgebietskörpers und schematische Darstellung der Niederschlagsabflussbeziehungen (nach DYCK\&PESCHKE, 1995)

Nachfolgend wird an einem Beispiel demonstriert, welchen Einflüssen die ungesättigte Zone auf Grund ihrer Einbindung in den Gebietswasserhaushalt unterliegt. Abb. 37 zeigt die Grundwasserstandsentwicklung von November 1980 bis Oktober 1997 (= Zeitraum der Lysimetermessungen) am Beispiel von zwei Meßstellen im Raum Naunhof in der Nähe der Parthe (südöstlich von Leipzig). Diese Messstellen repräsentieren den Hauptgrundwasserleiter des Einzugsgebietes, für den die Parthe die Vorflut bildet. Zeitgleich dargestellt sind die Wasserstände der Parthe am Pegel Naunhof.

Bei ebenen Standorten, wie hier in der Partheniederung, spielt der Abfluß an der Geländeoberfläche (Sättigungsflächenabfluss) eine untergeordnete Rolle. Nur im Fall von Starkregen und hoher Vorfeuchte (hohe Grundwasserstände) wird ein erheblicher Anteil des Niederschlages innerhalb kurzer Zeit im Vorfluter konzentriert und führt dort sehr schnell zu erhöhten Abflüssen. Kurzzeitig überflutet die Parthe das Gelände, wie Abb. 37 zeigt (s. Zeitraum 1979/80, 1987 und 1994).

Im übrigen Zeitraum bewirken die hydrogeologischen Bedingungen im Einzugsgebiet, dass die Parthe vorwiegend im Oberlauf gespeist wird und dann im Raum Naunhof in die Bodenund Grundwasserzone infiltriert. Reicht der Zustrom aus dem Oberlauf nicht aus, fällt das Gewässer auch mehrere Monate trocken. Auf diese Weise führt u. a. ein bereits als hypodermischer und/oder Abfluß an der Geländeoberfläche definierter Anteil des Niederschlages schließlich doch noch zu einer Aufhöhung des Bodenwasservorrates und zur Grundwasserneubildung. 


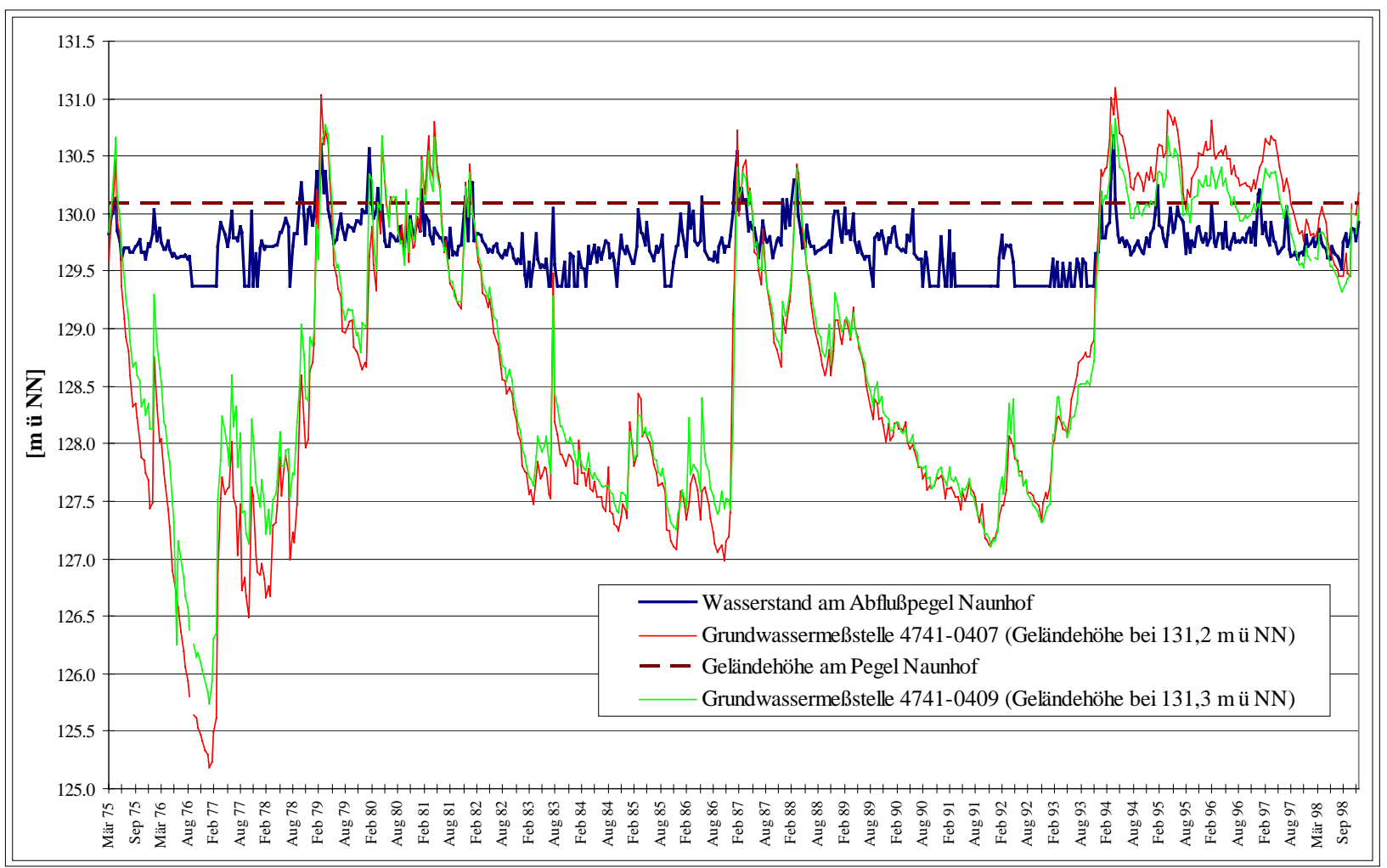

Abb. 37: Grundwasserstandsentwicklung und Wasserstände der Parthe am Pegel Naunhof von März 1975 bis Oktober 1998

Zusammenfassend wird deutlich, dass eine rein stationäre und standortbezogene Betrachtung des Bodenwasserhaushaltes nicht vorgenommen werden sollte. Auf diese Problematik verweist u. a. HÖLTING (1997) als er davor warnt, punktuell auf Basis von Vegetations- und Klimadaten sowie pedologischer Parameter gewonnene Sickerwassermengen mittels GIS (Geographisches Informationssystem) auf die Fläche zu übertragen und der Grundwasserneubildung gleich zu setzen.

\subsubsection{Verwendung der Lysimeteruntersuchungen im Einzugsgebiet der Parthe}

Ziel der nachfolgenden Untersuchungen ist ein Vergleich zwischen dem für die Einzugsgebietsfläche ermittelten Gesamtabfluss und den im vergleichbarem Zeitraum 1981-97 gemessenen Abflüssen am Gebietspegel Thekla. Die überwiegende Anzahl der Lysimeterböden wurde im Einzugsgebiet der Parthe gewonnen. Deshalb war es möglich, dass mittels Lysimetermessungen ermittelte „Gesamtdargebot“ (unter Berücksichtigung der anstehenden Böden und der Flächenutzung) auf die Einzugsgebietsfläche zu extrapolieren.

Rd. $74 \%$ der Flächen werden landwirtschaftlich genutzt, wobei die drei Versickerungstypen (Tope) a, b und c mit jeweils $5 \%, 28 \%$ und $41 \%$ vertreten sind Abb. 38. Rd. $14 \%$ der Einzugsgebietsfläche sind Wald. Der hohe Bebauungsanteil von $8 \%$ resultiert aus dem Flächenanteil der Stadt Leipzig, der aber erst unterhalb des Bezugspegels Thekla in die Parthe entwässert. Der Pegel Thekla hat ein Einzugsgebiet von $314 \mathrm{~km}^{2}$. Das sind $87 \%$ des $366 \mathrm{~km}^{2}$ großen Parthegebietes.

Um das Abflussverhalten im Einzugsgebiet besser beurteilen und mit den mittels Lysimeter ermittelten Standortabflüssen vergleichen zu können, wurden die am Pegel Thekla seit 1951 gemessenen Durchflüsse der Parthe (Tageswerte) analysiert. Mittels Ganglinienseparation nach dem Verfahren DIGFA (SCHWARZE, 1985) wurden drei Abflusskomponenten identifiziert: 
- Direktabfluss (RO)

- kurzfristiger Basisabfluss (RH) und

- langfristiger Basisabfluss $(R G)$

und prozentual zum Gebietsniederschlag in Beziehung gesetzt (MELLENTIN \& HAFERKORN, 1999).

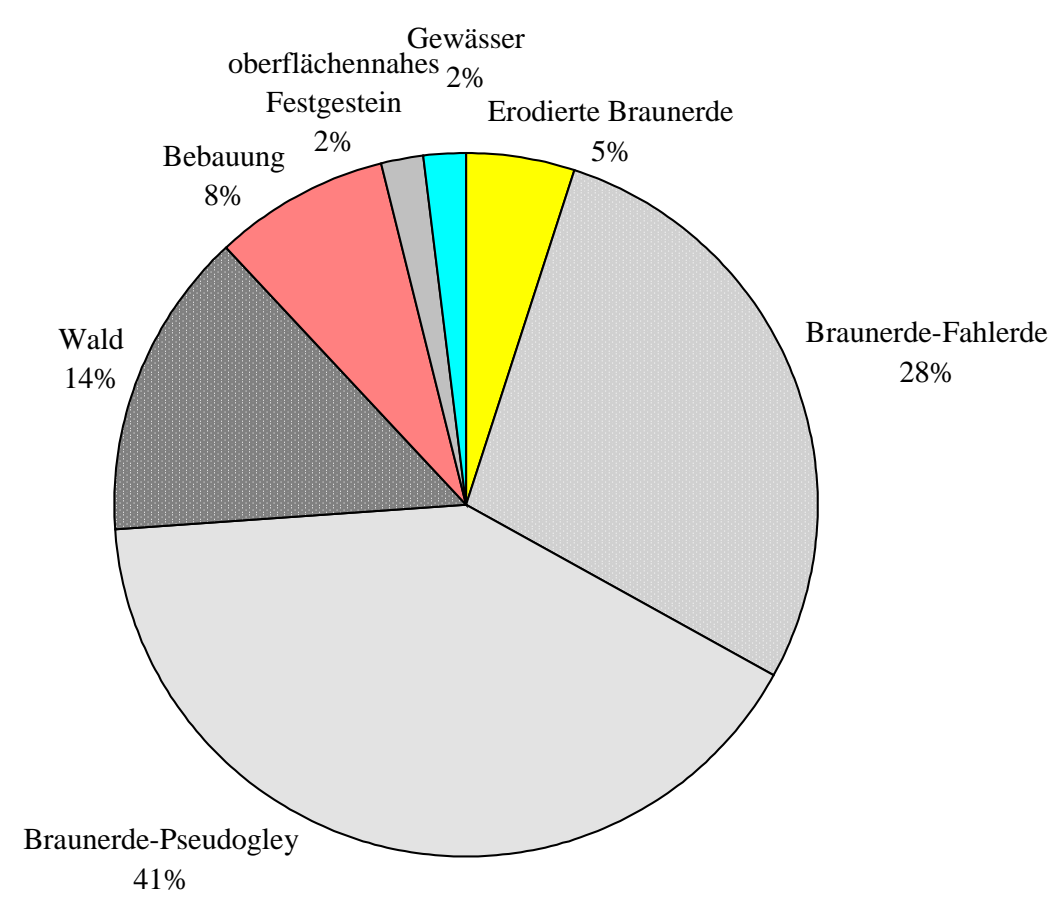

Abb. 38: Flächennutzung und Böden der landwirtschaftlichen Nutzflächen im Einzugsgebiet der Parthe

Tab. 45 und Abb. 39 zeigen die mittleren Wasserhaushaltskomponenten für den Vergleichszeitraum von 1981-97, ausgehend von einem korrigierten Gebietsniederschlag von $660 \mathrm{~mm}$. Die Gebietsverdunstung resultiert bei der Ganglinienseparation als Restgröße in einer Höhe von rd. $540 \mathrm{~mm}$. Damit Vergleiche möglich werden, erfolgte eine Umrechnung aller Komponenten in $\mathrm{mm} /$ Zeiteinheit.

Tab. 45: Mittlere Wasserhaushaltskomponenten im Einzugsgebiet der Parthe - Bezugspegel Thekla (1981-97)

\begin{tabular}{|l|c|c|}
\hline \multicolumn{1}{|c|}{$\begin{array}{c}\text { Wasserhaushalts- } \\
\text { komponenten } \\
\text { [mm/Jahr] }\end{array}$} & $\begin{array}{c}\text { Einzugsgebiet } \\
\text { (Abflussbildung lt. } \\
\text { Gl.-Separation) }\end{array}$ & $\begin{array}{c}\text { Lysimeter } \\
\text { (Abflussbildung in den } \\
\text { Lysimetern und Hochrechnung } \\
\text { auf die Einzugsgebietsfläche) }\end{array}$ \\
\hline Niederschlag & 660 & 655 \\
Verdunstung & 540 & 528 \\
\hline Direktabfluss & 21 & - \\
Kurzfristiger Basisabfluss & 30 & - \\
Langfristiger Basisabfluss & 42 & 127 \\
GW-Entnahme der WW & 27 & $\mathbf{1 2 7}$ \\
Naunhof & & $\mathbf{9 3 ( 1 2 0 * )}$ \\
\hline Summe der Abflussbildung & & \\
\hline
\end{tabular}

$\mathrm{GW}=$ Grundwasser

* Abflussbildung + GW-Entnahme 


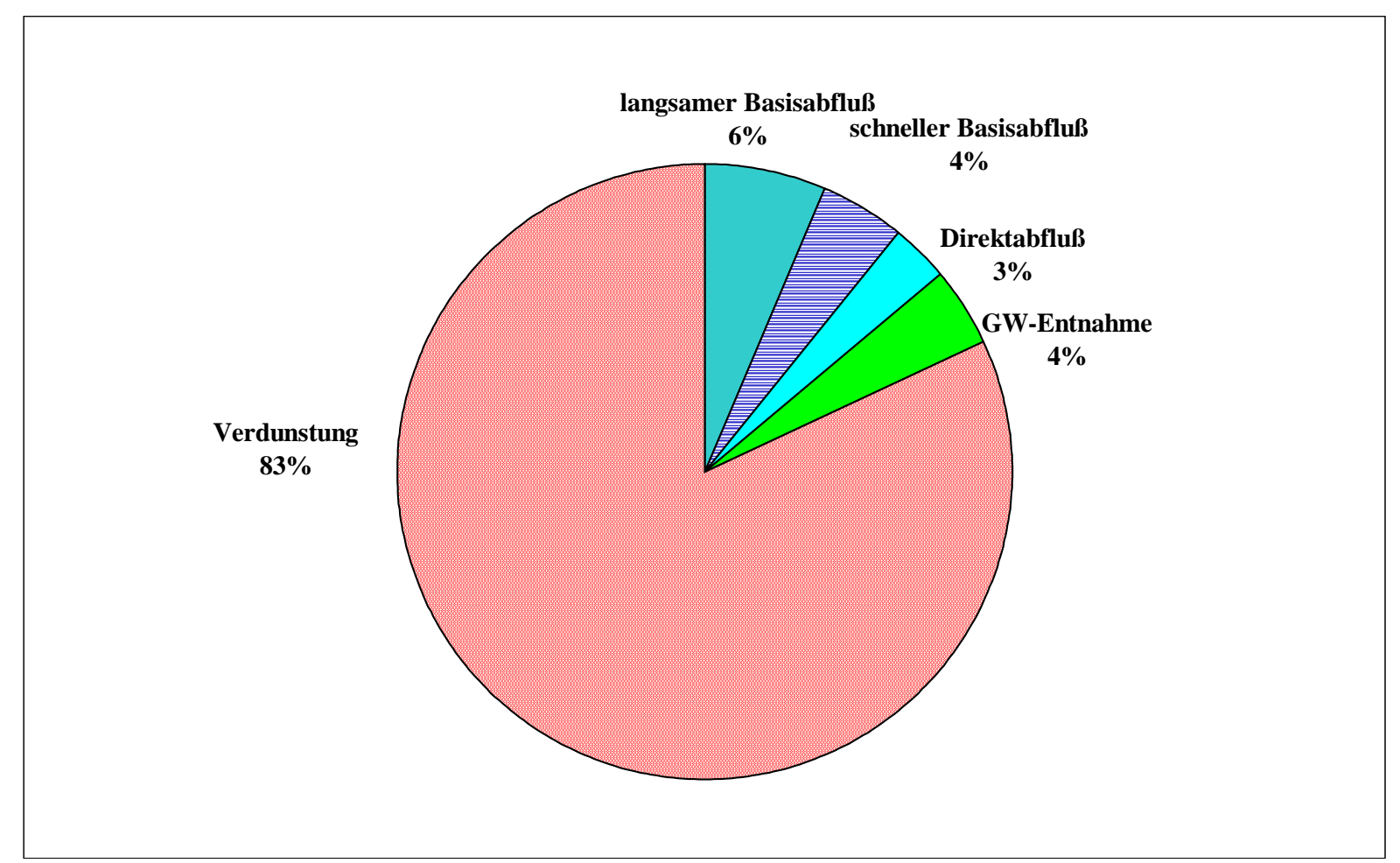

Abb. 39: Mittlere Wasserhaushaltskomponenten am Pegel Thekla/Parthe der Jahre 1981-97

Der langfristige Basisabfluss stellt mit $42 \mathrm{~mm} / \mathrm{Jahr}$ die wichtigste grundwasserbürtige Abflusskomponente im Einzugsgebiet der Parthe. Herkunftsraum sind die mächtigen Muldeschotter, die im Parthegebiet den Hauptgrundwasserleiter bilden. Aus dem Grundwasserleiter entnehmen die Wasserwerke (WW) Naunhof erhebliche Wassermengen, die zu $75 \%$ außerhalb des Parthegebietes im Stadtgebiet Leipzig genutzt werden. Mit $27 \mathrm{~mm} / \mathrm{Jahr}$ ist diese Grundwasserentnahme ein wesentlicher Teil der Gesamtbilanz und wird deshalb berücksichtigt. Inwiefern sie vollständig dem langfristigen Basisabfluss zuzurechnen ist, bleibt ungeklärt.

Der langfristige Basisabfluss ist auf Grund des großen Retentions- und Translationsraumes im Einzugsgebiet die einzige kontinuierliche - auch in Trockenzeiten fließende - Komponente. Beim Standortwasserhaushalt ist dieser Umsatzraum vergleichsweise sehr gering und es kann kein horizontaler Austausch erfolgen. Deshalb kommt es beim Standortwasserhaushalt zu einer erheblichen zeitlichen Umverteilung der Abflussbildung (Abb. 40.

Kurzfristiger Basisabfluss entstammt vorwiegend den oberen lokalen Grundwasserleitern, die gut entwässerbar sind und geringe Umsatzräume haben, so dass diese Abflusskomponenten bereits großen Schwankungen unterliegt. Hinsichtlich des Abflussregimes am Standort handelt es sich vorwiegend um verzögerten bodeninneren lateralen Abfluß, der bei geschichteten Böden und Bodensättigung insbesondere im Februar, März und April entsteht und der im Lysimeter nur vertikal zum Abfluss kommen kann.

Verzögerter Direktabfluss entsteht als bodeninnerer lateraler Abfluss, der überwiegend nur bei Vorflutnähe abflußwirksam wird. Schneller Direktabfluss ist Oberflächenlandabfluss von versiegelten sowie gewässer- und grundwassernahen Bereichen (Sättigungsflächen). Nur er ist echtes Ereigniswasser, welches ohne bedeutsame Retention zum Abfluss gelangt. 


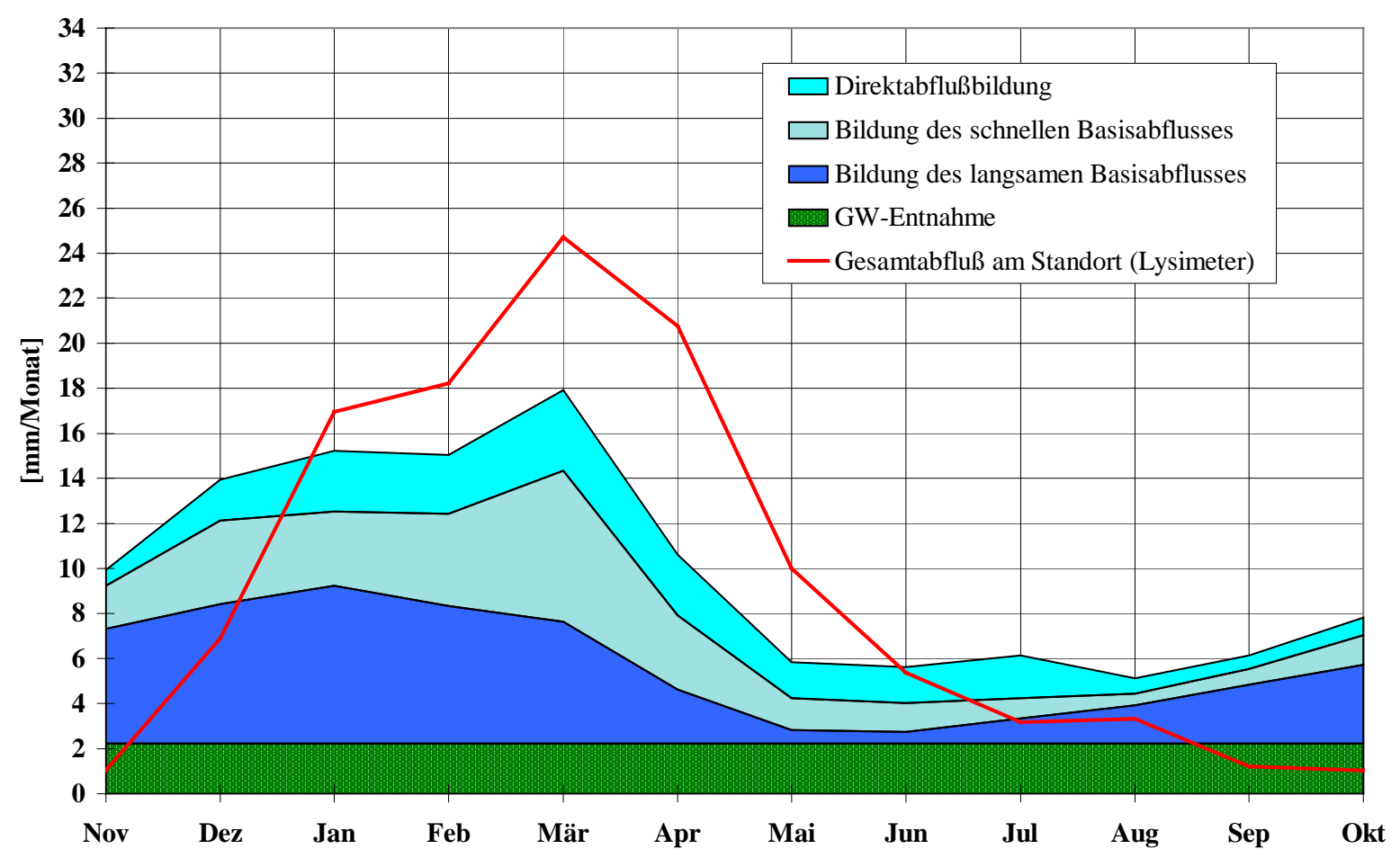

Abb. 40: Vergleich der mittleren Abflussbildung am Standort (Lysimetermessungen) und im Einzugsgebiet der Parthe (Reihe 1981-97)

Die beiden Abflussanteile des Direktabflusses können nur bei einer zeitlichen Auflösung von Stundenwerten voneinander unterschieden werden.

Der Direktabfluss kann mittels Lysimeter nicht ermittelt werden. Mit einem Anteil von nur rd. $3 \%$ am Gebietsniederschlag spielt er als Abflusskomponente eine untergeordnete Rolle. Nur bei hohen Niederschlagsintensitäten und hohen Bodenwassergehalten ist Direktabfluss in größerem Umfang an der Abflussbildung beteiligt.

Mittels Lysimeter werden alle Abflusskomponenten summarisch als Gesamtabfluss gemessen. Aus den vergleichenden Untersuchungen zwischen Abflussbildung (im Sinne von Auffüllung und Entleerung der entsprechenden Speicher) am Standort und im Einzugsgebiet lassen sich folgende Schlussfolgerungen ableiten:

- Auch im Einzugsgebiet bestimmt die hohe Ausschöpfung des Bodenwasserspeichers durch die Verdunstung den Wasserhaushalt. Nur 13 \% (mit GW-Entnahme $18 \%$ ) des fallenden Niederschlages kommen zur Abflussbildung. Der grundwasserbürtige Abfluss ist die wichtigste Abflusskomponente.

- Die semiariden Bedingungen des Untersuchungsgebietes führen in Gebieten mit flurnahen Grundwasserständen im Sommerhalbjahr zu einer erheblichen Zehrung aus dem Grundwasser.

- Durch die Wirkung des Grundwasserspeichers kommt es zwischen Standort und Einzugsgebiet zu erheblichen Unterschieden in der innerjährlichen Abflussdynamik. Mengenmäßig kann eine Übereinstimmung erzielt werden. 
Besonders beim Vergleich zwischen diffusen Stoffeinträgen (z. B. Nitrat) infolge landwirtschaftlicher Bewirtschaftung und der Höhe der Frachten die das Einzugsgebiet mit der Vorflut verlassenden, gewinnen Fließweg, Fließ- und Verweilzeiten des Wassers und seine Herkunftsräume an Bedeutung. Wie mittels Ganglinienseparation am Beispiel des Parthegebietes gezeigt werden konnte, spielt neben dem Herkunftsraum „ungesättigte Zone“ auch die gesättigte Zone als Transport- und Umsatzraum eine entscheidende Rolle.

Der auf Basis von langjährigen Mittelwerten herbeigeführte Vergleich zwischen den Abflüssen am Gebietspegel Thekla und dem auf Basis von Standortuntersuchungen (Lysimeter) ermittelten Gebietsabfluss ergab in der Bilanz eine gute Übereinstimmung, zeigte aber Unterschiede im innerjährlichen Gang der Abflussbildung.

\subsection{Zeitliche Repräsentanz der Messreihen von 1981 bis 1997}

Bereits in Kap. 3.3 wurde darauf hingewiesen, dass im Verlauf der Untersuchungsperiode 1981-97 Extremwerte auftraten, die in den klimatologischen Bezugsreihen 1951-80 und 196190 nicht zu verzeichnen waren.

Zur Einordnung der Untersuchungsergebnisse in das langjährige meteorologische und hydrologische Regime des Untersuchungsgebietes werden mittlere klimatologische Monats- und Jahreswerte ausgewählter Stationen des DWD miteinander verglichen (Tab. 46.

Tab. 46: Niederschlag und Lufttemperatur von ausgewählten Stationen des DWD

\begin{tabular}{|c|c|c|c|c|}
\hline$\frac{\text { Stati }}{[\mathrm{m}}$ & $\frac{\text { nslage }}{\text { NN] }}$ & $\underline{\text { Reihe }}$ & $\frac{\text { Niederschlag* }}{[\mathrm{mm} / \mathrm{Jahr}]}$ & $\frac{\text { Lufttemperatur }}{\left[{ }^{\circ} \mathrm{C}\right]}$ \\
\hline \multirow[t]{4}{*}{ Wittenberg } & 105 & $1901-50$ & 542 & 8.4 \\
\hline & & $1951-80$ & 576 & 8.6 \\
\hline & & $1961-90$ & 563 & 8.7 \\
\hline & & 1981-97 & 556 & 9.1 \\
\hline \multirow[t]{3}{*}{ Halle-Kröllwitz } & 96 & $1951-80$ & 476 & 9.0 \\
\hline & & $1961-90$ & 462 & 9.1 \\
\hline & & 1981-97 & 452 & 9.3 \\
\hline \multirow[t]{4}{*}{ Leipzig-Schkeuditz } & 131 & $1901-50$ & 516 & 8.5 \\
\hline & & $1951-80$ & 529 & 8.6 \\
\hline & & $1961-90$ & 503 & 8.8 \\
\hline & & 1981-97 & 513 & 9.2 \\
\hline \multirow[t]{4}{*}{ Oschatz } & 150 & $1901-50$ & 599 & 8.4 \\
\hline & & $1951-80$ & 583 & 8.4 \\
\hline & & $1961-90$ & 572 & 8.6 \\
\hline & & 1981-97 & 570 & 9.0 \\
\hline
\end{tabular}

*Niederschlag unkorrigiert

Dabei wird ersichtlich, dass sich der Zeitraum von 1981-97 im gesamten Untersuchungsgebiet durch ein erhöhtes Energieangebot von den Mittelwerten der anderen Beobachtungsreihen unterscheidet. Die Jahresmittel der Lufttemperaturen lassen im Vergleich der vieljährigen Reihen seit 1901 eine eindeutige Zunahme erkennen. 
Darüber hinaus zeigt die Untersuchungsperiode 1981-97 im Vergleich zu den Reihen 1951-80 und 1901-50 eine Verringerung der Sommerniederschläge, dafür aber höhere Niederschläge im Winterhalbjahr Abb. 41. Diese Aussagen decken sich mit den Untersuchungsergebnissen von RAPP \& SCHOONWIESE (1996). Die von ihnen erstellten ,Trendkarten für 1891 - 1990 zeigen verbreitet eine signifikante Zunahme der Jahresniederschlagssummen um durchschnittlich $9 \%$. Besonders deutlich ist dieser Überschuss im Herbst und Winter mit $+16 \%$ bis +19 $\%$ ausgefallen. Dagegen besteht im Sommer der letzten Jahrzehnte eine Neigung zum Niederschlagsrückgang, so dass eine jahreszeitliche Umverteilung des Niederschlages stattgefunden haben muss."

Diese Entwicklung kann für das Untersuchungsgebiet bestätigt werden. Abb. 41 zeigt die Entwicklung am Beispiel der Station Schkeuditz des DWD.

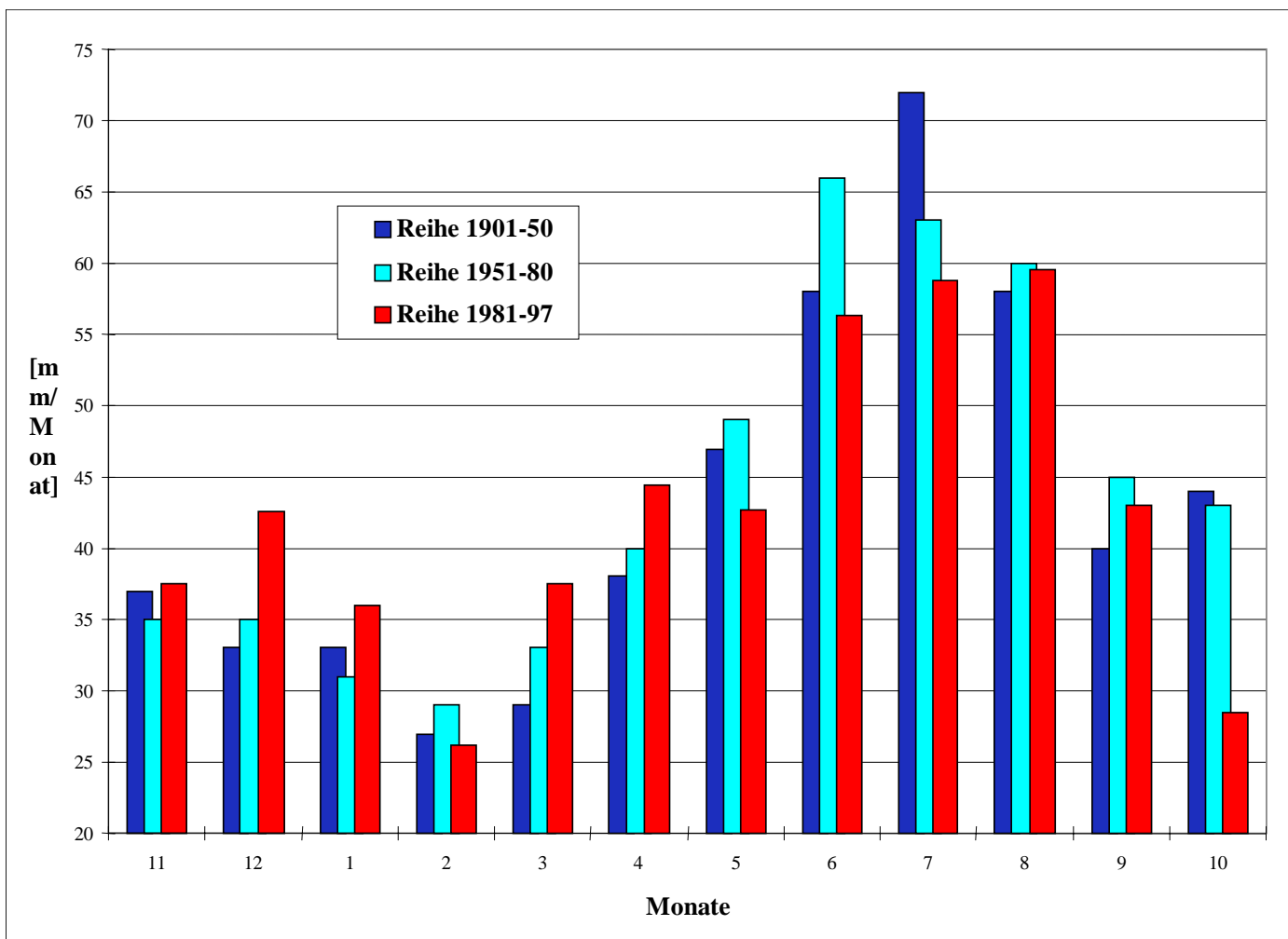

Abb. 41: Langjährige Monatsmittelwerte des Niederschlages in [mm/Monat] verschiedener Reihen am Beispiel der Station Leipzig-Schkeuditz des DWD

Mit Bezug auf die Lufttemperatur schreiben RAPP \& SCHÖNWIESE (1996) weiter: „Seit 1891 nahm die Lufttemperatur in Deutschland - jahreszeitlich nuanciert - um durchschnittlich 0,8 K zu. Zwischen 1961 und 1990 war im Winter die Erwärmung sogar noch intensiver und betrug im Mittel aller analysierten Stationen 1,7 K.“

Die Verschiebung der innerjährlichen Verteilung der Niederschläge und die zunehmende Erwärmung gewinnen Einfluss auf den Wasserhaushalt, wenn sie zur erhöhten Verdunstung und einer relevanten Veränderung der Abflusshöhe führen.

der verschiedenen Böden

Wie die Untersuchungen zur Kapazität der Bodenwasserspeicher auf Standorten mit flurfernen Grundwasserständen gezeigt haben (Kap. 3.4.1), ist bei zunehmender Trockenheit in den Sommermonaten (außer bei den Lößböden) nicht mit einer Erhöhung der Bodenwasserausschöpfung zu rechnen. Eher deutet sich ein Rückgang der tatsächlichen Verdunstung im Sommerhalbjahr infolge Wassermangel an (=Rückgang der Ernteerträge), 
wie MÜLLER et al. (1991) mit Modellrechnungen zur realen Verdunstung am Standort Halle nachwiesen. Die erhöhten Winterniederschläge werden zu einer erhöhten Abflussbildung beitragen.

Mit einer Zunahme der Verdunstung (und einem Rückgang der Abflüsse) ist in Regionen mit Parabraunerden und Schwarzerden auf tiefgründigem Löß (auf Grund sehr hoher pflanzenverfügbarer Bodenwasservorräte) und auf Standorten, die bedarfsgerecht aus dem Grundwasser versorgt werden, zu rechnen.

Die integrale Wirkung von möglichen Veränderungen zeigt sich im Oberflächenwasserabfluss der Einzugsgebiete, der sowohl Ausdruck des Klimageschehens, der Landnutzungsänderung als auch der speziellen Hydrogeologie des Gebietes ist.

An Hand von vieljährig beobachteten Grundwasser- und Oberflächenwassermessstellen läßt sich prüfen, ob der Untersuchungszeitraum 1981-97 bereits alle klimatisch bedingten Extremjahre enthält. So ist dem Verlauf der Ganglinien in Abb. 42 zu entnehmen, dass in den Jahren 1940-41, 1961 und 1966 vermutlich deutlich höhere Abflüsse auftraten als im Untersuchungszeitraum 1981-97 registriert werden konnten. Dagegen repräsentieren die Jahre von 1988-91 eine gebietstypische Trockenperiode, wie sie bereits in den Jahren 1931-34, 1950-52 und 1975-77 zu verzeichnen war.

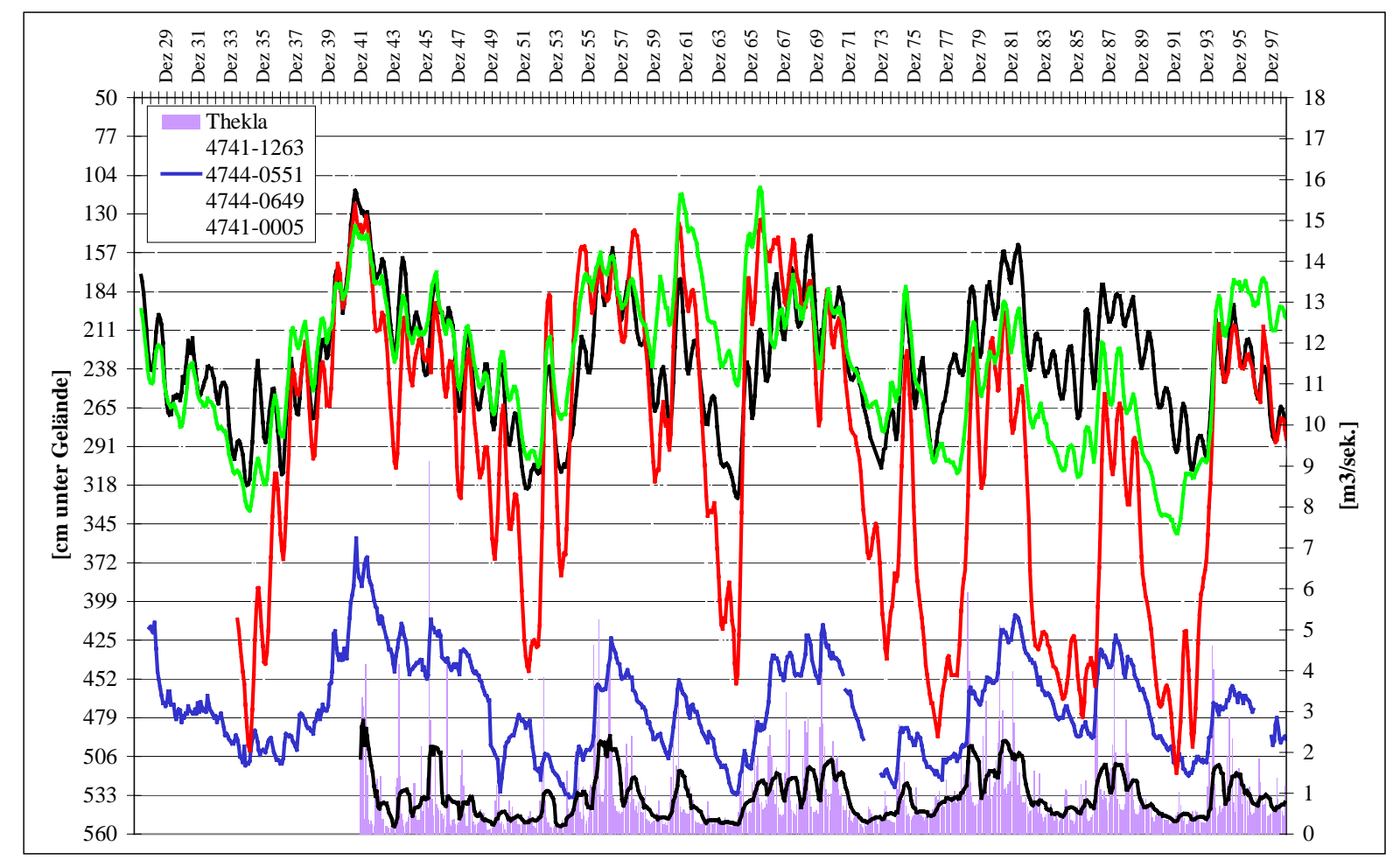

Abb. 42: Abfluss am Pegel Thekla/Parthe und Grundwasserstandsentwicklung am Beispiel ausgewählter Messstellen

Zusammenfassend wird festgestellt, dass die Jahre 1981-97 im Vergleich zu den Reihen 1901-50 und 1951-80 durch

- ein höheres Strahlungsangebot (erhöhte Lufttemperaturen) und

- eine etwas veränderte innerjährliche Verteilung der Niederschläge gekennzeichnet sind. 
Die Frage nach den Auswirkungen weiterer möglicher Klimaänderungen auf den Verlauf der Wasserhaushaltsgrößen wird u. a. nur durch weiterführende langjährige Messungen, so auch Lysimeteruntersuchungen, zu beantworten sein wird.

\subsection{Diskussion der Ergebnisse}

Im Ergebnis einer Varianzanalyse zur Wirkung bodenphysikalischer und -morphologischer Eigenschaften natürlicher landwirtschaftlich genutzter Böden auf die Wasserhaushaltsgrößen Verdunstung und Sickerwasserbildung wurden acht analysierte Bodentypen zu vier PedoHydroptopen zusammen gefasst. Das führte zu der Schlussfolgerung, dass der Bodenwasserhaushalt von Böden mit flurfernen Grundwasserständen bei gleicher Witterung und Bewirtschaftung primär durch die Ausgangssedimente geprägt wird. D. h. ähnliche Bodentypen weisen trotz Unterschiede in der Horizontabfolge in Bezug auf ihren Bodenwasserhaushalt keine Unterschiede auf, die über die natürliche Variabilität der Standorte hinausgehen. Aus diesen Erkenntnissen kann ein Ansatz für Bodenwasserhaushaltsuntersuchungen in unterschiedlichen Skalen resultieren. So erfordern Untersuchungen im Mikround Mesoskale die separate Berücksichtigung der einzelnen Bodentypen, während beim Übergang zum Makroskale (Skalen nach DIEKKRÜGER, 1997) eine vereinfachende Betrachtungsweise im Sinne der o. g. Pedo-Hydropope ausreichend ist.

Die Bildung von „Flächen gleichen Bilanztyps“ wird auch von SCHEUERER (1998) beschrieben. Mit Hilfe einer Clusteranalyse unterteilt er ein ackerbaulich genutztes Untersuchungsgebiet mit 19 Bodenformen in Scheyern in 7 größere Flächeneinheiten mit ähnlicher Bilanz. Kriterium für die Zusammenfassung von Bodenformen waren hier allerdings neben Transpiration und Sickerwasserbildung auch laterale Abflusskomponenten (Oberflächen- und Zwischenabfluss). Die Bedeutung der lateralen Flüsse fanden sich in der Charakterisierung der einzelnen Klassen wieder (z. B. Zuflussgebiet, Kuppenlage, Tallage). Die Klassen, in denen laterale Flüsse keine dominierende Rolle spielen, stimmen mit den hier definierten Topen (Versickerungstypen) überein.

Auf Grundlage von Untersuchungsergebnissen aus dem $360 \mathrm{~km}^{2}$ großen Einzugsgebiet der Parthe war es möglich, einen gebietsbezogenen Vergleich zwischen gemessenen und auf Grundlage von Standortuntersuchungen (Lysimetermessungen) ermittelten Abflüssen durchzuführen. In der Gesamtbilanz konnte (unter Berücksichtigung des Grundwasserexports für die Trinkwasserversorgung von Leipzig) eine gute Übereinstimmung erzielt werden. Zur weiteren Präzisierung der Aussagen wurden die Ergebnisse der Ganglinienseparation (MELLENTIN \& HAFERKORN, 1999) genutzt. Beim Vergleich zwischen gebietsintegralen und auf Grundlage von Standortuntersuchungen ermittelten Abflüssen zeigten sich nun deutliche Unterschiede im innerjährlichen Gang. Diese Unterschiede im zeitlichen Verlauf der Abflusskomponenten verweisen auf das unterschiedliche Systemverhalten des Einzugsgebietes im Vergleich zum Standort. Von einer Regionalisierung hydrologischer Größen kann also erst dann gesprochen werden, ,wenn der laterale Transport berechnet wird und dabei Übertragungsbedingungen von einem Rasterpunkt (bzw. Fläche) zum nächsten berücksichtigt werden, das heißt, auch hierbei räumliche Zusammenhänge einbezogen werden“ (KLEEBERG \& CEMUS, 1992).

Zur Beurteilung der Repräsentativität der ermittelten Bodenwasserhaushaltsgrößen interessieren nicht nur die klimatischen Bedingungen im Untersuchungszeitraum, sondern auch die Frage, ob der Untersuchungszeitraum 1981-97 bereits alle gebietstypischen Extreme enthält. Nur wenn dies der Fall ist, sind die zum Bodenwasserhaushalt getroffenen Aussagen (= statistischen Parameter, Korrelation und Regressionsbeziehungen) repräsentativ. Hier 
zeigte sich am Beispiel ausgewählter anthropogen unbeeinflußter Grundwasserstandsganglinien, dass in den Jahren 1940-41, 1961 und 1966 vermutlich deutlich höhere Niederschläge auftraten als im Untersuchungszeitraum 1981-97 registriert werden konnten. Dagegen repräsentieren die Jahre von 1988-91 eine gebietstypische Trockenperiode, wie sie bereits in den Jahren 1931-34, 1950-52 und 1975-77 zu verzeichnen war. Desweiteren wurde festgestellt, dass sich der Untersuchungszeitraum 1981-97 von den Reihen 1901-50 und 195180 durch eine erhöhte potentielle Verdunstung und eine etwas veränderte innerjährliche Verteilung der Niederschläge unterscheidet.

Obwohl der Zeitraum 1981-97 aus hydrologischer Sicht eine interessante Spannbreite von sehr trockenen bis zu sehr feuchten Jahren beinhaltet, zeigte sich bei Untersuchungen z. B. zur Verdunstungsleistung und Ertragsbildung einzelner Kulturen, dass die entsprechenden Beobachtungsjahre nicht alle am Standort Brandis möglichen Witterungsabläufe widerspiegeln. Die Anbaujahre für Zuckerrüben und Kartoffeln waren vergleichsweise zu feucht, nur die Jahre mit Wintergetreide enthalten einige sehr trockene Jahre. In Bezug auf die angebauten Kulturen war die Anzahl der Untersuchungsjahre also nicht ausreichend. 


\section{Schlussfolgerungen}

Mittels Lysimeter wurden der Bodenwasserhaushalt und der Stickstoffaustrag über das Sickerwasser von acht typischen Böden Mitteldeutschlands (mit jeweils drei Wiederholungen) untersucht. Gegenstand der Untersuchungen waren sowohl Standorte mit Sandlöß über Schmelzwassersanden, Sandlöß über Geschiebelehm als auch Standorte mit Geschiebemergel und tiefgründigem Löß.

Alle Böden unterlagen gleichen Einflüssen in Bezug auf Bewirtschaftung, Witterung und Klima (Leipziger Tieflandsbucht). Die für den Untersuchungszeitraum von 1981-97 ermittelten Ergebnisse führten zu folgenden Schlußfolgerungen:

\section{- Zur Lysimetertechnik}

Die Genauigkeit und Repräsentativität von Lysimetermessungen resultiert aus der aufgabenbezogenen baulichen Ausführung und messtechnischen Ausstattung der Lysimeter. In Bezug auf die Brandiser Anlage zeigten sich zwei Probleme:

1. Um Stauwassereinflüsse zu vermeiden, darf die Kapillarwasserzone, die sich über der Grundfläche des Lysimeterbehälters aufbaut, nicht in die verdunstungsbeeinflußte Zone hinaufreichen. Auf Grund der Schichtung der Böden und der erreichten Ausschöpfungstiefen kann bei den Lößböden ein Einfluß der Bodenplatte nicht ausgeschlossen werden, da die tiefste Lage der Wasserscheide bis zur Schichtgrenze Löß-Schmelzwassersand bei ca. 2,0 2,2 m unter Gelände (einzelne Wurzelbahnen bis 1,7 m) reicht und eine Anlieferung von Stauwasser aus dem Bereich der Bodenplatte erfolgen kann. Der Einfluß der Bodenplatte auf die Wasserhaushaltsgrößen bzw. die tatsächlich erforderliche Lysimetergröße kann im Rahmen weitererführender Arbeiten mittels Modellrechnungen bei Variation der unteren Berandung ermittelt werden.

2. Die mit Hellmann-Messern (1 Gerät in $1 \mathrm{~m}$ Höhe $=$ Standardaufstellung, 2 Geräte in Bodenniveau) ermittelten Niederschläge dienten der Berechnung der Lysimeterverdunstung. Dazu musste möglichst genau bestimmt werden, wie viel Niederschlag auf die Bodenoberflächen der 24 verschiedenen Lysimeter gelangte. Dies erforderte relativ aufwendige Niederschlagskorrekturen und bereitete insbesondere bei Starkregen und Schneeverwehungen erhebliche Probleme. Abhilfe kann durch kontinuierliche Wägung der Lysimeter geschaffen werden. Dann ist es möglich, den Niederschlag auf Grundlage der Gewichtsänderung (unter Annahme, dass während des Niederschlagsereignisses keine Verdunstung stattfindet) direkt am Lysimeter zu bestimmen. Über technische und finanzielle Realisierungsmöglichkeiten wird derzeit nachgedacht.

Die acht verschiedenen Lysimeterböden wurden im Einzugsgebiet der Parthe, sowie im Raum Oschatz (Mittelsächsisches Lößhügelland), Wittenberg (Fläming) und Etzdorf östlich von Halle (Mitteldeutsches Schwarzerdegebiet) entnommen. Repräsentative Meßwerte und eine Rückübertragung der Ergebnisse auf die Herkunftsfläche sind nur möglich, wenn Untersuchungsstandort und Herkunftsfläche der Böden keine die Wasserbilanz und den Stoffhaushalt beeinflussenden Unterschiede aufweisen.

Untersuchungen zu klimatischen Unterschieden zwischen Brandis und den Herkunftsflächen ergaben, dass nur die Herkunftsfläche Etzdorf (Löß-Schwarzerde), im Regenschatten des Harzes, mit einer klimatischen Wasserbilanz von rd. $-200 \mathrm{~mm} / \mathrm{Jahr}$ wesentliche Abweichungen zu Brandis aufweist. Infolge der größeren Sickerwasserbildung ist in der Löß- 
Schwarzerde am Lysimeterstandort Brandis ein erhöhter, standortuntypischer Stickstoffumsatz infolge Mineralisation zu verzeichnen.

Probleme dieser Art lassen sich vermeiden, wenn die Lysimeter, wie im Fall des hessischen Lysimetermessnetzes (KLAUSING, 1992) auf den Herkunftsfläche verbleiben, deren Auswahl in der Regel nach gewässerkundlichen und naturräumlichen Gesichtspunkten erfolgt. Von Nachteil ist der relativ hohe Aufwand beim Betrieb eines „Lysimetermeßnetzes“ zumal tatsächlich (in der Lysimetermethode begründet) eine Verbindung zwischen dem Wasserhaushalt im Lysimeter und den hydrogeologischen (gewässerkundlichen und naturräumlichen) Gegebenheiten am Lysimeterstandort nicht mehr besteht.

\section{- Zur Genauigkeit der klimatische Wasserbilanz (kWB)}

Eine Übereinstimmung zwischen der im „Löß“-Lysimeter gemessenen potentiellen Bodenwasserausschöpfung und dem mittels klimatischer Wasserbilanz (kWB) ermittelten Wasserdefizit ist nur zu erreichen, wenn der Berechnung der kWB der korrigierte d. h. der tatsächlich auf die Bodenoberfläche gelangte Niederschlag zu Grunde gelegt wird.

Von Einfluß auf die Genauigkeit der kWB ist darüberhinaus die Wahl des Verfahrens zur Berechnung der potentiellen Verdunstung. Vergleichsrechnungen u. a. mit den Verfahren von TURC/IVANOV, HAUDE und der Gras-Referenzverdunstung ergaben bei kleinen Zeitintervallen (Tages- und Dekadenwerten) große Unterschiede. Die größten Abweichungen zeigten die Berechnungen nach HAUDE im Verlauf sehr trockener aber auch im Verlauf sehr feuchter Perioden. Deshalb sollte dieses Verfahren vor allem bei kleinen Zeitintervallen nicht verwendet werden.

Zur Nivellierung der unterschiedlichen Berechnungsansätze führt der DWD derzeit die von der FAO als Standard empfohlene „Gras-Referenzverdunstung“ auf Basis der PENMANMONTEITH-Beziehung ein, die auch in dieser Arbeit zur Beschreibung des Verdunstungsanspruchs der Atmosphäre verwendet wurde.

\section{- Ermittlung der Kapazität der Bodenwasserspeicher mittels Lysimeter}

Auf Basis der Lysimetermessungen war es möglich, für verschiedene Böden bei wechselndem Bewuchs, die pflanzenverfügbare Bodenwassermenge zu ermitteln und mit Werten, die auf Grundlage von bodenkundlichen Erhebungen (Körnung, effektive Lagerungsdichte, Humusund Skelettgehalt, Bodenart) aus der Bodenkundlichen Katieranleitung (KA 4, 1994) abgeleitet wurden, zu vergleichen.

Es konnten Abhängigkeiten der nutzbaren Feldkapazität im effektiven Wurzelraum (nFK $\left.\mathrm{We}_{\mathrm{W}}\right)$ sowohl von den Standortbedingungen (pflanzenverfügbare Bodenwassermenge der Lößböden $>400 \mathrm{~mm}$ ), als auch Auswirkungen von Bewirtschaftungsmaßnahmen (Beispiel Lysimetergruppe 11) auf das Wasserspeichervermögen nachgewiesen werden. Die in Einzeljahren in Abhängigkeit von der klimatischen Wasserbilanz, der angebauten Kultur und vom Witterungsverlauf ermittelten tatsächlichen Bodenwasserentnahmen, liefern wesentliche Ergänzungen zu bereits vorhandenen Literaturwerten.

\section{- Verdunstung und Wasserverbrauch der Pflanzen}

Auf Grund des hohen Verdunstungsanspruchs der Atmosphäre und der hohen Sommerniederschläge werden auch auf den Böden mit geringer Wasserspeicherkapazität im Mittel der Jahre rd. $75 \%$ des Jahresniederschlages verdunstet. Auf Grund ihres hohen Wasserbereitstellungsvermögens realisieren die Lößböden (in Abhängigkeit von der Bewirtschaftung) selbst im 
Verlauf von Trockenjahren eine kulturartenspezifische maximale Verdunstung, so daß die Berechnung von Bestandskoeffizienten erfolgen konnte. Ein Vergleich zu Literaturwerten führte zu dem Schluß, daß deren kritiklose Anwendung für aktuelle Einzeljahre zu großen Berechnungsfehlern führt, da die tatsächliche Bestandsentwicklung in der Regel stark von den in der Literatur angegebenen Mittelwerten abweicht. Mit den vorliegenden Untersuchungsergebnissen können die Literaturangaben mit aktuellen, standorttypischen Werten für verschiedene Fruchtarten untersetzt werden.

Die limitierte Wasserversorgung der Pflanzen auf den Böden mit geringer und mittlerer $\mathrm{nFK}_{\mathrm{We}}$ wirkte sich bei allen Fruchtarten auch auf die Ertragsbildung aus und führte zu einer ungünstigen Wasserausnutzung. Prinzipiell konnte gezeigt werden: je limitierter die Versorgung der Pflanzen mit Bodenwasser, um so ungünstiger die Evapotranspirationskoeffizienten. Für die ermittelten Evapotranspirationskoeffizienten konnte eine gute Übereinstimmung mit Untersuchungsergebnissen festgestellt werden, die unter vergleichbaren Bedingungen ermittelt wurden. Da es sich dabei sowohl um Feld- als auch um Lysimeterversuche handelte, bestätigte sich, dass mittels Lysimeter geeigneter Bauart realistische Verdunstungswerte und Erträge erbracht werden können.

Aus den Messwerten wägbarer Lysimeter können reale Verdunstung und gleichzeitig der entsprechende Verlauf der Bodenfeuchte (= relativer Bodenwasservorrat in $\% \mathrm{nFK}_{\mathrm{We}}$ ) ermittelt werden. Liegt auch eine Dokumentation der phänologischen Stadien vor, kann die Reaktion der Pflanzen (in den einzelnen Aufwuchsstadien) auf den aktuellen Zustand der Atmosphäre und die Wasser- und Nährstoffentwicklung in den verschiedenen Böden detailliert analysiert und beschrieben werden. Das ist eines der wichtigsten Resultate von Lysimeteruntersuchungen. Im Rahmen dieser Arbeit konnte nur mit einem Beispiel auf diese Möglichkeiten verwiesen werden, so dass die Untersuchungen an dieser Stelle weitergeführt werden müssen.

\section{- Jahresniederschlag und Sickerwasserbildung}

Größten Einfluß auf die Sickerwasserbildung im betrachteten Bilanzjahr haben die Bodenwasserausschöpfung (in Abhängigkeit von Fruchtart und Witterung) und die Höhe der (vergleichsweise geringen) Niederschläge im Anschluß an die Vegetationsperiode. Je geringer die Bodenwasserdefizite am Ende des Sommerhalbjahres und je höher die Niederschläge in den darauffolgenden Herbst- und Wintermonaten, um so eher beginnt die Sickerwasserperiode und um so höher sind die Sickerwassermengen. Aus diesem jährlichen Wechselspiel zwischen Niederschlagshöhe und Bodenwasserdefizit resultiert eine hohe Variabilität der jährlichen Sickerwassermengen. Es besteht kein unmittelbarer Zusammenhang zwischen Wasserverbrauch der Pflanzen und Höhe der Sickerwassermenge und nur ein mäßiger Zusammenhang zwischen Sickerwassermenge und Jahresniederschlag. Von Nachteil für die Sickerwasserbildung ist der hohe Anteil von Sommerniederschlägen am Jahresniederschlag, da diese nahezu vollständig verdunsten.

Hervorzuheben ist die Sickerwasserleistung (bis zu $300 \mathrm{~mm}$ in Feuchtjahren) der Geschiebelehmböden. Dem Moränenlehm wird in der Regel eine hohe Schutzfunktion für das Grundwasser zugeschrieben. Dies trifft, auf Grund ihrer Entstehungsgeschichte, für die hier untersuchten Böden nicht zu. 


\section{- Notwendigkeit langer Untersuchungszeiträume}

Wie am Beispiel ausgewählter anthropogen unbeeinflußter Grundwasserstandsganglinien gezeigt werden konnte, enthielt der Zeitraum 1981-97 aus hydrologischer Sicht eine interessante Spannbreite von sehr trockenen bis zu sehr feuchten Jahren. Für die Untersuchungen zur Verdunstungsleistung und Ertragsbildung einzelner Kulturen war die Anzahl der Beobachtungsjahre zu gering. Die Anbaujahre für Zuckerrüben und Kartoffeln waren vergleichsweise zu feucht und nur der Anbau von Winterweizen erfolgte sowohl in Trockenals auch in Feuchtjahren. Aus diesem Grund ist die Gültigkeit der Nomogramme die auf Basis einzelner Fruchtarten erstellt wurden eingeschränkt, und muß durch weitere Beobachtungsjahre untersetzt werden.

\section{- Wasserhaushaltsbilanzen verschiedener Böden unter landwirtschaftlicher Nutzung}

Im Zusammenhang mit der Bildung von Jahres- und Halbjahresbilanzen (Anlage 8) stellte sich die Frage nach einem geeigneten Bilanzzeitraum, da im 3 m mächtigen Bodenprofil, vor allem in Sommerhalbjahren, zeitgleich zwei Prozesse ablaufen: Der Wasservorrat in den unteren Schichten wird durch auslaufendes Sickerwasser reduziert, während den oberen Bodenschichten bereits Wasser durch die Verdunstung entzogen wird. Im Interesse der Vergleichbarkeit mit anderen hydrologischen Daten wurde der 1. November als Jahresbeginn gewählt.

Die Reihe 1981-97 beinhaltet das Trockenjahr 1982 und die Trockenperiode von 1989-92 sowie die sich anschließenden feuchten Jahre 1993 und 1994. Anhand der Jahresmittelwerte der Vorratsänderung $(\Delta \mathrm{SB})$ ist festzustellen, dass am Ende der 17jährigen Messphase vorübergehende, über mehrjährige Trockenperioden existierende Defizite nahezu wieder ausgeglichen wurden.

Auf Grund des relativ hohen Verdunstungsanspruchs der Atmosphäre, zeigt sich die Verdunstung in der Wasserhaushaltsbilanz der Böden als die große Verlustgröße. Mit Hilfe von Pflanzen mit großer Verdunstungsleistung auf Substraten mit hohem pflanzenverfügbaren Wasser- und Nährstoffvorrat können die Wasserverluste noch erheblich gesteigert werden, wie sich am Bespiel der Lößböden zeigte. Hohe Verdunstungsverluste sind $u$. a. erwünscht, um Vernässungsflächen auf Braunkohlenkippen zu reduzieren oder um auf Deponieabdeckungen die Sickerwassermengen zu minimieren.

\section{- Stickstoff-Saldo und Stickstoff-Austrag mit dem Sickerwasser}

Mit Berechnungen zur jährlichen Austauschhäufigkeit von Bodenwasser (Bodenlösung) im effektiven Wurzelraum und zur Verlagerungsgeschwindigkeit in der Dränwasserzone wurde gezeigt, dass witterungs- und bodenbedingte Effekte gegenüber dem nutzungsbedingten Verlagerungsrisiko eindeutig überwiegen.

Mit Kenntnis des (vorwiegend geringen) Wasserumsatzes in der Bodenzone und der langen Verweilzeit der Bodenlösung in der Dränzone wurde verständlich, warum für einzelne Jahre kein unmittelbarer Zusammenhang zwischen N-Saldo und der Höhe der N-Austräge über das Sickerwasser besteht. Deutlich enger zeigte sich der Zusammenhang zwischen mittlerer jährlicher Stickstoff-Konzentration und Sickerwassermenge. Auch dies ist ein Hinweis darauf, dass die Aussagekraft von jährlichen N-Salden wesentlich von den aktuellen hydrologischen Bedingungen beeinflußt wird.

Werden dagegen langjährige Mittelwerte von N-Saldo und N-Austrag über das Sickerwassers gegenüber gestellt, treten wieder nutzungsbedingte Effekte in den Vordergrund. Ziel einer 
grundwasserschonenden Landbewirtschaftung sollten möglichst geringe N-Salden sein. So dienen alle ökologisch vertretbaren Maßnahmen, die bei geringem Wasserverbrauch der Pflanzen zu hohen, stabilen Erträgen und geringen umweltbelastenden N-Austrägen führen, letztlich auch dem Gewässerschutz.

\section{- Zur Bildung von vier Pedo-Hydrotopen (=Versickerungstypen)}

Die hier vorgestellten Untersuchungen an verschiedenen Böden wurden unter gleichen Klimaund Witterungsbedingungen und gleicher Bewirtschaftung durchgeführt. Somit bestand die Möglichkeit zur Identifikation einzelner Einflussfaktoren auf den Bodenwasserhaushalt. Mittels Varianzanalyse wurde geprüft, wie signifikant sich Wasserhaushaltsgrößen verschiedener Böden tatsächlich voneinander unterscheiden.

Im Ergebnis der Untersuchungen konnte festgestellt werden, dass der Bodenwasserhaushalt von Böden mit flurfernen Grundwasserständen bei gleicher Witterung und Bewirtschaftung, primär durch die Ausgangsgesteine geprägt wird. D. h. ähnliche Bodentypen weisen, trotz Abweichungen in der Horizontabfolge, in Bezug auf ihren Bodenwasserhaushalt keine Unterschiede auf, die über die natürliche Variabilität der Standorte hinausgehen. Deshalb konnten die acht analysierten Bodentypen zu vier Pedo-Hydrotopen zusammen gefasst werden, wobei der Begriff der „hydrologischen Wirksamkeit“ (hier im Sinne der PedoHydrotopen) auch den Anteil des Wasserhaushaltes an der Bodenfruchtbarkeit mit einschließt.

Mit diesen Erkenntnissen vereinfacht sich die Übertragung von Standortuntersuchungen auf die Fläche. Erfordern Untersuchungen im Mikro- und Mesoskale die separate Berücksichtigung von einzelnen Bodentypen, so ist beim Übergang zum Makroskale eine vereinfachende Betrachtungsweise im Sinne der abgeleiteten Pedo-Hydrotope ausreichend.

\section{- Kontrolle der Untersuchungsergebnisse zum Bodenwasserhaushalt über Gebietswerte des Wasserhaushaltes am Beispiel des Parthegebietes}

Um einen gebietsbezogenen Vergleich zwischen gemessenen und auf Grundlage von Standortuntersuchungen ermittelten Abflüssen vornehmen $\mathrm{zu}$ können, wurde das auf Grundlage der Lysimetermessungen ermittelte „Gesamtdargebot“" auf die Fläche des $360 \mathrm{~km}^{2}$ großen Parthegebietes extrapoliert. Für diesen Vergleich standen die Ergebnisse einer Ganglinienseparation für den Abflußpegel Thekla zur Verfügung.

Im Mittel der Jahre konnte bilanzmäßig eine gute Übereinstimmung erzielt werden. Die Unterschiede die sich im innerjährlichen Gang der Abflussbildung zeigten, verweisen erwartungsgemäß auf das, im Vergleich zum Standort, unterschiedliche Systemverhalten des Einzugsgebietes. Auf Grund dieser Unterschiede erfordern vor allem Untersuchungen zum wassergebundenen Stofftransport und -umsatz eine in sich geschlossene Betrachtung des Wasserhaushaltes im Einzugsgebiet, denn nur mit der Kenntnis von Herkunftsräumen, Fließwegen und Verweilzeiten können Beziehungen zwischen Ursache (antrophogene Stoffeinträge über den Boden) und Wirkung (Stoffkonzentrationen im Boden-, Grund- und Oberflächenwasser) hergestellt werden. 


\section{Literaturverzeichnis}

ALLEN, R.G.; SMITH, M.; PERRIER, A. u. PEREIRA, L.S. (1994): An update for definition of reference evapotranspiration. ICID Bulletin 43, No.2, 1-34.

ALY, I. (1988): Vergleich verschiedener Methoden zur Berechnung der Bodenfeuchte eines Zuckerrüben- und Weizenbestandes auf dem Versuchsfeld Zöberitz. Dissertation, Landwirtschaftliche Fakultät für Naturwissenschaften der Martin-Luther-Universität Halle/Wittenberg.

AMBERGER, A.; SCHWEIGER, P. (1978): Substanzproduktion und Sickerwassermengen verschiedener Böden in einem langjährigen Lysimeterversuch. Bayr Landw. Jahrb. 55.

BÄTZ, G .; DÖRFEL, H.; FUCHS, A.; THOMAS, E. (1982): Einführung in die Methodik des Feldversuchs. VEB Deutscher Landwirtschaftsverlag, Berlin.

BECKER, K.-W. (1994): Der klimatologische und pedohydrologische Vergleich Lysimeter Freiland als Grundlage der Datenübertragung. BAL Gumpenstein.

BfG (1997): Wasserhaushaltsverfahren. DFG-Projekt GL 242/1-2. Zwischenbericht (unveröff.). Bundesanstalt für Gewässerkunde, AS Berlin.

BONTA, J. V.; EDWARDS, W. M.; OWENS, L. B.; HAROLD, L. (1989): Hydrologie and Water Quality Studies of Agricultural Watersheds. Ohio Agricultural Research and Development Center.

BÖTTCHER, J.; FREDE, H. G.; MEYER, B. (1983): Chlorid- und Nitratverlagerung in LössLysimetern bei unterschiedlichen Grundwasserständen. Mitt. Dtsch. Bodenkundl. Ges. 38.

BOUWER, W.; GÄTH, H.; FREDE, G. (1997): Vergleich dreier Instrumentarien zur Abschätzung und Kontrolle der nutzungsbedingten Nitratauswaschung auf auswaschungsgefährdeten Standorten. Z. f. Kulturtechnik und Landentwicklung, 38, 154-160.

DENMEAD, O. T.; SHAW, R. H. (1962): Availability of soil water to plants as affected by soil moisture content and meteorological conditions. Agron. J. 54: 385-390.

DFG (1987): Anthropogene Einflüsse auf den lokalen und regionalen Bodenwasserhaushalt. Hrsg. von R. Mull, VCH Verlagsgesellschaft mdH, Weinheim.

DIEKKRÜGER, B. (1992): Standort- und Gebietsmodelle. Landschaftsökologie und Umweltforschung, H. 19, Institut für Geographie und Geoökologie, TU Braunschweig.

DIN 4049 (1994): Teil 1: Hydrologie - Begriffe zur quantitativen Hydrologie. Deutsches Institut für Normung.

DISSE, M. (1995): Modellierung der Verdunstung und der Grundwasserneubildung in ebenen Einzugsgebieten. IHW Universität Karlsruhe (TH), Heft 53.

DÖRHÖFER, G.; JOSOPAIT, V. (1980): Eine Methode zur flächendifferenzierten Ermittlung der Grundwasserneubildungsrate. Geol. Jahrbuch C 27, Hannover.

DÖRING, J. (1994): Ergebnisse des Vergleichs der Daten zur Lufttemperatur und Sonnenscheindauer zwischen Brandis und Leipzig-Schkeuditz bzw. Oschatz. Arbeitsbericht (unveröffentlicht), Landwirtschaftliche Fakultät der Martin-Luther-Universität Halle/Wittenberg.

DÖRING, J.; MÜLLER, J.; JÖRN, M.; NEUBERT, S.; PANNICKE, I.; WEDEKIND, G.; SCHURIGT, J. (1995): Klimatische Kennzeichnung des mitteldeutschen Schwarzerde- 
gebietes. In Strategien zur Regeneration belasteter Agrarökosysteme des mitteldeutschen Schwarzerdegebietes. B. G. Teubner Verlagsgesellschaft Stuttgart ` Leipzig.

DUNGER, V. (1999): Dokumentation des Modells BOWAM zur Simulation des Wasserhaushaltes in der wasserungesättigten Bodenzone. TU Bergakademie Freiberg.

DUTELOFF, T. (1996): Überlegungen zur Übertragbarkeit der Brandiser Lysimeteruntersuchungen. Kurzbericht (unveröff.). Sächsisches Landesamt für Umwelt und Geologie.

DUYNISVELD, W. (1984): Entwicklung und Anwendung von Simulationsmodellen für den Wasserhaushalt und den Transport von gelösten Stoffen in wasserungesättigten Böden. Dissertation, Technische Universität Berlin.

DVWK (1994): Verminderung des Stickstoffaustrages aus landwirtschaftlich genutzten Flächen in das Grundwasser - Grundlagen und Fallbeispiele. Schriften Nr. 106. Kommissionsvertrieb Wirtschafts- und Verlagsgesellschaft Gas und Wasser mbH, Bonn.

DVWK (1996): Ermittlung der Verdunstung von Land- und Wasserflächen. Merkblatt 238. Kommissionsvertrieb Wirtschafts- und Verlagsgesellschaft Gas und Wasser mbH, Bonn.

DYCK, S.; PESCHKE, G. (1995): Grundlagen der Hydrologie. Verlag für Bauwesen Berlin.

EHLERS, W. (1989): Beziehungen zwischen Wurzelsystem-Parametern und der Wasseraufnahme von Kulturpflanzen, Mitteilgn. Dtsch. Bodenkundl. Gesellsch. 59/1.

EHLERS, W. (1996): Wasser in Boden und Pflanze. Eugen Ulmer GmbH \& Co., Stuttgart.

EISSMANN, L. (1970): Geologie des Bezirkes Leipzig, Eine Übersicht. Natura regionis Lipsiensis, Heft 1, Naturwissenschaftliches Museum Leipzig.

ETZENBERG, CH. (1998): Zur Regionalisierung der Abflußbildung - Bestimmung flächenspezifischer Abflußbeträge aus Gebietseigenschaften. Dissertation, Internationales Hochschulinstitut Zittau.

EULENSTEIN, F.; WURBS A. (1994): Konzepte und Modellvorhaben zum Grundwasserschutz in den neuen Bundesländern. KTBL-Arbeitspapier 206: Strategien zur Verminderung der Nitratauswaschung in Wasserschutzgebieten, 183-192.

FREDE, H.-G.; DABBERT, H.-G. (Hrsg.) (1999): Handbuch zum Gewässerschutz in der Landwirtschaft. Ecomed-Verlagsgesellschaft, Landsberg.

FUNK, R. (1993): Verlagerung und Abbau von Nitrat in tiefere Bodenschichten süddeutscher Ackerstandorte. Dissertation. Universität München.

GALL, H.; BURCK, M.; KLITZSCH, S.; ZACHOW, B. (1994): Ergebnisse der Lysimetermessungen in Groß Lüsewitz aus pflanzenbaulicher Sicht. Wasser und Boden, Heft 10.

GENID, A. (1982): Wasser-Haushalt von Löss in Lysimetern. Dissertation. Fachbereich Agrarwissenschaften der Georg-August-Universität Göttingen.

GERIES, H. (1989): Nitrat unter Acker in mächtigen Lössdecken mit tiefem Grundwasserstand im Klimaraum Südniedersachsen: Tiefen-Verlagerung, Datierung, Umsatz und Einbindung in den Gashaushalt. Diss. Institut für Bodenwissenschaft der Georg-AugustUniversität Göttingen.

GERMANN, P. (1981): Untersuchungen über den Bodenwasserhaushalt im hydrologischen Einzugsgebiet Rietholzbach. Mitteilungen der Versuchsanstalt für Wasserbau, Hydrologie und Glaziologie Nr. 51, ETH Zürich. 
GLUGLA, G. (1993): Eine Variante des Blockmodells VERMO zur Berechnung aktueller Werte des Bodenwasserhaushalts. Programmbeschreibung (unveröff). Bundesanstalt für Gewässerkunde, Außenstelle Berlin.

GLUGLA, G. (1998): Anforderungen an Anlagen mit wägbaren Lysimetern und an Beobachtungen zur Bestimmung des Wasser- und Nährstoffhaushaltes, (unveröff.). DVWKFachausschuß 1.11 ,Verdunstung“.

GOLDSCHMIDT, J. (1950): Das Klima von Sachsen. Abhandlungen des Meteorologischen Dienstes der DDR, Nr. 3, Akademie Verlag Berlin.

GOLF, W. (1982): Niederschlagskorrektur und Wasserbilanz. Zeitschrift für Meteorologie, Heft 32.

GOLF, W. (1993): Unterlagen zur Vorlesung Wasserhaushalt. Institut für Hydrologie der TU Dresden.

GRAHMANN, R. (1943): Abhandlung des Sächsischen Geologischen Landesamt, Heft 16.

GÜK 400 (1992): Geologische Übersichtskarte Sachsen 1 : 400 000. Sächsisches Landesamt für Umwelt und Geologie.

GÜNTER, R. (1987): Entwicklung einer Wasserhaushaltsmeßstation auf der Basis wägbarer Lysimeter sowie Ergebnisse zum Wasserverbrauch und zur Wasserbilanz von Winterweizen, Sommergerste und Zuckerrüben auf einer Löß-Braunschwarzerde. Diss., Universität Jena.

GÜNTHER, R. (1999): Vergleich von berechneten Verdunstungswerten und Lysimetermessungen. DVWK- Veranstaltung vom 23. bis 24. November in Magdeburg.

GÜNTHER, R.; KNOBLAUCH, S. (1993): Zur Wasser- und Nährstoffbilanz auf einer landwirtschaftlich intensiv genutzten Löß-Braunschwarzerde (Ergebnisse 10-jähriger Lysimeteruntersuchungen). Mittlgn. Dtsch. Bodenkundl. Gesellsch. 71.

GUTSER, R.; HAUK, S. (1994): Pflanzenbauliche Maßnahmen zur Verringerung des Stickstoffaustrages aus landwirtschaftlich genutzten Flächen. DVWK Schriften 106.

GUTSER, R.; HEYN, J.; AMBERGER, A.; BRÜNE, H. (1987): Zur Stickstoff- und Mineralstoffauswaschung aus Lößböden - Ergebnisse von Lysimeterversuchen in Darmstadt und Weihenstephan. Landwirtschaftl. Forschung 40 (4).

HAFERKORN, U.; MANSEL, H.; KUHN, K. (1999): Einsatz eines gekoppelten Grund- und Oberflächenwassermodells am Beispiel des Partheeinzugsgebietes. Zeitschrift Wasser und Abfall. Heft 11.

HANSEL, N.; SCHÄFER, U. (1970): Die Doppelsummenanalyse Wasserwirtschaft Wassertechnik 20. Jahrg., 1970, H.5, S. 145-150.

HARTGE, K. H.; HORN, R. (1991): Einführung in die Bodenphysik. Ferdinad Enke Verlag, Stuttgart.

HAUDE, W. (1955): Zur Bestimmung der Verdunstung auf möglichst einfache Weise. Mitteilung des Deutschen Wetterdienstes, Nr. 11 (Bd. 2), Bad Kissingen.

HELLEKES, R. (1985): Analyse des Bodenwasserhaushaltes im Bereich Mönchengladbach Besondere Mitteilungen zum Dt. Gewässerkundlichen Jahrbuch, Nr. 47.

HENNINGS, H.; SCHEFFER, B. (1999): Landwirtschaft und Grundwasserqualität. Literaturstudie. Niedersächsisches Landesamt für Bodenforschung, Bremen. 
HERSEMANN, H. (1997): Simulationsrechnungen mit dem Modell NUBALYS und Vergleich mit Lysimetermessungen der Station Brandis. Arbeitsbericht (unveröff.). Staatliche Landwirtschaftliche Untersuchungs- und Forschungsanstalt Karlsruhe-Augustenberg.

HÖLTING, B.; HAERTLÉ, T.; HOHBERGER K.-H.; NACHTIGALL, K. H.; VILLINGER, E.; WEINZIERL, W.; WROBEL, J.-P. (1995): Konzept zur Ermittlung der Schutzfunktion der Grundwasserüberdeckung. Geologisches Jahrbuch, Reihe C, Hannover.

HÖLTING, B. (1997): Modellrechnungen zur Grundwasserneubildung. Zeitschrift der Fachsektion Hydrogeologie 1/97.

HOSANG, J. (1995): Wasser- und Stoffhaushalt von Lößboden im Niederen Sundgau (Region Basel). Physiogeographica. Basler Beiträge zur Physiogeographie Band 19, Basel.

JANKIEWICZ, P. (1998): Erläuterung der an Lysimeterdaten durchzuführenden Korrekturen. Bericht (unveröff.). Bundesanstalt für Gewässerkunde, AS Berlin.

JORDAN, E. (1978): Wasserhaushaltsuntersuchungen in der Gleidinger Senke. Mitteilgn. Dtsch. Bodenkundl. Gesellsch. 26, 143-152.

KA4 (1994): Bodenkundliche Kartieranleitung. E. Schweizerbart'sche Verlagsbuchhandlung, Hannover.

KATZUR, J.; MROSKO, A. (1983): Einfluß der Sickerwassermengen auf die Höhe des NAustrages aus einer Sand-Rosterde. Z. Wasserwirtschaftstechnik H. 1.

KATZUR, J.; TÖLLE, R.; HOFFMANN, E. (1989): Langzeituntersuchungen zur Nauswaschungsminimierenden Wirkung der Nitrifizide bei Harnstoffdüngung in grundwasserfreien Lysimetern ohne Unterdruck. Arch. Acker- Pflanzenbau Bodenkd. 33.

KEESE, U., KRÖNERT, R., STEINHARDT, U., KNAPPE, S. (1996): Charakteristik des Einzugsgebietes Parthe. In: UFZ-Bericht Nr. 21.

KEESE, U.; KNAPPE, S. (1995): Einfluß unterschiedlicher Lysimetertiefen auf Wasser- und Stoffhaushalt grundwasserferner Standorte am Beispiel von zwei Sandlößböden - Ergebnisse aus der Lysimeterstation Brandis. BAL Gumpenstein.

KEESE, U.; KNAPPE, S. (1996): Problemstellung und allgemeine Angaben zu vergleichenden Untersuchungen zwischen Lysimetern und ihren Herkunftsflächen am Beispiel von drei typischen Böden Mitteldeutschlands unter landwirtschaftlicher Nutzung. Arch. Acker-Pfl. Boden, 40.

KEESE, U.; NITSCHE, C.; KNAPPE, S.; WALDSCHMIDT, U. (1997): Vergleichende bodenphysikalische Untersuchungen zwischen Lysimetern und ihren Herkunftsflächen am Beispiel von drei typischen Böden Mitteldeutschlands unter landwirtschaftlicher Nutzung. I. Mitteilung: Ermittlung bodenphysikalischer Kenngrößen. Arch. Acker-Pfl. Boden, 41.

KLAGHOFER, E. (1991): Bodenphysikalische Aspekte bei der Erfassung von gelösten Stoffen mit Hilfe von Lysimetern. Bericht über die 1. Lysimetertagung, BAL Gumpenstein.

KLAUSING, O. (1992): Ergebnisse der Lysimeter-, Bodenfeuchte- und Verdunstungsmessungen 1971-90 in Hessen. Schriftenreihe der Hessischen Landesanstalt für Umwelt, Wiesbaden.

KLEEBERG, H.-B.; CEMUS, J. (1992): Regionalisierung hydrologischer Daten Definitionen. In: Regionalisierung in der Hydrologie, Hrsg. von Kleeberg, Weinheim.

KNAPPE, S., MORITZ, CH., KEESE, U. (1994): Grundwasserneubildung und N-Austrag über Sickerwasser bei intensiver Landnutzung - Lysimeteruntersuchungen an acht Bodenformen in der Anlage Brandis. Arch. Acker- Pflanzenbau Bodenkd. 38. 
KNAPPE, S.; KEESE, U.; KALBITZ, K. (1997): Lysimeteruntersuchungen zur Wirkung von Flächenstillegungsmaßnahmen auf den Stickstoffaustrag und den Nitratgehalt von Sickerwasser von vier Bodenformen. BAL Gumpenstein.

KNOBLAUCH, S.; ROTH, D.; PFLEGER, I. (1999): Beziehung zwischen N-Saldo, $\mathrm{N}_{\min }$ im Boden, Nitratgehalt im Sickerwasser und N-Austrag bei unterschiedlichen Ackerstandorten Thüringens. BAL Gumpenstein.

KOITZSCH, R. (1977): Schätzung der Bodenfeuchte aus meteorologischen Daten, Bodenund Pflanzenparametern mit einem Mehrschichtenmodell. Zeitschrift für Meteorolgie, Bd. 27, H. 5, 302-306.

KOITZSCH, R. (1980): Simulation des Bodenfeuchteverlaufes unter Berücksichtigung der Wasserbewegung durch Pflanzenbestände. Archiv Acker- und Pflanzenbau und Bodenkunde, Berlin 24/11, 717-725.

KOITZSCH, R; DZINGEL, M. (1983): Entwicklung eines Verfahrens zur Schätzung von Tageswerten der aktuellen Evapotranspiration von Zuckerrüben aus meteorologischen Standarddaten, Boden- und Pflanzenparametern. Forschungsbericht. Forschungsinstitut für Agrarmeteorologie, Müncheberg.

KÖRSCHENS, M.; MAHN, E.-G. (Hrsg.), (1995): Strategien zur Regeneration belasteter Agrarsysteme des mitteldeutschen Schwarzerdegebietes. B. G. Teubner Verlagsgesellschaft Stuttgart · Leipzig.

KTBL - Arbeitspapier 206: Strategien zur Verminderung der Nitratauswaschung in Wasserschutzgebieten, 143-155.

KUNDLER, P. und Autorenkollektiv (1989): Erhöhung der Bodenfruchtbarkeit. VEB Deutscher Landwirtschaftsverlag Berlin.

KUNKEL, R.; WENDLAND, F. (1998): Der Landschaftswasserhaushalt im Flußeinzugsgebiet der Elbe. Foschungszentrum Jülich GmbH.

KUNTZE, H.; ROESCHMANN, G.; SCHWERDTFEGER, G. (1994): Bodenkunde. Verlag Eugen Ulmer, Stuttgart.

LARCHER, W. (1994): Ökophysiologie der Pflanzen. 5. Aufl., UTB.

LfUG; IBGW (1994): Sächsisches Landesamt für Umwelt und Geologie (LfUG) und Ingenieurbüro für Grundwasser (IBGW) Leipzig GmbH (1994): Hydrogeologisches Modell für den Raum des Parthegebietes (PART).

LIEBSCHER, H. J. (1970): Eine neue, wägbare und registrierende Lysimeteranlage in Niedermending (Eifel). Dt. Gewässerkundl. Mitt..

LIEDKE, H.; MARCINEK, J. (Hrsg.) (1995): Physische Geographie Deutschlands. Justus Perthes Verlag, Gotha.

LINKE, M. (1991): Beurteilung der Standortbedingungen in den früheren ostdeutschen und den ehemaligen bundesdeutschen Gebieten unter dem Aspekt der Getreideproduktion. Z. Meteorol. 41, H.2, 142-147.

MANNSFELD, K.; RICHTER, H. (Hrsg.) (1995): Naturräume in Sachsen. Forschungen zur deutschen Landeskunde 238.

MELLENTIN, U.; HAFERKORN, U. (1999): Bericht zur Ganglinienseparation an Oberflächenwassermeßstellen der Parthe und des Schnellbaches. Arbeitsbericht (unveröff.). Staatliche Umweltbetriebsgesellschaft, Lysimeterstation Brandis. 
MEYNEN, E.; SCHMITHÜSEN, J. (Hrsg.) (1953 bis 1962): Handbuch der naturräumlichen Gliederung Deutschlands. Bundesanstalt für Landeskunde und Raumforschung, Selbstverlag Bad Godesberg.

MORITZ, CH.; SPENGLER, R.; SÄMISCH, G. (1991): Die Basislysimeterstation Brandis bei Leipzig - Einrichtung und erste Untersuchungsergebnisse. Deutsche Gewässerkundliche Mitteilungen 35. 1991 H. 5/6.

MÜLLER, G. (Hrsg.) (1989): Bodenkunde. VEB Deutscher Landwirtschaftsverlag, Berlin.

MÜLLER, J.; KRÜGER, R.; SCHELLIN, H.-G. (1991): Zum Trend der Evapotranspiration im Zeitraum 1901-1985. - Eine Untersuchung auf der Grundlage von Modellrechnungen für einen Standort im mitteldeutschen Raum. Deutscher Wetterdienst intern, Nr. 41.

MÜLLER, L.; DANNOWSKI, R.; SCHINDLER, U.; EULENSTEIN, F.; MEISSNER, R. (1996): Gebietsabflüsse aus Agrarlandschaften Nordost- und Mitteldeutschlands. Arch. Acker-Pfl. Boden (40), S. 345-362.

MÜLLER, S.; HANSCHMANN, A.; HEINRICH, L.; BRIX, B. (1991): Sickerwasser und Nitrataustrag - Lysimeteruntersuchungen für Sand-, Lehm- und Lößböden unter einheitlichen Bedingungen. Arch. Acker- und Pflanzenbau Bodenkd. 35.

MÜNCH, A. (1994): Wasserhaushaltsberechnungen für Mittelgebirgseinzugsgebiete unter Berücksichtigung einer sich ändernden Landnutzung. Dissertation, Fakultät für Geo-, Forstund Hydrowissenschaften der TU Dresden.

NATURRÄUME (1986): Naturräume der sächsischen Bezirke. Sächsische Heimatblätter, Sonderdruck aus den Heften 4/5.

OLBERTZ, M. (1957): Über die am Standort des Kulturbodens erfaßbaren Größen des Wasserhaushaltes. Wiss. Abhandlung der AdL, Nr. 23, Berlin.

OLBRISCH, H.-D. (1975): Beitrag zur Methodik der Datenanalyse bei Lysimeteruntersuchungen. Mitteilungen Institut für Wasserbau und Wasserwirtschaft. RheinischWestfälische Technische Hochschule Aachen.

OLIKOVA, I. S.; SYCHEVA, S. A. (1996): Water Regime of Virgin Chernozems in the Central Russian Upland Ist Changes. Eurasian Soil Science. Vol. 29, No. 5.

PESCHKE, G. (1999): Vorwort in SEIDLER, CH. (1999): Ursachen und Charakterisierung eines eingeschränkten Wasserhaushaltes sowie seine Auswirkungen auf das Pflanzenwachstum. Dissertation, Internationales Hochschulinstitut Zittau.

PESTER, L. (1978): Idealprofile für geotechnische Arbeiten in der Braunkohlenindustrie. Neue Bergbautechnik. 8. Jg. Heft 6.

PFISTER, C. (1999): Wetternachhersage. Verlag Paul Haupt, Bern, Stuttgart, Wien.

PRETSCHOLD, H.-H. (1963): Die hydrogeologische Kartierung des Parthegebietes. Unveröffentlichter Forschungsbericht der Wasserwirtschaftsdirektion Saale - Weiße Elster, Halle.

PROKSCH, W. (1990): Lysimeterauswertungen zur flächendifferenzierten Ermittlung mittlerer Grundwasserneubildungsraten. Besondere Mitteilungen zum Deutschen Gewässerkundlichen Jahrbuch, Nr. 55. Bundesanstalt für Gewässerkunde in Koblenz.

RAPP, J.; SCHÖNWIESE, CH.-D. (1996): Atlas der Niederschlags- und Temperaturtrends in Deutschland 1891-1990. Frankfurter Geowissenschaftliche Arbeiten, Serie B, Meteorologie und Geophysik, Band 5, Frankfurt/Main. 
RENGER, M. (1992): Bestimmung der Bodenwasserhaushaltskomponenten. DVGWSchriftenreihe Wasser 72, 283-298.

RENGER, M.; STREBEL, O.; GIESEL, W. (1974): Beurteilung bodenkundlicher, kulturtechnischer und hydrologischer Fragen mit Hilfe von klimatischer Wasserbilanz und bodenphysikalischen Kennwerten. Z. f. Kulturtechnik und Flurbereinigung H. 15.

RENGER, M.; WESSOLEK, G. (1990): Auswirkungen von Grundwasserabsenkungen und Nutzungsänderungen auf die Grundwasserneubildung. Mitt. Inst. für Wasserwesen, Bundeswehr Hochschule München, Band 38 B.

RENGER, M.; WESSOLEK, G. (1996): Berechnung der Verdunstungsjahressummen einzelner Jahre. In: DVWK-Merkblätter zur Wasserwirtschaft, Heft 32 (8), Bonn.

RICHTER, D. (1995): Ergebnisse methodischer Untersuchungen zur Korrektur des systematischen Meßfehlers des Hellmann-Niederschlagsmessers. Bericht des Deutschen Wetterdienstes, Nr. 194.

RICHTER, U.; HARRACH, T.; SCHMÜCKER, M. (1994): Abschätzung der Nitratverlagerung für repräsentative Testflächen in drei Hessischen Wasserschutzgebieten.

RIJTEMA, P. E. (1965): An analysis of actual evapotranspiration. Agricultural Research Reports No. 659, Wageningen.

RODDA, J. (1971): The precipitation measurement paradox - The instrument accuracy problem. WMO/IHD Report No. 16, Genf.

ROTH, D.; GÜNTHER, R.; ROTH, R. (1988): Transpirationskoeffizienten und Wasserausnutzungsraten landwirtschaftlicher Fruchtarten 1. Mitteilung: Transpirationskoeffizienten und Wasserausnutzungsraten von Getreide, Hackfrüchten und Silomais unter Feldbedingungen und in Gefäßversuchen. Arch. Acker- Pflanzenbau Bodenkd., Berlin 32 H. 6.

ROTH, D.; GÜNTHER, R.; ROTH, R. (1993): Der Einfluß unterschiedlicher Wasserversorgung auf die Wassernutzung landwirtschaftlicher Fruchtarten. Z. f. Kulturtechnik und Landentwicklung 34, Heft 2.

ROTH, D.; GÜNTHER, R.; KNOBLAUCH, S. (1994): Technische Anforderungen an Lysimeteranlagen als Voraussetzung für die Übertragbarkeit von Lysimeterergebnissen auf landwirtschaftliche Nutzflächen. BAL Gumpenstein.

ROTH, D.; KNOBLAUCH, S.; HEROLD, L.; PFLEGER, I. (1995): Untersuchungen zur Sickerwasserbildung und zum Stickstoffaustrag auf Ackerböden Thüringens. BAL Gumpenstein.

RUSSOW, R., KNAPPE, S.; FÖRSTEL, H. (1996): Vergleich der Wasser- und Anionenbewegung in einem landwirtschaftlich genutzten Sandlöß-Braunerde- und LößSchwarzerde-Boden an Hand von Lysimeteruntersuchungen. BAL Gumpenstein.

SAMBALE, C.; PESCHKE, G. (2000): Vegetationsbedingte Verdunstungsunterschiede und ihre Auswirkungen auf die hydrologische Modellierung. Wasser \& Boden, 52.

SÄMISCH, G. (1990): Jahresarbeitsberichte 1981-90 (unveröff.). Staatliche Umweltbetriebsgesellschaft, Lysimeterstation Brandis.

SCHEFFER, F.; SCHACHTSCHABEL, P. (1992): Lehrbuch der Bodenkunde. Ferdinand Enke Verlag, Stuttgart.

SCHEUERER, W. (1998): Flächendifferenzierte quasidreidimensionierte Simulation des Bodenwasserhaushaltes während der Vegetationsperiode unter einheitlicher Nutzung in Scheyern. Herbert Utz Verlag-Wissenschaft, München. 
SCHILLING, D.; BANNORTH, H.; SCHLICHT, H.(1965): Natürliche Standorteinheiten der landwirtschaftlichen Produktion in der DDR. Landwirtschaftsrat der DDR.

SCHILLING, G. (Hrsg.) (1982): Pflanzenernährung und Düngung, Teil 1 - Pflanzenernährung. VEB Deutscher Landwirtschaftsverlag, Berlin.

SCHÖNERMARK, E. (1973): Methodische Untersuchungen zur Berechnung der Globalstrahlung für das Gebiet der DDR. Zeitschrift für Meteorologie, Bd. 23, Heft 9/10.

SCHRÖDER (1980): Empfehlungen zum Bau und Betrieb von Lysimeterstationen. DVWKRegeln zur Wasserwirtschaft 114. Verlag Paul Parey, Berlin, Hamburg.

SCHRÖDTER, H. (1985): Verdunstung. Springer Verlag, Berlin, Heidelberg, New York, Tokyo.

SCHWARZE, R. (1985): Gegliederte Analyse und Synthese des Niederschlag-AbflußProzesses von Einzugsgebieten. Dissertation, Fakultät für Bau-Wasser-und Forstwesen der TU Dresden.

SEIDLER, CH. (1999): Ursachen und Charakterisierung eines eingeschränkten Wasserhaushaltes sowie seine Auswirkungen auf das Pflanzenwachstum. Dissertation, Internationales Hochschulinstitut Zittau.

SEVERIN, K. (1982): Wassehaushalt von Löss und Sand in Grundwasserlysimetern. Dissertation. Landwirtschaftliche Fakultät der Georg-August-Universität Göttingen.

SML (1995): Umweltgerechte Landwirtschaft im Feistaat Sachsen. Freistaat Sachsen, Staatsministerium für Landwirtschaft und Fortsten.

SOKOLLEK, V. (1983): Der Einfluß der Bodennutzung auf den Wasserhaushalt kleiner Einzugsgebiete in unteren Mittelgebirgslagen. Diss. Uni Gießen.

SPENGLER, R. (1973): Beiträge zur Ermittlung der Grundwasserneubildung und des Grundwasserdargebots im Lockergesteinsbereich, dargestellt am Parthegebiet. Dissertation, Fakultät für Naturwissenschaften der Martin-Luther-Universität Halle/Wittenberg.

SPENGLER, R. (1975): Beiträge zur Ermittlung des Grundwasserhaushaltes im Lockergesteinsbereich. Wissenschaftliche Zeitschrift der Martin-Luther-Universität HalleWittenberg 24.

SPONAGEL, H. (1980): Zur Bestimmung der realen Evapotranspiration landwirtschaftlicher Kulturpflanzen. Geologisches Jahrbuch (Reihe F) 9, Schweizerbartsche Verlagsbuchhandlung, Stuttgart.

THOMAS, M.; HAASE, J. (1970): Geländeklimatische Untersuchungen im Einzugsgebiet Parthe. Unveröffentlichter Forschungsbericht der Sektion Geographie der Martin-LutherUniversität Halle.

WALTHER, W.; SCHEFFER, B.; TEICHGRÄBER, B. (1985): Ergebnisse langjähriger Lysimeter-, Drän- und Saugkerzenversuche zur Stickstoffauswaschung bei landbaulich genutzten Böden und Bedeutung für die Belastung des Grundwassers. Veröffentlichungen des Instituts für Stadtbauwesen, Technische Universität Braunschweig.

WEINZIERL, W. (1984): Wasserhaushalt und Ertrag landwirtschaftlicher Kulturpflanzen ausgewählter Löß-Standorte im Raum Göttingen. Diss. Institut für Bodenwissenschaften der Georg-August-Universität Göttingen.

WEISE, K.; WENDLING, U. (1975): Bodenkundliche und meteorologische Parameter zur Einschätzung der Beregnungsbedürftigkeit grundwasserferner Böden. Archiv für Acker- und Pflanzenbau und Bodenkunde, 19. Band, Heft 1. 
WENDLAND, F.; ALBERT, H.; BACH, M.; SCHMIDT, R. (1993): Atlas zum Nitratstrom in der Bundesrepublik Deutschland. Springer Verlag, Heidelberg.

WENDLING, U. (1991): Schätzmethoden der Verdunstung landwirtschaftl. Bestände nach den Ansätzen von Penman und Turc. Arch. Acker-Pflanzenbau Bodenkunde, Berlin 35/4, 251-257.

WENDLING, U. (1995): Berechnung der Gras-Referenzverdunstung mit der FAO-PenmanMonteith-Beziehung. Wasserwirtschaft 85 (12).

WENDLING, U.; MÜLLER, J. (1984): Entwicklung eines Verfahrens zur rechnerischen Abschätzung der Verdunstung im Winter. Zeitschrift Meteorol. 34.

WOHLRAB, B.; ERNSTBERGER, H.; MEUSER, A.; SOKOLLEK, V. (1992): Landschaftswasserhaushalt. Verlag Paul Parey, Berlin und Hamburg.

ZEPP, H. (1987): Wasserhaushalt und Verlagerung wasserlöslicher Stoffe in Lößdecken des Taunus-Vorlandes. Arbeiten zur Rheinischen Landeskunde. H 11. 


\section{Abkürzungen und Formelzeichen}

\begin{tabular}{|c|c|c|}
\hline Symbol & Beschreibung & Einheit \\
\hline$\theta$ & Wassergehalt im Boden & Vol.- $\%$ \\
\hline$\lambda$ & latente Verdunstungswärme & Joule/kg \\
\hline$\gamma$ & Psychrometerkonstante & $0,655 \mathrm{hPa} / \mathrm{K}$ \\
\hline$\theta_{\mathrm{FK}}$ & Bodenwassergehalt bei einer Saugspannung von $5-16 \mathrm{kPa}$ (Feldkapazität) & Vol.-\% \\
\hline$\Psi_{\mathrm{m}}$ & Saug- bzw. Wasserspannung (Matrixpotential) im Boden & $\mathrm{cm} \mathrm{WS,} \mathrm{kPa,} \mathrm{bar}$ \\
\hline$\theta_{\text {PWP }}$ & Bodenwassergehalt bei einer Saugspannung von 1,5 MPa (PWP) & Vol.- $\%$ \\
\hline A & Fläche, z.B. eines Einzugsgebietes & $\mathrm{km}^{2}$ \\
\hline $\mathrm{C} / \mathrm{N}-$ & $\mathrm{C}_{\text {org }} / \mathrm{N}_{\text {org }}-$ Verhältnis & - \\
\hline $\mathrm{C}_{\text {org }}$ & organische Kohlenstoff-Komponenten & - \\
\hline$c_{p}$ & spezifische Wärme der Luft bei konstantem Druck & $1005 \mathrm{Joule} / \mathrm{kg} / \mathrm{K}$ \\
\hline$c_{v}$ & $\begin{array}{l}\text { Variationskoeffizient } c_{v}=\left(s_{x} / x\right) \cdot 100 \% \text { (Standardabweichung in Relation zum } \\
\text { arithmetischen Mittelwert } \mathrm{x} \text { ) }\end{array}$ & 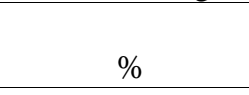 \\
\hline $\mathrm{e}_{2 \mathrm{~m}}$ & Tagesmittel des Dampfdruckes der Luft in 2 m Höhe & $\mathrm{hPa}$ \\
\hline$E_{2 m}$ & temperaturabhängiger Sättigungsdampfdruck der Luft in $2 \mathrm{~m}$ Höhe & $\mathrm{hPa}$ \\
\hline $\mathrm{E}_{2 \mathrm{~m}}-\mathrm{e}_{2 \mathrm{~m}}$ & Sättigungsdefizit der Luft mit Wasserdampf in $2 \mathrm{~m}$ Höhe & $\mathrm{hPa}$ \\
\hline ETK & Evapotranspirations-Koeffizient & $1 / \mathrm{kg}$ \\
\hline $\mathrm{ET}_{0}$ & Gras-Referenzverdunstung von ALLEN et al. (1994) & $\mathrm{mm}$ \\
\hline $\mathrm{ET}_{\text {Lys } 9}$ & mittels Lysimetergruppe 9 gemessene (maximale) Verdunstung & $\mathrm{mm}$ \\
\hline $\begin{array}{l}\mathrm{ET}_{\max } \\
\text { ETP }_{\text {Bestand }}\end{array}$ & landnutzungsabhängige maximale Verdunstung & $\mathrm{mm}$ \\
\hline $\begin{array}{l}\text { ETP, } \\
\text { ETP }_{\text {Gras }}\end{array}$ & potentielle Verdunstung als maximale Verdunstung von Gras & $\mathrm{mm}$ \\
\hline ETR & reale Verdunstung (Evapotranspiration) & $\mathrm{mm}$ \\
\hline FK & Feldkapazität einer Bodenschicht & $\mathrm{mm} / \mathrm{dm}$ \\
\hline $\mathrm{FK}_{\text {Drän. }}$ & Feldkapazität der Dränwasserzone & $\mathrm{mm} / \mathrm{dm}$ \\
\hline FP & Feinporen $(<0,0002 \mathrm{~mm} \varnothing)$ & $\% \mathrm{PV}$ \\
\hline $\mathrm{G}$ & Bodenwärmestrom & $\mathrm{W} / \mathrm{m}^{2}$ \\
\hline $\mathrm{GP}_{\mathrm{e}}$ & enge Grobporen $(0,01-0,05 \mathrm{~mm} \varnothing)$ & $\% \mathrm{PV}$ \\
\hline $\mathrm{GP}_{\mathrm{w}}$ & weite Grobporen (>0,05 mm Ø) - Luftkapazität & $\% \mathrm{PV}$ \\
\hline GW & Grundwasser & - \\
\hline GWL & Grundwasserleiter & - \\
\hline GWN & Grundwasserneubildung & $\mathrm{mm}$ \\
\hline INF & Infiltration & $\mathrm{mm}$ \\
\hline KA & kapillare Aufstiegsrate & $\mathrm{mm} / \mathrm{d}$ \\
\hline $\mathrm{k}_{\mathrm{c}}$ & Bestandskoeffizient & - \\
\hline $\mathrm{k}_{\mathrm{f}}-$ Wert & Gesättigte hydraulische Leitfähigkeit (Durchlässigkeitsbeiwert) & $\mathrm{m} \cdot \mathrm{s}^{-1}$ \\
\hline kWB & klimatische Wasserbilanz & $\mathrm{Mm}$ \\
\hline $\mathrm{Ld}$ & $\begin{array}{l}\text { effektive Lagerungsdichte von Böden mit Gefügeeinfluß bei Berücksichtigung des } \\
\text { Tongehaltes }\end{array}$ & $\mathrm{g} / \mathrm{cm}^{3}$ \\
\hline LK, $\mathrm{n}_{\mathrm{e}}$ & Luftkapazität (PV minus FK), entwässerbare Porosität & Mm, Vol.- \%, \\
\hline MP & Mittelporen $(0,0002-0,02 \mathrm{~mm} \varnothing)$ & $\% \mathrm{PV}$ \\
\hline $\mathrm{N}$ & Stickstoff als Bodeneintrag, Entzug oder Austrag & $\mathrm{kg} / \mathrm{ha}$ \\
\hline $\mathrm{N}_{\mathrm{an}}$ & anorganische Stickstoff-Komponenten & - \\
\hline nFK & nutzbare Feldkapazität (FK minus PWP) einer Bodenschicht & $\mathrm{mm} / \mathrm{dm}$ \\
\hline
\end{tabular}




\begin{tabular}{|c|c|c|}
\hline $\mathrm{nFK}_{\mathrm{we}}$ & nutzbare Feldkapazität im effektiven Wurzelraum & $\mathrm{mm}$ \\
\hline $\mathrm{N}_{\text {org }}$ & organische Stickstoff-Komponenten & - \\
\hline NStE & Natürliche Standorteinheiten & - \\
\hline $\mathrm{P}$ & Niederschlag nach Hellmann in $1 \mathrm{~m}$ Höhe & $\mathrm{mm}$ \\
\hline pF-Kurven & Beziehung zwischen Wassergehalt $(\theta)$ im Boden und Saugspannung $(\Psi)$ & - \\
\hline $\mathrm{P}_{\text {korr }}$ & korrigierter Niederschlag & $\mathrm{mm}$ \\
\hline $\mathrm{p}_{\mathrm{L}}$ & Dichte trockener Luft & $1,293 \mathrm{~kg} / \mathrm{m}^{3}$ \\
\hline PSM & Pflanzenschutzmittel & - \\
\hline $\mathrm{PV}, \mathrm{n}_{\mathrm{ges}}$ & Gesamtporenvolumen, Porosität, Gesamtporosität & $\mathrm{mm}, \mathrm{Vol} .-\%$ \\
\hline $\mathrm{p}_{\mathrm{w}}$ & Dichte von Wasser & $10^{3} \mathrm{~kg} / \mathrm{m}^{3}$ \\
\hline PWP & permaneter Welkepunkt einer Bodenschicht & $\mathrm{mm} / \mathrm{dm}$ \\
\hline Q & Durchfluß am Meßquerschnitt im Vorfluter & $\mathrm{m}^{3} \cdot \mathrm{s}^{-1}$ \\
\hline $\mathrm{Q}_{\mathrm{F}}$ & Wasserförderung eines Grundwasserwerkes & $1 \cdot \mathrm{s}^{-1}$ \\
\hline $\mathrm{R}$ & im Einzugsgebiet flächenhaft gebildeter Abfluß & $\mathrm{mm}$ \\
\hline $\mathrm{R}_{0}$ & extraterrestrische Strahlung & Joule $* \mathrm{~cm}^{-2}$ \\
\hline$r_{a}$ & aerodynamischer Widerstand (abhängig von der Bestandshöhe) & $\mathrm{s} / \mathrm{m}$ \\
\hline RG & Grundwasserabfluß, auch Basisabfluß oder grundwasserbürtiger Abfluß & $\mathrm{mm}$ \\
\hline $\mathrm{R}_{\mathrm{G}}$ & Globalstrahlung & Joule $* \mathrm{~cm}^{-2}$ \\
\hline RH & bodeninnerer, lateraler (hypodermischer) Abfluß & $\mathrm{mm}$ \\
\hline $\mathrm{R}_{\mathrm{n}}$ & Strahlungsbilanz am Erdboden (abhängig von der Albedo) & $\mathrm{W} / \mathrm{m}^{2}$ \\
\hline $\mathrm{RO}$ & kurzzeitiger, nicht gw-wirksamer Landoberflächenabfluß & $\mathrm{mm}$ \\
\hline $\mathrm{R}^{2}$ & Bestimmtheitsmaß $\mathrm{R}^{2}=\mathrm{r}^{2} * 100 \%$ & $\%$ \\
\hline $\mathrm{r}$ & Korrelationskoeffizient & - \\
\hline$r_{s}$ & Stomatawiderstand (abhängig vom Bodenwasservorrat) & $\mathrm{s} / \mathrm{m}$ \\
\hline S & Änderung des Sättigungsdampfdruckes mit der Temperatur & $\mathrm{hPa} / \mathrm{K}$ \\
\hline S & tatsächliche Sonnenscheindauer des Tages & $\mathrm{h}$ \\
\hline $\mathrm{S}_{0}$ & astronomisch mögliche Sonnenscheindauer des Tages & $\mathrm{h}$ \\
\hline$\Delta \mathrm{SB}$ & Bodenwasservorratsänderung & $\mathrm{mm}$ \\
\hline SB & tatsächlich jährlich entnommene Bodenwassermenge & $\mathrm{mm}$ \\
\hline $\mathrm{SB}_{\max / \min }$ & höchste/geringste sommerliche Bodenwasserentnahme im Untersuchungszeitraum & $\mathrm{mm}$ \\
\hline $\mathrm{S}^{*}$ & Versickerung aus der verdunstungsbeeinflußten Zone in die Dränwasserzone & $\mathrm{mm}$ \\
\hline SW & Sickerwasser in $3 \mathrm{~m}$ Tiefe & $\mathrm{mm}$ \\
\hline $\mathrm{s}_{\mathrm{x}}$ & Standardabweichung & $\mathrm{mm}$ \\
\hline $\mathrm{T}_{14}$ & Lufttemperatur in 2 m Höhe um 14 Uhr & ${ }^{\circ} \mathrm{C}$ \\
\hline $\mathrm{T}_{2 \mathrm{~m}}$ & Tagesmittel der Lufttemperatur in 2 m Höhe & ${ }^{\circ} \mathrm{C}$ \\
\hline TDR & Time Domain Reflectometry (Bodenfeuchtemeßverfahren) & - \\
\hline TM & Trockenmasseerträge & $\mathrm{dt} / \mathrm{ha}$ \\
\hline $\mathrm{U}_{14}$ & Luftfeuchte zum Mittagstermin 14.30 Uhr MEZ in 2 m Höhe & $\%$ \\
\hline $\mathrm{U}_{2 \mathrm{~m}}$ & Tagesmittel der Luftfeuchte in $2 \mathrm{~m}$ Höhe & $\%$ \\
\hline $\mathrm{v}_{2 \mathrm{~m}}$ & Windgeschwindigkeit in $2 \mathrm{~m}$ Höhe & $\mathrm{m} / \mathrm{s}$ \\
\hline VG & Verlagerungsgeschwindigkeit & $\mathrm{dm} / \mathrm{a}$ \\
\hline VS & Verlagerungsstrecke & $\mathrm{dm}$ \\
\hline $\mathrm{We}_{\mathrm{eff}}$ & Effektive Durchwurzelungstiefe & $\mathrm{cm}$ \\
\hline $\mathrm{We}$ & $\begin{array}{l}\text { Ausschöpfungstiefe - entspricht bei fehlender kapillarer Wasserlieferung aus dem } \\
\text { Unterboden der Wurzeltiefe }\end{array}$ & $\mathrm{cm}$ \\
\hline $\mathrm{We}_{\text {aktuell }}$ & Auschöpfungstiefe im zeitlichen Verlauf & $\mathrm{cm}$ \\
\hline $\mathrm{We}_{\max / \min }$ & größste/geringste Ausschöpfungstiefe & $\mathrm{cm}$ \\
\hline
\end{tabular}




\section{Verzeichnis der Abbildungen}

Abb. 1: Lage der Lysimeterstation Brandis und weiterer wägbarer Lysimeteranlagen der Bundesrepublik Deutschland (aus DVWK, 1996) ............................................................ 8

Abb. 2: Standortwasserhaushalt: Schematische, auf ein $3 \mathrm{~m}$ tiefes Lysimeter bezogene Darstellung der ungesättigten Zone am Beispiel eines ungeschichteten Bodenprofils ............11

Abb. 3: Einzellysimeter mit Wägeeinrichtung, Sickerwasserauslauf, Saugkerzen, Tensiometern, TDR-Sonden und Sonden zur Bodenluftentnahme ..................................... 13

Abb. 4: Filterrohr für den Sickerwasserablauf am Lysimeterboden .................................... 15

Abb. 5: Vergleich der in verschiedenen Tiefen gemessenen Bodentemperaturen mit der im Lysimeterkeller gemessenen Lufttemperatur am Beispiel der Jahre 1997-99

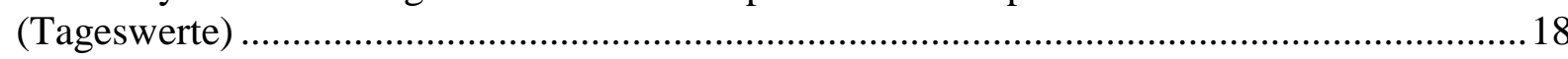

Abb. 6: Doppelsummenanalyse am Beispiel der Lysimetergruppe 5 (erodierte Braunerde) und Lysimetergruppe 10 (Löß-Schwarzerde) auf Basis der Monatswerte der Jahre 1981-97

Abb. 7: Skizze zur Lage der Naturräume in Sachsen (ergänzt aus MEYNEN \&

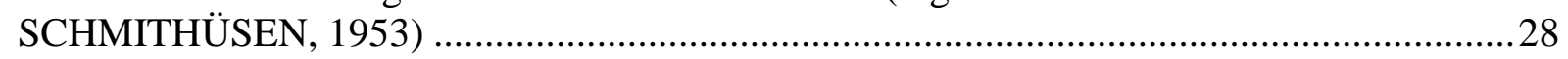

Abb. 8: Klimastufen- und gebiete (aus „Naturräume der sächsischen Bezirke“, 1986)..........33

Abb. 9: Monatswerte von unkorrigiertem Niederschlag und potentieller Verdunstung nach TURC für ausgewählte Klimastationen des DWD und Brandis (Reihe 1981-97) ..........34

Abb. 10: Beispiel für die Ermittlung des effektiven Wurzelraumes ( $\left.\mathrm{We}_{\mathrm{eff}}\right)$ aus Feldkapazität, permanentem Welkepunkt und aktuellem Wassergehalt, dargestellt für den Herbst eines Trockenjahres auf einem Boden mit Feinsand (aus KA4, 1995 und SCHEFFER \& SCHACHTSCHABEL, 1992) ....................................................... 40

Abb. 11: Mittlere Anzahl der Tage mit messbarem Niederschlag, sowie potentielle Verdunstung (ETP) und Niederschlagsregime der Station Brandis (Reihe 1981-97, korrigierte Niederschläge) [Angaben in $\mathrm{mm}$ ] ............................................................47

Abb. 12: Regressionsgerade: Dekadenwerte der potentiellen Verdunstung von TURC/IVANOW (Gl. 6 und Gl. 7) und WENDLING nach TURC (Gl. 9) .........................51

Abb. 13: Regressionsgerade: Dekadenwerte der Gras-Referenzverdunstung und der potentiellen Verdunstung von WENDLING nach PENMAN (Gl. 5) ................................52

Abb. 14: Summenkurve der Dekadenwerte des korrigierten Niederschlages und der mittels verschiedener Verfahren ermittelten Verdunstung [mm].

Abb. 15: Niederschlag und potentielle Verdunstung der Winter- und Sommerhalbjahre am Standort Brandis (Minimum, Mittelwert und Maximum der Reihe 1981-97) ..................55

Abb. 16: Jahreswerte der klimatischen Wasserbilanz am Standort Brandis in [mm/a]..........56

Abb. 17: Niederschlag (korrigiert) und Gras-Referenzverdunstung der Winter- und Sommerhalbjahre am Standort Brandis ....................................................................56

Abb. 18: Vergleich zwischen potentiellem Bodenwasserbedarf und tatsächlicher jährlicher Bodenwasserentnahme [mm/a] .............................................................. 61

Abb. 19: Verlauf der Bodenwasserausschöpfung am Beispiel von Zuckerrüben 1981 und 1990 
Abb. 20: Mittlere Jahresverdunstung (1981-97) in Abhängigkeit vom pflanzenverfügbaren Bodenwasservorrat für Wintergetreide, Kartoffeln, Zuckerrüben und Gras/Kleesowie Mittel der Jahre 1981-97 (ohne Klee) unter den klimatischen Bedingungen am Standort Brandis ........................................................................... 70

Abb. 21: Beziehung zwischen Trockenmasseerträgen und Jahresverdunstung am Beispiel von Wintergetreide, Gras/Klee, Kartoffeln und Zuckerrüben am

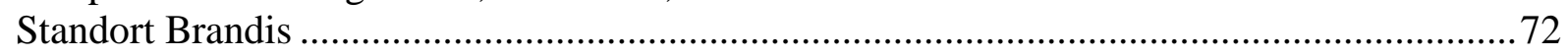

Abb. 22: Einfluss von Niederschlag und potentieller Verdunstung im Sommerhalbjahr (P/ETP) auf die Verdunstungsleistung (ETR/ETP) von Wintergetreide, Gras/Klee, Kartoffeln und Zuckerrüben in Abhängigkeit von der Bodenart .........................................74

Abb. 23: Beziehung zwischen realer und potentieller Verdunstung im Winterhalbjahr (Nov. - April) [ mm] (Halbjahresmittel über alle 8 Lysimeterböden) .................................75

Abb. 24: Prozentuale Abweichung zwischen der nach RENGER \& WESSOLEK (1990)....77

Abb. 25: Mittlere Monatssummen der potentiellen Verdunstung nach TURC, der Gras-Referenzverdunstung und der realen Verdunstung der untersuchten Böden am Standort Brandis bei landwirtschaftlicher Bewirtschaftung (Reihe 1981-97)........................79

Abb. 26: Vergleich der SPONAGEL-Faktoren mit den mittels Lysimetermessungen in Brandis ermittelten Faktoren am Beispiel von Kartoffeln im Jahr 1996 (Dekadenwerte).......88

Abb. 27: Jahressummen der Sickerwasserbildung, unterteilt in Sommer- und Wintersumme [mm]....

Abb. 28: Abhängigkeit der Sickerwassermengen vom Niederschlag [mm/Jahr] (Beginn des Jahres am 1. April).....

Abb. 29: Beziehung zwischen Sickerwasserrate aus dem Boden und der nutzbaren Feldkapazität im effektiven Wurzelraum $\left(\mathrm{nFK}_{\mathrm{We}}\right)$ für unterschiedliche Klimagebiete

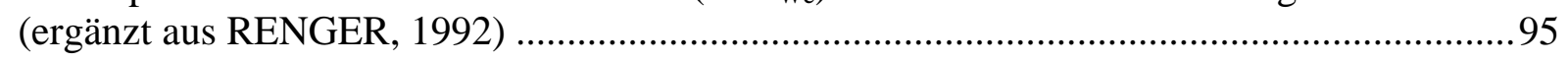

Abb. 30: Mittlerer Jahresgang von Bodenwasservorratsänderung (dSB), Niederschlag $\left(\mathrm{P}_{\text {korr }}\right)$, Gras-Referenzverdunstung $\left(\mathrm{ETP}_{\mathrm{Gras}}\right)$, klimatischer Wasserbilanz $(\mathrm{kWB}$, realer Verdunstung und Grundwasserneubildung am Standort Brandis (Reihe 1981-97) ..... 102

Abb. 31: Jahresmittel 1981-92 der Frischmasseabfuhr und N-Salden der verschiedenen Lysimetergruppen .......................................................................................................... 105

Abb. 32: Jahresmittel 1981-92 der N-Salden und der N-Austräge über das Sickerwasser der verschiedenen Lysimetergruppen (s. Tab. 33) ........................................................ 112

Abb. 33: Gegenüberstellung von N-Eintrag, N-Entzug und N-Austrag am Beispiel von ausgewählten Lysimeterböden (Reihe 1980-96)............................................................ 113

Abb. 34: Nitratkonzentration im Sickerwasser (Jahresmittelwerte) in Abhängigkeit

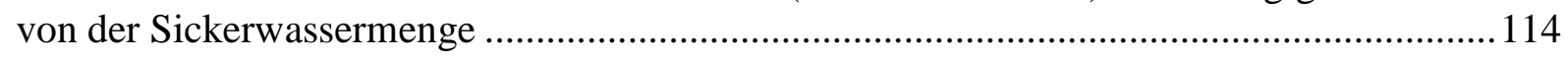

Abb. 35: Schematische Darstellung der Geltungsbereiche geowissenschaftlicher Übersichtskarten des Sächsischen Landesamtes für Geologie (DUTELOFF, 1996) ............120

Abb. 36: Gebietswasserhaushalt: Vertikale Gliederung eines Einzugsgebietskörpers und schematische Darstellung der Niederschlagsabflussbeziehungen (nach DYCK\&PESCHKE, 1995).......

Abb. 37: Grundwasserstandsentwicklung und Wasserstände der Parthe am Pegel Naunhof von März 1975 bis Oktober $1998 . .$. 
Abb. 38: Flächennutzung und Böden der landwirtschaftlichen Nutzflächen im Einzugsgebiet der Parthe .

Abb. 39: Mittlere Wasserhaushaltskomponenten am Pegel Thekla/Parthe

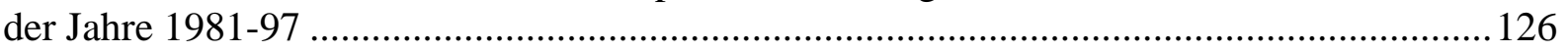

Abb. 40: Vergleich der mittleren Abflussbildung am Standort (Lysimetermessungen) und im Einzugsgebiet der Parthe (Reihe 1981-97) ........................................................... 127

Abb. 41: Langjährige Monatsmittelwerte des Niederschlages in [mm/Monat] verschiedener Reihen am Beispiel der Station Leipzig-Schkeuditz des DWD 129

Abb. 42: Abfluss am Pegel Thekla/Parthe und Grundwasserstandsentwicklung

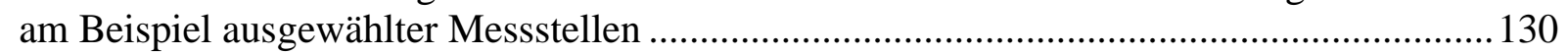




\section{Verzeichnis der Tabellen}

Tab. 1: Fruchtfolge, Stickstoff-(N)-Einträge und Niederschläge der Jahre 1980-98 ............. 17

Tab. 2: Standardabweichung der Verdunstung der Einzellysimeter vom Gruppenmittel

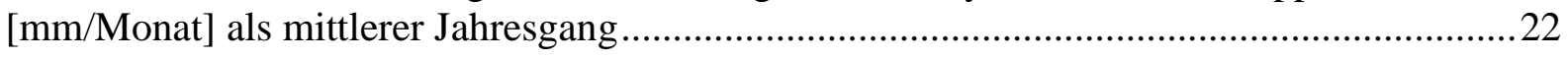

Tab. 3: Korrekturwerte des bodengleichen Niederschlages nach RICHTER (1995)............23

Tab. 4: Unkorrigierter und korrigierter Niederschlag [mm/a] am Standort Brandis

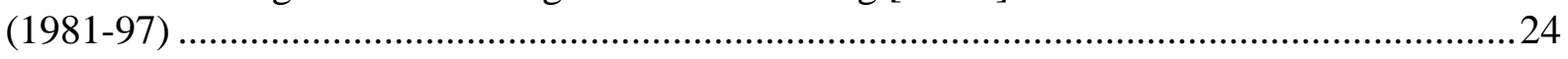

Tab. 5: Zusammenstellung charakteristischer Werte der Wasserbindung [Vol.\%]..............26

Tab. 6: Bodenform, Entnahmeorte und Profilaufbau der Brandiser Lysimeter .....................32

Tab. 7: Monatsmittelwerte ausgewählter Klimagrößen für die Jahre 1981-97 von Brandis und Klimastationen des DWD (Hydrologisches Jahr; Niederschlag unkorrigiert)....34

Tab. 8: Relative Häufigkeit monatlicher Niederschlagssummen in Prozent und ihre jahreszeitliche Differenzierung für ausgewählte Stationen (Wasserhaushaltsjahre 1981-96)

Tab. 9: Anzahl der Bodentypen und Wiederholungen je Pedo-Hydrotop.

Tab. 10: Korrigierter Niederschlag [mm] am Standort Brandis und statistische Maßzahlen (Wasserhaushaltsjahre 1981-97)....

Tab. 11: Berechnete und mittels Lysimeter gemessene Jahresverdunstung, bezogen auf die Gras-Referenzverdunstung (=100\%) am Standort Brandis in verschiedenen

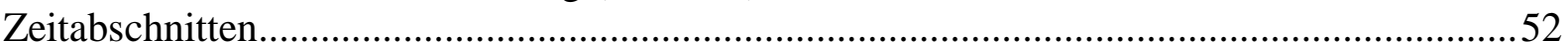

Tab. 12: Niederschlag, potentielle Verdunstung und klimatische Wasserbilanz der Jahre 1981-97 am Standort Brandis [mm/a]

Tab. 13: Bodenwasserentnahme in Abhängigkeit von den Fruchtarten, ohne Rotklee (Reihenfolge nach der Entnahmemenge) ...............................................................

Tab. 14: Boden- und kulturartenspezifische Bodenwasserentnahme [mm] ..........................58

Tab. 15: Statistische Kennwerte der tatsächlichen Bodenwasserentnahme für die Böden der Station Brandis im Vergleich zu nFK ${ }_{w e}$ und We nach KA4 (1994) ................59

Tab. 16: Höhe des Bodenwasserdefizits bei den Lößböden zu Beginn der Ausschöpfung (a), am 1.4. des Jahres (b), Höhe der jährlichen Bodenwasserentnahme (c) und Wasserdefizit - ermittelt auf Basis der kWB - zu Beginn der Ausschöpfung (d) in [mm] am Standort Brandis ...................................................59

Tab. 17: Maximale, minimale und durchschnittliche Entnahmetiefen von Bodenwasser ......62

Tab. 18: Ausschöpfungstiefen [cm] der verschiedenen Kulturarten (Anzahl der Anbaujahre).....

Tab. 19: Jahreswerte der Gras-Referenzverdunstung und der realen Verdunstung für die 8 verschiedenen Böden (Gruppenmittel) in Reihung nach der Vegetation

Tab. 20: Vergleich zwischen mittels Lysimeter gemessener und nach RENGER\&WESSOLEK (1990) berechneter Jahresverdunstung in [mm] und in [\% Abweichung] der Jahre 1981-97 ..................................................................................... 77 
Tab. 21: Mittlere Monatswerte der potentiellen Verdunstung nach TURC, der GrasReferenzverdunstung und der realen Verdunstung in Abhängigkeit von Bodenart und Vegetation-abgeleitet aus den Lysimetermessungen der Station Brandis (Reihe 1981-97).....80 Tab. 22: Bestandskoeffizienten kc $\left(\mathrm{ETR}_{\mathrm{Lög}} / \mathrm{ETP}_{\mathrm{Gras}}\right)$ im Vergleich zu Angaben ....................82

Tab. 23: Höhe der Verdunstung im Verlauf der Vegetationsperiode (Aufgang bis Ernte), Trockenmasseerträge und Evapotranspirationskoeffizienten der Jahre 1981-97 für acht verschiedene Böden der Station Brandis ......................................................................... 84

Tab. 24: Dekadenwerte der Verdunstung von Kartoffeln im Jahr 1996 (April bis September)

Tab. 25: Jahreswerte (November - Oktober) 1981-97 der Sickerwasserbildung für 8 verschiedenen Böden (Gruppenmittel) unter Reihung nach der Vegetation in [mm]

Tab. 26: Charakterisierung der Wasserhaushaltsjahre 1981-97 in Abhängigkeit von der Bodenart

Tab. 27: Mittel der Sickerwasserbildung für die Untersuchungsjahre 1981-97 und maximale bzw. minimale Sickerwasserbildung für die acht Lysimetergruppen ....

Tab. 28: Häufigkeitsverteilung für den Beginn der jährlichen Sickerwasserperiode auf der Basis der Monatswerte der 17 Abflußjahre 1981-97....

Tab. 29: Häufigkeitsverteilung für den Eintritt des Maximums der jährlichen Sickerwasserperiode auf der Basis der Monatswerte der 17 Abflußjahre 1981-97

Tab. 30: Mittlerer Jahresgang der Sickerwasserbildung und des Niederschlages [mm] für Standorte mit Sand, Geschiebelehm und Löß (Wasserhaushaltsjahre 1981-97) ...............98

Tab. 31: Mittlere Abweichung zwischen gemessener und berechneter Sickerwasserbildung und Bestimmtheitsmaß $\left(\mathrm{R}^{2}\right)$ für die Übereinstimmung in den Einzeljahren in [\%] - Beginn des Bilanzjahres am 1. Oktober ............................................................ 99

Tab. 32: Vergleich zwischen nach RENGER \& WESSOLEK (1990) berechneten und gemessenen Sickerwassermengen sowie Niederschlag $\left(\mathrm{P}_{\text {korr }}\right)$ und Gras-Referenzverdunstung $\left(\mathrm{ETP}_{\mathrm{Gras}}\right)$ - Beginn des Bilanzjahres am 1. April ....................................... 100

Tab. 33: N-Entzüge durch das Erntegut verschiedener Fruchtarten auf acht verschiedenen Böden [kg/ha/Jahr] (Die Lysimetergruppen sind nach fallender Sickerwasserleistung gereiht.)

Tab. 34: Mittlere N-Salden in [kg/ha/Jahr](Böden in Reihung nach abnehmender Sickerwasserleistung)

Tab. 35: Mittlere Austauschhäufigkeit des Bodenwassers bei Feldkapazität im effektiven Wurzelraum [\%/Jahr] - Reihe 1981-97 ......................................................... 107

Tab. 36: Durchschnittliche Verlagerungsgeschwindigkeit [dm/Jahr] und Verweilzeit des Sickerwassers in der Dränwasserzone der Lysimeter - Reihe 1981-97 ....

Tab. 37: Austauschhäufigkeit des Bodenwassers bei Feldkapazität im effektiven Wurzelraum [\%/Jahr] am Beispiel der Lysimetergruppe 5 (D3) in den Jahren 1981-97 ....... 109

Tab. 38: Austauschhäufigkeit des Bodenwassers bei Feldkapazität im effektiven Wurzelraum [\%/Jahr] am Beispiel der Lysimetergruppe 7 (D4) in den Jahren 1981-97 ....... 109

Tab. 39: Austauschhäufigkeit des Bodenwassers bei Feldkapazität im effektiven Wurzelraum [\%/Jahr] am Beispiel der Lysimetergruppe 9 (Lö3) in den Jahren 1981-97 ..... 110 
Tab. 40: N-Auswaschungsverluste über das Sickerwasser in $3 \mathrm{~m}$ Tiefe [kg/ha/Jahr] (Reihung nach abnehmender Sickerwasserleistung)....

Tab. 41: Nach Jahresmittelwerten der Sickerwasserbildung (SW) geordnete Angaben zur Verdunstung, zu Trockenmasse-Erträgen, Stickstoffentzügen durch die Pflanze und Höhe der Stickstoffausträge über das Sickerwasser der Einzellysimeter (Abflussjahre 1981-94) .. 116

Tab. 42: Anzahl der Monate mit signifikanten Unterschieden der Verdunstung und Sickerwasserbildung zwischen den Lysimetergruppen (Ergebnisse der Varianzanalyse von 168 Monaten je Einzellysimeter der Jahre 1981-94) ................................................. 117

Tab. 43: Langjährige Jahresmittelwerte der Sickerwasserbildung (SW), der realen Verdunstung (ETR), der Trockenmasse-Erträge (TM), Stickstoffentzüge durch die Pflanze (N-Entzug) und die Höhe der Stickstoffausträge über das Sickerwasser (N-Austrag) der Versickerungstypen (Wasserhaushaltsjahre 1981-94) ..............................118

Tab. 44: Definition relevanter Pedo-Hydrotope im Untersuchungsgebiet ...........................119

Tab. 45: Mittlere Wasserhaushaltskomponenten im Einzugsgebiet der Parthe -

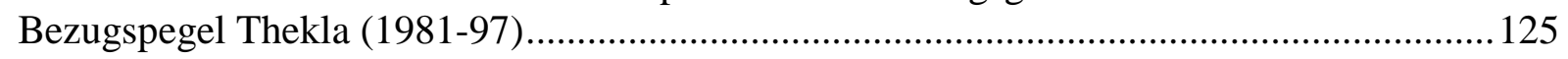

Tab. 46: Niederschlag und Lufttemperatur von ausgewählten Stationen des DWD ............128 


\section{Anlagen}

1

2

3

4

5

6

7

8
Stickstoffentzüge durch die Ackerfrüchte und jährlich über das Sickerwasser in $3 \mathrm{~m}$ Tiefe ausgetragene Stickstofffrachten der wägbaren Lysimeter der Station Brandis (Reihe 1981-97).

Beschreibung der acht Bodenprofile aus der Zeit der Lysimetergewinnung

Tabellarische Zusammenstellung der Ergebnisse bodenphysikalischer Untersuchungen aus der Zeit der Lysimetergewinnung

Vergleich zwischen gemessenen und berechneten Dekadenwerten der Verdunstung am Beispiel der Jahre 1993,1996 und 1997.

Verlauf der Wasserhaushaltsgrößen am Beispiel einer erodierten Braunerde und einer Löß-Schwarzerde

Zur Definition von Trocken- und Feuchtjahren in Abhängigkeit von Niederschlag und Grundwasserneubildung für vier Versicherungstypen (Pedo-Hydrotope)

Aktuelle Monatswerte der Grundwasserneubildung im Vergleich zu den langjährigen Monatsmittelwerten für vier Versicherungstypen (PedoHydrotope)

Wasserhauahaltsbilanzen der Jahre 1981-97 für 4 Versickerungstypen (Pedo-Hydrotope) unter landwirtschaftlicher Nutzung 
Anlage 1: Stickstoffentzüge über die Pflanze in [kg/ha] (Gruppenmittel)

\begin{tabular}{|c|c|c|c|c|c|c|c|c|c|}
\hline \multirow[t]{2}{*}{ Jahr } & \multirow[t]{2}{*}{ Fruchtart } & \multicolumn{8}{|c|}{ Lysimetergruppe } \\
\hline & & Ly 5 & Ly 4 & Ly 8 & Ly 1 & Ly 7 & Ly 11 & Ly 9 & Ly 10 \\
\hline & & Typ a & \multicolumn{2}{|c|}{ Typ b } & \multicolumn{3}{|c|}{ Typ c } & \multicolumn{2}{|c|}{ Typ d } \\
\hline 1980 & Mais & 97 & 124 & 139 & 125 & 159 & 165 & 178 & 148 \\
\hline 1981 & Zuckerrüben & 111 & 201 & 127 & 214 & 200 & 218 & 240 & 423 \\
\hline 1982 & Winterweizen & 90 & 122 & 132 & 147 & 169 & 106 & 250 & 216 \\
\hline 1983 & Wintergerste & 117 & 126 & 123 & 141 & 125 & 150 & 165 & 158 \\
\hline 1984 & Wel. Weidelgras & 118 & 130 & 133 & 142 & 139 & 168 & 155 & 175 \\
\hline 1985 & Kartoffeln & 73 & 89 & 118 & 103 & 79 & 128 & 161 & 158 \\
\hline 1986 & Winterweizen & 94 & 115 & 115 & 130 & 158 & 114 & 192 & 180 \\
\hline 1987 & Kartoffeln & 131 & 128 & 157 & 141 & 136 & 158 & 180 & 169 \\
\hline 1988 & Winterweizen & 72 & 93 & 104 & 102 & 153 & 124 & 195 & 171 \\
\hline 1989 & Wintergerste & 124 & 136 & 124 & 135 & 152 & 155 & 231 & 219 \\
\hline 1990 & Zuckerrüben & 155 & 210 & 142 & 209 & 175 & 250 & 229 & 333 \\
\hline 1991 & Winterweizen & 101 & 138 & 126 & 136 & 167 & 149 & 165 & 178 \\
\hline 1992 & Wintergerste & 50 & 84 & 63 & 136 & 87 & 86 & 68 & 114 \\
\hline Mittel & $1980-92$ & 103 & 130 & 123 & 143 & 146 & 152 & 185 & 203 \\
\hline 1993 & Grünbrache & 21 & 26 & 21 & 39 & 25 & 33 & 32 & 36 \\
\hline 1994 & Grünbrache & 19 & 35 & 23 & 37 & 38 & 29 & 31 & 24 \\
\hline 1995 & Rotklee & 52 & 104 & 48 & 87 & 61 & 55 & 111 & 168 \\
\hline 1996 & Kartoffel & 133 & 196 & 135 & 149 & 125 & 163 & 205 & 189 \\
\hline 1997 & Sommerweizen & 34 & 61 & 65 & 74 & 84 & 76 & 130 & 116 \\
\hline 1998 & Winterroggen & & & & & & & & \\
\hline 1999 & Wintergerste & & & & & & & & \\
\hline Mittel & 1993-97 & 52 & 84 & 58 & 77 & 67 & 71 & 102 & 107 \\
\hline
\end{tabular}


Fortsetzung Anlage 1: Stickstoffausträge über das Sickerwasser in [kg/ha] (Gruppenmittel)

\begin{tabular}{|c|c|c|c|c|c|c|c|c|c|}
\hline \multirow[t]{2}{*}{ Jahr } & \multirow[t]{2}{*}{ Fruchtart } & \multicolumn{8}{|c|}{ Lysimetergruppe } \\
\hline & & Ly 5 & Ly 4 & Ly 8 & Ly 1 & Ly 7 & Ly 11 & Ly 9 & Ly 10 \\
\hline & & Typ a & \multicolumn{2}{|c|}{ Typ b } & \multicolumn{3}{|c|}{ Typ c } & \multicolumn{2}{|c|}{ Typ d } \\
\hline 1980 & Mais & - & - & - & - & - & - & - & - \\
\hline 1981 & Zuckerrüben & 88 & 108 & 81 & 97 & 58 & 0 & 37 & 280 \\
\hline 1982 & Winterweizen & 60 & 49 & 37 & 29 & 29 & 11 & 3 & 22 \\
\hline 1983 & Wintergerste & 47 & 43 & 33 & 28 & 14 & 25 & 0 & 0 \\
\hline 1984 & Wel. Weidelgras & 14 & 18 & 10 & 14 & 10 & 13 & 1 & 0 \\
\hline 1985 & Kartoffeln & 17 & 22 & 11 & 12 & 8 & 20 & 12 & 67 \\
\hline 1986 & Winterweizen & 61 & 24 & 24 & 17 & 13 & 33 & 8 & 107 \\
\hline 1987 & Kartoffeln & 98 & 71 & 78 & 74 & 32 & 142 & 8 & 93 \\
\hline 1988 & Winterweizen & 56 & 58 & 41 & 67 & 29 & 118 & 15 & 79 \\
\hline 1989 & Wintergerste & 34 & 34 & 21 & 26 & 7 & 47 & 0 & 9 \\
\hline 1990 & Zuckerrüben & 21 & 14 & 10 & 7 & 2 & 13 & 0 & 0 \\
\hline 1991 & Winterweizen & 25 & 18 & 11 & 8 & 2 & 8 & 0 & 0 \\
\hline 1992 & Wintergerste & 35 & 24 & 19 & 12 & 2 & 26 & 0 & 0 \\
\hline Mittel & $1980-92$ & 46 & 40 & 31 & 33 & 17 & 38 & 7 & 55 \\
\hline 1993 & Grünbrache & 32 & 19 & 17 & 14 & 1 & 25 & 0 & 0 \\
\hline 1994 & Grünbrache & 20 & 48 & 21 & 35 & 5 & 52 & 3 & 9 \\
\hline 1995 & Rotklee & 16 & 9 & 6 & 5 & 3 & 18 & 2 & 9 \\
\hline 1996 & Kartoffel & 16 & 2 & 5 & 0,2 & 1,4 & 2 & - & - \\
\hline 1997 & Sommerweizen & 105 & 6 & 14 & 6 & 5 & 9 & 0,2 & - \\
\hline 1998 & Winterroggen & & & & & & & & \\
\hline 1999 & Wintergerste & & & & & & & & \\
\hline Mittel & 1993-97 & 38 & 17 & 13 & 12 & 14 & 21 & 1 & 9 \\
\hline
\end{tabular}




\section{Lysimetergruppe 5}

\section{Erodierte Braunerde geringer Entwicklungstiefe aus Sandlöß über kiesführendem Fluvisand}

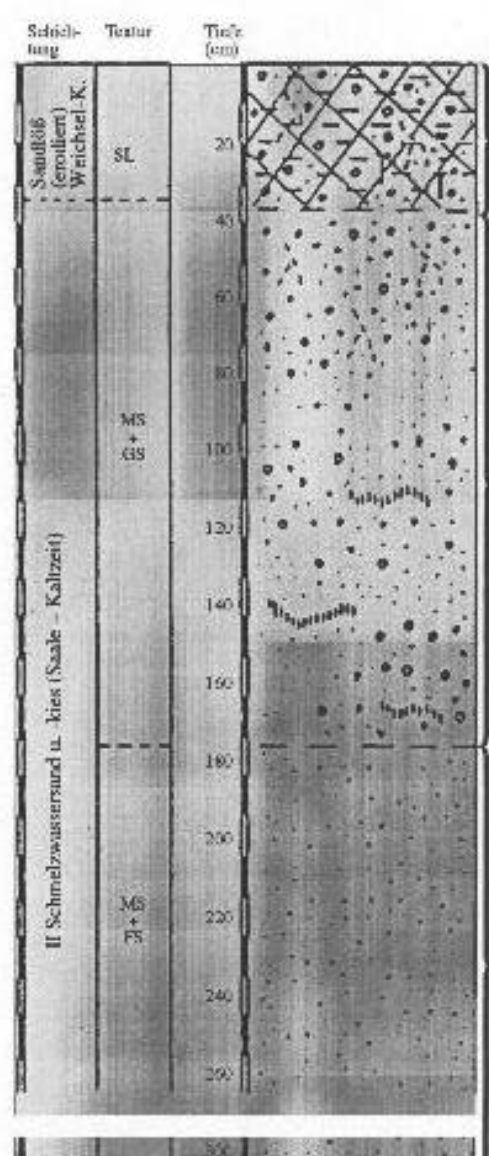

Bv - Ap 0 - 35 cm: dunkelbrauner (10 YR 3/3), humoser, kiesiger, sandiger Lehm, Krümelgefüge, mäßig dicht, durchwurzelt, Horizont gestört, an der Basis lokal $5 \mathrm{~cm}$ mächtiger $\mathrm{Br}$.-Horizont erhalten, scharf begrenzt, $2.1 \%$ Humus

II ICv $35-175 \mathrm{~cm}$ : hellbräunlich bis grauer $(2.5$ Y $6 / 2)$, stark kiesiger Mittelsand und Grobsand, Einzelkorngefüge, locker mit kiesärmeren Partien; im oberen Horizontbereich einzelne Wurzeln enthalten, einzelne Rostbänder, geschichtet, scharf begrenzt

II ICn 175 - $300 \mathrm{~cm}$ : hellbräunlichgrauer (2.5 Y 6/2) Mittelsand und Feinsand, Einzelkorngefüge, locker, geschichtet

Anlage 2: Seite 1 
Lysimetergruppe 4:

\section{Braunerde-Fahlerde mittlerer Entwicklungstiefe aus Sandlöß über kiesführendem Fluvisand}

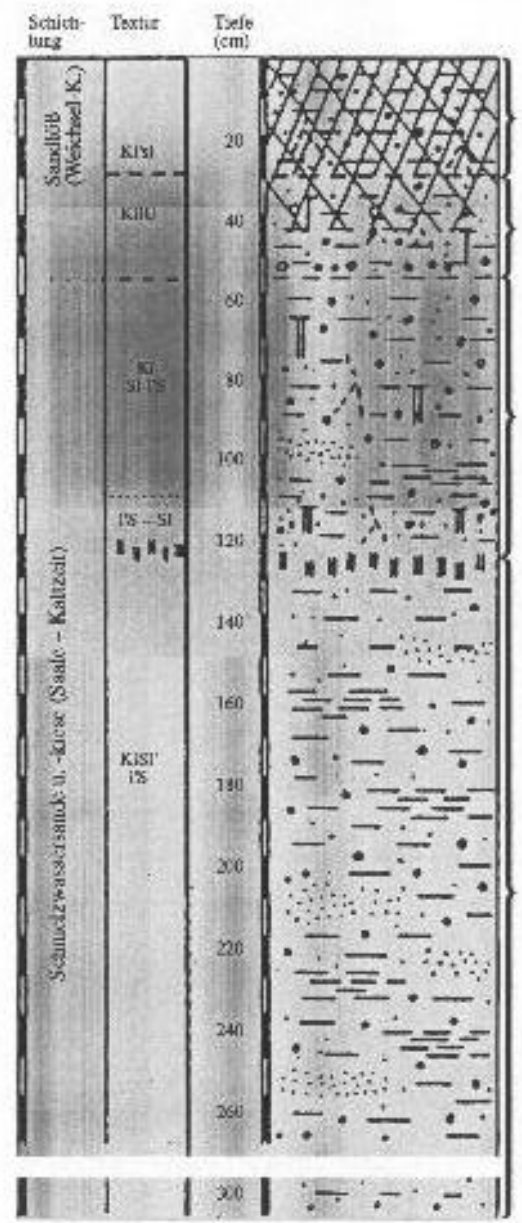

Ap 0 - $30 \mathrm{~cm}$ : dunkelbrauner (10 YR 3/3), humoser, schwach kiesiger, sandiger Lehm, Bröckelgefüge, mäßig dicht, durchwurzelt, scharf begrenzt, $2.3 \%$ Humus

Bv - Ael 30 - 55 cm: gelblichbrauner bis dunkelbrauner (10 YR 5/4 4/4), sehr schw. humoser, kiesiger (2 - 5 Vol.\%) lehmiger Schluff, Bröckelgefüge, mäßig dicht, Wurzel- u. Wurmgänge, auf der Basis Häufung von Kiesen, scharf begrenzt, $0.5 \%$ Humus

II Bbt 55 - 120 cm: dunkelbrauner (7.5 YR 4/4), kiesiger ( 30 Vol. \%) schwach lehmiger bis lehmiger Sand, Einzelkorn- bis Bröckelgefüge, kiesreiche u. kiesärmere Partien wechseln ab, bänderartig durch Tonsubstrat verkittet, z. T. stark verdichtet, einzelne Wurzeln.

Im Liegenden d. Horizonts tritt z. T. eine Fließerdschicht auf in der helle Sandbänder $u$. braune Lehmbänder abwechseln, mit deutl. Kryoturbationen durchsetzt (taschenartig ausgebildet - verlagertes Geschiebelehmmaterial?), $0.07 \%$ Humus

II Bbt - ICv 120 - $300 \mathrm{~cm}$ : dunkelbrauner (7.5 YR 4/4) und hellolivbrauner ( 2.5 Y $5 / 6$ ), (Zwischenlagen, bänderartig), kiesiger anlehmiger Sand bis schwachlehmiger Sand, Einzelkorn-, z, T. Bröckelgefüge, locker, z. T. verdichtet, Tonanreicherungsbänder auch geschichtet, Feinsandbänder u. braune Sandbänder, z. T. stark verkittete Partien, uneinheitl. Kornzusammensetzung, 0.04\% Humus

Anlage 2: Seite 2 


\section{Lysimetergruppe 8:}

\section{Parabraunerde - Braunerde mittlerer Entwicklungstiefe aus Sandlöß über tiefem, kiesführendem Moränenlehm}

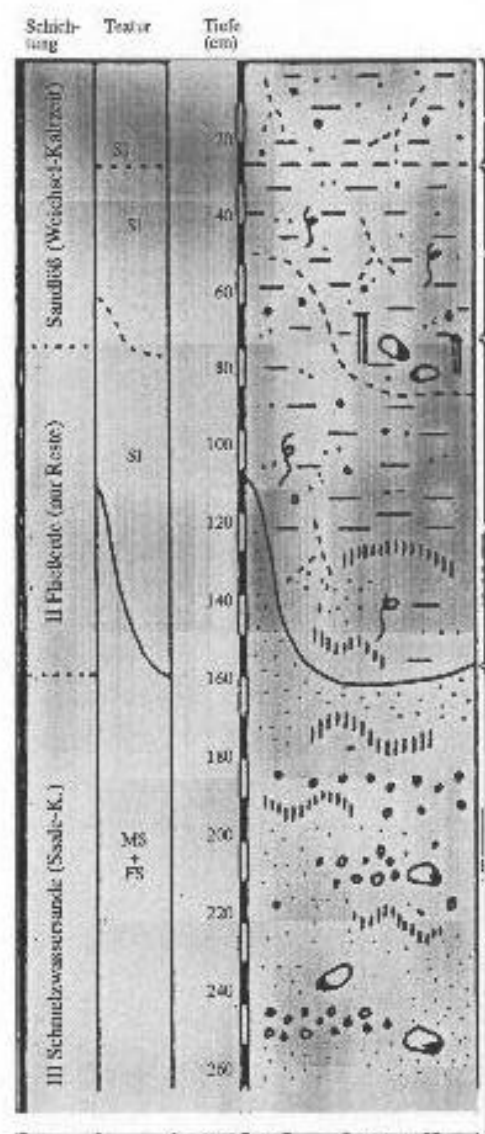

Ap 0 - 25 cm: dunkelgelblicher bis brauner (10 YR 4/4) humoser, schwach kiesiger sandiger Lehm, Krümelgefüge, locker durchwurzelt, scharf begrenzt, $1.6 \%$ Humus

Al - Bv 25 - 70 cm: gelblichbrauner ( 10 YR $5 / 6$ ) sehr schwach humoser, sand. Lehm, Bröckelgefüge, mäßig fest, $\mathrm{m}$. Feinwurzeln u. Wurzelröhren, Regenwurmgänge. Dieser Horizont z. T. bloß bis $45 \mathrm{~cm}$ mächtig, z. T. bis $90 \mathrm{~cm}$ taschenartig reichend. In den Taschen sind im unteren Teil Tonbeläge erkennbar.

gelbl. brauner (10 YR 5/4) kiesiger $(2-3$ Vol \%) sand. Lehm, Bröckelgefüge, mäßig fest, Horizont nur lokal in Taschen. An der Basis (von $70 \mathrm{~cm}$ ) Häufung von Steinen (keine durchgehende Steinsohle), scharf begrenzt, $0.4 \%$ Humus

II Bbt 70 - $150 \mathrm{~cm}$ : dunkelbr. (7.5 YR 4/4) kiesiger (5 - 7 Vol\%) sand. Lehm, Bröckelgefüge, mäßig fest gelagert, sand. u. lehmige Partien wechseln, braune, tonreiche Bänder, die z. T. stärker verdichtet sind, Horizont nur taschenartig erhalten, Regenwurmgänge, Feinwurzeln, Taschen setzen z. T. erst. $30 \mathrm{~cm}$ unter der Sandlößdecke an, uneinheitlich

III Bbt - ICv 150 - $300 \mathrm{~cm}$ : olivgrauer bis olivfarb. ( 5 Y $5 / 2-6 / 2$ ) Mittel- und Feinsand, Einzelkorngefüge, locker, mit Tonanreicherungsbändern $(2 \mathrm{~cm})$, nach unten abnehmend, Bänder schneiden Schichtung, mit Grobsandlagen, einzelne Steine 
Lysimetergruppe 1:

Lessivierter Braunerde-Pseudogley mittlerer Entwicklungstiefe über fossilem Gley im nahen Untergrund aus Sandlöß über kiesführendem Moränenlehm

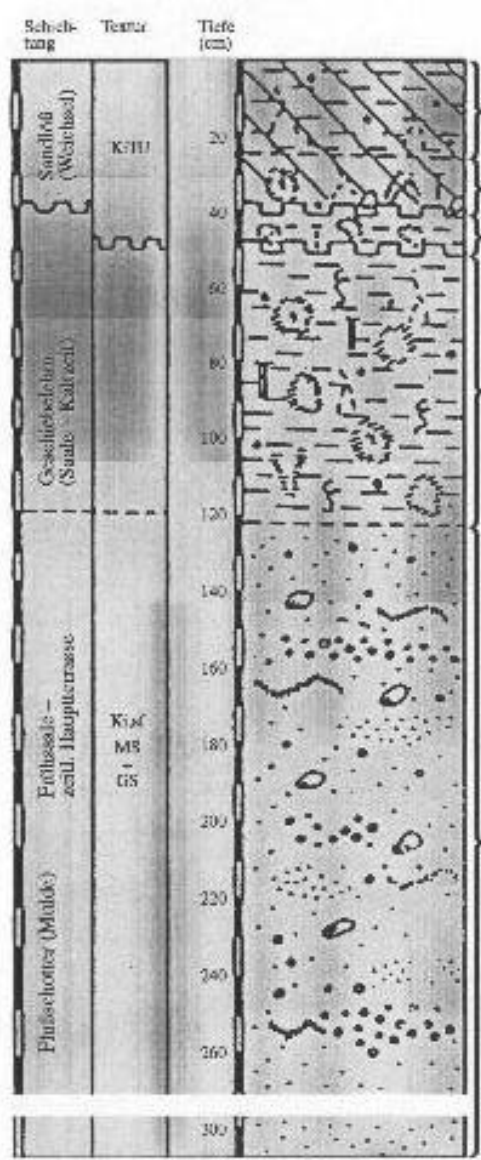

Ap 0 - $25 \mathrm{~cm}$ : schwarzer (10 YR 2/1), stark humoser, schw. kiesiger, lehmiger Schluff, Bröckelgefüge, mäßig dicht, durchwurzelt, scharf begrenzt, $4.1 \%$ Humus

Bv - Sw 25 - $40 \mathrm{~cm}$ : brauner (10 YR 1/3), schwach humoser, schw. kiesiger (2 Vol,\%) lehmiger Schluff, Bröckelgefüge, mäßig dicht, rostfleckig u, rostadrig, Wurm- u. Wurzelgänge enthaltend, schw. durchwurzelt, an der Basis Stein- u. Kiesanhäufungen, verzahnter Übergang, $1.5 \%$ Humus

Al -Sw 40-50 cm: fahlbrauner bis gelblichbrauner (10 YR 6/3 + 5/8), sehr schw. humoser, schw. kiesiger, lehmiger Schluff, Bröckelgefüge mäßig dicht, gebleichte Bereiche u. z. T. stark rostfleckige Bereiche, Wurzelröhren, Sandlößkeile ragen in diesen Horizont, einzelne Wurzeln, verzahnter Ubergang, $0.5 \%$ Humus

II Bt-Sd 50 - 120 cm: olivgrauer ( 5 Y 5/2) und gelblichbrauner (10 YR $5 / 8)$, schwach kiesiger $(\approx 2 \mathrm{Vol} . \%)$ Lehm, Grobpolyedergefüge, sehr dicht, intensiv rost- u. graufleckig, grau gebleicht, senkrechte Kluffflächen sind grau gebleicht, Wurzelbahnen, Regenwurmgänge, scharf begrenzt, $0.2 \%$ Humus

III fGo 120 - $300 \mathrm{~cm}$ : rostig-brauner, kiesiger, steiniger ( $70-80$ Vol.\%) Mittel- u. Grobsand, Einzelkorngefüge, locker, Kiese gut gerundet und horizontal geschichtet, mit Sandzwischenlagerungen, Rostbänder, z. T. durch Fe verkittet, $0.08 \%$ Humus.

Anlage 2: Seite 4 


\section{Lysimetergruppe 7:}

\section{Braunerde Pseudogley mittlerer Entwicklungstiefe aus Sandlöß über kiesführendem Moränenlehm}

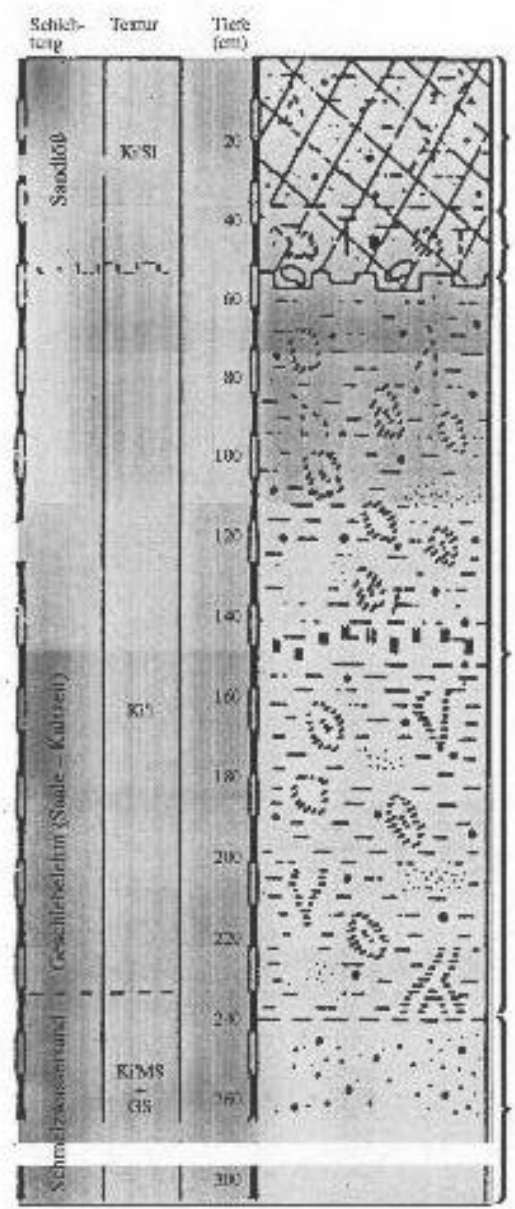

Ap 0 - 35 cm: sehr dunkelgraubrauner (10 YR 4/3 - 3/2), humoser, sehr schwach kiesiger, sandiger Lehm, Bröckel- bis Krümelgefüge, locker, durchwurzelt, scharf begrenzt, $2.2 \%$ Humus

Bv - Sw 35 - 50 cm: brauner bis graugebleichter (10 YR 5/3), schwach humoser, schwachkiesiger Sand, Lehm, Feinbröckelgefüge, mäßig dicht, rostfleckig, Fe-Mn-Konkretionen bis $1 \mathrm{~cm} \varnothing$, grau gebleicht, mit Feinwurzeln und homosen Wurzelbahnen: $z$. T. Steinhäufungen an der Basis, verzahnter Übergang, $0.9 \%$ Humus

II Sd 50 - $135 \mathrm{~cm}$ : intensiv brauner (7.5 YR 5/6) und grauer ( 5 Y $5 / 1$ ) sehr schwach kiesiger Sand, Lehm, Bröckelgefüge, sehr dicht, intensiv rostfleckig und graugebleicht, mit Feinwurzelbahnen auf Klüften, mit rostbraunen Sandlinsen, allmählicher Übergang, $0.2 \%$ Humus

II Sd 135 - 220 cm: gelblichbrauner ( 10 YR 5/8) und hellbräunlichgrauer ( 2.5 Y 6/2), sehr schwach kiesiger Lehm, Bröckelgefüge, sehr dicht, zäh, intensiv rostfleckig, mit grauen Bleichadern und rostbraunen Sandlinsen, scharf begrenzt

III ICn 220 - $300 \mathrm{~cm}$ : hellbrauner (10 YR 6/3), schwach kiesiger Mittelsand und Grobsand, Einzelkorngefüge, locker geschichtet

Anlage 2: Seite 5 


\section{Pseudovergleyte Braunerde - Fahlerde mittlerer Entwicklungstiefe aus Geschiebedecksand über Geschiebemergel}

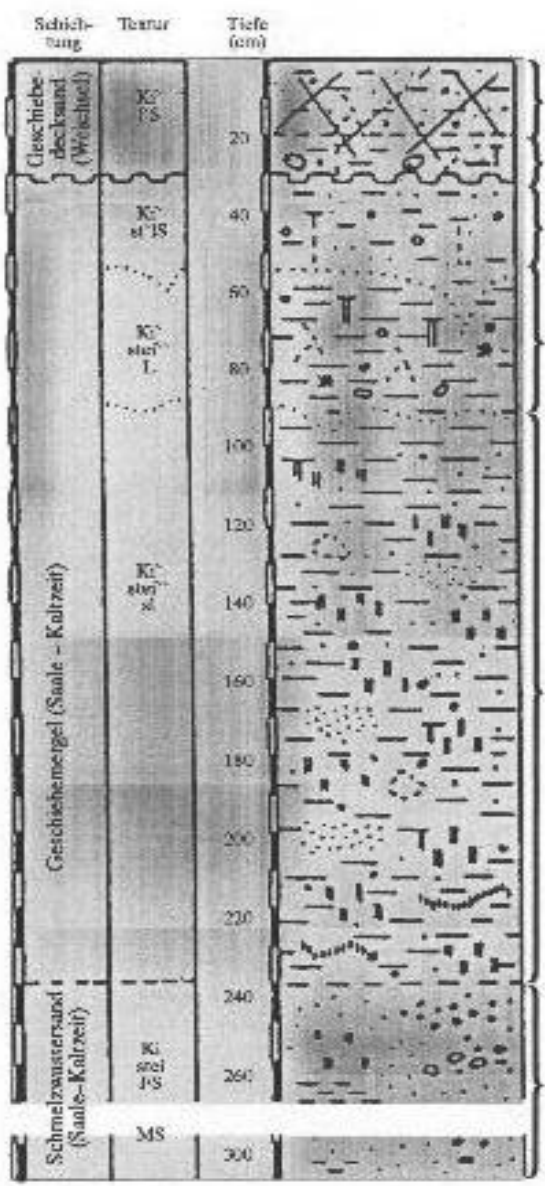

Ap 0 - $20 \mathrm{~cm}$ : brauner bis dunkelbr. (10 YR 4/3), schwach hum., sehr schwach kies., schwach lehm. Sand, Bröckelgefüge, mäßig dicht, schwach durchwurzelt, Wurzelreste, Strohreste, scharf begrenzt, $1.4 \%$ Humus

Bv -Ael 20 - $35 \mathrm{~cm}$ : gelbl.-brauner bis dkl-gelb-br. (10 YR 5/4-4/4) sehr schwach hum., sehr schwach kies., schwach lehm. Sand, Bröckelg., mäßig dicht, s. schw. durchwurz., mit gelbl. Flecken, an der Basis lock. Steinanreich., z. T. hoher, z. T. tiefer verzahnter Übergang, $0.4 \%$ Humus

II Ael 35 - 55 cm: hellgelblich-br. bis hellbr. (10 YR 6/3-6/4), sehr schwach kiesiger, sehr schwach steiniger lehmiger Sand, Einzelkorngefüge, locker, Horizont reicht keilartig ins liegende (Eiskeile), z. T. nur schwach ausgeprägt, z. T. fehlend, Kryoturbationshorizont, $0.1 \%$ Humus

II Swd - Bt 55 - $90 \mathrm{~cm}$ brauner bis dkl. br. (7.5 YR 4/4), sehr schwach kies., sehr schwach stein. Lehm, Polyedergefüge, dicht, z. T. Säulengefüge, schwach durchwurzelt, humose Wurzelbahnen, deutl. Tonbeläge auf den Gefügekörpern, m. einzelnen Sandlinsen, scharf begrenzt, Untergrenze wellig. z. T. tiefer (taschenartig) reichend, ist der Geschiebemergel etwas sandiger, dann tiefer entkalkt, $\mathrm{m}$. kies- $\mathrm{u}$. steinreichen Partien, mit sandigen Bändern, einz. kleine Fe-Mn-Konkretionen enth., $0.2 \%$ Humus

II Sd - lcCv 90 - $\mathbf{2 4 0}$ cm: brauner ( 7.5 YR 5/4 - 4/4), sehr schwach kies., sehr schwach stein. sand. Lehm, Plattengefüge, dicht, $\mathrm{m}$. weißgrauen Kalkausscheid. (horiz.), Kalkreste, mit Sandlinsen, m. zerfallenen Geschieben, scharf begrenzt, an der Basis Roststreifen, stark karbonath.

III leCn 240 - $300 \mathrm{~cm}$ : sehr mattbraun (10 YR 7/3 - 6/3) kiesig, steinig (unterschiedl. Gehalt) Fein- u. Mittelsand $\mathrm{m}$, hohem Skelettanteil, z. T. auch skelettfrei, geschichtet (Kies- u. Steinbänder), Lehmbänder und linsen, mäßig karbonathaltig, Einzelkorngefüge, locker 


\section{Parabraunerde mittlerer Entwicklungstiefe aus Löß}

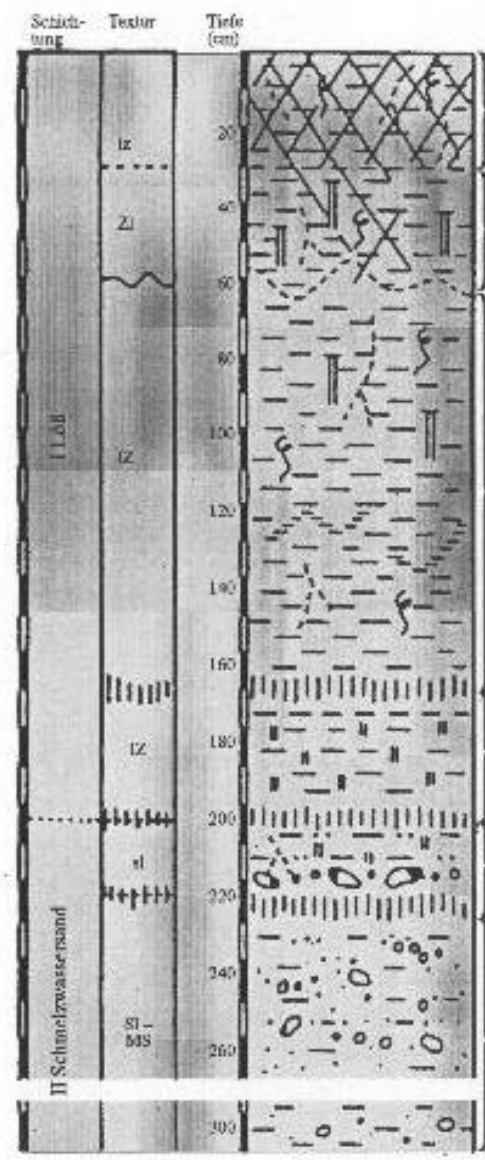

Ap 0 - $30 \mathrm{~cm}$ : dunkler, gelblich-brauner (10 YR 4/4), humoser, lehmiger Schluff, Bröckelgefüge, mäßig dicht, durchwurzelt, Tiergänge, Wurzelgänge, scharf begrenzt, $2.5 \%$ Humus

Bt 30 - 60 cm: dunkelbrauner (7.5 YR 4/4) Schlufflehm, Bröckelgefüge, oberhalb etwas plattig, schwach porös, schwach durchwurzelt, Tier- u. Wurzelgänge, Feinwurzeln, Tierlosung, verzahnter Übergang, Tonbeläge, $0.6 \%$ Humus

Bt - ICv 60 - 170 cm: fahlbr. bis dunkelbrauner (10 YR 6/3 - 7,5 YR 4/4) lehm. Schluff, Plattengefüge, mäßig dicht, schwach porös, einzelne Wurzeln u. Wurzelbahnen, im oberen Teil noch Tonbeläge, Lamellenfleckenzone (hellere und dunkelbraune Bänder wechseln), Wurmgänge mit Tierlosung, allmählicher Übergang, $0,3 \%$ Humus

IcCn 170 - 205 cm: gelbl.-brauner (10 YR 5/6) lehmiger Schluff, Gefüge mäßig dicht, allmählicher Übergang stark karbonathaltig, $0.4 \%$ Humus

II leCn 205 - 225 cm: gelbl.-brauner (10 YR 5/4) sandiger Lehm, Bröckelgefüge, Übergangsbereich sand. LöB, Geschiebemergelreste enthaltend, Kiese u. Steine sohlenartig angereichert, Feinwurzeln, rotstreifig, stark karbonathaltig, $0.2 \%$ Humus

III ICn 225 - $300 \mathrm{~cm}$ : hellbraun bis hellgelbl. (10 YR 6/3-6/4) steiniger, kiesiger lehmiger Sand bis Mittelsand, Einzelkorngefüge, Kiesanteil, unterschiedlich geschichtet, $0.03 \%$ Humus

Anlage 2: Seite 7 


\section{Lysimetergruppe 10:}

\section{Schwarzerde mittlerer Entwicklungstiefe aus Löß}

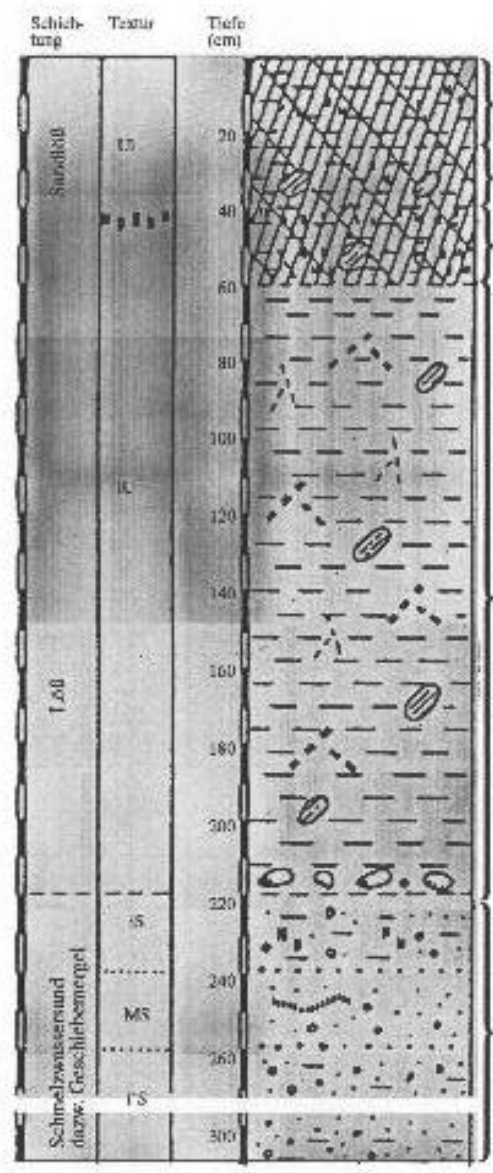

Ap 0 - 25 cm: sehr dunkelbr. (10 YR 2/2), humoser Schlufflehm, Krümelgefüge, mäßig dicht, einzelne Wurzeln, in Spuren karbonathaltig, untersetzte Stalldungreste, scharf begrenzt, 3.1\% Humus

rAh 25 - 45 cm: sehr dunkelbr. (10 YR 2/2), humoser Schlufflehm, Krümelgefüge, locker, porös, schw. durchwurzelt, in Spuren karbonathaltig, Krotowinen, Hamstergänge u. -baue, allmähl. Überg., 2.4\% Humus

rAh-IcCv 45 - $60 \mathrm{~cm}$ : braun bis dunkelbr. (10 YR 5/4), schw, humoser lehmiger Schluff, Krümelgefüge, locker, porös, einzelne Wurzeln enthaltend, stark karbonathaltig, mit Krotowinen u. Regenwurmgängen u. Wurzelbahnen, scharf begrenzt, $1.3 \%$ Humus

lcCv 60 - $220 \mathrm{~cm}$ : gelbl.-brauner (10 YR 5/4 - 5/6) lehmiger Schluff, Schwammgefüge, mäßig dicht, porös, karbonatreich, im unteren Teil des Horizonts nur noch stark karbonathaltig, Wurzelbahnen bis $160 \mathrm{~cm}$; Krotwinen, Kalkpseudomycel, einzelne Feinwurzeln enthaltend, Steinsohle an der Basis, Löß wird im unteren Teil sandiger u. rötlich-braun scharf begrenzt, $0.6 \%$ Humus

II ICn 220 - $300 \mathrm{~cm}$ : fahloliv bis fahlgelb (5Y 7/3 - 6/3), sehr schwach kiesiger Mittelsand, Einzelkorngefüge, karbonatfrei, z. T. braune Bänder; dunkelbr. (7.5 YR 5/6), sehr stark kiesiger, schwach lehm. Sand, Einzelkorngefüge, locker, Kies ca. 70 Vol.\% - Mittelkies, kleinflächig wechselnd; dunkelbr. (7.5 YR 4/4), kiesiger, stark lehm. Sand, Bröckelgefüge, nur z. T. ausgebildet (Geschiebemergelreste); mäBig, $0.2 \%$ Humus 
Anlage 3: Bodendaten aus der Zeit der Lysimetergewinnung

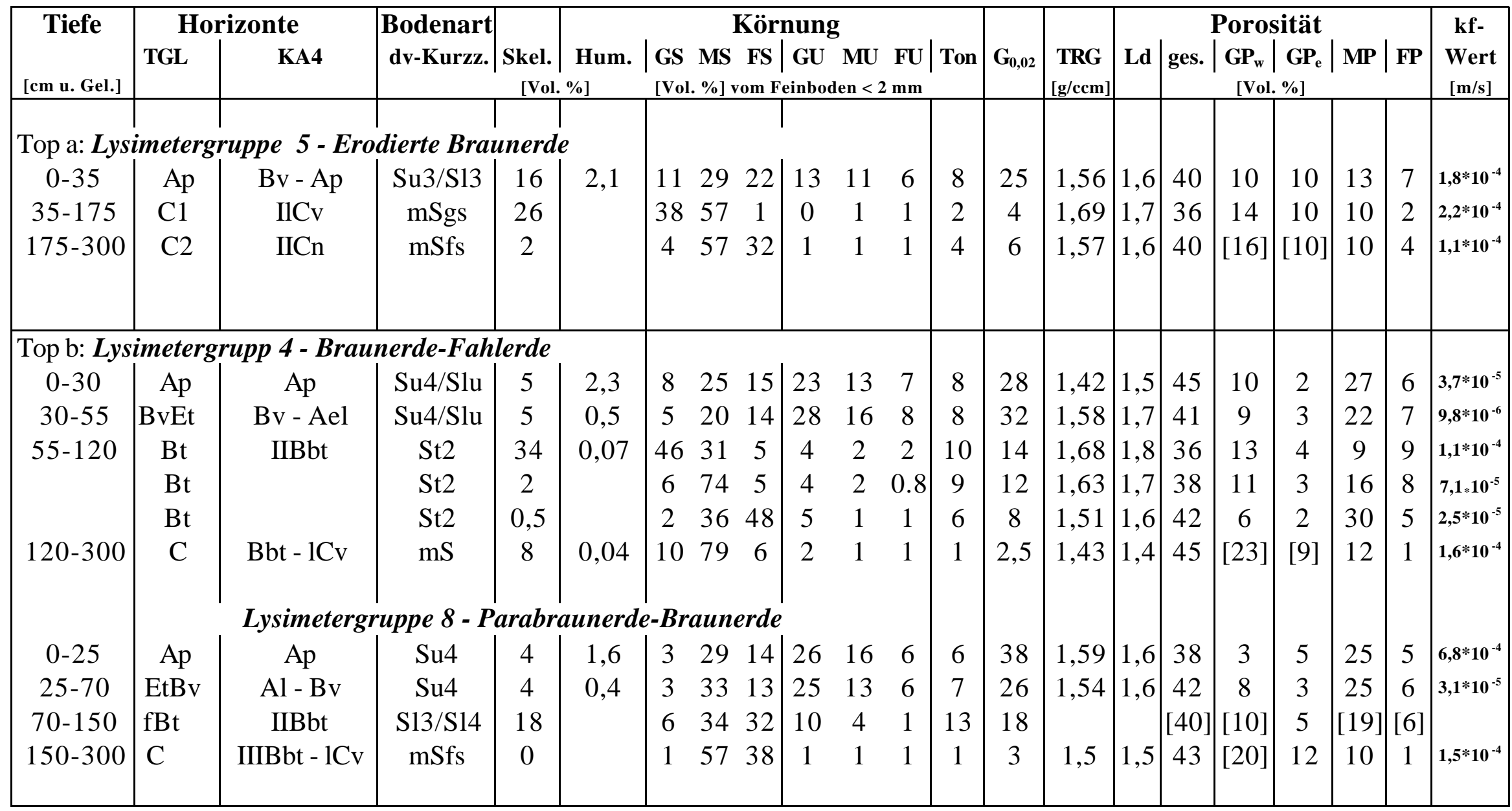


Fortsetzung Anlage 3

\begin{tabular}{|c|c|c|c|c|c|c|c|c|c|c|c|c|c|c|c|c|c|c|c|c|}
\hline \multirow{2}{*}{\begin{tabular}{|c|} 
Tiefe \\
[cm u. Gel.] \\
\end{tabular}} & \multicolumn{2}{|c|}{ Horizonte } & \multirow{2}{*}{$\begin{array}{c}\text { Bodenart } \\
\text { dv-Kurzz. }\end{array}$} & \multirow[b]{2}{*}{$\begin{array}{r}\text { Skel. } \\
{[\text { Vol. }}\end{array}$} & \multicolumn{7}{|c|}{ Körnung } & \multirow[b]{2}{*}{$\mathbf{G}_{0,02}$} & \multirow[b]{2}{*}{$\begin{array}{c}\text { TRG } \\
{[\mathrm{g} / \mathrm{ccm}]}\end{array}$} & \multicolumn{6}{|c|}{ Porosität } & \multirow{2}{*}{\begin{tabular}{|c} 
kf- \\
Wert \\
{$[\mathrm{m} / \mathrm{s}]$} \\
\end{tabular}} \\
\hline & TGL & KA4 & & & $\begin{array}{l}\text { Hum. } \\
\%]\end{array}$ & & $\begin{array}{l}\text { MS FS } \\
\text { 1. \%] vom F }\end{array}$ & \begin{tabular}{|l}
$\mid \mathbf{G U}$ \\
einboc
\end{tabular} & $\begin{array}{l}\text { MU } \\
\text { len < }\end{array}$ & $\begin{array}{l}\mathrm{FU} \\
\mathrm{mm}\end{array}$ & | Ton & & & Ld & ges. & $\begin{array}{r}\mathbf{G P}_{\mathrm{w}} \\
{[\mathrm{Vo}} \\
\end{array}$ & $\begin{array}{c}\mathbf{G P}_{\mathrm{e}} \\
\%]\end{array}$ & MP & FP & \\
\hline \multicolumn{21}{|c|}{ Top c: Lysimetergruppe 1- lessivierter Braunerde-Pseudogley } \\
\hline $0-25$ & Ap & Ap & Jlls & 5 & 4,1 & 6 & 1417 & 26 & 18 & 8 & 11 & 37 & $\mid 1,45$ & $\mid 1,5$ & 43 & 3 & 2 & 29 & 9 & $8,5 * 10$ \\
\hline $25-40$ & Bvg & $\mathrm{Br}_{\mathrm{x}}$ & & 7 & 1 , & 2 & 1618 & 28 & 18 & 8 & 10 & 36 & 1,58 & 1,7 & 39 & 4 & 2 & 25 & 8 & $*$ \\
\hline 40 & Etg & & & 9 & 0 & 4 & 1417 & 26 & 19 & 8 & 2 & 39 & & & & & & & & \\
\hline $50-120$ & $\mathrm{Bt} / \mathrm{rG}$ & $\mathrm{u}$ & & 8 & 0,2 & 8 & $\begin{array}{ll}30 & 20\end{array}$ & 11 & 6 & 4 & 20 & 30 & 1,74 & 1,9 & 34 & 2 & 3 & 11 & 18 & $3,1 * 10-$ \\
\hline \multirow[t]{2}{*}{$120-300$} & rGo & 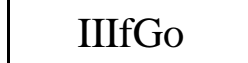 & & 73 & 0,08 & 40 & 494 & 2 & 1 & 1 & 2 & 4 & & & 30 & 20 & 5 & 4 & 1 & $5,0 * 10-$ \\
\hline & \multicolumn{12}{|c|}{ Lysimetergruppe 7 - Braunerde-Pseudogley } & & & & & & & & \\
\hline $0-35$ & Ap & $A p$ & & 2 & 2,2 & 5 & 2514 & 25 & 13 & 8 & 10 & 31 & 1,68 & 1,8 & 35 & 2 & 17 & 7 & 9 & $1,2 * 10$ \\
\hline $35-$ & BvEg & 2 & & 2 & 0 , & 3 & 2315 & 25 & 15 & 9 & 10 & 34 & 1,58 & 1,7 & 40 & 5 & 6 & 20 & 8 & $1,0 * 10$ \\
\hline $50-1$ & $\mathrm{Bg} 1$ & & & 4 & 0,2 & 8 & 2619 & 11 & 6 & 5 & 25 & 36 & 1,83 & 2,1 & 31 & 1 & 3 & 8 & 19 & $8,1 * 10-$ \\
\hline $135-$ & $\mathrm{Bg} 2$ & & & 3 & & 2 & 2318 & 17 & 8 & 7 & 25 & 40 & 1,87 & 2,1 & 29 & 0 & 5 & 3 & {$[21$} & $2,0 * 10$ \\
\hline \multirow[t]{2}{*}{$220-300$} & $\mathrm{C}$ & III $1 \mathrm{Cn}$ & $\mathrm{m}$ & 24 & & 33 & $\begin{array}{ll}56 & 5\end{array}$ & 3 & 1 & 1 & 1 & 3 & 1,68 & 1,7 & 36 & 16 & 8 & 11 & 1 & $1,5 * 10$ \\
\hline & \multicolumn{12}{|c|}{ Lysimetergruppe 11 - pseudovergleyte Braunerde-Fahlerde } & & & & & & & & \\
\hline $0-20$ & Ap & $\Delta \mathrm{n}$ & $\mathrm{Su} 2 / \mathrm{Su} 3$ & 5 & 1,4 & 6 & 4026 & 16 & 5 & 3 & 4 & 12 & 1,67 & 1,7 & 36 & 6 & 4 & 22 & 4 & $7,5 * 10-$ \\
\hline $20-35$ & EtBv & $-A$ & & 4 & 0, & 7 & 4422 & 14 & 6 & 4 & 3 & 13 & & & 36 & 6 & 4 & 22 & 4 & \\
\hline $35-$ & $\mathrm{E}$ & & & 9 & 0 & 10 & $\begin{array}{ll}41 & 32\end{array}$ & 10 & 2 & 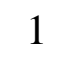 & 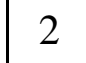 & - & & & 34 & 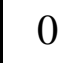 & 5 & 16 & 4 & \\
\hline $55-90$ & $(\mathrm{~g}) \mathrm{Bt}$ & ( & & 4 & 0,2 & 6 & 2625 & 11 & 5 & 4 & 23 & 32 & 1,83 & 2,0 & 31 & 0 & 3 & 2 & 18 & $7,9 * 10-$ \\
\hline $90-240$ & Cc1 & 110 & & 4 & & 5 & 4427 & 13 & 7 & 8 & 15 & 30 & 1,86 & 2,0 & 29 & 2 & 3 & 10 & 14 & $2,5 * 10$ \\
\hline $240-300$ & $\mathrm{Cc} 2$ & III Z(16)SlcC & $\mathrm{mSfs}$ & & & 2 & $44 ? 48$ & 5 & 0 & 0 & 0 & 0 & & & 40 & 20 & 8 & 12 & 0 & $1,0 * 10$ \\
\hline
\end{tabular}


Fortsetzung Anlage 3

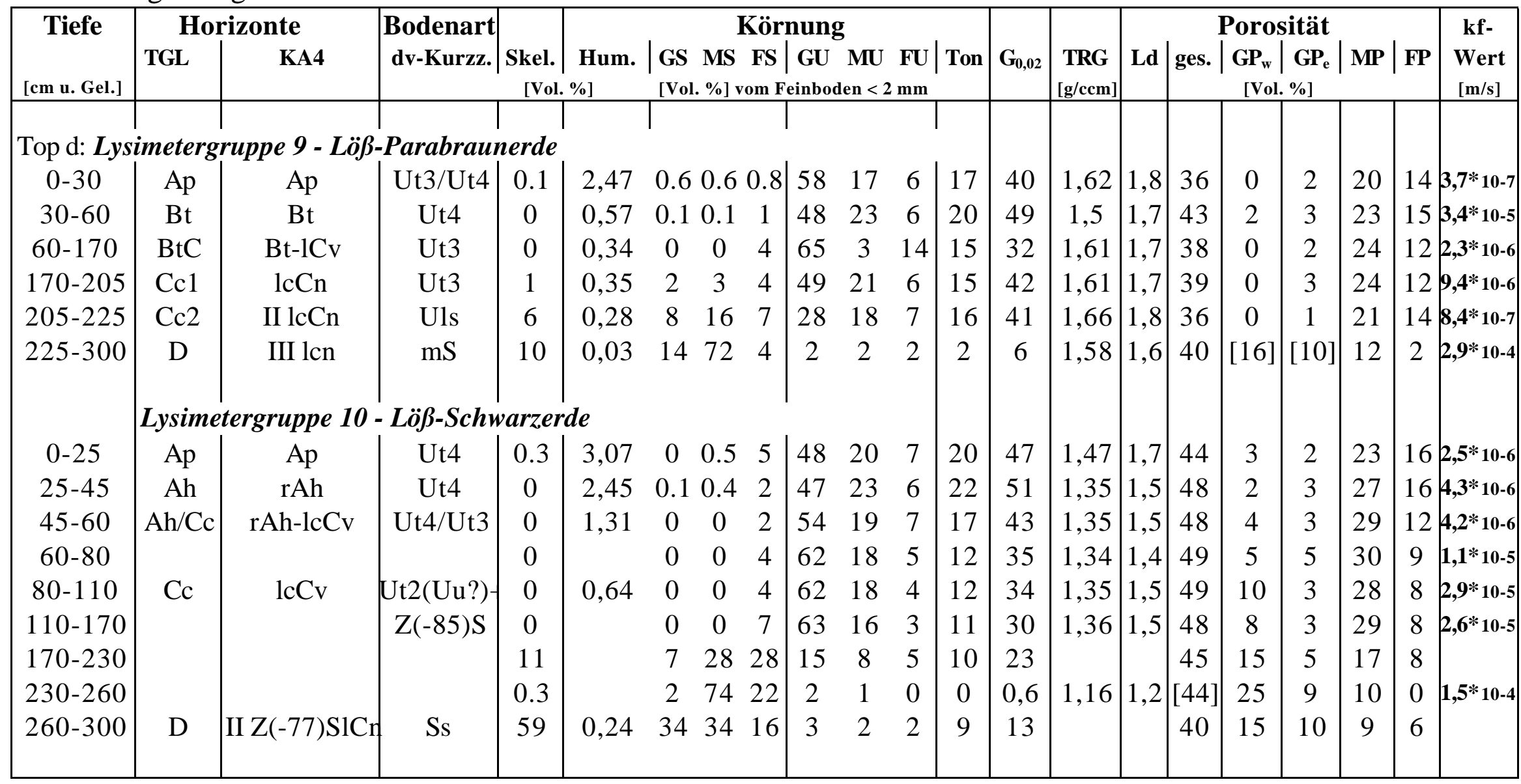




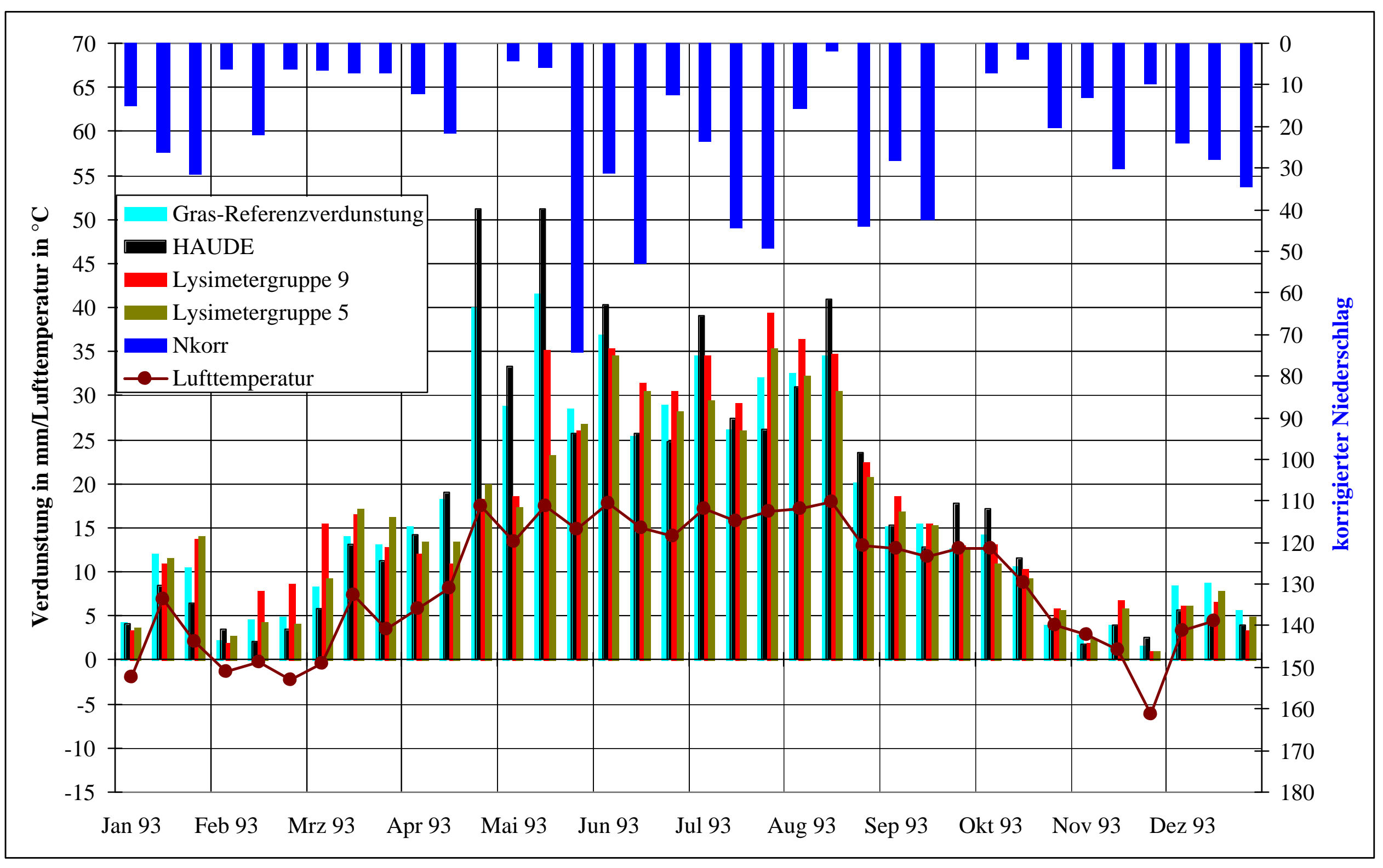

Anlage 4a: Dekadenwerte von Niederschlag, Lufttemperatur und Verdunstung im Jahr 1993 (Bewuchs: Grünbrache) 


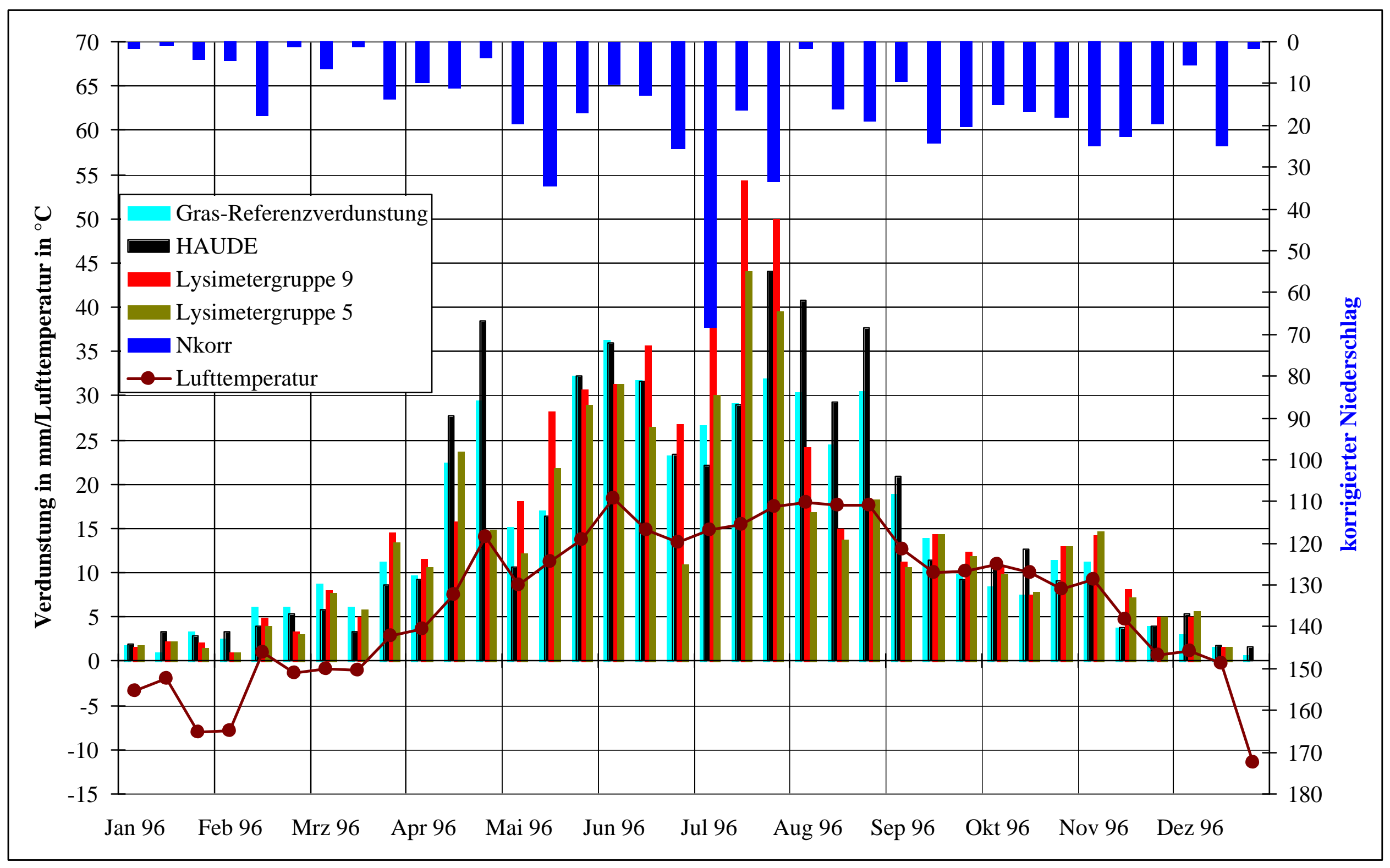

Anlage 4b: Dekadenwerte von Niederschlag, Lufttemperatur und Verdunstung im Jahr 1996 (Bewuchs: Kartoffel) 


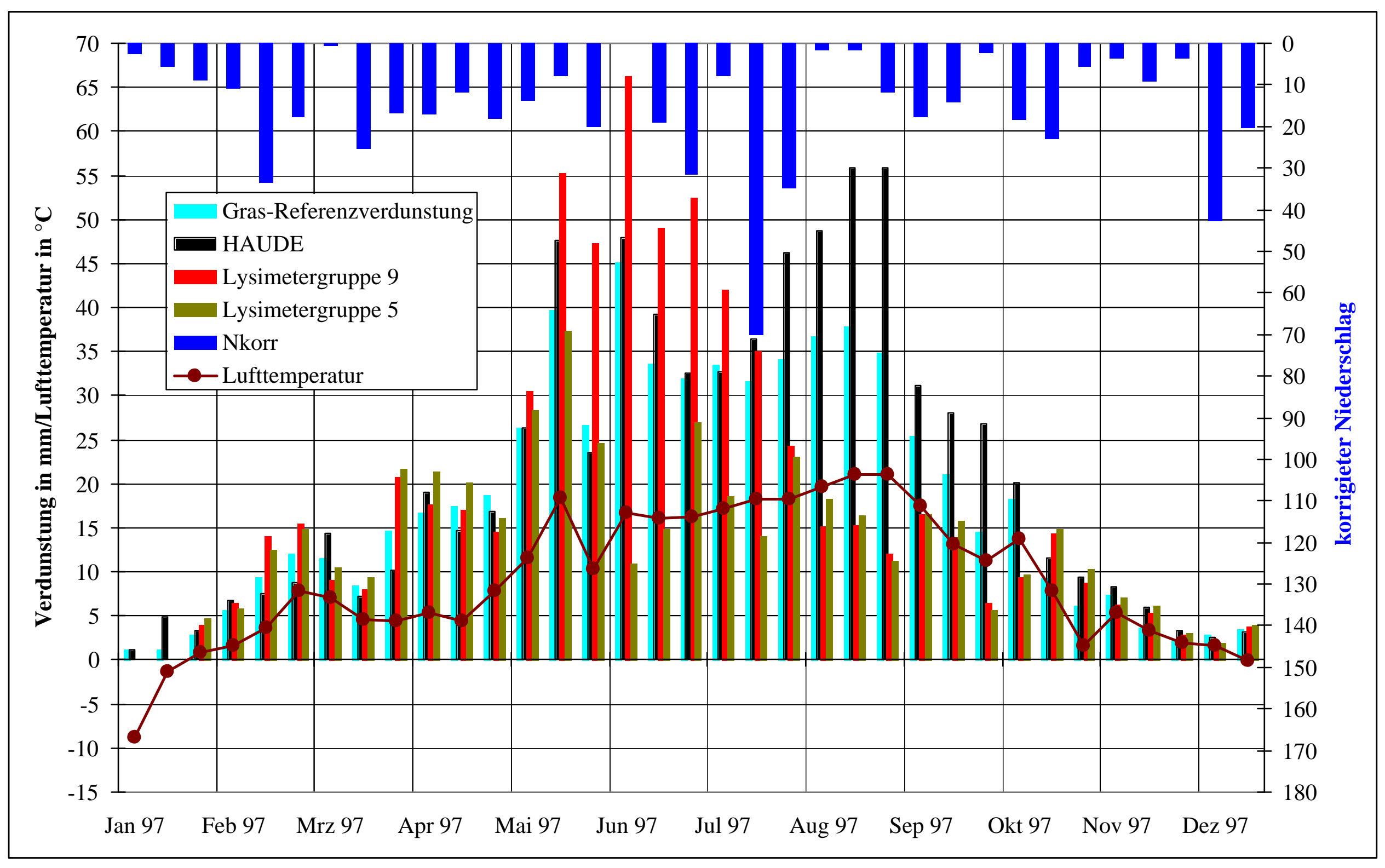

Anlage 4c: Dekadenwerte von Niederschlag, Lufttemperatur und Verdunstung im Jahr 1997 (Bewuchs: Sommerweizen) 

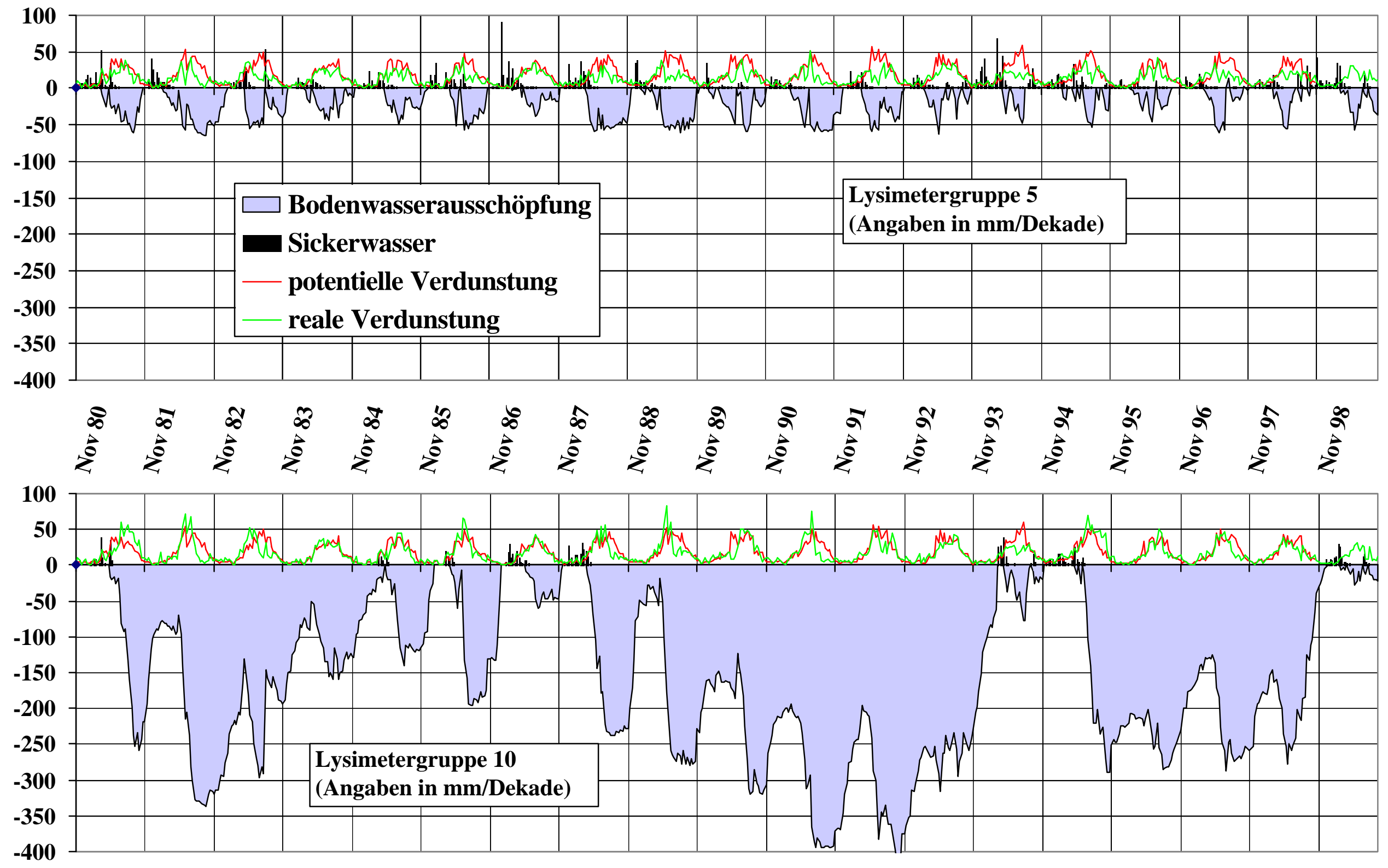

Anlage 5: Verlauf der Bodenwasserhaushaltsgrößen am Beispiel einer erodierten Braunerde und einer Löß-Schwarzerde 
Definition von Trocken- und Feuchtjahren - Versickerungstyp a

(Sandlöß über Schmelzwassersanden)

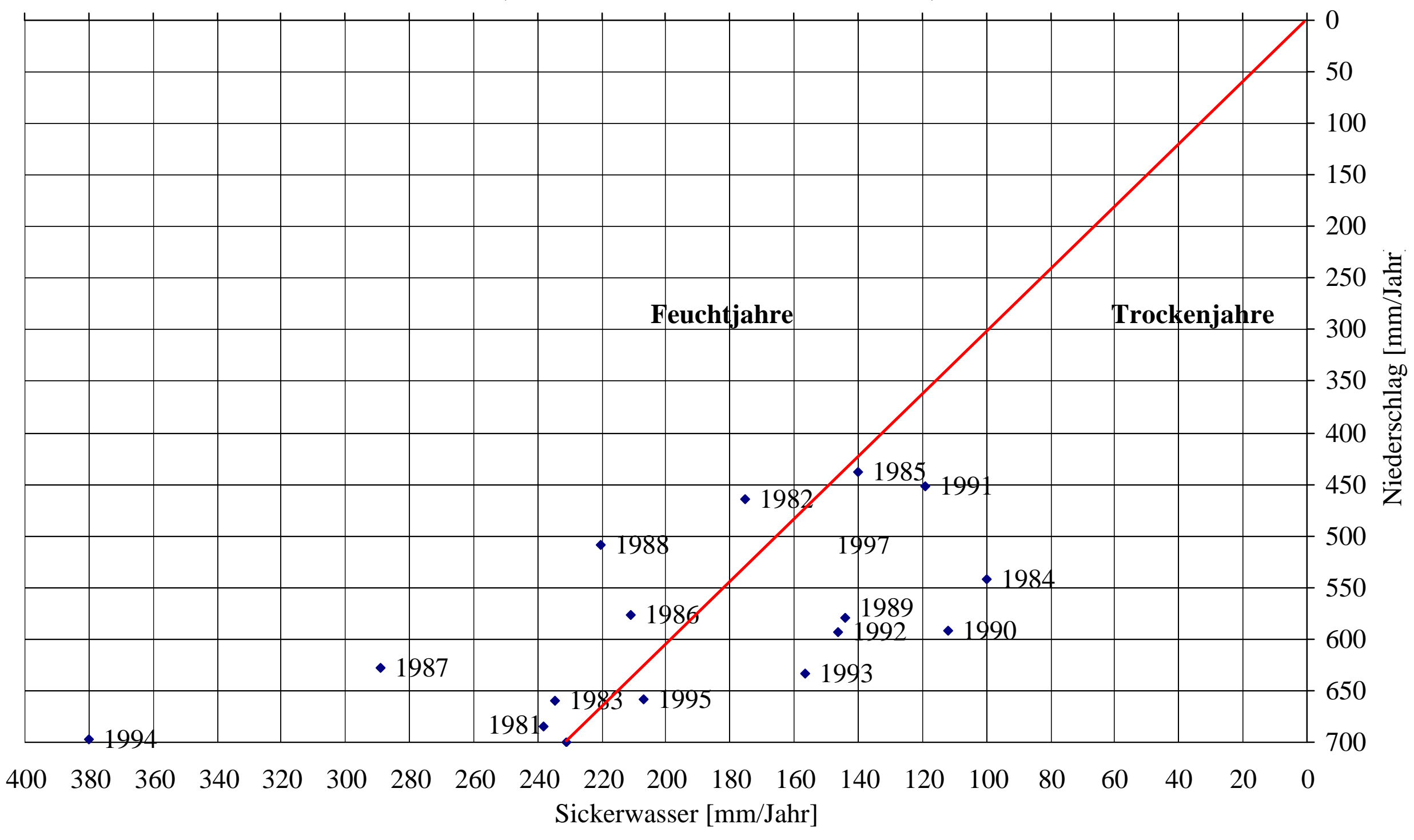

Anlage 6: Seite 1 


\section{Definition von Trocken- und Feuchtjahren - Versickerungstyp b \\ (Sandlöß über gebänderten Sanden)}

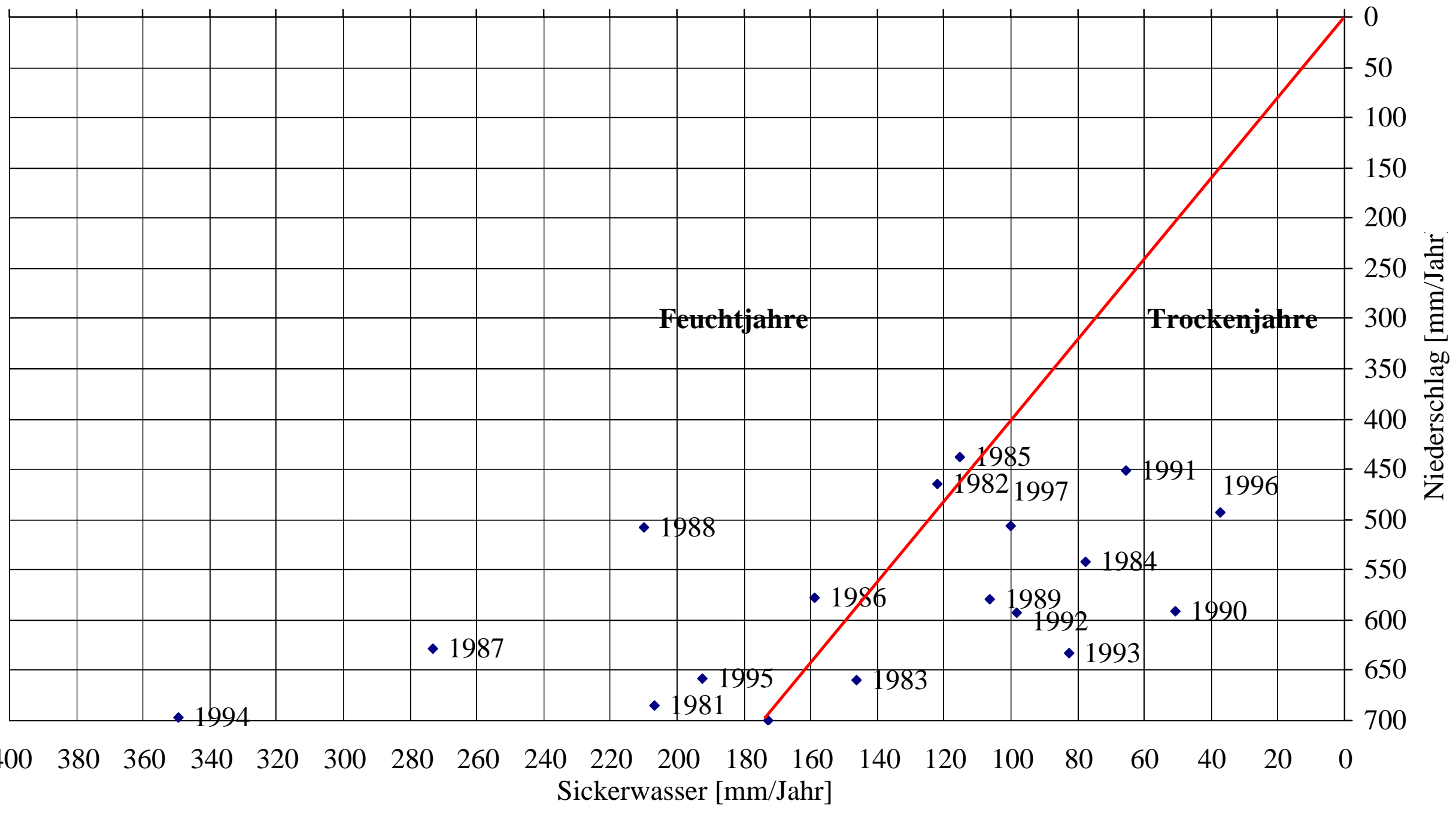


Definition von Trocken- und Feuchtjahren - Versickerungstyp c (Sandlöß über Geschiebelehm)

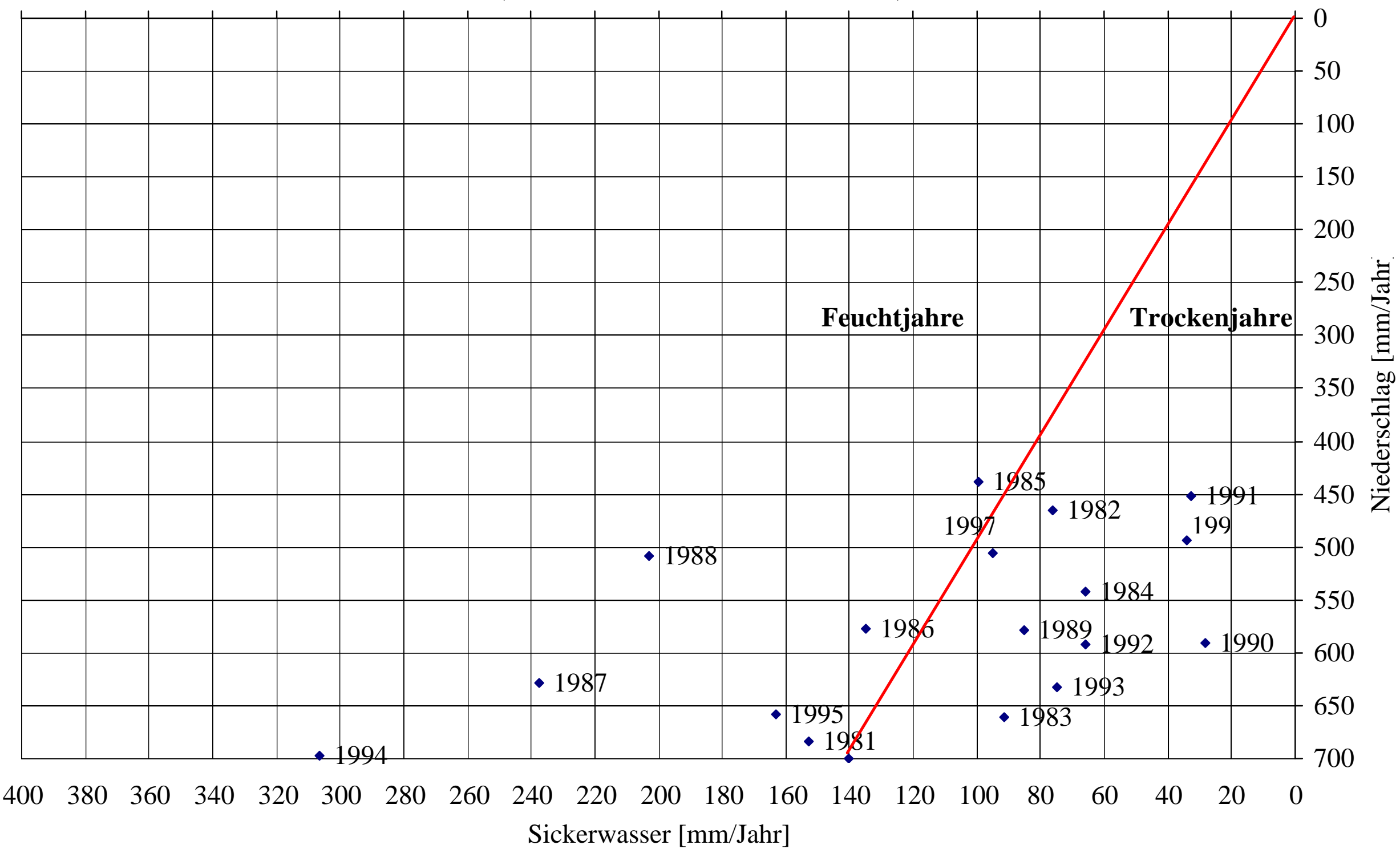




\section{Definition von Trocken- und Feuchtjahren - Versickerungstyp d}

(Tiefgründiger Löß)

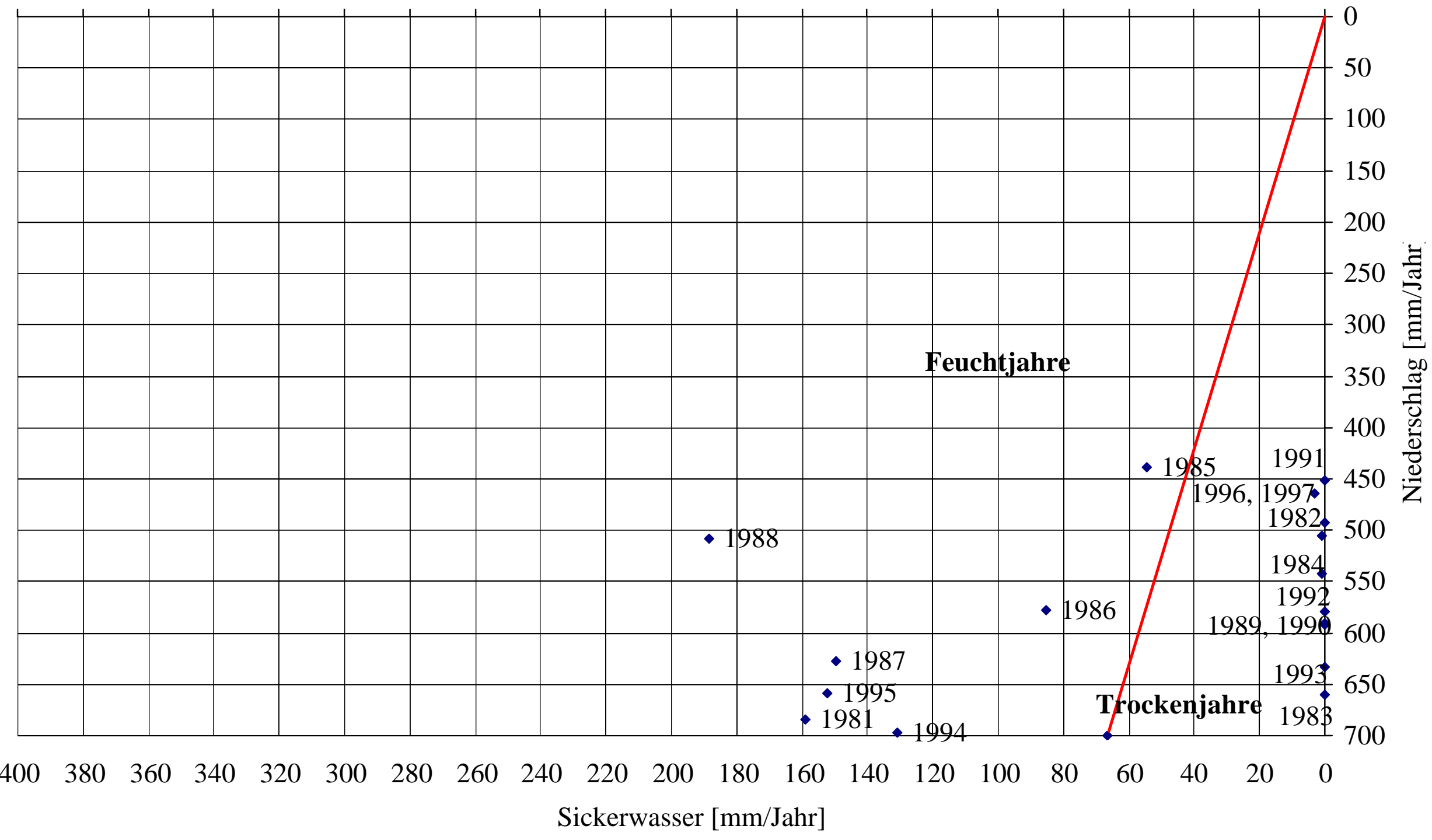


Sickerwasser in 3 m Tiefe von Nov. 1980 bis Okt. 1999 (Monatssummen in mm)

Bodenart: Sandlöß über gut durchlässigen Schmelzwassersanden

(Lysimetergruppe 5)

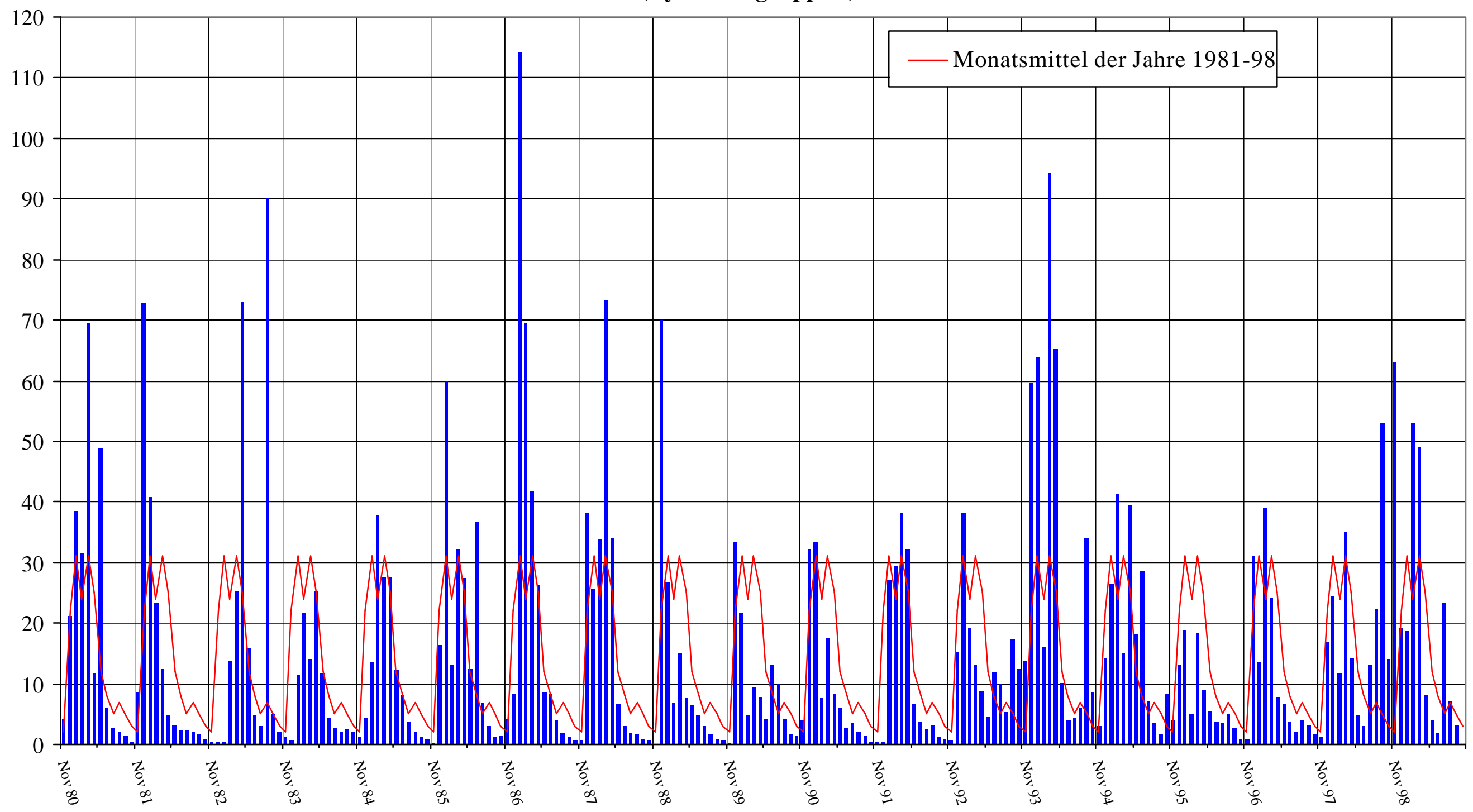


Sickerwasser in 3 m Tiefe - Monatsummen von Nov. 1980 bis Okt. 1999 in [mm]

Bodenart: Sandlöß über Fließerde und lehmhaltigen Schmelzwassersanden

(Lysimetergruppe 4 und 8)

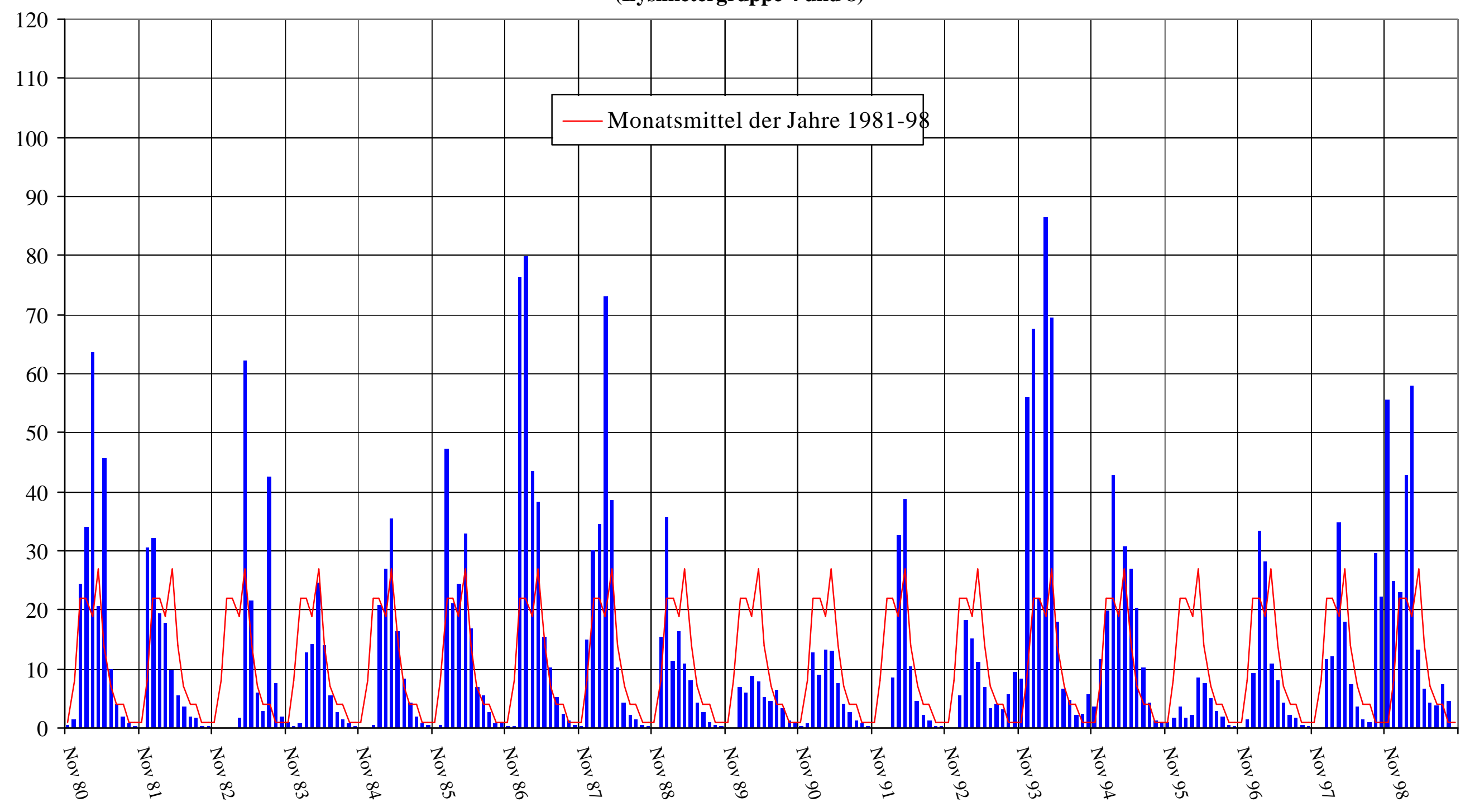


Sickerwasser in 3 m Tiefe - Monatssummen von Nov. 1980 bis Okt. 1999 in [mm]

Bodenart: Sandlöß oder Geschiebedecksand über teils stark verdichtetem Geschiebelehm (Lysimetergruppe 1, 7 und 11)

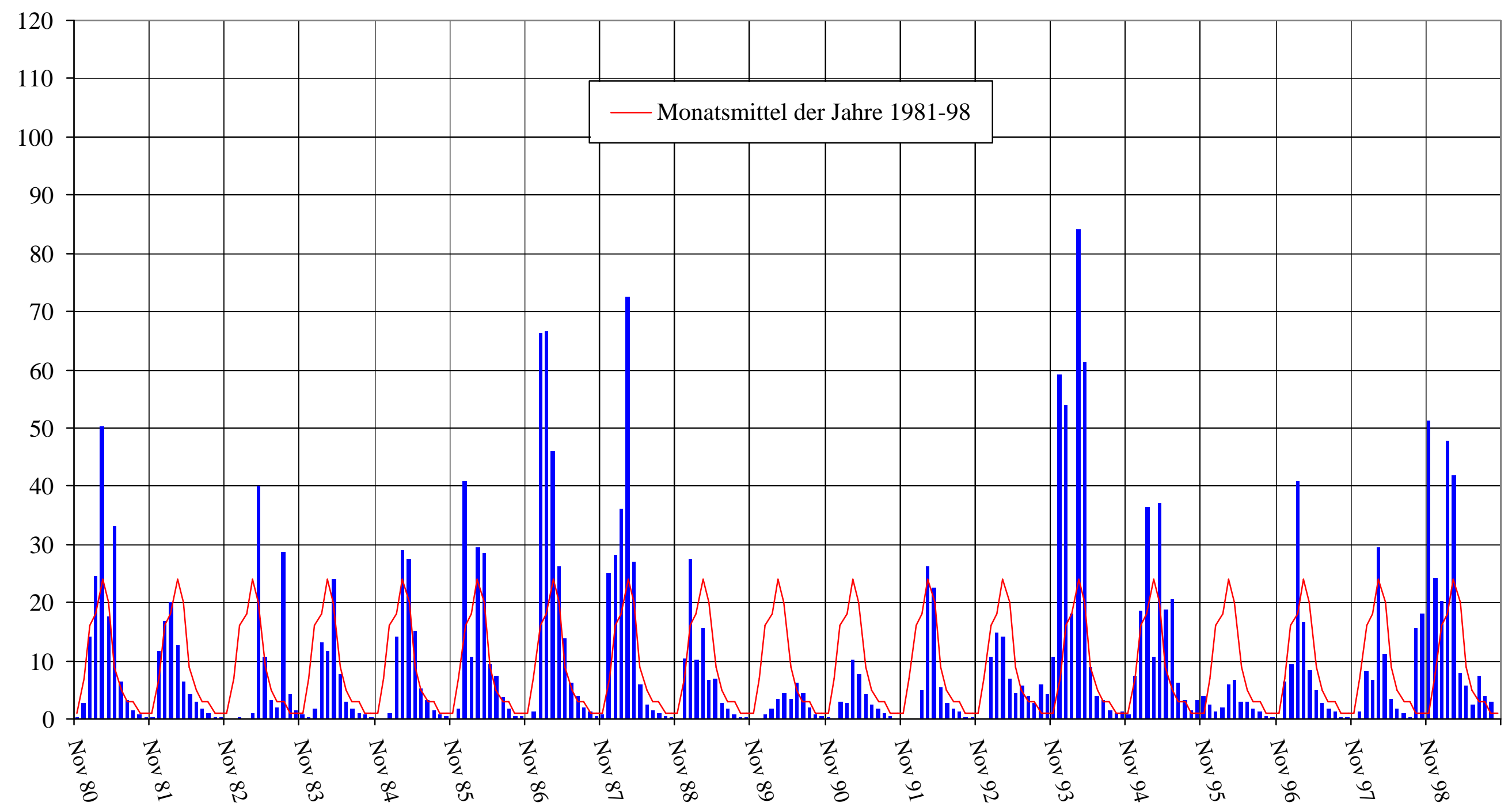


Sickerwasser in 3 m Tiefe - Monatssummen von Nov. 1980 bis Okt. 1999 in [mm]

Bodenart: Mehr als $2 \mathrm{~m}$ mächtiger Löß über Schmelzwassersanden

(Lysimetergruppe 9 und 10)

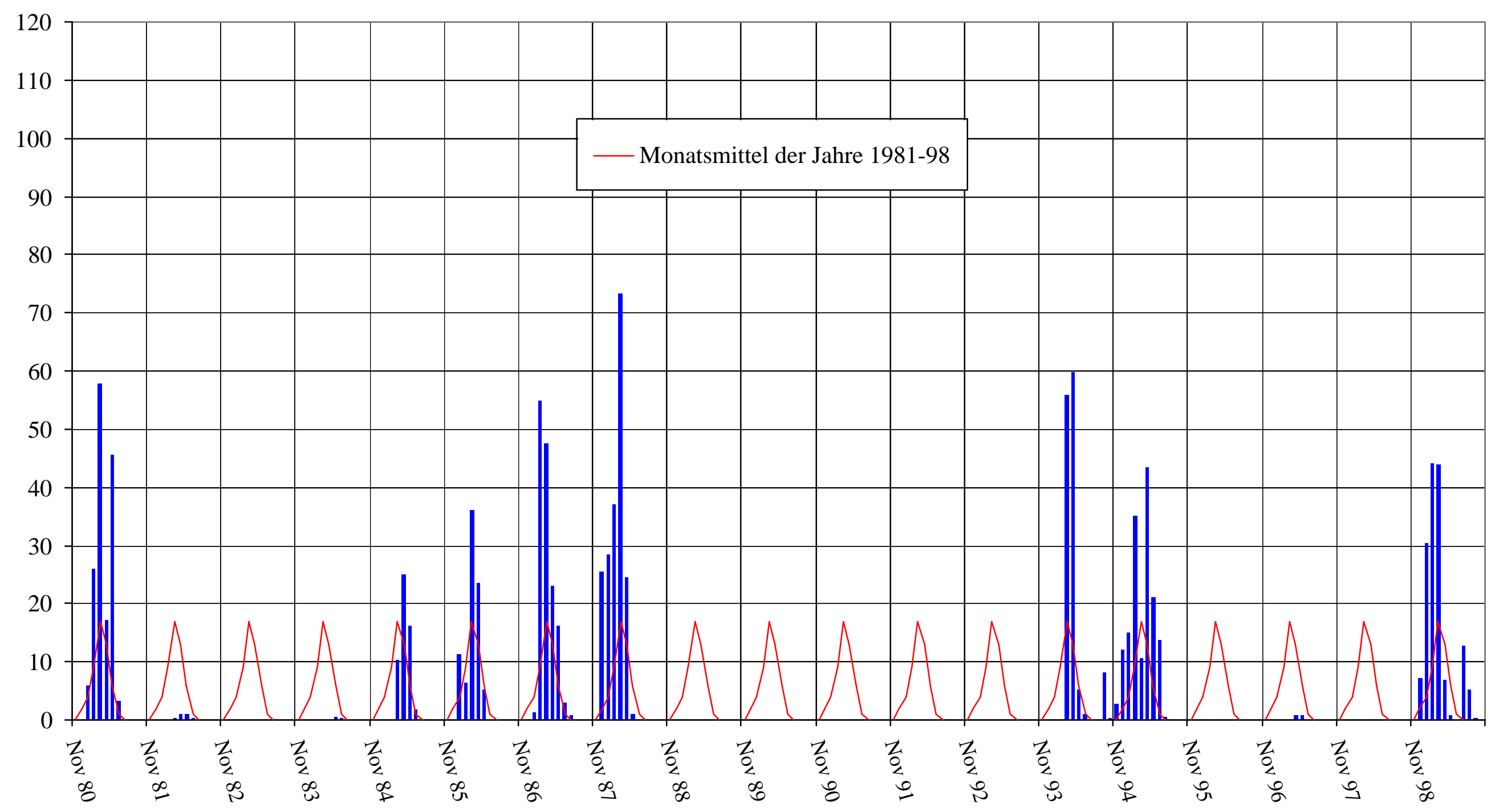


Anlage 8: Wasserhaushaltsbilanzen der Jahre 198197 für 4 Versickerungstypen unter landwirtschaftlicher Nutzung in [mm/Jahr]

$\begin{array}{ll}\mathrm{P}_{\text {korr }} & \text { - korrigierter Niederschlag } \\ \text { ETP }_{\text {Gras }} & \text { - Gras-Refernzverdunstung } \\ \text { KWB } & \text { - klimatische Wasserbilanz } \\ \text { ETR } & \text { - reale Verdunstung } \\ \text { SW } & \text { - Sickerwasser } \\ \Delta \text { SB } & \text { - Vorratsänderung im Lysimeter }\end{array}$

Top: a (Sand-Lysimetergruppe 5)

\begin{tabular}{|l|cccccc|ccccccc|c|c|c|}
\hline Monat & $\mathbf{1 1}$ & $\mathbf{1 2}$ & $\mathbf{1}$ & $\mathbf{2}$ & $\mathbf{3}$ & $\mathbf{4}$ & $\mathbf{5}$ & $\mathbf{6}$ & $\mathbf{7}$ & $\mathbf{8}$ & $\mathbf{9}$ & $\mathbf{1 0}$ & $\mathbf{W i}$ & So & Jahr \\
\hline P $_{\text {korr }}$ & 56 & 57 & 43 & 37 & 47 & 59 & 53 & 72 & 68 & 70 & 52 & 37 & 299 & 352 & 652 \\
\hline ETP $_{\text {Gras }}$ & 17 & 12 & 15 & 17 & 35 & 57 & 88 & 93 & 108 & 93 & 54 & 33 & 153 & 470 & 623 \\
kWB & $\mathbf{3 9}$ & $\mathbf{4 5}$ & $\mathbf{2 8}$ & $\mathbf{2 0}$ & $\mathbf{1 2}$ & $\mathbf{2}$ & $\mathbf{- 3 4}$ & $\mathbf{- 2 1}$ & $\mathbf{- 4 0}$ & $\mathbf{- 2 4}$ & $\mathbf{- 2}$ & $\mathbf{4}$ & $\mathbf{1 4 7}$ & $\mathbf{- 1 1 8}$ & $\mathbf{2 9}$ \\
\hline ETR & 19 & 14 & 13 & 15 & 36 & 52 & 72 & 70 & 73 & 56 & 45 & 29 & 149 & 344 & 493 \\
SW & 2 & 22 & 31 & 24 & 31 & 25 & 12 & 8 & 5 & 7 & 5 & 3 & 136 & 40 & 175 \\
$\Delta$ SB & 34 & 22 & -1 & -2 & -20 & -19 & -30 & -6 & -9 & 6 & 3 & 6 & 15 & -31 & -17 \\
\hline
\end{tabular}

Top: b (gebänderte Sande- Lysimetergrupp 4 und 8)

\begin{tabular}{|l|cccccc|ccccccc|c|c|c|}
\hline Monat & $\mathbf{1 1}$ & $\mathbf{1 2}$ & $\mathbf{1}$ & $\mathbf{2}$ & $\mathbf{3}$ & $\mathbf{4}$ & $\mathbf{5}$ & $\mathbf{6}$ & $\mathbf{7}$ & $\mathbf{8}$ & $\mathbf{9}$ & $\mathbf{1 0}$ & $\mathbf{W i}$ & So & Jahr \\
\hline P $_{\text {korr }}$ & 56 & 58 & 44 & 37 & 47 & 59 & 54 & 72 & 68 & 70 & 52 & 37 & 302 & 353 & 655 \\
\hline ETP & & 17 & 12 & 15 & 17 & 35 & 57 & 88 & 93 & 108 & 93 & 54 & 33 & 153 & 470 & 623 \\
kWB & $\mathbf{4 0}$ & $\mathbf{4 6}$ & $\mathbf{2 9}$ & $\mathbf{2 0}$ & $\mathbf{1 3}$ & $\mathbf{2}$ & $\mathbf{- 3 4}$ & $\mathbf{- 2 1}$ & $\mathbf{- 4 1}$ & $\mathbf{- 2 3}$ & $\mathbf{- 2}$ & $\mathbf{4}$ & $\mathbf{1 4 9}$ & $\mathbf{- 1 1 7}$ & $\mathbf{3 2}$ \\
\hline ETR & 19 & 13 & 13 & 16 & 36 & 55 & 87 & 84 & 81 & 59 & 45 & 28 & 152 & 384 & 536 \\
SW & 1 & 8 & 22 & 22 & 19 & 27 & 14 & 7 & 4 & 4 & 1 & 1 & 109 & 32 & 141 \\
$\Delta$ SB & 37 & 38 & 9 & -1 & -17 & -23 & -48 & -19 & -17 & 7 & 6 & 7 & 41 & -63 & -22 \\
\hline
\end{tabular}

Top: c (Geschiebelehm- - Lysimetergruppe 1, 7 und 11)

\begin{tabular}{|l|cccccc|ccccccc|c|c|c|}
\hline Monat & $\mathbf{1 1}$ & $\mathbf{1 2}$ & $\mathbf{1}$ & $\mathbf{2}$ & $\mathbf{3}$ & $\mathbf{4}$ & $\mathbf{5}$ & $\mathbf{6}$ & $\mathbf{7}$ & $\mathbf{8}$ & $\mathbf{9}$ & $\mathbf{1 0}$ & $\mathbf{W i}$ & So & Jahr \\
\hline P $_{\text {korr }}$ & 56 & 58 & 44 & 37 & 47 & 59 & 54 & 72 & 68 & 71 & 52 & 37 & 301 & 354 & 655 \\
\hline ETP & & 17 & 12 & 15 & 17 & 35 & 57 & 88 & 93 & 108 & 93 & 54 & 33 & 153 & 470 & 623 \\
kWB & $\mathbf{3 9}$ & $\mathbf{4 5}$ & $\mathbf{2 9}$ & $\mathbf{2 0}$ & $\mathbf{1 3}$ & $\mathbf{2}$ & $\mathbf{- 3 4}$ & $\mathbf{- 2 1}$ & $\mathbf{- 4 0}$ & $\mathbf{- 2 3}$ & $\mathbf{- 2}$ & $\mathbf{4}$ & $\mathbf{1 4 8}$ & $\mathbf{- 1 1 7}$ & $\mathbf{3 2}$ \\
\hline ETR & 20 & 14 & 14 & 17 & 37 & 57 & 91 & 92 & 85 & 62 & 47 & 30 & 158 & 406 & 564 \\
SW & 1 & 7 & 16 & 18 & 24 & 20 & 9 & 5 & 3 & 3 & 1 & 1 & 87 & 22 & 108 \\
$\Delta$ SB & 35 & 37 & 13 & 3 & -14 & -18 & -47 & -25 & -20 & 6 & 5 & 7 & 57 & -74 & -18 \\
\hline
\end{tabular}

Top: d (Löß- Lysimetergruppe 9 und 10)

\begin{tabular}{|l|cccccc|ccccccc|c|c|c|}
\hline Monat & $\mathbf{1 1}$ & $\mathbf{1 2}$ & $\mathbf{1}$ & $\mathbf{2}$ & $\mathbf{3}$ & $\mathbf{4}$ & $\mathbf{5}$ & $\mathbf{6}$ & $\mathbf{7}$ & $\mathbf{8}$ & $\mathbf{9}$ & $\mathbf{1 0}$ & $\mathbf{W i}$ & So & Jahr \\
\hline P $_{\text {korr }}$ & 57 & 59 & 45 & 38 & 48 & 59 & 54 & 72 & 68 & 71 & 52 & 37 & 305 & 354 & 659 \\
\hline ETP $_{\text {Gras }}$ & 17 & 12 & 15 & 17 & 35 & 57 & 88 & 93 & 108 & 93 & 54 & 33 & 153 & 470 & 623 \\
kWB & $\mathbf{4 0}$ & $\mathbf{4 6}$ & $\mathbf{3 0}$ & $\mathbf{2 0}$ & $\mathbf{1 3}$ & $\mathbf{2}$ & $\mathbf{- 3 4}$ & $\mathbf{- 2 1}$ & $\mathbf{- 4 0}$ & $\mathbf{- 2 3}$ & $\mathbf{- 2}$ & $\mathbf{4}$ & $\mathbf{1 5 2}$ & $\mathbf{- 1 1 6}$ & $\mathbf{3 6}$ \\
\hline ETR & 20 & 13 & 14 & 16 & 35 & 51 & 97 & 116 & 110 & 74 & 52 & 31 & 149 & 480 & 629 \\
SW & 0 & 2 & 4 & 9 & 17 & 13 & 6 & 1 & 0 & 0 & 0 & 0 & 45 & 8 & 54 \\
$\Delta$ SB & 37 & 43 & 27 & 12 & -4 & -4 & -50 & -45 & -42 & -4 & 0 & 7 & 110 & -134 & -24 \\
\hline
\end{tabular}




\section{Danksagung}

Stellvertretend für alle, die zum Gelingen dieser Arbeit beigetragen haben, möchte ich mich ganz herzlich bedanken bei:

- Herrn Prof. Meyer für die Überlassung des Themas und die konsequente Förderung der Arbeit,

- Herrn Prof. Ehlers für die Übernahme des Korreferates und die kritischen Hinweise zur inhaltlichen Gestaltung der Arbeit,

- Herrn Dr. Becker für die stete freundliche Beratung und die Durchsicht des Manuskriptes,

- dem Landesamt für Umwelt und Geologie (LfUG) für die Bereitstellung der Daten und insbesondere den Mitarbeitern der Referate Boden und Grundwasser für die fachliche Unterstützung,

- Herrn Dr. Knappe (Umweltforschungszentrum Leipzig-Halle) für die unendlich vielen geduldigen Gespräche, die sehr zum Gelingen der Arbeit beitrugen,

- Herrn Dr. Glugla und Frau Jankiewicz (Bundesanstalt für Gewässerkunde - BfG) für die langjährige Diskussionsbereitschaft und Hilfe bei der Datenaufbereitung,

- Frau Dr. Warnstorff (Arbeitsgruppe Biometrie und Agrarinformatik der Landwirtschaftlichen Fakultät der Universität Halle-Wittenberg) für die Durchführung der Varianzanalyse und Beratung zur Interpretation der Ergebnisse,

- Herrn Dr. Moritz als ehemaligen langjährigen wissenschaftlichen Begleiter der Station Brandis für seine Anmerkungen im Manuskript,

- Frau Thaler und meinen Freunden Ingrid und Peter für die Anregungen aus der Sicht artverwandter Wissensgebiete und ihre Mitwirkung bei der technischen Fertigstellung der Arbeit sowie

- meinen Kollegen aus der Lysimeterstation Brandis für die vielen großen und kleinen Hilfeleistungen und die Geduld mit der sie diese Arbeit begleiteten.

Nicht zuletzt meiner Familie für ihre Unterstützung und ihr Verständnis für meine häufige „geistige“ und körperliche Abwesenheit. 


\section{Lebenslauf}

\section{Ausbildung}

1969 - 72 Abitur und Facharbeiterausbildung an der Betriebsschule des Reichsbahnamtes Leipzig

1972 - 76 Direktstudium "Hydrologie" an der Technischen Universität Dresden, Sektion Wasserwesen

1981 - 82 Postgraduales Studium "Grundwasser" an der Technischen Universität Dresden

\section{Berufliche Tätigkeiten}

1976 - 87 Hydrologin in der Oberflußmeisterei Leipzig (Abflußmessungen, Bearbeitung hydrologischer und hydrogeologischer Gutachten, Hochwasservorhersage unter Anwendung von Hochwasservorhersagemodellen, Grundwassermeßdienst, Mitarbeit beim Aufbau und der Anwendung von Grundwasserleitermodellen im Bergbaugebiet um Leipzig)

1987 - 91 wissenschaftliche Mitarbeiterin in der Forschungsgruppe der Wasserwirtschafts-direktion Saale-Werra (Aufbereitung und Anwendung von Methoden zur Bilanzierung des Wasserhaushaltes in bergbaubeeinflußten Gebieten)

1991 - 94 Mitarbeiterin im Referat Grundwasser des Sächsischen Landesamtes für Umwelt und Geologie und Leiterin der Lysimeterstation Brandis

1994 - nach Gründung der Staatlichen Umweltbetriebsgesellschaft Leiterin der Lysimeterstation Brandis und Stellvertreterin des Geschäftsbereichsleiters Meßnetzbetrieb Wasser

\section{Privat}

Geboren am 25. 3. 1953 als Ulrike Saß in Rostock.

Heirat 1977 (Namensänderung zu U. Keese)

und Geburt des Sohnes Martin am 14. 6. 1977, geschieden 1983.

1998 Heirat und Namensänderung zu U. Haferkorn. 